This report was prepared as an account of work sponsored by an agency of the United States INEL-95/0381

Government. Neither the United States Government nor any agency thereof, nor any of their employees, makes any warranty, express or implied, or assumes any legal liability or responsibility for the accuracy, completeness, or usefulness of any information, apparatus, product, or process disclosed, or represents that its use would not infringe privately owned rights. Reference herein to any specific commercial product, process, or service by trade name, trademark, manufacturer, or otherwise does not necessarily constitute or imply its endorsement, recommendation, or favoring by the United States Government or any agency thereof. The views and opinions of authors expressed herein do not necessarily state or reflect those of the United States Government or any agency thereof.

\title{
Radiological, Physical, and Chemical Characterization of Additional Alpha Contaminated and Mixed Low- Level Waste for Treatment at the Advanced Mixed Waste Treatment Project
}

D. P. Hutchison

Published July 1995

Idaho National Engineering Laboratory Lockheed Idaho Technologies Company Idaho Falls, Idaho 83415

Prepared for the

U.S. Department of Energy

Office of Environmental Management

Under DOE Idaho Operations Office

Contract DE-AC07-94ID13223 


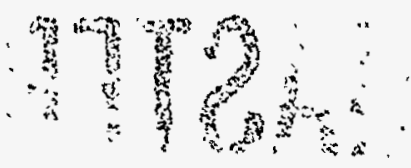




\section{DISCLAIMER}

Portions of this document may be illegible in electronic image products. Images are produced from the best available original document. 


\begin{abstract}
This document provides physical, chemical, and radiological descriptive information for a portion of mixed waste that is potentially available for private sector treatment. The format and contents are designed to provide treatment vendors with preliminary information on the characteristics and properties for additional candidate portions of the Idaho National Engineering Laboratory (INEL) and offsite mixed wastes not covered in the two previous characterization reports for the INELstored low-level alpha-contaminated and transuranic wastes. This report defines the waste, provides background information, briefly reviews the requirements of the Federal Facility Compliance Act (P.L. 102-386), and relates the Site Treatment Plans developed under the Federal Facility Compliance Act to the waste streams described herein. Each waste is summarized in a Waste Profile Sheet with text, charts, and tables of waste descriptive information for a particular waste stream. A discussion of the availability and uncertainty of data for these waste streams precedes the characterization descriptions.
\end{abstract}




\section{CONTENTS}

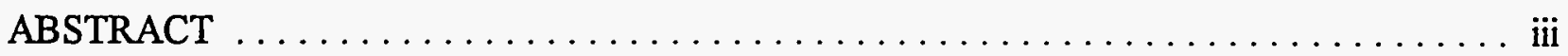

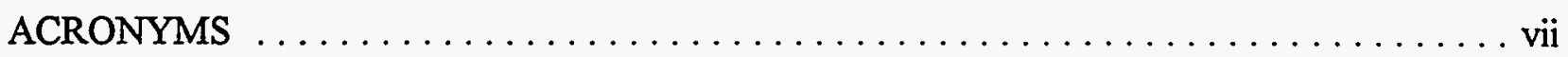

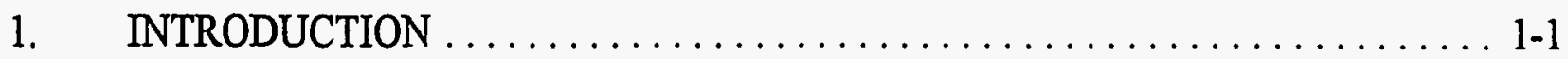

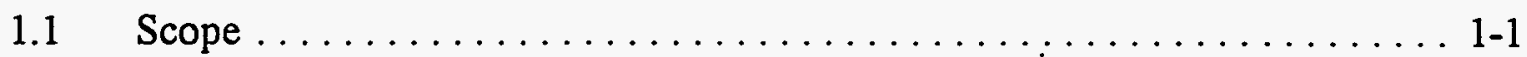

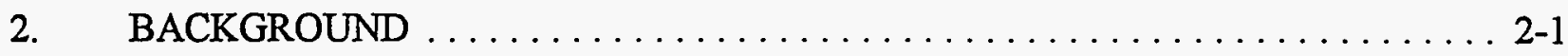

$2.1 \quad$ Federal Facility Compliance Act $\ldots \ldots \ldots \ldots \ldots \ldots \ldots \ldots \ldots . \ldots \ldots$

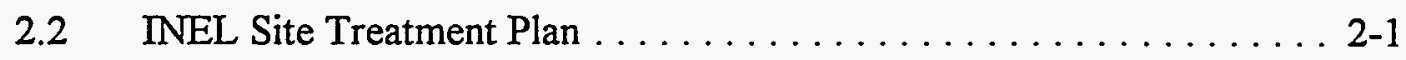

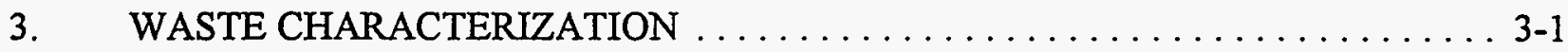

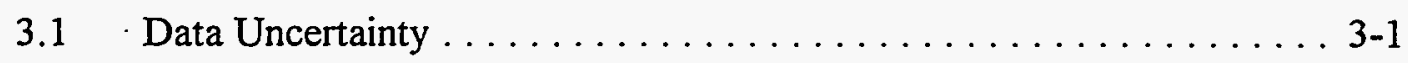

3.2 INEL Waste Stream Profile Sheet Format . . . . . . . . . . 3-6

4. INEL AND OFFSITE WASTE STREAM PROFILES NOT IDENTIFIED

IN THE DOCUMENTS $\ldots \ldots \ldots \ldots \ldots \ldots \ldots \ldots \ldots \ldots \ldots \ldots, 4-1$

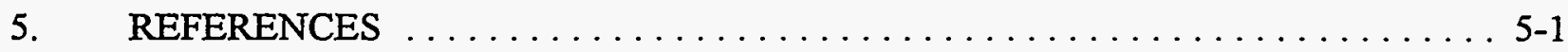

\section{TABLES}

3-1. Preliminary listing of INEL waste for AMWTP $\ldots \ldots \ldots \ldots \ldots \ldots \ldots \ldots \ldots \ldots \ldots .2$

3-2. Preliminary listing of offsite waste for AMWTP $\ldots \ldots \ldots \ldots \ldots \ldots \ldots \ldots \ldots \ldots \ldots$ 


\section{ACRONYMS}

AdvMWTP

AMLLW

$\mathrm{CH}$

DOE

EPA

FFCAct

FR

IMWI

INEL

IWPF

LDR

MLLW

MTRU

PSTP

QA/QC

RCRA

STP

TRU

USC

WAC

WERF

WIPP
Advanced Mixed Waste Treatment Project

Alpha-contaminated mixed low-level waste

Contact-handled

U.S. Department of Energy

U.S. Environmental Protection Agency

Federal Facilities Compliance Act

Federal Register

Idaho Mixed Waste Information database

Idaho National Engineering Laboratory

Idaho Waste Processing Facility

Land disposal restrictions

Mixed low-level waste

Mixed transuranic waste

Proposed Site Treatment Plan

Quality assurance/quality control

Resource Conservation and Recovery Act

Site Treatment Plan

Transuranic

United States Code

Waste Acceptance Criteria

Waste Experimental Reduction Facility

Waste Isolation Pilot Plant 


\section{Radiological, Physical, and Chemical Characterization of Additional Alpha Contaminated and Mixed Low-Level Waste for Treatment at the Advanced Mixed Waste Treatment \\ Project}

\section{INTRODUCTION}

This document provides physical, chemical and radiological descriptive information for a portion of mixed waste that is potentially available for private sector treatment. The format and contents of this document have been designed to provide treatment vendors with preliminary information concerning the characteristics and properties for portions of the Idaho National Engineering Laboratory (INEL) and offsite mixed waste. Specifically, this document contains a description of additional waste streams not covered in the two characterization documents for the INEL stored Low-Level Alpha Contaminated and Transuranic Waste. See Section 1.1 for the scope of the waste covered in this document.

This report is divided into four sections. Section 1 contains a definition of the waste addressed in this document. Section 2 provides background on this waste. Section 2 also includes a brief overview of the requirements of the Federal Facility Compliance Act (P. L. 102386, the FFC Act) and how the Site Treatment Plans developed under the FFC Act relate to the waste streams covered in this document. Section 3 provides a summary of the waste streams addressed in this document and includes a summary of the format and contents of the Waste Profile sheets for those wastes. Each Waste Profile Sheet consists of text, charts and tables of waste descriptive information for a particular waste stream. Also covered in section 3 is a discussion regarding the uncertainty and availability of data for these waste streams. Section 4 contains the Waste Profile Sheets for additional waste stored and generated at the INEL and waste stored and generated at offsite facilities, but not described in the two previous waste characterization documents cited below.

\subsection{Scope}

The waste covered in this document is a portion of the waste streams that are potentially available for treatment by the private sector and include those waste streams not addressed in the documents: Radiological, Physical, and Chenical Characterization of Low-Level Alpha Contaminated Wastes Stored at the Idaho National Engineering laboratory for Use in Conducting Feasibility Studies for Treatment Services ${ }^{1}$ and Radiological, Physical, and Chemical Characterization of Transuranic Wastes Stored at the Idaho National Engineering laboratory for Use in Conducting Feasibility Studies for Treatment Services ${ }^{2}$, dated April 5, 1994

Waste streams covered in this document include mixed low-level waste (MLLW), alpha mixed low-level waste (A-MLLW), and mixed transuranic (MTRU) waste stored and generated at the INEL and stored and generated offsite. Section 3 contains a list of these waste streams. These waste streams have been identified under the Site Treatment Plans (STP) developed under the FFC Act as requiring treatment to meet the Land Disposal Restrictions (LDR) under the Resource Conservation and Recovery Act (RCRA). A majority of these waste streams are identified in the Idaho National Engineering Laboratory Proposed Site Treatment Plan (INEL 
PSTP), dated March $1995 .{ }^{3}$ Some of the waste streams addressed in this document were identified as potentially treatable at the INEL after the publication of the INEL PSTP and are not addressed in that document. Those new waste streams will be included in the next revision to the INEL PSTP. 


\section{BACKGROUND}

This section contains a brief overview of the requirements of the Federal Facility Compliance Act (P. L. 102-386, the FFC Act) and how the Site Treatment Plans developed under the FFC Act relate to the waste streams covered in this document.

\subsection{Federal Facility Compliance Act}

The U. S. Department of Energy (DOE) is required to prepare a plan for developing treatment capacities and technologies for each facility at which the DOE generates or stores mixed waste, pursuant to Section 3021(b) of RCRA, 42 Unites States Code (USC) Section 6939 c(b), as amended by Section 105(a) of the FFC Act. Upon submission of a plan to the appropriate regulatory agency, the FFC Act requires the recipient agency to solicit and consider public comments and approve, approve with modification, or disapprove the plan within six months. Upon approval of a plan, the regulatory agency must issue an order requiring compliance with the approved plan.

\subsection{INEL Site Treatment Plan}

On April 6, 1993, DOE published a Federal Register (FR) notice (58 FR 17875) describing its proposed process for developing the plans as required under the FFC Act. In that FR notice, DOE proposed to develop Site Treatment Plans (STP) in three phases, including a conceptual, draft and final STP. In March 1995, DOE delivered the INEL PSTP to the Idaho Department of Health and Welfare. Upon approval or approval with modifications by the State of Idaho, the INEL PSTP will become the Final INEL STP.

All of the mixed waste stored and projected to be generated in the future at the INEL is covered in the INEL PSTP. Also included in the INEL PSTP are those offsite wastes identified as potentially being treated at the INEL. The INEL PSTP provides treatment plans for the mixed waste from the INEL and for some of the offsite waste. The INEL PSTP includes both existing and planned treatment facilities at the INEL and includes detailed treatment plans for each waste stream.

The INEL PSTP identified the Advanced Mixed Waste Treatment Project as providing the required treatment for a large portion of the waste at the INEL and was also identified as providing the required treatment for a number of offsite mixed waste streams. In the INEL PSTP, the Advanced Mixed Waste Treatment Project was identified as providing treatment for some of the mixed low-level and all of the alpha mixed low-level waste to LDR treatment standards and the mixed transuranic waste as required to meet the Waste Isolation Pilot Plant Waste Acceptance Criteria (WIPP WAC) and transportation requirements. The INEL identified two options for the Advanced Mixed Waste Treatment Project: (1) design and construction of a DOE facility (formerly called the Idaho Waste Processing Facility) and (2) privatization of this treatment. Subsequent to publication of the INEL PSTP, the DOE decided to pursue the privatization of the treatment, including treatment of the TRU waste. 
All of the waste destined to be treated at the Advanced Mixed Waste Treatment Project is identified in the INEL PSTP ${ }^{3}$ in Appendix A, Table A-3. Note: For consistency of treatment plans between the INEL PSTP and the INEL Draft Site Treatment Plan, the Advanced Mixed Waste Treatment Project was identified as the Idaho Waste Processing Facility (IWPF) in the INEL PSTP Table A-3. The waste in the INEL PSTP covered in this document include:

- The waste destined to be treated at the Contact Handled $(\mathrm{CH})$ treatment units at the IWPF with the exclusion of the MTRU and A-MLLW streams identified in the documents Radiological, Physical, and Chemical Characterization of Low-Level Alpha Contaminated Wastes Stored at the Idaho National Engineering laboratory for Use in Conducting Feasibility Studies for Treatment Services ${ }^{1}$ and Radiological, Physical, and Chemical Characterization of Transuranic Wastes Stored at the Idaho National Engineering laboratory for Use in Conducting Feasibility Studies for Treatment Services ${ }^{2}$, dated April 5, 1994

- The waste identified as treatable at the Waste Reduction Operations Complex (WROC) mercury retort unit, and

- The offsite waste destined to be treated at the WROC mercury amalgamation unit.

The offsite waste identified in the INEL PSTP as treatable at the WROC mercury amalgamation unit, and the INEL and offsite waste destined to be treated at the WROC mercury retort unit is also included in this document. That waste has been included in this document since it is a relatively small volume, and should the waste be treatable at the Advanced Mixed Waste Treatment Project, the WROC mercury amalgamation and mercury retort units will not be constructed.

Also subsequent to publication of the INEL PSTP, several additional offsite waste streams have been identified to be treated at the Advanced Mixed Waste Treatment Project or at the WROC mercury amalgamation or mercury retort units. These additional waste streams are also included in this document. Some of the treatment plans for the newly-identified waste streams from Los Alamos National Laboratory are identified for treatment at the INEL Waste Experimental Reduction Facility (WERF) Incinerator. They are also included in this document since the alpha contamination of these waste streams is not known and the WERF Incinerator is limited on the amount of alpha contamination it can accept.

During development of the Site Treatment Plans, several offsite DOE facilities identified INEL as the second and third option for treatment of their waste streams. Table 9-3 in the INEL PSTP $^{3}$ lists those waste streams. Since these waste streams have not been identified as being treated at the INEL as the primary option, the IMWI database does not contain the profile information for these waste streams. Therefore, they are not included in Table 3-2 or in the waste stream profile forms in Section 4. The majority of the waste streams were identified as requiring treatment at the Advanced Mixed Waste Treatment Project and should be considered as potential treatable waste streams. The volume of this waste is approximately $350 \mathrm{~m}^{3}$ of waste in storage and up to $5,400 \mathrm{~m}^{3}$ of waste estimated to be generated in the future. 


\section{WASTE CHARACTERIZATION}

The waste streams from the INEL addressed in this document are identified in Table 3-1. The waste streams from offsite are identified in Table 3-2. Table 3-1 and 3-2 include a listing of the waste stream numbers, waste stream name, the current amount of waste in storage and the amount of waste estimated to be generated from 1995 through 1999.

\subsection{Data Uncertainty}

The data in this document comes from the Idaho Mixed Waste Information (IMWI) database. The IMWI database was utilized for development of the INEL PSTP and represents a compilation of data on each of the mixed waste streams destined to be treated at the INEL treatment facilities or offsite.

A majority of the waste identified in this document has been in storage for several years. It should be noted that much of the process used at the INEL and other DOE facilities to characterize this waste originated several years before treatment of these wastes was strongly considered. Current methods of waste identification, characterization, documentation and auditing were not in place at the time the majority of these waste were generated and placed into storage.

This report presents data currently available at the INEL concerning these wastes. Until further characterization of these materials is undertaken, it is likely that the extent of error in these data, or their overall accuracy and completeness, will not be fully known. Therefore, caution should be exercised in the use and interpretation of data contained in this report, as well as other documents describing these wastes.

At the time of publication of this document, the available data for the offsite waste streams is limited. The DOE updates the national Mixed Waste Inventory Report on an annual basis. The data updating effort for 1995 has been initiated and the data are expected to be available by October 1995. Additional data for the offsite waste will be obtained from that data collection effort and will be augmented with information from the offsite generators as it can be obtained.

The waste stream volumes identified in Tables 3-1 and 3-2 and the waste profile sheets in Section 4 are the container volumes and not the actual volumes of the waste materials. The actual volumes of the waste materials therefore are assumed less that the volumes listed. It should also be noted that the volumes listed here are the volumes for the total waste stream. As 
Table 3-1: Preliminary Listing of INEL waste for AMWTP.

\begin{tabular}{|c|c|c|c|}
\hline Waste Stream ID & Waste Stream Name & $\begin{array}{c}\text { Current } \\
\text { Storage } \\
\text { Volume (m3) }\end{array}$ & $\begin{array}{c}95-99 \text { 5-Year } \\
\text { Generation } \\
\text { (m3) }\end{array}$ \\
\hline \multicolumn{4}{|l|}{ MLLW: } \\
\hline ID-CPP-301 & LIQUID ACID/MERCURY MIXED WASTE & 0.27 & 7.00 \\
\hline ID-CPP-302 & LIQUID HIGH CHLORIDE CORROSIVE MW & 2.30 & 7.00 \\
\hline ID-CPP-304 & CONTAMINATED DEBRIS & 128.80 & 500.00 \\
\hline ID-CPP-504 & NON-DEBRIS SOLIDS & 0.09 & 7.74 \\
\hline ID-INL-220 & ACTIVATED CARBON LLMW & 0.34 & 0.00 \\
\hline ID-INL-267 & PWTU SPENT FILTERS & 0.42 & 1.26 \\
\hline ID-INL-268 & PWTU SPENT RESINS & 0.22 & 0.00 \\
\hline $\mathbb{D}-\mathbb{N} L-270$ & HEAVY METAL CONTAMINATED SOLIDS & 0.19 & 0.00 \\
\hline ID-PBF-153 & TAN/IET HOT WASTE SLUDGE & 2.29 & 0.00 \\
\hline ID-TAN-254 & HTRE-III TREATMENT SLUDGE & 0.83 & 0.00 \\
\hline \multirow[t]{2}{*}{ ID-TRA-127 } & TRA SCINTILLATION COCKTAILS (ALPHA <10) & 0.32 & 0.00 \\
\hline & INEL - MLLW: & 136.07 & 523.00 \\
\hline \multicolumn{4}{|l|}{ a-MLLW: } \\
\hline \multirow[t]{2}{*}{ ID-TAN-163 } & TAN DECON HEAVY METAL SOLIDS AND DEBRIS & 0.32 & 0.00 \\
\hline & INEL - a-MLLW: & 0.32 & 0.00 \\
\hline \multicolumn{4}{|l|}{ MTRU: } \\
\hline CH-ANL-503T & \multirow{2}{*}{$\begin{array}{l}\text { TRU WASTE USED PRE-FLTERS } \\
\text { ALHC UPGRADE DECON DEBRIS }\end{array}$} & 0.91 & 0.85 \\
\hline \multirow[t]{3}{*}{ CH-ANL-505T } & & 1.18 & 0.03 \\
\hline & INEL - MTRU: & 2.08 & 0.88 \\
\hline & Total INEL: & 138.48 & 523.88 \\
\hline
\end{tabular}


Table 3-2: Preliminary Listing of Off Site waste for AMWTP.

\begin{tabular}{|c|c|c|c|}
\hline Waste Stream D & Waste Stream Name & $\begin{array}{c}\text { Current } \\
\text { Storage } \\
\text { Volume }(\mathrm{m} 3)\end{array}$ & $\begin{array}{l}\text { 95-99 5-Year } \\
\text { Generation } \\
\text { (m3) }\end{array}$ \\
\hline \multicolumn{4}{|l|}{ MLLW: } \\
\hline BN-W007 & MERCURY WASTE & 0.04 & 0.06 \\
\hline BT-W005 & LEAD AND CHROM. BASED PAINT CHIPS & 0.10 & 0.10 \\
\hline BT-W008 & MERCURY CONTAINING WASTE & 0.00 & 0.02 \\
\hline BT-W009 & VOC CONTAMINATED SOIL & 0.63 & 0.00 \\
\hline BT-W010 & WASTE OIL WITH HEAVY METALS AND PCBS & 0.26 & 0.00 \\
\hline BT-W012 & VOC \& PCB CONTAMINATED DEBRIS & 1.68 & 0.42 \\
\hline BT-W013 & VOC \& PCB CONTAMINATED SOIL & 0.84 & 0.00 \\
\hline BT-W020 & BRASS AND BRONZE & 0.00 & 0.05 \\
\hline BT-W028 & VOC AND PCB CONTAMINATED WATER & 2.10 & 0.63 \\
\hline BT-W029 & VOC CONTAMINATED SEDIMENT/SLUDGE & 0.42 & 0.63 \\
\hline BT-W030 & VOC CONTAMINATED DEBRIS & 0.21 & 0.21 \\
\hline BT-W031 & VOC AND PCB CONTAMINATED SLUDGE & 2.73 & 1.05 \\
\hline DP-W079 & METALLIC MERCURYMERCURY PRODUCTS & 2.79 & 4.80 \\
\hline DP-W114 & RQ, WASTE MERCURY, METALLIC ORM-B & 0.73 & 0.00 \\
\hline ET-W009 & PAINT CHIPS & 0.64 & 0.00 \\
\hline ET-W019 & CHROME SALT CORES & 2.45 & 0.00 \\
\hline ET-W020 & LABORATORY ANALYTICAL REAGENT WASTE & 0.15 & 0.00 \\
\hline ET-W023 & ELEMENTAL MERCURY & 0.0002 & 0.00 \\
\hline ET-W026 & CRUSHED MERCURY LIGHT BULBS & 0.10 & 0.00 \\
\hline GA-W003 & SVA: Pb CONTAMINATED SLUDGE & 1.47 & 0.00 \\
\hline GA-W007 & HOT CELL D\&D: Pb SHOT & 0.21 & 0.00 \\
\hline GA-W013 & HOT CELL D\&D: Pb BRICKS & 1.04 & 0.00 \\
\hline KA-W001 & MISC. LABORATORY CHEMICALS W/O METALS & 0.00 & 2.00 \\
\hline KA-W015 & SOILS & 0.00 & 21.00 \\
\hline KA-W018 & Hg CONTAMINATED ORGANICS & 0.00 & 1.00 \\
\hline KA-W019 & Hg CONTAMINATED INORGANICS & 0.10 & 0.30 \\
\hline KA-W020 & ELEMENTAL Hg & 0.02 & 0.08 \\
\hline KA-W021 & PCB CONTAMINATED WASTE & 0.00 & 0.15 \\
\hline KK-W013 & SOILS & 0.00 & 15.90 \\
\hline KK-W014 & Hg CONTAMINATED ORGANICS & 0.00 & 0.20 \\
\hline KK-W015 & Hg CONTAMINATED INORGANICS & 0.00 & 0.20 \\
\hline KK-W016 & ELEMENTAL Hg & 0.00 & 0.0010 \\
\hline KK-W017 & PCB CONTAMINATED WASTE & 0.00 & 2.00 \\
\hline KW-W009 & SOILS & 0.00 & 5.88 \\
\hline KW-W010 & Hg CONTAMINATED ORGANICS & 0.00 & 0.05 \\
\hline $\mathrm{KW}-\mathrm{W} 011$ & Hg CONTAMINATED INORGANICS & 0.00 & 0.50 \\
\hline KW-W012 & ELEMENTAL Hg & 0.00 & 0.0010 \\
\hline LA-W901 & IPA WASTES & 15.89 & 0.01 \\
\hline LA-W902 & SCINTILLATION VIALS & 2.47 & 4.00 \\
\hline LA-W904 & SOIL WITH HEAVY METALS & 10.53 & 2.00 \\
\hline LA-W905 & ER SOILS & 39.32 & 0.00 \\
\hline
\end{tabular}


Table 3-2: (continued).

\begin{tabular}{|c|c|c|c|}
\hline Waste Stream ID & Waste Stream Name & $\begin{array}{c}\text { Current } \\
\text { Storage } \\
\text { Volume }(\mathrm{m} 3)\end{array}$ & $\begin{array}{l}95-995 \text {-Year } \\
\text { Generation } \\
\text { (m3) }\end{array}$ \\
\hline LA-W906 & AQUEOUS ORGANIC WASTES & 1.65 & $\overline{0.50}$ \\
\hline LA-W907 & HALOGENATED ORGANIC LIQUIDS & 16.58 & 5.50 \\
\hline LA-W908 & NONHALOGENATED ORGANIC LIQUIDS & 14.34 & 10.00 \\
\hline LA-W910 & PCB WASTES WITH RCRA COMPONENTS & 0.74 & 0.20 \\
\hline LA-W911 & ORGANIC-CONTAMINATED COMBUSTIBLE SOLIDS & 28.32 & 7.00 \\
\hline LA-W912 & COMBUSTIBLE DEBRIS & 13.82 & 1.50 \\
\hline LA-W913 & AQUEOUS WASTES WITH HEAVY METALS & 1.85 & 1.00 \\
\hline LA-W914 & CORROSIVE SOLUTIONS & 1.36 & 0.50 \\
\hline LA-W915 & AQUEOUS CYANDEES, NITRATES, CHROMATES & 0.13 & 0.01 \\
\hline LA-W919 & ORGANIC-CONTAMINATED NONCOMBUST SOLIDS & 7.82 & 8.00 \\
\hline LA-W920 & ELEMENTAL MERCURY & 0.50 & 0.05 \\
\hline LA-W921 & ACTIVATED OR INSEPARABLE LEAD & 15.60 & 1.00 \\
\hline LA-W922 & NONCOMBUSTIBLE DEBRIS & 5.62 & 3.00 \\
\hline LA-W923 & INORGANIC SOLID OXIDIZERS & 0.20 & 0.05 \\
\hline LA-W925 & MERCURY WASTES - TBD & 18.30 & 25.50 \\
\hline LB-W002 & BASIC AQUEOUS LIQUIDS - LOW ALPHA & 1.00 & 0.81 \\
\hline LB-W005 & BLOCKS \& SHEET LEAD - CONTAMINATEDD & 0.43 & 0.00 \\
\hline LB-W006 & LIQUID INDUCED MERCURY & 0.11 & 0.00 \\
\hline LB-W011 & ACIDIC SOLIDS w/METALS - HIGH ALPHA & 0.00 & 0.00 \\
\hline LB-W014 & LIQUID/SOLIDS CONTAINING SOLVENTS \& OIL & 0.00 & 0.00 \\
\hline LB-W017 & ORGANIC SCINTILATION FLUIDS- HIGH ALPHA & 0.00 & 0.00 \\
\hline LB-W018 & AQUEOUS/SOLID OXIDIZERS - HIGH ALPHA & 0.00 & 0.00 \\
\hline LB-W019 & DEBRIS CONTAMINATED W/ORGANIC-HIGH ALPHA & 0.00 & 0.00 \\
\hline LB-W101 & AQUEOUS ORGANIC LIQUIDS & 0.0050 & 0.00 \\
\hline LB-W111 & AQUEOUS LIQUIDS & 0.01 & 0.00 \\
\hline LL.W003 & LOW LEVEL MIXED INORGANIC TRASH-1 & 8.70 & 7.00 \\
\hline LL-W006 & LOW LEVEL MIXED SCRAP METAL & 15.20 & 5.00 \\
\hline LL-W007 & ELEMENTAL LEAD & 3.90 & 5.00 \\
\hline LL-W015 & INORGANIC DEBRIS & 3.00 & 15.00 \\
\hline LL-W017 & LOW LEVEL MIXED INORGANIC TRASH-3 & 50.70 & 50.00 \\
\hline LL-W021 & LAB PACKS WITH METALS & 0.80 & 1.50 \\
\hline LL-W024 & LIQUID MERCURY WASTE & 0.09 & 0.05 \\
\hline MI-W011 & MATERIALS CONTAINING PCBS & 0.11 & 0.62 \\
\hline MI-W013 & ORGANIC PROCESS RESIDUES & 0.00 & 1.06 \\
\hline MU-W001 & DECON \& FAILED EQUIP DEBRIS W/ Cd \& Ag & 1.00 & 4.00 \\
\hline OR-W007 & BULK LIQUID MERCURY & 1.00 & 0.00 \\
\hline PO-W006 & WASTE HG, METALLIC & 1.00 & 1.05 \\
\hline PO-W061 & MERCURY SOLIDS & 10.50 & 10.00 \\
\hline PS-W004 & LIQUID WITH F-LISTED SOLVENTS & 0.25 & 0.00 \\
\hline PS-W006 & SOLIDIFIED LIQUID WITH ACETONE & 0.84 & 0.00 \\
\hline PS-W007 & DEBRIS WITH HEAVY METALS AND PCB & 3.11 & 0.50 \\
\hline PS-W009 & PAINT THINNER WITH BUTYL ALCOHOL & 0.62 & 0.00 \\
\hline
\end{tabular}


Table 3-2: (continued).

\begin{tabular}{|c|c|c|c|}
\hline Waste Stream ID & Waste Stream Name & $\begin{array}{c}\text { Current } \\
\text { Storage } \\
\text { Volume (m3) }\end{array}$ & $\begin{array}{c}95-99 \text { 5-Year } \\
\text { Generation } \\
\text { (m3) }\end{array}$ \\
\hline PS-W011 & DEBRIS WITH TCLP METALS/F LISTED SOLVENT & 0.19 & 0.00 \\
\hline PS-W012 & CHROMIUM \& LEAD BASED PAINT CHIPS W/PCB & 0.03 & 0.23 \\
\hline PS-W019 & FILTERS W/ASBESTOS AND DIOCTYL PHTHALATE & 2.18 & 2.18 \\
\hline PS-W020 & COMPRESSED FIL TERS W/DIOCTYL PHTHALATES & 2.70 & 0.00 \\
\hline RF-W017 & PCB LIQUIDS/LLM & 39.01 & 0.00 \\
\hline RF-W027 & PAINTS/LLM & 1.15 & 0.00 \\
\hline RF-W049 & MISCELLANEOUS LIQUIDS/LLM & 1.94 & 27.23 \\
\hline RF-W083 & EXCESS CHEMICALS ORGANOMETALLIC LAB PACK & 40.46 & 13.49 \\
\hline RF-W085 & EXCESS CHEMICALS NON-LABPACKS W/D009/LLM & 4.92 & 13.49 \\
\hline RF-W086 & EXCESS CHEMICALS NON-LAB PACKS-OTHER/LLM & 2.00 & 13.49 \\
\hline SR-W014 & TRITIATED MERCURY & 0.30 & 0.10 \\
\hline \multirow[t]{2}{*}{ SR-W068 } & LIQUID ELEMENTAL MERCURY & 0.10 & 0.20 \\
\hline & Off Site - MLLD & 411.10 & 299.05 \\
\hline
\end{tabular}

a-MLLW:

LB-W012

BASIC SOLIDS w/METALS - HIGH ALPHA

Off Site - a-MLLW:

\begin{tabular}{rr}
0.00 & 0.00 \\
\hline 0.00 & 0.00 \\
\hline 411.10 & 299.05
\end{tabular}


part of the treatment planning for the INEL PSTP, each waste stream was evaluated and a treatment train identified. This treatment train included pretreatment and post-treatment steps such as segregation and sizing, or stabilization following incineration. Accordingly, the waste volumes found in the INEL PSTP Appendix A, Table A-3 is the volume of the waste ready for the treatment step. Therefore, the volume of waste included in this document may differ from that listed in the MNEL PSTP since the volumes in the INEL PSTP may include only the portion destined for that treatment step and include volume change factors appropriate for the waste matrix and treatment step.

\subsection{INEL Waste Stream Profile Sheet Format}

The INEL waste stream profile sheets found in Section 4 are, for the most part, selfexplanatory. The waste stream profile sheets are from the IMWI system. Each profile sheet contains 12 sections. Each of the 12 sections is listed below and, as necessary, clarification on the contents for a specific item in a section is provided.

Section A: Identification and Description

Content Code: Only applicable for MTRU and A-MLLW and is a historical waste categorization number

FFCA ID: The number of the waste stream for the national Mixed Waste Inventory Report

Section B: Generation Site/Process Description

Section C: Projected Waste Generation

Section D: Storage Inventory

Units: Container Volume units $(\mathrm{CF}=\mathrm{Cubic}$ Feet, $\mathrm{CM}=$ Cubic Meters, $\mathrm{GAL}=$ Gallons)

G. Fac.: The facility where the individual container or waste stream was generated.

Section E: Radiation Characteristics

Section F: TRU Alpha Activity Distribution

Section G: Radionuclide Concentrations

Basis Code(s): The basis for the radionuclide concentration data. The codes are listed as footnotes A through $\mathrm{J}$ on the form.

Section H: Matrix Characteristics

Primary Matrix Code: The primary matrix code assigned to the waste stream.

The code and description are as identified in the DOE Waste Treatability Group Guidance, dated January 1995.'

Section I: Matrix Composition

Code: The matrix code(s) describing the matrix composition of the waste stream.

The code and description are as identified in the DOE Waste Treatability Group Guidance, dated January 1995. ${ }^{1}$

Section J: Hazardous Constituents

Code: The EPA hazardous waste code

Treatment subcategory: The treatment subcategory as identified in the RCRA

LDR regulations 
Basis: The basis for the characterization of the hazardous constituents. The codes are:

A - Process knowledge based on general knowledge

B - Process knowledge based upon verification of existing process records and documents.

C - Sampling and analysis data of uncertain or undocumented quality

D - Quantitative data, obtained using approved QA protocols (low degree of certainty)

E - Quantitative data, obtained using approved QA protocols (high degree of certainty)

F - Analysis performed using SW-846 or similar EPA protocols and QAMS-005 QA/QC

1 - Grab Sample

2 - Composite Sample

3 - Batch Sample

Conc. Type: Identification is the concentration of hazardous constituents was based on total analysis of TCLP

Section K: Polychlorinated Biphenyls (PCBs)

Section L: Treatment Plans

Opt. \#: The option number for the treatment plan as identified in the INEL PSTP.

Vol.\%:The volume percent of the waste stream represented by the media name category.

JT: Indicates a just-in-time processing step. 'Yes' indicates that waste should not be treated for a preliminary step until the capacity for the following step is available.

SC: Indicates split unit capacity between media types. This indicator is essential for waste streams where a treatment step splits the waste stream into two parts. 'Yes' forces the process simulation to split the capacity of treatment steps prior to the split of the waste stream between the media percent of the component parts.

CIF: Indicates the Capacity Impact Factor. This factor allows waste-specific adjustment to the treatment unit's defined process rate. ( $1=$ no change, 2 $=$ unit's process rate for handling this waste will be reduced by $1 / 2,3=$ unit's process rate reduced by $1 / 3$, etc.) That is, some waste streams require longer process times to be fully treated. Reducing the processing rate will allow the waste stream more time for treatment. 
4. INEL and Offsite Waste Stream Profiles Not Identified in the documents: Radiological, Physical, and Chemical Characterization of Low-Level Alpha Contaminated Wastes Stored at the Idaho National Engineering laboratory for Use in Conducting Feasibility Studies for Treatment Services ${ }^{1}$ and Radiological, Physical, and Chemical Characterization of Transuranic Wastes Stored at the Idaho National Engineering laboratory for Use in Conducting Feasibility Studies for Treatment Services, ${ }^{2}$ dated April 5, 1994 


\section{ID CPPP-301 How ContactiH}

\section{A. IDENTIFICATION AND DESCRIPTION}

Waste Stream ID: ID-CPP-301

W. S. Name:

LIQUID ACID/MERCURY MIXED WASTE

Content Code:

FFCA ID:

$$
\text { * }
$$

IN-W119

\section{Waste Stream Description:}

Liquid low-level acid/mercury mixed waste from analytical lab that has a high concentration of

sulfates. Until LDR concerns are met, waste is stored in Satellite Accumulation areas and

transferrred to CPP-1617. Generated to support regulatory activites.

\section{LIQÜID AGIDMMERCURY MIXED WASTE}

iD-CP̈P

Still-Gen - Active

\section{B. GENERATION SITE/PROCESS DESCRIPTION}

The following questions, concerning the generation site and process, should be answered even if the waste stream is no longer generated.

Name of Site Generating Waste (e.g., INEL, Rocky Flats, etc.): Idaho National Engineering Laboratory

Location of activities (Area and Building): ICPP-684, ICPP-602

Operations (functions) performed in building:

Routine analytical procedures are performed which support a number of activities at ICPP.

Description of Process Generating Waste:

Waste stream generated from laboratory activities. 


\section{iD.CPP-301 \\ LWW Contact-H \\ C. PROJECTED WASTE GENERATION}

Is this waste stream still generated?

If yes, when is the expected generation termination date?

\section{OYes ONo}

Current Projections

\begin{tabular}{|r|r|r|r|r|l|}
\hline Est. Date & \multicolumn{1}{|c|}{ Start Year } & Interval & \multicolumn{1}{|c|}{ Vol (m3) } & Mass $(\mathrm{kg})$ & \multicolumn{1}{|c|}{ Source of Estimate } \\
\hline $6 / 9 / 94$ & 1994 & 1 & 1.40 & $1,400.00$ & 1994 DSTP \\
\hline $6 / 3 / 94$ & 1995 & 1 & 1.40 & $1,400.00$ & 1994-DSTP \\
\hline $6 / 3 / 94$ & 1996 & 1 & 1.40 & $1,400.00$ & 1994 DSTP \\
\hline $6 / 3 / 94$ & 1997 & 1 & 1.40 & 1.400 .00 & 1994 DSTP \\
\hline $6 / 3 / 94$ & 1998 & 1 & 1.40 & $1,400.00$ & 1994 DSTP \\
\hline $6 / 3 / 94$ & 1999 & 1 & 1.40 & $1,400.00$ & 1994 DSTP \\
\hline
\end{tabular}

Historical Projections

\begin{tabular}{|r|r|r|r|r|l|}
\hline Est. Date & \multicolumn{1}{|c|}{ Start Year } & \multicolumn{1}{|c|}{ Interval } & \multicolumn{1}{|c|}{ Vol $(\mathrm{m} 3)$} & \multicolumn{1}{c|}{ Mass $(\mathrm{kg})$} & \multicolumn{1}{c|}{ Source of Estimate } \\
\hline $6 / 3 / 94$ & 2000 & 5 & 7.00 & $7,000.00$ & $1994-\mathrm{DSTP}$ \\
\hline $6 / 3 / 94$ & 2005 & 10 & 14.00 & $14,000.00$ & $1994-\mathrm{DSTP}$ \\
\hline $11 / 1 / 93$ & 1993 & 1 & 0.42 & 419.80 & $1993-\mathrm{FFCA}$ \\
\hline $11 / 1 / 93$ & 1994 & 1 & 0.42 & 419.80 & $1993-\mathrm{FFCA}$ \\
\hline $11 / 1 / 93$ & 1995 & 1 & 0.42 & 419.80 & $1993-\mathrm{FFCA}$ \\
\hline $11 / 1 / 93$ & 1996 & 1 & 0.42 & 419.80 & $1993-\mathrm{FFCA}$ \\
\hline $11 / 1 / 93$ & 1997 & 1 & 0.42 & 419.80 & $1993-\mathrm{FFCA}$ \\
\hline $11 / 1 / 93$ & 1998 & 5 & 2.10 & $2,099.00$ & $1993-$ FFCA \\
\hline $11 / 1 / 93$ & 2003 & 20 & 6.30 & $8,396.00$ & $1993-$ FFCA \\
\hline $12 / 1 / 92$ & 1992 & 5 & 2.10 & $2,099.00$ & $1992-$ FFCA \\
\hline
\end{tabular}


D. STORAGE INVENTORY

\begin{tabular}{|l|l|l|l|r|r|r|r|r|r|}
\hline Container ID & Stor. Loc. & \# of Cont. & Type & Total Vol. & Units & Weight (Ibs) & G. Fac. & Date Rec. & Waste Description \\
\hline & & & & 0.3 & CM & 496 & & & Inventory stored at CPP-1617 as of 12/31/94. \\
\hline \multicolumn{8}{|c|}{$0.27 \mathrm{m3}$} \\
\hline
\end{tabular}

\section{Waste Removed From Storage:}




\section{D.CPP-301 \\ Llw: Contact-H}

E. RADIATION CHARACTERISTICS

Radioactive Waste Type:

\begin{tabular}{|ll|}
\hline OlLW & Oa-LLW $\bigcirc$ tRU \\
OHLW & O Non-Rad
\end{tabular}

Mixed Waste:

\begin{tabular}{|l|}
\hline OYes O No \\
\hline O Contact O Remote \\
\hline
\end{tabular}

Radionuclide Contamination Accessiblity:

External Surface

Internal Surface

Dispersed Through Matrix

\begin{tabular}{|c|c|c|}
\hline OYes & O No & Unknown \\
\hline Yyes & O No & Unknown \\
\hline $\mathrm{OYe}$ & No & $\mathrm{Nn}$ \\
\hline
\end{tabular}

Activity Levels

Transuranic Alpha Activity:

Uranium/Thorium Alpha Activity:

Beta/Gamma Activity:

Surface Neutron Activity:

Total Activity:

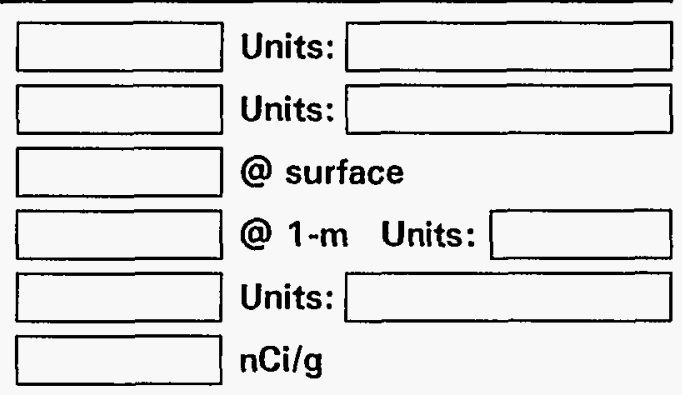

1D-CPP-301 Stiil-Gen : Active

\section{F. TRU ALPHA ACTIVITY DISTRIBUTION}

This waste stream is not identified as a TRU or a-LLW. 
TD-CPP. 301

LIOUID ACIDIMERCURY MIXED WASTE

LlW... Contact-H

\section{G. RADIONUCLIDE CONCENTRATIONS}

\begin{tabular}{|l|l|l|l|l|}
\cline { 2 - 5 } \multicolumn{1}{c|}{} & \multicolumn{3}{c|}{ Specific Activity } & \multicolumn{1}{c}{} \\
\hline Isotope & Typical & Lower Limit & Upper Limit & Units \\
\hline Ru-106 & & & & \\
\hline Sb-125 & & & & \\
\hline Co-60 & & & & \\
\hline Eu-154 & & & & \\
\hline Ce-152 & & & & \\
\hline Ce-134 & & & & \\
\hline
\end{tabular}

A. Passive-Active Neutron (PAN)

B. Segmented Gamma Scan (SGS)

C: Passive-Active Neutron/Segmented Gamma Scan (PAN/SGS)

D. Radiochemical Analysis

E. Mass Spectrometry

\begin{tabular}{|l|l|}
\hline Basis Code(s) & Basis Desc. (if other) \\
\hline D & \\
\hline D & \\
\hline D & \\
\hline D & \\
\hline D & \\
\hline D & \\
\hline D & \\
\hline
\end{tabular}

F. Accountability

G. Calorimetry

H. Multiple Energy Gamma Analysis (MEGAS)

1. Process Knowledge

J. Other 
ID.CPP.301

LiW ContactH

H. MATRIX CHARACTERISTICS

Primary Matrix Code: $L 1110$

Description: Aqueous Liquids/Slurries: Wastewaters: Acidic Wastewaters:

Combustibility:

Combustible (>90\%)

Mixed $(10 \%-90 \%)$

(- Noncombustible (<10\%)

\section{MATRIX COMPOSITION}

\begin{tabular}{|c|c|c|c|c|c|c|}
\hline & & & \multicolumn{4}{|c|}{ Composition } \\
\hline Code & Matrix Description & Material & Typical & LL & UL. & Units \\
\hline$L 1110$ & Aqueous Liquids/Slurries.Wastewaters.Acidic Wastewaters. & Acidic Wastewater & 100 & 100 & 100 & \%vol \\
\hline
\end{tabular}
Still-Gen Active
ID-CPP-301 
D.CPP-301

LOUID ACIDIMERCURY MIXËD WÄSTE

ID:CPP-301

HWW Contacth

\section{J. HAZARDOUS CONSTITUENTS}

\begin{tabular}{|c|c|c|c|c|c|c|c|c|}
\hline \multirow[b]{2}{*}{ Code } & \multirow[b]{2}{*}{ Hazardous Waste Description } & \multirow[b]{2}{*}{ Treatment Subcategory } & & \multirow[b]{2}{*}{ Basis } & \multirow[b]{2}{*}{ Conc. Type } \\
\hline & & & Typical & $\mathbf{L L}$ & UL & Units & & \\
\hline $\mathrm{DO02}$ & Corrosive & Corrosive characteristic waste that are manag & & & & & $A ; F$ & Total \\
\hline D004 & Arsenic & & & & & & $A ; F$ & TCLP \\
\hline 0005 & Barium & & & & & & $A ; F$ & TCLP \\
\hline D006 & Cadmium & . & & & & & A;F & TCLP \\
\hline D007 & Chromium & Chromium (Total) & & & & & $A ; F$ & TCLP \\
\hline $\mathrm{D008}$ & Lead & & & & & & $A ; F$ & TCLP \\
\hline D010 & Selenium & & & & & & $A ; F$ & TCLP \\
\hline 0011 & Silver & & & & & & $A ; F$ & TCLP \\
\hline$D 018$ & Benzene & Benzene managed in non-CWA/non-CWA-equi & & & & & A & Total \\
\hline 0019 & Carbon tetrachloride & Carbon Tetrachloride managed in non-CWA/no & & & & & A & Total \\
\hline$\overline{D 021}$ & Chlorobenzene & Chlorobenzene managed in non-CWA/non-CW & & . & & & A & Total \\
\hline$\overline{0022}$ & Chloroform & Chloroform managed in non-CWA/non-CWA-e & & & & & A & Total \\
\hline DO26 & Cresol & Cresols (Total) managed in non-CWA/non-CW & & & & & A & Total \\
\hline 0032 & Hexachlorobenzene & Hexachlorobenzene managed in non-CWA/non & & & & & A & Total \\
\hline$\overline{D 034}$ & Hexachloroethane & Hexachloroethane managed in non-CWA/non- & & & & & $A$ & Total \\
\hline 0035 & Methyl ethyl ketone & Methyle ethyl ketone managed in non-CWA/no & & & & & A & Total \\
\hline 0036 & Nitrobenzene & Nitrobenzene managed in non-CWA/non-CWA- & & & & & A & Total \\
\hline 0038 & Pyridine & Pyridino managed in non-CWA/non-CWA-equiv & & & & & A & Total \\
\hline 0039 & Tetrachloroethylene & Tetrachloroethylene managed in non-CWA/non & & & & & A & Total \\
\hline D040 & Trichloroethylene & Trichloroethylene managed in non-CWA/non-C & & & & & A & Total \\
\hline F001 & Spent halogenated solvents used in degreasing & 1,1,1-Trichloroethane & & & & & $A$ & Total \\
\hline F001 & Spent halogenated solvents used in degreasing & Carbon tetrachloride & & & & & A & Total \\
\hline F001 & Spent halogenated solvents used in degreasing & Methylene chloride & & & & & A & Total \\
\hline F001 & Spent halogenated solvents used in degreasing & Tetrachloroethylene & & & & & A & Total \\
\hline FOO1 & Spent halogenated solvents used in degreasing & Trichloroethylene & & & & & A & Total \\
\hline F002 & Spent halogenated solvents & o-Dichlorobenzene & & & & & A & Total \\
\hline FO02 & Spent halogenated solvents & 1,1,1-Trichloroethane & & & & & $A$ & Total \\
\hline FOO2 & Spent halogenated solvents & Methylene chloride & & & & & A & Total \\
\hline F002 & Spent halogenated solvents & Tetrachloroethylene & & & & & A & Total \\
\hline
\end{tabular}


ID-cPp. 301

LLWW

F002

F005

F005

F005

FO05

F005

F005

F005

P005

P012

$\mathrm{PO22}$

P024

P027

028

P030

P031

P056

P073

P075

077

P098

$\mathrm{P} 104$

P105

P106

P113

P116

P119

P120

บ003

U004

U007

U009

U012

U014

U019

$\mathrm{U} 02 \mathrm{O}$

U032

7/27/95

Allyl alcohol

Benzyl chloride

Cyanogen

Fluorine

p-Nitroaniline

Silver cyanide

Sodium azide

Thallic oxide

Acetonitrile

Acetophenone

Acrylamido

Acrylonitrile

Aniline

Auramine

Benzene

LIQUID ACIDIMERCURY MIXED WASTE

ID CPP 301

\section{Contact-H}

Spent halogenated solvents

Spent non-halogenated solvents

Spent non-halogenated solvents

Spent non-halogenated solvents

Spent non-halogenated solvents

Spent non-halogenated solvents

Spent non-halogenated solvents

Spent non-halogenated solvents

Arsenic trioxide

Carbon disulfide

p-Chloroaniline

3-Chloropropionitrile

Cyanides (Soluble salts and complexes)

Nickel carbonyl

Nicotine and Salts

Potassium cyanide

Sodium cyanide

Thiosemicarbazide

Ammonium vanadate

Vanadium pentoxide

Benzenesulfonyl chloride

Calcium chromate

Chromium (total)

Waste Stream Profile Report - Idaho Mixed Waste Information (IMWII System

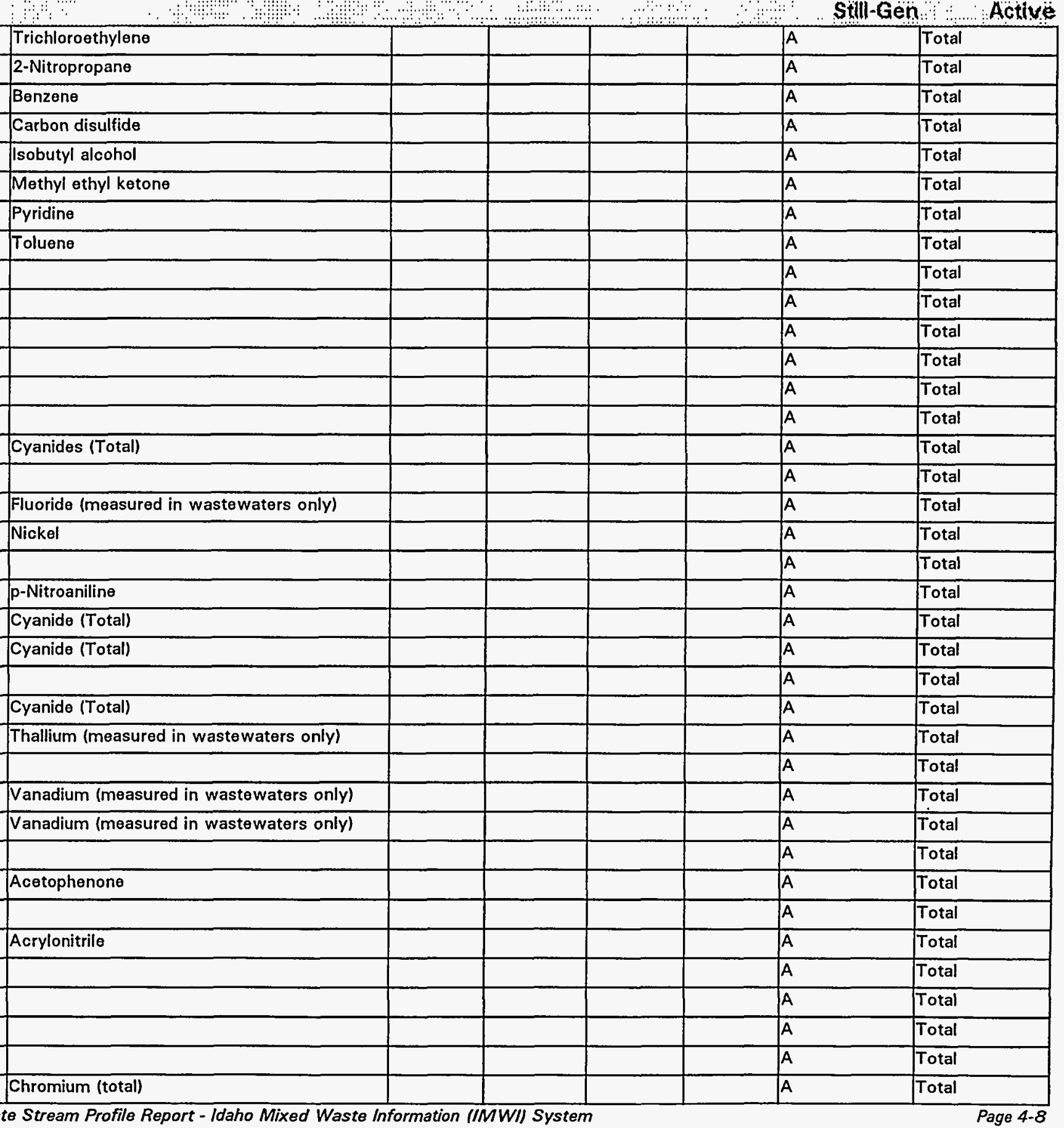




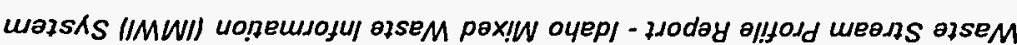

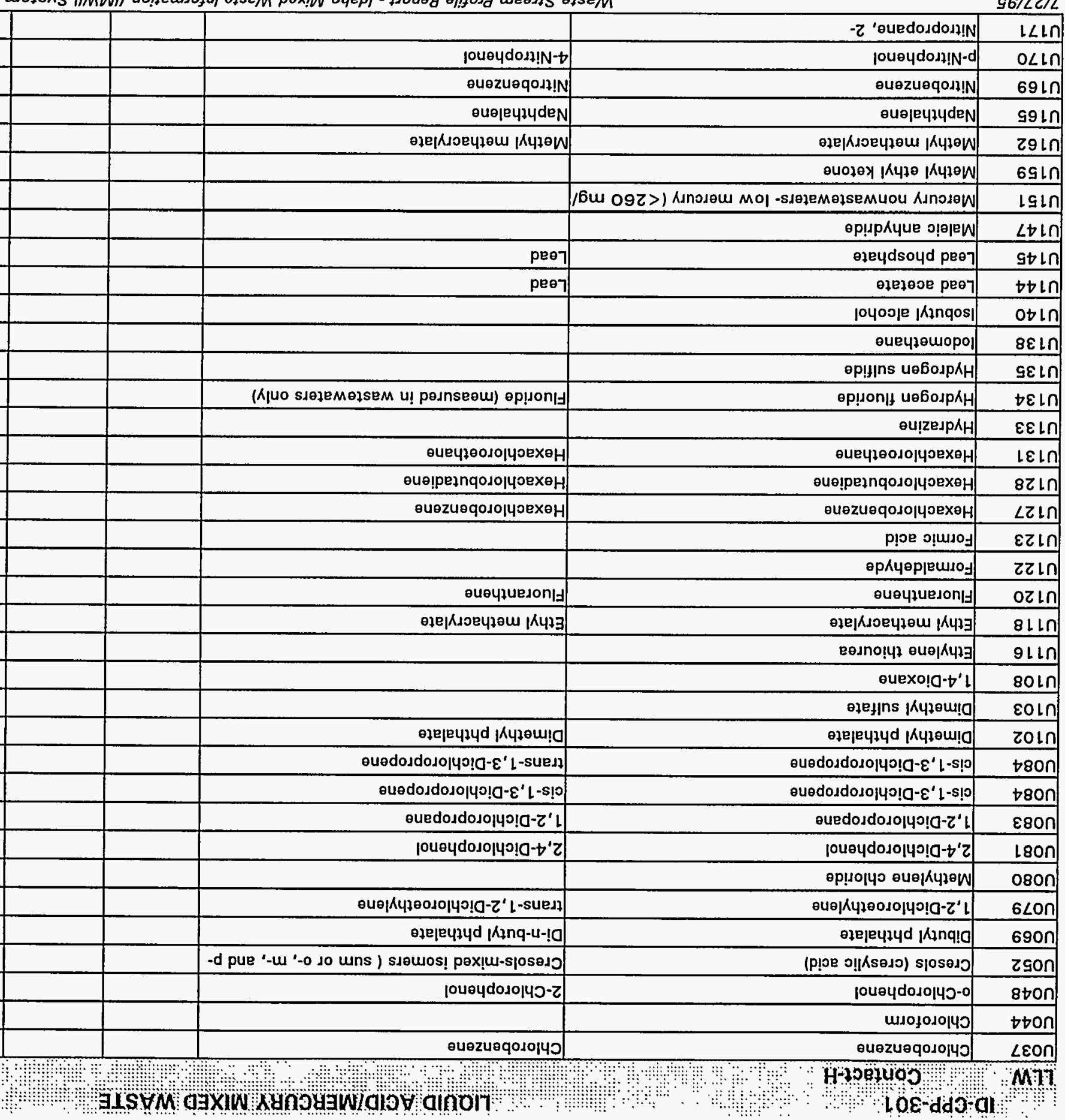


1D-CPP. 301

LIQUID ACID/MIERCURY MIXED WASTE

IDMCPP-301 : :

Liw

Cöntact-H

Still-Gen

Active

\begin{tabular}{|l|l|l|}
\hline U182 & Paraldehyde \\
\hline U188
\end{tabular}

\begin{tabular}{|l|l|}
\hline U188 & Phenol \\
\hline
\end{tabular}

U190

U191

U196

Phthallic anhydride

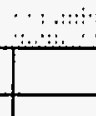

2-Picoline

Pyridine

\begin{tabular}{|l|l}
\hline U201 Resorcinol \\
\hline
\end{tabular}

\begin{tabular}{|l|l}
\hline U204 & Selenioum dioxide \\
\hline
\end{tabular}

\begin{tabular}{l|l}
\hline de & Selenium \\
\hline
\end{tabular}

\begin{tabular}{|l|l|}
\hline$U 207$ & $1,2,4,5$-Tetrachlorobenzene \\
\hline
\end{tabular}

\begin{tabular}{l|l}
\hline U208 & $1,1,1,2$-Tetrachloroethane \\
\hline U2
\end{tabular}

\begin{tabular}{|l|l|}
\hline $\mathrm{U} 210$ & Tetrachloroethylene \\
\hline $\mathrm{U} 211$
\end{tabular}

U211

Carbon tetrachloride

\begin{tabular}{|l|l}
\hline U215 & Thallium (I) carbonate \\
\hline
\end{tabular}

U217

U218

Thallium (I) nitrate

Thioacetamide

\begin{tabular}{|l|l|}
\hline U219 & Thiourea \\
\hline
\end{tabular}

U220

U225

U226

U227

Toluene

Bromoform

1,1,1-Trichloroethane

1,1,2-Trichloroethane

Trichloroethylene

\begin{tabular}{|l|l|}
\hline U239 & Xylenes \\
\hline
\end{tabular}

\begin{tabular}{|l|l}
\hline U328 & O-Toluidine
\end{tabular}

LIQUID ACIPIMERCURY MIXED WASTE

\begin{tabular}{|l|l|}
\hline \multicolumn{2}{|c|}{ Still Gen } \\
\hline A & Total \\
\hline A & Total \\
\hline A & Total \\
\hline A & Total \\
\hline A & Total \\
\hline A & Total \\
\hline A & Total \\
\hline A & Total \\
\hline A & Total \\
\hline A & Total \\
\hline A & Total \\
\hline A & Total \\
\hline A & Total \\
\hline A & Total \\
\hline A & Total \\
\hline A & Total \\
\hline A & Total \\
\hline A & Total \\
\hline A & Total \\
\hline A & Total \\
\hline A & Total \\
\hline A & Total \\
\hline
\end{tabular}


: ID:CPP 301

LLW Contact

K. POLYCHLORINATED BIPHENYLS (PCBS)

Is this waste stream PCB contaminated?

\section{OYes O No O Unknown}

If yes, what portion of the waste is $\mathrm{PCB}$ contaminated (volume \%):

If $<100 \%$, can the PCB contaminated waste be segregated and treated separately?

What is the PCB concentration in ppm?

Y Yes No Unknown

If only a portion of the waste is $P C B$ contaminated, report values for contaminated portion.

\section{TREATMENT PLANS}

\begin{tabular}{|c|c|c|c|c|c|c|c|c|c|c|c|}
\hline $\begin{array}{c}\text { Opt. } \\
\#\end{array}$ & $\begin{array}{c}\text { Media Type } \\
\text { (If more than one) }\end{array}$ & $\begin{array}{c}\text { Matrix } \\
\text { Code }\end{array}$ & $\begin{array}{l}\text { Vol. \% } \\
\text { IIf }\end{array}$ & Step & JIT & sc & CIF & $\begin{array}{l}\text { Trans. } \\
\text { Miles }\end{array}$ & $\begin{array}{l}\text { Facility } \\
\text { Abbr. }\end{array}$ & Unit Name & Comments \\
\hline 1 & & $L 1110$ & & $\bar{a}$ & () & 0 & $1.00 E+00$ & & AMWTF & $\mathrm{CH}$ - Opening \& Sorting & \\
\hline & & & & $\bar{b}$ & 0 & 0 & $1.00 E+00$ & & AMWTF & $\mathrm{CH}$ - Incineration/Thermal Desorption & \\
\hline & & & & c & 0 & $\bar{O}$ & $1.00 \mathrm{E}+00$ & & AMWTF & $\mathrm{CH}$ - Vitrification & \\
\hline & & & & d & 0 & 0 & $1.00 E+00$ & & SCDF & Disposal - Contact Handled & \\
\hline
\end{tabular}

Note: Where provided, media percentages are rough estimates used to facilitate treatment planning. They do not necessarily imply an accurate knowledge of waste stream composition, and were not derived for use outside this conceptual planning exercise.

Describe any special or unique technical concerns related to the treatment of this waste stream that would impact the use or implementation of standard hazardous waste treatment methods. 


\section{B. GENERATION SITE/PROCESS DESCRIPTION}

\section{A. IDENTIFICATION AND DESCRIPTION}

Waste Stream ID: ID-CPP-302

W. S. Name:

Content Code:

FFCA ID:

UIQUID HIGH CHLORIDE CORROSIVE MW

Waste generated from analytical lab procedures at CPP-684 and CPP-602. High chloride liquid low level corrosive mixed waste cannot be disposed of to the CPP liquid waste stream, because it will cause excessive corrosion of pipes.
The following questions, concerning the generation site and process, should be answered oven if the waste stream is no longer generated.

Name of Site Generating Waste (e.g., INEL, Rocky Flats, etc.): Idaho National Engineering Laboratory

Location of activities (Area and Building):

Waste generated from analytical lab procedures at CPP-684 and CPP602.

Operations (functions) performed in building: Laboratory activities.

Description of Process Generating Waste: Laboratory activities. 
DDCPP 302

LiW $\quad$ Contact H

\section{PROJECTED WASTE GENERATION}

Is this waste stream still generated?

OYes ONo

If yes, when is the expected generation termination date?

Current Projections

\begin{tabular}{|r|r|r|r|r|l|}
\hline Est. Date & \multicolumn{1}{|c|}{ Start Year } & \multicolumn{1}{|c|}{ Interval } & \multicolumn{1}{|c|}{ Vol (m3) } & \multicolumn{1}{c|}{ Mass $(\mathrm{kg})$} & Source of Estimate \\
\hline $6 / 9 / 94$ & 1994 & 1 & 1.40 & $1,400.00$ & $1994-\mathrm{DSTP}$ \\
\hline $6 / 3 / 94$ & 1995 & 1 & 1.40 & $1,400.00$ & $1994-\mathrm{DSTP}$ \\
\hline $6 / 3 / 94$ & 1996 & 1 & 1.40 & $1,400.00$ & $1994-\mathrm{DSTP}$ \\
\hline $6 / 3 / 94$ & 1997 & 1 & 1.40 & $1,400.00$ & $1994-\mathrm{DSTP}$ \\
\hline $6 / 3 / 94$ & 1998 & 1 & 1.40 & $1,400.00$ & $1994 \mathrm{DSTP}$ \\
\hline $6 / 3 / 94$ & 1999 & 1 & 1.40 & $1,400.00$ & $1994 \mathrm{DSTP}$ \\
\hline
\end{tabular}

Historical Projections

\begin{tabular}{|r|r|r|r|r|l|}
\hline Est. Date & \multicolumn{1}{|c|}{ Start Year } & \multicolumn{1}{|c|}{ Interval } & \multicolumn{1}{|c|}{ Vol $(\mathbf{m} 3)$} & Mass $(\mathbf{k g})$ & Source of Estimate \\
\hline $6 / 3 / 94$ & 2000 & 5 & 7.00 & $7,000.00$ & $1994-D S T P$ \\
\hline $6 / 3 / 94$ & 2005 & 10 & 14.00 & $14,000.00$ & $1994-D S T P$ \\
\hline $11 / 1 / 93$ & 1993 & 1 & 0.42 & 419.80 & $1993-F F C A$ \\
\hline $11 / 1 / 93$ & 1994 & 1 & 0.42 & 419.80 & $1993-F F C A$ \\
\hline $11 / 1 / 93$ & 1995 & 1 & 0.42 & 419.80 & $1993-F F C A$ \\
\hline $11 / 1 / 93$ & 1996 & 1 & 0.42 & 419.80 & $1993-F F C A$ \\
\hline $11 / 1 / 93$ & 1997 & 1 & 0.42 & 419.80 & $1993-F F C A$ \\
\hline $11 / 1 / 93$ & 1998 & 5 & 2.10 & $2,099.00$ & $1993-F F C A$ \\
\hline $11 / 1 / 93$ & 2003 & 20 & 8.40 & $8,396.00$ & $1993-F F C A$ \\
\hline $12 / 1 / 92$ & 1992 & 5 & 2.10 & $2,099.00$ & $1992-F F C A$ \\
\hline
\end{tabular}




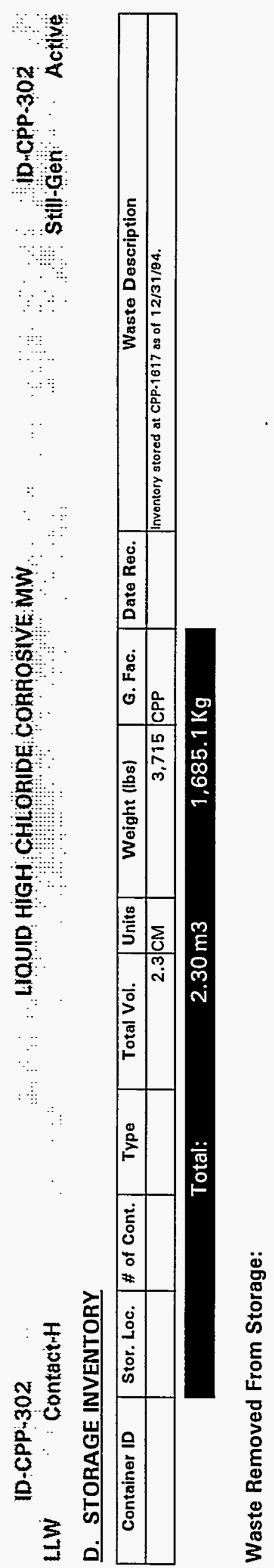




\section{E. RADIATION CHARACTERISTICS}

Radioactive Waste Type:

\begin{tabular}{ll} 
OllW & Oa-LLW $\bigcirc$ TRU \\
OhlW & Onon-Rad \\
\hline
\end{tabular}

Mixed Waste:

Handling:

\begin{tabular}{|l|}
\hline OYes O No \\
\hline O Contact O Remote \\
\hline
\end{tabular}

Radionuclide Contamination Accessiblity:

\begin{tabular}{rlrl|} 
External Surface & OYes & O No & O Unknown \\
Internal Surface & OYes & O No & O Unknown \\
\cline { 2 - 4 } Dispersed Through Matrix & OYes & O No & O Unknown
\end{tabular}

\section{Activity Levels}

Transuranic Alpha Activity: Uranium/Thorium Alpha Activity: Beta/Gamma Activity:

Surface Neutron Activity: Total Activity:

$\square$ Units: $\square$
$\square$ Units: $\square$
@ surface
$\square$ @ 1-m Units: $\square$
$\square$ Units: $\square$
$\square$ nCi/g

\section{F. TRU ALPHA ACTIVITY DISTRIBUTION}

This waste stream is not identified as a TRU or a-LLW. 

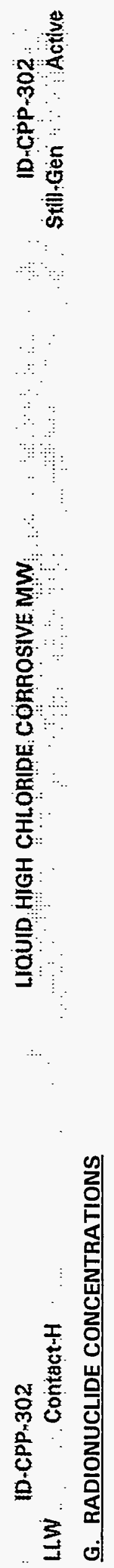


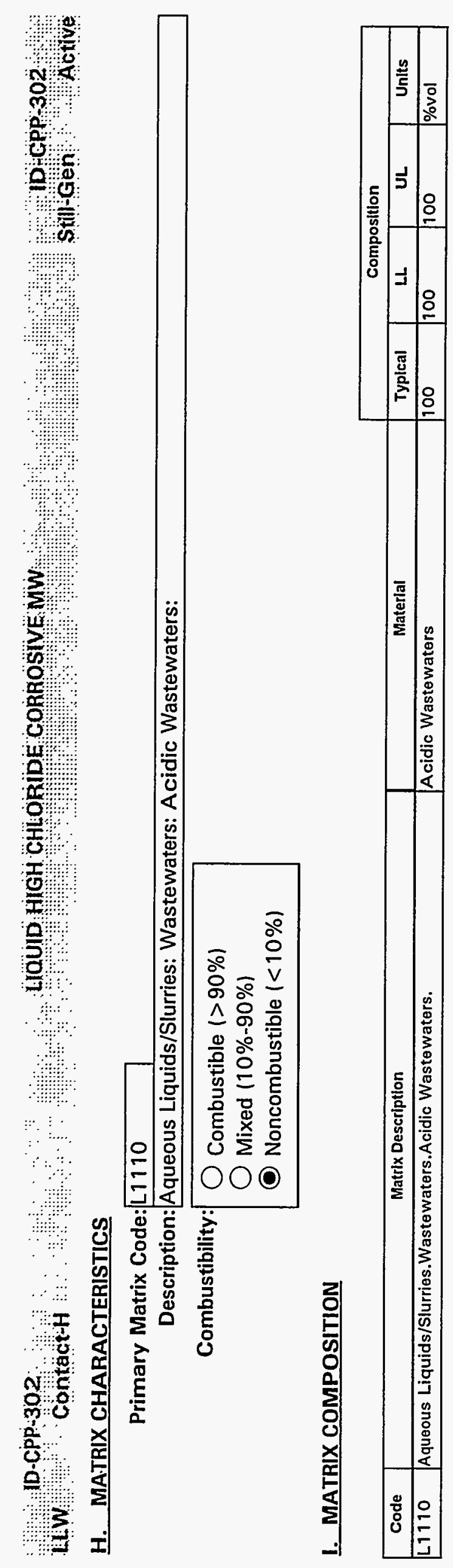


ID $C$ CPP -302

LLW: Contagt-H

\section{J. HAZARDOUS CONSTITUENTS}

\begin{tabular}{|c|c|c|c|c|c|c|c|c|}
\hline & & & \multicolumn{4}{|c|}{ Concentration } & \multirow[b]{2}{*}{ Basis } & \multirow[b]{2}{*}{ Conc. Type } \\
\hline Code & Hazardous Waste Description & Treatment Subcategory & Typical & $\mathbf{L L}$ & UL & Units & & \\
\hline $\mathrm{DO02}$ & Corrosive & Corrosive characteristic waste that are manag & & & & & $A ; F$ & Total \\
\hline D004 & Arsenic & & & & & & $A ; F$ & TCLP \\
\hline D005 & Barium & & & & & & $A ; F$ & TCLP \\
\hline D006 & Cadmium & & & & & & $A ; F$ & TCLP \\
\hline D007 & Chromium & Chromium (Total) & & & & & $A ; F$ & TCLP \\
\hline D008 & Lead & & & & & & $A ; F$ & TCLP \\
\hline D009 & Mercury & Low mercury $<260 \mathrm{mg} / \mathrm{kg}$ total mercury & & & & & $\mathrm{A}: \mathrm{F}$ & TCLP \\
\hline D010 & Selenium & & & & & & $A ; F$ & TCLP \\
\hline D011 & Silver & & & & & & $A ; F$ & TCLP \\
\hline D018 & Benzene & Benzene managed in non-CWA/non-CWA-equi & & & & & A & Total \\
\hline D019 & Carbon tetrachloride & Carbon Tetrachloride managed in non-CWA/no & & & & & A & Total \\
\hline $\mathrm{DO21}$ & Chlorobenzene & Chlorobenzene managed in non-CWA/non-CW & & & & & A & Total \\
\hline D022 & Chloroform & Chloroform managed in non-CWA/non-CWA-e & & & & & A & Total \\
\hline DO26 & Cresol & Cresols (Total) managed in non-CWA/non-CW & & & & & A & Total \\
\hline D028 & 1,2-Dichloroethane & 1,2-Dichloroethane managed in non-CWA/non- & & & & & A & Total \\
\hline D032 & Hexachlorobenzene & Hexachlorobenzene managed in non-CWA/non & & & & & A & Total \\
\hline D034 & Hexachloroethane & Hexachloroethane managed in non-CWA/non- & & & & & A & Total \\
\hline D035 & Methyl ethyl ketone & Methyle ethyl ketone managed in non-CWA/no & & & & & A & Total \\
\hline D036 & Nitrobenzene & Nitrobenzene managed in non-CWA/non-CWA- & & & & & A & Total \\
\hline D038 & Pyridine & Pyridine managed in non-CWA/non-CWA-equiv & & & & & A & Total \\
\hline$D 039$ & Tetrachloroethylene & Tetrachloroethylene managed in non-CWA/non & & & & & A & Total \\
\hline $\mathrm{DO40}$ & Trichloroethylene & Trichloroethylene managed in non-CWA/non-C & & & & & A & Total \\
\hline F001 & Spent halogenated solvents used in degreasing & 1,1,1-Trichloroethane & & & & & A & Total \\
\hline F001 & Spent halogenated solvents used in degreasing & Carbon tetrachloride & & & & & A & Total \\
\hline Fo01 & Spent halogenated solvents used in degreasing & Methylene chloride & & & & & A & Total \\
\hline F001 & Spent halogenated solvents used in degreasing & Tetrachloroethylene & & & & & A & Total \\
\hline Fo01 & Spent halogenated solvents used in degreasing & Trichloroethylene & & & & & A & Total \\
\hline FOO2 & Spent halogenated solvents & o-Dichlorobenzene & & & & & A & Total \\
\hline FOO2 & Spent halogenated solvents & 1,1,1-Trichloroethane & & & & & A & Total \\
\hline FOO2 & Spent halogenated solvents & 1,1,2-Trichloroethane & & & & & A & Total \\
\hline FOO2 & Spent halogenated solvents & Chlorobenzene & & & & & A & Total \\
\hline F002 & Spent halogenated solvents & Methylene chloride & & & & & A & Total \\
\hline FOO2 & Spent halogenated solvents & Tetrachloroethylene & & & & & A & Total \\
\hline
\end{tabular}

\section{LIQUID HIGH CHLORIDE CORROSIVE MWW}

ID-CPP +302

Still-Gen $\because$ Active
Waste Stream Profile Report - Idaho Mixed Waste Information (IMWI) System

Page 4-18 
ID $=\mathrm{CPP}+302$

LUQUD HIGH CḦLORIDE CORROSIVE MWW

$1 D-C P P 302$

HWW ContactH

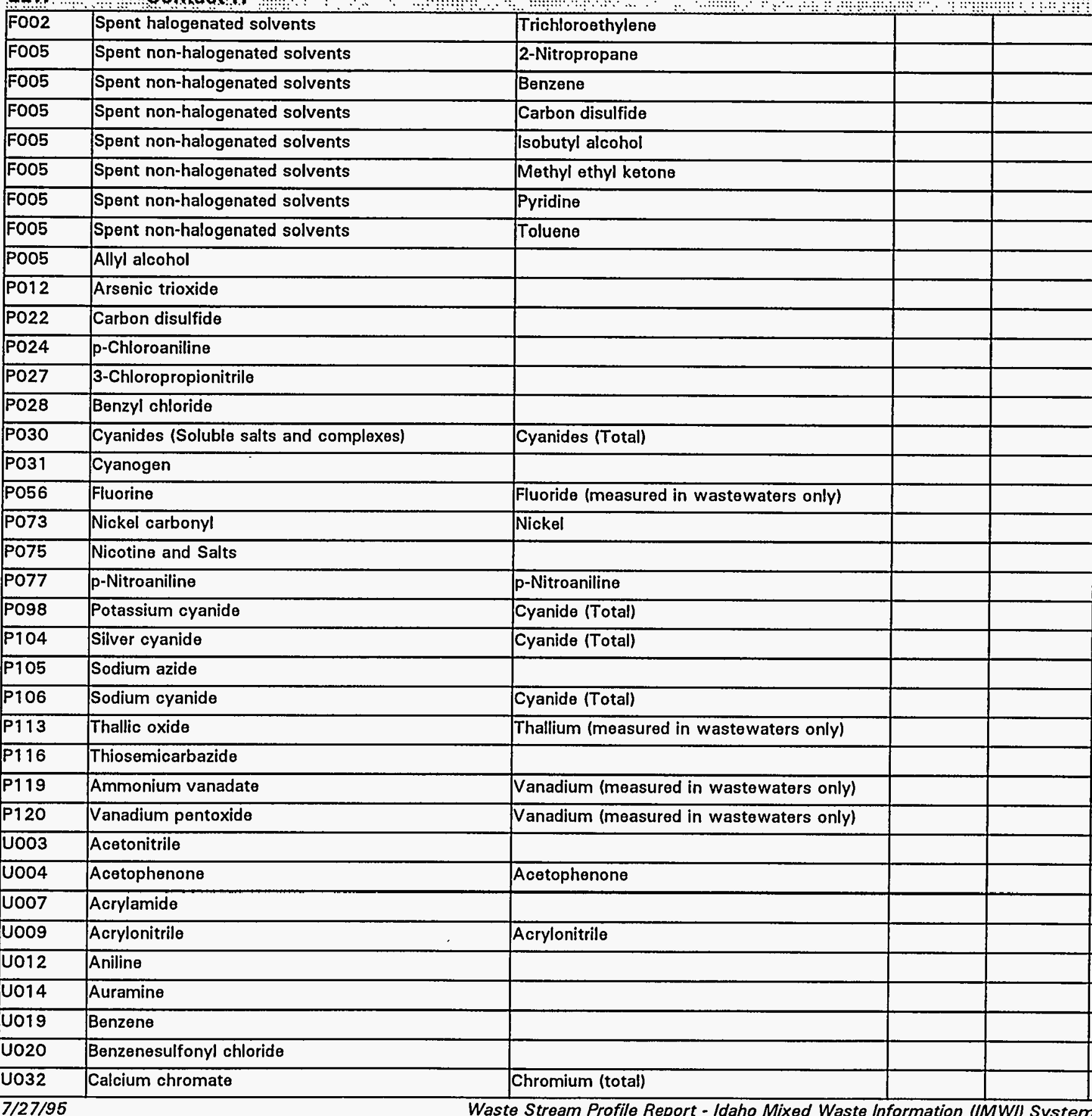

Stilligen Active Total

\begin{tabular}{|l|l|l}
\hline & & A \\
\hline & & A \\
\hline & & A \\
\hline & & A \\
\hline & & A \\
& & A \\
\hline
\end{tabular}

\begin{tabular}{|l|l|}
\hline$A$ & Total \\
\hline$A$ & Total \\
\hline
\end{tabular}


ID-CPP -302

LIQUID HIGH CHLOR̈IDE CORROSIVE MW

$1 \mathrm{D}-\mathrm{CPP}-302$

Hiw:

Contact

U037 Chlorobenzene

\begin{tabular}{|l|l|}
\hline U044 & Chloroform \\
\hline
\end{tabular}

\begin{tabular}{|l|l}
\hline 0048 & o-Chlorophenol
\end{tabular}

\begin{tabular}{ll}
\hline 052 & Cresols (cresylic acid)
\end{tabular}

\begin{tabular}{|l|l|}
\hline 0069 & Dibutyl phthalate \\
\hline
\end{tabular}

\begin{tabular}{|l|l|}
\hline U079 & 1,2-Dichloroethylene \\
\hline
\end{tabular}

Methylene chloride

2,4-Dichlorophenol

1,2-Dichloropropane

cis-1,3-Dichloropropene

cis-1,3-Dichloropropene

Dimethyl phthalate

Dimethyl sulfate

1,4-Dioxane

\begin{tabular}{|l|l}
\hline U116 Ethylene thiourea \\
\hline U118
\end{tabular}

\begin{tabular}{|l|l}
\hline U118 & Ethyl methacrylate \\
\hline U120
\end{tabular}

U120 Fluoranthene

\begin{tabular}{|l|l}
\hline U122 & Formaldehyde \\
\hline
\end{tabular}

U123 Formic acid

\begin{tabular}{|l|l}
\hline U127 Hexachlorobenzene \\
\hline U128
\end{tabular}

\begin{tabular}{|l|l}
\hline U128 & Hexachlorobutadiene \\
\hline
\end{tabular}

\begin{tabular}{|l|l|}
\hline U131 & Hexachloroethane \\
\hline V133
\end{tabular}

U133 Hydrazine

\begin{tabular}{|l|l}
\hline U134 Hydrogen fluoride \\
\hline
\end{tabular}

........... Chlorobenzene

(2)

U135

U138

U140

U144

U145

U147

U151

U159

U162

U165

U169

U170

Hydrogen sulfide

lodomethane

Isobutyl alcohol

Lead acetate

Lead phosphate

Maleic anhydride

Mercury nonwastewaters- low mercury $(<260 \mathrm{mg}$ )

Methyl ethyl ketone

\section{Methyl methacrylate}

Naphthalene

\begin{tabular}{l|l}
\hline U171 Nitropropane, 2- \\
\hline
\end{tabular}

2-Chlorophenol

Cresols-mixed isomers ( sum or $\mathrm{o}^{-}, \mathrm{m}-$, and $\mathrm{p}$ -

Di-n-butyl phthalate

trans-1,2-Dichloroethylene

2,4-Dichlorophenol

1,2-Dichloropropane

cis-1,3-Dichloropropene

trans-1,3-Dichloropropene

Dimethyl phthalate

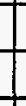

\section{Ethyl methacrylate}

Fluoranthene

Hexachlorobenzene

Hexachlorobutadiene

Hexachloroethane

Fluoride (measured in wastewaters only)

-

Lead

Lead

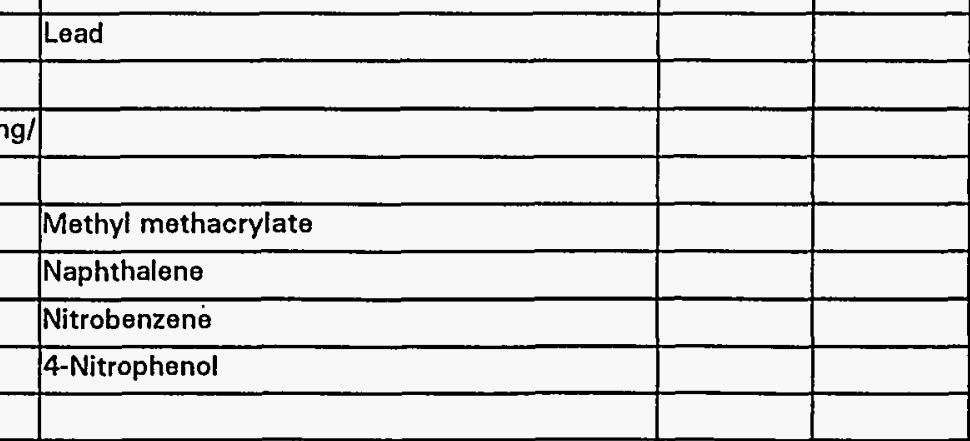

Waste Stream Profile Report - Idaho Mixed Waste Information (IMWI) System
Still-Ger in Active

\begin{tabular}{|l|l|}
\hline A & Total \\
\hline A & Total \\
\hline A & Total \\
\hline A & Total \\
\hline A & Total \\
\hline A & Total \\
\hline A & Total \\
\hline A & Total \\
\hline A & Total \\
\hline A & Total \\
\hline & Total \\
\hline A & Total \\
\hline A & Total \\
\hline A & Total \\
\hline A & Total \\
\hline A & Total \\
\hline A & Total \\
\hline A & Total \\
\hline A & Total \\
\hline A & Total \\
\hline A & Total \\
\hline A & Total \\
\hline A & Total \\
\hline A & Total \\
\hline A & Total \\
\hline A & Total \\
\hline A & Total \\
\hline A & Total \\
\hline A & Total \\
\hline A & Total \\
\hline A & Total \\
\hline A & Total \\
\hline A & Total \\
\hline A & Total \\
\hline
\end{tabular}


ID:CPP.302

MQUUID HIGH CHLORIDE CORROSIVEMW

ID $C P P=302$

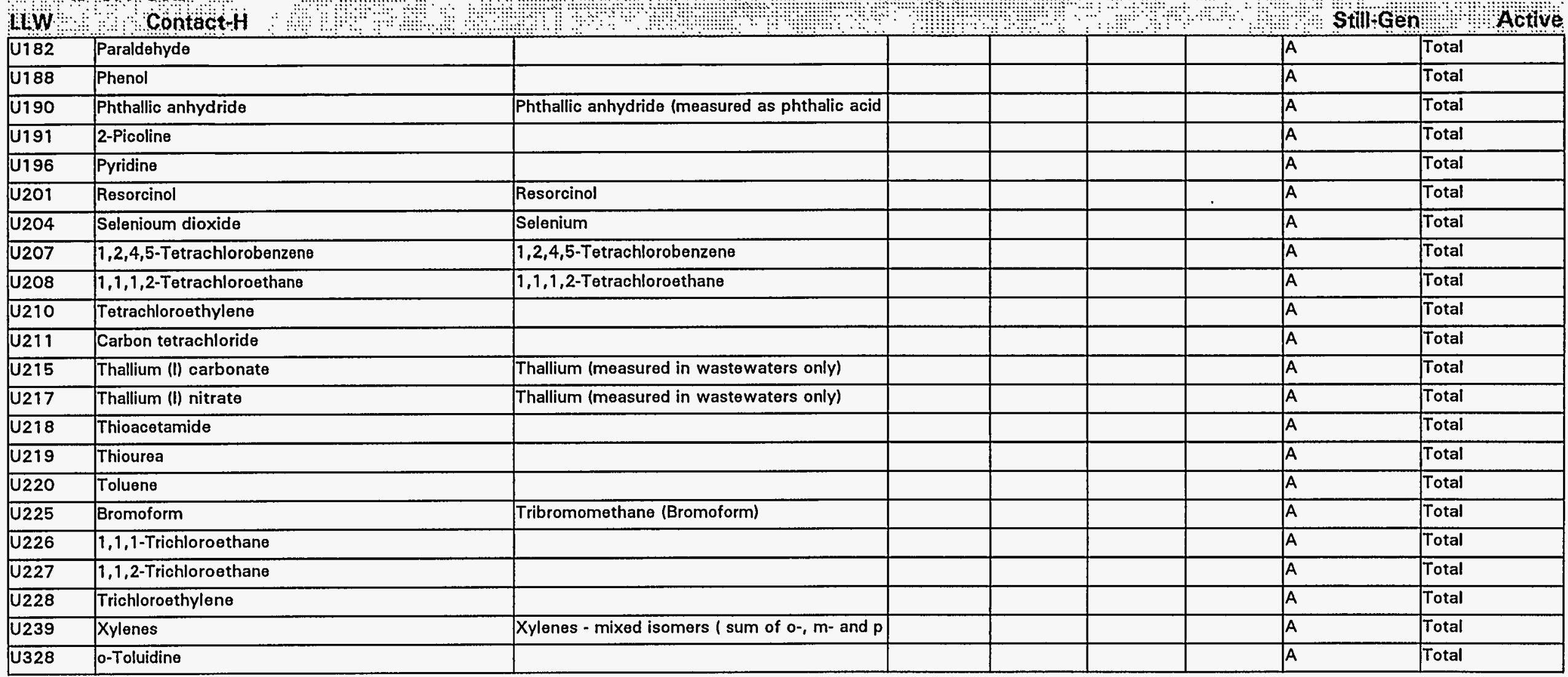


K. POLYCHLORINATED BIPHENYLS (PCBS)
Is this waste stream PCB contaminated?
OYes
No
Unknown

If yes, what portion of the waste is PCB contaminated (volume \%):

If $<100 \%$, can the PCB contaminated waste be segregated and treated separately?

What is the PCB concentration in ppm?

\section{TREATMENT PLANS}

\begin{tabular}{|c|c|c|c|c|c|c|c|c|c|c|c|}
\hline $\begin{array}{c}\text { Opt. } \\
\#\end{array}$ & $\begin{array}{c}\text { Media Type } \\
\text { (If more than one) }\end{array}$ & $\begin{array}{l}\text { Matrix } \\
\text { Code }\end{array}$ & $\begin{array}{l}\text { Vol. \% } \\
\text { lif }\end{array}$ & Step & JIT & SC & CIF & $\begin{array}{l}\text { Trans. } \\
\text { Miles }\end{array}$ & $\begin{array}{l}\text { Facility } \\
\text { Abbr. }\end{array}$ & Unit Name & Comments \\
\hline \multirow[t]{4}{*}{1} & & L.1110 & & $\mathbf{a}$ & 0 & O & $1.00 E+00$ & & AMWTF & $\mathrm{CH}$ - Opening \& Sorting & \\
\hline & & & & b & 0 & 0 & $1.00 E+00$ & & AMWTF & $\mathrm{CH}$ - Incineration/Thermal Desorption & \\
\hline & & & & c & 0 & 0 & $1.00 E+00$ & & AMWTF & $\mathrm{CH}$ - Vitrification & \\
\hline & & & & d & 0 & 0 & $1.00 \mathrm{E}+\mathrm{OO}$ & & SCDF & Disposal - Contact Handled & \\
\hline
\end{tabular}

Note: Where provided, media percentages are rough estimates used to facilitate treatment planning. They do not necessarily imply an accurate knowledge of waste stream composition, and were not derived for use outside this conceptual planning exercise.

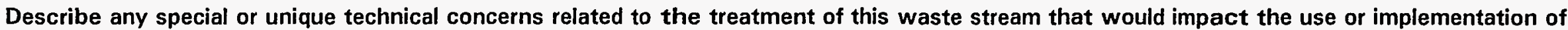
standard hazardous waste treatment methods. 


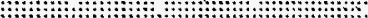

\section{A. IDENTIFICATION AND DESCRIPTION}

Waste Stream ID: ID-CPP-304

W. S. Name: CONTAMINATED DEBRIS

\section{Content Code:}

FFCA ID:

*

IN-W122

\section{Waste Stream Description}

Debris from contruction, maintenance, routine operations, etc. - paper, rubber, metal, bricks,

concrete, plastics, HEPA filter, etc. Can be categorized into combustible, non-porous and porous materials.

\section{B. GENERATION SITE/PROCESS DESCRIPTION}

The following questions, concerning the generation site and process, should be answered even if the waste stream is no longer generated.

Name of Site Generating Waste (e.g., INEL, Rocky Flats, etc.): Idaho National Engineering Laboratory

Location of activities (Area and Building):

Debris from construction, maintenance, and routine operations at the ICPP.

Operations (functions) performed in building:

Description of Process Generating Waste:

Waste stream generated from maintenance, production, and construction. 
LWW RemoterH

\section{PROJECTED WASTE GENERATION}

Is this waste stream still generated?

If yes, when is the expected generation termination date?

\section{OYes ONo}

Current Projections

\begin{tabular}{|r|r|r|r|r|l|}
\hline Est. Date & \multicolumn{1}{|c|}{ Start Year } & Interval & Vol $(\mathbf{m} 3)$ & \multicolumn{1}{|c|}{ Mass $(\mathbf{k g})$} & Source of Estimate \\
\hline $6 / 9 / 94$ & 1994 & 1 & 100.00 & $100,000.00$ & 1994 DSTP \\
\hline $6 / 3 / 94$ & 1995 & 1 & 100.00 & $100,000.00$ & 1994 DSTP \\
\hline $6 / 3 / 94$ & 1996 & 1 & 100.00 & $100,000.00$ & 1994 DSTP \\
\hline $6 / 3 / 94$ & 1997 & 1 & 100.00 & $100,000.00$ & 1994 DSTP \\
\hline $6 / 3 / 94$ & 1998 & 1 & 100.00 & $100,000.00$ & 1994 -DSTP \\
\hline $6 / 3 / 94$ & 1999 & 1 & 100.00 & $100,000.00$ & 1994 DSTP \\
\hline
\end{tabular}

Historical Projections

\begin{tabular}{|c|c|c|c|c|c|}
\hline Est. Date & Start Year & Interval & Vol (m3) & Mass (kg) & Source of Estimate \\
\hline $6 / 3 / 94$ & 2000 & 5 & 500.00 & $500,000.00$ & 1994-DSTP \\
\hline $6 / 3 / 94$ & 2005 & 10 & $1,000,00$ & $1,000,000.00$ & 1994-DSTP \\
\hline $11 / 1 / 93$ & 1993 & 1 & 400.00 & $399,646.20$ & 1993-FFCA \\
\hline $11 / 1 / 93$ & 1994 & 1 & 400.00 & $399,646.20$ & 1993-FFCA \\
\hline $11 / 1 / 93$ & 1995 & 1 & 400.00 & $399,646.20$ & 1993-FFCA \\
\hline $11 / 1 / 93$ & 1996 & 1 & 400.00 & $399,646.20$ & 1993-FFCA \\
\hline $11 / 1 / 93$ & 1997 & 1 & 400.00 & $399,646,20$ & 1993-FFCA \\
\hline 11/1/93 & 1998 & 5 & $2,000.00$ & $1,998,231.00$ & 1993-FFCA \\
\hline $11 / 1 / 93$ & 2003 & 20 & $8,000.00$ & $7,992,924.00$ & 1993-FFCA \\
\hline $12 / 1 / 92$ & 1992 & 5 & $2,000.00$ & $1,998,231.00$ & 1992-FFCA \\
\hline
\end{tabular}




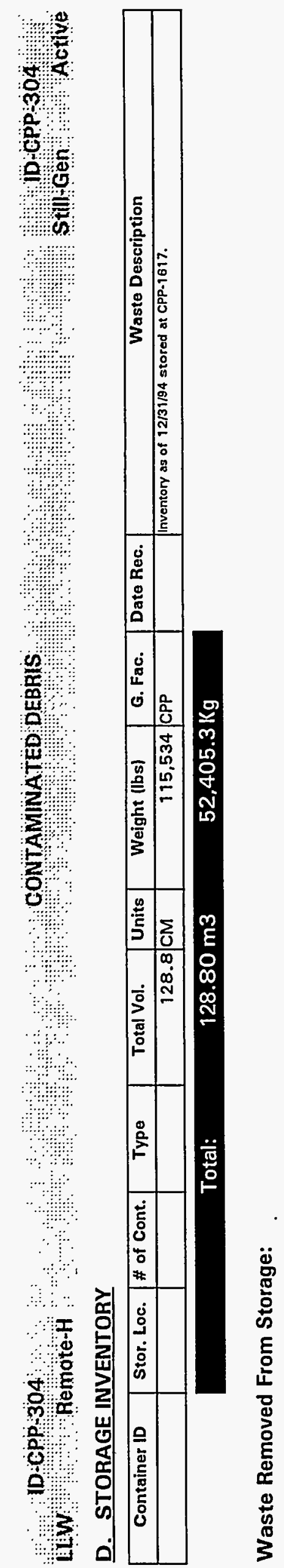




\section{ID.CPP.304 \\ LiW RemoterH \\ E. RADIATION CHARACTERISTICS}

Radioactive Waste Type:

\begin{tabular}{|ll|}
\hline OlLW & Oallw $\bigcirc$ TRU \\
OHLW & O Non-Rad \\
\hline
\end{tabular}

Mixed Waste:

Handling:

\begin{tabular}{|l|}
\hline OYes O No \\
\hline OContact \\
\hline
\end{tabular}

Radionuclide Contamination Accessiblity:

\begin{tabular}{rlrl|} 
External Surface & OYes & O No & OUnknown \\
\cline { 3 - 5 } Internal Surface & OYes & O No & OUnknown \\
\cline { 2 - 5 } Dispersed Through Matrix & OYes & O No & OUnknown \\
\cline { 2 - 3 } & &
\end{tabular}

\section{Activity Levels}

Transuranic Alpha Activity: Uranium/Thorium Alpha Activity:

Beta/Gamma Activity:

Surface Neutron Activity:

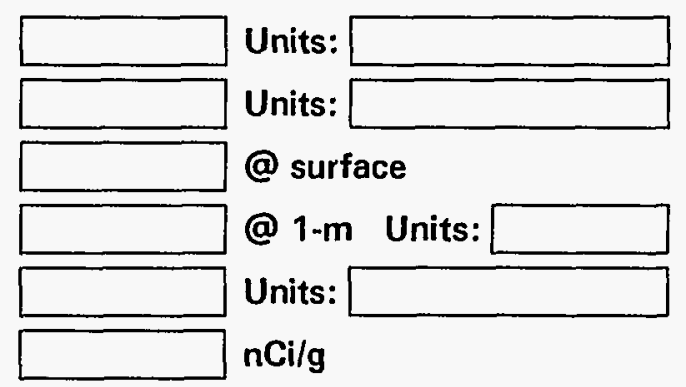

Total Activity: $\mathrm{nCl} / \mathrm{g}$
$10-C P P-304$ Stiil-Gen : Active

\section{F. TRU ALPHA ACTIVITY DISTRIBUTION}

This waste stream is not identified as a TRU or a-LLW. 


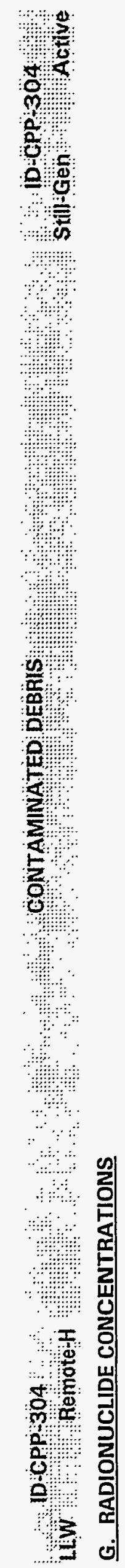




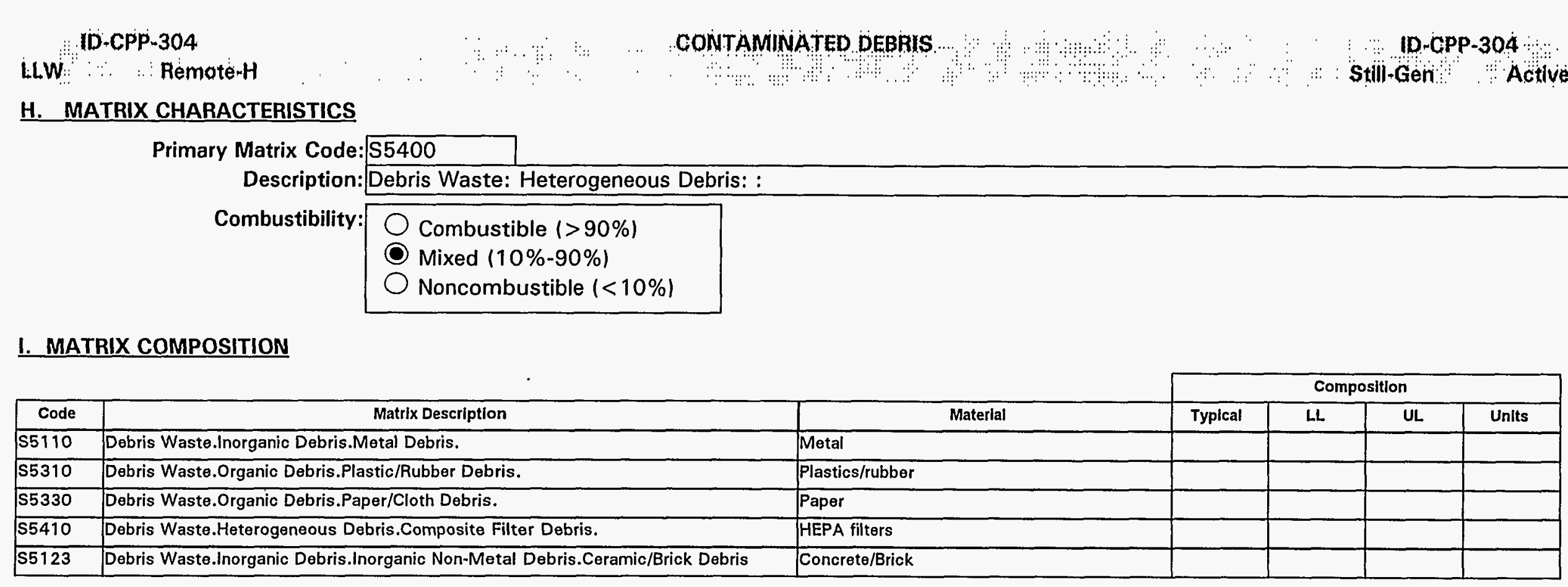




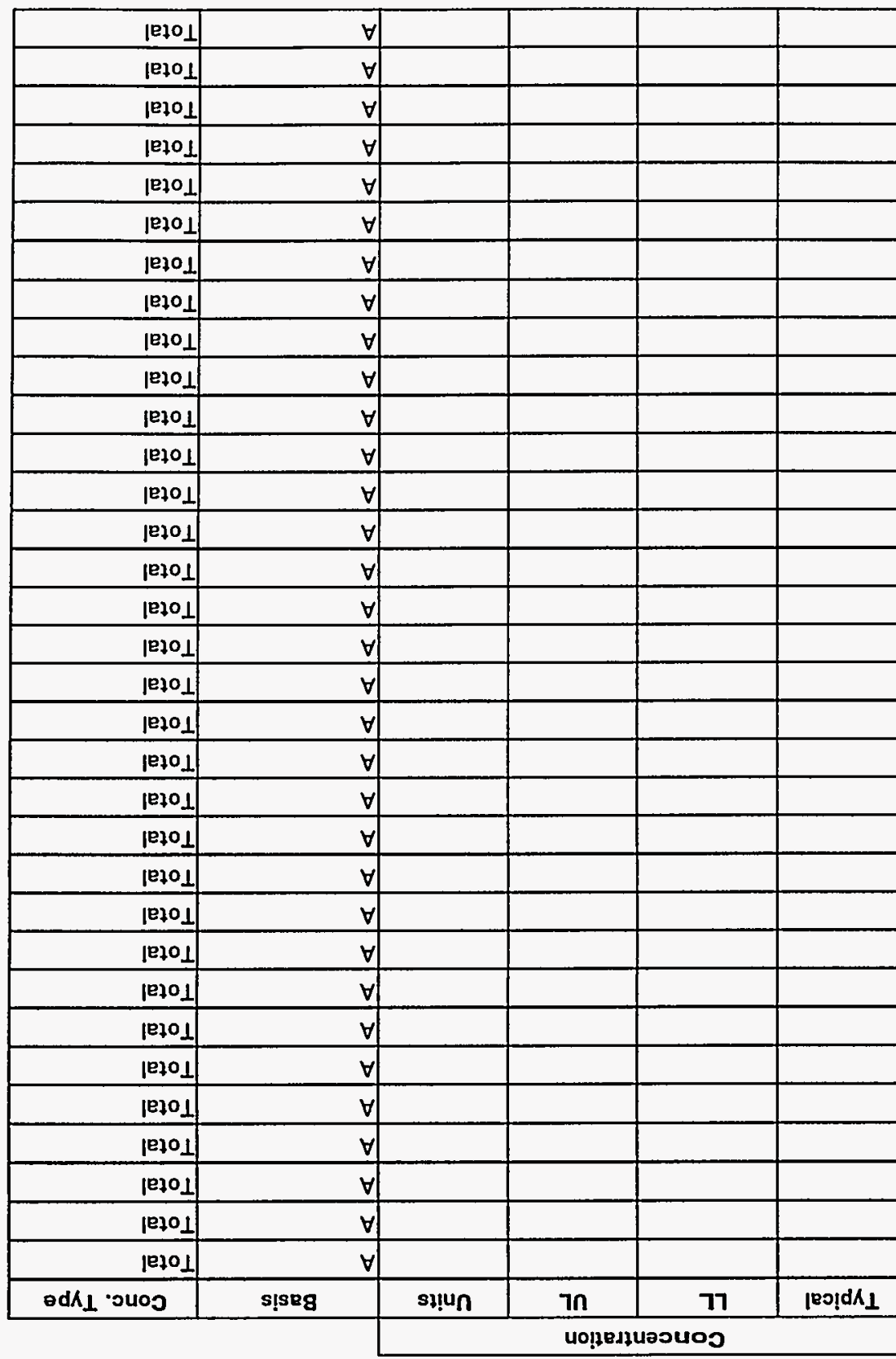

\begin{tabular}{|c|c|c|}
\hline 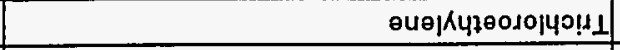 & sjuesjos pejeuebojey jueds & 200- \\
\hline 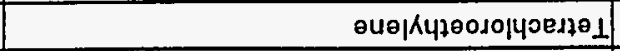 & słuesjos pejeue6ojey zueds & 200 \\
\hline 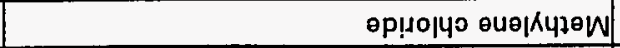 & sluespos peleue6ojey jueds & $200 \mathrm{3}$ \\
\hline өuezuequdojuว & sjuenjos pajeuéojey jueds & $200 \pm$ \\
\hline 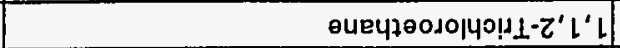 & słuesjos peleuebo|ey jueds & 2007 \\
\hline 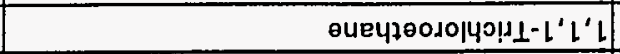 & słuenjos pełeue6ojey queds & 200 \\
\hline өuezueqosolyग!a-0 & squenjos pezeuebojey zueds & 200 \\
\hline 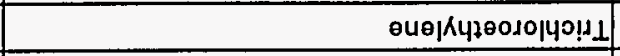 & Bu!seed6ep u! pesn słueл|os pe\}euebojey quods & $100 \mathrm{H}$ \\
\hline 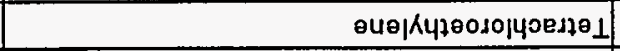 & Gu!seed6ep u! pesn słuesjos pejeuebojey łueds & 100 \\
\hline 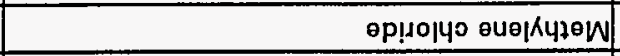 & Gu!seed6ep u! pesn siuenjos peleue6ojey queds & $100 \mathrm{H}$ \\
\hline 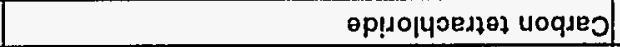 & 6u!seed6ep u! pesn słuesjos pejeuebojey lueds & $100 \mathrm{H}$ \\
\hline өue4ว & 6u!sees6ep u! pesn sfuesjos pejeue6ojey queds & $100 \pm$ \\
\hline 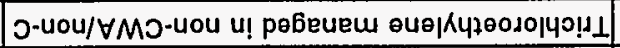 & 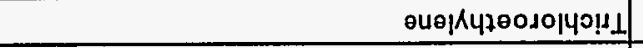 & otod \\
\hline 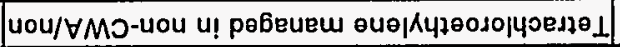 & 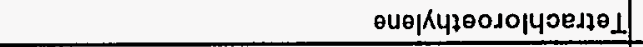 & $6 \varepsilon 00$ \\
\hline 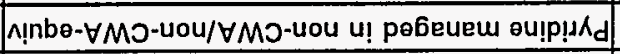 & ou!p! $! \wedge_{d}$ & $8 \varepsilon 0$ \\
\hline - $\forall M O-$-uou/ $\forall M O$-uou u! pebeueu өuezueqod!!N & euezueqod!!N & $9 \varepsilon 00$ \\
\hline 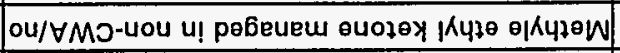 & 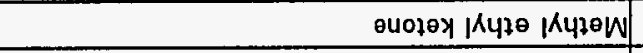 & sqoo \\
\hline 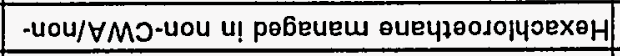 & 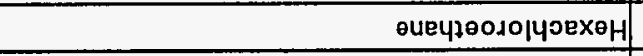 & $t \varepsilon 00$ \\
\hline uou/ $\forall M O$-uou u! pebeueu enezueqosolyoexeH & өuezueqosolyoexeH & $z \varepsilon 00$ \\
\hline 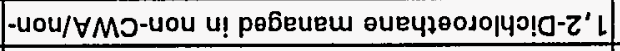 & 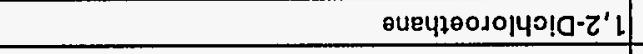 & 8200 \\
\hline 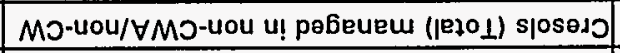 & 10sedo & 9200 \\
\hline$\theta-\forall M O-$-uou/ $\forall M O$-uou u! pe6eueu usojodo|บว & ш10,0د0140 & 2200 \\
\hline MO-uou/ $\forall M O-$-uou u! pe6eueu әuezueqodolyว & enezueqojoluo & 1200 \\
\hline 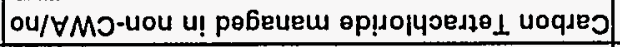 & 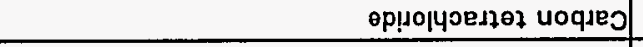 & 6100 \\
\hline \multirow[t]{3}{*}{ !nbe- $\forall M O$-uou/ $\forall M O$-uou u! pebeueu euezueg } & ouezueg & 8100 \\
\hline & JөN!!S & 1.100 \\
\hline & 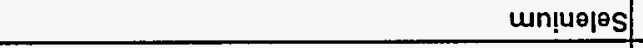 & 0100 \\
\hline \multirow[t]{2}{*}{ 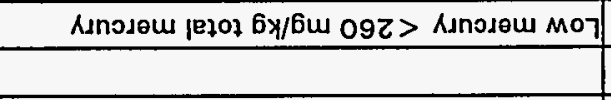 } & Aunosow & 6000 \\
\hline & peo7 & 8000 \\
\hline \multirow[t]{4}{*}{ 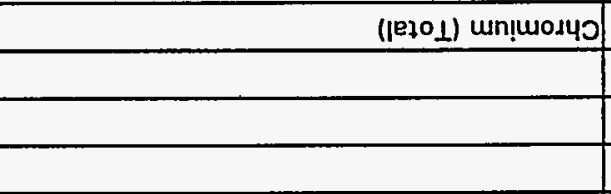 } & un!̣uosyo & $\angle 000$ \\
\hline & un!̣upes & 9000 \\
\hline & un!ıeg & 9000 \\
\hline & ग!uәsı & $\downarrow 000$ \\
\hline 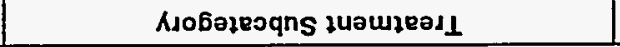 & 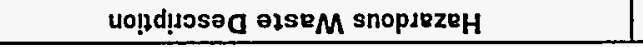 & өpos \\
\hline
\end{tabular}

SINERIIISNOJ SNOQY甘VZVH T 
Lliw:

\section{Remote-H}

Spent non-halogenated solvents Spent non-halogenated solvents

Spent non-halogenated solvents Spent non-halogenated solvents Spent non-halogenated solvents Spent non-halogenated solvents Spent non-halogenated solvents

Allyl alcohol

Arsenic trioxide

Carbon disulfide

p-Chloroaniline

3-Chlaropropionitrile Benzyl chloride

Cyanides (Soluble salts and complexes)

Cyanogen

Fluorine

Nickel carbonyl

Nicotine and Salts

p-Nitroaniline

Potassium cyanide

Silver cyanide

Sodium azide

Sodium cyanide

Thallic oxide

Thiosemicarbazide

Ammonium vanadate

Vanadium pentoxide

Acetonitrile

Acetophenone

Acrylamide

Acrylonitrile

Aniline

Auramine

Benzene

Benzenesulfonyl chloride

Calcium chromate

Chlorobenzene

\section{GONTAMINATED DEBRIS}

StillGen Active

2-Nitropropane

Benzene

Carbon disulfide

isobutyl alcohol

Methyl ethyl ketone

Pyridine

Toluene

1

\section{Cyanides (Total)}

Fluoride (measured in wastewaters only) Nickel

p-Nint

p-Nitroaniline

Cyanide (Total)

Cyanide (Total)

Cyanide (Total)

Thallium (measured in wastewaters only)

Vanadium (measured in wastewaters only)

Vanadium (measured in wastewaters only)

Acetophenone

\begin{tabular}{|l|l|l|l|l|l|}
\hline Acetophenone & & & & & A \\
\hline Acrylonitrile & & & & & A \\
\hline & & & & & A \\
\hline & & & & & \\
\hline & & & & & A \\
\hline Chromium (total) & & & & & A \\
\hline Chlorobenzene & & & & & A \\
\hline
\end{tabular}

Waste Stream Profile Report - Idaho Mixed Waste Information (IMWII) System

\begin{tabular}{|l|l|}
\hline A S Stll Gen & Total \\
\hline A A tive \\
\hline A & Total \\
\hline A & Total \\
\hline A & Total \\
\hline A & Total \\
\hline A & Total \\
\hline A & Total \\
\hline A & Total \\
\hline A & Total \\
\hline A & Total \\
\hline A & Total \\
\hline A & Total \\
\hline A & Total \\
\hline A & Total \\
\hline A & Total \\
\hline A & Total \\
\hline A & Total \\
\hline A & Total \\
\hline A & Total \\
\hline A & Total \\
\hline A & Total \\
\hline A & Total \\
\hline A & Total \\
\hline A & Total \\
\hline A & Total \\
\hline A & Total \\
\hline A & Total \\
\hline A & Total \\
\hline A & Total \\
\hline A & Total \\
\hline A & Total \\
\hline A & Total \\
\hline A & Total \\
\hline A & Total \\
\hline A & Total \\
\hline A & Total \\
\hline A & Total \\
\hline
\end{tabular}

Page 4-30 
ID CPP 304

GONTAMINATED DEBRIS

iD CPP 304

Hiw

Remote-

Stili-gen n Active

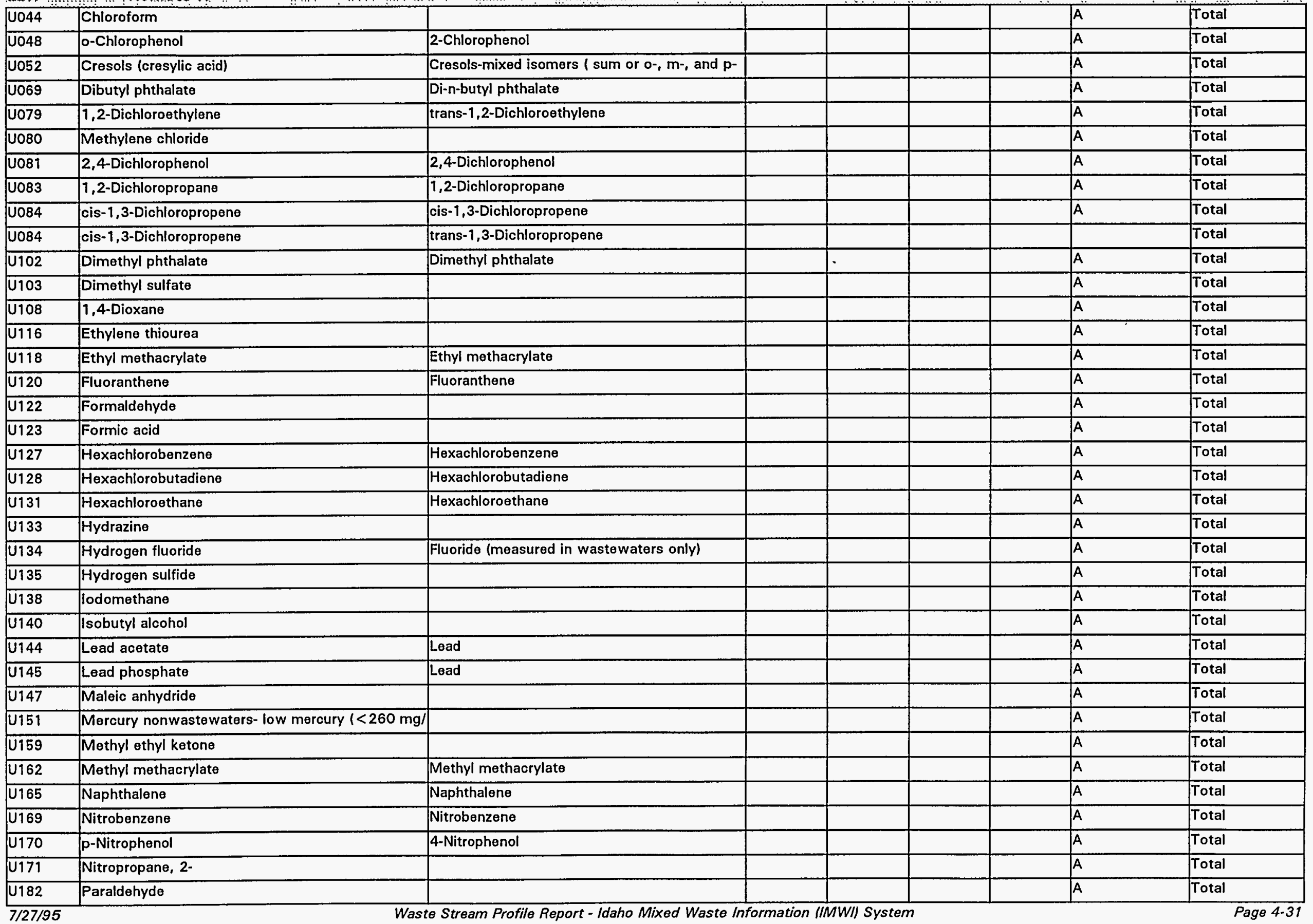




\section{ID.CPP.304}

CONTAMINATED DEBRIS

1D $\mathrm{CPP}: 304$

LLW

RemöterH

CONTAMINATED DEBAIS

U188 Phon

\begin{tabular}{l|l}
\hline U190 Phthallic anhydrid \\
\hline
\end{tabular}

U191 2-Picoline

\begin{tabular}{|l|l|}
\hline U196 & Pyridine \\
\hline
\end{tabular}

\begin{tabular}{|l|l}
\hline U201 Resorcinol \\
\hline
\end{tabular}

\begin{tabular}{|l|l}
\hline U204 Selenioum dioxide \\
\hline
\end{tabular}

U207

U208

1,2,4,5-Tetrachlorobenzene

1,1,1,2-Tetrachloroethane

Tetrachloroethylene

U211

Carbon tetrachloride

Thallium (I) carbonate

Thallium (I) nitrate

Thioacetamide

Thiourea

Toluene

Bromoform

1,1,1-Trichloroethan $\theta$

1,1,2-Trichloroethane

Trichloroethylene

Xylenes

\begin{tabular}{l|l}
\hline U328 & o-Toluidine \\
\hline
\end{tabular}

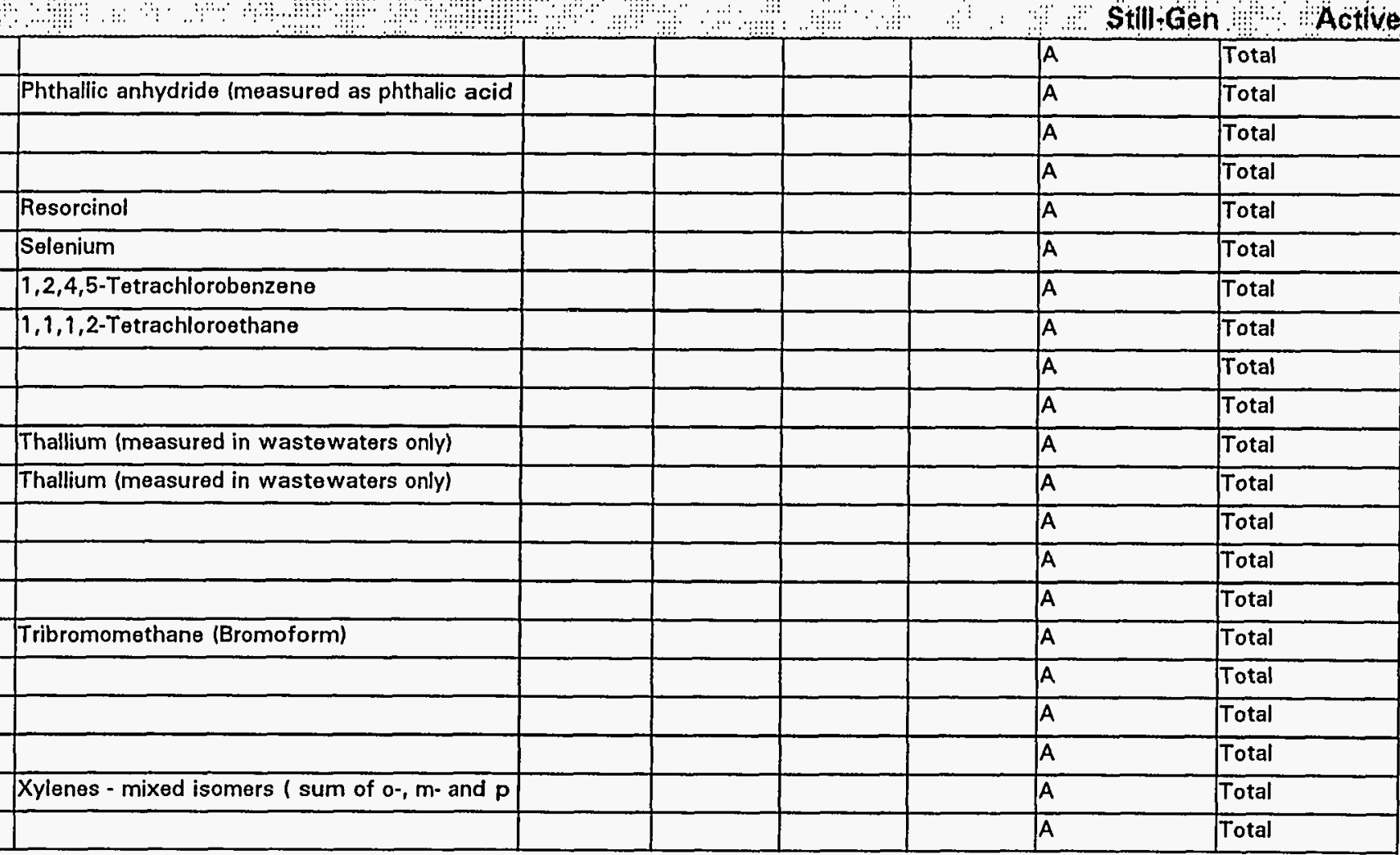


ID-CPp 304

\section{K. POLYCHLORINATED BIPHENYLS (PCBS)}

Is this waste stream PCB contaminated? OYes $O$ No $O$ Unknown

If yes, what portion of the waste is PCB contaminated (volume \%):

If $<100 \%$, can the PCB contaminated waste be segregated and treated separately? $O$ Yes ONo O Unknown

What is the PCB concentration in ppm?

If only a portion of the waste is PCB contaminated, report values for contaminated portion.

\section{TREATMENT PLANS}

\begin{tabular}{|c|c|c|c|c|c|c|c|c|c|c|c|}
\hline $\begin{array}{c}\text { Opt. } \\
\#\end{array}$ & $\begin{array}{c}\text { Media Type } \\
\text { (If more than one) }\end{array}$ & $\begin{array}{c}\text { Matrix } \\
\text { Code }\end{array}$ & $\begin{array}{l}\text { Vol. \% } \\
\text { (If }\end{array}$ & Step & JiT & sc & CIF & $\begin{array}{l}\text { Trans. } \\
\text { Miles }\end{array}$ & $\begin{array}{l}\text { Facility } \\
\text { Abbr. }\end{array}$ & Unit Name & Comments \\
\hline \multirow[t]{19}{*}{1} & $\mathrm{CH}$-porous-debris & S5120 & 59.000 & $\mathbf{a}$ & 0 & 0 & $1.00 \mathrm{E}+00$ & & CPP659 & Segregation & \\
\hline & & & & b & $O$ & 0 & $1.00 E+00$ & & AMWTF & CH-Opening \& Sorting & \\
\hline & & & & c & 0 & 0 & $1.00 \mathrm{E}+00$ & & AMWTF & $\mathrm{CH}$ - Sizing & \\
\hline & & & & $\bar{d}$ & 0 & 0 & $1.00 E+00$ & & AMWTF & CH - Incineration/Thermal Desorption & \\
\hline & & & & e & 0 & 0 & $1.00 E+00$ & & AMWTF & $\mathrm{CH}$ - Vitrification & \\
\hline & & & & $\bar{f}$ & $O$ & 0 & $1.00 E+00$ & & SCDF & Disposal - Contact Handled & \\
\hline & RH-comb/porous-debris & $\mathbf{S 5 3 0 0}$ & 20.000 & $\bar{a}$ & 0 & 0 & $1.00 E+00$ & & CPP659 & Segregation & \\
\hline & & & & b & 0 & 0 & $1.00 E+00$ & & AMWTF & RH-Opening \& Sorting & \\
\hline & & & & c & $O$ & 0 & $1.00 E+00$ & & AMWTF & RH - Sizing & \\
\hline & & & & d & 0 & 0 & $1.00 \mathrm{E}+00$ & & AMWTF & RH - Incineration/Thermal Desorption & \\
\hline & & & & e & 0 & 0 & $1.00 E+00$ & & AMWTF & RH - Vitrification & \\
\hline & & & & $\bar{f}$ & 0 & 0 & $1.00 \mathrm{E}+00$ & & SCDF & Disposal - Remote Handled & \\
\hline & $\mathrm{CH}$-combustibles & $\$ 5300$ & 16.000 & $\mathbf{a}$ & 0 & 0 & $1.00 E+00$ & & CPP659 & Segregation & \\
\hline & & & & $\bar{c}$ & $\mathrm{O}$ & 0 & $1.00 E+00$ & & WERF & Incineration - Controlled Air Incinerator & \\
\hline & & & & d & $\bar{O}$ & 0 & $1.00 \mathrm{E}+00$ & & WERF & Stabilization - Portland Cement & \\
\hline & & & & e & $\mathrm{O}$ & $\bar{O}$ & $1.00 E+00$ & & SCDF & Disposal - Contact Handled & \\
\hline & $\mathrm{CH} / \mathrm{RH}$-nonporous debri & $\$ 5400$ & 5.000 & $\bar{a}$ & 0 & 0 & $1.00 E+00$ & & CPP659 & Segregation & \\
\hline & & & & $\mathbf{b}$ & O & 0 & $1.00 E+00$ & & CPP659 & Decontamination - Water Washing System & \\
\hline & & & & c & 0 & 0 & $1.00 \mathrm{E}+00$ & & RWMC & Disposal - Contact-Handled & \\
\hline
\end{tabular}

Note: Where provided, media percentages are rough estimates used to facilitate treatment planning. They do not necessarily imply an accurate knowledge of waste stream composition, and were not derived for use outside this conceptual planning exercise.

Describe any special or unique technical concerns related to the treatment of this waste stream that would impact the use or implementation of standard hazardous waste treatment methods. 


\section{A. IDENTIFICATION AND DESCRIPTION}

Waste Stream ID: ID-CPP-504

W. S. Name: NON-DEBRIS SOLIDS

Content Code:

FFCA ID: $\quad$ IN-N381

\section{Waste Stream Description:}

Consists of materials $<60 \mathrm{~mm}$ that are generated from construction, maintenance, etc. Includes broken glass, kitty litter, concrete chips and floor sweepings.

\section{B. GENERATION SITE/PROCESS DESCRIPTION}

The following questions, concerning the generation site and process, should be answered even if the waste stream is no longer generated.

Name of Site Generating Waste (e.g., INEL, Rocky Flats, etc.): Idaho National Engineering Laboratory

Location of activities (Area and Building): CPP

Operations (functions) performed in building:

Description of Process Generating Waste:

Waste stream generated from construction and maintenance activities. 


\section{PROJECTED WASTE GENERATION}

Is this waste stream still generated?

If yes, when is the expected generation termination date?

Current Projections

\begin{tabular}{|r|r|r|r|r|l|}
\hline Est. Date & Start Year & \multicolumn{1}{|c|}{ Interval } & \multicolumn{1}{|c|}{ Vol $(\mathrm{m} 3)$} & Mass $(\mathrm{kg})$ & Source of Estlmate \\
\hline $6 / 9 / 94$ & 1995 & 1 & 1.56 & $1,554.00$ & 1994 DSTP \\
\hline $6 / 9 / 94$ & 1996 & 1 & 1.56 & $1,554.00$ & $1994-\mathrm{DSTP}$ \\
\hline $6 / 9 / 94$ & 1997 & 1 & 1.50 & $1,500.00$ & 1994 DSTP \\
\hline $6 / 9 / 94$ & 1998 & 1 & 1.56 & $1,554.00$ & $1994-D S T P$ \\
\hline $6 / 9 / 94$ & 1999 & 1 & 1.56 & $1,554.00$ & 1994 DSTP \\
\hline
\end{tabular}

\section{Historical Projections}

\begin{tabular}{|r|r|r|r|r|l|}
\hline Est. Date & \multicolumn{1}{|c|}{ Start Year } & Interval & \multicolumn{1}{|c|}{ Vol $(\mathrm{m} 3)$} & \multicolumn{1}{c|}{ Mass $(\mathrm{kg})$} & \multicolumn{1}{c|}{ Source of Estimate } \\
\hline $6 / 9 / 94$ & 2000 & 5 & 7.74 & $7,716.00$ & 1994 DSTP \\
\hline $6 / 9 / 94$ & 2005 & 10 & 15.48 & $15,432.00$ & 1994 DSTP \\
\hline
\end{tabular}




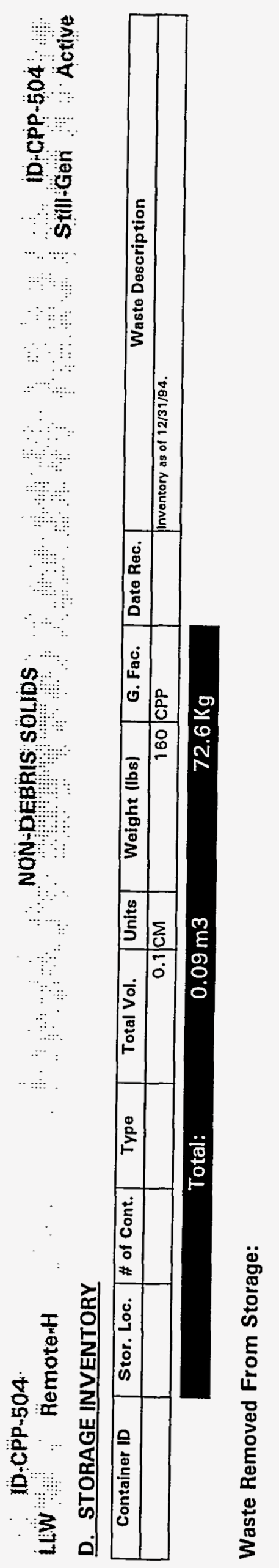




\section{E. RADIATION CHARACTERISTICS}

Radioactive Waste Type:

\begin{tabular}{|ll|}
\hline O lLW & O a-llw O tRU \\
OhLW & O Non-Rad \\
\hline
\end{tabular}

Mixed Waste:

Handling:

\begin{tabular}{|l|}
\hline OYes O No \\
\hline OContact \\
\hline
\end{tabular}

Radionuclide Contamination Accessiblity:

External Surface

Internal Surface

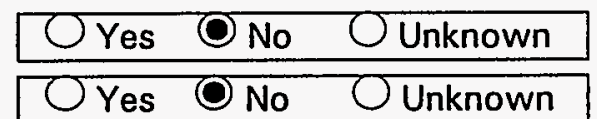

Dispersed Through Matrix

OYes O No OUnknown

\section{Activity Levels}

Transuranic Alpha Activity:

Uranium/Thorium Alpha Activity:

Beta/Gamma Activity:

Surface Neutron Activity:

Total Activity:

\begin{tabular}{l}
\hline \\
\hline \\
\hline \\
Units: \\
\hline \\
@ @ surface \\
\hline \\
@ 1-m Units: $\square$ \\
Units: $\square$ \\
nCi/g
\end{tabular}

\section{F. TRU ALPHA ACTIVITY DISTRIBUTION}

This waste stream is not identified as a TRU or a-LLW. 


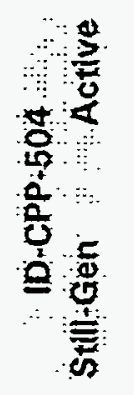




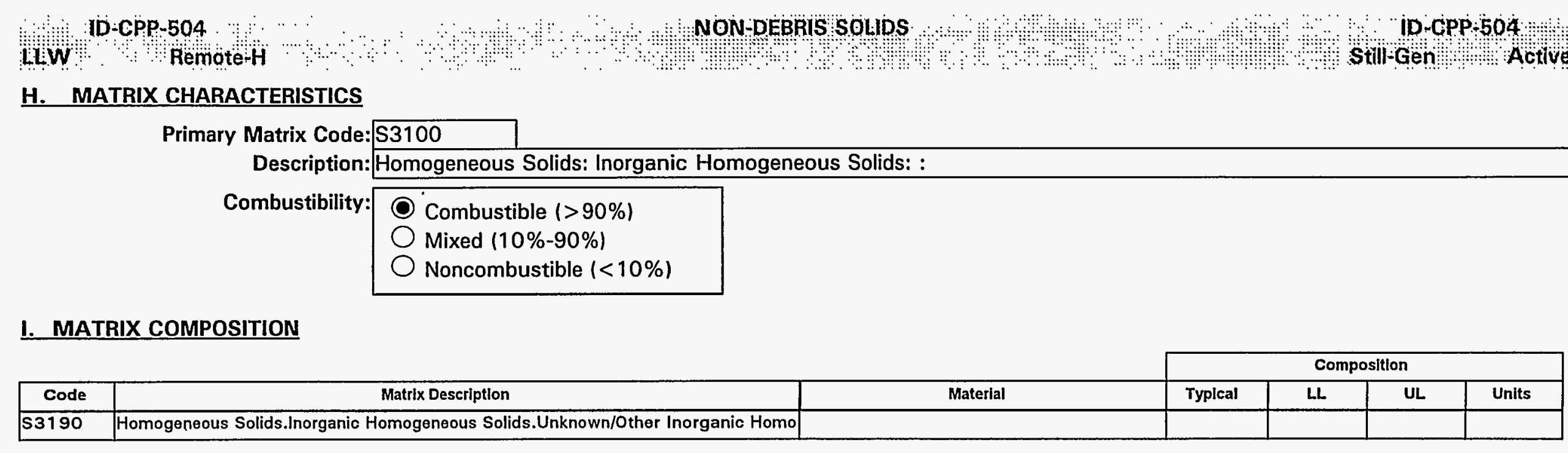




\begin{tabular}{|c|c|c|c|c|c|c|c|c|}
\hline piol & $\forall$ & & & & & 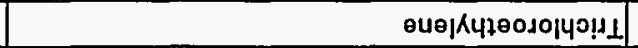 & sjuenjos pejeue6ojey jueds & 2001 \\
\hline ploL & $\forall$ & & & & & 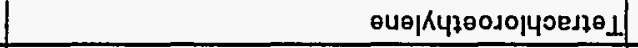 & siuenjos pejeuebojey jueds & $200 \mathrm{~J}$ \\
\hline [EłO] & $\forall$ & & & & & өр!10|чо өนөןкцдоW & słuenjos pejeuebojey jueds & 2001 \\
\hline |etol & $\forall$ & & & & & өuezueqojo|40 & sfuenjos pejeuebojey jueds & 2001 \\
\hline |etol & $\forall$ & & & & & 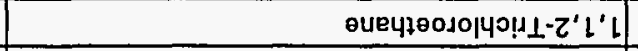 & słuenjos pojeue6ojey jueds & 2005 \\
\hline 1EłOL & $\forall$ & & & & & өueपว & słuenjos pejeue6ojey jueds & 200J \\
\hline 18101 & $\forall$ & & & & & өuezuəqoso|บэ!a-0 & słuenjos pejeuebojey jueds & $200 \pm$ \\
\hline jejoL & $\forall$ & & & & & 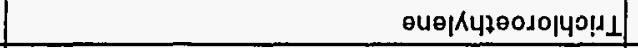 & Gu!seadbep u! pesn słuenjos pejeuebojey jueds & 1001 \\
\hline 1eto & $\forall$ & & & & & 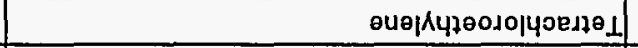 & Бu! seod6ep u! pesn słuenjos pejeuebo|eч jueds & LOOJ \\
\hline 1ejol & $\forall$ & & & & & 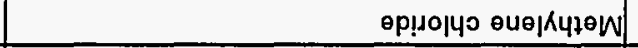 & bu!seedbep u! pesn słuenjos pejeuebojey jueds & $100 \pm$ \\
\hline piol & $\forall$ & & & & & өp!10|чоestel uoqreo & 6u!seasbep u! posn słuenjos pejeuebojey jueds & 100J \\
\hline 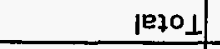 & $\forall$ & & & & & จนеप1 & Gu!seadbep u! pesn słuenjos pejeueboley jueds & 1001 \\
\hline |EłOL & $\forall$ & & & & & 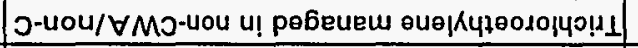 & 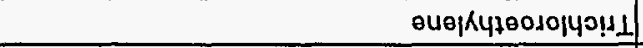 & $0+00$ \\
\hline 1ejol & $\forall$ & & & & & 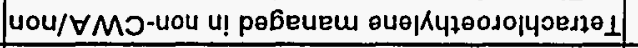 & 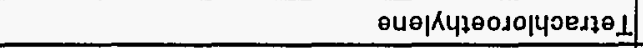 & $6 \varepsilon 00$ \\
\hline plol & $\forall$ & & & & & A!nbe- $\forall M$ M-uou/ $\forall M O$-uou u! pebeueu eu!p!s $\Lambda_{d}$ & ou!p!n $\Lambda_{d}$ & $8 \varepsilon 00$ \\
\hline 1etol & $\forall$ & & & & & $-\forall M O-$-uou/ $\forall M O$-uou u! pe6euru euezuaqoגi!N & ouezueqodz!n & $9 \varepsilon 00$ \\
\hline 18701 & $\forall$ & & & & & 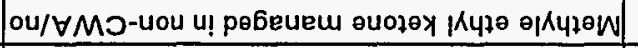 & 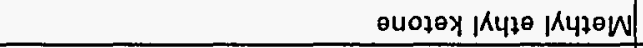 & gعo0 \\
\hline pło & $\forall$ & & & & & 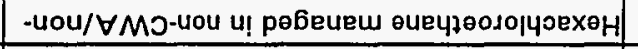 & 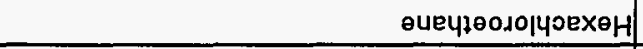 & $\nabla \varepsilon 00$ \\
\hline jeło上 & $\forall$ & & & & & 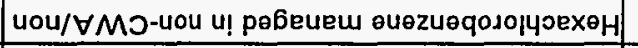 & өuezueqosolyoexeH & $2 \varepsilon 00$ \\
\hline ןlelo & $\forall$ & & & & & 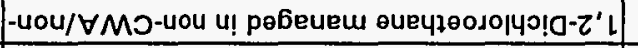 & 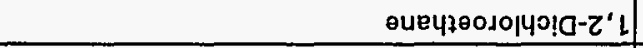 & 8200 \\
\hline 1ejol & $\forall$ & & & & & 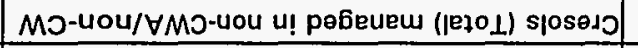 & josesp & 9200 \\
\hline 1e101 & $\forall$ & & & & & 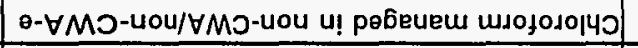 & usојодоцว & 2200 \\
\hline jejo上 & $\forall$ & & & & & MO-uou/ $\forall M O$-uou u! pebeueu euezueq010140 & өuezueqosolu० & 1200 \\
\hline fejol & $\forall$ & & & & & 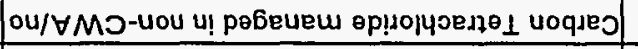 & 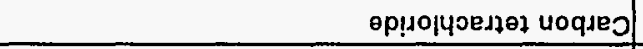 & 6100 \\
\hline 1ejol| & $\forall$ & & & & & !nbe- $\forall M O$-uou/ $\forall M O$-uou u! pe6euem өuezueg & өuezueg & 8100 \\
\hline $1810_{1}$ & $\forall$ & & & & & & دөл!!S & 1100 \\
\hline 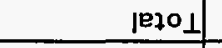 & $\forall$ & & & & & & un!̣uejes & 0100 \\
\hline peto & $\forall$ & & & & & 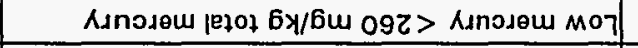 & Annosow & 6000 \\
\hline 1ejo1 & $\forall$ & & & & & & peo] & 8000 \\
\hline |ejol & $\forall$ & & & & & (1Е101) un!mod40 & un!modū & 2000 \\
\hline [e101) & $\forall$ & & & & & & un!̣upeo & 9000 \\
\hline 1ejo1 & $\forall$ & & & & & & un!ıeg & 9000 \\
\hline |eło1 & $\forall$ & & & & & & ọ!uesı $\forall$ & 6000 \\
\hline$\theta \mathrm{dk}_{1} \cdot$ ouos & s!̣seg & sı!un & $7 n$ & 77 & peo!d $\Lambda_{\perp}$ & Alobejeogns zueurzed1 & uo!̣d!̣ıseg elsem snopsezeH & өpoj \\
\hline
\end{tabular}

SINGRIIISNOJ SNOAYZZVH T

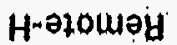

tosindda-al 


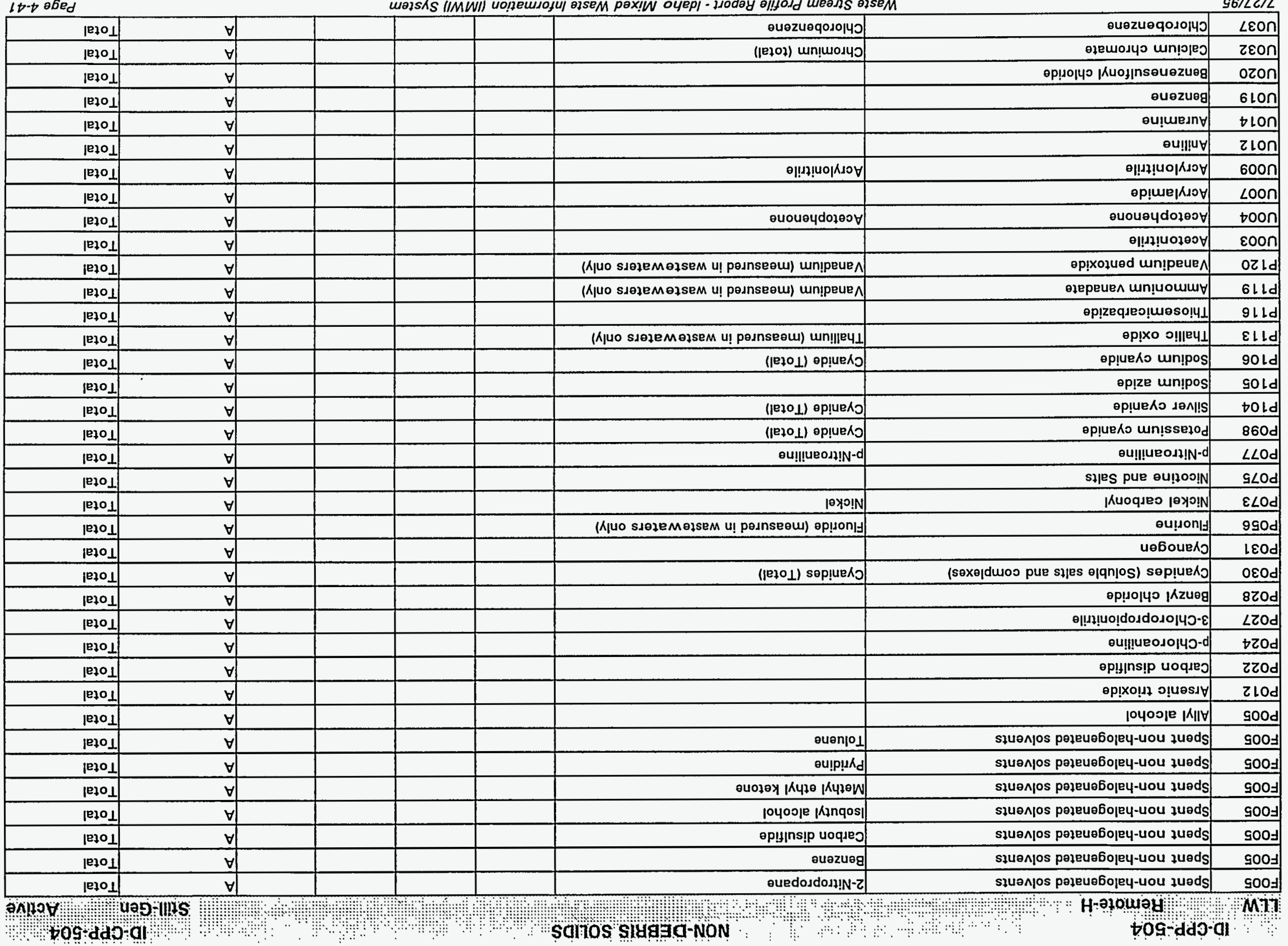


10 CPP- 504

LiL:W

\begin{tabular}{l|l}
\hline U048 & o-Chlorophenol
\end{tabular}

\begin{tabular}{|l|l}
\hline U052 & Cresols (cresylic acid) \\
\hline
\end{tabular}

\begin{tabular}{|l|l}
\hline U069 Dibutyl phthalate \\
\hline
\end{tabular}

\begin{tabular}{|l|l}
\hline U079 & 1,2-Dichloroethylene \\
\hline
\end{tabular}

\begin{tabular}{|l|l}
\hline U080 & Methylene chloride \\
\hline
\end{tabular}

\begin{tabular}{l|l}
\hline U081 2,4-Dichlorophenol \\
\hline UO83
\end{tabular}

\begin{tabular}{ll} 
U083 1,2-Dichloropropane \\
\hline
\end{tabular}

U084 cis-1,3-Dichloropropene

\begin{tabular}{l|l}
\hline U084 cis-1,3-Dichloropropene \\
\hline U102
\end{tabular}

\begin{tabular}{l|l}
\hline U102 & Dimethyl phthalate
\end{tabular}

U103 Dimethyl sulfate

U108

U116

U118

U120

U122

U123

U127

U128

U131

U133

U134

U135

U138

1140

U144

U145

U147

U151

U159

U162

U165

U169

U170

U171

U182

$7 / 27 / 95$
1,4-Dioxane

Ethylene thiourea

Ethyl methacrylate

Fluoranthene

Formaldehyde

Formic acid

Hexachlorobenzene

Hexachlorobutadiene

Hexachloroethane

Hydrazine

Hydrogen fluoride

Hydrogen sulfide

lodomethane

Isobutyl alcohol

Lead acetate

Lead phosphate

Maleic anhydride

Mercury nonwastewaters- low mercury $(<260 \mathrm{mg} /$

Methyl ethyl ketone

Methyl methacrylate

Naphthalene

Nitrobenzene

p-Nitrophenol

Nitropropane, 2

Paraldehyde

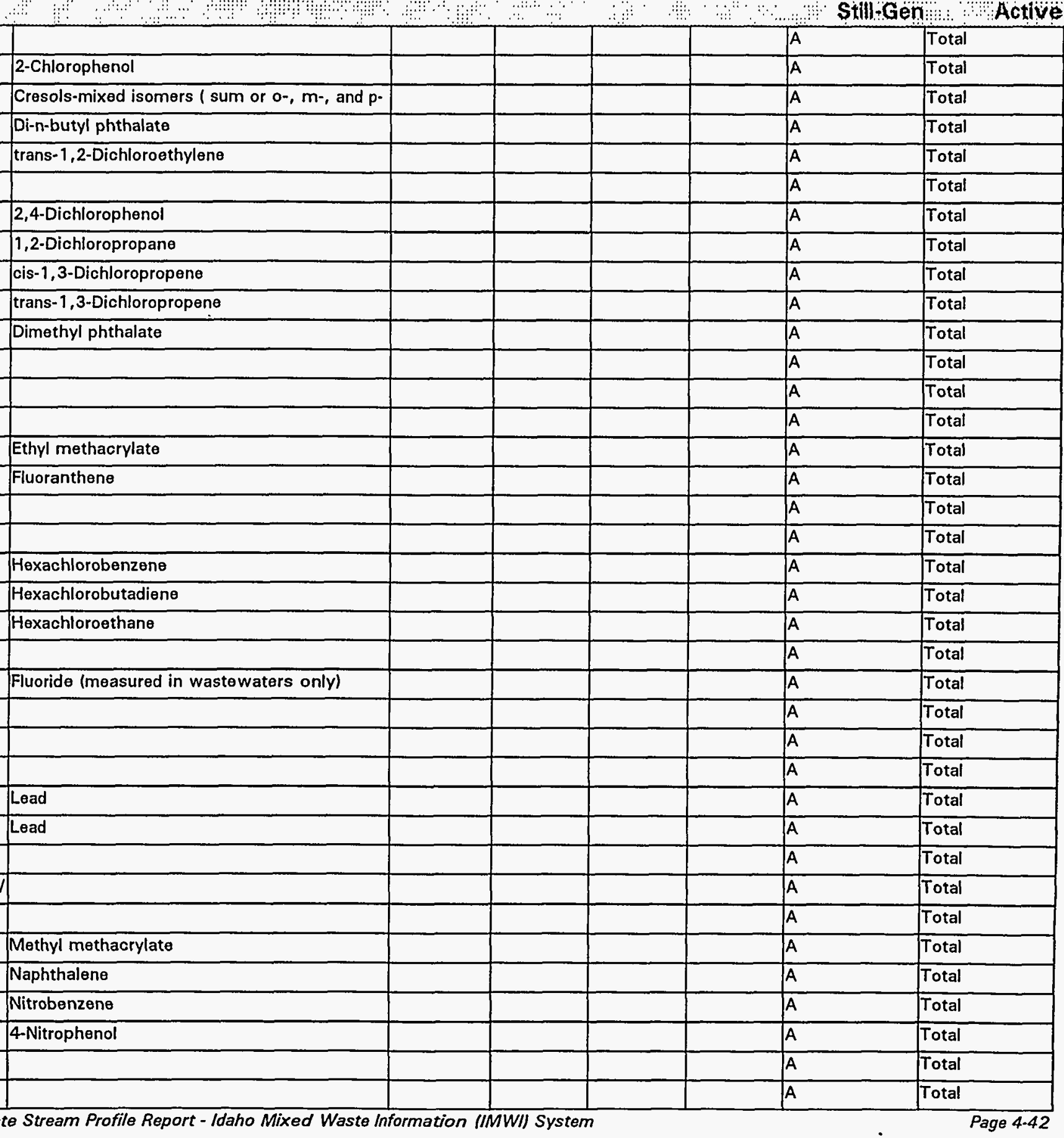


ID-CPP-504

NÖN-DEBRIS SOLIDS

ID

LWW RemóterH

\begin{tabular}{|l|l|}
\hline U188 & Phenol \\
\hline
\end{tabular}

Phthallic anhydride

2-Picoline

\begin{tabular}{|l|l}
\hline U196 & Pyridine \\
\hline
\end{tabular}

\begin{tabular}{|l|l}
\hline U201 & Resorcinol \\
\hline
\end{tabular}

Selenioum dioxide

1,2,4,5-Tetrachlorobenzene

1,1,1,2-Tetrachloroethane

㑭

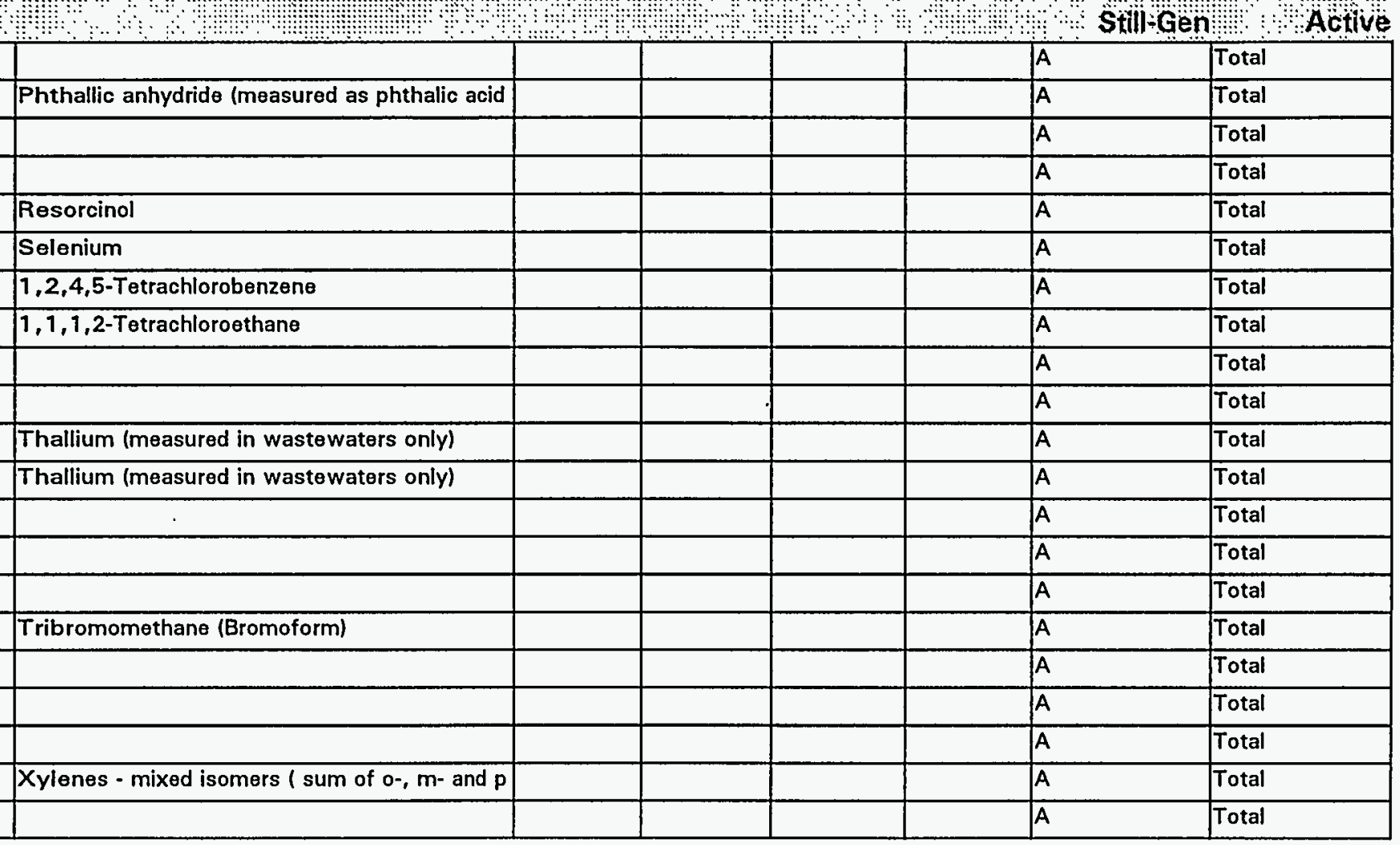




\section{K. POLYCHLORINATED BIPHENYLS (PCBS)}

Is this waste stream PCB contaminated?

OYes O No O Unknown

If yes, what portion of the waste is PCB contaminated (volume \%):

If $<100 \%$, can the PCB contaminated waste be segregated and treated separately?

What is the PCB concentration in ppm?

If only a portion of the waste is $P C B$ contaminated, report values for contaminated portion.

\section{TREATMENT PLANS}

\begin{tabular}{|c|c|c|c|c|c|c|c|c|c|c|c|}
\hline $\begin{array}{c}\text { Opt. } \\
\#\end{array}$ & $\begin{array}{c}\text { Media Type } \\
\text { (If more than one) }\end{array}$ & $\begin{array}{c}\text { Matrix } \\
\text { Code }\end{array}$ & $\begin{array}{l}\text { Vol. \% } \\
\text { (If }\end{array}$ & Step & JIT & sc & CIF & $\begin{array}{l}\text { Trans. } \\
\text { Miles }\end{array}$ & $\begin{array}{l}\text { Facility } \\
\text { Abbr. }\end{array}$ & Unit Name & Comments \\
\hline \multirow[t]{10}{*}{1} & $\mathrm{CH}$ & $\mathbf{5 3 1 0 0}$ & 50.000 & a & (O) & 0 & $1.00 E+00$ & & AMWTF & $\mathrm{CH}$ - Opening \& Sorting & \\
\hline & & & & b & 0 & 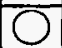 & $1.00 E+00$ & & AMWTF & $\mathrm{CH}$ - Sizing & \\
\hline & & & & c & $\bar{O}$ & D & $1.00 E+00$ & & AMWTF & $\mathrm{CH}$ - Incineration/Thermal Desorption & \\
\hline & & & & d & O & $\bar{O}$ & $1.00 E+00$ & & AMWTF & $\mathrm{CH}$ - Vitrification & \\
\hline & & & & e & 0 & ) & $1.00 E+00$ & & SCDF & Disposal - Contact Handled & \\
\hline & $\overline{\mathrm{RH}}$ & S3100 & 50.000 & $\mathbf{a}$ & (O) & $\bar{O}$ & $1.00 E+00$ & & AMWTF & RH - Opening \& Sorting & \\
\hline & & & & $\mathbf{b}$ & 0 & $\bar{O}$ & $1.00 E+00$ & & AMWTF & RH - Sizing & \\
\hline & & & & c & O & O & $1.00 E+00$ & & AMWTF & RH - Incineration/Thermal Desorption & \\
\hline & & & & d & 0 & $\bar{O}$ & $1.00 E+00$ & & AMWTF & $\mathrm{RH}$ - Vitrification & \\
\hline & & & & e & O & 0 & $1.00 E+00$ & & SCDF & Disposal - Remote Handled & \\
\hline
\end{tabular}

Note: Where provided, media percentages are rough estimates used to facilitate treatment planning. They do not necessarily imply an accurate knowledge of waste stream composition, and were not derived for use outside this conceptual planning exercise.

Describe any special or unique technical concerns related to the treatment of this waste stream that would impact the use or implementation of standard hazardous waste treatment methods. 
$10+1020$

HWW OWO Contact

\section{PROJECTED WASTE GENERATION}

Is this waste stream still generated?

OYes $O$ No

If yes, when is the expected generation termination date?

$12 / 31 / 96$

Current Projections

\begin{tabular}{|r|r|r|r|r|c|}
\hline Est. Date & Start Year & Interval & \multicolumn{1}{|c|}{ Vol (m3) } & Mass (kg) & Source of Estlmate \\
\hline $5 / 23 / 95$ & 1995 & 3 & 0.42 & 416.00 & $1995-M W R$ \\
\hline
\end{tabular}

Historical Projections

\begin{tabular}{|r|r|r|r|r|l|}
\hline \multicolumn{1}{|c|}{ Est. Date } & \multicolumn{1}{|c|}{ Start Year } & \multicolumn{1}{|c|}{ Interval } & \multicolumn{1}{c|}{ Vol (m3) } & \multicolumn{1}{c|}{ Mass $(\mathrm{kg})$} & \multicolumn{1}{c|}{ Source of Estimate } \\
\hline $6 / 1 / 94$ & 1998 & 1 & 0.42 & 416.00 & $1994-\mathrm{DSTP}$ \\
\hline $11 / 1 / 93$ & 1993 & 1 & 0.42 & 416.00 & $1993-F F C A$ \\
\hline $11 / 1 / 93$ & 1994 & 1 & 0.42 & 416.00 & $1993-F F C A$ \\
\hline $11 / 1 / 93$ & 1995 & 1 & 0.42 & 416.00 & $1993-F F C A$ \\
\hline $11 / 1 / 93$ & 1996 & 1 & 0.42 & 416.00 & $1993-F F C A$ \\
\hline $11 / 1 / 93$ & 1997 & 1 & 0.42 & 416.00 & $1993-F F C A$ \\
\hline $12 / 1 / 92$ & 1992 & 5 & 2.10 & $2,080.00$ & $1992-F F C A$ \\
\hline
\end{tabular}




\section{LiWw: Contact-H}

\section{A. IDENTIFICATION AND DESCRIPTION}

Waste Stream ID: ID-INL-220

W. S. Name:

Content Code:

FFCA ID:

\section{ACTIVATED CARBON LLMW}

Waste stream is activated carbon that has become "spent" by absorbing contaminants to capacity and is no longer applicable to regeneration. This waste stream is generated by

treatment of aqueous or gaseous phase waste streams.

\section{B. GENERATION SITE/PROCESS DESCRIPTION}

The following questions, concerning the generation site and process, should be answered even if the waste stream is no longer generated.

Name of Site Generating Waste (e.g., INEL, Rocky Flats, etc.): Idaho National Engineering Laboratory

Location of activities (Area and Building):

PBF, PWTU, and TAN Water Treatment Unit.

Operations (functions) performed in building:

Treatment of aqueous mixed wastes. Ground water pump and treat unit.

Description of Process Generating Waste:

Treatment of aqueous wastes to remove dissolved organic

contaminants. Removal of dissolved and inter mixed organics. 
ID INIL 220

ACTIVATED CAR̈BÖN LLNIW

LiWW Gontact

ACTIVATED CARBON LLMW

\section{STORAGE INVENTORY}

\begin{tabular}{|c|c|c|c|c|c|c|c|c|c|}
\hline Container ID & Stor. Loc. & \# of Cont. & Type & Total Vol. & Units & Weight (lbs) & G. Fac. & Date Rec. & Waste Description \\
\hline INEL4759A & MWSF & & DF & 90.0 & GAL & 480 & TAN/ERP & $3 / 27 / 95$ & ACTIVATED CARBON FILTER MEDIA WITCE AND PCE \\
\hline \multicolumn{10}{|c|}{ Total: } \\
\hline Container ID & Stor. Loc. & \# of Cont. & Type & Total Vol. & Units & Weight (lbs) & G. Fac. & Date Rec. & Waste Description \\
\hline INEL4759 & MWSF & & $\overline{D M}$ & 55.0 & GAL & 480 & TAN/ERP & $10 / 2 / 91$ & $3 / 22 / 95$ ACTIVATED CARBON W/TCE\&PCE \\
\hline
\end{tabular}




\section{E. RADIATION CHARACTERISTICS}

Radioactive Waste Type:

\begin{tabular}{|ll|}
\hline OllW & Oa-LlW $\bigcirc$ tru \\
OhLW & O Non-Rad \\
\hline
\end{tabular}

Mixed Waste:

Handling:

\begin{tabular}{|l|}
\hline OYes ONo \\
\hline O Contact O Remote \\
\hline
\end{tabular}

Radionuclide Contamination Accessiblity:

External Surface

Internal Surface

Dispersed Through Matrix

\begin{tabular}{|}
\begin{tabular}{|ccc|}
\hline OYes & Ono & O Unknown \\
\hline OYes & O No & O Unknown \\
\hline OYes & O No & O Unknown \\
\hline
\end{tabular}
\end{tabular}

\section{Activity Levels}

Transuranic Alpha Activity:

Uranium/Thorium Alpha Activity:

\begin{tabular}{l}
$\square$ Units: $\square$ \\
$\square$ Units: $\square$ \\
\hline
\end{tabular}

Beta/Gamma Activity:

$0.1 @$ surface

$<0.1 @ 1-\mathrm{m}$ Units: $\mathrm{mR} / \mathrm{hr}$

Surface Neutron Activity: Units:

Total Activity: $1.37 \mathrm{E}-07 \mathrm{nCi} / \mathrm{g}$ 


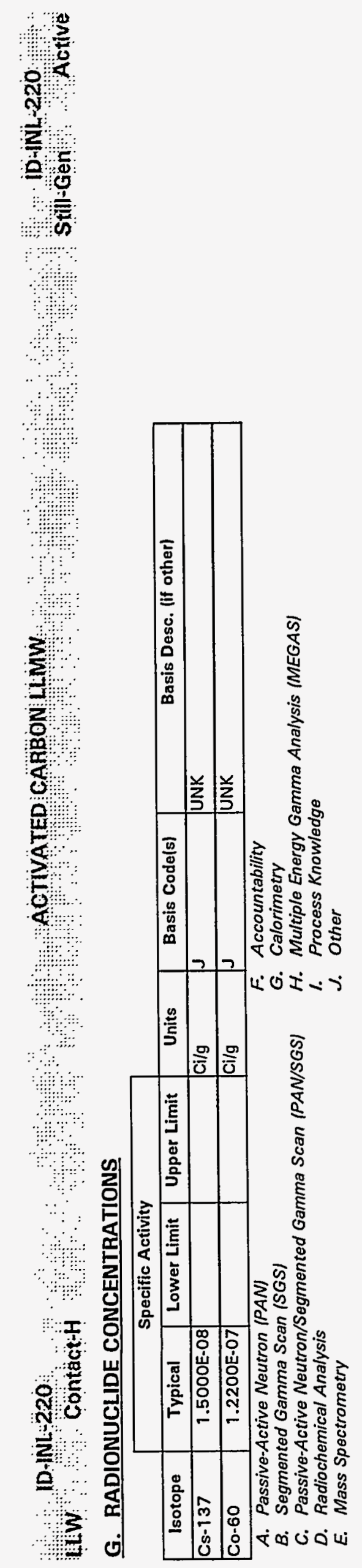

ह

ָั 
ID-INL-220

LiW $\cdots$, Contact-H

H. MÁTRIX CHARACTERISTICS

Primary Matrix Code: $\mathbf{S 3 1 1 8}$

Description: Homogeneous Solids: Inorganic Homogeneous Solids: Inorganic Particulates: Activated Carbon

Combustibility

Combustible (>90\%)

Mixed $(10 \%-90 \%)$

Noncombustible $(<10 \%)$

\section{MATRIX COMPOSITION}

\begin{tabular}{|c|c|c|c|c|c|c|}
\hline & & & \multicolumn{4}{|c|}{ Composition } \\
\hline Code & Matrix Description & Materlal & Typical & LL & UL & Units \\
\hline 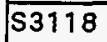 & Homogeneous Solids.Inorganic Homogeneous Solids.Inorganic Particulates.Activated & Spent Activated Carbon & & & & \\
\hline
\end{tabular}




\section{ID INL-220}

ACTIVATED CARBON LIMW

ID-INL-2ZOO

LWW Contagt H

ACIIVATED CARBON LIMW

Stillogen Gol Active

\section{J. HAZARDOUS CONSTITUENTS}

\begin{tabular}{|c|c|c|c|c|c|c|c|c|}
\hline \multirow{2}{*}{ Code } & \multirow[b]{2}{*}{ Hazardous Waste Description } & \multirow[b]{2}{*}{ Treatment Subcategory } & \multicolumn{4}{|c|}{ Concentration } & \multirow[b]{2}{*}{ Basis } & \multirow[b]{2}{*}{ Conc. Type } \\
\hline & & & Typical & $\mathbf{L L}$ & UL. & Units & & \\
\hline 0002 & Corrosive & Corrosive characteristic waste that are manag & & & & & A & Total \\
\hline D004 & Arsenic & & & & & & $A$ & Total \\
\hline 005 & Barium & & & & & & A & Total \\
\hline D006 & Cadmium & & & & & & $\bar{A}$ & Total \\
\hline$\overline{D 007}$ & Chromium & Chromium (Total) & & & & & A & Total \\
\hline$D 008$ & Lead & & & & & & A & Total \\
\hline 0009 & Mercury & Low mercury $<260 \mathrm{mg} / \mathrm{kg}$ total mercury & & & & & A & Total \\
\hline 0010 & Selenium & & & & & & A & Total \\
\hline$\overline{D 011}$ & Silver & & & & & & A & Total \\
\hline D012 & Endrin & & & & & & A & Total \\
\hline$\overline{0012}$ & Endrin & Endrin aldehyde & & & & & A & Total \\
\hline$\overline{D 013}$ & Lindane & alpha-BHC & & & & & A & Total \\
\hline$D 013$ & Lindane & beta-BHC & & & & & A & Total \\
\hline$\overline{D 013}$ & Lindane & gamma-BHC (Lindano) & & & & & A & Total \\
\hline D014 & Methoxychlor & & & & & & A & Total \\
\hline$D 015$ & Toxaphene & & & & & & A & Total \\
\hline$\overline{D 016}$ & $2,4-D$ & 2,4-D (2,4-Dichlorophenoxyacetic acid) & & & & & A & Total \\
\hline 0017 & 2,4,5-TP (Silvex) & & & & & & A & Total \\
\hline 0018 & Benzene & Benzene managed in non-CWA/non-CWA-equi & & & & & A & Total \\
\hline$\overline{D 019}$ & Carbon tetrachloride & Carbon Tetrachloride managed in non-CWA/no & & & & & A & Total \\
\hline $\mathrm{D020}$ & Chlordane & Chlordane managed in non-CWA/non-CWA-eq & & & & & A & Total \\
\hline$\overline{D 021}$ & Chlorobenzene & Chlorobenzene managed in non-CWA/non-CW & & & & & $\bar{A}$ & Total \\
\hline$\overline{D 022}$ & Chloroform & Chloroform managed in non-CWA/non-CWA-e & & & & & A & Total \\
\hline D023 & o-Cresol & o-Cresol managed in non-CWA/non-CWA-equi & & & & & $\bar{A}$ & Total \\
\hline D024 & m-Cresol & $\mathrm{m}$-Cresol managed in non-CWA/non-CWA-equi & & & & & $\bar{A}$ & Total \\
\hline$\overline{\mathrm{DO25}}$ & p-Cresol & $\mathrm{p}$-Cresol managed in non-CWA/non-CWA-equi & & & & & A & Total \\
\hline D026 & Cresol & Cresols (Total) managed in non-CWA/non-CW & & & & & A & Total \\
\hline D029 & 1,1-Dichloroethylene & 1,1-Dichloroethylene managed in non-CWA/no & & & & & A & Total \\
\hline$\overline{D 030}$ & 2,4-Dinitrotoluene & 2,4-Dinitrotoluene managed in non-CWA/non- & & & & & A & Total \\
\hline D031 & Heptachlor & Heptachlor managed in non-CWA/non-CWA-eq & & & & & A & Total \\
\hline
\end{tabular}


ID.INL -220

ACTIVATED CARBON LLIMW

ID-INL-220:

HLW Contact H

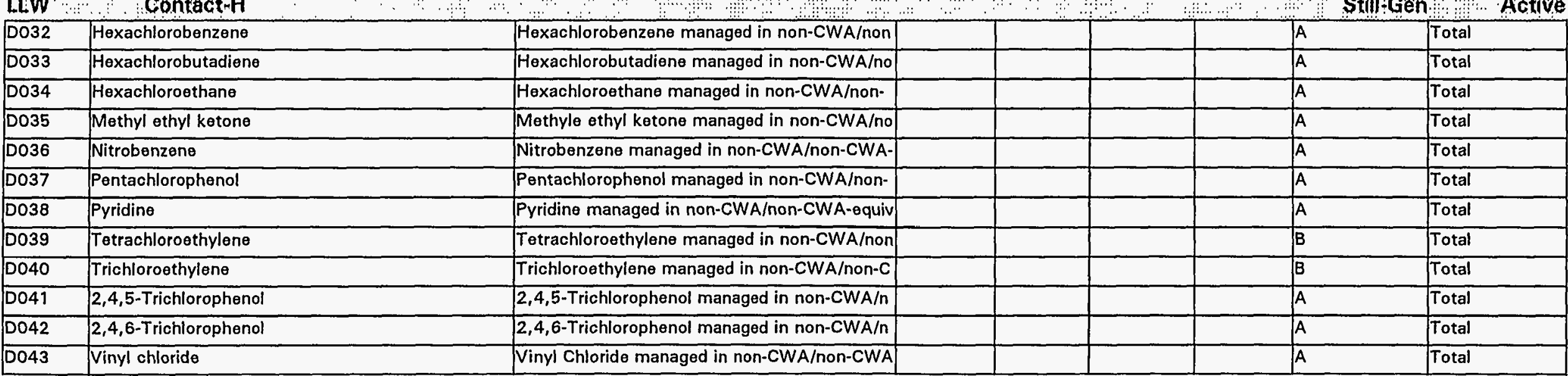


LWW Contact H

\section{K. POLYCHLORINATED BIPHENYLS (PCBS)}

Is this waste stream PCB contaminated?

\section{OYes}

O No

If yes, what portion of the waste is PCB contaminated (volume \%):

If $<100 \%$, can the PCB contaminated waste be segregated and treated separately?

OYes ONo OUnknown

What is the PCB concentration in ppm?

If only a portion of the waste is PCB contaminated, report values for contaminated portion.

\section{TREATMENT PLANS}

\begin{tabular}{|c|c|c|c|c|c|c|c|c|c|c|c|}
\hline $\begin{array}{l}\text { Opt. } \\
\#\end{array}$ & $\begin{array}{c}\text { Media Type } \\
\text { (If more than one) }\end{array}$ & $\begin{array}{c}\text { Matrix } \\
\text { Code }\end{array}$ & $\begin{array}{l}\text { Vol. \% } \\
\text { IIf }\end{array}$ & Step & JIT & sc & ClF & $\begin{array}{l}\text { Trans. } \\
\text { Miles }\end{array}$ & $\begin{array}{l}\text { Facility } \\
\text { Abbr. }\end{array}$ & Unit Name & Comments \\
\hline \multirow[t]{4}{*}{1} & & $\$ 3118$ & & $\bar{a}$ & O & 0 & $1.00 E+00$ & & WROC & Hg Retort & \\
\hline & & & & $\mathbf{b}$ & O & $\mathrm{O}$ & $1.00 E+00$ & & WERF & Incineration - Controlled Air Incinerator & \\
\hline & & & & c & $\mathrm{O}$ & $\mathrm{O}$ & $1.00 E+00$ & & WERF & Stabilization-Portland Cement & \\
\hline & & & & d & $\bar{O}$ & $\mathrm{O}$ & $1.00 \mathrm{E}+00$ & & RWMC & Disposal - Contact-Handled & \\
\hline
\end{tabular}

Note: Where provided, media percentages are rough estimates used to facilitate treatment planning. They do not necessarily imply an accurate knowledge of waste stream composition, and were not derived for use outside this conceptual planning exercise.

Describe any special or unique technical concerns related to the treatment of this waste stream that would impact the use or implementation of standard hazardous waste treatment methods.

None. 


ID-INL-267
A. IDENTIFICATION AND DESCRIPTION
Waste Stream ID: ID-INL-267
W. S. Name:
Content Code:
FFCA ID:
Waste Stream Description:
$\begin{aligned} & \text { This waste consists of filters that have been removed from the PWTU, equipment, etc. waste } \\ & \text { filters generated from various activities at the INEL. }\end{aligned}$

\section{B. GENERATION SITE/PROCESS DESCRIPTION}

The following questions, concerning the generation site and process, should be answered even if the waste stream is no longer generated.

Name of Site Generating Waste (e.g., INEL, Rocky Flats, etc.): Idaho National Engineering Laboratory

Location of activities (Area and Building): PBF PWTU

Operations (functions) performed in building: Treatment of aqueous mixed low level wastes.

Description of Process Generating Waste:

The PWTU Filters remove suspended solids before the liquid is treated by carbon absorption or ion exchange. The filters are replaced prior to treatment of each waste stream. 
$\therefore:$ ID-INL-267

Hiw Contagth

PWTU SPENT FILTERS

\section{PROJECTED WASTE GENERATION}

Is this waste stream still generated?

If yes, when is the expected generation termination date?

Current Projections

\begin{tabular}{|r|r|r|r|r|l|}
\hline Est. Date & \multicolumn{1}{|c|}{ Start Year } & Interval & \multicolumn{1}{|c|}{ Vol (m) } & Mass (kg) & Source of Estimate \\
\hline $3 / 1 / 95$ & 1995 & 1 & $\mathbf{0 . 4 2}$ & 89.04 & Generator Update \\
\hline $3 / 1 / 195$ & 1996 & 1 & $\mathbf{0 . 4 2}$ & 89.04 & Generator Update \\
\hline $5 / 23 / 95$ & 1997 & 1 & $\mathbf{0 . 4 2}$ & 89.04 & 1995-FFCA \\
\hline
\end{tabular}

Historical Projections

\begin{tabular}{|r|r|r|r|r|l|}
\hline Est. Date & \multicolumn{1}{|c|}{ Start Year } & \multicolumn{1}{|c|}{ Interval } & \multicolumn{1}{|c|}{ Vol (m3) } & \multicolumn{1}{c|}{ Mass $(\mathrm{kg})$} & Source of Estimate \\
\hline $6 / 1 / 94$ & 1998 & 1 & 1.00 & 212.00 & $1994-D S T P$ \\
\hline $11 / 1 / 93$ & 1993 & 1 & 1.00 & 212.00 & $1993-F F C A$ \\
\hline $11 / 1 / 93$ & 1994 & 1 & 1.00 & 212.00 & $1993-F F C A$ \\
\hline $11 / 1 / 93$ & 1995 & 1 & 1.00 & 212.00 & $1993-F F C A$ \\
\hline $11 / 1 / 93$ & 1996 & 1 & 1.00 & 212.00 & $1993-F F C A$ \\
\hline $11 / 1 / 93$ & 1997 & 1 & 1.00 & 212.00 & $1993-F F C A$ \\
\hline $12 / 1 / 92$ & 1992 & 5 & 5.00 & $1,060.00$ & $1992-F F C A$ \\
\hline
\end{tabular}


ID-INL-267

LiW :.... Contact-H

D. STORAGE INVENTORY

\begin{tabular}{|c|c|c|c|c|c|c|c|c|c|}
\hline Container ID & Stor. Loc. & \# of Cont. & Type & Total Vol. & Units & Weight (lbs) & G. Fac. & Date Rec. & Waste Description \\
\hline INEL5479 & MWSF & & $\overline{\mathrm{DM}}$ & 55.0 & $\widehat{\mathrm{GAL}}$ & 193 & TAN/WRO & $2 / 1 / 93$ & PWTU FILTERS WITH LEAD AND MERCURY \\
\hline INEL6760 & MWSF & & DM & 2.0 & $\overline{\text { GAL }}$ & 10 & EGG-PWTU & $10 / 27 / 93$ & PWTU FILTERS CONTAMINATED WITH MERCURY AND LEAD \\
\hline INEL6839 & MWSF & & $\overline{D M}$ & 55.0 & $\overline{G A L}$ & 178 & TAN 607 & $12 / 12 / 94$ & $\begin{array}{l}\text { POLYPROPYLENE FLLTERS CONTAMINATED WITH CADMIUM AND TRACE } \\
\text { BADIONUCUDES }\end{array}$ \\
\hline
\end{tabular}

Waste Removed From Storage: 
Radioactive Waste Type:

\begin{tabular}{|ll|}
\hline OlLW & Oa-LLW O TRU \\
OHLW & O Non-Rad
\end{tabular}

This waste stream is not identified as a TRU or a-LLW.

Mixed Waste:

\begin{tabular}{|l|}
\hline OYes ONo \\
\hline O Contact ORemote \\
\hline
\end{tabular}

Radionuclide Contamination Accessiblity:

\begin{tabular}{|c|c|c|c|}
\hline External Surface & OYes & ONo & OUnknown \\
\hline Internal Surface & OYes & ONo & OUnknown \\
\hline ersed Through Matrix & OYes & ONo & OUnknown \\
\hline
\end{tabular}

\section{Activity Levels}

Transuranic Alpha Activity:

Uranium/Thorium Alpha Activity: Beta/Gamma Activity:

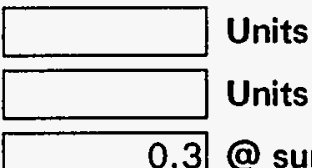

(

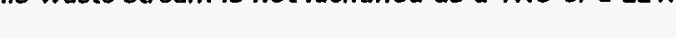

Surface Neutron Activity:
Total Activity:

0.1 @-m Units: $m R / h r$

$$
\begin{aligned}
& \text { Units: } \\
& \mathrm{nCi} / \mathrm{g}
\end{aligned}
$$


Contact-H

$\because \quad \ldots$

\section{G. RADIONUCLIDE CONCENTRATIONS}

\begin{tabular}{|c|c|c|c|c|c|c|}
\hline & \multicolumn{3}{|c|}{ Specific Activity } & \multirow[b]{2}{*}{ Units } & \multirow[b]{2}{*}{ Basis Code(s) } & \multirow[b]{2}{*}{ Basis Desc. (if other) } \\
\hline Isotope & Typical & Lower Limit & Upper Limit & & & \\
\hline Eu-152 & $2.1000 \mathrm{E}-11$ & & & $\mathrm{Ci} / \mathrm{g}$ & J & Gamma Spec \\
\hline Cs-137 & $8.3000 \mathrm{E}-12$ & & & $\mathrm{Ci} / \mathrm{g}$ & $j$ & Gamma Spec \\
\hline Co-60 & $2.2000 \mathrm{E}-10$ & & & $\mathrm{Ci} / \mathrm{g}$ & $j$ & Gamma Spec \\
\hline $\mathrm{Ag}-108 \mathrm{~m}$ & $2.9000 \mathrm{E}-12$ & & & $\mathrm{Ci} / \mathrm{g}$ & $J$ & Gamma Spec \\
\hline $\begin{array}{l}\text { A. Passiv } \\
\text { B. Segme } \\
\text { C. Passiv } \\
\text { D. Radioc } \\
\text { E. Mass }\end{array}$ & $\begin{array}{l}\text { otive Neutron } \\
\text { d Gamma Scar } \\
\text { ctive Neutron/S } \\
\text { ical Analysis } \\
\text { trometry }\end{array}$ & $\begin{array}{l}\text { PAN) } \\
\text { n (SGS) } \\
\text { Segmented G }\end{array}$ & Scan $I P A$ & SGSI & $\begin{array}{l}\text { F. Accountability } \\
\text { G. Calorimetry } \\
\text { H. Multiple Energy } \\
\text { l. Process Knowlec } \\
\text { J. Other }\end{array}$ & Iamma Analysis (MEGAS) \\
\hline
\end{tabular}




\section{H. MATRIX CHARACTERISTICS}

Primary Matrix Code: $\$ 5410$

Description: Debris Waste: Heterogeneous Debris: Composite Filter Debris:

Combustibility:

Combustible $(>90 \%)$

Mixed (10\%-90\%)

Noncombustible $(<10 \%)$

\section{MATRIX COMPOSITION}

\begin{tabular}{|c|c|c|c|c|c|c|}
\hline & & & \multicolumn{4}{|c|}{ Composition } \\
\hline Code & Matrix Description & Material & Typlcal & LL & UL & Units \\
\hline$\$ 5410$ & Debris Waste.Heterogeneous Debris.Composite Filter Debris. & Filters & & & & \\
\hline
\end{tabular}


ID INL-267

\section{PWTU SPENT FILTERS}

1D-INL-267

LWW Contact-H

\section{PWTU SPENT FITHS}

\section{J. HAZARDOUS CONSTITUENTS}

\begin{tabular}{|c|c|c|c|c|c|c|c|c|}
\hline \multicolumn{3}{|c|}{ (2) } & \multicolumn{4}{|c|}{ Concentration } & \multirow[b]{2}{*}{ Basis } & \multirow[b]{2}{*}{ Conc. Type } \\
\hline Code & Hazardous Waste Description & Treatment Subcategory & Typical & LL & UL & Units & & \\
\hline$\longdiv { D 0 0 4 }$ & Arsenic & & & & & & F2 & Total \\
\hline$\overline{D 005}$ & Barium & & & & & & F2 & Total \\
\hline D006 & Cadmium & & & & & & F2 & Total \\
\hline$\overline{D 007}$ & Chromium & Chromium (Total) & & 0 & 0.03 & $\% w t$ & F2 & Total \\
\hline$D 008$ & Lead & & & 6.7 & 65 & $\% w t$ & F2 & Total \\
\hline$\overline{D 009}$ & Mercury & High-mercury organic subcategory $(>=260 \mathrm{~m}$ & & 0.3 & & $\% w t$ & F2 & Total \\
\hline$\longdiv { 0 0 1 0 }$ & Selenium & & & & & & F2 & Total \\
\hline$\overline{D 011}$ & Silver & & & & & & F2 & Total \\
\hline
\end{tabular}




\section{ID-INL-267}

\section{PWTTU: SPENTT FILTERS}

HWW Contact

\section{K. POLYCHLORINATED BIPHENYLS (PCBS)}

Is this waste stream PCB contaminated? OYes $O$ No $O$ Unknown

If yes, what portion of the waste is PCB contaminated (volume \%):

If $<100 \%$, can the PCB contaminated waste be segregated and treated separately? $O$ Yes ONo O Unknown

What is the PCB concentration in ppm? If only a portion of the waste is PCB contaminated, report values for contaminated portion.

\section{TREATMENT PLANS}

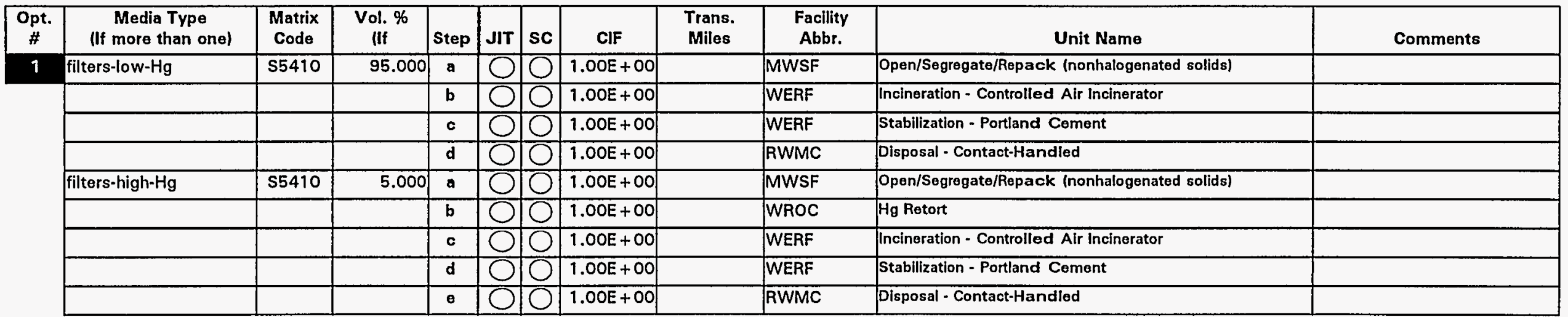

Note: Where provided, media percentages are rough estimates used to facilitate treatment planning. They do not necessarily imply an accurate knowledge of waste stream composition, and were not derived for use outside this conceptual planning exercise.

Describe any special or unique technical concerns related to the treatment of this waste stream that would impact the use or implementation of standard hazardous waste treatment methods. 


\section{A. IDENTIFICATION AND DESCRIPTION}

Waste Stream ID: ID-INL-268

W. S. Name:

Content Code:

FFCA ID:

\section{PWTU SPENT RESINS}

\section{Waste Stream Description:}

This waste consists of spent ion exchange resins and various other zeolite media. Waste stream is generated through various laboratory and treatment processes at the INEL.

\section{B. GENERATION SITE/PROCESS DESCRIPTION}

The following questions, concerning the generation site and process, should be answered even if the waste stream is no longer generated.

Name of Site Generating Waste (e.g., INEL, Rocky Flats, etc.): Idaho National Engineering Laboratory

Location of activities (Area and Building): PWTU and TRA Laboratories.

Operations (functions) performed in building:

Treatment of aqueous mixed low level waste. Mixed waste treatability studies.

Description of Process Generating Waste:

lon exchange resins are used to remove metal ions from water. Used resins may be mixed if the metal does not bind to the resin properly. Treatability studies are used to select resins prior to treatment. Occassionally a resin fails to properly bind hazardous metals. 
LeW Contacth

\section{PROJECTED WASTE GENERATION}

Is this waste stream still generated?

OYes ONo

If yes, when is the expected generation termination date?

Current Projections

\begin{tabular}{|r|r|r|r|r|r|}
\hline Est. Date & Start Year & Interval & \multicolumn{1}{|c|}{ Vol (m3) } & Mass (kg) & Source of Estimate \\
\hline $5 / 23 / 95$ & 1995 & 3 & 0.57 & $1,132.80$ & $1995-\mathrm{MWR}$ \\
\hline
\end{tabular}

\section{Historical Projections}

\begin{tabular}{|r|r|r|r|r|l|}
\hline \multicolumn{1}{|c|}{ Est. Date } & \multicolumn{1}{|c|}{ Start Year } & \multicolumn{1}{|c|}{ Interval } & \multicolumn{1}{|c|}{ Vol $(\mathbf{m} 3)$} & \multicolumn{1}{c|}{ Mass $(\mathrm{kg})$} & \multicolumn{1}{c|}{ Source of Estimate } \\
\hline $6 / 1 / 94$ & 1998 & 1 & 0.02 & 40.00 & 1994 DSTP \\
\hline $6 / 1 / 94$ & 1999 & 5 & 0.12 & 200.00 & $1994-\mathrm{DSTP}$ \\
\hline $11 / 1 / 93$ & 1993 & 1 & 0.02 & 40.00 & $1993-\mathrm{FFCA}$ \\
\hline $11 / 1 / 93$ & 1994 & 1 & 0.02 & 40.00 & $1993-\mathrm{FFCA}$ \\
\hline $11 / 1 / 93$ & 1995 & 1 & 0.02 & 40.00 & $1993-\mathrm{FFCA}$ \\
\hline $11 / 1 / 93$ & 1996 & 1 & 0.02 & 40.00 & $1993-\mathrm{FFCA}$ \\
\hline $11 / 1 / 93$ & 1997 & 1 & 0.02 & 40.00 & $1993-\mathrm{FFCA}$ \\
\hline $11 / 1 / 93$ & 1998 & 5 & 0.02 & 40.00 & $1993-\mathrm{FFCA}$ \\
\hline $11 / 1 / 93$ & 2003 & 20 & 0.02 & 40.00 & $1993-\mathrm{FFCA}$ \\
\hline $12 / 1 / 92$ & 1992 & 5 & 0.12 & 200.00 & $1992-\mathrm{FFCA}$ \\
\hline
\end{tabular}




\section{ID.INL-268}

LiW

\section{STORAGE INVENTORY}

\begin{tabular}{|c|c|c|c|c|c|c|c|c|c|}
\hline Container ID & Stor. Loc. & \# of Cont. & Type & Total Vol. & Units & Weight (lbs) & G. Fac. & Date Rec. & Waste Description \\
\hline INEL5477A & MWSF & & DM & 55.0 & GAL & 146 & TAN & $3 / 27 / 95$ & ION EXCHANGE RESIN WITH LEAD AND MERCURY FROM THE TAEATMENT OF \\
\hline INEL6752 & MWSF & & DM & 2.0 & GAL. & 10 & TAN/WRO & $10 / 27 / 93$ & SOLID ION EXCHANGE RESIN, OXIDE \\
\hline
\end{tabular}

Waste Removed From Storage:

\begin{tabular}{|c|c|c|c|c|c|c|c|c|c|c|}
\hline Container ID & Stor. Loc. & \# of Cont. & Type & Total Vol. & Units & Weight (lbs) & G. Fac. & Date Rec. & Date Out & Waste Description \\
\hline INEL5477 & MWSF & & DM & 55.0 & GAL & 146 & TAN & $2 / 1 / 93$ & $3 / 22 / 95$ & ION EXCHANGE RESIN WITH LEAD AND MERCURY \\
\hline
\end{tabular}




\section{E. RADIATION CHARACTERISTICS}

Radioactive Waste Type:

\begin{tabular}{|ll|}
\hline Ollw & Oa-llW O tRU \\
OhlW & O Non-Rad
\end{tabular}

Mixed Waste:

\begin{tabular}{|l|}
\hline O Yes O No \\
\hline O Contact O Remote \\
\hline
\end{tabular}

Radionuclide Contamination Accessiblity:

External Surface

Internal Surface

Dispersed Through Matrix

\begin{tabular}{|c|c|c|}
\hline OYes & ONo & OUnknown \\
\hline OYes & ONo & O Unknown \\
\hline OYes & ONo & OUnknown \\
\hline
\end{tabular}

\section{Activity Levels}

Transuranic Alpha Activity:

Uranium/Thorium Alpha Activity: Beta/Gamma Activity:

Surface Neutron Activity:

Total Activity:

\begin{tabular}{|c|c|c|}
\hline & Units: & \\
\hline & Units: & \\
\hline 0.4 & @ surf & ace \\
\hline 0.1 & @ 1-m & Units: $\mathrm{mR} / \mathrm{hr}$ \\
\hline & Units: & \\
\hline & $\mathrm{nCi} / \mathrm{g}$ & \\
\hline
\end{tabular}

\section{F. TRU ALPHA ACTIVITY DISTRIBUTION}

This waste stream is not identified as a TRU or a-LLW. 
12410

abpa/mouy ssasold

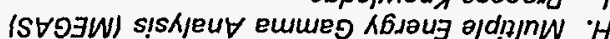

Anowisoles

AIIIIqE) UnOOOY

\begin{tabular}{|c|c|c|c|c|c|c|}
\hline \multicolumn{4}{|c|}{ Al!!!qeiunoso $\forall$ i] } & \multicolumn{3}{|c|}{ 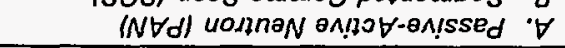 } \\
\hline oods emueg & $r$ & $6 / ! 0$ & & & $\angle 0 \cdot \exists 00+9^{\circ} z$ & $09-00$ \\
\hline & 1 & & & & & $\angle E l-s J$ \\
\hline & 1 & & & & & $\lfloor\varepsilon|-|$ \\
\hline & 1 & & & & & $d \exists W$ \\
\hline & 1 & & & & & $06^{-1 S}$ \\
\hline oods eurmes & $r$ & $6 / !$ & & & $60-30000^{\circ} 1$ & $w_{80 l-6 \forall}$ \\
\hline (10420 f!) .0800 s!s8g & (s)epoo s!seg & sụun & น & มุแ! & jeo!d/ 1 & odozos| \\
\hline
\end{tabular}

Anauronjoads ssew Anourasoads ssem

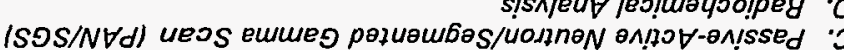

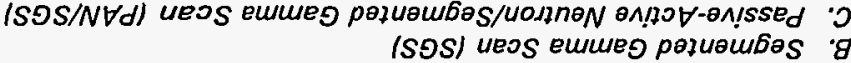

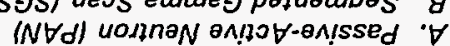

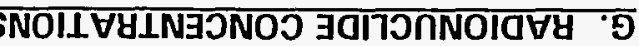

anipo:

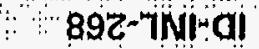

SNISAY ING̈S กIMA
H.30\%7uoj

$89 \mathrm{C}^{-1 N \mathrm{NI} \text { al }}$ 
liw WontactiH

H. MATRIX CHARACTERISTICS

Primary Matrix Code: $\mathbf{S 3 1 1 5}$

Description: Homogeneous Solids: Inorganic Homogeneous Solids: Inorganic Particulates: Ion Exchange Media

\section{Combustibility: $\bigcirc$ Combustible $(>90 \%)$ \\ Mixed $(10 \%-90 \%)$ \\ O Noncombustible (<10\%)}

\section{MATRIX COMPOSITION}

\begin{tabular}{|c|c|c|c|c|c|c|}
\hline & & & \multicolumn{4}{|c|}{ Composition } \\
\hline Code & Matrix Description & Material & Typical & LL & UL & Units \\
\hline$\$ 3115$ & Homogeneous Solids.Inorganic Homogeneous Solids.Inorganic Particulates.Ion Excha & Miscellaneous Resins & & & & \\
\hline
\end{tabular}




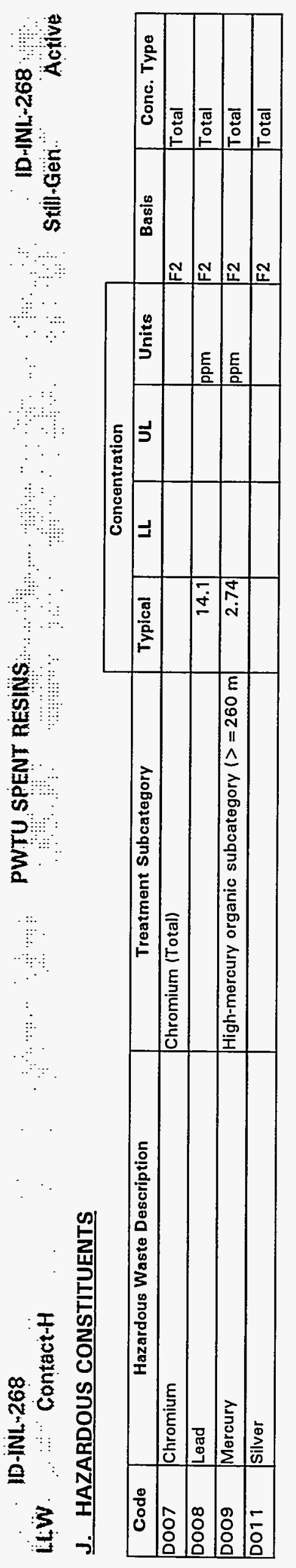




\section{K. POLYCHLORINATED BIPHENYLS (PCBS)}

\section{Is this waste stream PCB contaminated? OYes $O$ No $O$ Unknown}

If yes, what portion of the waste is PCB contaminated (volume \%):

\section{If $<100 \%$, can the PCB contaminated waste be segregated and treated separately?}

What is the PCB concentration in ppm?

\section{TREATMENT PLANS}

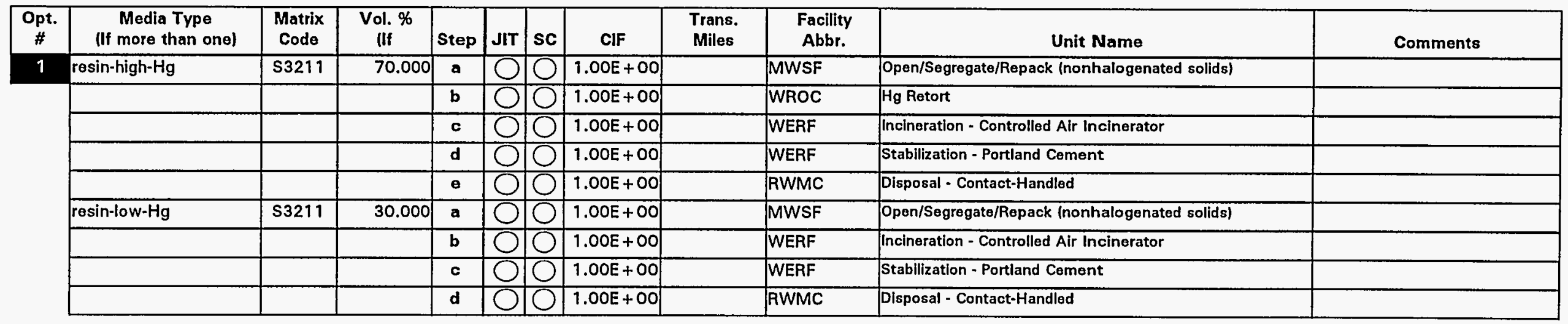

Note: Where provided, media percentages are rough estimates used to facilitate treatment planning. They do not necessarily imply an accurate knowledge of waste stream composition, and were not derived for use outside this conceptual planning exercise.

Describe any special or unique technical concerns related to the treatment of this waste stream that would impact the use or implementation of standard hazardous waste treatment methods.

None. 


\section{ID-INL-270 \\ LLW Contact H}

\section{A. IDENTIFICATION AND DESCRIPTION}

Waste Stream ID: ID-INL-270

W. S. Name:

Content Code:

FFCA ID:

HEAVY METAL CONTAMINATED SOLIDS

IN-W075

\section{Waste Stream Description:}

This waste stream consists of sludges, characterization samples, treatment test residues, and other miscellaneous solids contaminated with toxicity characteristic and radioactive constituents. No longer analyzing mixed low level samples.

\section{HEAVY METAL CONTAMINATED SOLIDS}

10 -1NL-270 Nótuen $:$ Active

\section{B. GENERATION SITE/PROCESS DESCRIPTION}

The following questions, concerning the generation site and process, should be answered even if the wasto stream is no longer generated.

Name of Site Generating Waste (e.g., INEL, Rocky Flats, etc.): Idaho National Engineering Laboratory

Location of activities (Area and Building):

Various

Operations (functions) performed in building: Various

Description of Process Generating Waste: Various 
ID-INL:270

LWW Contact H

HEAVY METAL COONTAMINAATED SÖLIODS

IB INIL -270

\section{PROJECTED WASTE GENERATION}

Is this waste stream still generated?

If yes, when is the expected generation termination date?

Current Projections

\section{Historical Projections}

\begin{tabular}{|r|r|r|r|r|l|}
\hline Est. Date & \multicolumn{1}{|c|}{ Start Year } & \multicolumn{1}{|c|}{ Interval } & \multicolumn{1}{|c|}{ Vol (m3) } & \multicolumn{1}{c|}{ Mass (kg) } & Source of Estimate \\
\hline $6 / 1 / 94$ & 1998 & 1 & 1.29 & 774.00 & $1994-D S T P$ \\
\hline $11 / 1 / 93$ & 1993 & 1 & 1.29 & 774.00 & $1993-F F C A$ \\
\hline $11 / 1 / 93$ & 1994 & 1 & 1.29 & 774.00 & $1993-F F C A$ \\
\hline $11 / 1 / 93$ & 1995 & 1 & 1.29 & 774.00 & $1993-F F C A$ \\
\hline $11 / 1 / 93$ & 1996 & 1 & 1.29 & 774.00 & $1993-F F C A$ \\
\hline $11 / 1 / 93$ & 1997 & 1 & 1.29 & 774.00 & $1993-F F C A$ \\
\hline $12 / 1 / 92$ & 1992 & 5 & 6.45 & $3,870.00$ & $1992-F F C A$ \\
\hline
\end{tabular}


ID-INL-270

LiLw : Contact-H

\section{STORAGE INVENTORY}

\begin{tabular}{|c|c|c|c|c|c|c|c|c|c|}
\hline Container ID & Stor. Loc. & \# of Cont. & Type & Total Vol. & Units & Weight (lbs) & G. Fac. & Date Rec. & Waste Description \\
\hline INEL5008 & MWSF & & $\overline{D M}$ & 5.0 & GAL & 9 & CFA-625 & $10 / 5 / 94$ & SOIL CONTAMINATED WITH CADMIUM, LEAD, AND TRACE RADIONUCLIDES \\
\hline INEL5793 & MWSF & & $\overline{D M}$ & 5.0 & GAL & 25 & EGG/ECL & $5 / 26 / 93$ & HEPA FILTER DEBRIS \& SOIL WIARSENIC,LEAD \& CADMIUM \\
\hline INEL5794 & MWSF & & $D M$ & $\overline{5.0}$ & $\overline{G A L}$ & 40 & EGG/ECL & $5 / 26 / 93$ & HEPA FILTER DEBRIS \& SOIL W/ARSENIC,LEAD \& CADMIUM \\
\hline INEL5795A & MWSF & & DM & 5.0 & GAL & 19 & EGG/ECL & $6 / 30 / 94$ & SOIL CONTAMINATED WITH CADMIUM AND LEAD \\
\hline INEL5796A & MWSF & & DM & 30.0 & GAL & 187 & EGG/ECL & $6 / 30 / 94$ & SOIL CONTAMINATED WITH CADMIUM AND LEAD \\
\hline
\end{tabular}

\section{Total:}

HEAVY METAL CONTAMINATED SOLIDS

$10-1 N L-270$ Not-Gen

\section{Waste Removed From Storage:}

\begin{tabular}{|c|c|c|c|c|c|c|c|c|c|}
\hline Container ID & Stor. Loc. & \# of Cont. & Type & Total Vol. & Units & Weight (lbs) & G. Fac. & Date Rec. Date Out & Waste Description \\
\hline INEL5795 & MWSF & & DM & & GAL & 19 & EGG/ECL & $5 / 26 / 93 \quad 6 / 27 / 94$ & SOIL CONTAMINATED WITH CADMIUM AND LEAD \\
\hline$\longdiv { I N E L 5 7 9 6 }$ & MWSF & & $\overline{D M}$ & 30.0 & GAL & 187 & EGG/ECL & $5 / 26 / 93 \quad 6 / 27 / 94$ & SOIL CONTAMINATED WITH CADMIUM AND LEAD \\
\hline
\end{tabular}


$\because \quad \because$ ID INL-270

HEAVY METAL CONTAMINATEO SOLIDS

HiW ContactiH

E. RADIATION CHARACTERISTICS

Radioactive Waste Type:

\begin{tabular}{|ll|}
\hline OlLW & Oa-LLW O TRU \\
O HLW & $\bigcirc$ Non-Rad \\
\hline
\end{tabular}

Mixed Waste:

\begin{tabular}{|l|}
\hline OYes O No \\
\hline O Contact O Remote \\
\hline
\end{tabular}

Radionuclide Contamination Accessiblity:

\begin{tabular}{|c|c|c|c|}
\hline External Surface & OYes & ONo & OUnknown \\
\hline Internal Surface & OYes & ONo & (O) Unknown \\
\hline d Through Mat & & No & in \\
\hline
\end{tabular}

Dispersed Through Matrix

\section{Activity Levels}

Transuranic Alpha Activity:

Uranium/Thorium Alpha Activity: Beta/Gamma Activity:

Surface Neutron Activity: Total Activity:

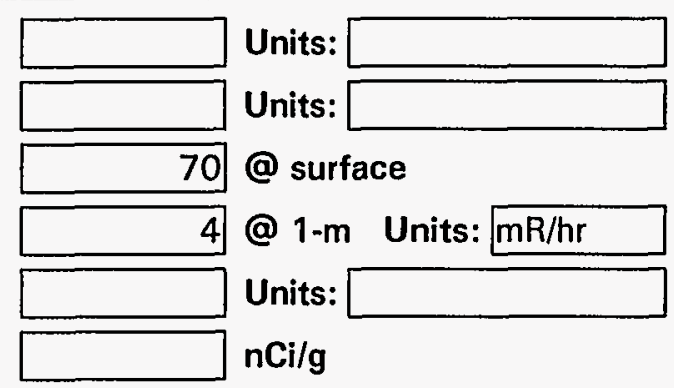

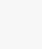

\section{F. TRU ALPHA ACTIVITY DISTRIBUTION}

This waste stream is not identified as a TRU or a-LLW. 
ID-INL-270

HEAVY METAL CONTAMINATED SOLIDS

ID-INL-270 LiW Contact-H

\section{G. RADIONUCLIDE CONCENTRATIONS}

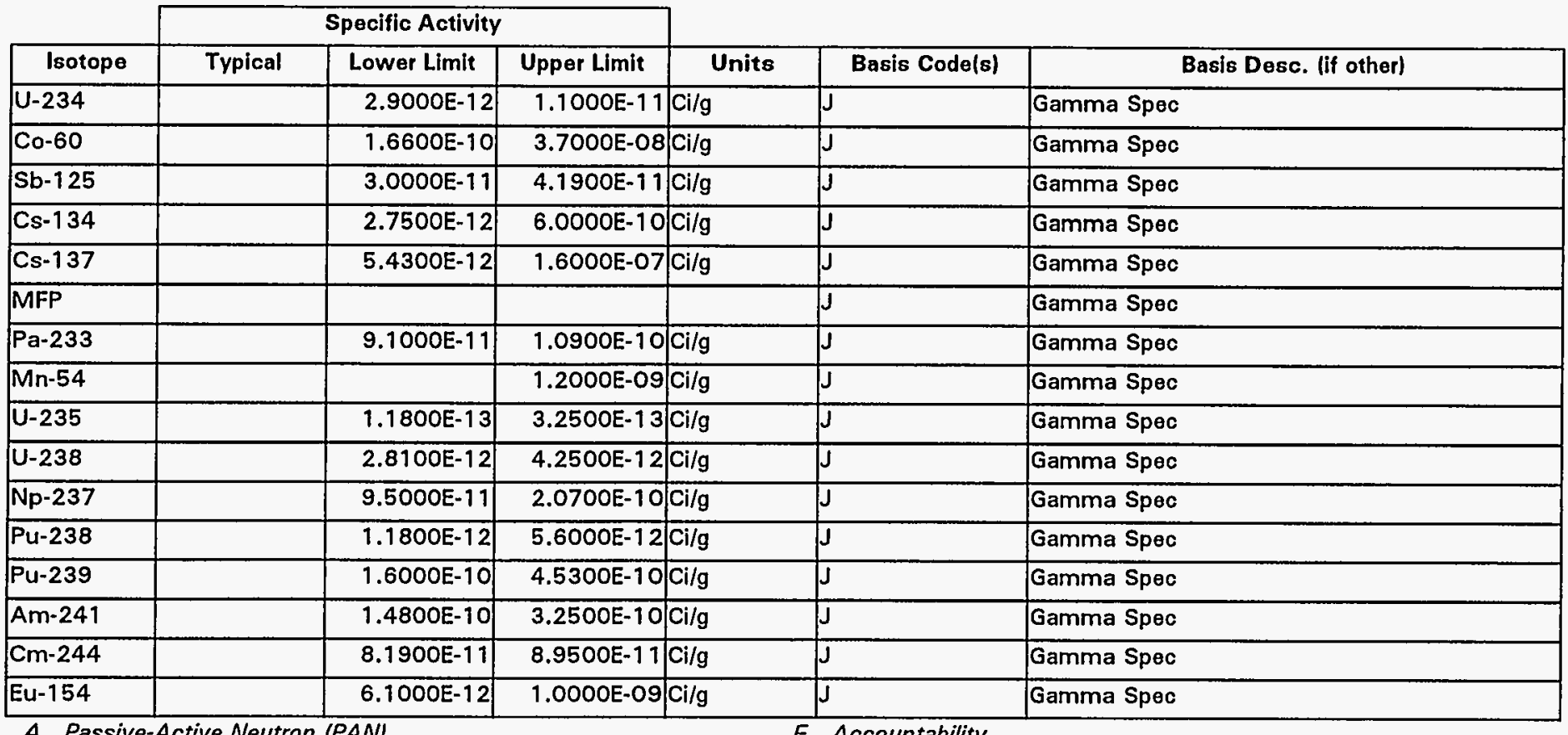

A. Passive-Active Neutron (PAN)

B. Segmented Gamma Scan (SGS)

C. Passive-Active Neutron/Segmented Gamma Scan (PAN/SGS)

D. Radiochemical Analysis

E. Mass Spectrometry

F. Accountability

G. Calorimetry

H. Multiple Energy Gamma Analysis (MEGAS)

l. Process Knowledge

J. Other

Other 
IDIINL: 270

LWW GontactH

\section{H. MATRIX CHARACTERISTICS}

Primary Matrix Code: $\$ 9000$

Description: Unknown/Other Solids: : :

Combustibility: $\bigcirc$ Combustible (>90\%)

Mixed $(10 \%-90 \%)$

O Noncombustible $(<10 \%)$

\section{MATRIX COMPOSITION}

\begin{tabular}{|c|c|c|c|c|c|c|}
\hline & & & & & & \\
\hline Code & Matrix Description & Material & Typlcal & $\overline{L L}$ & UL & Units \\
\hline$\$ 4100$ & Soil/Gravel.Soill.. & Soil $w / P b$ & & & & \\
\hline 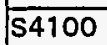 & Soil/Gravel.Soil.. & Soil $w / C d$ & & & & \\
\hline$\widehat{55122}$ & Debris Waste.Inorganic Debris.Inorganic Non-Motal Debris.Glass Debris & Glass & & & & \\
\hline 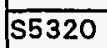 & Debris Waste.Organic Debris.Wood Debris. & Wood & & & & \\
\hline$\$ 4200$ & Soil/Gravel.Soil/Debris.. & Soil & & & & \\
\hline$\$ 5330$ & Debris Waste.Organic Debris.Paper/Cloth Debris. & Paper and cloth & & & & \\
\hline$\widehat{55410}$ & Debris Waste.Heterogeneous Debris.Composite Filter Debris. & HEPA Filter & & & & \\
\hline
\end{tabular}




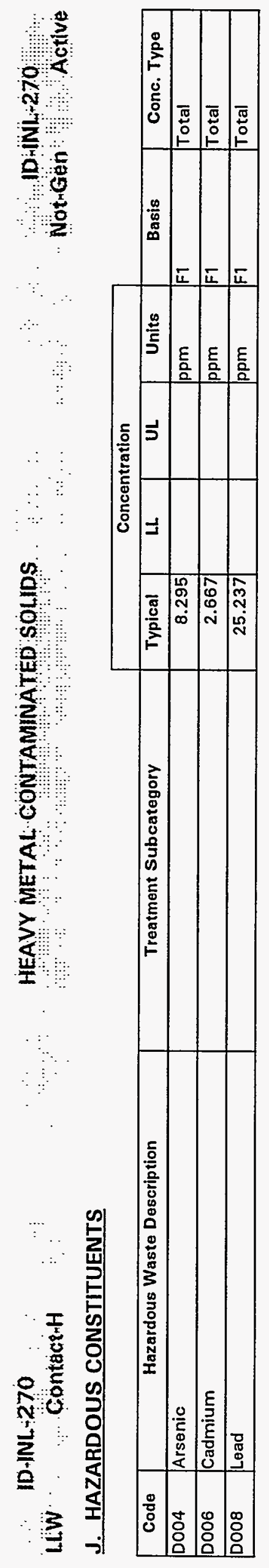

0
0
8
8
0

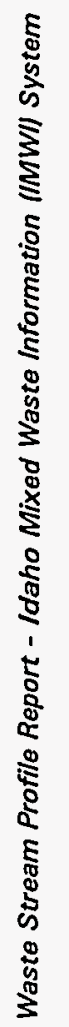

ฌิ 


\section{ID INL-270}

\section{K. POLYCHLORINATED BIPHENYLS (PCBS)}

\section{Is this waste stream PCB contaminated? OYes $O$ No $O$ Unknown}

If yes, what portion of the waste is PCB contaminated (volume \%):

If $<100 \%$, can the PCB contaminated waste be segregated and treated separately?

What is the PCB concentration in ppm?

Yes No Unknown

\section{TREATMENT PLANS}

\begin{tabular}{|c|c|c|c|c|c|c|c|c|c|c|c|}
\hline $\begin{array}{l}\text { Opt. } \\
\#\end{array}$ & $\begin{array}{c}\text { Media Type } \\
\text { (If more than one) }\end{array}$ & $\begin{array}{c}\text { Matrix } \\
\text { Code }\end{array}$ & $\begin{array}{l}\text { Vol. \% } \\
\text { (If }\end{array}$ & Step & JIT & sc & CIF & $\begin{array}{l}\text { Trans. } \\
\text { Miles }\end{array}$ & $\begin{array}{l}\text { Facility } \\
\text { Abbr. }\end{array}$ & Unit Name & Comments \\
\hline \multirow[t]{9}{*}{1} & low-Hg & 54100 & 95.000 & $\bar{a}$ & O & 0 & $1.00 E+00$ & & MWSF & Open/Segregate/Repack (nonhalogenated sollds) & \\
\hline & & & & $\mathbf{b}$ & 0 & O & $1.00 E+00$ & & WERF & Incineration - Controlled Air Incinerator & \\
\hline & & & & c & 0 & O & $1.00 E+00$ & & WERF & Stabilization - Portland Cement & \\
\hline & & & & d & 0 & 0 & $1.00 E+00$ & & RWMC & Disposal - Contact-Handled & \\
\hline & high-Hg & $\$ 4100$ & 5.000 & a & 0 & 0 & $1.00 \mathrm{E}+00$ & & MWSF & Open/Segregate/Repack (nonhalogenated solids) & \\
\hline & & & & $\mathbf{b}$ & 0 & $D$ & $1.00 E+00$ & & WROC & Hg Retort & \\
\hline & & & & c & 0 & 0 & $1.00 \mathrm{E}+00$ & & WERF & Incineration - Controlled Air Incinerator & \\
\hline & & & & $\bar{d}$ & 0 & 0 & $1.00 \mathrm{E}+00$ & & WERF & Stabilization - Portland Cement & \\
\hline & & & & $\bar{e}$ & 0 & 0 & $1.00 \mathrm{E}+00$ & & RWMC & Disposal - Contact-Handled & \\
\hline
\end{tabular}

Note: Where provided, media percentages are rough estimates used to facilitate treatment planning. They do not necessarily imply an accurate knowledge of waste stream composition, and were not derived for use outside this conceptual planning exercise.

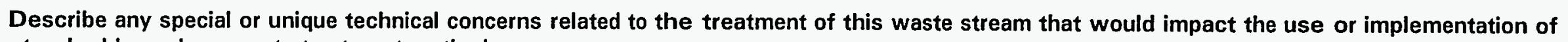
standard hazardous waste treatment methods.

None. 


ID-PBF-153
A.W IDENTIFICATION AND DESCRIPTION
Waste Stream ID: ID-PBF-153
W. S. Name:
Content Code:
FFCA ID:
Waste Stream Description:
Pin-W033
Piping, sludge, dirt, water, Hg and vermiculite from D\&D of IET radioactive waste piping system.

\section{B. GENERATION SITE/PROCESS DESCRIPTION}

The following questions, concerning the generation site and process, should be answered even if the waste stream is no longer generated.

Name of Site Generating Waste (e.g., INEL, Rocky Flats, etc.): Idaho National Engineering Laboratory

Location of activities (Area and Building): Waste Engineering Development Facility (WEDF)

Operations (functions) performed in building: Segregation, sizing and decontamination.

Description of Process Generating Waste:

Decontamination of TAN/IET wastes. 
Is this waste stream still generated?

If yes, when is the expected generation termination date?

Current Projections

Historical Projections 


\section{STORAGE INVENTORY}

\begin{tabular}{|c|c|c|c|c|c|c|c|c|c|}
\hline Container ID & Stor. Loc. & \# of Cont. & Type & Total Vol. & Units & Weight (lbs) & G. Fac. & Date Rec. & Waste Description \\
\hline INEL556A & MWSF & & DM & 55.0 & GAL & 625 & PER612 & $5 / 1 / 89$ & MERCURY CONTAMINATED SOIL/SLUDGE FROM IET D\&D \\
\hline INEL558A & MWSF & & DM & 55.0 & GAL & 625 & PER612 & $5 / 1 / 89$ & MERCURY CONTAMINATED SOIL/SLUDGE FROM IET D\&D \\
\hline INEL559 & MWSF & & $\mathrm{DM}$ & 55.0 & $\overline{G A L}$ & 570 & WEDF & $6 / 18 / 87$ & MERCURY CONTAMINATED SOIL/SLLUDGE FROM IET D\&D \\
\hline INEL632A & MWSF & & DM & 55.0 & $\overline{G A L}$ & 420 & PER612 & $5 / 1 / 89$ & MERCUAY CONTAMINATED SOIL/SLUDGE FROM IET D\&ID \\
\hline INEL658A & MWSF & & $D M$ & 55.0 & GAL & 400 & PER612 & $5 / 1 / 89$ & MERCURY CONTAMINATED SOIL/SLUDGE FROM IET D\&D \\
\hline INEL708 & MWSF & & DM & 55.0 & $\overline{G A L}$ & 670 & PER612 & $5 / 1 / 89$ & MERCURY CONTAMINATED SOLL/SLUDGE FROM IET D\&D \\
\hline INEL709 & MWSF & & DM & 55.0 & $\overline{\mathrm{GAL}}$ & 630 & PER612 & $5 / 1 / 89$ & MERCURY CONTAMINATED SOIL/SLUDGE FROM IET D\&D \\
\hline INEL710 & MWSF & & DM & 55.0 & GAL & 650 & PER612 & $5 / 1 / 89$ & MERCURY CONTAMINATED SOIL/SLUDGE FROM IET D\&D \\
\hline INEL711 & MWSF & & DM & 55.0 & GAL & 650 & PER612 & $5 / 1 / 89$ & MERCURY CONTAMINATED SOIL/SLUDGE FROM IET D\&D \\
\hline INEL922 & MWSF & & $\mathrm{DM}$ & 55.0 & GAL & 607 & WEDF & $6 / 16 / 87$ & MERCURY CONTAMINATED SOIL/SLUDGE FROM IET D\&D \\
\hline INEL941 & MWSF & & $D M$ & 55.0 & GAL & 605 & WEDF & $6 / 16 / 87$ & MERCURY CONTAMINATED SOIL/SLUDGE FROM IET D\&D \\
\hline
\end{tabular}

Total: $\quad 2.29 \mathrm{m3} \quad 2,926.6 \mathrm{Kg}$

Waste Removed From Storage:

\begin{tabular}{|c|c|c|c|c|c|c|c|c|c|c|}
\hline Container ID & Stor. Loc. & \# of Cont. & Type & Total Vol. & Units & Weight (lbs) & G. Fac. & Date Rec. & Date Out & Waste Description \\
\hline INEL556 & MWSF & & DM & 55.0 & GAL & 625 & WEDF & $6 / 78 / 87$ & $1 / 17 / 89$ & SLUDGE (DIRT, WATER, MERCURY, VERMICULITE) PIPE WISLUDGE \\
\hline INEL557 & MWSF & & DM & 55.0 & GAL & 420 & WEDF & $6 / 18 / 87$ & $1 / 17 / 89$ & SLUDGE (DIRT, WATER, MERCURY, VERMICULITE) PIPE W/SLUDGE \\
\hline INEL558 & MWSF & & DM & 55.0 & GAL & 625 & WEDF & $6 / 18 / 87$ & $1 / 17 / 89$ & SLUDGE (DIRT, WATER, MERCURY, VERMICULITE) PIPE WISLUDGE \\
\hline INEL583 & MWSF & & DM & 55.0 & GAL & 375 & WEDF & $8 / 20 / 87$ & $1 / 17 / 89$ & TAN MERCURY CONTAMINATED CONCAETE WASTE MATERIAL \\
\hline INEL584 & MWSF & & DM & 55.0 & GAL & 325 & WEDF & $8 / 20 / 87$ & $1 / 17 / 89$ & TAN MERCURY CONTAMINATED CONCRETE WASTE MATERIAL \\
\hline INEL585 & MWSF & & DM & 55.0 & GAL & 358 & WEDF & $8 / 20 / 87$ & $1 / 17 / 89$ & TAN MERCURY CONTAMINATED CONCRETE WASTE MATERIAL \\
\hline INEL586 & MWSF & & DM & 55.0 & GAL & 342 & WEDF & $8 / 20 / 87$ & $1 / 17 / 89$ & TAN MERCURY CONTAMINATED CONCRETE WASTE MATERIAL \\
\hline INEL595 & MWSF & & DM & 55.0 & $\overline{G A L}$ & 420 & WEDF & $7 / 7 / 87$ & $1 / 17 / 89$ & TAN MERCURY CONTAMINATED CONCRETE WASTE MATERIAL \\
\hline INEL596 & MWSF & & DM & 55.0 & GAL & 350 & WEDF & $7 / 7 / 87$ & $1 / 17 / 89$ & TAN MERCURY CONTAMINATED CONCRETE WASTE MATERIAL \\
\hline INEL597 & MWSF & & $D M$ & 55.0 & $\widehat{G A L}$ & 350 & WEDF & $7 / 7 / 87$ & $1 / 17 / 89$ & TAN MERCURY CONTAMINATED CONCRETE WASTE MATERIAL \\
\hline INEL598 & MWSF & & $D M$ & 55.0 & GAL & 380 & WEDF & $7 / 7 / 87$ & $1 / 17 / 89$ & TAN MERCURY CONTAMINATED CONCRETE WASTE MATERIAL \\
\hline INEL631 & MWSF & & DM & 55.0 & GAL & 430 & WEDF & $6 / 16 / 87$ & $1 / 17 / 89$ & TAN MERCURY CONTAMINATED RINSE SOLUTION \\
\hline INEL632 & MWSF & & $\mathrm{DM}$ & 55.0 & GAL & 420 & WEDF & $6 / 16 / 87$ & $1 / 17 / 89$ & TAN MERCURY CONTAMINATED RINSE SOLUTION \\
\hline INEL633 & MWSF & & $\mathrm{DM}$ & 55.0 & GAL & 320 & WEDF & $6 / 16 / 87$ & $1 / 17 / 89$ & MERCURY CONTAMINATED SOIL/SLUDGE FROM IET D\&D \\
\hline INEL658 & MWSF & & DM & 55.0 & $\overline{\text { GAL }}$ & 400 & WEDF & $6 / 16 / 87$ & $1 / 17 / 89$ & TAN MERCURY CONTAMINATED CONCRETE WASTE MATERIAL \\
\hline INEL659 & MWSF & & $\overline{D M}$ & 55.0 & GAL & 200 & WEDF & $6 / 16 / 87$ & $1 / 17 / 89$ & MERCURY CONTAMINATED SOILISLUDGE FROM IET D\&D \\
\hline$\longdiv { \text { INEL660 } }$ & MWSF & & DM & 55.0 & GAL & 440 & WEDF & $6 / 16 / 87$ & $1 / 17 / 89$ & MERCURY CONTAMINATED SOILSLLUDGE FROM IET D\&D \\
\hline INEL661 & MWSF & & DM & 55.0 & GAL & 460 & WEDF & $6 / 16 / 87$ & $1 / 17 / 89$ & MERCURY CONTAMINATED SOIL/SLUDGE FROM IET D\&D \\
\hline
\end{tabular}




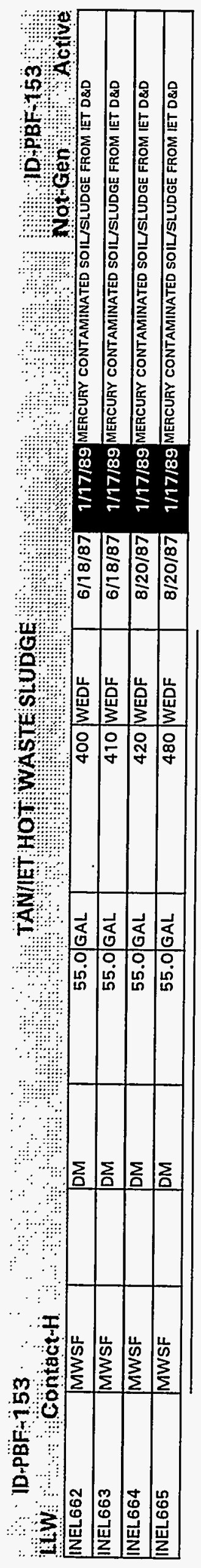




\section{E. RADIATION CHARACTERISTICS}

Radioactive Waste Type:

\begin{tabular}{|ll|}
\hline OlLW & Oa-LLW $\bigcirc$ TRU \\
OHLW & ONon-Rad \\
\hline
\end{tabular}

Mixed Waste:

Handling:

\begin{tabular}{|l|}
\hline O Yes ONo \\
\hline O Contact O Remote \\
\hline
\end{tabular}

Radionuclide Contamination Accessiblity:

External Surface OYes O No O Unknown

Internal Surface $O$ Yes $O$ No $O$ Unknown

Dispersed Through Matrix

OYes ONo

\section{Activity Levels}

Transuranic Alpha Activity: Uranium/Thorium Alpha Activity:

Beta/Gamma Activity:

Surface Neutron Activity:

Total Activity:

\begin{tabular}{|c|c|c|}
\hline Transuranic Alpha Activity: & 0 & Units: $\mathrm{nCi} / \mathrm{g}$ \\
\hline Uranium/Thorium Alpha Activity: & 0 & Units: $n \mathrm{ni} / \mathrm{g}$ \\
\hline Beta/Gamma Activity: & & @ surface \\
\hline & & @ 1-m Units: \\
\hline Surface Neutron Activity: & 0 & Units: $\mathrm{n} / \mathrm{cm} 2 / \mathrm{s}$ \\
\hline Total Activity: & & $\mathrm{nCi} / \mathrm{g}$ \\
\hline
\end{tabular}

\section{F. TRU ALPHA ACTIVITY DISTRIBUTION}

This waste stream is not identified as a TRU or a-LLW. 


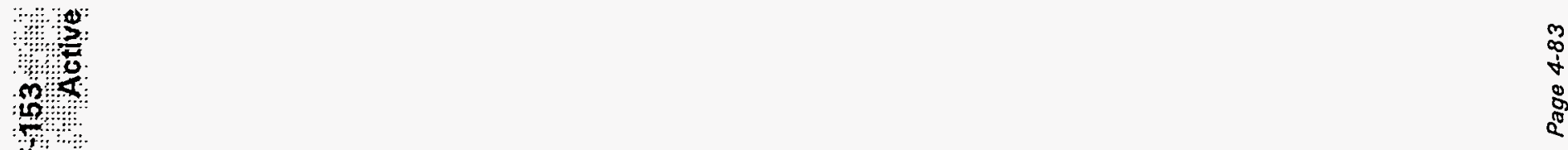

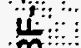

\%

:

正

2

:

.

:

i

.....

?

0

?

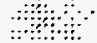

$\because$

ư

o.

3

뿐

为

3

5

Eis

Z

8

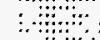

$\because$

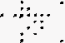

.

$\because$

$\because$

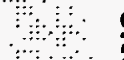

$\because \therefore$

의

$\therefore$....

군

$\therefore . .0$

IT

. W

ํㅡㅇ

T. 0

it :

a.

음 


\section{ID $-\mathrm{PBF}+153$}

\section{TAN/ETT HÖT WASTE SLUDGE}

ID.PBF-153:

LWW Contact-H

H. MATRIX CHARACTERISTICS

Primary Matrix Code: $\$ 5420$

Description: Debris Waste: Heterogeneous Debris: Predominantly Inorganic Debris:

\section{Combustibility: $\bigcirc$ Combustible $(>90 \%)$ \\ Mixed (10\%-90\%) \\ O Noncombustible $(<10 \%)$}

\section{MATRIX COMPOSITION}

\begin{tabular}{|c|c|c|c|c|c|c|}
\hline \multirow[b]{2}{*}{ Code } & \multirow[b]{2}{*}{ Matrix Description } & \multirow[b]{2}{*}{ Material } & \multicolumn{4}{|c|}{ Composition } \\
\hline & & & Typical & LL & UL & Units \\
\hline 53113 & Homogeneous Solids.Inorganic Homogeneous Solids.Inorganic Particulates.Inorganic & Vermiculite & & & & \\
\hline S5111 & Debris Waste.Inorganic Debris.Metal Debris.Metal Debris w/o Pb or Cd & Piping & & & & \\
\hline$\$ 4100$ & Soil/Gravel.Soil.. & Dirt & & & & \\
\hline 55121 & Debris Waste.Inorganic Debris.Inorganic Non-Metal Debris.Concrete Debris & Concrete Debris & & & & \\
\hline
\end{tabular}


ID PBF 15S3 O TANIET HÖT WASTE SLUDGE

Not Gen 1 PB

Uiw Contact

J. HAZARDOUS CONSTITUENTS

\begin{tabular}{|c|c|c|c|c|c|c|c|c|}
\hline & & & \multicolumn{4}{|c|}{ Concentration } & & \\
\hline Code & Hazardous Waste Description & Treatment Subcategory & Typical & $\mathbf{L L}$ & UL & Units & Basis & Conc. Type \\
\hline 0009 & Mercury & High-mercury inorganic subcategory $(>=260$ & & & & & C & Total \\
\hline
\end{tabular}




\section{K. POLYCHLORINATED BIPHENYLS (PCBS)}

Is this waste stream PCB contaminated? OYes $O$ No $O$ Unknown

If yes, what portion of the waste is PCB contaminated (volume \%):

If $<100 \%$, can the PCB contaminated waste be segregated and treated separately?

What is the PCB concentration in ppm?

If only a portion of the waste is PCB contaminated, report values for contaminated portion.

\section{TREATMENT PLANS}

\begin{tabular}{|c|c|c|c|c|c|c|c|c|c|c|c|}
\hline $\begin{array}{c}\text { Opt. } \\
\#\end{array}$ & $\begin{array}{c}\text { Media Type } \\
\text { (If more than one) }\end{array}$ & $\begin{array}{c}\text { Matrix } \\
\text { Code }\end{array}$ & $\begin{array}{l}\text { Vol. \% } \\
\text { IIf }\end{array}$ & Step & JIT & sc & CIF & $\begin{array}{l}\text { Trans. } \\
\text { Miles }\end{array}$ & $\begin{array}{l}\text { Facility } \\
\text { Abbr. }\end{array}$ & Unit Name & Comments \\
\hline 1 & & S3120 & & $\bar{a}$ & 0 & 0 & $1.00 E+00$ & & WROC & Hg Rotort & \\
\hline & & & & b & 0 & 0 & $1.00 E+00$ & & WERF & Stabilization - Portland Cement & \\
\hline & & & & c & 0 & 0 & $1.00 E+00$ & & AWMC & Disposal - Contact-Handled & \\
\hline
\end{tabular}

Note: Where provided, media percentages are rough estimates used to facilitate treatment planning. They do not necessarily imply an accurate knowledge of waste stream composition, and were not derived for use outside this conceptual planning exercise.

Describe any special or unique technical concerns related to the treatment of this waste stream that would impact the use or implementation of standard hazardous waste treatment methods.

Contact handled. 


\section{A. IDENTIFICATION AND DESCRIPTION}

Waste Stream ID: ID-TAN-254

\section{W. S. Name:}

\section{Content Code:}

FFCA ID:

\begin{tabular}{|l|}
\hline HTRE-III TREATMENT SLUDGE \\
\hline * \\
\hline IN-W059 \\
\hline
\end{tabular}

\section{Waste Stream Description:}

Filtered mercury sulfide sludge from the treatment of TAN mercury contaminated rinse solution

that came from decontamination activities at HTRE-III. Waste stream is $90 \%$ aqueous and

approximately $10 \% \mathrm{Hg}$ sludge.

\section{B. GENERATION SITE/PROCESS DESCRIPTION}

The following questions, concerning the generation site and process, should be answered even if the waste stream is no longer generated.

Name of Site Generating Waste (e.g., INEL, Rocky Flats, etc.): Idaho National Engineering Laboratory

Location of activities (Area and Building):

Test Area North (TAN), Portable Water Treatment Unit

Operations (functions) performed in building:

Mixed waste treatment.

Description of Process Generating Waste:

Aqueous waste treatment utilizing filteration, ion exchange, and carbon absorbtion. 


\section{ID-TAN-254}

\section{LLW . Contact-H}

C. PROJECTED WASTE GENERATION

Is this waste stream still generated?

If yes, when is the expected generation termination date?

Current Projections
ID-TAN-254:

NotmGen : Active 
D. STORAGE INVENTORY

\begin{tabular}{|l|l|l|l|r|l|r|r|r|r|}
\hline \multicolumn{1}{|c|}{ Container ID } & Stor. Loc. & \# of Cont. & Type & Total Vol. & Units & Weight (lbs) & G. Fac. & Date Rec. & Waste Description \\
\hline INEL5801A & MWSF & & DM & 55.0 & GAL & 435 & TAN/D\&D & $6 / 30 / 94$ & HTRE-3 FLLTERED SLUDGE WITH LEAD AND MERCUAY \\
\hline INEL5802 & MWSF & & DM & 55.0 & GAL & 440 & TAN/D\&D & $9 / 17 / 92$ & HTRE-3 FLLTERED SLUDGE WITH LEAD AND MERCURY \\
\hline INEL5803 & MWSF & & DM & 55.0 & GAL & 460 & TAN/D\&D & $9 / 17 / 92$ & HTRE-3 FLLTERED SLUDGE WITH LEAD AND MERCUAY \\
\hline INEL5804 & MWSF & & DM & 55.0 & GAL & 450 & TAN/D\&D & $9 / 17 / 92$ & HTRE-3 FLLTERED SLUDGE WITH LEAD AND MERCURY \\
\hline
\end{tabular}

Waste Removed From Storage:

\begin{tabular}{|c|c|c|c|r|c|r|r|r|r|}
\hline Container ID & Stor. Loc. & \# of Cont. & Type & Total Vol. & Units & Weight (lbs) & G. Fac. & Date Rec. & Date Out \\
\hline INEL5801 & MWSF & & DM & 55.0 & GAL & 435 & TAN/D\&D & $9 / 17 / 92$ & 6/27/94 HTRE-3 FILTERED SLUDGE WITH LEAD AND MERCURY \\
\hline
\end{tabular}




\section{E. RADIATION CHARACTERISTICS}

Radioactive Waste Type:

\begin{tabular}{|ll|}
\hline O LLW & Oa-LLW O TRU \\
OHLW & O Non-Rad \\
\hline
\end{tabular}

Mixed Waste:

\begin{tabular}{|l|}
\hline Yes $O$ No \\
\hline Contact \\
\hline Remote
\end{tabular}

Handling: $O$ Contact $O$ Remote

Radionuclide Contamination Accessiblity:

\begin{tabular}{rlrl} 
External Surface & $O$ Yes & O No & O Unknown \\
Internal Surface & OYes & O No & O Unknown \\
\cline { 2 - 4 } Dispersed Through Matrix & OYes & O No & O Unknown \\
\cline { 2 - 3 }
\end{tabular}

\section{Activity Levels}

Transuranic Alpha Activity: Uranium/Thorium Alpha Activity:

Beta/Gamma Activity:

\begin{tabular}{rl|}
\hline .18 & Units: $\mathrm{nCi} / \mathrm{g}$ \\
\hline 0 & Units: $\mathrm{nCi} / \mathrm{g}$ \\
\hline 2 & $@$ surface \\
\hline 0.4 & $@ 1-\mathrm{m}$ Units: $\mathrm{mR} / \mathrm{hr}$ \\
\hline
\end{tabular}

Surface Neutron Activity:

0 Units: $\mathrm{n} / \mathrm{cm} 2 / \mathrm{s}$

Total Activity: $62.2 \mathrm{nCi} / \mathrm{g}$

\section{F. TRU ALPHA ACTIVITY DISTRIBUTION}

This waste stream is not identified as a TRU or a-LLW. 
D-TAN-254

HTRE-II TREATMENT SLLUDGE

Liw ContactiH

\section{G. RADIONUCLIDE CONCENTRATIONS}

\begin{tabular}{|c|c|c|c|c|c|c|}
\hline & \multicolumn{3}{|c|}{ Specific Activity } & \multirow[b]{2}{*}{ Units } & \multirow[b]{2}{*}{ Basis Code(s) } & \multirow[b]{2}{*}{ Basis Desc. lif other } \\
\hline Isotope & Typical & Lower Limit & Upper Limit & & & \\
\hline Sr-90 & $3.4000 \mathrm{E}-08$ & & & $\mathrm{Ci} / \mathrm{g}$ & j & UNK \\
\hline Cs-137 & $2.8000 \mathrm{E}-08$ & & & $\mathrm{Ci} / \mathrm{g}$ & $\mathrm{J}$ & UNK \\
\hline$A m-241$ & $1.8000 \mathrm{E}-10$ & & & $\mathrm{Ci} / \mathrm{g}$ & $j$ & UNK \\
\hline $\begin{array}{l}\text { A. Passiv } \\
\text { B. Segme } \\
\text { C. Passiv } \\
\text { D. Radioc } \\
\text { E. Mass }\end{array}$ & $\begin{array}{l}\text { tive Neutron } \\
\text { d Gamma Sca } \\
\text { tive Neutron } \\
\text { jical Analysis } \\
\text { trometry }\end{array}$ & $\begin{array}{l}\text { PAN) } \\
\text { (SGS) } \\
\text { Segmented Ga }\end{array}$ & la Scan $(P)$ & SGSI & $\begin{array}{l}\text { Accountability } \\
\text { 3. Calorimetry } \\
\text { \%. Multiple Energy } \\
\text { Process Knowle } \\
\text { \%. Other }\end{array}$ & $\begin{array}{l}\text { Samma Analysis (MEGAS) } \\
\text { lge }\end{array}$ \\
\hline
\end{tabular}




\section{H. MATRIX CHARACTERISTICS}

Primary Matrix Code: S3121

Description: Homogeneous Solids: Inorganic Homogeneous Solids: Inorganic Sludges: Wastewater Treatment Sludges

Combustibility: $\bigcirc$ Combustible (>90\%)

Mixed $(10 \%-90 \%)$

O Noncombustible $(<10 \%)$

\section{MATRIX COMPOSITION}

\begin{tabular}{|c|c|c|c|c|c|c|}
\hline & & & \multicolumn{4}{|c|}{ Composition } \\
\hline Code & Matrix Description & Materlal & Typlcal & LL & UL & Units \\
\hline $\mathbf{S 3 1 2 1}$ & Homogeneous Solids.Inorganic Homogeneous Solids.Inorganic Sludges.Wastewater & HTRE-III Treatment Sludge & & & & \\
\hline
\end{tabular}


HWW ANA

$\because+4)$ Not Gen active

\section{J. HAZARDOUS CONSTITUENTS}

\begin{tabular}{|c|c|c|c|c|c|c|c|c|}
\hline & & & \multicolumn{4}{|c|}{ Concentration } & & \\
\hline Code & Hazardous Waste Description & Treatment Subcategory & Typical & $\mathbf{L L}$ & UL & Units & Basis & Conc. Type \\
\hline$\overline{D 008}$ & Lead & & 21.6 & & & $\% w t$ & $E$ & Total \\
\hline$\overline{D 009}$ & Mercury & High-mercury inorganic subcategory $(>=260$ & 5.5 & & & $\% w t$ & $E$ & Total \\
\hline
\end{tabular}




\section{$\therefore$ ID-TAN-254 \\ LiLw Cöntact-H \\ K. POLYCHLORINATED BIPHENYLS (PCBS)}

HTRE-IIITREATMENT SLUDGE

ID - TAN -254

Is this waste stream PCB contaminated?

Y Yes

No

Unknown

If yes, what portion of the waste is PCB contaminated (volume \%):

If $<100 \%$, can the PCB contaminated waste be segregated and treated separately?

What is the PCB concentration in ppm?

If only a portion of the waste is PCB contaminated, report values for contaminated portion.

\section{TREATMENT PLANS}

\begin{tabular}{|c|c|c|c|c|c|c|c|c|c|c|c|}
\hline $\begin{array}{c}\text { Opt. } \\
\#\end{array}$ & $\begin{array}{c}\text { Media Type } \\
\text { (If more than one) }\end{array}$ & $\begin{array}{c}\text { Matrix } \\
\text { Code }\end{array}$ & $\begin{array}{c}\text { Vol. } \% \\
\text { IIf }\end{array}$ & Step & JIT & sc & CIF & $\begin{array}{l}\text { Trans. } \\
\text { Miles }\end{array}$ & $\begin{array}{l}\text { Facility } \\
\text { Abbr. }\end{array}$ & Unit Name & Comments \\
\hline \multirow[t]{3}{*}{1} & & $\$ 3121$ & & $a$ & 0 & 0 & $1.00 E+00$ & & WROC & Hg Retort & \\
\hline & & & & $\mathbf{b}$ & 0 & 0 & $1.00 E+00$ & & WERF & Stabilization - Portland Cement & \\
\hline & & & & c & 0 & 0 & $1.00 E+00$ & & RWMC & Disposal - Contact-Handled & \\
\hline
\end{tabular}

Note: Where provided, media percentages are rough estimates used to facilitate treatment planning. They do not necessarily imply an accurate knowledge of waste stream composition, and were not derived for use outside this conceptual planning exercise.

Describe any special or unique technical concerns related to the treatment of this waste stream that would impact the use or implementation of standard hazardous waste treatment methods.

A treatability variance is required unless Mercury retorting is used. 


\section{A. IDENTIFICATION AND DESCRIPTION}

Waste Stream ID: ID-TRA-127

W. S. Name:

TRA SCINTILLATION COCKTAILS (ALPHA <10)

Content Code:

FFCA ID:

$$
\text { IN-W021 }
$$

\section{Waste Stream Description:}

Waste consists of scintillation cocktails and solvent extraction waste generated from

experiments. This waste is $49 \%$ water and $49 \%$ xylene. It is contaminated with low-level alpha

emitters and some beta, gamma activity due to radiotracers. In addition to the radionuclides

reported, the total actinides are $3.9 \mathrm{E}-9 \mathrm{Ci} / \mathrm{g}$.

\section{B. GENERATION SITE/PROCESS DESCRIPTION}

The following questions, concerning the generation site and process, should be answered even if the waste stream is no longer generated.

Name of Site Generating Waste (e.g., INEL, Rocky Flats, etc.): Idaho National Engineering Laboratory

Location of activities (Area and Building):

Test Reactor Area

Operations (functions) performed in building: Laboratory Operations

Laboratory experiments.
Description of Process Generating Waste: 


\section{ID-TRA +127 \\ Contac:-H \\ C. PROJECTED WASTE GENERATION}

Is this waste stream still generated?

If yes, when is the expected generation termination date? 


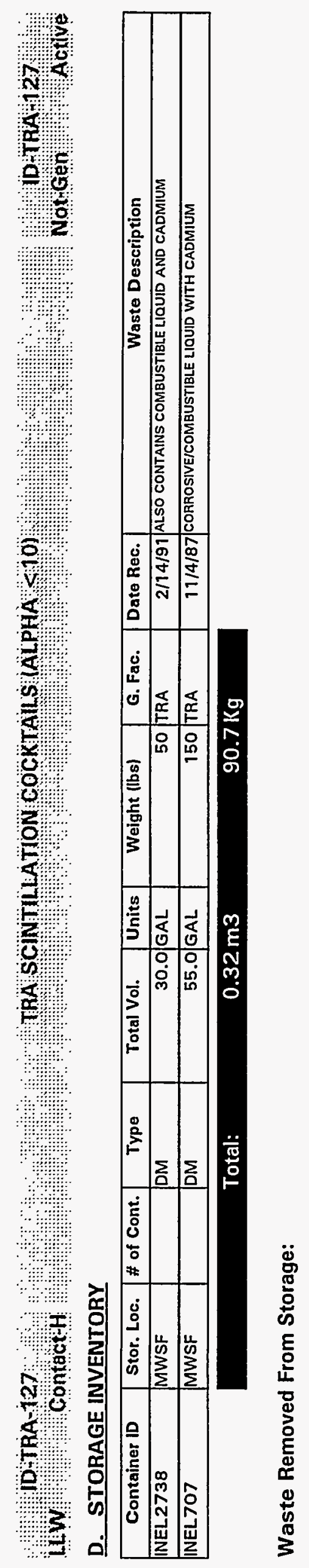


LWW: ID-TRA 127

\section{E. RADIATION CHARACTERISTICS}

Radioactive Waste Type:

\begin{tabular}{|ll|}
\hline O LLW & Oa-LLW O TRU \\
OHLW & ONon-Rad \\
\hline
\end{tabular}

Mixed Waste:

Handling:

\begin{tabular}{|l|}
\hline Yes ONo \\
\hline Contact O Remote
\end{tabular}

Radionuclide Contamination Accessiblity:

External Surface OYes $O$ No OUnknown

Internal Surface $O$ Yes $O$ No O Unknown

Dispersed Through Matrix

OYes

\section{Activity Levels}

Transuranic Alpha Activity:

Uranium/Thorium Alpha Activity:

Beta/Gamma Activity:

Surface Neutron Activity:

Total Activity:

\begin{tabular}{|c|c|c|}
\hline & Units: & \\
\hline & Units: & \\
\hline$<200$ & \multicolumn{2}{|c|}{ @ surface } \\
\hline & @ 1-m & Units: $m R / h r$ \\
\hline & Units: & \\
\hline & $\mathrm{nCi} / \mathrm{g}$ & \\
\hline
\end{tabular}

\section{F. TRU ALPHA ACTIVITY DISTRIBUTION}

This waste stream is not identified as a TRU or a-LLW. 
IDTTRA 127

TRA SC̈INTLLATIONNCOGKTALS IALPHA $<10$

LWW ContactH

$\because$

(n)

\section{G. RADIONUCLIDE CONCENTRATIONS}

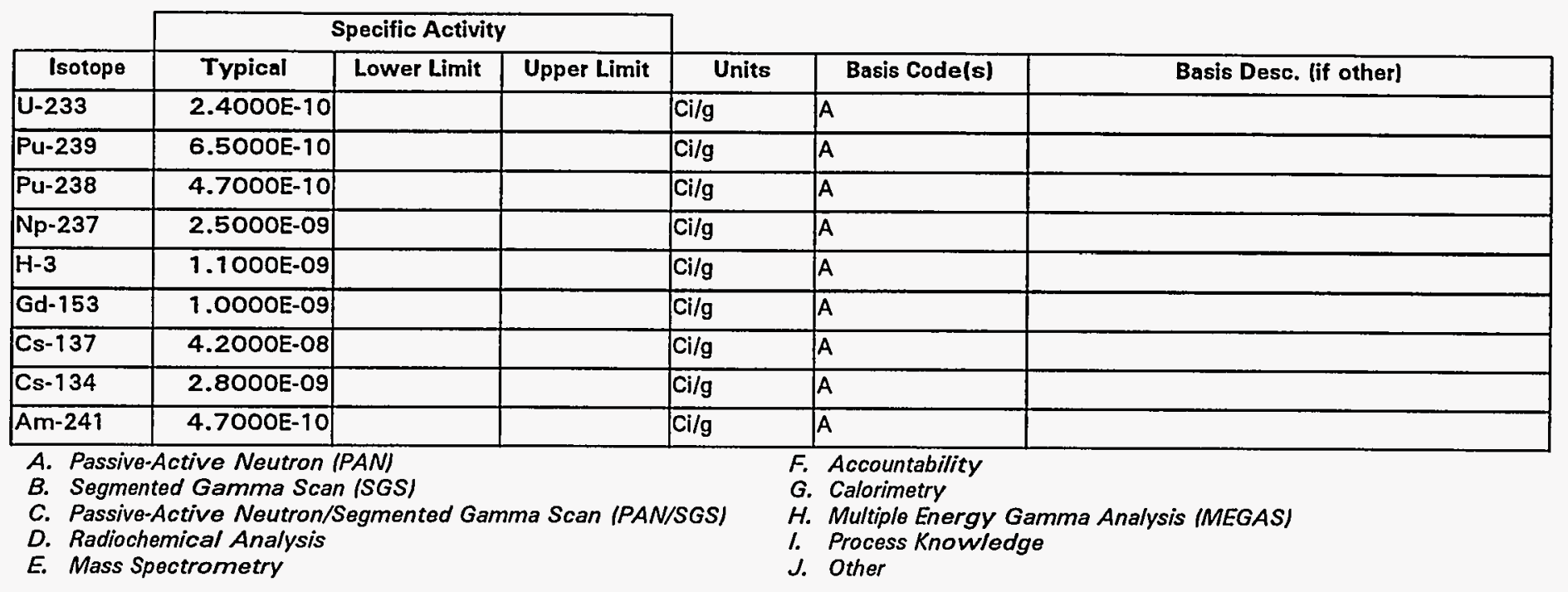




\section{H. MATRIX CHARACTERISTICS}

Primary Matrix Code: $L 2120$

Description: Organic Liquids: Aqueous/Organic Liquids: Aqueous/Nonhalogenated Organic Liquids:

Combustibility:

Combustible (>90\%)

Mixed (10\%-90\%)

Noncombustible $(<10 \%)$

\section{MATRIX COMPOSITION}

\begin{tabular}{|c|c|c|c|c|c|c|}
\hline & & & \multicolumn{4}{|c|}{ Composition } \\
\hline Code & Matrix Description & Material & Typlcal & LL & UL & Units \\
\hline$\lcm{2120}$ & Organic Liquids.Aqueous/Organic Liquids.Aqueous/Nonhalogenated Organic Liquids. & Xylene & 49 & & & $\%$ vol \\
\hline$L 2120$ & Organic Liquids.Aqueous/Organic Liquids.Aqueous/Nonhalogenated Organic Liquids. & Water & 49 & & & $\%$ vol \\
\hline
\end{tabular}


ID TRA-127

Uliw GontactH

TRA SCINTLLATION COCKTALS LALPHA $\&$ IO)

J. HAZARDOUS CONSTITUENTS

\begin{tabular}{|c|c|c|c|c|c|c|c|c|}
\hline & & & \multicolumn{4}{|c|}{ Concentration } & \multirow[b]{2}{*}{ Basis } & \multirow[b]{2}{*}{ Conc. Type } \\
\hline Code & Hazardous Waste Description & Treatment Subcategory & Typical & $\mathbf{L L}$ & $\mathbf{U L}$ & Units & & \\
\hline$\overline{D 002}$ & Corrosive & Corrosive characteristic waste that are manag & & & & & c & Total \\
\hline$D 006$ & Cadmium & & 7.5 & & & $\mathrm{ppm}$ & $\bar{c}$ & Total \\
\hline$\overline{D 022}$ & Chloroform & Chloroform managed in non-CWA/non-CWA-8 & 282 & & & ppm & c & Total \\
\hline
\end{tabular}




\section{ID-TRA $\div 127$}

\section{LiW Contact H}

TRA SCINTILLATION COCKTAILS (ALIPHA $<10)$

ID-TAA-127:

K. POLYCHLORINATED BIPHENYLS (PCBS)
Is this waste stream PCB contaminated?
OYes
ONo
Unknown

If yes, what portion of the waste is PCB contaminated (volume \%):

If $<100 \%$, can the PCB contaminated waste be segregated and treated separately?

What is the PCB concentration in ppm?

\section{TREATMENT PLANS}

\begin{tabular}{|c|c|c|c|c|c|c|c|c|c|c|c|}
\hline $\begin{array}{c}\text { Opt. } \\
\#\end{array}$ & $\begin{array}{c}\text { Media Type } \\
\text { (If more than one) }\end{array}$ & $\begin{array}{c}\text { Matrix } \\
\text { Code } \\
\end{array}$ & $\begin{array}{c}\text { Vol. } \% \\
\text { IIf }\end{array}$ & Step & JIT & sc & CIF & $\begin{array}{l}\text { Trans. } \\
\text { Miles } \\
\end{array}$ & $\begin{array}{l}\text { Facility } \\
\text { Abbr. }\end{array}$ & Unit Name & Comments \\
\hline 1 & & $\mathrm{~L} 2100$ & & $\bar{a}$ & ( & 0 & $1.00 E+00$ & & AMWTF & $\mathrm{CH}$ - Opening \& Sorting & \\
\hline & & & & $\mathbf{b}$ & 0 & 0 & $1.00 E+00$ & & AMWTF & $\mathrm{CH}$ - Incineration/Thermal Desorption & \\
\hline & & & & c & 0 & O & $1.00 \mathrm{E}+00$ & & AMWTF & CH - Vitrification & \\
\hline & & & & d & 0 & 0 & $1.00 E+00$ & & SCDF & Dlsposal - Contact Handled & \\
\hline
\end{tabular}

Note: Where provided, media percentages are rough estimates used to facilitate treatment planning. They do not necessarily imply an accurate knowledge of waste stream composition, and were not derived for use outside this conceptual planning exercise.

Describe any special or unique technical concerns related to the treatment of this waste stream that would impact the use or implementation of standard hazardous waste treatment methods.

Alpha Containment. 


\section{IÖ-TAN-163}

a-liWW Contact

\section{A. IDENTIFICATION AND DESCRIPTION}

Waste Stream ID: ID-TAN-163

W. S. Name:

Content Code:

FFCA ID:

\begin{tabular}{|l|}
\hline TAN DECON HEAVY METAL SOLIDS AND DEBRIS \\
\hline IN-W039 \\
\hline
\end{tabular}

\section{Waste Stream Description:}

This waste stream is dry solids (appears as sand) water rinsed from contaminated materials.

The waste is from decontamination and decommissioning of a decontamination facility. High

alpha content. 

ID T TAN -1.63
a-LiW Contact-H
C. PROJECTED WASTE GENERATION

Is this waste stream still generated?

If yes, when is the expected generation termination date?

Current Projections

\section{Historical Projections}


ID-TAN-163

TAN DECON HEAVYMETAL SOLIDS AND DEBRIS

a-LiW Contagt H

D. STORAGE INVENTORY

\begin{tabular}{|c|c|c|c|c|c|c|c|c|c|}
\hline Container ID & Stor. Loc. & $\#$ of Cont. & Type & Total Vol. & Units & Weight l(bs) & G. Fac. & Date Rec. & Waste Description \\
\hline INEL2324 & MWSF & & DM & 85. & GAL & 580 & TAN & $4 / 19 / 90$ & DRYIPODERED SLUDGE WITH METALS \\
\hline
\end{tabular}

Waste Removed From Storage: 


\section{E. RADIATION CHARACTERISTICS}

\section{F. TRU ALPHA ACTIVITY DISTRIBUTION}

Radioactive Waste Type:

\begin{tabular}{|ll|}
\hline OllW & $O_{\text {a-LLW }} \bigcirc \mathrm{TRU}$ \\
OhLW & $\bigcirc_{\text {Non-Rad }}$ \\
\hline
\end{tabular}

Mixed Waste:

\begin{tabular}{|l|}
\hline O Yes O No \\
\hline O Contact O Remote \\
\hline
\end{tabular}

Handling:

Contact O Remote

Radionuclide Contamination Accessiblity:

\begin{tabular}{rlrlr|} 
External Surface & OYes & O No & OUnknown \\
Internal Surface & OYes & O No & O Unknown \\
\cline { 2 - 5 } Dispersed Through Matrix & OYes & O No & O Unknown \\
\cline { 2 - 3 } &
\end{tabular}

\section{Activity Levels}

Transuranic Alpha Activity: $2.1 \mathrm{E}+1$ Units: $\mathrm{nCi} / \mathrm{g}$ Uranium/Thorium Alpha Activity: $4.8 \mathrm{E}-2$ Units: $\mathrm{nCi} / \mathrm{g}$

Beta/Gamma Activity: $<200$ @ surface

r<50 @ 1-m Units: mR/hr

Surface Neutron Activity:

Total Activity: Units: $\mathrm{nCi} / \mathrm{g}$ 
ID TAN 163

alliW Contact-H

ID TAN 163

\section{G. RADIONUCLIDE CONCENTRATIONS}

\begin{tabular}{|c|c|c|c|c|}
\hline & \multicolumn{3}{|c|}{ Specific Activity } & \multirow[b]{2}{*}{ Units } \\
\hline Isotope & Typical & Lower Limit & Upper Limit & \\
\hline $\mathrm{U}-238$ & $3.6300 \mathrm{E}-11$ & & & $\mathrm{Ci} / \mathrm{g}$ \\
\hline U-235 & $1.1300 \mathrm{E}-11$ & & & $\mathrm{Ci} / \mathrm{g}$ \\
\hline $\mathrm{U}-234$ & $4.4500 \mathrm{E}-10$ & & & $\mathrm{Ci} / \mathrm{g}$ \\
\hline Sr-90 & $7.6000 \mathrm{E}-09$ & & & $\mathrm{Ci} / \mathrm{g}$ \\
\hline Pu-239 & $1.5000 \mathrm{E}-08$ & & & $\mathrm{Ci} / \mathrm{g}$ \\
\hline$P u-238$ & 3.7000E-09 & & & $\mathrm{Ci} / \mathrm{g}$ \\
\hline$K-40$ & 1.1000 E- 14 & & & $\mathrm{Ci} / \mathrm{ml}$ \\
\hline Eu-155 & $4.4900 \mathrm{E}-15$ & & & $\mathrm{Ci} / \mathrm{ml}$ \\
\hline Eu-154 & $4.9900 \mathrm{E}-15$ & & & $\mathrm{Ci} / \mathrm{ml}$ \\
\hline Eu-152 & $2.9500 \mathrm{E}-15$ & & & $\mathrm{Ci} / \mathrm{ml}$ \\
\hline Cs-134 & $7.2400 \mathrm{E}-15$ & & & $\mathrm{Ci} / \mathrm{ml}$ \\
\hline Co-60 & $4.0000 \mathrm{E}-13$ & & & $\mathrm{Ci} / \mathrm{ml}$ \\
\hline \multicolumn{5}{|l|}{$\mathrm{Cm}-244$} \\
\hline$A M-241$ & 4.7000E-09 & & & $\mathrm{Ci} / \mathrm{g}$ \\
\hline $\mathrm{Ag}-10 \mathrm{Bm}$ & 7.0700E-15 & & & $\mathrm{Ci} / \mathrm{ml}$ \\
\hline
\end{tabular}

A. Passive-Active Neutron (PAN)

B. Segmented Gamma Scan (SGS)

C. Passive-Active Neutron/Segmented Gamma Scan (PAN/SGS)

D. Radiochemical Analysis

E. Mass Spectrometry

\begin{tabular}{|l|l|}
\hline \multicolumn{1}{|c|}{ Basis Code(s) } & \\
\hline$D ; H$ & \\
\hline$D ; H$ & \\
\hline$D ; H$ & \\
\hline$D ; H$ & \\
\hline$D ; H$ & \\
\hline$D ; H$ & \\
\hline$D ; H$ & \\
\hline$D ; H$ & \\
\hline$D ; H$ & \\
\hline$D ; H$ & \\
\hline$D ; H$ & \\
\hline$D ; H$ & \\
\hline$D ; H$ & \\
\hline$D ; H$ & \\
\hline$D ; H$ & \\
\hline
\end{tabular}

F. Accountability

G. Calorimetry

H. Multiple Energy Gamma Analysis (MEGAS)

l. Process Knowledge

J. Other

(a)

$$
\text { r }
$$




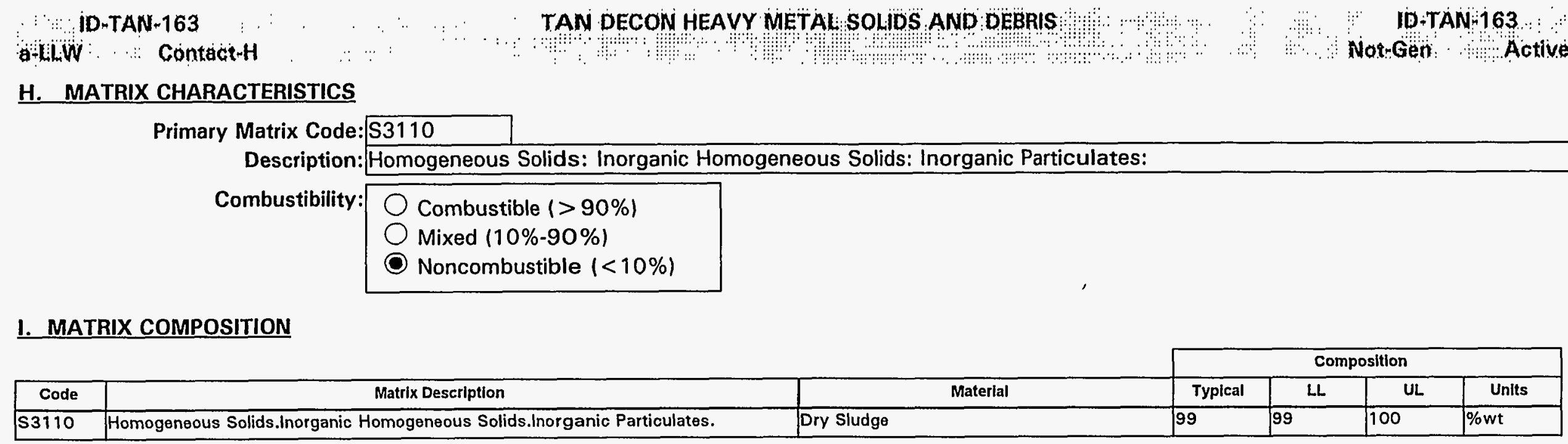


IDTANAN-163

TAN DECON HEAVY METAL SOLIDS ANOD DEBRIS

G:LWW Contact H

\section{J. HAZARDOUS CONSTITUENTS}

\begin{tabular}{|c|c|c|c|c|c|c|c|c|}
\hline Code & Hazardous Waste Description & Treatment Subcategory & Typical & LL & UL & Units & Basis & Conc. Type \\
\hline$\overline{D 005}$ & Barium & & 266 & & & $\mathrm{mg} / \mathrm{kg}$ & $\overline{\mathrm{D} 2}$ & Total \\
\hline D006 & Cadmium & & 84.4 & & & $\mathrm{mg} / \mathrm{kg}$ & $\mathrm{D2}$ & Total \\
\hline$D 007$ & Chromium & Chromium (Total) & 864 & & & $\mathrm{mg} / \mathrm{kg}$ & $\overline{D 2}$ & Total \\
\hline$\overline{D 008}$ & Lead & & 158 & . & & $\mathrm{mg} / \mathrm{kg}$ & $\mathrm{D2}$ & Total \\
\hline D009 & Mercury & Low mercury $<260 \mathrm{mg} / \mathrm{kg}$ total mercury & 240 & & & $\mathrm{mg} / \mathrm{kg}$ & $\overline{D 2}$ & Total \\
\hline D011 & Silver & & 74.4 & & & $\mathrm{mg} / \mathrm{kg}$ & $\overline{\mathrm{D} 2}$ & Total \\
\hline
\end{tabular}




\section{ID-TAN-163}

\section{aLiLW Contact-H}

K. POLYCHLORINATED BIPHENYLS (PCBS)

Is this waste stream PCB contaminated?

OYes O No O Unknown

If yes, what portion of the waste is PCB contaminated (volume \%):

If $<100 \%$, can the PCB contaminated waste be segregated and treated separately?

What is the PCB concentration in ppm?

\section{TREATMENT PLANS}

\begin{tabular}{|c|c|c|c|c|c|c|c|c|c|c|c|}
\hline $\begin{array}{c}\text { Opt. } \\
\#\end{array}$ & $\begin{array}{c}\text { Media Type } \\
\text { (If more than one) }\end{array}$ & $\begin{array}{c}\text { Matrix } \\
\text { Code }\end{array}$ & $\begin{array}{l}\text { Vol. \% } \\
\text { lif }\end{array}$ & Step & JiT & sc & CIF & $\begin{array}{l}\text { Trans. } \\
\text { Miles }\end{array}$ & $\begin{array}{l}\text { Facility } \\
\text { Abbr. }\end{array}$ & Unit Name & Comments \\
\hline 1 & & S3110 & & $\mathbf{a}$ & 0 & 0 & $1.00 E+00$ & & AMWTF & $\mathrm{CH}$ - Opening \& Sorting & \\
\hline & & & & $\mathbf{b}$ & () & 0 & $1.00 E+00$ & & AMWTF & $\mathrm{CH}$ - Sizing & \\
\hline & & & & c & O & 0 & $1.00 E+00$ & & AMWTF & $\mathrm{CH}$ - Incineration/Thermal Desorption & \\
\hline & & & & d & 0 & $\mathrm{O}$ & $1.00 E+00$ & & AMWTF & CH - Vitrification & \\
\hline & & & & e & 0 & 0 & $1.00 E+00$ & & TRANS & Transport - TRUPACT & \\
\hline & & & & $f$ & 0 & 0 & $1.00 E+00$ & & WIPP & Disposal & \\
\hline
\end{tabular}

Note: Where provided, media percentages are rough estimates used to facilitate treatment planning. They do not necessarily imply an accurate knowledge of waste stream composition, and were not derived for use outside this conceptual planning exercise.

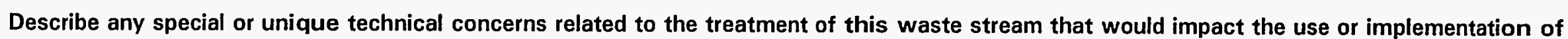
standard hazardous waste treatment methods. 


\section{ind}

Waste Stream ID: CH-ANL-503T

\section{W. S. Name:}

Content Code:

FFCA ID:

\section{TRU WASTE USED PRE-FILTERS}

\section{Waste Stream Description:}

This waste stream consists of metal or wood framed pre-filters. Pre filters are $2^{\prime} \times 2^{\prime} \times 0.5^{\prime}$.

The filters have screen mesh covering high efficiency filtering media. The concentration of radioisotopes and RCRA metals varies in each filter. These filters were generated from the deontamination of the analytical hot cells in 1993 and 1994.

\section{B. GENERATION SITE/PROCESS DESCRIPTION}

The following questions, concerning the generation site and process, should be answered even if the waste stream is no longer generated.

Name of Site Generating Waste (e.g., INEL, Rocky Flats, etc.): Idaho National Engineering Laboratory - Argonne National Laboratory West

Location of activities (Area and Building):

Bldg ANL-752, Analytical Laboratory

Operations (functions) performed in building:

Typical laboratory operations, analytical and radiochemistry. The facility also houses and experimental fuels casting lab, and areas for small-scale testing of experimental processes related to nuclear fuels fabrication. Wastes were generated primarily from hot cell refurbishment.

Description of Process Generating Waste:

Pre-filters are used to control airborne radioactive particulate emissions from hot cells, hot repair areas, analytical lab hoods and other radioactively contaminated systems. Refurbishment of hot cells and hot repair areas has generated lead, cadmium, and chromium dusts from paint removal, stainless steel cutting, and fuel experiment dissolution decontamination. The filters trap the metals in the filter media in concentrations high enough to classify the filters as mixed waste. 


\section{PROJECTED WASTE GENERATION}

Is this waste stream still generated?

If yes, when is the expected generation termination date?

\section{OYes ONo}

Current Projections

\begin{tabular}{|c|c|c|c|c|c|}
\hline Est. Date & Start Year & Interval & $\operatorname{Vol}(\mathrm{m} 3)$ & Mass (kg) & Source of Estimate \\
\hline $4 / 26 / 95$ & 1995 & 1 & 0.17 & 72.00 & 1995-MWR \\
\hline $4 / 26 / 95$ & 1996 & 1 & 0.17 & 72.00 & 1995-MWR \\
\hline $4 / 26 / 95$ & 1997 & 1 & 0.17 & 72.00 & 1995-MWR \\
\hline $4 / 26 / 95$ & 1998 & 1 & 0.17 & 72.00 & 1995-MWR \\
\hline $4 / 26 / 95$ & 1999 & 1 & 0.17 & 72.00 & 1995-MWR \\
\hline \multicolumn{6}{|c|}{ Historical Projections } \\
\hline Est. Date & Start Year & Interval & Vol (m3) & Mass (kg) & Source of Estimate \\
\hline 2/4/94 & 1994 & 1 & 0.09 & 21.00 & 1994-DSTP \\
\hline
\end{tabular}




\section{CH-ANL-503T}

TRU W Remote:H

TRU WASTE USED PRE-FILTERS

CHAANL-5O3T

\section{STORAGE INVENTORY}

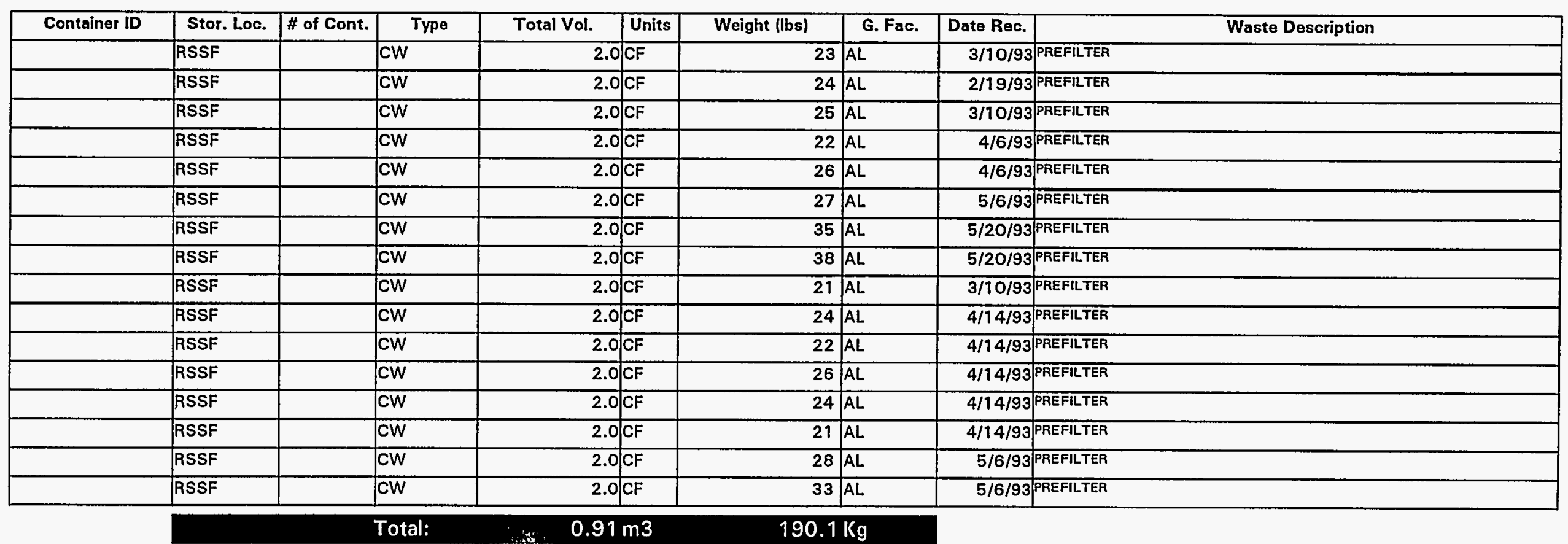

Waste Removed From Storage: 


\section{CH-ANL-503T \\ Thiu Remote-H \\ E. RADIATION CHARACTERISTICS}

Radioactive Waste Type:

\begin{tabular}{|ll|}
\hline OLLW & Oa-LLW O TRU \\
O HLW & ONon-Rad \\
\hline
\end{tabular}

Mixed Waste:

Handling

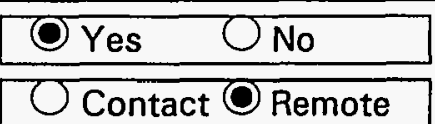

Radionuclide Contamination Accessiblity:

External Surface

Internal Surface

Dispersed Through Matrix

\begin{tabular}{|c|c|c|}
\hline OYes & No & OUnknown \\
\hline OYes & ONo & OUnknown \\
\hline OYes & ONo & OUnknown \\
\hline
\end{tabular}

\section{Activity Levels}

Transuranic Alpha Activity:

Uranium/Thorium Alpha Activity:

Beta/Gamma Activity

Surface Neutron Activity:

Total Activity:

\begin{tabular}{|c|c|c|}
\hline $5.3 E-17$ & \multicolumn{2}{|c|}{ Units: $\mathrm{Ci} / \mathrm{g}$} \\
\hline & Units: [ & \\
\hline 5188 & \multicolumn{2}{|c|}{ @ surface } \\
\hline 510 & @ 1-m & Units: $\mathrm{mR} / \mathrm{hr}$ \\
\hline & Units: [ & \\
\hline & $\mathrm{nCi} / \mathrm{g}$ & \\
\hline
\end{tabular}

\section{F. TRU ALPHA ACTIVITY DISTRIBUTION}


: $\because:$

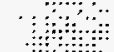

$\because$

$\therefore \therefore$

$\therefore$

\#

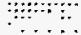

$\because$

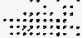

$\because$

$\therefore$

D.:

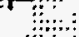

\%

. $\therefore$

- $\therefore$

$\therefore$

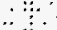

$\because$

$\because$ 


\section{H. MATRIX CHARACTERISTICS}

Primary Matrix Code: S5410

Description: Debris Waste: Heterogeneous Debris: Composite Filter Debris:

Combustibility:

O Combustible (>90\%)

Mixed $(10 \%-90 \%)$

Noncombustible $(<10 \%)$

\section{MATRIX COMPOSITION}

\begin{tabular}{|c|c|c|c|c|c|c|}
\hline & & & \multicolumn{4}{|c|}{ Composition } \\
\hline Code & Matrix Description & Materlal & Typlcal & LL & UL & Units \\
\hline$\$ 5410$ & Debris Waste.Heterogeneous Debris.Composite Filter Debris. & Dirt/Dust & 5 & & & $\%$ vol \\
\hline$\$ 5410$ & Debris Waste.Heterogeneous Debris.Composite Filter Debris. & Stainless Steel & 5 & & & $\%$ vol \\
\hline$\$ 5410$ & Debris Waste.Heterogeneous Debris.Composite Filter Debris. & Plastic & 4 & & & $\%$ vol \\
\hline$\$ 5410$ & Debris Waste.Heterogeneous Debris.Composite Filter Debris. & Wood Housing & 80 & & & $\%$ vol \\
\hline$\$ 5410$ & Debris Waste.Heterogeneous Debris.Composite Filter Debris. & Filter Media & 6 & & & $\%$ vol \\
\hline
\end{tabular}




\begin{tabular}{|c|c|c|c|c|c|c|c|}
\hline 1ełol & 10 & ven $7 "+911$ & $6.0 \varepsilon$ & & & peo7 & 8000 \\
\hline peol & 10 & $y^{\prime} 6 \mathrm{n} \mid \angle \cdot \angle L 6 L$ & 8.9 & & (|в201) un!mod40 & wn!̣เมرบว & $\angle 000$ \\
\hline |ejol| & 10 & $y / 6 n \mid 9: 8 L$ & $2 \cdot 0$ & & & wn!upes & 9000 \\
\hline ed $\Lambda_{\perp} \cdot$ ouos & s!seg & sụun & 77 & |ro!d $\Lambda_{\perp}$ & 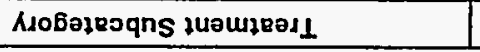 & uo!nd!ysea ofse $M$ snopjezeH & өроэ \\
\hline
\end{tabular}


CH-ANL-503T

Thü Memate-H

K. POLYCHLORINATED BIPHENYLS (PCBS)

Is this waste stream PCB contaminated?

\section{OYes O No O Unknown}

If yes, what portion of the waste is PCB contaminated (volume \%):

If $<100 \%$, can the PCB contaminated waste be segregated and treated separately?

What is the PCB concentration in ppm?

If only a portion of the waste is $P C B$ contaminated, report values for contaminated portion.

\section{TREATMENT PLANS}

\begin{tabular}{|c|c|c|c|c|c|c|c|c|c|c|c|}
\hline $\begin{array}{c}\text { Opt. } \\
\#\end{array}$ & $\begin{array}{c}\text { Media Type } \\
\text { (If more than one) }\end{array}$ & $\begin{array}{l}\text { Matrix } \\
\text { Code }\end{array}$ & $\begin{array}{l}\text { Vol. \% } \\
\text { IIf }\end{array}$ & Step & JIT & sc & CIF & $\begin{array}{l}\text { Trans. } \\
\text { Miles }\end{array}$ & $\begin{array}{l}\text { Facility } \\
\text { Abbr. }\end{array}$ & Unit Name & Comments \\
\hline \multirow[t]{8}{*}{1} & Filter Media & $\mathbf{S 5 4 1 0}$ & 95.000 & $\bar{a}$ & 0 & 0 & $1.00 E+00$ & & CPP659* & Extraction - HEPA Filter Leach & \\
\hline & & & & $\mathbf{b}$ & 0 & 0 & $1.00 E+00$ & & RWMC & Disposal - Remote-Handled - & \\
\hline & Wood Frames & $\mathbf{5 5 3 2 0}$ & 5.000 & $\bar{a}$ & $\mathbf{0}$ & $\bar{T}$ & $1.00 E+00$ & & AMWTF & $\mathrm{CH}$ - Opening \& Sorting & \\
\hline & & & & $\mathbf{b}$ & $\mathbf{O}$ & D & $1.00 E+00$ & & AMWTF & $\mathrm{CH}$ - Sizing & \\
\hline & & & & c & () & $J$ & $1.00 E+00$ & & AMWTF & $\mathrm{CH}$ - Incineration/Thermal Desorption & \\
\hline & & & & d & 8 & O & $1.00 E+00$ & & AMWTF & $\mathrm{CH}$ - Vitrification & \\
\hline & & & & $\bar{e}$ & 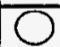 & O & $1.00 E+00$ & & TRANS & Transport - TRUPACT & \\
\hline & & & & $f$ & $\bar{x}$ & 0 & $1.00 E+00$ & & WIPP & Disposal & \\
\hline
\end{tabular}

Note: Where provided, media percentages are rough estimates used to facilitate treatment planning. They do not necessarily imply an accurate knowledge of waste stream composition, and were not derived for use outside this conceptual planning exercise.

Describe any special or unique technical concerns related to the treatment of this waste stream that would impact the use or implementation of standard hazardous waste treatment methods. 


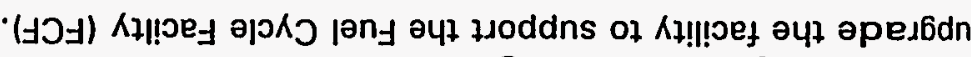

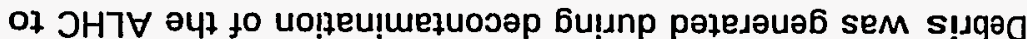

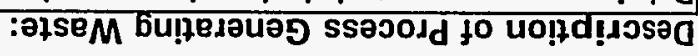

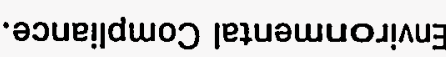

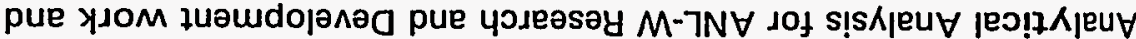

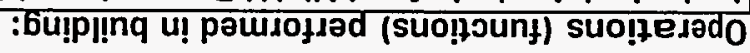

Z9L 6р19 ‘әред6d :(бu!p!!ng pue ead

$7 \mathrm{~s} \partial \mathrm{M}$

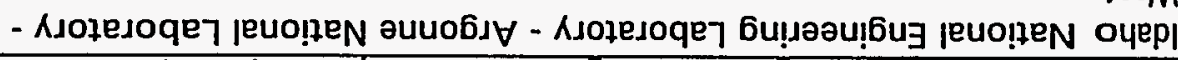

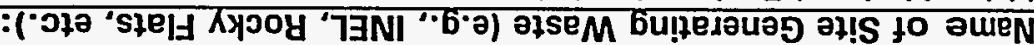

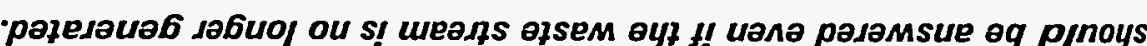

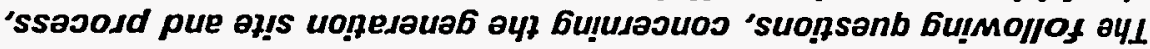

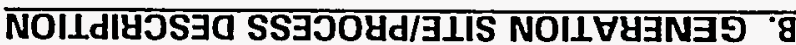

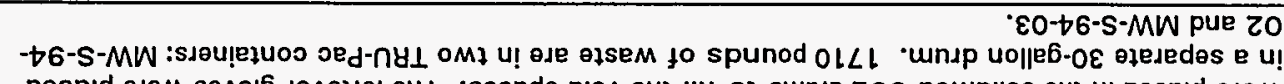

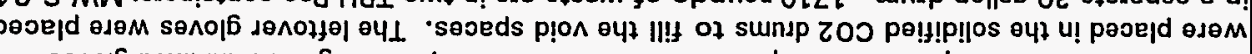

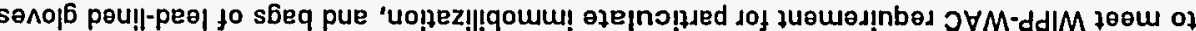

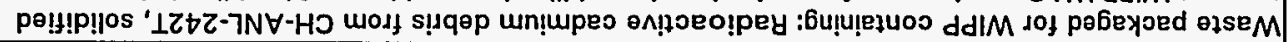

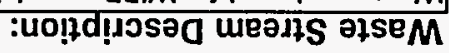

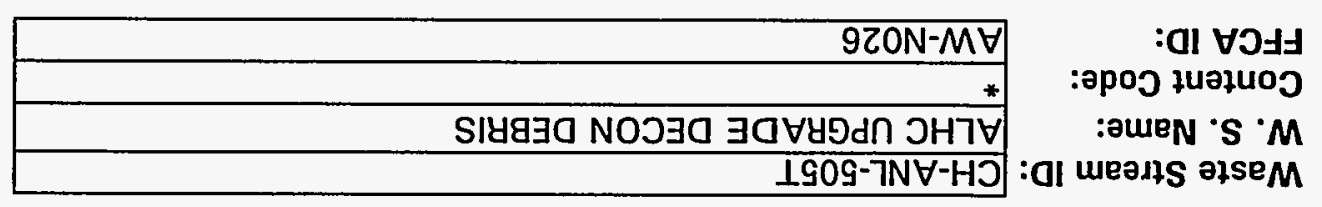


CH-ANL-505T

Thu $\therefore$ Contact-H

C. PROJECTED WASTE GENERATION

Is this waste stream still generated?

If yes, when is the expected generation termination date?
ALHC UPGRADE DECON DEBRIS

CHANL 5O5T: Still-Gen Active

\section{Current Projections}

\begin{tabular}{|r|r|r|r|r|l|}
\hline Est. Date & Start Year & Interval & \multicolumn{1}{|c|}{ Vol (m3) } & \multicolumn{1}{|c|}{ Mass (kg) } & Source of Estlmate \\
\hline $4 / 3 / 95$ & 1995 & 1 & 0.01 & 4.54 & $1995-\mathrm{MWR}$ \\
\hline $4 / 3 / 95$ & 1996 & 1 & 0.01 & 4.54 & $1995-\mathrm{MWR}$ \\
\hline $4 / 3 / 95$ & 1997 & 1 & 0.01 & 4.54 & $1995-\mathrm{MWMR}$ \\
\hline \multicolumn{5}{|c}{ Historical Projections } \\
\hline
\end{tabular}


CHANL-505T

TRU Contäctì

ALHC UPGRADE DECON DEBRIS

D. STORAGE INVENTORY

\begin{tabular}{|c|c|c|c|c|c|c|c|}
\hline Container ID & Stor. Loc. & \# of Cont. & Type & Total Vol. & Units & Weight (lbs) & G. Fac \\
\hline & RSSF & & $\mathrm{CM}$ & 41.6 & CF & 1,710 & ANL-W \\
\hline
\end{tabular}

\section{Waste Removed From Storage:}


CH+ANL-505T TRU:........ Contact-H

E. RADIATION CHARACTERISTICS

ALHC UPGRADE DEECON DEBRIS

CHAANL-505T

Radioactive Waste Type:

\begin{tabular}{|ll|}
\hline OLLW & Oa-LLW O TRU \\
O HLW & $\bigcirc$ Non-Rad \\
\hline
\end{tabular}

Mixed Waste:

Handling:

\begin{tabular}{|l|}
\hline O Yes O No \\
\hline O Contact O Remote
\end{tabular}

Radionuclide Contamination Accessiblity:

External Surface

Internal Surface

Dispersed Through Matrix

\begin{tabular}{|c|c|c|}
\hline (C) Yes & ONo & OUnknown \\
\hline OYes & ONo & OUnknown \\
\hline OYes & ONo & OUnknown \\
\hline
\end{tabular}

\section{Activity Levels}

Transuranic Alpha Activity: Uranium/Thorium Alpha Activity:

Uranium/Thorium Alpha Activity:
Beta/Gamma Activity:

4.001E-6 Units: $\mathrm{Ci} / \mathrm{g}$

6.13E-8 Units: $\mathrm{Ci} / \mathrm{g}$

$<200$ @ surface

Surface Neutron Activity: $<20$ @ 1-m Units: $\mathrm{mR} / \mathrm{hr}$

Total Activity:

$<1$ Units: $\mathrm{n} / \mathrm{cm} 2 / \mathrm{s}$ $4062 \mathrm{nCi} / \mathrm{g}$ 
CH:ANL-505T

TRU : ContactiH

\section{G. RADIONUCLIDE CONCENTRATIONS}

\begin{tabular}{|c|c|c|c|c|}
\hline & & pecific Activity & & \\
\hline Isotope & Typical & Lower Limit & Upper Limit & Units \\
\hline$M n-54$ & $1.1900 \mathrm{E}-11$ & & & $\mathrm{Ci} / \mathrm{g}$ \\
\hline$\overline{U-238}$ & $5.5700 E-12$ & $3.1600 E-12$ & $7.9700 \mathrm{E}-12$ & $\mathrm{Ci} / \mathrm{g}$ \\
\hline U-235 & $4.3200 E-11$ & $2.3400 \mathrm{E}-11$ & $6.2900 E-11$ & $\mathrm{Ci} / \mathrm{g}$ \\
\hline Pu-239 & $1.9800 E-07$ & $8.0400 E-08$ & $3.1500 E-07$ & $\mathrm{Ci} / \mathrm{g}$ \\
\hline Am-241 & 2.4900E-06 & $1.2900 \mathrm{E}-06$ & $3.6900 \mathrm{E}-06$ & $\mathrm{Ci} / \mathrm{g}$ \\
\hline $\mathrm{Y}-90$ & $4.0000 E-06$ & $3.3500 \mathrm{E}-06$ & $1.5700 \mathrm{E}-05$ & $\mathrm{Ci} / \mathrm{g}$ \\
\hline Sr-90 & $4.0000 E-06$ & $3.3500 \mathrm{E}-06$ & $1.5700 \mathrm{E}-05$ & $\mathrm{Ci} / \mathrm{g}$ \\
\hline Eu-154 & $1.5000 \mathrm{E}-08$ & $3.3100 \mathrm{E}-09$ & $6.4200 \mathrm{E}-08$ & $\mathrm{Ci} / \mathrm{g}$ \\
\hline Eu-155 & $3.7000 \mathrm{E}-08$ & $7.5100 \mathrm{E}-09$ & $1.8800 \mathrm{E}-07$ & $\mathrm{Ci} / \mathrm{g}$ \\
\hline $\mathrm{Ce}-144$ & $7.3000 \mathrm{E}-08$ & $2.1600 \mathrm{E}-08$ & $5.2600 \mathrm{E}-08$ & $\mathrm{Ci} / \mathrm{g}$ \\
\hline Cs-134 & $4.3000 \mathrm{E}-08$ & $1.1900 \mathrm{E}-08$ & $1.2900 \mathrm{E}-07$ & $\mathrm{Ci} / \mathrm{g}$ \\
\hline Cs-137 & $1.8000 \mathrm{E}-06$ & $9.3300 \mathrm{E}-07$ & $7.7700 E-06$ & $\mathrm{Ci} / \mathrm{g}$ \\
\hline Rh-106 & $4.7700 E-07$ & $6.3000 \mathrm{E}-08$ & $1.4900 E-07$ & $\mathrm{Ci} / \mathrm{g}$ \\
\hline $2 r-95$ & $1.6000 \mathrm{E}-08$ & $1.6000 \mathrm{E}-08$ & $3.0000 E-08$ & $\mathrm{Ci} / \mathrm{g}$ \\
\hline Co-60 & $1.1400 E-07$ & $5.0000 \mathrm{E}-09$ & $1.4000 \mathrm{E}-07$ & $\mathrm{Ci} / \mathrm{g}$ \\
\hline
\end{tabular}

A. Passive-Active Neutron (PAN)

B. Segmented Gamma Scan (SGS)

C. Passive-Active Neutran/Segmented Gamma Scan (PAN/SGS)

D. Radiochemical Analysis

E. Mass Spectrometry

ALHC UPGRAOEE DECONDEBRIS

\begin{tabular}{|l|l|}
\hline Basis Code(s) & \\
\hline D & \\
\hline D & \\
\hline D & \\
\hline D & \\
\hline D & \\
\hline D & \\
\hline D & \\
\hline D & \\
\hline D & \\
\hline D & \\
\hline D & \\
\hline D & \\
\hline D & \\
\hline D & \\
\hline D & \\
\hline
\end{tabular}

F. Accountability

G. Calorimetry

H. Multiple Energy Gamma Analysis (MEGAS)

1. Process Knowledge

J. Other 


\section{H. MATRIX CHARACTERISTICS}

Primary Matrix Code: $\mathbf{S 5 4 0 0}$

Description: Debris Waste: Heterogeneous Debris: :

Combustibility: $\bigcirc$ Combustible (>90\%)

Mixed (10\%-90\%)

Noncombustible $(<10 \%)$

\section{MATAIX COMPOSITION}

\begin{tabular}{|c|c|c|c|c|c|c|}
\hline & & & \multicolumn{4}{|c|}{ Composition } \\
\hline Code & Matrix Description & Material & Typlcal & LL & UL & Units \\
\hline 53150 & Homogeneous Solids.Inorganic Homogeneous Solids.Solidified Homogeneous Solids. & solidified process residues & 36 & & & $\%$ vol \\
\hline$\$ 5330$ & Debris Waste.Organic Debris.Paper/Cloth Debris. & mops & 40 & & & $\%$ vol \\
\hline$\$ 5312$ & Debris Waste.Organic Debris.Plastic/Rubber Debris.Halogenated Plastic Debris & Halogenated Plastics & 1 & & & $\%$ vol \\
\hline$\$ 5313$ & Debris Waste.Organic Debris.Plastic/Rubber Debris.Nonhalogenated Plastic Debris & Plastics & 1 & & & $\%$ ovol \\
\hline 55311 & Debris Waste.Organic Debris.Plastic/Rubber Debris.Leaded Gloves/Aprons & Leaded Gloves & 22 & & & $\%$ vol \\
\hline
\end{tabular}


CHEANL-505T

TRU Contact $-H$

ALHC UPGRADE DECON DEBRIS

J. HAZARDOUS CONSTITUENTS

\begin{tabular}{|c|c|c|c|c|c|c|c|c|}
\hline & & & \multicolumn{4}{|c|}{ Concentration } & \multirow[b]{2}{*}{ Basis } & \multirow[b]{2}{*}{ Conc. Type } \\
\hline Code & Hazardous Waste Description & Treatment Subcategory & Typical & $\overline{\mathbf{L L}}$ & UL & Units & & \\
\hline$\overline{D 006}$ & Cadmium & & 20 & 1 & & $\mathrm{pm}$ & F1 & Total \\
\hline$\overline{D 007}$ & Chromium & Chromium (Total) & 100 & 32 & 300 & $\mathrm{ppm}$ & F1 & Total \\
\hline$\overline{D 008}$ & Lead & & 100 & 82 & 650000 & $\mathrm{ppm}$ & A & Total \\
\hline$\overline{D 008}$ & Lead & Radioactive lead solids & & & & & B & Total \\
\hline
\end{tabular}




\section{CH-ANL-505T}

Thu " Contactuh

\section{K. POLYCHLORINATED BIPHENYLS (PCBS)}

Is this waste stream PCB contaminated?

\section{OYes 0 No $\bigcirc$ Unknown}

If yes, what portion of the waste is PCB contaminated (volume \%):

If $<100 \%$, can the PCB contaminated waste be segregated and treated separately? OYes O No OUnknown

What is the PCB concentration in ppm?

If only a portion of the waste is $P C B$ contaminated, report values for contaminated portion.

\section{TREATMENT PLANS}

\begin{tabular}{|c|c|c|c|c|c|c|c|c|c|c|c|}
\hline $\begin{array}{c}\text { Opt. } \\
\#\end{array}$ & $\begin{array}{c}\text { Media Type } \\
\text { (If more than one) }\end{array}$ & $\begin{array}{l}\text { Matrix } \\
\text { Code }\end{array}$ & $\begin{array}{l}\text { Vol. \% } \\
\text { lif }\end{array}$ & Step & JIT & sc & CIF & $\begin{array}{l}\text { Trans. } \\
\text { Miles }\end{array}$ & $\begin{array}{l}\text { Facility } \\
\text { Abbr. }\end{array}$ & Unit Name & Comments \\
\hline 1 & & S5400 & & $\overline{\mathbf{a}}$ & 0 & 0 & $1.00 E+00$ & & TRANS & Transport - TRUPACT & \\
\hline & & & & b & 0 & 0 & $1.00 \mathrm{E}+00$ & & WIPP & Disposal & \\
\hline
\end{tabular}

Note: Where provided, media percentages are rough estimates used to facilitate treatment planning. They do not necessarily imply an accurate knowledge of waste stream composition, and were not derived for use outside this conceptual planning exercise.

Describe any special or unique technical concerns related to the treatment of this waste stream that would impact the use or implementation of standard hazardous waste treatment methods.

This is a TRU waste packaged to meet the WIPP WAC. Particulate materials were solidified for immobilization. Treatment is not planned. 
BNWWOOP

LUW Contact

\section{A. IDENTIFICATION AND DESCRIPTION}

Waste Stream ID: BN-W007

W. S. Name:

Content Code:

FFCA ID:

\section{MERCURY WASTE}

BN-W007

\section{Waste Stream Description:}

Stream consists primarily of bulk liquid mercury. It also may contian Mercury contaminated equipment, broken manometers and thermometers, and compounds containing mercury.

\section{B. GENERATION SITE/PROCESS DESCRIPTION}

The following questions, concerning the generation site and process, should be answered even if the waste stream is no longer generated.

Name of Site Generating Waste (e.g., INEL, Rocky Flats, etc.): Brookhaven National Laboratory

Location of activities (Area and Building):

These wastes are generated site wide, primarily from the Chemistry, Biology, Applied Sciences, and Medical Departments.

Operations (functions) performed in building: Analytical work performed for a variety of research experiments is responsible for the generation of most of this waste stream. A wide range of contaminants and radioactive materials may be present as this work is varied and frequently changing.

Description of Process Generating Waste: 


\section{LWw : Contact-H}

\section{PROJECTED WASTE GENERATION}

Is this waste stream still generated?

If yes, when is the expected generation termination date?

OYes ONo

Current Projections

\begin{tabular}{|r|r|r|r|r|r|}
\hline Est. Date & Start Year & \multicolumn{1}{|c|}{ Interval } & \multicolumn{1}{|c|}{ Vol $(\mathrm{m} 3)$} & \multicolumn{1}{c|}{ Mass $(\mathrm{kg})$} & \multicolumn{1}{|c|}{ Source of Estimate } \\
\hline $11 / 30 / 93$ & 1993 & 1 & 0.02 & 270.00 & $1993-\mathrm{FFCA}$ \\
\hline $11 / 30 / 93$ & 1994 & 1 & 0.02 & 270.00 & $1993-\mathrm{FFCA}$ \\
\hline $11 / 30 / 93$ & 1995 & 1 & 0.02 & 270.00 & $1993-\mathrm{FFCA}$ \\
\hline $11 / 30 / 93$ & 1996 & 1 & 0.02 & 270.00 & $1993-\mathrm{FFCA}$ \\
\hline $11 / 30 / 93$ & 1997 & 1 & 0.02 & 270.00 & $1993-\mathrm{FFCA}$ \\
\hline
\end{tabular}

Historical Projections 
:abejots wody panowəy әңseM

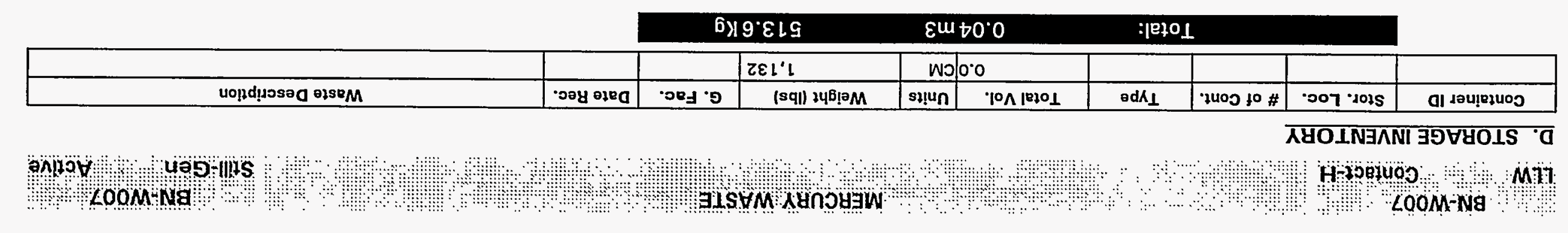


BN-WWOO7

$$
\text { LLW Contact-H }
$$

\section{E. RADIATION CHARACTERISTICS}

Radioactive Waste Type:

\begin{tabular}{|ll|}
\hline O LLW & Oa-LLW $\bigcirc$ TRU \\
O HLW & O Non-Rad \\
\hline
\end{tabular}

Mixed Waste:

Handling:

\begin{tabular}{|l|}
\hline OYes O No \\
\hline O Contact O Remote \\
\hline
\end{tabular}

Radionuclide Contamination Accessiblity:

External Surface

Internal Surface

Dispersed Through Matrix

\begin{tabular}{|}
\begin{tabular}{|ccc|}
\hline OYes & O No & OUnknown \\
\hline OYes & O No & OUnknown \\
\hline O Yes & O No & O Unknown \\
\hline
\end{tabular}
\end{tabular}

\section{Activity Levels}

Transuranic Alpha Activity: Uranium/Thorium Alpha Activity:

Beta/Gamma Activity:

Surface Neutron Activity: Total Activity:

\begin{tabular}{|c|c|c|}
\hline & Units: & \\
\hline & Units: & \\
\hline & @ surfa & \\
\hline & @1-m & Units: $\mathrm{mR} / \mathrm{hr}$ \\
\hline & Units: & \\
\hline & $\mathrm{nCi} / \mathrm{g}$ & \\
\hline
\end{tabular}

BN-WOOT Still:Gen Active

\section{F. TRU ALPHA ACTIVITY DISTRIBUTION}

This waste stream is not identified as a TRU or a-LLW. 
дачзо $\mathrm{C}$

obpolmouy ssojold \%

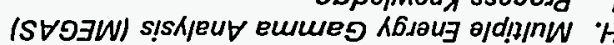

Anoumpoles

A.!!!qezunoos $\because$

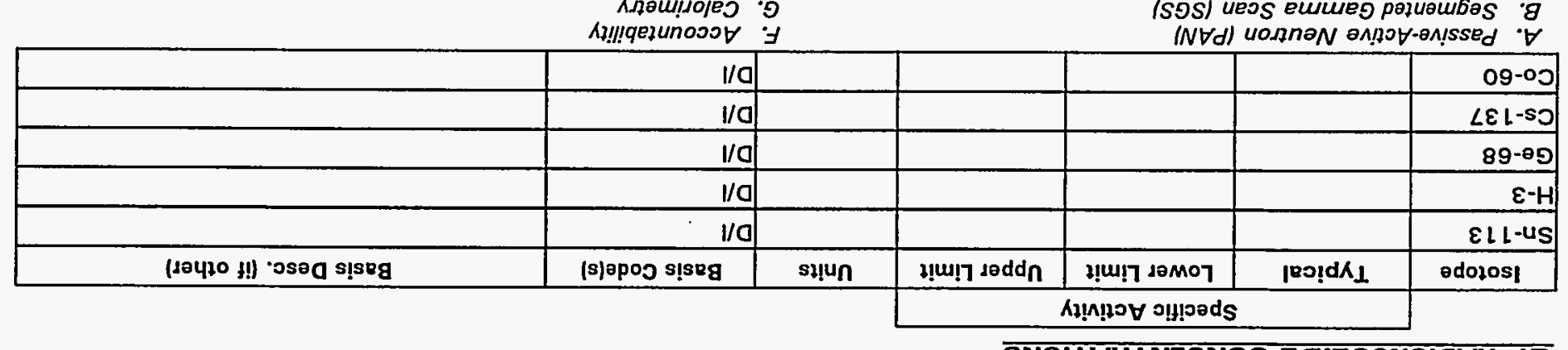

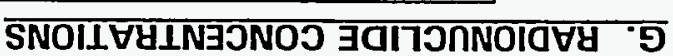

Knawonoods ssew $\exists$

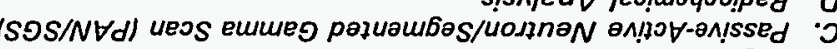

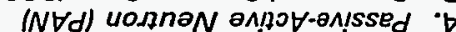

\section{$\because$ H} $\angle O O M N E$ s!sA/eut jeoluayooipey (SOS) ueos eumes pajuambas 'a 


\section{BN-WOO? \\ ContactrH \\ H. MATRIX CHARACTERISTICS}

Primary Matrix Code: X7100

Description: Special Waste: Elemental Mercury: :

Combustibility: $\bigcirc$ Combustible (>90\%)

Mixed (10\%-90\%)

Noncombustible $(<10 \%)$

\section{MATRIX COMPOSITION}

\begin{tabular}{|c|c|c|c|c|c|c|}
\hline & & & \multicolumn{4}{|c|}{ Composition } \\
\hline Code & Matrix Description & Materlal & Typical & LL & UL & Units \\
\hline 55120 & Debris Waste.Inorganic Debris.Inorganic Non-Metal Debris. & & & & & \\
\hline$x 7100$ & Special Waste.Elemental Mercury.. & & & & & \\
\hline
\end{tabular}




\section{J. HAZARDOUS CONSTITUENTS}

Still-Gen Active

\begin{tabular}{|c|c|c|c|c|c|c|c|c|}
\hline \multirow{2}{*}{\multicolumn{3}{|c|}{ 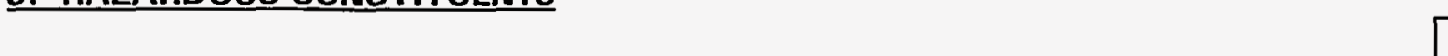 }} & \multirow{2}{*}{\multicolumn{4}{|c|}{ Concentration }} & \multirow[b]{3}{*}{ Basis } & \multirow[b]{3}{*}{ Conc. Type } \\
\hline & & & & & & & & \\
\hline Code & Hazardous Waste Description & Treatment Subcategory & Typical & $\mathbf{L L}$ & UL & Units & & \\
\hline D009 & Mercury & Elemental mercury contaminated with radioact & & & & & & Total \\
\hline$\overline{D 009}$ & Mercury & High mercury $>=260 \mathrm{mg} / \mathrm{kg}$ total mercury & 20 & $\overline{1}$ & & bvol & $\mathrm{B}$ & Total \\
\hline
\end{tabular}




\section{K. POLYCHLORINATED BIPHENYLS (PCBS)}

\section{Is this waste stream PCB contaminated? OYes $O$ No $O$ Unknown}

If yes, what portion of the waste is PCB contaminated (volume \%):

If $<100 \%$, can the PCB contaminated waste be segregated and treated separately? OYes ONo O Unknown

What is the PCB concentration in ppm?

If only a portion of the waste is $P C B$ contaminated, report values for contaminated portion.

\section{TREATMENT PLANS}

\begin{tabular}{|c|c|c|c|c|c|c|c|c|c|c|c|}
\hline $\begin{array}{c}\text { Opt. } \\
\#\end{array}$ & $\begin{array}{c}\text { Media Type } \\
\text { (If more than one) }\end{array}$ & $\begin{array}{c}\text { Matrix } \\
\text { Code }\end{array}$ & $\begin{array}{l}\text { Vol. \% } \\
\text { IIf }\end{array}$ & Step & JIT & sc & ClF & $\begin{array}{l}\text { Trans. } \\
\text { Miles }\end{array}$ & $\begin{array}{l}\text { Facility } \\
\text { Abbr. }\end{array}$ & Unit Name & Comments \\
\hline \multirow[t]{4}{*}{1} & & $\times 7100$ & & $\bar{a}$ & O) & 0 & $1.00 E+00$ & & TRANS & Transport - LLW & \\
\hline & & & & b & 0 & 0 & $1.00 E+00$ & & AMWTF & $\mathrm{CH}$ - Opening \& Sorting & \\
\hline & & & & c & 0 & 0 & $1.00 E+00$ & & AMWTF & $\mathrm{CH}$ - Amalgamation & \\
\hline & & & & d & 0 & O & $1.00 E+00$ & & SCDF & Disposal - Contact Handled & \\
\hline
\end{tabular}

Note: Where provided, media percentages are rough estimates used to facilitate treatment planning. They do not necessarily imply an accurate knowledge of waste stream composition, and were not derived for use outside this conceptual planning exercise.

Describe any special or unique technical concerns related to the treatment of this waste stream that would impact the use or implementation of standard hazardous waste treatment methods. 
suo!̣ejado vo!̣eu!mełuoja

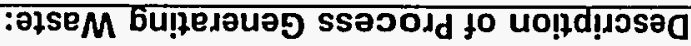

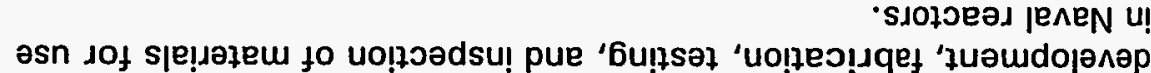

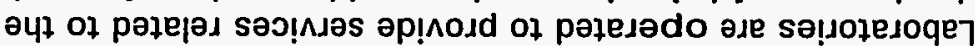

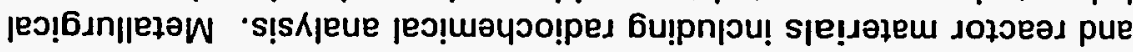

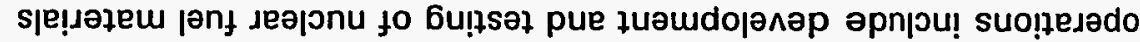

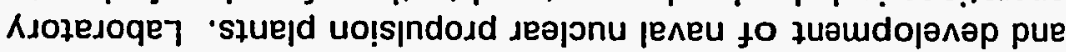

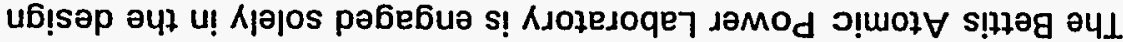

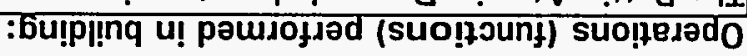

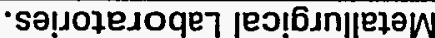

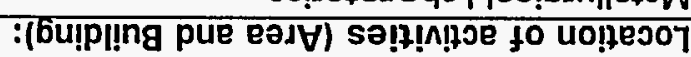

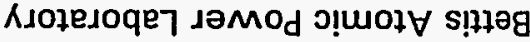

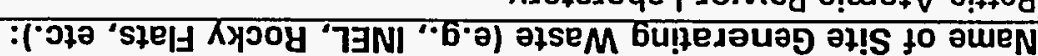

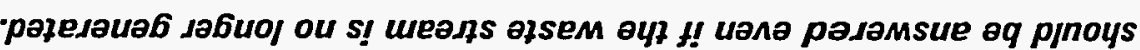
'ssavosd pue ał!s uonesauab ay? 6u!usaouos 'suopsanb fu!mo/lof ayL

\section{NOIIdIYUS}

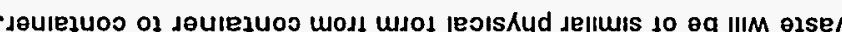

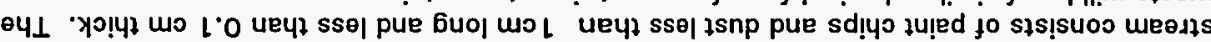

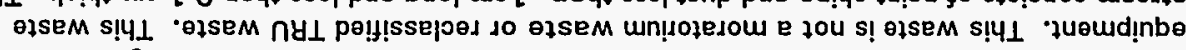

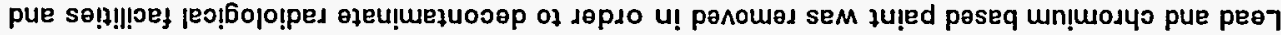

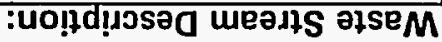

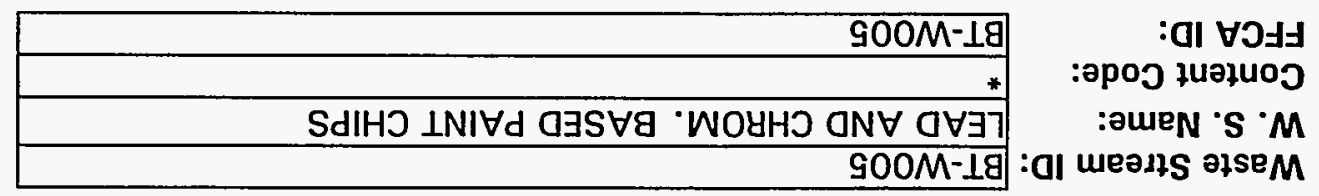

NOIIdIYOS

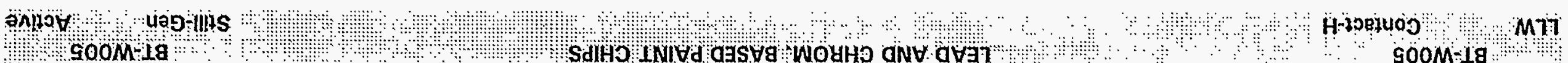



BT-WOOS
LLW ContactiH
C. PROJECTED WASTE GENERATION

LEAD AND CHROM. BASED PAINT CHIPS

Is this waste stream still generated?

If yes, when is the expected generation termination date?

\begin{tabular}{|}
\begin{tabular}{|c|r|r|r|r|r|}
\hline \multicolumn{5}{|c|}{ Current Projections } \\
\hline Est. Date & Start Year & Interval & Vol (m3) & Mass (kg) & Source of Estlmate \\
\hline $11 / 15 / 94$ & 1997 & 1 & 0.10 & & 1994 DSTP \\
\hline
\end{tabular}
\end{tabular}

Historical Projections 


\section{STORAGE INVENTORY}

\begin{tabular}{|c|c|c|c|c|c|c|c|c|c|}
\hline Container ID & Stor. Loc. & \# of Cont. & Type & Total Vol. & Units & Weight (lbs) & G. Fac. & Date Rec. & Waste Description \\
\hline & & & & & $\mathrm{CM}$ & & & & \\
\hline
\end{tabular}

Waste Removed From Storage: 


\section{BT-W005 \\ LLW , Contact H \\ E. RADIATION CHARACTERISTICS}

Radioactive Waste Type:

\begin{tabular}{|ll|}
\hline OlLW & $\bigcirc$ a-LLW $\bigcirc$ TRU \\
OHLW & $\bigcirc$ Non-Rad \\
\hline
\end{tabular}

Mixed Waste:

Handling:

\begin{tabular}{l} 
OYes ONo \\
\hline O Contact O Remote
\end{tabular}

Radionuclide Contamination Accessiblity:

External Surface $O$ Yes $O_{\text {No }}$ OUnknown

Internal Surface OYes O No OUnknown

Dispersed Through Matrix

O Yes ONo OUnknown

\section{Activity Levels}

Transuranic Alpha Activity:

Uranium/Thorium Alpha Activity:

Beta/Gamma Activity:

Surface Neutron Activity:

Total Activity:

\begin{tabular}{|c|c|}
\hline $3 \mathrm{E}-12$ & Units: $\mathrm{Ci} / \mathrm{g}$ \\
\hline $7 E-10$ & Units: $\mathrm{Ci} / \mathrm{g}$ \\
\hline$<0.1$ & @ surface \\
\hline$<0.1$ & @ 1-m Units: $\mathrm{mR} / \mathrm{hr}$ \\
\hline & Units: [ \\
\hline 2 & $\mathrm{nCi} / \mathrm{g}$ \\
\hline
\end{tabular}

\section{F. TRU ALPHA ACTIVITY DISTRIBUTION}

This waste stream is not identified as a TRU or a-LLW. 


\section{BTTWOOS}

LEAD ANDD CHROM, BASED PAINT CHIPS

LLW : Contáct H

BT:WOOD

Still-Gen Active

G. RADIONUCLIDE CONCENTRATIONS

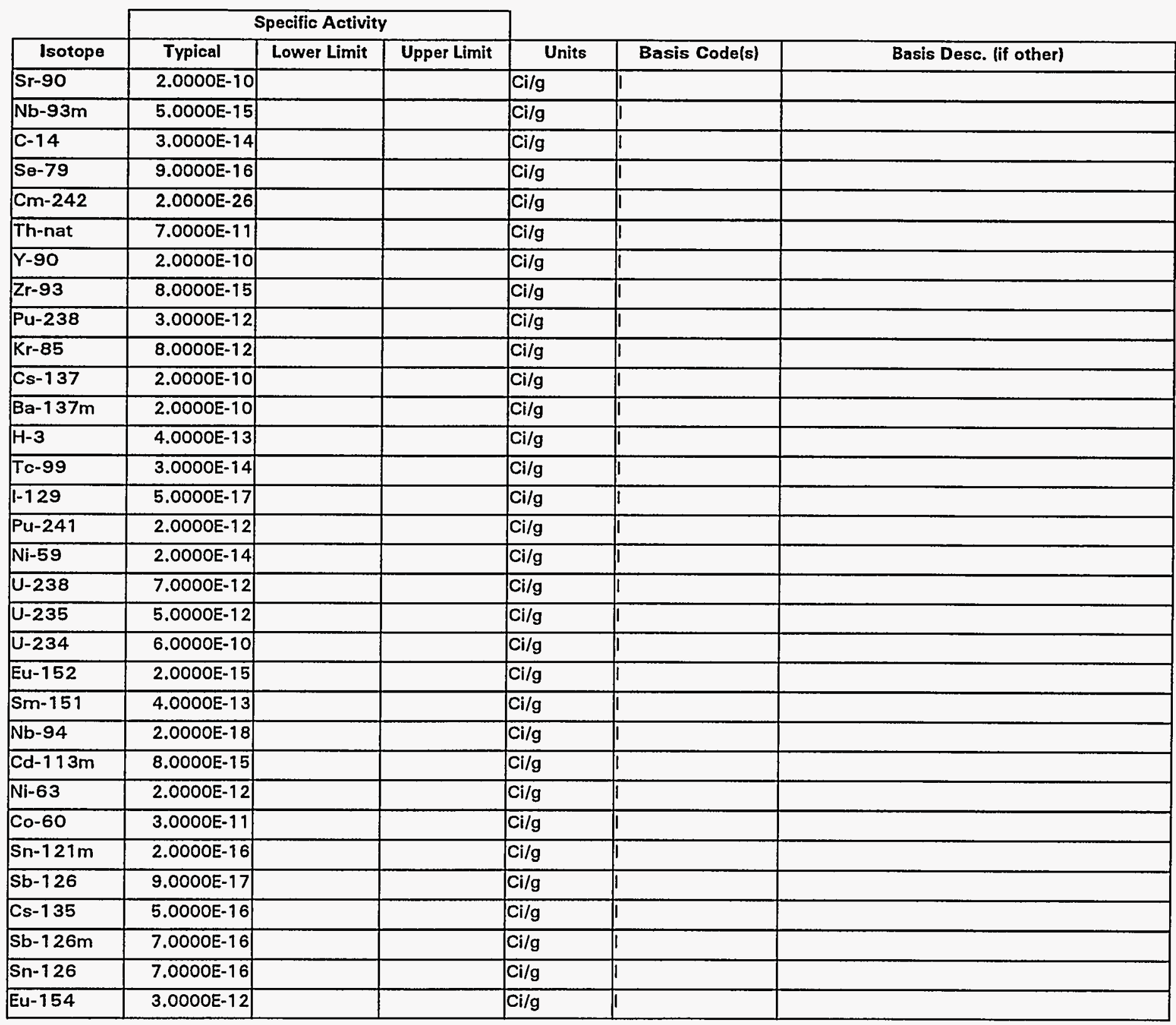




\section{BT-W005}

Liw Contäct-H

A. Passive-Active Neutron (PAN)

B. Segmented Gamma Scan (SGS)

C. Passive-Active Neutron/Segmented Gamma Scan (PAN/SGS)

D. Radiochemical Analysis

E. Mass Spectrometry

\section{LEAD AND CHROM, BASED PAINT CHIPS}
Accountability
G. Calorimetry
H. Multiple Energy Gamma Analysis (MEGAS)
1. Process Knowledge
J. Other

BT:-WOO5: Still-Gen Active 
H. MATRIX CHARACTERISTICS

Primary Matrix Code: $\mathbf{S 3 1 3 1}$

Description: Homogeneous Solids: Inorganic Homogeneous Solids: Paint Waste: Paint Chips/Solids

Combustibility:

Combustible (>90\%)

Mixed (10\%-90\%)

Noncombustible $(<10 \%)$

\section{MATRIX COMPOSITION}

\begin{tabular}{|c|c|c|c|c|c|c|}
\hline & & & \multicolumn{4}{|c|}{ Composition } \\
\hline Code & Matrix Description & Material & Typlcal & LL & UL & Units \\
\hline$\longdiv { \mathbf { S 3 1 3 1 } }$ & Homogeneous Solids.Inorganic Homogeneous Solids.Paint Waste.Paint Chips/Solids & & 100 & 100 & 100 & \%vol \\
\hline
\end{tabular}




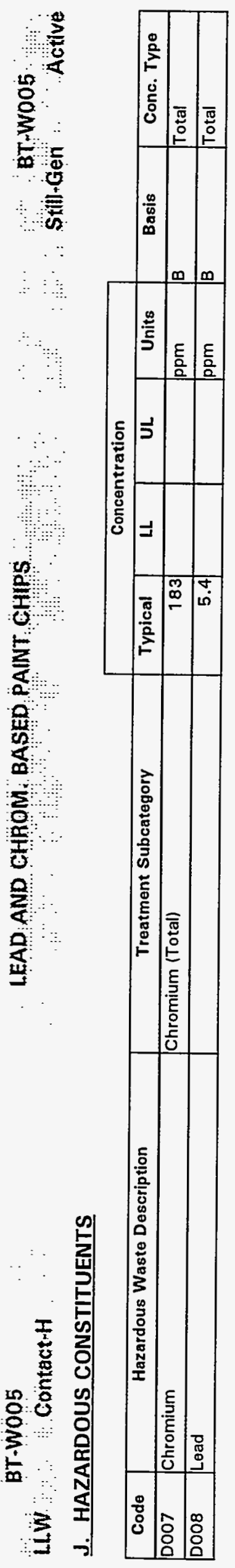

$\frac{7}{7}$
5
8
0

ह

$\stackrel{n}{N}$ 


\section{K. POLYCHLORINATED BIPHENYLS (PCBS)}

\section{Is this waste stream PCB contaminated? OYes $O$ No $O$ Unknown}

If yes, what portion of the waste is $\mathrm{PCB}$ contaminated (volume \%):

If $<100 \%$, can the PCB contaminated waste be segregated and treated separately? $O$ Yes $\bigcirc$ No O Unknown

What is the PCB concentration in ppm?

If only a portion of the waste is $P C B$ contaminated, report values for contaminated portion.

\section{TREATMENT PLANS}

\begin{tabular}{|c|c|c|c|c|c|c|c|c|c|c|c|}
\hline $\begin{array}{c}\text { Opt. } \\
\#\end{array}$ & $\begin{array}{c}\text { Media Type } \\
\text { (If more than one) }\end{array}$ & $\begin{array}{c}\text { Matrix } \\
\text { Code }\end{array}$ & $\begin{array}{c}\text { Vol. \% } \\
\text { (If }\end{array}$ & Step & JIT & sc & CIF & $\begin{array}{l}\text { Trans. } \\
\text { Miles }\end{array}$ & $\begin{array}{l}\text { Facility } \\
\text { Abbr. }\end{array}$ & Unit Name & Comments \\
\hline \multirow[t]{5}{*}{1} & & S3131 & & $\bar{a}$ & O & $\bar{O}$ & $1.00 E+00$ & & TRANS & Transport - LLW & \\
\hline & & & & $\mathbf{b}$ & $\mathcal{O}$ & 0 & $1.00 E+00$ & & AMWTF & $\mathrm{CH}$ - Opening \& Sorting & \\
\hline & & & & $\bar{d}$ & 0 & 0 & $1.00 E+00$ & & AMWTF & $\mathrm{CH}$ - Incineration/Thermal Desorption & \\
\hline & & & & e & 0 & 0 & $1.00 E+00$ & & AMWTF & $\mathrm{CH}$ - Vitrification & \\
\hline & & & & $f$ & 0 & $\mathrm{O}$ & $1.00 E+00$ & & SCDF & Disposal - Contact Handled & \\
\hline
\end{tabular}

Note: Where provided, media percentages are rough estimates used to facilitate treatment planning. They do not necessarily imply an accurate knowledge of waste stream composition, and were not derived for use outside this conceptual planning exercise.

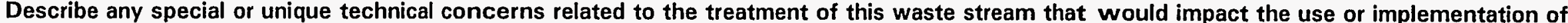
standard hazardous waste treatment methods. 


\section{A. IDENTIFICATION AND DESCRIPTION}

Waste Stream ID: BT-W008

W. S. Name:

Content Code:

FFCA ID:

MERCURY CONTAINING WASTE

\section{Waste Stream Description:}

Cloth, metal, plastic, PVC, and glass waste containing small amounts of hardened mercury residue. This waste stream is currently stored in one container. Homogeneity of waste is unavailable. This waste is not a moratorium or reclassified TRU waste.

\section{B. GENERATION SITE/PROCESS DESCRIPTION}

The following questions, concerning the generation site and process, should be answered oven if the waste stream is no longer generated.

Name of Site Generating Waste (e.g., INEL; Rocky Flats, etc.): Bettis Atomic Power Laboratory

Location of activities (Area and Building):

Materials Evaluation Laboratory (MEL) and Metallurgical Laboratories.

Operations (functions) performed in building:

The Bettis Atomic Power Laboratory is engaged solely in the design and development of naval nuclear propulsion plants. Laboratory operations include development and testing of nuclear fuel materials and reactor materials including radiochemical analysis. The Materials Evaluation Laboratory (MEL) consists of shielded rooms and support facilities for the physical, chemical, and metallurgical testing of radioactive material specimens. Metallurgical Laboratories are operated to provide services related to the development, fabrication, testing, and inspection of materials for use in Naval reactors.

Description of Process Generating Waste:

Equipment repair and decontamination and decommissioning. 
BTTW008

Liw Wo contact-H

C. PROJECTED WASTE GENERATION

Is this waste stream still generated?

If yes, when is the expected generation termination date?

Current Projections

\section{MERGURY CONTAINING WASTE}

Current Projections

\begin{tabular}{|c|r|r|r|l|}
\hline Est. Date & \multicolumn{1}{|l|}{ Start Year } & Interval & \multicolumn{1}{|c|}{ Vol (m3) } & Mass (kg) \\
\hline $11 / 15 / 94$ & 1997 & 1 & 0.02 & \\
\hline
\end{tabular}

Historical Projections

\section{B̈TWÖ̈8}

NateGen Active 

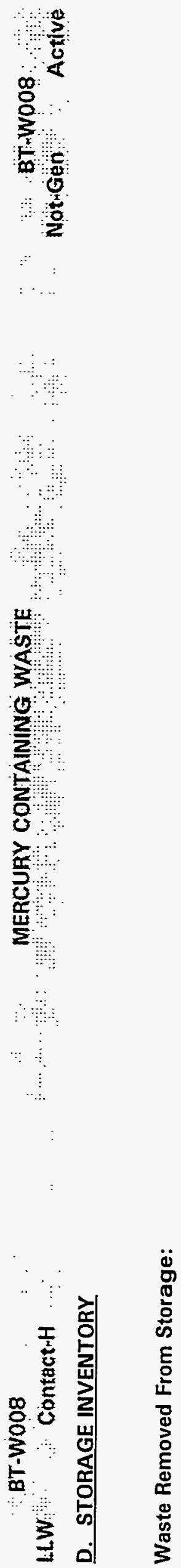


\section{E. RADIATION CHARACTERISTICS}

Radioactive Waste Type:

\begin{tabular}{|ll}
\hline OlLW & $\bigcirc$ a-LlW $\bigcirc$ TRU \\
OHLW & $\bigcirc$ Non-Rad
\end{tabular}

Mixed Waste:

Handling:

\begin{tabular}{|l|}
\hline O Yes O No \\
\hline O Contact O Remote
\end{tabular}

Radionuclide Contamination Accessiblity:

External Surface

Internal Surface

Dispersed Through Matrix

\begin{tabular}{|c|c|c|}
\hline OYes & O No & OUnknown \\
\hline OYes & ONo & O Unknown \\
\hline OYes & ONo & 00 \\
\hline
\end{tabular}

\section{Activity Levels}

Transuranic Alpha Activity: Uranium/Thorium Alpha Activity:

Beta/Gamma Activity:

Surface Neutron Activity:

Total Activity:

\begin{tabular}{rl|}
\hline $4 \mathrm{E}-12$ & Units: $\mathrm{Ci} / \mathrm{g}$ \\
\hline $2 \mathrm{E}-13$ & Units: $\mathrm{Ci} / \mathrm{g}$ \\
\hline$<0.1$ & @ surface \\
\hline$<0.1$ & @ 1-m Units: $\mathrm{mR} / \mathrm{hr}$ \\
\hline & Units: \\
\hline
\end{tabular}
$5 \mathrm{nCi} / \mathrm{g}$
This waste stream is not identified as a TRU or a-LLW. 
BT-W008

\section{LiW Contacth}

\section{G. RADIONUCLIDE CONCENTRATIONS}

\begin{tabular}{|c|c|c|c|c|c|c|}
\hline & & pecific Activit & & & & \\
\hline Isotope & Typical & Lower Limit & Upper Limit & Units & Basis Code(s) & Basis Desc. (if other) \\
\hline Ba-137m & $2.0000 \mathrm{E}-11$ & & & $\mathrm{Ci} / \mathrm{g}$ & 1 & \\
\hline $5 \theta-79$ & $9.0000 \mathrm{E}-16$ & & & $\mathrm{Ci} / \mathrm{g}$ & 1 & \\
\hline C-14 & $3.0000 E-14$ & & & $\mathrm{Ci} / \mathrm{g}$ & 1 & \\
\hline $\mathrm{Zr}=93$ & $8.0000 \mathrm{E}-15$ & & & $\mathrm{Ci} / \mathrm{g}$ & 1 & \\
\hline $\mathrm{Cd}-113 \mathrm{~m}$ & $2.0000 \mathrm{E}-14$ & & & $\mathrm{Ci} / \mathrm{g}$ & $\pi$ & \\
\hline Sm-151 & $4.0000 \mathrm{E}-13$ & & & $\mathrm{Ci} / \mathrm{g}$ & i & \\
\hline Th-232 & $2.0000 \mathrm{E}-14$ & & & $\mathrm{Ci} / \mathrm{g}$ & 1 & \\
\hline Pu-238 & $4.0000 E-12$ & & & $\mathrm{Ci} / \mathrm{g}$ & 1 & \\
\hline $\mathrm{Nb}-93 \mathrm{~m}$ & $4.0000 E-15$ & & & $\mathrm{Ci} / \mathrm{g}$ & 1 & \\
\hline $\mathrm{Kr}-85$ & $2.0000 \mathrm{E}-11$ & & & $\mathrm{Ci} / \mathrm{g}$ & 1 & \\
\hline$Y-90$ & 2.0000E-10 & & & $\mathrm{Ci} / \mathrm{g}$ & 1 & \\
\hline Sr-90 & $2.0000 \mathrm{E}-10$ & & & $\mathrm{Ci} / \mathrm{g}$ & 1 & \\
\hline Cs-137 & $2.0000 E-10$ & & & $\mathrm{Ci} / \mathrm{g}$ & 1 & \\
\hline $\mathrm{H}-3$ & $2.0000 \mathrm{E}-12$ & & & $\mathrm{Ci} / \mathrm{g}$ & 1 & \\
\hline Tc-99 & $3.0000 \mathrm{E}-14$ & & & $\mathrm{Ci} / \mathrm{g}$ & 1 & \\
\hline $1-129$ & $5.0000 \mathrm{E}-17$ & & & $\mathrm{Ci} / \mathrm{g}$ & 1 & \\
\hline Eu-154 & $3.0000 \mathrm{E}-10$ & & & $\mathrm{Ci} / \mathrm{g}$ & 1 & \\
\hline Cs-134 & $3.0000 \mathrm{E}-11$ & & & $\mathrm{Ci} / \mathrm{g}$ & 1 & \\
\hline $\mathrm{U}-238$ & $2.0000 \mathrm{E}-15$ & & & $\mathrm{Ci} / \mathrm{g}$ & 1 & \\
\hline$U-234$ & $2.0000 \mathrm{E}-13$ & & & $\mathrm{Ci} / \mathrm{g}$ & 1 & \\
\hline U-235 & $2.0000 \mathrm{E}-15$ & & & $\mathrm{Ci} / \mathrm{g}$ & 1 & \\
\hline Sb-126m & $2.0000 \mathrm{E}-19$ & & & $\mathrm{Ci} / \mathrm{g}$ & $\pi$ & \\
\hline $\mathrm{Ni}-59$ & 5.0000E-12 & & & $\mathrm{Ci} / \mathrm{g}$ & 1 & \\
\hline Eu-152 & $2.0000 \mathrm{E}-10$ & & & $\mathrm{Ci} / \mathrm{g}$ & I & \\
\hline Pu-241 & $2.0000 \mathrm{E}-12$ & & & $\mathrm{Ci} / \mathrm{g}$ & 1 & \\
\hline $5 n-126$ & 7.0000 E- 16 & & & $\mathrm{Ci} / \mathrm{g}$ & 1 & \\
\hline $\mathrm{Cm}-242$ & $4.0000 E-19$ & & & $\mathrm{Ci} / \mathrm{g}$ & 1 & \\
\hline $\mathrm{Fe}-55$ & $2.0000 \mathrm{E}-10$ & & & $\mathrm{Ci} / \mathrm{g}$ & 1 & \\
\hline $\mathrm{Ni}-63$ & $6.0000 \mathrm{E}-10$ & & & $\mathrm{Ci} / \mathrm{g}$ & 1 & \\
\hline Sb-125 & $2.0000 \mathrm{E}-09$ & & & $\mathrm{Ci} / \mathrm{g}$ & 1 & \\
\hline Co-60 & $3.0000 \mathrm{E}-10$ & & & $\mathrm{Ci} / \mathrm{g}$ & 1 & \\
\hline Cs-135 & $5.0000 \mathrm{E}-16$ & & & $\mathrm{Ci} / \mathrm{g}$ & 1 & \\
\hline $\mathrm{Pm}-147$ & $3.0000 \mathrm{E}-11$ & & & $\mathrm{Ci} / \mathrm{g}$ & $\pi$ & \\
\hline
\end{tabular}


BT WOOO8

LLW Wontagt H

\begin{tabular}{|l|l|}
\hline $\mathrm{Nb}-94$ & $5.0000 \mathrm{E}-20$ \\
\hline
\end{tabular}

MERCURY CONTAINING WASTE

A. Passive-Active Neutron (PAN)

B. Segmented Gamma Scan (SGS)

C. Passive-Active Neutron/Segmented Gamma Scan (PAN/SGS)

D. Radiochemical Analysis

1

E. Mass Spectrometry

F. Accountability

G. Calorimetry

H. Multiple Energy Gamma Analysis (MEGAS)

1. Process Knowledge

J. Other 


\section{H. MATRIX CHARACTERISTICS}

Primary Matrix Code: $\mathbf{S 5 4 0 0}$

Description: Debris Waste: Heterogeneous Debris: :

Combustibility:

Combustible (>90\%)

Mixed $(10 \%-90 \%)$

Noncombustible $(<10 \%)$

\section{MATRIX COMPOSITION}

\begin{tabular}{|c|c|c|c|c|c|c|}
\hline \multirow[b]{2}{*}{ Code } & \multirow[b]{2}{*}{ Matrix Description } & \multirow[b]{2}{*}{ Materlal } & \multicolumn{4}{|c|}{ Composition } \\
\hline & & & Typical & $\mathrm{LL}$ & UL & Units \\
\hline 55122 & Debris Waste.Inorganic Debris.Inorganic Non-Metal Debris.Glass Debris & Glass & 15 & & & $\% w t$ \\
\hline 55119 & Debris Waste.Inorganic Debris.Metal Debris.Unknown/Other Metal Debris & Metal & 65 & & & $\% w t$ \\
\hline $\mathbf{5 5 3 1 2}$ & Debris Waste.Organic Debris.Plastic/Rubber Debris.Halogenated Plastic Debris & PVC & 10 & & & $\% w t$ \\
\hline$\$ 5330$ & Debris Waste.Organic Debris.Paper/Cloth Debris. & Cloth & 10 & & & $\% w t$ \\
\hline
\end{tabular}


BTT-WOOB:

HWW Contäct H

MERCURY CÖNTAINING WASTE

J. HAZARDOUS CONSTITUENTS

\begin{tabular}{|c|c|c|c|c|c|c|c|c|}
\hline \multirow{2}{*}{\multicolumn{3}{|c|}{. }} & \multirow{2}{*}{\multicolumn{4}{|c|}{ Concentration }} & \multirow{3}{*}{ Basis } & \multirow[b]{3}{*}{ Conc. Type } \\
\hline & & & & & & & & \\
\hline Code & Hazardous Waste Description & Treatment Subcategory & Typical & $\mathrm{LL}$ & $\mathbf{U L}$ & Units & & \\
\hline D008 & Lead & & 7 & & & $\% w t$ & $B$ & Total \\
\hline D009 & Mercury & High mercury $>=260 \mathrm{mg} / \mathrm{kg}$ total mercury & 6 & & & $\% w t$ & $B$ & Total \\
\hline
\end{tabular}




\section{LLW Contact H}

\section{K. POLYCHLORINATED BIPHENYLS (PCBS)}
Is this waste stream PCB contaminated?
OYes
O No
Unknown

If yes, what portion of the waste is PCB contaminated (volume \%):

If $<100 \%$, can the PCB contaminated waste be segregated and treated separately?

What is the PCB concentration in ppm?

If only a portion of the waste is PCB contaminated, report values for contaminated portion.

\section{TREATMENT PLANS}

\begin{tabular}{|c|c|c|c|c|c|c|c|c|c|c|c|}
\hline Opt. & $\begin{array}{c}\text { Media Type } \\
\text { (If more than one) }\end{array}$ & $\begin{array}{c}\text { Matrix } \\
\text { Code }\end{array}$ & $\begin{array}{l}\text { Vol. \% } \\
\text { IIf }\end{array}$ & Step & JIT & sc & CIF & $\begin{array}{l}\text { Trans. } \\
\text { Miles }\end{array}$ & $\begin{array}{l}\text { Facility } \\
\text { Abbr. }\end{array}$ & Unit Name & Comments \\
\hline \multirow[t]{5}{*}{1} & & 55400 & & $\overline{\mathbf{a}}$ & 0 & $\mathrm{O}$ & $1.00 \mathrm{E}+00$ & & TRANS & Transport - LLW & \\
\hline & & & & $\bar{b}$ & 0 & $\mathrm{O}$ & $1.00 E+00$ & & WROC & Sizing & \\
\hline & & & & c & 0 & $\bar{O}$ & $1.00 E+00$ & & WROC & Hg Retort & \\
\hline & & & & $\bar{d}$ & 0 & $\mathrm{O}$ & $1.00 E+00$ & & WERF & Stabilization - Portland Cement & \\
\hline & & & & e & 0 & $\bar{O}$ & $1.00 \mathrm{E}+00$ & & SCDF & Disposal - Contact Handled & \\
\hline
\end{tabular}

Note: Where provided, media percentages are rough estimates used to facilitate treatment planning. They do not necessarily imply an accurate knowledge of waste stream composition, and were not derived for use outside this conceptual planning exercise.

Describe any special or unique technical concerns related to the treatment of this waste stream that would impact the use or implementation of standard hazardous waste treatment methods. 


\section{B. GENERATION SITE/PROCESS DESCRIPTION}

The following questions, concerning the generation site and process, should be answered even if the waste stream is no longer generated.

Waste Stream

W. S. Name:

Content Code:

FFCA ID:

\section{VOC CONTAMINATED SOIL}

\section{Waste Stream Description:}

Waste consists of soil, contaminated with VOCs. Waste will be of similar form from container to container. Waste is not a moratorium waste or reclassified TRU waste.
Name of Site Generating Waste (e.g., INEL, Rocky Flats, etc.): Bettis Atomic Power Laboratory

Location of activities (Area and Building): Inactive Waste Site and areas near some radiological facilities.

Operations (functions) performed in building:

Bettis Atomic Power Laboratory is engaged solely in the design and development of naval nuclear propulsion plants. Laboratory operations include development and testing of nuclear fuel materials and reactor materials including radiochemical analysis.

\section{Description of Process Generating Waste:}

Soil samples from borings. 
C. PROJECTED WASTE GENERATION

OYes O No

If yes, when is the expected generation termination date? 


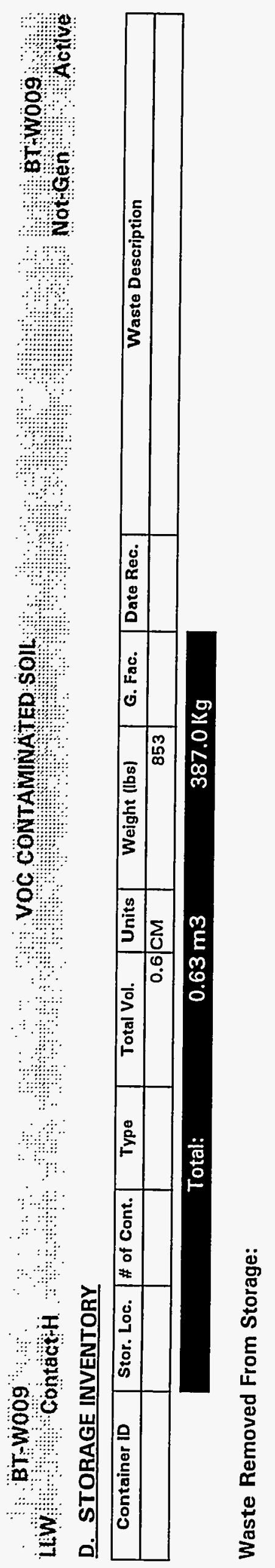

ह 


\section{LiW: \\ BT-W009 \\ Contact-H \\ E. RADIATION CHARACTERISTICS}

Radioactive Waste Type:

\begin{tabular}{|ll|}
\hline Ollw & Oa-llw O TRU \\
OhLW & O Non-Rad \\
\hline
\end{tabular}

Mixed Waste:

\begin{tabular}{|l|}
\hline O Yes O No \\
\hline O Contact O Remote \\
\hline
\end{tabular}

Radionuclide Contamination Accessiblity:

\begin{tabular}{|c|c|c|c|}
\hline External Surface & OYes & No & OUnknown \\
\hline Internal Surface & OYes & O No & Unknown \\
\hline d Through Ma & OYes & ONo & OUnknown \\
\hline
\end{tabular}

Dispersed Through Matrix

\section{Activity Levels}

Transuranic Alpha Activity: Uranium/Thorium Alpha Activity:

Beta/Gamma Activity:

8E-14 Units: $\mathrm{Ci} / \mathrm{g}$

5E-11 Units: Ci/g

$<0.1$ @ surface

\begin{tabular}{l}
$\square<0.1$ @ $1-\mathrm{m}$ Units: $\mathrm{mR} / \mathrm{hr}$ \\
\hline
\end{tabular}

Surface Neutron Activity:

Total Activity: $0.2 \mathrm{nCi} / \mathrm{g}$

\section{VOC CONTAMINÁTED SOIL}

BT WW09

\section{F. TRU ALPHA ACTIVITY DISTRIBUTION}

This waste stream is not identified as a TRU or a-LLW. 
BTWOOO9

YOC CONTAMINATED SÖL

LLW Contact

Active

\section{G. RADIONUCLIDE CONCENTRATIONS}

\begin{tabular}{|c|c|c|c|c|c|c|}
\hline & & pecific Activit & & & & \\
\hline Isotope & Typical & Lower Limit & Upper Limit & Units & Basis Code(s) & Basis Desc. (if other) \\
\hline Cs-135 & $2.0000 E-16$ & & & $\mathrm{Ci} / \mathrm{g}$ & D & \\
\hline $\mathrm{Nb}-93 \mathrm{~m}$ & $3.0000 E-14$ & & & $\mathrm{Ci} / \mathrm{g}$ & $\bar{D}$ & \\
\hline$S b-126 m$ & $2.0000 \mathrm{E}-16$ & & & $\mathrm{Ci} / \mathrm{g}$ & D & \\
\hline$C d-113 m$ & $2.0000 \mathrm{E}-15$ & & & $\mathrm{Ci} / \mathrm{g}$ & $D$ & \\
\hline $1-129$ & $2.0000 \mathrm{E}-17$ & & & $\overline{\mathrm{Ci}} / \mathrm{g}$ & $D$ & \\
\hline $\mathrm{U}-235$ & $5.0000 \mathrm{E}-13$ & & & $\mathrm{Ci} / \mathrm{g}$ & $D$ & \\
\hline Se-79 & $3.0000 E-16$ & & & $\mathrm{Ci} / \mathrm{g}$ & $D$ & \\
\hline Kr-85 & $2.0000 \mathrm{E}-12$ & & & $\mathrm{Ci} / \mathrm{g}$ & $D$ & \\
\hline Sn-126 & $2.0000 \mathrm{E}-16$ & & & $\mathrm{Ci} / \mathrm{g}$ & $D$ & \\
\hline Th-232 & $5.0000 \mathrm{E}-14$ & & & $\mathrm{Ci} / \mathrm{g}$ & D & \\
\hline U-234 & $5.0000 \mathrm{E}-11$ & & & $\mathrm{Ci} / \mathrm{g}$ & D & \\
\hline $\mathrm{H}-3$ & $1.0000 \mathrm{E}-13$ & & & $\mathrm{Ci} / \mathrm{g}$ & D & \\
\hline U-238 & $6.0000 \mathrm{E}-13$ & & & $\mathrm{Ci} / \mathrm{g}$ & $D$ & \\
\hline $\mathrm{Sm}-151$ & $1.0000 \mathrm{E}-13$ & & & $\mathrm{Ci} / \mathrm{g}$ & $D$ & \\
\hline Tc-99 & $8.0000 E-15$ & & & $\mathrm{Ci} / \mathrm{g}$ & D & \\
\hline Zr-93 & $2.0000 \mathrm{E}-15$ & & & $\mathrm{Ci} / \mathrm{g}$ & D & \\
\hline Ba-137m & $4.0000 \mathrm{E}-11$ & & & $\mathrm{Ci} / \mathrm{g}$ & D & \\
\hline $\mathrm{Cm}-242$ & $4.0000 \mathrm{E}-27$ & & & $\mathrm{Ci} / \mathrm{g}$ & $D$ & \\
\hline Pu-241 & 2.0000 E-13 & & & $\mathrm{Ci} / \mathrm{g}$ & $D$ & \\
\hline $\mathrm{Ni}-59$ & $3.0000 \mathrm{E}-15$ & & & $\mathrm{Ci} / \mathrm{g}$ & $D$ & \\
\hline$S n-121 m$ & $3.0000 \mathrm{E}-17$ & & & $\mathrm{Ci} / \mathrm{g}$ & $D$ & \\
\hline Sr-90 & $4.0000 \mathrm{E}-11$ & & & $\mathrm{Ci} / \mathrm{g}$ & $D$ & \\
\hline $\mathrm{Sb}-126$ & $3.0000 \mathrm{E}-17$ & & & $\mathrm{Ci} / \mathrm{g}$ & $D$ & \\
\hline C-14 & $2.0000 \mathrm{E}-13$ & & & $\mathrm{Ci} / \mathrm{g}$ & $D$ & \\
\hline Co-60 & $8.0000 \mathrm{E}-12$ & & . & $\mathrm{Ci} / \mathrm{g}$ & $\bar{D}$ & \\
\hline $\mathrm{Nb}-94$ & $2.0000 \mathrm{E}-20$ & & & $\mathrm{Ci} / \mathrm{g}$ & $\bar{D}$ & \\
\hline Eu-152 & $4.0000 \mathrm{E}-16$ & & & $\mathrm{Ci} / \mathrm{g}$ & $\mathrm{D}$ & \\
\hline Eu-154 & $8.0000 \mathrm{E}-13$ & & & $\mathrm{Ci} / \mathrm{g}$ & D & \\
\hline Ni-63 & $3.0000 \mathrm{E}-13$ & & & $\mathrm{Ci} / \mathrm{g}$ & D & \\
\hline Fe-55 & $2.0000 \mathrm{E}-12$ & & & $\mathrm{Ci} / \mathrm{g}$ & $D$ & \\
\hline Pu-238 & $8.0000 E-14$ & & & $\mathrm{Ci} / \mathrm{g}$ & D & \\
\hline Cs-137 & $4.0000 \mathrm{E}-11$ & & & $\mathrm{Ci} / \mathrm{g}$ & $D$ & \\
\hline$\overline{Y-90}$ & $4.0000 \mathrm{E}-11$ & & & $\mathrm{Ci} / \mathrm{g}$ & $D$ & \\
\hline
\end{tabular}



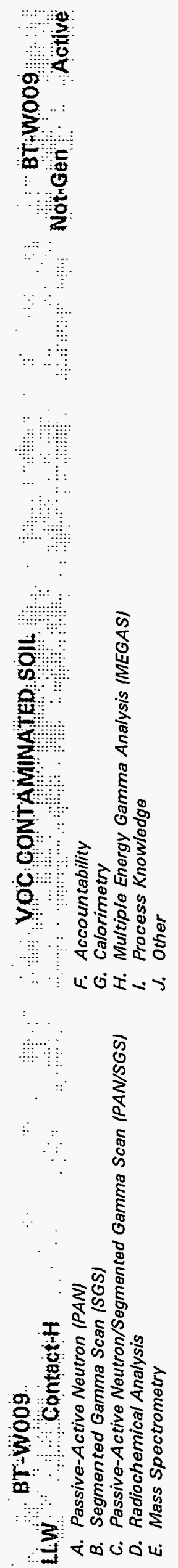


\section{H. MATRIX CHARACTERISTICS}

Primary Matrix Code: $\mathbf{S 4 1 0 0}$

Description: Soil/Gravel: Soil: :

Combustibility: $\bigcirc$ combustible (>90\%)

Mixed $(10 \%-90 \%)$

Noncombustible $(<10 \%)$

\section{MATRIX COMPOSITION}

\begin{tabular}{|c|c|c|c|c|c|c|}
\hline & & & \multicolumn{4}{|c|}{ Composition } \\
\hline Code & Matrix Description & Material & Typical & LL & UL & Units \\
\hline 54100 & Soil/Gravel.Soil.. & & 100 & 100 & 100 & $\%$ vol \\
\hline
\end{tabular}




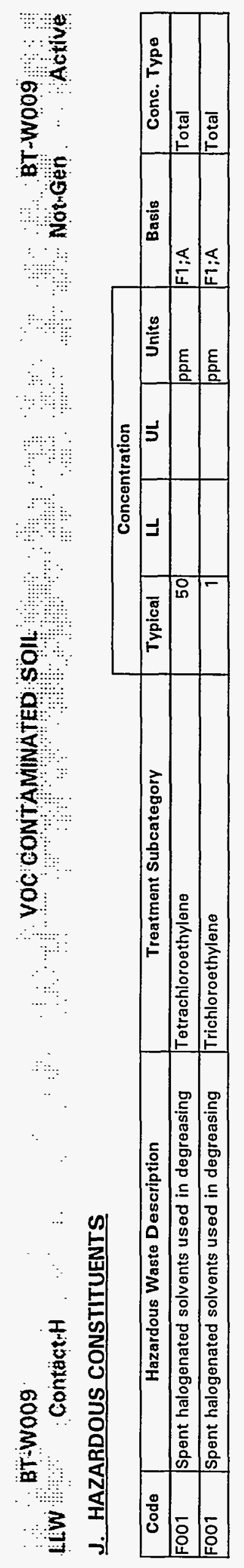




\section{K. POLYCHLORINATED BIPHENYLS (PCBS)}

Is this waste stream PCB contaminated? $O$ Yes $O$ No $O$ Unknown

If yes, what portion of the waste is PCB contaminated (volume \%): 100

If $<100 \%$, can the PCB contaminated waste be segregated and treated separately?

What is the PCB concentration in ppm?

$<32$

If only a portion of the waste is $P C B$ contaminated, report values for contaminated portion.

\section{TREATMENT PLANS}

\begin{tabular}{|c|c|c|c|c|c|c|c|c|c|c|c|}
\hline $\begin{array}{l}\text { Opt. } \\
\#\end{array}$ & $\begin{array}{c}\text { Media Type } \\
\text { (If more than one) }\end{array}$ & $\begin{array}{c}\text { Matrix } \\
\text { Code }\end{array}$ & $\begin{array}{l}\text { Vol. \% } \\
\text { IIf }\end{array}$ & Step & JIT & sc & CIF & $\begin{array}{l}\text { Trans. } \\
\text { Miles }\end{array}$ & $\begin{array}{l}\text { Facility } \\
\text { Abbr. }\end{array}$ & Unit Name & Comments \\
\hline 1 & & $\$ 4100$ & & $\bar{a}$ & () & 0 & $1.00 E+00$ & & TRANS & Transport - LLW & \\
\hline & & & & $\bar{b}$ & $\mathbf{O}$ & 0 & $1.00 \mathrm{E}+00$ & & AMWTF & CH - Opening \& Sorting & \\
\hline & & & & c & $\mathbf{O}$ & 0 & $1.00 \mathrm{E}+00$ & & AMWTF & $\mathrm{CH}$ - Sizing & \\
\hline & & & & $\bar{d}$ & 0 & 0 & $1.00 E+00$ & & AMWTF & $\mathrm{CH}$ - Incineration/Thermal Desorption & \\
\hline & & & & e & 0 & 0 & $1.00 \mathrm{E}+00$ & & AMWTF & $\mathrm{CH}$ - Vitrification & \\
\hline & & & & $f$ & 0 & 0 & $1.00 \mathrm{E}+00$ & & SCDF & Disposal - Contact Handled & \\
\hline
\end{tabular}

Note: Where provided, media percentages are rough estimates used to facilitate treatment planning. They do not necessarily imply an accurate knowledge of waste stream composition, and were not derived for use outside this conceptual planning exercise.

Describe any special or unique technical concerns related to the treatment of this waste stream that would impact the use or implementation of standard hazardous waste treatment methods. 


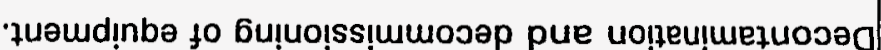

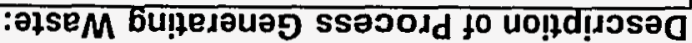

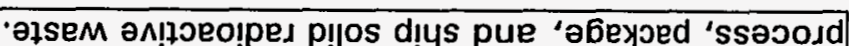

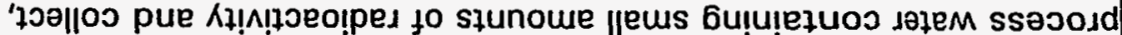

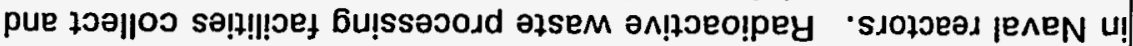

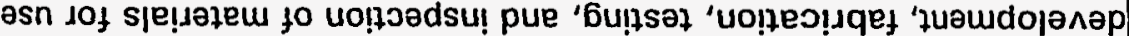

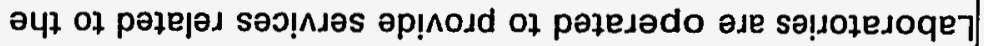

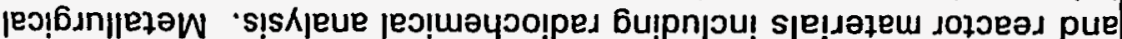

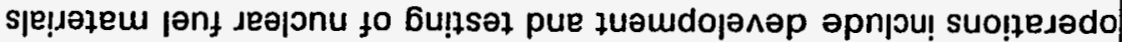

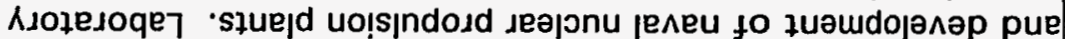

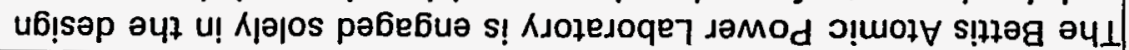
:бu!p!!nq u! pausołsad (suoltount) suoljesado

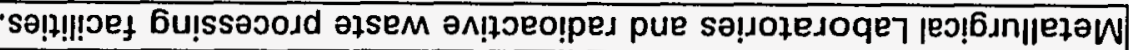

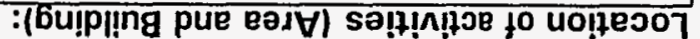

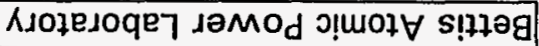

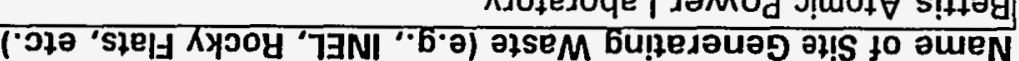

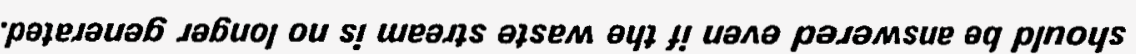

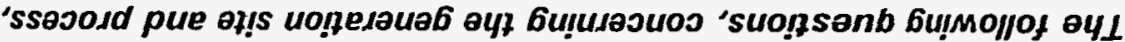

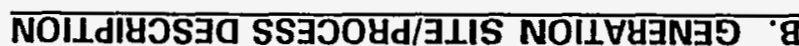

' $\mathrm{H} \perp$ po!j!ssejoed Jo olsem un!

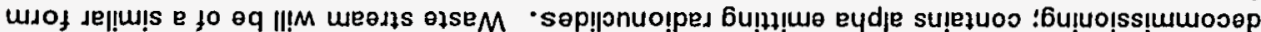

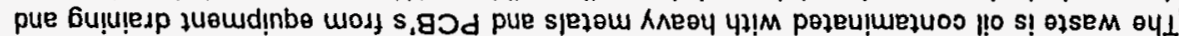

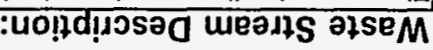

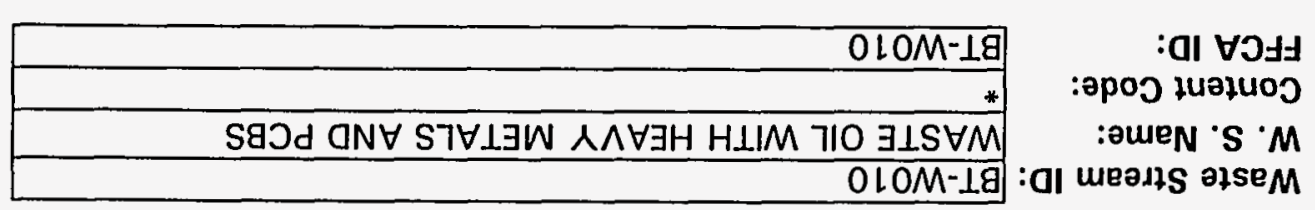

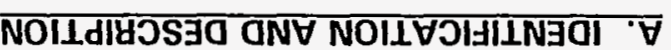

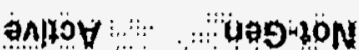

OLOM 19

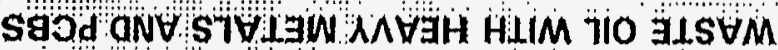

H-3017uOS

aLOM-La 
If yes, when is the expected generation termination date?

Current Projections

\section{Historical Projections}




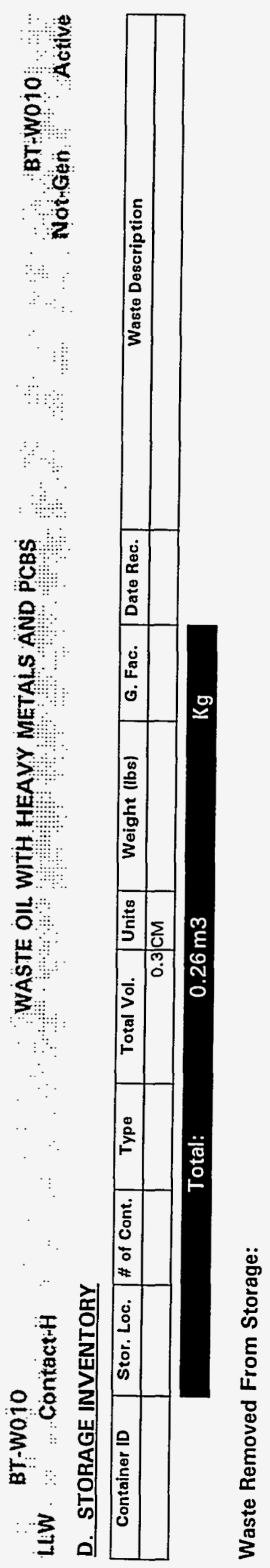




\section{F. TRU ALPHA ACTIVITY DISTRIBUTION}

Radioactive Waste Type:

\begin{tabular}{|ll|}
\hline O lLW & Oa-LlW OtRU \\
OhLW & O Non-Rad \\
\hline
\end{tabular}

This waste stream is not identified as a TRU or a-LLW.

Mixed Waste:

Handling:

\begin{tabular}{|l|}
\hline O Yes O No \\
\hline O Contact O Remote \\
\hline
\end{tabular}

Radionuclide Contamination Accessiblity:

\begin{tabular}{rlrl|} 
External Surface & OYes & O No & O Unknown \\
\cline { 3 - 4 } Internal Surface & OYes & O No & OUnknown \\
\cline { 2 - 4 } Dispersed Through Matrix & O Yes & O No & O Unknown \\
\cline { 2 - 3 } & &
\end{tabular}

\section{Activity Levels}

Transuranic Alpha Activity: Uranium/Thorium Alpha Activity:

Beta/Gamma Activity:

Surface Neutron Activity: Total Activity: 6E-8 Units: $\mathrm{Ci} / \mathrm{g}$

\begin{tabular}{rl|}
\hline $6 \mathrm{E}-8$ & Units: $\mathrm{Ci} / \mathrm{g}$ \\
\hline $6 \mathrm{E}-8$ & Units: $\mathrm{Ci} / \mathrm{g}$ \\
\hline$<3$ & $@$ surface \\
\hline$<0.1$ & $@ 1-\mathrm{m}$ Units: $\mathrm{mR} / \mathrm{hr}$ \\
\hline & Units: \\
\hline
\end{tabular}
$67 \mathrm{nCi} / \mathrm{g}$ 
BT-W010

\section{LLW .... ContactrH}

G. RADIONUCLIDE CONCENTRATIONS

\begin{tabular}{|c|c|c|c|c|c|c|}
\hline & \multicolumn{3}{|c|}{ Specific Activity } & \multirow[b]{2}{*}{ Units } & \multirow[b]{2}{*}{ Basis Code(s) } & \multirow[b]{2}{*}{ Basis Desc. (if other) } \\
\hline Isotope & Typical & Lower Limit & Upper Limit & & & \\
\hline MFP & $2.0000 E-11$ & & & $\mathrm{Ci} / \mathrm{g}$ & $D$ & \\
\hline Th-nat & $3.0000 \mathrm{E}-10$ & & & $\mathrm{Ci} / \mathrm{g}$ & $D$ & \\
\hline$U-232$ & 7.0000E-09 & & & $\mathrm{Ci} / \mathrm{g}$ & D & \\
\hline $\mathrm{U}-233$ & $6.0000 E-08$ & & & $\mathrm{Ci} / \mathrm{g}$ & $D$ & \\
\hline
\end{tabular}
A. Passive-Active Neutron (PAN)
F. Accountability
B. Segmented Gamma Scan (SGS)
G. Calorimetry
H. Multiple Energy Gamma Analysis (MEGAS)
l. Process Knowledge
J. Other
D. Radiochemical Analysis
E. Mass Spectrometry

WASTE OIL WITH HEAVY METALS AND PCB̈S

BT-WO10 NotGer Active 


\section{BT:WO10}

HiWW ContacteH

H. MATRIX CHARACTERISTICS

Primary Matrix Code: L2220

Description: Organic Liquids: Pure Organic Liquids: Nonhalogenated Pure Organic Liquids:

Combustibility: $\bigcirc$ Combustible (>90\%)

Mixed (10\%-90\%)

Noncombustible $(<10 \%)$

\section{MATRIX COMPOSITION}

\begin{tabular}{|c|c|c|c|c|c|c|}
\hline & & & \multicolumn{4}{|c|}{ Composition } \\
\hline Code & Matrix Description & Material & Typical & LL & UL. & Units \\
\hline L2220 & Organic Liquids.Pure Organic Liquids.Nonhalogenated Pure Organic Liquids. & Oils & 100 & 100 & 100 & $\% w t$ \\
\hline
\end{tabular}




\section{BT-W010} LiW: :- Contact J. HAZARDOUS CONSTITUENTS

\begin{tabular}{|c|c|c|c|c|c|c|c|c|}
\hline & & & \multicolumn{4}{|c|}{ Concentration } & & \\
\hline Code & Hazardous Waste Description & Treatment Subcategory & Typical & LL & UL & Units & Basis & Conc. Type \\
\hline D006 & Cadmium & & 4.2 & & & ppm & $F 1 ; A$ & Total \\
\hline D008 & Lead & & 31.4 & & & ppm & $F 1 ; A$ & Total \\
\hline$D 010$ & Selenium & & 1.4 & & & $\mathrm{ppm}$ & $F 1 ; A$ & Total \\
\hline
\end{tabular}

WASTE OIL WITH HEAVY METALS ANO PCBS O BTWWO

.......... Notuen Active 


\section{K. POLYCHLORINATED BIPHENYLS (PCBS)}

Is this waste stream PCB contaminated?

O Yes O No O Unknown

If yes, what portion of the waste is PCB contaminated (volume \%): 16

If $<100 \%$, can the PCB contaminated waste be segregated and treated separately? $O$ Yes O No O Unknown

What is the PCB concentration in ppm?

74

If only a portion of the waste is PCB contaminated, report values for contaminated portion.

\section{TREATMENT PLANS}

\begin{tabular}{|c|c|c|c|c|c|c|c|c|c|c|c|}
\hline $\begin{array}{c}\text { Opt. } \\
\#\end{array}$ & $\begin{array}{c}\text { Media Type } \\
\text { (If more than one) }\end{array}$ & $\begin{array}{c}\text { Matrix } \\
\text { Code }\end{array}$ & $\begin{array}{c}\text { Vol. \% } \\
\text { (If }\end{array}$ & Step & |JIT & sc & CIF & $\begin{array}{l}\text { Trans. } \\
\text { Miles }\end{array}$ & $\begin{array}{l}\text { Facility } \\
\text { Abbr. }\end{array}$ & Unit Name & Comments \\
\hline \multirow[t]{6}{*}{1} & & L2220 & & $\bar{a}$ & $\mathcal{O}$ & O & $1.00 \mathrm{E}+00$ & & TRANS & Transport - LLW & \\
\hline & & & & $\mathbf{b}$ & 0 & O & $1.00 \mathrm{E}+00$ & & AMWTF & $\mathrm{CH}$ - Opening \& Sorting & \\
\hline & & & & c & 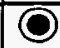 & $\bar{O}$ & $1.00 E+00$ & & AMWTF & $\mathrm{CH}$ - Sizing & \\
\hline & & & & $\mathbf{d}$ & $\mathrm{O}$ & $\overline{0}$ & $1.00 \mathrm{E}+00$ & & AMWTF & $\mathrm{CH}$ - Incineration/Thermal Desorption & \\
\hline & & & & e & 0 & $\overline{0}$ & $1.00 \mathrm{E}+00$ & & AMWTF & CH - Vitrification & \\
\hline & & & & $\mathbf{f}$ & 0 & 0 & $1.00 E+00$ & & SCDF & Disposal - Contact Handled & \\
\hline
\end{tabular}

Note: Where provided, media percentages are rough estimates used to facilitate treatment planning. They do not necessarily imply an accurate knowledge of waste stream composition, and were not derived for use outside this conceptual planning exercise.

Describe any special or unique technical concerns related to the treatment of this waste stream that would impact the use or implementation of standard hazardous waste treatment methods. 


\section{$\therefore$ BT-W012 \\ i.iw. \\ Contactit}

VOC \& PCB:CONTAMINATED DEB̈RIS

\section{A. IDENTIFICATION AND DESCRIPTION}

Waste Stream ID: BT-W012

W. S. Name:

Content Code:

FFCA ID:

\begin{tabular}{|l|}
\hline VOC \& PCB CONTAMINATED DEBRIS \\
\hline BT-W012 \\
\hline
\end{tabular}

\section{Waste Stream Description:}

Debris removed from storm sewer system. Waste is non-homogeneous. It includes sediment, rock, brick, cement clock, plastic, herculite, PVC bags, cloth, rubber, glass, and metal. Waste will be segregated and repackaged before shipment. Waste also contains some liquid. None of the drums are reclassified TRU wastes. This waste is not a moratorium waste or reclassified TRU.

\section{B. GENERATION SITE/PROCESS DESCRIPTION}

The following questions, concerning the generation site and process, should be answered even if the waste stream is no longer generated.

Name of Site Generating Waste (e.g., INEL, Rocky Flats, etc.): Bettis Atomic Power Laboratory

Location of activities (Area and Building):

Manhole sediment and debris and areas near some Radiological Facilities.

Operations (functions) performed in building:

The Bettis Atomic Power Laboratory is engaged solely in the design and development of naval nuclear propulsion plants. Laboratory operations include development and testing of nuclear fuel materials and reactor materials including radiochemical analysis.

\section{Description of Process Generating Waste:}

Cleanout of part of storm sewer system.

\section{BT-WO12}

Still-Gein Active 
BTWO12

YOC \& RCB CONTAMINATED DEBRIS

UWW Contact H

C. PROJECTED WASTE GENERATION

Is this waste stream still generated?

If yes, when is the expected generation termination date?

Current Projections

\begin{tabular}{|r|r|r|r|l|l|}
\hline Est. Date & Start Year & Interval & Vol (m3) & Mass (kg) & Source of Estimate \\
\hline $3 / 30 / 95$ & 1995 & 5 & 0.42 & & Generator Update \\
\hline \multicolumn{5}{|c|}{ Historical Projections } \\
\hline
\end{tabular}

\begin{tabular}{|c|r|r|r|r|r|}
\hline Est. Date & Start Year & Interval & Vol $(\mathrm{m} 3)$ & Mass $(\mathrm{kg})$ & Source of Estimate \\
\hline $11 / 1 / 93$ & 1995 & 1 & 2.10 & & $1993-\mathrm{FFCA}$ \\
\hline
\end{tabular}




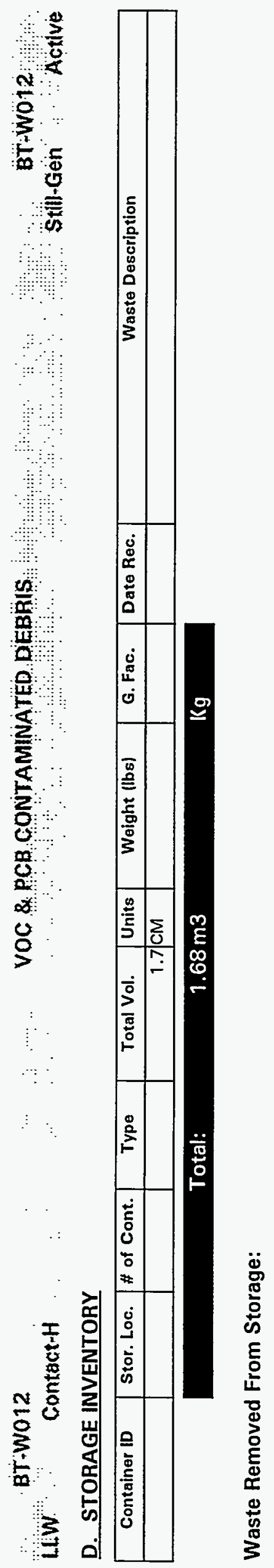




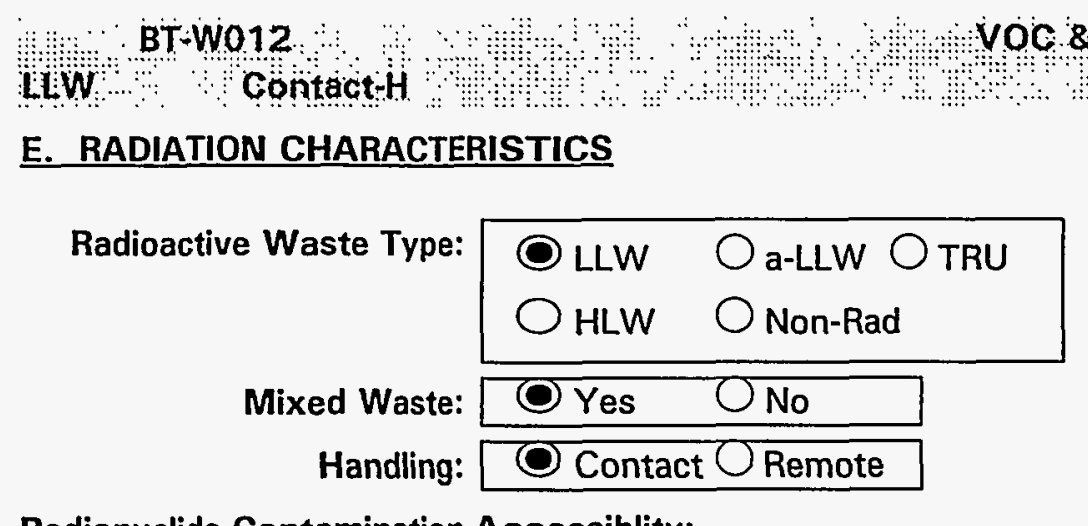

Radionuclide Contamination Accessiblity:

External Surface

Internal Surface

Dispersed Through Matrix

\begin{tabular}{|c|c|c|}
\hline OYes & ONo & OUnknown \\
\hline OYes & ONo & OUnknown \\
\hline OYes & ONo & OUnt \\
\hline
\end{tabular}

Activity Levels

Transuranic Alpha Activity:

Uranium/Thorium Alpha Activity: 8E-14 Units: $\mathrm{Ci} / \mathrm{g}$

Beta/Gamma Activity:

Surface Neutron Activity:

Total Activity:

\section{F. TRU ALPHA ACTIVITY DISTRIBUTION}

This waste stream is not identified as a TRU or a- $L L W$.

\begin{tabular}{|c|c|}
\hline $8 \mathrm{E}-14$ & Units: $\mathrm{Ci} / \mathrm{g}$ \\
\hline $5.1 \mathrm{E}-11$ & Units: $\mathrm{Ci} / \mathrm{g}$ \\
\hline$<0.1$ & @ surface \\
\hline$<0.1$ & @ 1-m Units: $\mathrm{mR} / \mathrm{hr}$ \\
\hline & Units: [ \\
\hline 0.2 & $\mathrm{nCi} / \mathrm{g}$ \\
\hline
\end{tabular}


BT-W012

LiWW Contact H

\section{G. RADIONUCLIDE CONCENTRATIONS}

Specific Activity

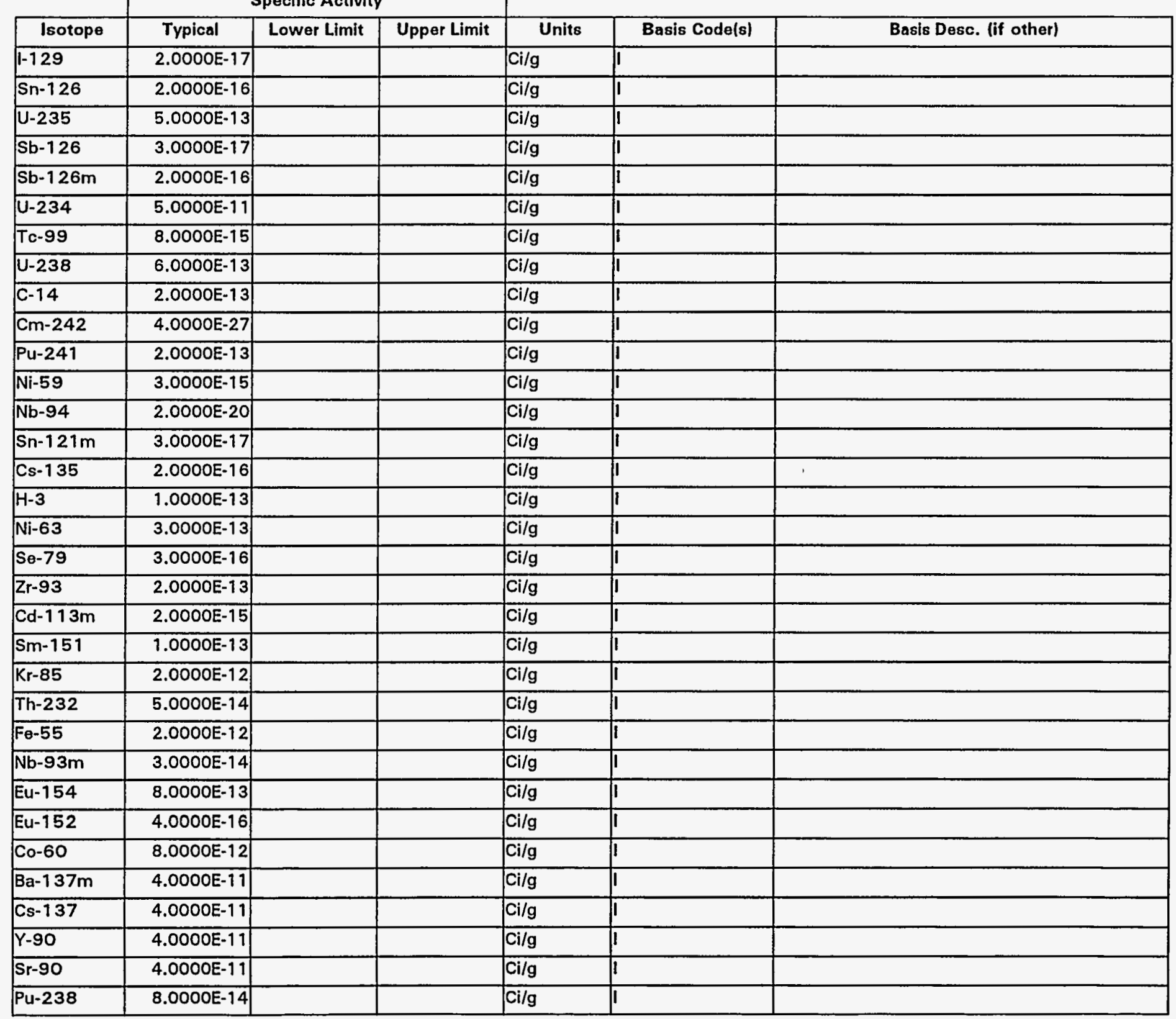

$7 / 27 / 95$
VOC \& PCB CONTAMINATED DEBRIS

BT:W012 Stili:Geñ : Active 


\section{BT WO12}

\section{LWW Contact H}

A. Passive-Active Neutron (PAN)

B. Segmented Gamma Scan (SGS)

C. Passive-Active Neutron/Segmented Gamma Scan (PAN/SGS

D. Radiochemical Analysis

E. Mass Spectrometry

F. Accountability

G. Calorimetry

G. Calorimetry

H. Multiple Energy Garm
I. Process Knowledge

J. Other 


\section{H. MATRIX CHARACTERISTICS}

Primary Matrix Code: 55490

Description: Debris Waste: Heterogeneous Debris: Unknown/Other Heterogeneous Debris:

Combustibility: $\begin{array}{ll}\bigcirc \text { Combustible }(>90 \%) \\ \bigcirc \text { Mixed }(10 \%-90 \%) \\ \bigcirc \text { Noncombustible }(<10 \%)\end{array}$

\section{MATRIX COMPOSITION}

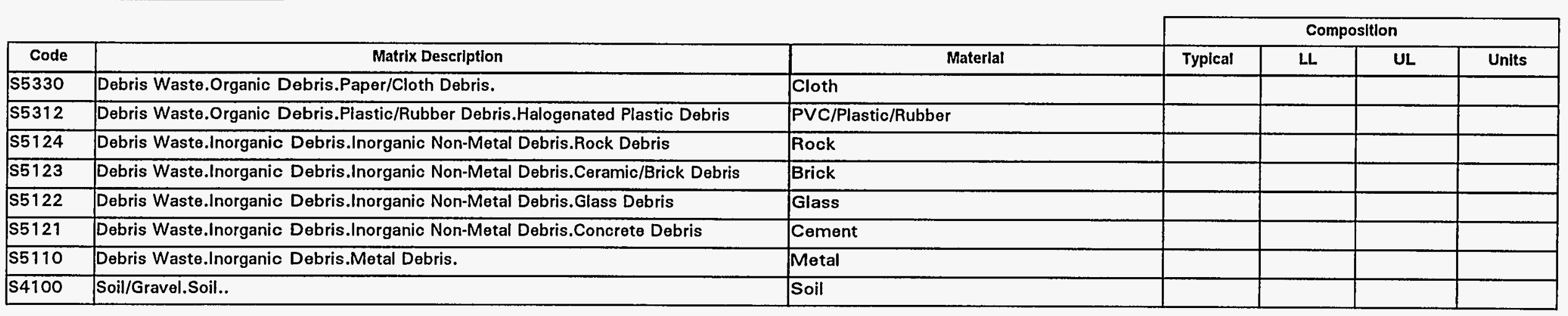


BT-WO12 LWW Contacth

VOG \& PCB CONTAMINATED DEBRIS

\section{J. HAZARDOUS CONSTITUENTS}

\begin{tabular}{|c|c|c|c|c|c|c|c|c|}
\hline & & & \multicolumn{4}{|c|}{ Concentration } & \multirow[b]{2}{*}{ Basis } & \multirow[b]{2}{*}{ Conc. Type } \\
\hline Code & Hazardous Waste Description & Treatment Subcategory & Typical & $\mathbf{L L}$ & UL & Units & & \\
\hline F001 & Spent halogenated solvents used in degreasing & Tetrachloroethylene & 1000 & 0.007 & 33000 & ppm & $\bar{A}$ & Total \\
\hline F002 & Spent halogenated solvents & Chlorobenzene & & $\overline{19}$ & 95 & ppm & A & Total \\
\hline F002 & Spent halogenated solvents & Methylene chloride & & 1.5 & 1100 & ppm & A & Total \\
\hline F002 & Spent halogenated solvents & Trichloroethylene & 20 & 0.028 & 230 & ppm & A & Total \\
\hline F003 & Spent non-halogenated solvents & Acetone & 0.6 & & & ppm & $A$ & Total \\
\hline FOO3 & Spent non-halogenated solvents & Ethyl benzene & 0.044 & & & ppm & $\bar{A}$ & Total \\
\hline F003 & Spent non-halogenated solvents & Xylenes - mixed isomers & & & & & $A$ & Total \\
\hline$\overline{F 005}$ & Spent non-halogenated solvents & Carbon disulfide & 0.01 & & & $\mathrm{ppm}$ & A & Total \\
\hline F005 & Spent non-halogenated solvents & Methyl ethyl ketone & & 0.12 & 0.17 & $\mathrm{ppm}$ & A & Total \\
\hline F005 & Spent non-halogenated solvents & Toluene & & 0.006 & 0.062 & $\mathrm{ppm}$ & A & Total \\
\hline
\end{tabular}




\section{LWW Contact H}

\section{K. POLYCHLORINATED BIPHENYLS (PCBS)}

Is this waste stream PCB contaminated?

O Yes O No $\bigcirc$ Unknown
contaminated (volume \%): 75

If yes, what portion of the waste is $\mathrm{PCB}$ contaminated (volume \%): 75

If $<100 \%$, can the PCB contaminated waste be segregated and treated separately?

What is the PCB concentration in ppm? $>500$

If only a portion of the waste is $P C B$ contaminated, report values for contaminated portion.

\section{TREATMENT PLANS}

\begin{tabular}{|c|c|c|c|c|c|c|c|c|c|c|c|}
\hline $\begin{array}{c}\text { Opt. } \\
\#\end{array}$ & $\begin{array}{c}\text { Media Type } \\
\text { (If more than one) }\end{array}$ & $\begin{array}{c}\text { Matrix } \\
\text { Code }\end{array}$ & $\begin{array}{l}\text { Vol. \% } \\
\text { (If }\end{array}$ & Step & JIT & sc & CIF & $\begin{array}{l}\text { Trans. } \\
\text { Miles }\end{array}$ & $\begin{array}{l}\text { Facility } \\
\text { Abbr. }\end{array}$ & Unit Name & Comments \\
\hline \multirow[t]{6}{*}{1} & & 55490 & & $\bar{a}$ & 0 & 0 & $1.00 E+00$ & & TRANS & Transport - LLW & \\
\hline & & & & $\mathbf{b}$ & $\mathbf{O}$ & 0 & $1.00 E+00$ & & AMWTF & $\mathrm{CH}$ - Opening \& Sorting & \\
\hline & & & & c & (O) & 0 & $1.00 E+00$ & & AMWTF & $\mathrm{CH}$ - Sizing & \\
\hline & & & & d & 0 & 0 & $1.00 \mathrm{E}+00$ & & AMWTF & $\mathrm{CH}$ - Incineration/Thermal Desorption & \\
\hline & & & & $\mathbf{e}$ & $O$ & $\bar{O}$ & $1.00 E+00$ & & AMWTF & $\mathrm{CH} \cdot$ Vitrification & \\
\hline & & & & $f$ & $O$ & $\bar{O}$ & $1.00 E+00$ & & SCDF & Disposal - Contact Handled & \\
\hline
\end{tabular}

Note: Where provided, media percentages are rough estimates used to facilitate treatment planning. They do not necessarily imply an accurate knowledge of waste stream composition, and were not derived for use outside this conceptual planning exercise.

Describe any special or unique technical concerns related to the treatment of this waste stream that would impact the use or implementation of standard hazardous waste treatment methods. 


\section{$\therefore \quad$ BT WWO13}

YOC \& PCB CONTAMINATED SÖIL

BT:W013

LWW Contact-H

\section{A. IDENTIFICATION AND DESCRIPTION}

\section{Waste Stream ID: BT-W013}

W. S. Name:

Content Code:

FFCA ID:

\section{VOC \& PCB CONTAMINATED SOIL}

BT-W013

Waste Stream Description:

Soil containing F listed solvents and PCB's; contains alpha emitting radionuclides. Waste is of similar form from container to container. This waste is not a moratorium waste or reclassified TRU.

\section{B. GENERATION SITE/PROCESS DESCRIPTION}

The following questions, concerning the generation site and process, should be answered even if the waste stream is no longer generated.

Name of Site Generating Waste (e.g., INEL, Rocky Flats, etc.): Bettis Atomic Power Laboratory

\section{Location of activities (Area and Building):}

Inactive Waste Site and areas near some radiological facilities.

Operations (functions) performed in building:

The Bettis Atomic Power Laboratory is engaged solely in the design and development of naval nuclear propulsion plants. Laboratory operations include development and testing of nuclear fuel materials and reactor materials including radiochemical analysis.

\section{Description of Process Generating Waste:}

Soils generated as part of facility investigation. 
BT-W013

LLW Cöntact-H

C. PROJECTED WASTE GENERATION

Is this waste stream still generated?

If yes, when is the expected generation termination date?

Current Projections

\section{VOC \& PCB CONTAMINATED SOIL:}

BT-W013 Natmen Active

OYes ONo

\section{Historical Projections}


D. STORAGE INVENTORY

\begin{tabular}{|l|c|c|c|c|c|c|c|c|c|}
\hline Container ID & Stor. Loc. & \# of Cont. & Type & Total Vol. & Units & Weight (lbs) & G. Fac. & Date Rec. & Waste Description \\
\hline & \multicolumn{7}{|c|}{0.8} & \multicolumn{3}{|c|}{$0.84 \mathrm{m3}$} & & & & \\
\hline
\end{tabular}

Waste Removed From Storage: 


\section{BT-W013 \\ LLW Contact-H \\ E. RADIATION CHARACTERISTICS}

Radioactive Waste Type:

\begin{tabular}{|ll|}
\hline OllW & Oa-LLW O TRU \\
OHLW & O Non-Rad \\
\hline
\end{tabular}

Mixed Waste:

Handling:

\begin{tabular}{|l|}
\hline OYes ONo \\
\hline O Contact O Remote \\
\hline
\end{tabular}

Radionuclide Contamination Accessiblity:

\begin{tabular}{|c|c|c|}
\hline External Surface $\bigcirc$ Yes & ONo & OUnknown \\
\hline Internal Surface $O$ Yes & O No & OUnknown \\
\hline Dispersed Through Matrix $\bigcirc$ Yes & ONo & OUnknown \\
\hline \multirow{3}{*}{$\begin{array}{r}\text { Transuranic Alpha Activity: } \\
\text { Uranium/Thorium Alpha Activity: } \\
\text { Beta/Gamma Activity: }\end{array}$} & & Units: \\
\hline & & Units: \\
\hline & 6 & @ surface \\
\hline Beta/Gamma ActIvIty: & $<0.1$ & @ 1-m Units: $\mathrm{mR} / \mathrm{hr}$ \\
\hline Surface Neutron Activity: & & Units: [ \\
\hline Total Activity: & & $\mathrm{nCi} / \mathrm{g}$ \\
\hline
\end{tabular}

VOC \& PCB CONTAMINATED SOIL

BT-W013

NötGën Active

\section{F. TRU ALPHA ACTIVITY DISTRIBUTION}

This waste stream is not identified as a TRU or a-LLW. 
BT-W013

VOC \& PGB CONTAMINATED SOIL

HeW oontactit

\section{G. RADIONUCLIDE CONCENTRATIONS}

\begin{tabular}{|l|c|c|c|c|c|c|}
\cline { 2 - 6 } \multicolumn{1}{c|}{ Isotope } & Typical & Lower Limit & Upper Limit & Units & Basis Code(s) & Basis Desc. (if other) \\
\hline Sr-90 & & $5.0000 \mathrm{E}-13$ & $2.3000 \mathrm{E}-11$ & $\mathrm{Ci} / \mathrm{g}$ & $\mathrm{D}$ & \\
\hline Cs-137 & $4.3000 \mathrm{E}-13$ & $2.9000 \mathrm{E}-13$ & $5.7000 \mathrm{E}-13$ & $\mathrm{Ci} / \mathrm{g}$ & $\mathrm{D}$ & \\
\hline U-nat & & & & & $\mathrm{D}$ & \\
\hline
\end{tabular}

A. Passive-Active Neutron (PAN)

F. Accountability

Segmented Gamma Scan (SGS)

G. Calorimetry

. Passive Active Neutron/Segmented Gamma Scan (PAN/SGS)

H. Multiple Energy Gamma Analysis (MEGAS

D. Radiochemical Analysis

1. Process Knowledge

E. Mass Spectrometry

J. Other 


\section{BT:W013}

LiW : Contact H

VOC \& PCB CONTAMINATED SOIL

BTHOWO13

H. MATRIX CHARACTERISTICS

Primary Matrix Code: $\mathbf{S 4 1 0 0}$

Description: Soil/Gravel: Soil: :

Combustibility: $\bigcirc$ Combustible (>90\%)

Mixed $(10 \%-90 \%)$

Noncombustible (<10\%)

\section{MATRIX COMPOSITION}

\begin{tabular}{|c|c|c|c|c|c|c|}
\hline & & & \multicolumn{4}{|c|}{ Composittion } \\
\hline Code & Matrix Description & Materlal & Typical & LL & UL & Units \\
\hline$\$ 4100$ & Soil/Gravel.Soil.. & Soils & 100 & 100 & 100 & $\%$ vol \\
\hline
\end{tabular}




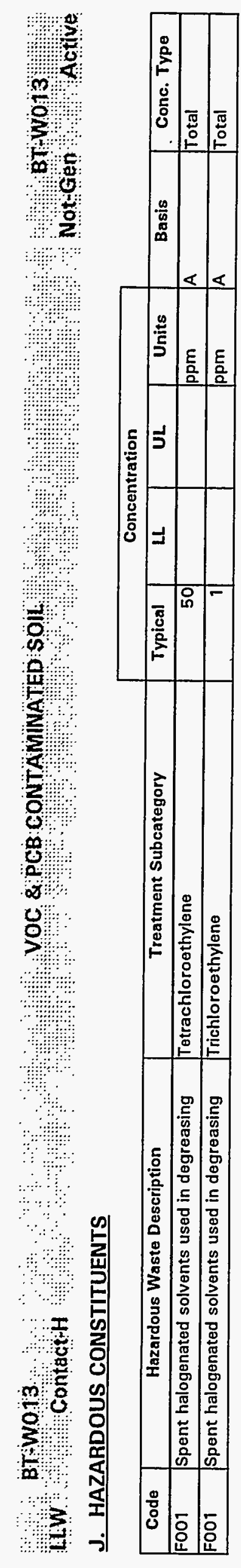




\section{BT:W013 \\ Contact H}

YOC \& PCB CONNTAMINATED SOIL

BT-WO13

K. POLYCHLORINATED BIPHENYLS (PCBS)

Is this waste stream PCB contaminated?

O Yes O No O Unknown

If yes, what portion of the waste is $\mathrm{PCB}$ contaminated (volume \%): 100

If $<100 \%$, can the PCB contaminated waste be segregated and treated separately?

What is the PCB concentration in ppm?

$<50$

If only a portion of the waste is $P C B$ contaminated, report values for contaminated portion.

\section{TREATMENT PLANS}

\begin{tabular}{|c|c|c|c|c|c|c|c|c|c|c|c|}
\hline $\begin{array}{l}\text { Opt. } \\
\#\end{array}$ & $\begin{array}{c}\text { Media Type } \\
\text { (If more than one) }\end{array}$ & $\begin{array}{l}\text { Matrix } \\
\text { Code }\end{array}$ & $\begin{array}{l}\text { Vol. \% } \\
\text { IIf }\end{array}$ & Step & JIT & sc & CIF & $\begin{array}{l}\text { Trans. } \\
\text { Miles }\end{array}$ & $\begin{array}{l}\text { Facility } \\
\text { Abbr. }\end{array}$ & Unit Name & Comments \\
\hline \multirow[t]{6}{*}{1} & & 54100 & & $\bar{a}$ & O) & $\bar{O}$ & $1.00 E+00$ & & TRANS & Transport - LLW & \\
\hline & & & & $\bar{b}$ & O) & 0 & $1.00 \mathrm{E}+00$ & & AMWTF & $\mathrm{CH}$ - Opening \& Sorting & \\
\hline & & & & $\bar{c}$ & $\mathcal{O}$ & 0 & $1.00 E+00$ & & AMWTF & $\mathrm{CH}$ - Sizing & \\
\hline & & & & d & 0 & $\bar{O}$ & $1.00 E+00$ & & AMWTF & $\mathrm{CH}$ - Incineration/Thermal Desorption & \\
\hline & & & & e & 0 & 0 & $1.00 E+00$ & & AMWTF & $\mathrm{CH}$ - Vitrification & \\
\hline & & & & $f$ & 0 & 0 & $1.00 E+00$ & & SCDF & Disposal - Contact Handled & \\
\hline
\end{tabular}

Note: Where provided, media percentages are rough estimates used to facilitate treatment planning. They do not necessarily imply an accurate knowledge of waste stream composition, and were not derived for use outside this conceptual planning exercise.

Describe any special or unique technical concerns related to the treatment of this waste stream that would impact the use or implementation of standard hazardous waste treatment methods. 
0

$\because \ldots$

\%

m

$\because$

$\because$

.

$\therefore$

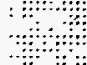

和

0

$\because$ :

:

औnon

:

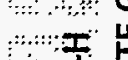

密

0

융웅

o:

$\frac{1}{\infty} \begin{aligned} & 3 \\ & \vdots\end{aligned}$

0
$z$

3

$\left[\begin{array}{l}0 \\ 2 \\ 0 \\ a \\ 2 \\ 0\end{array}\right]$

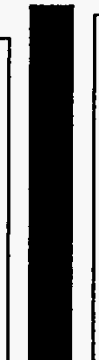




BLW BT-WO20
A. IDENTIFICATION AND DESCRIPTION
Waste Stream ID:
WT-W020
W. S. Name:
Content Code:
FFCA ID:
WaSS AND BRONZE
Waste Stream Description:
Brass and bronze valves, items, and fittings. This mixed waste stream has not been generated.
This waste is not a moratorium waste or reclassified TRU waste.

BT-W020

Still:Gen : Active

\section{B. GENERATION SITE/PROCESS DESCRIPTION}

The following questions, concerning the generation site and process, should be answered even if the waste stream is no longer generated.

Name of Site Generating Waste (e.g., INEL, Rocky Flats, etc.): Bettis Atomic Power Laboratory

Location of activities (Area and Building): Metallurgical Laboratories and radioactive waste processing facilities.

Operations (functions) performed in building:

The Bettis Atomic Power Laboratory is engaged solely in the design and development of naval nuclear propulsion plants. Laboratory operations include development and testing of nuclear fuel materials and reactor materials including radiochemical analysis.

Description of Process Generating Waste:

This mixed waste stream has not yet been generated. 
$\because$ BT-WOZ20 HW Contage H

D. STORAGE INVENTORY

Waste Removed From Storage:
BRASS AND BRONZE

BTWWO2O

Still-Gèn Actlue 
BT-W020

LiW $\quad$ Cóntact-H

E. RADIATION CHARACTERISTICS

Radioactive Waste Type:

\begin{tabular}{|ll|}
\hline OLLW & Oa-LLW $\bigcirc$ TRU \\
OHLW & $\bigcirc$ Non-Rad \\
\hline
\end{tabular}

BRASS AND BR̈ONZE

BT-WiO20

Still:Gen : Active

\section{F. TRU ALPHA ACTIVITY DISTRIBUTION}

This waste stream is not identified as a TRU or a-LLW.
Mixed Waste:

\begin{tabular}{|l|}
\hline O Yes $O$ No \\
\hline Contact O Remote
\end{tabular}

Handling: O Contact $\bigcirc$ Remote

Radionuclide Contamination Accessiblity:

External Surface

Internal Surface

Dispersed Through Matrix

\begin{tabular}{|lll|}
\hline Yes & O No & O Unknown \\
\hline OYes & O No & O Unknown \\
\hline OYes & O No & OUnknown \\
\hline
\end{tabular}

\section{Activity Levels}

Transuranic Alpha Activity: Uranium/Thorium Alpha Activity:

Beta/Gamma Activity:

Surface Neutron Activity:

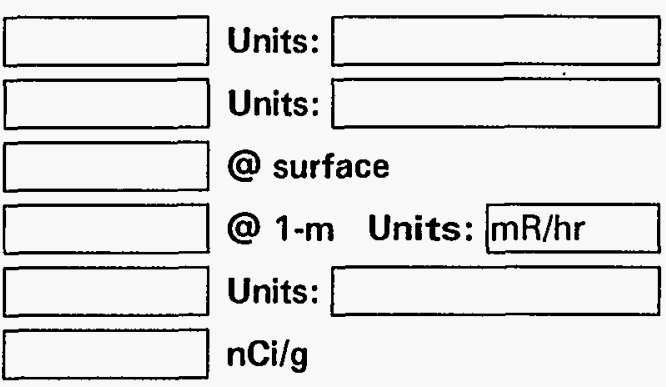

Total Activity: 
BT WO2O

HWW ContactH

\section{G. RADIONUCLIDE CONCENTRATIONS}

\begin{tabular}{|c|c|c|c|c|c|c|}
\hline & & pecific Activit & & & & \\
\hline Isotope & Typical & Lower Limit & Upper Limit & Units & Basis Code(s) & Basis Desc. (if other) \\
\hline Se-79 & & & & & 1 & \\
\hline $\mid-129$ & & & & & T & \\
\hline Tc-99 & & & & & 1 & \\
\hline $\mathrm{H}-3$ & & & & & $i$ & \\
\hline Cs-137 & & & & & 1 & \\
\hline Sr-90 & & & & & 1 & \\
\hline$Y-90$ & & & & & 1 & \\
\hline $\mathrm{Ba}-137 \mathrm{~m}$ & & & & & 1 & \\
\hline Cs-134 & & & & & I & \\
\hline Pm-147 & & & & & 1 & \\
\hline Kr-85 & & & & & $T$ & \\
\hline $\mathrm{Sm} \cdot 151$ & & & & & 1 & \\
\hline$C-14$ & & & & & 1 & \\
\hline $2 \mathrm{r}-93$ & & & & & 1 & \\
\hline $\mathrm{C}_{\theta-144}$ & & & & & 1 & \\
\hline $\mathrm{Nb}-93 \mathrm{~m}$ & & & & & 1 & \\
\hline $5 n-126$ & & & & & 1 & \\
\hline Cs-135 & & & & & 1 & \\
\hline $5 n-121 m$ & & & & & $L$ & \\
\hline $\mathrm{Nb}-94$ & & & & & $L$ & \\
\hline Pu-238 & & & & & 1 & \\
\hline Pu-241 & & & & & 1 & \\
\hline $\mathrm{Cm}-242$ & & & & & 1 & \\
\hline Co-60 & & & & & 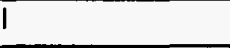 & \\
\hline $\mathrm{Ni}-63$ & & & & & 1 & \\
\hline Ni-59 & & & & & I & \\
\hline Eu-152 & & & & & I & \\
\hline Eu-154 & & & & & I & \\
\hline Pr-144 & & & & & I & \\
\hline $\mathrm{Cd}-113 \mathrm{~m}$ & & & & & I & \\
\hline
\end{tabular}

A. Passive-Active Neutron (PAN)

F. Accountability

B. Segmented Gamma Scan (SGS)

G. Calorimetry

H. Multiple Energy Gamma Analysis IMEGASI

I. Process Knowledge

J. Other

Waste Stream Profile Report - Idaho Mixed Waste Information (IMWII System

D. Radiochemical Analysis

7/27/95
BT: WOZ̈O

Still-Geñ an Active

Page 4-191 

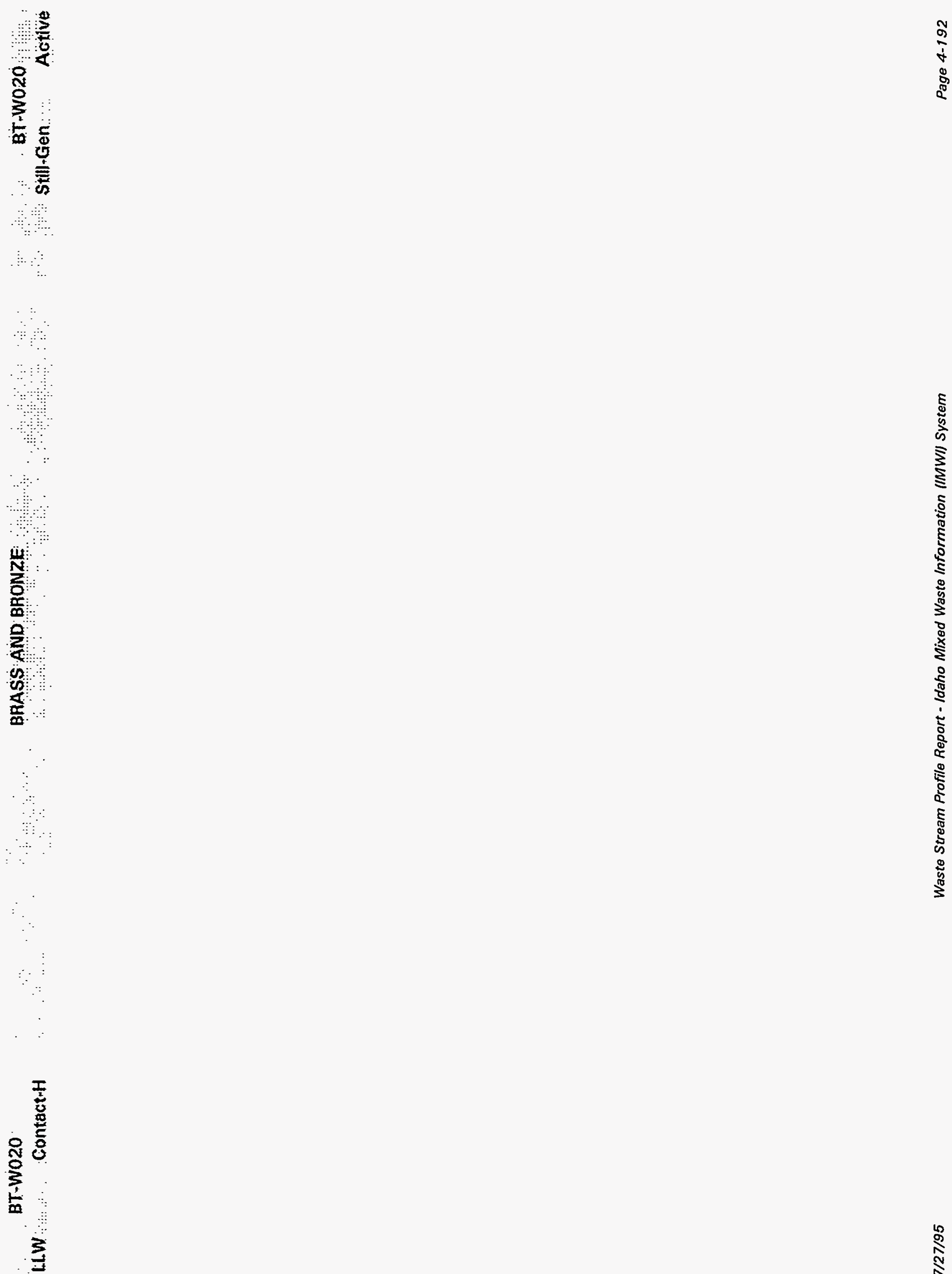
HWW Contact

H. MATRIX CHARACTERISTICS

Primary Matrix Code: $\mathbf{5} 5110$

Description: Debris Waste: Inorganic Debris: Metal Debris:

Combustibility: $\bigcirc$ Combustible $(>90 \%)$

Mixed (10\%-90\%)

Noncombustible $(<10 \%)$

\section{MATRIX COMPOSITION}

\begin{tabular}{|c|c|c|c|c|c|c|}
\hline & & & \multicolumn{4}{|c|}{ Composition } \\
\hline Code & Matrix Description & Material & Typical & LL & UL & Units \\
\hline$\$ 5110$ & Debris Waste.Inorganic Debris.Metal Debris. & & 100 & 100 & 100 & $\%$ vol \\
\hline
\end{tabular}




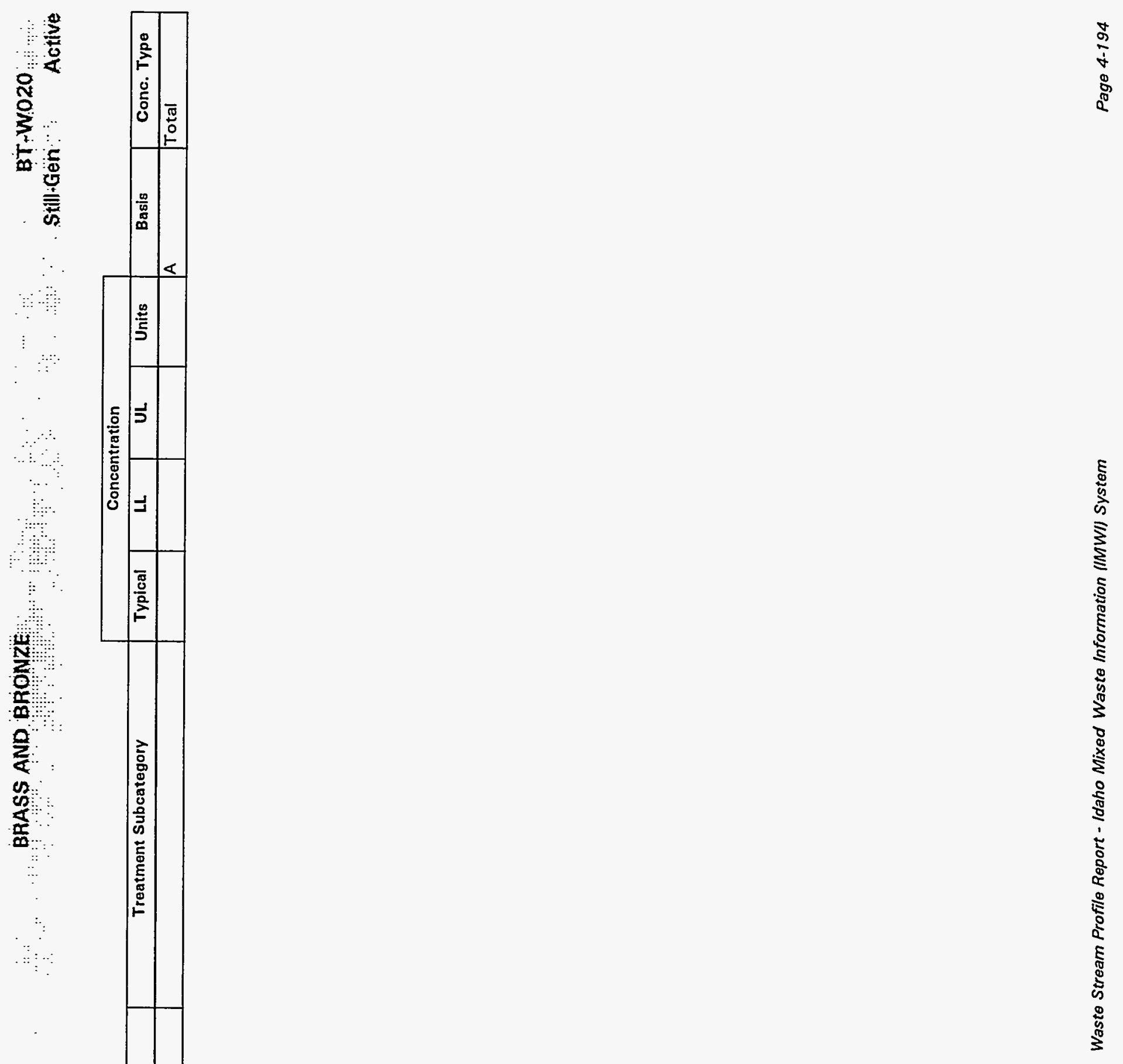

है 


\section{BTWWO2O}

HiW Contact H

\section{K. POLYCHLORINATED BIPHENYLS (PCBS)}

\section{Is this waste stream PCB contaminated? OYes $O$ No $O$ Unknown}

If yes, what portion of the waste is $\mathrm{PCB}$ contaminated (volume \%):

If $<100 \%$, can the PCB contaminated waste be segregated and treated separately? OYes O No O Unknown

What is the PCB concentration in ppm? If only a portion of the waste is PCB contaminated, report values for contaminated portion.

\section{TREATMENT PLANS}

\begin{tabular}{|c|c|c|c|c|c|c|c|c|c|c|c|}
\hline $\begin{array}{c}\text { Opt. } \\
\#\end{array}$ & $\begin{array}{c}\text { Media Type } \\
\text { (If more than one) }\end{array}$ & $\begin{array}{c}\text { Matrix } \\
\text { Code }\end{array}$ & $\begin{array}{l}\text { Vol. \% } \\
\text { (If }\end{array}$ & Step & JIT & Sc & ClF & $\begin{array}{l}\text { Trans. } \\
\text { Miles }\end{array}$ & $\begin{array}{l}\text { Facility } \\
\text { Abbr. }\end{array}$ & Unit Name & Comments \\
\hline 1 & & $\$ 5110$ & & $\mathbf{a}$ & 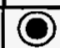 & 0 & $1.00 E+00$ & & TRANS & Transport - LLW & \\
\hline & & & & b & O & 0 & $1.00 E+00$ & & AMWTF & $\mathrm{CH}$ - Opening \& Sorting & \\
\hline & & & & c & 0 & 0 & $1.00 \mathrm{E}+00$ & & AMWTF & $\mathrm{CH}$ - Decontamination & \\
\hline & & & & d & 0 & 0 & $1.00 E+00$ & & AMWTF & $\mathrm{CH}$ - Sizing & \\
\hline & & & & e & 0 & 0 & $1.00 E+00$ & & AMWTF & CH - Macroencapsulation/Stabilization & \\
\hline & & & & $\bar{f}$ & 0 & 0 & $1.00 E+00$ & & SCDF & Disposal - Contact Handled & \\
\hline
\end{tabular}

Note: Where provided, media percentages are rough estimates used to facilitate treatment planning. They do not necessarily imply an accurate knowledge of waste stream composition, and were not derived for use outside this conceptual planning exercise.

Describe any special or unique technical concerns related to the treatment of this waste stream that would impact the use or implementation of standard hazardous waste treatment methods. 
BT-WO28

LLW

\section{Contactin}

\section{A. IDENTIFICATION AND DESCRIPTION}

Waste Stream ID: BT-W028

W. S. Name:

Content Code:

FFCA ID: $\quad$ BT-W028

\section{VOC AND PCB CONTAMINATED WATER}

Waste Stream Description:

Contaminated water from storm sewer system. Waste is homogeneous. This waste is not a moratorium waste or reclassified TRU waste.

\section{VOC AND PCB CONTAMINATED WATER}

\section{BT-W028}

Nötigèn Active

\section{B. GENERATION SITE/PROCESS DESCRIPTION}

The following questions, concerning the generation site and process, should be answered even if the waste stream is no longer generated.

Name of Site Generating Waste (e.g., INEL, Rocky Flats, etc.): Bettis Atomic Power Laboratory

Location of activities (Area and Building):

Manhole and yarddrains.

Operations (functions) performed in building:

The Bettis Atomic Power Laboratory is engaged solely in the design and development of naval nuclear propulsion plants. Laboratory operations include development and testing of nuclear fuel materials and reactor materials including radiochemical analysis.

\section{Description of Process Generating Waste:}

Cleanout of part of storm sewer system. 
BT-WÖ28

LlWW : Contact $+\mathrm{H}$

C. PROJECTED WASTE GENERATION

Is this waste stream still generated?

If yes, when is the expected generation termination date?

Current Projections

\begin{tabular}{|r|r|r|r|r|l|}
\hline Est. Date & Start Year & Interval & Vol (m3) & Mass $(\mathrm{kg})$ & Source of Estimate \\
\hline $3 / 30 / 95$ & 1995 & 5 & 0.63 & & Generator Update \\
\hline
\end{tabular}

Historical Projections 


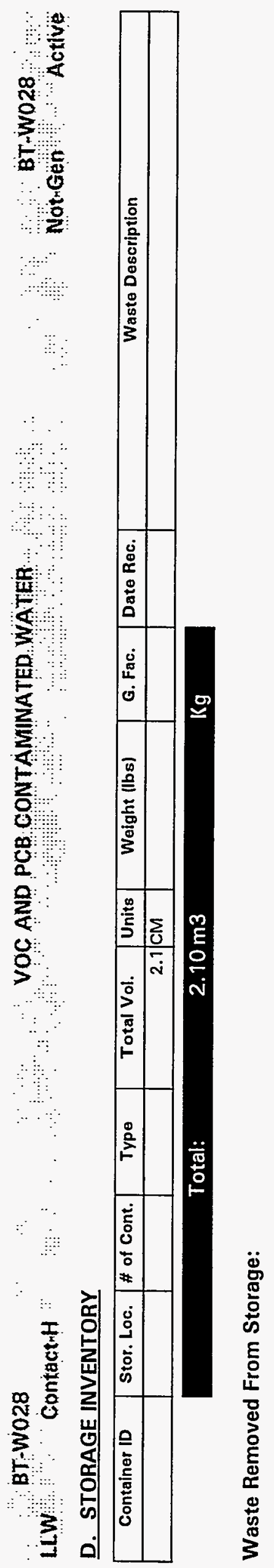




\section{BT-Woz}

\section{E. RADIATION CHARACTERISTICS}

Radioactive Waste Type:

\begin{tabular}{|ll|}
\hline O LLW & Oa-LLW $\bigcirc$ TRU \\
OHLW & O Non-Rad \\
\hline
\end{tabular}

Mixed Waste:

Handling:

\begin{tabular}{|l|}
\hline OYes O No \\
\hline O Contact O Remote \\
\hline
\end{tabular}

Radionuclide Contamination Accessiblity:

External Surface $O$ Yes $O$ No OUnknown

Internal Surface $O$ Yes $O$ No OUnknown

Dispersed Through Matrix $O$ Yes O No O Unknown

\section{Activity Levels}

Transuranic Alpha Activity:

Uranium/Thorium Alpha Activity:

Beta/Gamma Activity:

Surface Neutron Activity:

Total Activity:

\begin{tabular}{|c|c|}
\hline $8 \mathrm{E}-16$ & Units: $\mathrm{Ci} / \mathrm{g}$ \\
\hline $5 E-14$ & Units: $\widehat{\mathrm{Ci} / \mathrm{g}}$ \\
\hline$<0.1$ & @ surface \\
\hline$<0.1$ & @ 1-m Units: $\mathrm{mR} / \mathrm{hr}$ \\
\hline & Units: [ \\
\hline $1.9 \mathrm{E}-4$ & $\mathrm{nCi} / \mathrm{g}$ \\
\hline
\end{tabular}

This waste stream is not identified as a TRU or a- $L L W$. 


\section{BT-W028}

\section{LiW: Contact}

\section{G. RADIONUCLIDE CONCENTRATIONS}

\section{Specific Activity}

\begin{tabular}{|c|c|c|c|c|c|c|}
\hline & & pecific Act & & & & \\
\hline Isotope & Typical & Lower Limit & Upper Limit & Units & Basis Code(s) & Basis Desc. (if other) \\
\hline $\mathrm{Cd}-113 \mathrm{~m}$ & $2.0000 \mathrm{E}-18$ & & & $\mathrm{Ci} / \mathrm{g}$ & 1 & \\
\hline Cs-137 & $3.0000 \mathrm{E}-14$ & & & $\mathrm{Ci} / \mathrm{g}$ & 1 & \\
\hline$S n-126$ & $2.0000 E-19$ & & & $\mathrm{Ci} / \mathrm{g}$ & I & \\
\hline $\mathrm{Nb}-93 \mathrm{~m}$ & $2.0000 E-19$ & & & $\mathrm{Ci} / \mathrm{g}$ & 1 & \\
\hline Se-79 & $2.0000 \mathrm{E}-19$ & & & $\mathrm{Ci} / \mathrm{g}$ & ti & \\
\hline Cs-135 & $1.0000 \mathrm{E}-19$ & & & $\overline{\mathrm{Ci} / \mathrm{g}}$ & 1 & \\
\hline$z r-93$ & $2.0000 \mathrm{E}-18$ & & & $\overline{\mathrm{Ci} / \mathrm{g}}$ & T & \\
\hline Sb-126 & $2.0000 E-20$ & & & $\mathrm{Ci} / \mathrm{g}$ & $\pi$ & \\
\hline Tc-99 & $7.0000 E-18$ & & & $\mathrm{Ci} / \mathrm{g}$ & T & \\
\hline $\mathrm{Sm}-151$ & $1.0000 \mathrm{E}-16$ & & & $\mathrm{Ci} / \mathrm{g}$ & $\pi$ & \\
\hline Eu-154 & $6.0000 \mathrm{E}-16$ & & & $\mathrm{Ci} / \mathrm{g}$ & 1 & \\
\hline Kr-95 & $2.0000 \mathrm{E}-15$ & & & $\mathrm{Ci} / \mathrm{g}$ & $\pi$ & \\
\hline$\longdiv { \gamma - 9 0 }$ & $3.0000 E-14$ & & & $\mathrm{Ci} / \mathrm{g}$ & $\pi$ & \\
\hline $\mathrm{Sr}-90$ & $3.0000 \mathrm{E}-14$ & & & $\mathrm{Ci} / \mathrm{g}$ & $\pi$ & \\
\hline $\mathrm{Ba}-137 \mathrm{~m}$ & $3.0000 E-14$ & & & $\mathrm{Ci} / \mathrm{g}$ & $\pi$ & \\
\hline Eu-152 & $3.0000 \mathrm{E}-19$ & & & $\mathrm{Ci} / \mathrm{g}$ & 1 & \\
\hline Pu-241 & $1.0000 \mathrm{E}-16$ & & & $\mathrm{Ci} / \mathrm{g}$ & 1 & \\
\hline Th-232 & $5.0000 E-15$ & & & $\mathrm{Ci} / \mathrm{g}$ & 1 & \\
\hline$\overline{U-238}$ & $5.0000 E-16$ & & & $\mathrm{Ci} / \mathrm{g}$ & $\pi$ & \\
\hline U-235 & $4.0000 E-16$ & & & $\mathrm{Ci} / \mathrm{g}$ & 1 & \\
\hline$U-234$ & $4.0000 \mathrm{E}-14$ & & & $\mathrm{Ci} / \mathrm{g}$ & $T$ & \\
\hline C-14 & $2.0000 \mathrm{E}-16$ & & & $\overline{C i / g}$ & 1 & \\
\hline Sb-126m & $2.0000 \mathrm{E}-19$ & & & $\mathrm{Ci} / \mathrm{g}$ & 1 & \\
\hline $\mathrm{H}-3$ & $8.0000 \mathrm{E}-17$ & & & $\overline{\mathrm{Ci} / \mathrm{g}}$ & 1 & \\
\hline Pu-238 & $8.0000 \mathrm{E}-16$ & & & $\mathrm{Ci} / \mathrm{g}$ & 1 & \\
\hline $\mathrm{Cm}-242$ & $4.0000 \mathrm{E}-32$ & & & $\mathrm{Ci} / \mathrm{g}$ & $\pi$ & \\
\hline Fe-55 & $9.0000 \mathrm{E}-16$ & & & $\mathrm{Ci} / \mathrm{g}$ & 1 & \\
\hline $\mathrm{Ni}-63$ & $3.0000 \mathrm{E}-16$ & & & $\mathrm{Ci} / \mathrm{g}$ & 1 & \\
\hline $\mathrm{Nb}-94$ & $1.0000 \mathrm{E}-23$ & & & $\mathrm{Ci} / \mathrm{g}$ & 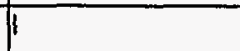 & \\
\hline Co-60 & $5.0000 \mathrm{E}-15$ & & & $\mathrm{Ci} / \mathrm{g}$ & 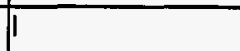 & \\
\hline $1-129$ & $1.0000 \mathrm{E}-20$ & & & $\overline{\mathrm{Ci} / \mathrm{g}}$ & 1 & \\
\hline $5 n-121 m$ & $2.0000 \mathrm{E}-20$ & & & $\mathrm{Ci} / \mathrm{g}$ & $t$ & \\
\hline Ni-59 & $3.0000 \mathrm{E}-18$ & & & $\mathrm{Ci} / \mathrm{g}$ & 7 & \\
\hline
\end{tabular}


JaYpO

abpalmouy ssavosd

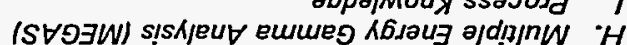
Rnawisojes : 1!!!!qejunooo $\forall$-y huawonoads ssew $\exists$ sishieu

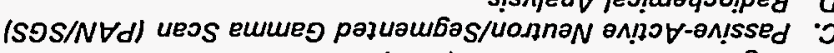
(SOS) ueos eumeg pajuaubas "8

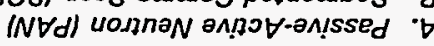




\section{BT-W028}

LLW: Contäct-H

H. MATRIX CHARACTERISTICS

Primary Matrix Code: $\lcm{1130}$

Description: Aqueous Liquids/Slurries: Wastewaters: Neutral Wastewaters:

\section{Combustibility: $\bigcirc$ Combustible $(>90 \%)$ \\ Mixed $(10 \%-90 \%)$ \\ Noncombustible $(<10 \%)$}

\section{MATRIX COMPOSITION}

\begin{tabular}{|c|c|c|c|c|c|c|}
\hline \multirow[b]{2}{*}{ Code } & \multirow[b]{2}{*}{ Matrix Description } & \multirow[b]{2}{*}{ Material } & \multicolumn{4}{|c|}{ Composition } \\
\hline & & & Typlcal & LL & UL & Units \\
\hline$\$ 3120$ & Homogeneous Solids.Inorganic Homogeneous Solids.Inorganic Sludges. & & $<1$ & & & $\%$ vol \\
\hline 11130 & Aqueous Liquids/Slurries.Wastewaters. Neutral WasteWaters. & & 99 & & & $\%$ vol \\
\hline
\end{tabular}


BT:WO28

HW $\because$ Contact

\section{J. HAZARDOUS CONSTITUENTS}

\begin{tabular}{|c|c|c|c|c|c|c|c|c|}
\hline & & & \multicolumn{4}{|c|}{ Concentration } & \multirow[b]{2}{*}{ Basis } & \multirow[b]{2}{*}{ Conc. Type } \\
\hline Code & Hazardous Waste Description & Treatment Subcategory & Typical & LL & UL & Units & & \\
\hline F001 & Spent halogenated solvents used in degreasing & Tetrachloroethylene & 1275 & & & $\mathrm{mg} / \mathrm{kg}$ & A & Total \\
\hline F001 & Spent halogenated solvents used in degreasing & Trichloroethylene & 69.4 & & & $\mathrm{mg} / \mathrm{kg}$ & A & Total \\
\hline$\overline{F 002}$ & Spent halogenated solvents & Chlorobenzene & 28.5 & & & $\mathrm{mg} / \mathrm{kg}$ & A & Total \\
\hline F002 & Spent halogenated solvents & Methylene chloride & 64.2 & & & $\mathrm{mg} / \mathrm{kg}$ & A & Total \\
\hline$\overline{F 003}$ & Spent non-halogenated solvents & Acetone & 0.6 & & & $\mathrm{mg} / \mathrm{kg}$ & A & Total \\
\hline
\end{tabular}


K. POLYCHLORINATED BIPHENYLS (PCBS)

Is this waste stream PCB contaminated?

OYes Ono O Unknown

If yes, what portion of the waste is PCB contaminated (volume \%): 100

If $<100 \%$, can the PCB contaminated waste be segregated and treated separately?

What is the PCB concentration in ppm? $<1500$ If only a portion of the waste is PCB contaminated, report values for contaminated portion.

\section{TREATMENT PLANS}

\begin{tabular}{|c|c|c|c|c|c|c|c|c|c|c|c|}
\hline Opt. & $\begin{array}{c}\text { Media Type } \\
\text { (If more than one) }\end{array}$ & $\begin{array}{l}\text { Matrix } \\
\text { Code }\end{array}$ & $\begin{array}{l}\text { Vol. \% } \\
\text { (If }\end{array}$ & Step & JIT & sc & CIF & $\begin{array}{l}\text { Trans. } \\
\text { Miles }\end{array}$ & $\begin{array}{l}\text { Facility } \\
\text { Abbr. }\end{array}$ & Unit Name & Comments \\
\hline 1 & & L1130 & & $\bar{a}$ & () & $\bar{O}$ & $1.00 E+00$ & & TRANS & Transport - LLW & \\
\hline & & & & $\mathbf{b}$ & ( & $\overline{0}$ & $1.00 \mathrm{E}+00$ & & AMWTF & $\mathrm{CH}$ - Opening \& Sorting & \\
\hline & & & & c & 0 & 0 & $1.00 E+00$ & & AMWTF & $\mathrm{CH}$ - Incineration/Thermal Desorption & liquid injection \\
\hline & & & & $\bar{d}$ & 0 & $\overline{0}$ & $1.00 \mathrm{E}+00$ & & AMWTF & $\mathrm{CH}$ - Vitrification & \\
\hline & & & & e & 0 & 0 & $1.00 E+00$ & & SCDF & Disposal - Contact Handled & \\
\hline
\end{tabular}

Note: Where provided, media percentages are rough estimates used to facilitate treatment planning. They do not necessarily imply an accurate knowledge of waste stream composition, and were not derived for use outside this conceptual planning exercise.

Describe any special or unique technical concerns related to the treatment of this waste stream that would impact the use or implementation of standard hazardous waste treatment methods. 
LWW BTWO29

\section{A. IDENTIFICATION AND DESCRIPTION}

Waste Stream ID: BT-W029

\section{W. S. Name:}

VOC CONTAMINATED SEDIMENT/SLUDGE

Content Code:

FFCA ID:

BT-W029

\section{Waste Stream Description:}

Contaminated sediment/sludge from storm sewer system. Waste is homogeneous. This waste is not a moratorium waste or reclassified TRU waste.
BT:WO29

\section{B. GENERATION SITE/PROCESS DESCRIPTION}

The following questions, concerning the generation site and process, should be answered even if the waste stream is no longer generated.

Name of Site Generating Waste (e.g., INEL, Rocky Flats, etc.): Bettis Atomic Power Laboratory

Location of activities (Area and Building):

Manholes and yarddrains.

Operations (functions) performed in building:

The Bettis Atomic Power Laboratory is engaged solely in the design and development of naval nuclear propulsion plants. Laboratory operations include development and testing of nuclear fuel materials and reactor materials including radiochemical analysis.

Description of Process Generating Waste:

Cleanout of part of storm sewer system. 
$\therefore$ BT:WO29

HWW ContactrH

C. PROJECTED WASTE GENERATION

Is this waste stream still generated?

If yes, when is the expected generation termination date?

Current Projections

\begin{tabular}{|r|r|r|r|r|r|}
\hline Est. Date & Start Year & Interval & \multicolumn{1}{|c|}{ Vol (m3) } & Mass (kg) & Source of Estlmate \\
\hline $11 / 15 / 94$ & 1997 & 1 & 0.63 & 800.00 & $1994-D S T P$ \\
\hline
\end{tabular}

Historical Projections

OYes ONo

\section{VOC CONTAMINATED SEDIMENTISLUDGE}

BT.WO29

Stlll Gen Acrive 
$\therefore$ BTWWO29

VOC CONTAMINATED SEDIMENTISLUDGE

D. STORAGE INVENTORY

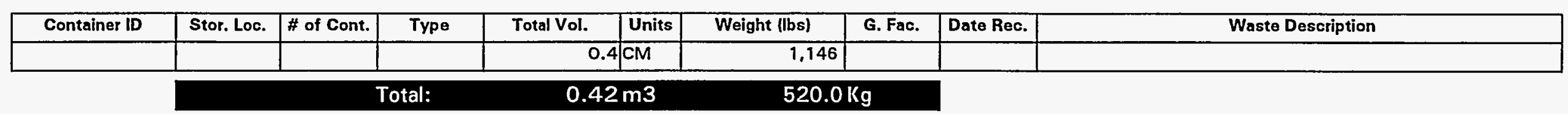

Waste Removed From Storage: 

LWW BT-WO29
VOC CONTAMINATED SEDIMENTISLUDGE

\section{E. RADIATION CHARACTERISTICS}

\section{F. TRU ALPHA ACTIVITY DISTRIBUTION}

\begin{tabular}{|c|c|}
\hline Radioactive Waste Type: & $\begin{array}{ll}\text { OLLW } & \text { Oa-LLW } \\
\text { OHLW } & \text { ONon-Rad }\end{array}$ \\
\hline Mixed Waste: & O Yes \\
\hline Handling: & (O) Contact ORemote \\
\hline
\end{tabular}

This waste stream is not identified as a TRU or a-LLW.

Radionuclide Contamination Accessiblity:

External Surface

Internal Surface

\begin{tabular}{|c|c|}
\hline OYes O No & Unknown \\
\hline No & Unnknown \\
\hline
\end{tabular}

Dispersed Through Matrix

OYes ONo O Unknown

\section{Activity Levels}

Transuranic Alpha Activity:

Uranium/Thorium Alpha Activity:

Beta/Gamma Activity:

Surface Neutron Activity:

\begin{tabular}{l}
\hline $2 \mathrm{E}-13$ Units: $\mathrm{Ci} / \mathrm{g}$ \\
\hline $1 \mathrm{E}-11$ Units: $\mathrm{Ci} / \mathrm{g}$ \\
\hline
\end{tabular}



$<0.1$ @ surface

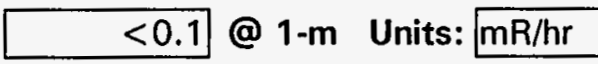

Total Activity: Units: $0.041 \mathrm{nCi} / \mathrm{g}$ 
BT-WOZ29

VOC CONTAMINIATED SEDIMENTTISLUDGE

\section{G. RADIONUCLIDE CONCENTRATIONS}

\begin{tabular}{|c|c|c|c|c|c|c|}
\hline \multirow{2}{*}{ Isotope } & \multicolumn{3}{|c|}{ Specific Activity } & \multirow[b]{2}{*}{ Units } & \multirow[b]{2}{*}{ Basis Code(s) } & \multirow[b]{2}{*}{ Basis Desc. (if other) } \\
\hline & Typical & Lower Limit & Upper Limit & & & \\
\hline$C d-113 m$ & $4.0000 E-16$ & & & $\mathrm{Ci} / \mathrm{g}$ & 1 & \\
\hline Cs-137 & $7.0000 E-12$ & & & $\mathrm{Ci} / \mathrm{g}$ & I & \\
\hline $5 n-126$ & $4.0000 \mathrm{E}-17$ & & & $\mathrm{Ci} / \mathrm{g}$ & $\pi$ & \\
\hline $\mathrm{Nb}-93 \mathrm{~m}$ & $4.0000 \mathrm{E}-17$ & & & $\overline{\mathrm{Ci} / \mathrm{g}}$ & t & \\
\hline$S \theta-79$ & $5.0000 \mathrm{E}-17$ & & & $\mathrm{Ci} / \mathrm{g}$ & 1 & \\
\hline Cs-135 & $3.0000 \mathrm{E}-17$ & & & $\mathrm{Ci} / \mathrm{g}$ & 1 & \\
\hline $2 r-93$ & $4.0000 \mathrm{E}-16$ & & & $\mathrm{Ci} / \mathrm{g}$ & 1 & \\
\hline$S b-126$ & $5.0000 \mathrm{E}-18$ & & & $\mathrm{Ci} / \mathrm{g}$ & 1 & \\
\hline Tc-99 & $2.0000 E-15$ & & & $\overline{\mathrm{Ci} / \mathrm{g}}$ & TI & \\
\hline $\mathrm{Sm}-151$ & $2.0000 E-14$ & & & $\mathrm{Ci} / \mathrm{g}$ & I & \\
\hline Eu-154 & $1.0000 E-13$ & & & $\overline{C i / g}$ & 1 & \\
\hline$\overline{K r-95}$ & $3.0000 E-13$ & & & $\mathrm{Ci} / \mathrm{g}$ & 1 & \\
\hline$\overline{Y-90}$ & $7.0000 \mathrm{E}-12$ & & & $\mathrm{Ci} / \mathrm{g}$ & 1 & \\
\hline Sr-90 & $7.0000 \mathrm{E}-12$ & & & $\mathrm{Ci} / \mathrm{g}$ & 1 & \\
\hline Ba-137m & $7.0000 \mathrm{E}-12$ & & & $\mathrm{Ci} / \mathrm{g}$ & 1 & \\
\hline Eu-152 & $7.0000 \mathrm{E}-17$ & & & $\mathrm{Ci} / \mathrm{g}$ & 1 & \\
\hline Pu-241 & $3.0000 \mathrm{E}-14$ & & & $\mathrm{Ci} / \mathrm{g}$ & 1 & \\
\hline Th-232 & $1.0000 \mathrm{E}-12$ & & & $\mathrm{Ci} / \mathrm{g}$ & 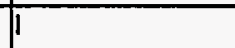 & \\
\hline U-238 & $1.0000 \mathrm{E}-13$ & & & $\mathrm{Ci} / \mathrm{g}$ & 1 & \\
\hline U-235 & $8.0000 E-14$ & & & $\mathrm{Ci} / \mathrm{g}$ & 1 & \\
\hline $\mathrm{U}-234$ & $9.0000 \mathrm{E}-12$ & & & $\mathrm{Ci} / \mathrm{g}$ & I & \\
\hline$\overline{C-14}$ & $3.0000 \mathrm{E}-14$ & & & $\mathrm{Ci} / \mathrm{g}$ & $\pi$ & \\
\hline Sb-126m & $3.0000 \mathrm{E}-17$ & & & $\mathrm{Ci} / \mathrm{g}$ & $\pi$ & \\
\hline $\mathrm{H}-3$ & $2.0000 \mathrm{E}-14$ & & & $\mathrm{Ci} / \mathrm{g}$ & 1 & \\
\hline Pu-238 & $2.0000 \mathrm{E}-13$ & & & $\mathrm{Ci} / \mathrm{g}$ & $\pi$ & \\
\hline $\mathrm{Cm}-242$ & $8.0000 E-30$ & & & $\mathrm{Ci} / \mathrm{g}$ & 1 & \\
\hline Fe-55 & $2.0000 \mathrm{E}-13$ & & & $\mathrm{Ci} / \mathrm{g}$ & 1 & \\
\hline $\mathrm{Ni}-63$ & 7.0000 E-14 & - & & $\mathrm{Ci} / \mathrm{g}$ & 1 & \\
\hline Nb-94 & 3.0000E-21 & & & $\mathrm{Ci} / \mathrm{g}$ & 1 & \\
\hline Co-60 & $1.0000 \mathrm{E}-12$ & & & $\mathrm{Ci} / \mathrm{g}$ & 1 & \\
\hline $1-129$ & $2.0000 E-18$ & & & $\mathrm{Ci} / \mathrm{g}$ & 1 & \\
\hline$S n-121 m$ & $5.0000 \mathrm{E}-18$ & & & $\mathrm{Ci} / \mathrm{g}$ & 1 & \\
\hline $\mathrm{Ni-59}$ & $7.0000 \mathrm{E}-16$ & & & $\mathrm{Ci} / \mathrm{g}$ & 1 & \\
\hline
\end{tabular}



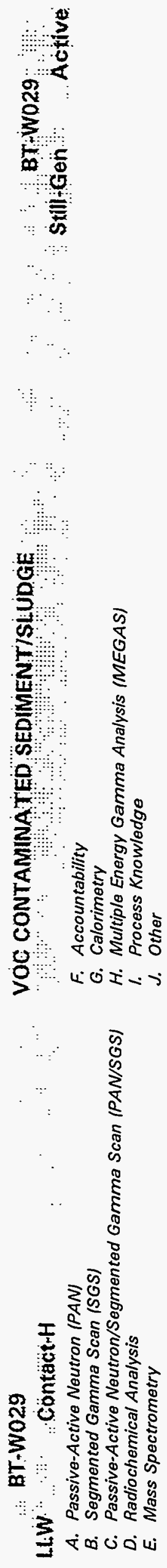
H. MATRIX CHARACTERISTICS

Primary Matrix Code: $\mathbf{S 3 1 2 0}$

Description: Homogeneous Solids: Inorganic Homogeneous Solids: Inorganic Sludges:

\section{Combustibility: $\bigcirc$ Combustible (>90\%) \\ Mixed $(10 \%-90 \%)$ \\ Noncombustible $(<10 \%)$}

\section{MATRIX COMPOSITION}

\begin{tabular}{|c|l|c|c|c|c|}
\cline { 2 - 6 } \multicolumn{2}{c|}{ Composition } \\
\hline Code & \multicolumn{1}{|c|}{ Matrix Description } & Material & UL & Units \\
\hline S3120 & Homogeneous Solids.Inorganic Homogeneous Solids.Inorganic Sludges. & LL & UL & 89 & \\
\hline
\end{tabular}




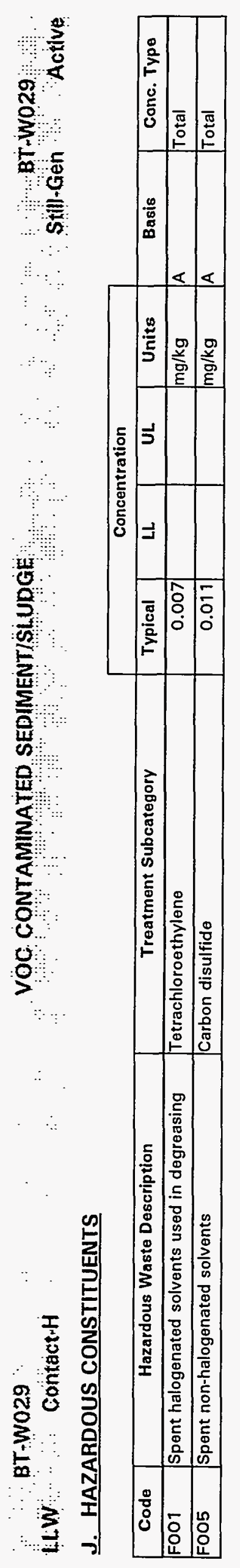




\section{LiW ContactH}

\section{VOC CONTAMNATED SEDIMENTISL DGE}

\section{K. POLYCHLORINATED BIPHENYLS (PCBS)}

Is this waste stream PCB contaminated?

Y Yes

No

\section{Unknown}

If yes, what portion of the waste is PCB contaminated (volume \%):

If $<100 \%$, can the PCB contaminated waste be segregated and treated separately?

What is the PCB concentration in ppm?

If only a portion of the waste is PCB contaminated, report values for contaminated portion.

\section{TREATMENT PLANS}

\begin{tabular}{|c|c|c|c|c|c|c|c|c|c|c|c|}
\hline $\begin{array}{l}\text { Opt. } \\
\#\end{array}$ & $\begin{array}{c}\text { Media Type } \\
\text { (If more than one) }\end{array}$ & $\begin{array}{c}\text { Matrix } \\
\text { Code }\end{array}$ & $\begin{array}{l}\text { Vol. \% } \\
\text { (If }\end{array}$ & Step & JIT & sc & ClF & $\begin{array}{l}\text { Trans. } \\
\text { Miles }\end{array}$ & $\begin{array}{l}\text { Facillty } \\
\text { Abbr. }\end{array}$ & Unit Name & Comments \\
\hline \multirow[t]{6}{*}{1} & & $\mathbf{S 3 1 2 0}$ & & a & 0 & 0 & $1.00 \mathrm{E}+00$ & & TRANS & Transport - LLW & \\
\hline & & & & b & 0 & O & $1.00 \mathrm{E}+00$ & & AMWTF & $\mathrm{CH}$ - Opening \& Sorting & \\
\hline & & & & c & $\mathbf{O}$ & O & $1.00 E+00$ & & AMWTF & $\mathrm{CH}$ - Sizing & \\
\hline & & & & d & 0 & 0 & $1.00 E+00$ & & AMWTF & $\mathrm{CH}$ - Incineration/Thermal Desorption & \\
\hline & & & & e & 0 & 0 & $1.00 E+00$ & & AMWTF & $\mathrm{CH}$ - Vitrification & \\
\hline & & & & $f$ & 0 & 0 & $1.00 E+00$ & & SCDF & Disposal - Contact Handled & \\
\hline
\end{tabular}

Note: Where provided, media percentages are rough estimates used to facilitate treatment planning. They do not necessarily imply an accurate knowledge of waste stream composition, and were not derived for use outside this conceptual planning exercise.

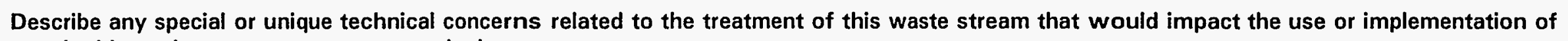
standard hazardous waste treatment methods. 


\section{L.WW}

BT-W030

A. IDENTIFICATION AND DESCRIPTION

Waste Stream ID: BT-W030

W. S. Name:

Content Code:

FFCA ID:

VOC CONTAMINATED DEBRIS

Contaminated debris from storm sewer system. Waste in non-homogeneous; it includes paper,

cloth, plastic, rubber, metal, glass, and brick. This waste is not a moratorium waste or

reclassified TRU waste.

\section{B. GENERATION SITE/PROCESS DESCRIPTION}

The following questions, concerning the generation site and process, should be answered even if the waste stream is no longer generated.

Name of Site Generating Waste (e.g., INEL, Rocky Flats, etc.): Bettis Atomic Power Laboratory

Location of activities (Area and Building):

Manholes and yarddrains.

Operations (functions) performed in building:

The Bettis Atomic Power Laboratory is engaged solely in the design and development of naval nuclear propulsion plants. Laboratory operations include development and testing of nuclear fuel materials and reactor materials including radiochemical analysis.

Description of Process Generating Waste:

Cleanout of part of storm sewer system. 
Hiw BT-Woso

C. PROJECTED WASTE GENERATION

Is this waste stream still generated?

If yes, when is the expected generation termination date?
VOC CONTAMINATED DEBRIS

BT: $\div$ WO30 Still-Gen Active

Current Projections

\begin{tabular}{|r|r|r|r|r|c|}
\hline Est. Date & \multicolumn{1}{|c|}{ Start Year } & Interval & Vol (m3) & \multicolumn{1}{c|}{ Mass $(\mathrm{kg})$} & Source of Estimate \\
\hline $11 / 15 / 94$ & 1997 & 1 & 0.21 & 50.00 & $1994-D S T P$ \\
\hline
\end{tabular}

\section{Historical Projections}




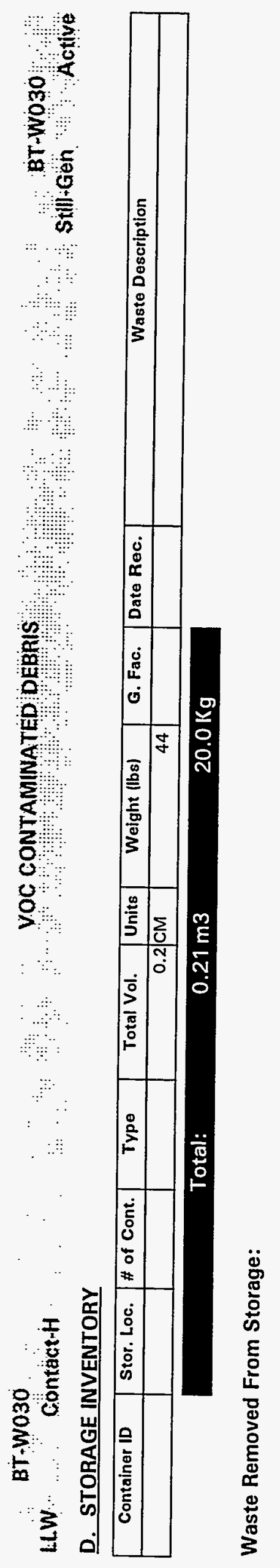

0
$\frac{1}{2}$
4
0
5
0

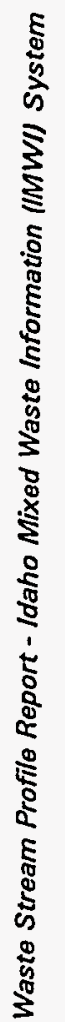




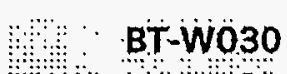

VOC CONTAMINATED DEBRIS

HLW: ContactH

\section{E. RADIATION CHARACTERISTICS}

Radioactive Waste Type:

\begin{tabular}{|ll|}
\hline O LLW & Oa-LLW O TRU \\
O HLW & O Non-Rad \\
\hline
\end{tabular}

Mixed Waste:

Handling:

\begin{tabular}{|l|}
\hline OYes O No \\
\hline O Contact \\
\hline
\end{tabular}

Radionuclide Contamination Accessiblity:

External Surface $O$ Yes $O$ No OUnknown

Internal Surface $O$ Yes $O$ No OUnknown

Dispersed Through Matrix

OYes O No OUnknown

\section{Activity Levels}

Transuranic Alpha Activity:

Uranium/Thorium Alpha Activity:

Beta/Gamma Activity:

Surface Neutron Activity:

Total Activity:

\begin{tabular}{rl|}
\hline $2 \mathrm{E}-13$ & Units: $\mathrm{Ci} / \mathrm{g}$ \\
\hline 1E-11 & Units: $\mathrm{Ci} / \mathrm{g}$ \\
\hline$<0.1$ & $@$ surface \\
\hline$<0.1$ & $@ 1-\mathrm{m}$ Units: $\mathrm{mR/hr}$ \\
\hline & Units: \\
\hline 0.041 & nCi/g
\end{tabular}

\section{F. TRU ALPHA ACTIVITY DISTRIBUTION}

This waste stream is not identified as a TRU or a-LLW, 


\section{LLW : Contacth}

\section{G. RADIONUCLIDE CONCENTRATIONS}

\begin{tabular}{|c|c|c|c|c|c|c|}
\hline & & pecific Activit & & & & \\
\hline Isotope & Typical & Lower Limit & Upper Limit & Units & Basis Code(s) & Basis Desc. lif otherl \\
\hline$C d-113 m$ & $4.0000 \mathrm{E}-16$ & & & $\mathrm{Ci} / \mathrm{g}$ & $i$ & \\
\hline Cs-137 & $7.0000 \mathrm{E}-12$ & & & $\mathrm{Ci} / \mathrm{g}$ & 1 & \\
\hline$S n-126$ & $4.0000 \mathrm{E}-17$ & & & $\mathrm{Ci} / \mathrm{g}$ & 1 & \\
\hline $\mathrm{Nb}-93 \mathrm{~m}$ & $4.0000 E-17$ & & & $\mathrm{Ci} / \mathrm{g}$ & 1 & \\
\hline Se-79 & $5.0000 \mathrm{E}-17$ & & & $\mathrm{Ci} / \mathrm{g}$ & 1 & \\
\hline Cs-135 & $3.0000 \mathrm{E}-17$ & & & $\mathrm{Ci} / \mathrm{g}$ & 1 & \\
\hline $2 r-93$ & 4.0000 E-16 & & & $\mathrm{Ci} / \mathrm{g}$ & $i$ & \\
\hline$S b-126$ & $5.0000 \mathrm{E}-18$ & & & $\mathrm{Ci} / \mathrm{g}$ & $\pi$ & \\
\hline Tc-99 & $2.0000 \mathrm{E}-15$ & & & $\mathrm{Ci} / \mathrm{g}$ & 1 & \\
\hline$S m-151$ & 2.0000 E-14 & & & $\mathrm{Ci} / \mathrm{g}$ & I & \\
\hline$E u-154$ & $1.0000 \mathrm{E}-13$ & & & $\mathrm{Ci} / \mathrm{g}$ & 1 & \\
\hline $\mathrm{Kr}-95$ & 3.0000E-13 & & & $\mathrm{Ci} / \mathrm{g}$ & i & \\
\hline$Y-90$ & $7.0000 \mathrm{E}-12$ & & & $\mathrm{Ci} / \mathrm{g}$ & $\pi$ & \\
\hline Sr-90 & $7.0000 \mathrm{E}-12$ & & & $\mathrm{Ci} / \mathrm{g}$ & 1 & \\
\hline$B a-137 m$ & $7.0000 \mathrm{E}-12$ & & & $\mathrm{Ci} / \mathrm{g}$ & 1 & \\
\hline Eu-152 & $7.0000 \mathrm{E}-17$ & & & $\mathrm{Ci} / \mathrm{g}$ & 1 & \\
\hline Pu-241 & $3.0000 \mathrm{E}-14$ & & & $\mathrm{Ci} / \mathrm{g}$ & $i$ & \\
\hline Th-232 & $1.0000 \mathrm{E}-12$ & & & $\mathrm{Ci} / \mathrm{g}$ & I & \\
\hline$U-238$ & $1.0000 \mathrm{E}-13$ & & & $\mathrm{Ci} / \mathrm{g}$ & 1 & \\
\hline U-235 & 8.0000 E-14 & & & $\mathrm{Ci} / \mathrm{g}$ & 1 & \\
\hline U-234 & $9.0000 \mathrm{E}-12$ & & & $\mathrm{Ci} / \mathrm{g}$ & 1 & \\
\hline C-14 & $3.0000 \mathrm{E}-14$ & & & $\mathrm{Ci} / \mathrm{g}$ & 1 & \\
\hline $\mathrm{Sb}-126 \mathrm{~m}$ & $3.0000 E-17$ & & & $\mathrm{Ci} / \mathrm{g}$ & 1 & \\
\hline $\mathrm{H}-3$ & $2.0000 E-14$ & & & $\mathrm{Ci} / \mathrm{g}$ & t & \\
\hline Pu-238 & $2.0000 \mathrm{E}-13$ & & & $\mathrm{Ci} / \mathrm{g}$ & 1 & \\
\hline $\mathrm{Cm}-242$ & $8.0000 \mathrm{E}-30$ & & & $\mathrm{Ci} / \mathrm{g}$ & 1 & \\
\hline Fe-55 & $2.0000 \mathrm{E}-13$ & & & $\mathrm{Ci} / \mathrm{g}$ & 1 & \\
\hline $\mathrm{Ni}-63$ & $7.0000 E-14$ & & & $\mathrm{Ci} / \mathrm{g}$ & 1 & \\
\hline Nb-94 & $3.0000 E-21$ & & & $\mathrm{Ci} / \mathrm{g}$ & 1 & \\
\hline Co-60 & $1.0000 \mathrm{E}-12$ & & & $\mathrm{Ci} / \mathrm{g}$ & 1 & \\
\hline $1-129$ & $2.0000 \mathrm{E}-18$ & & & $\mathrm{Ci} / \mathrm{g}$ & 1 & . \\
\hline Sn-121m & $5.0000 \mathrm{E}-18$ & & & $\mathrm{Ci} / \mathrm{g}$ & 1 & \\
\hline $\mathrm{Ni}-59$ & $7.0000 \mathrm{E}-16$ & & & $\mathrm{Ci} / \mathrm{g}$ & I & \\
\hline
\end{tabular}



s!SK/EU $\forall$ jes!urayoo!pey K’!!!qejunooob : 
VOC CONTAMINATED DEBRIS

BT-WO30:

Primary Matrix Code: 55490

Description: Debris Waste: Heterogeneous Debris: Unknown/Other Heterogeneous Debris:

Combustibility:

Combustible $(>90 \%)$

Mixed $(10 \%-90 \%)$

Noncombustible $(<10 \%)$

\section{MATRIX COMPOSITION}

\begin{tabular}{|c|c|c|c|c|c|c|}
\hline \multirow[b]{2}{*}{ Code } & \multirow[b]{2}{*}{ Matrix Description } & \multirow[b]{2}{*}{ Materlal } & \multicolumn{4}{|c|}{ Composition } \\
\hline & & & Typical & $\mathrm{LL}$ & UL & Units \\
\hline$\$ 5123$ & Debris Waste.Inorganic Debris.Inorganic Non-Metal Debris.Ceramic/Brick Debris & Brick & 16 & & & $\% w t$ \\
\hline $\mathbf{S 5 1 2 2}$ & Debris Waste.Inorganic Debris.Inorganic Non-Metal Debris.Glass Debris & Glass & 7 & & & $\% w t$ \\
\hline$\overline{55119}$ & Debris Waste.Inorganic Debris.Metal Debris.Unknown/Other Metal Debris & Metal & 5 & & & $\% w t$ \\
\hline$\$ 5312$ & Debris Waste.Organic Debris.Plastic/Rubber Debris. Halogenated Plastic Debris & Rubber & 12 & & & $\% w t$ \\
\hline S5312 & Debris Waste.Organic Debris.Plastic/Rubber Debris.Halogenated Plastic Debris & Plastic & 21 & & & $\% w t$ \\
\hline$\$ 5330$ & Debris Waste.Organic Debris.Paper/Cloth Debris. & Cloth & 30 & & & $\% w t$ \\
\hline 55330 & Debris Waste.Organic Debris.Paper/Cloth Debris. & Paper & 9 & & & $\% w t$ \\
\hline
\end{tabular}




\section{J. HAZARDOUS CONSTITUENTS}

\begin{tabular}{|c|c|c|c|c|c|c|c|c|}
\hline & & & \multicolumn{4}{|c|}{ Concentration } & & \\
\hline Code & Hazardous Waste Description & Treatment Subcategory & Typical & $\mathbf{L L}$ & UL & Units & Basis & Conc. Type \\
\hline F001 & Spent halogenated solvents used in degreasing & Tetrachloroethylene & 0.007 & & & $\mathrm{mg} / \mathrm{kg}$ & A & Total \\
\hline FO05 & Spent non-halogenated solvents & Carbon disulfide & 0.011 & & & $\mathrm{mg} / \mathrm{kg}$ & A & Total \\
\hline
\end{tabular}




\section{BT-W030}

\section{VOC CONTAMINATED DEBRIS}

\section{LWW ContactiH}

\section{K. POLYCHLORINATED BIPHENYLS (PCBS)}

Is this waste stream PCB contaminated?

OYes O No O Unknown

If yes, what portion of the waste is $\mathrm{PCB}$ contaminated (volume \%):

If $<100 \%$, can the PCB contaminated waste be segregated and treated separately?

What is the PCB concentration in ppm?

If only a portion of the waste is $P C B$ contaminated, report values for contaminated portion.

\section{TREATMENT PLANS}

\begin{tabular}{|c|c|c|c|c|c|c|c|c|c|c|c|}
\hline Opt. & $\begin{array}{c}\text { Media Type } \\
\text { (If more than onel }\end{array}$ & $\begin{array}{l}\text { Matrix } \\
\text { Code }\end{array}$ & $\begin{array}{l}\text { Vol. \% } \\
\text { IIf }\end{array}$ & Step & JIT & sc & CIF & $\begin{array}{l}\text { Trans. } \\
\text { Miles }\end{array}$ & $\begin{array}{l}\text { Facility } \\
\text { Abbr. }\end{array}$ & Unit Name & Comments \\
\hline 1 & & 55490 & & $\overline{\mathbf{a}}$ & $\mathbf{0}$ & 0 & $1.00 \mathrm{E}+00$ & & TRANS & Transport - LLW & \\
\hline & & & & $\mathbf{b}$ & $\mathbf{O}$ & 0 & $1.00 E+00$ & & AMWTF & $\mathrm{CH}-$ Opening \& Sorting & \\
\hline & & & & c & O & 0 & $1.00 E+00$ & & AMWTF & $\mathrm{CH}$ - Sizing & \\
\hline & & & & d & 0 & 0 & $1.00 \mathrm{E}+00$ & & AMWTF & $\mathrm{CH}$ - Incineration/Thermal Desorption & \\
\hline & & & & e & 0 & 0 & $1.00 E+00$ & & AMWTF & $\mathrm{CH}$ - Vitrification & \\
\hline & & & & $f$ & 0 & 0 & $1.00 E+00$ & & SCDF & Disposal - Contact Handled & \\
\hline
\end{tabular}

Note: Where provided, media percentages are rough estimates used to facilitate treatment planning. They do not necessarily imply an accurate knowledge of waste stream composition, and were not derived for use outside this conceptual planning exercise.

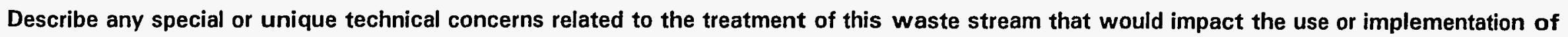
standard hazardous waste treatment methods. 


\section{A. IDENTIFICATION AND DESCRIPTION}

Waste Stream ID: BT-W031

W. S. Name:

Content Code:

FFCA ID:

\section{VOC AND PCB CONTAMINATED SLUDGE}

\section{Waste Stream Description:}

Contaminated sludge from storm sewer system. Waste is homogeneous. This waste is not a moratorium waste or reclassified TRU waste. 


\section{PROJECTED WASTE GENERATION}

Is this waste stream still generated? If yes, when is the expected generation termination date?

Current Projections

\begin{tabular}{|r|r|r|r|r|l|}
\hline Est. Date & Start Year & Interval & Vol (m3) & Mass (kg) & Source of Estlmate \\
\hline $3 / 30 / 95$ & 1995 & 5 & 1.05 & & Generator Update \\
\hline
\end{tabular}

Historical Projections 
BTWO31

VOC AND PCB CONTAMNATED SLUDGE

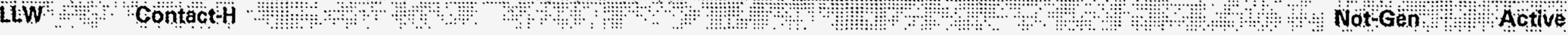

D. STORAGE INVENTORY

\begin{tabular}{|c|c|c|c|c|c|c|c|c|c|}
\hline Container ID & Stor. Loc. & \# of Cont. & Type & Total Vol. & Units & Weight (lbs) & G. Fac. & Date Rec. & Waste Description \\
\hline & & & & 2.7 & $\mathrm{CM}$ & 7,275 & & & \\
\hline
\end{tabular}

Waste Removed From Storage: 


\section{E. RADIATION CHARACTERISTICS}

Radioactive Waste Type:

\begin{tabular}{|ll|}
\hline OLLW & $\bigcirc$ a-LLW $\bigcirc$ TRU \\
HLW & $\bigcirc$ Non-Rad \\
\hline
\end{tabular}

Mixed Waste:

\begin{tabular}{|l|}
\hline O Yes ONo \\
\hline O Contact O Remote \\
\hline
\end{tabular}

Handling:

Radionuclide Contamination Accessiblity:

External Surface

Internal Surface

Dispersed Through Matrix

\begin{tabular}{|c|c|c|}
\hline OYes & ONo & OUnknown \\
\hline OYes & ONo & OUnknown \\
\hline OYes & No & OUn \\
\hline
\end{tabular}

\section{Activity Levèels}

Transuranic Alpha Activity: Uranium/Thorium Alpha Activity: Beta/Gamma Activity:

2E-13 Units: $\mathrm{Ci} / \mathrm{g}$

$1 \mathrm{E}-11$ Units: $\mathrm{Ci} / \mathrm{g}$
$<0.1$ @ surface $<0.1$ 1-m Units: $\mathrm{mR} / \mathrm{hr}$

Surface Neutron Activity: Units:

Total Activity: $0.045 \mathrm{nCi} / \mathrm{g}$

\section{F. TRU ALPHA ACTIVITY DISTRIBUTION}

This waste stream is not identified as a TRU or a-LLW. 


\section{BTWWO31}

HLW ContacotH

VOC AND PCB GONTAMINATED SLUDGE

BT:WO 31

\section{G. RADIONUCLIDE CONCENTRATIONS}

\begin{tabular}{|c|c|c|c|c|c|c|}
\hline & & pecific Activit & & & & \\
\hline Isotope & Typical & Lower Limit & Upper Limit & Units & Basis Code(s) & Basis Desc. (if other) \\
\hline$\overline{C d}-113 \mathrm{~m}$ & $4.0000 \mathrm{E}-16$ & & & $\mathrm{Ci} / \mathrm{g}$ & 1 & \\
\hline Cs-137 & $8.0000 \mathrm{E}-12$ & & & $\mathrm{Ci} / \mathrm{g}$ & 1 & \\
\hline $5 n-126$ & $4.0000 \mathrm{E}-17$ & & & $\mathrm{Ci} / \mathrm{g}$ & $i$ & \\
\hline $\mathrm{Nb}-93 \mathrm{~m}$ & $4.0000 E-17$ & & & $\mathrm{Ci} / \mathrm{g}$ & 1 & \\
\hline $5 e-79$ & $5.0000 \mathrm{E}-17$ & & & $\mathrm{Ci} / \mathrm{g}$ & 1 & \\
\hline$C s-135$ & $3.0000 \mathrm{E}-17$ & & & $\mathrm{Ci} / \mathrm{g}$ & 1 & \\
\hline $2 r-93$ & $5.0000 \mathrm{E}-16$ & & & $\mathrm{Ci} / \mathrm{g}$ & 1 & \\
\hline Sb-126 & $5.0000 \mathrm{E}-18$ & & & $\mathrm{Ci} / \mathrm{g}$ & I & \\
\hline Tc-99 & $2.0000 \mathrm{E}-15$ & & & $\mathrm{Ci} / \mathrm{g}$ & 1 & \\
\hline $5 m-151$ & 2.0000 E-14 & & & $\mathrm{Ci} / \mathrm{g}$ & 1 & \\
\hline$E u-154$ & $1.0000 \mathrm{E}-13$ & & & $\mathrm{Ci} / \mathrm{g}$ & 1 & \\
\hline Kr-95 & $4.0000 \mathrm{E}-13$ & & & $\mathrm{Ci} / \mathrm{g}$ & 1 & \\
\hline$\gamma-90$ & $8.0000 E-12$ & & & $\mathrm{Ci} / \mathrm{g}$ & 1 & \\
\hline Sr-90 & $8.0000 \mathrm{E}-12$ & & & $\mathrm{Ci} / \mathrm{g}$ & 1 & \\
\hline$B a-137 m$ & $8.0000 \mathrm{E}-12$ & & & $\mathrm{Ci} / \mathrm{g}$ & 1 & \\
\hline Eu-152 & $8.0000 \mathrm{E}-17$ & & & $\mathrm{Ci} / \mathrm{g}$ & 1 & \\
\hline $\mathrm{Pu}-241$ & $3.0000 E-14$ & & & $\mathrm{Ci} / \mathrm{g}$ & 1 & \\
\hline Th-232 & $1.0000 \mathrm{E}-12$ & & & $\mathrm{Ci} / \mathrm{g}$ & 1 & \\
\hline$U-238$ & $1.0000 \mathrm{E}-13$ & & & $\mathrm{Ci} / \mathrm{g}$ & 1 & \\
\hline $\mathrm{U}-235$ & $9.0000 \mathrm{E}-14$ & & & $\mathrm{Ci} / \mathrm{g}$ & 1 & \\
\hline$U-234$ & $1.0000 \mathrm{E}-11$ & & & $\mathrm{Ci} / \mathrm{g}$ & 1 & \\
\hline$c-14$ & $4.0000 \mathrm{E}-14$ & & & $\mathrm{Ci} / \mathrm{g}$ & 1 & \\
\hline Sb- $126 \mathrm{~m}$ & $4.0000 \mathrm{E}-17$ & & & $\mathrm{Ci} / \mathrm{g}$ & 1 & \\
\hline $\mathrm{H}-3$ & $2.0000 \mathrm{E}-14$ & & & $\mathrm{Ci} / \mathrm{g}$ & 1 & \\
\hline$P u-238$ & $2.0000 \mathrm{E}-13$ & & & $\mathrm{Ci} / \mathrm{g}$ & 1 & \\
\hline$C m-242$ & $9.0000 \mathrm{E}-30$ & & & $\mathrm{Ci} / \mathrm{g}$ & 1 & \\
\hline Fe-55 & $2.0000 \mathrm{E}-13$ & & & $\mathrm{Ci} / \mathrm{g}$ & 1 & \\
\hline$\overline{N i-63}$ & $8.0000 \mathrm{E}-14$ & & & $\mathrm{Ci} / \mathrm{g}$ & $\pi$ & \\
\hline $\mathrm{Nb}-94$ & $3.0000 \mathrm{E}-21$ & & & $\mathrm{Ci} / \mathrm{g}$ & $\pi$ & \\
\hline Co-60 & $1.0000 \mathrm{E}-12$ & & & $\mathrm{Ci} / \mathrm{g}$ & 1 & \\
\hline $1-129$ & $3.0000 \mathrm{E}-18$ & & & $\mathrm{Ci} / \mathrm{g}$ & 1 & \\
\hline$S n-121 m$ & $6.0000 \mathrm{E}-18$ & & & $\mathrm{Ci} / \mathrm{g}$ & 1 & \\
\hline $\mathrm{Ni}-59$ & $8.0000 E-16$ & & & $\mathrm{Ci} / \mathrm{g}$ & $\pi$ & \\
\hline
\end{tabular}

Specific Activity

-93m

Cs-135

Sb-126

$5.0000 \mathrm{E}-16$

$2.0000 \mathrm{E}-15$

Eu-154

$1.0000 \mathrm{E}-13$

Kr-95

$4.0000 \mathrm{E}-13$

8.0000E-12

8.0000E-12

Ba-137m

$3.0000 \mathrm{E}-14$

$1.0000 \mathrm{E}-12$

Th-232

U-235

9.0000E-14

C-14

Sb-126m 


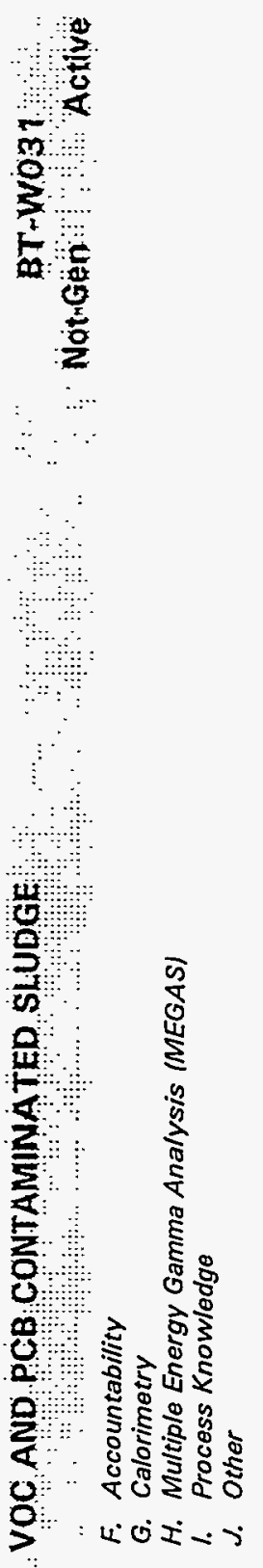

$\therefore \quad$ के

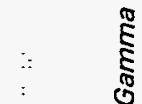

$\because$ ऊ

रू हू

工 ล ह

造

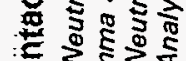

ल

3 0.3000

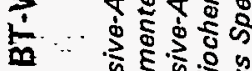

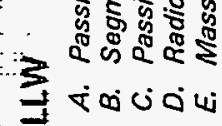




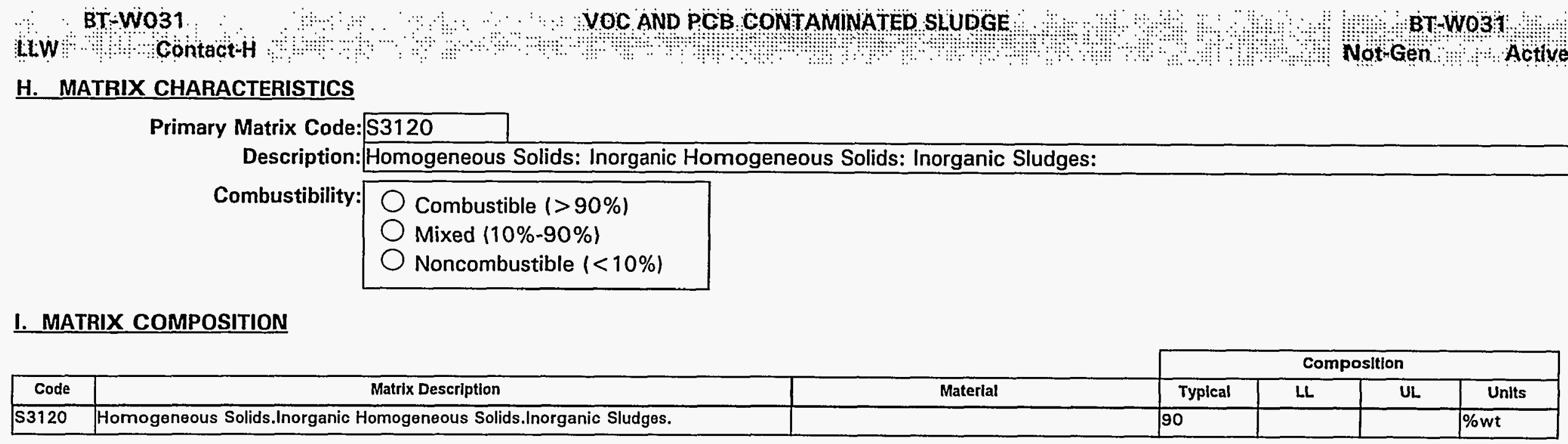


VOC AND PCB CONTAMMATED SLUDGE

Notigen Active

\section{J. HAZARDOUS CONSTITUENTS}

\begin{tabular}{|c|c|c|c|c|c|c|c|c|}
\hline \multirow[b]{2}{*}{ Code } & \multirow[b]{2}{*}{ Hazardous Waste Description } & \multirow[b]{2}{*}{ Treatment Subcategory } & \multicolumn{4}{|c|}{ Concentration } & \multirow[b]{2}{*}{ Basis } & \multirow[b]{2}{*}{ Conc. Type } \\
\hline & & & Typical & LL & $\mathbf{U L}$ & Units & & \\
\hline F001 & Spent halogenated solvents used in degreasing & Tetrachloroethylene & 1275 & & & $\mathrm{mg} / \mathrm{kg}$ & A & Total \\
\hline F001 & Spent halogenated solvents used in degreasing & Trichloroethylene & 69.4 & & & $\mathrm{mg} / \mathrm{kg}$ & A & Total \\
\hline FO02 & Spent halogenated solvents & Chlorobenzene & 28.5 & & & $\mathrm{mg} / \mathrm{kg}$ & A & Total \\
\hline FO02 & Spent halogenated solvents & Methylene chloride & 64.2 & & & $\mathrm{mg} / \mathrm{kg}$ & $A$ & Total \\
\hline FOO3 & Spent non-halogenated solvents & Acetone & 0.6 & & & $\mathrm{mg} / \mathrm{kg}$ & A & Total \\
\hline
\end{tabular}




\section{$\therefore$ BT-WO31}

VOC AND PCB CONTAMINATED SLUDGE

LuW o Contact

\section{K. POLYCHLORINATED BIPHENYLS (PCBS)}

\section{Is this waste stream PCB contaminated? O Yes O No $O$ Unknown}

If yes, what portion of the waste is PCB contaminated (volume \%): 100

If $<100 \%$, can the PCB contaminated waste be segregated and treated separately?

OYes O No O Unknown

What is the PCB concentration in ppm? $\quad<1500$ If only a portion of the waste is PCB contaminated, report values for contaminated portion.

\section{TREATMENT PLANS}

\begin{tabular}{|c|c|c|c|c|c|c|c|c|c|c|c|}
\hline $\begin{array}{c}\text { Opt. } \\
\#\end{array}$ & $\begin{array}{c}\text { Media Type } \\
\text { (If more than one) }\end{array}$ & $\begin{array}{c}\text { Matrix } \\
\text { Code }\end{array}$ & $\begin{array}{l}\text { Vol. \% } \\
\text { Iff }\end{array}$ & Step & JIT & sc & CIF & $\begin{array}{l}\text { Trans. } \\
\text { Miles }\end{array}$ & $\begin{array}{l}\text { Facility } \\
\text { Abbr. }\end{array}$ & Unit Name & Comments \\
\hline 1 & & $\$ 3120$ & & $\bar{a}$ & 0 & 0 & $1.00 E+00$ & & TRANS & Transport - LLW & \\
\hline & & & & $\mathbf{b}$ & 0 & $\mathrm{O}$ & $1.00 E+00$ & & AMWTF & $\mathrm{CH}$ - Opening \& Sorting & \\
\hline & & & & $\overline{\mathbf{c}}$ & () & 0 & $1.00 E+00$ & & AMWTF & $\mathrm{CH}$ - Sizing & \\
\hline & & & & $\bar{d}$ & 0 & 0 & $1.00 \mathrm{E}+00$ & & AMWTF & $\mathrm{CH}$ - Incineration/Thermal Desorption & \\
\hline & & & & $\bar{e}$ & 0 & 0 & $1.00 \mathrm{E}+00$ & & AMWTF & $\mathrm{CH}$ - Vitrification & \\
\hline & & & & $f$ & 0 & 0 & $1.00 E+00$ & & SCDF & Disposal - Contact Handled & \\
\hline
\end{tabular}

Note: Where provided, media percentages are rough estimates used to facilitate treatment planning. They do not necessarily imply an accurate knowledge of waste stream composition, and were not derived for use outside this conceptual planning exercise.

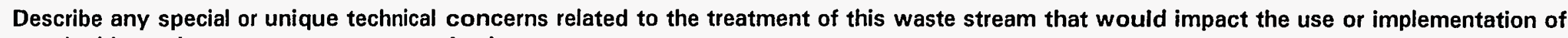
standard hazardous waste treatment methods. 


\section{DPP.W079}

LiWW ContactaH

\section{A. IDENTIFICATION AND DESCRIPTION}

Waste Stream ID: DP-W079

W. S. Name:

METALLIC MERCURY/MERCURY PRODUCTS

Content Code:

FFCA ID:

DP-W079

Waste Stream Description:

Metallic mercury/mercury products.

\section{METALLIC MERCURYIMERCURY PRODUCTS}

DP WO79:

Still-Gen Active

\section{B. GENERATION SITE/PROCESS DESCRIPTION}

The following questions, concerning the generation site and process, should be answered even if the waste stream is no longer generated.

Name of Site Generating Waste (e.g., INEL, Rocky Flats, etc.): K-25 Site

Location of activities (Area and Building):

Operations (functions) performed in building:

Description of Process Generating Waste: 
DP.WO79

LWW ôntact

C. PROJECTED WASTE GENERATION

Is this waste stream still generated?

OYes ONo

If yes, when is the expected generation termination date?

Current Projections

\begin{tabular}{|r|r|r|r|r|l|}
\hline Est. Date & \multicolumn{1}{|c|}{ Start Year } & Interval & \multicolumn{1}{|c|}{ Vol (m3) } & Mass $(\mathrm{kg})$ & \multicolumn{1}{|c|}{ Source of Estlmate } \\
\hline $3 / 22 / 94$ & 1993 & 1 & 1.60 & $2,400.00$ & 1993 -FFCA \\
\hline $3 / 22 / 94$ & 1994 & 1 & 1.60 & $2,400.00$ & 1993 -FFCA \\
\hline $3 / 22 / 94$ & 1995 & 1 & 1.60 & $2,400.00$ & $1993-F F C A$ \\
\hline $3 / 22 / 94$ & 1996 & 1 & 1.60 & $2,400.00$ & $1993-F F C A$ \\
\hline $3 / 22 / 94$ & 1997 & 1 & 1.60 & $2,400.00$ & $1993-F F C A$ \\
\hline
\end{tabular}

Historical Projections

\begin{tabular}{|r|r|r|r|r|l|}
\hline Est. Date & \multicolumn{1}{|c|}{ Start Year } & \multicolumn{1}{|c|}{ Interval } & \multicolumn{1}{|c|}{ Vol (m3) } & \multicolumn{1}{c|}{ Mass (kg) } & Source of Estimate \\
\hline $3 / 22 / 94$ & 1998 & 5 & 8.00 & $12,000.00$ & $1993-F F C A$ \\
\hline $3 / 22 / 94$ & 2003 & 20 & 32.00 & $48,000.00$ & $1993-F F C A$ \\
\hline
\end{tabular}




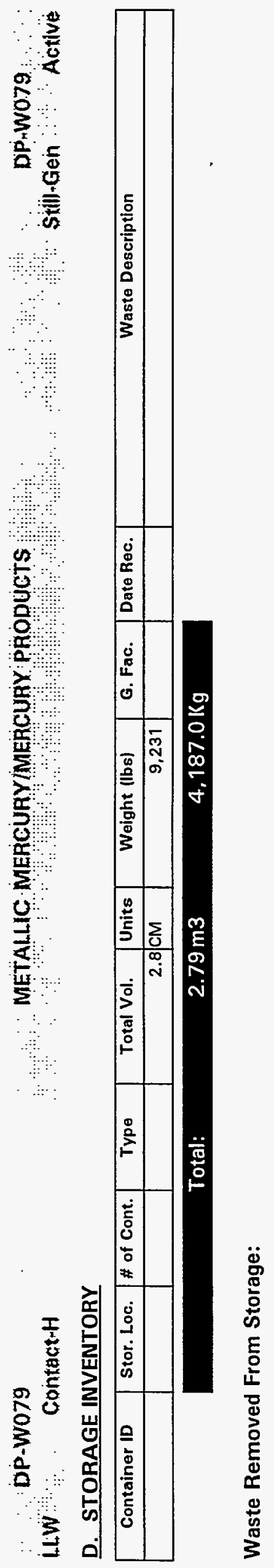




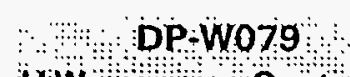
LLW Contact H

\section{E. RADIATION CHARACTERISTICS}

Radioactive Waste Type:

\begin{tabular}{|ll|}
\hline O LLW & O a-LLW O TRU \\
O HLW & O Non-Rad \\
\hline
\end{tabular}

Mixed Waste:

Handling:

\begin{tabular}{|l|}
\hline O Yes O No \\
\hline O Contact O Remote \\
\hline
\end{tabular}

Radionuclide Contamination Accessiblity:

\begin{tabular}{|c|c|c|}
\hline External Surface $\bigcirc$ Yes & O No & OUnknown \\
\hline Internal Surface $\bigcirc$ Yes & ONo & Unknown \\
\hline Dispersed Through Matrix $\mathrm{O}_{\mathrm{Yes}}$ & Ono & Unknown \\
\hline \multicolumn{3}{|c|}{ Activity Levels } \\
\hline \multirow{2}{*}{$\begin{array}{l}\text { Transuranic Alpha Activity: } \\
\text { Uranium/Thorium Alpha Activity: }\end{array}$} & $<1 E-8$ & Units: $\mathrm{Ci} / \mathrm{g}$ \\
\hline & & Units: \\
\hline \multirow[t]{2}{*}{ Beta/Gamma Activity: } & & @ surface \\
\hline & & @ 1-m Units: \\
\hline Surface Neutron Activity: & & Units: \\
\hline Total Activity: & & $\mathrm{nCi} / \mathrm{g}$ \\
\hline
\end{tabular}

\section{F. TRU ALPHA ACTIVITY DISTRIBUTION}

This waste stream is not identified as a TRU or a-LLW. 

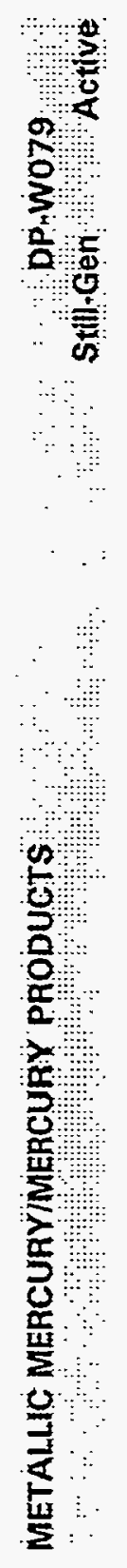

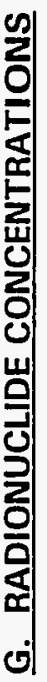




\section{DP WO79 M METALLCMERCURYMERCURY PRODUCTS}

How :

H. MATRIX CHARACTERISTICS

Primary Matrix Code: X7100

Description: Special Waste: Elemental Mercury: :

Combustibility: $\bigcirc$ Combustible $(>90 \%)$

Mixed $(10 \%-90 \%)$

Noncombustible $(<10 \%)$

\section{MATRIX COMPOSITION}

\begin{tabular}{|c|c|c|c|c|c|}
\cline { 2 - 6 } \multicolumn{1}{c|}{ Material } & \multicolumn{1}{c|}{ Composition } & Typlcal & LL & UL. & Units \\
\hline Code & \multicolumn{1}{|c|}{ Matrlx Description } & Elemental Mercury & & & \\
\hline
\end{tabular}




\section{J. HAZARDOUS CONSTITUENTS}

\begin{tabular}{|c|c|c|c|c|c|c|c|c|}
\hline & & & \multicolumn{4}{|c|}{ Concentration } & & \\
\hline Code & Hazardous Waste Description & Treatment Subcategory & Typical & $\mathbf{L L}$ & UL & Units & Basis & Conc. Typo \\
\hline D009 & Mercury & High mercury $>=260 \mathrm{mg} / \mathrm{kg}$ total mercury & & & & & $A ; C$ & Total \\
\hline U151 & Mercury (High mercury > $260 \mathrm{mg} / \mathrm{kg}$ ) & & & & & & $\overline{A ; C}$ & Total \\
\hline
\end{tabular}




\section{$\therefore$ DP:W079}

\section{UWW ContactiH}

\section{K. POLYCHLORINATED BIPHENYLS (PCBS)}

\section{Is this waste stream PCB contaminated? $O$ Yes $O$ No $O$ Unknown}

If yes, what portion of the waste is PCB contaminated (volume \%):

If $<100 \%$, can the PCB contaminated waste be segregated and treated separately?

What is the PCB concentration in ppm?

If only a portion of the waste is PCB contaminated, report values for contaminated portion.

\section{TREATMENT PLANS}

\begin{tabular}{|c|c|c|c|c|c|c|c|c|c|c|c|}
\hline Opt. & $\begin{array}{c}\text { Media Type } \\
\text { (If more than one) }\end{array}$ & $\begin{array}{c}\text { Matrix } \\
\text { Code }\end{array}$ & $\begin{array}{l}\text { Vol. \% } \\
\text { (If }\end{array}$ & Step & JIT & sc & CIF & $\begin{array}{l}\text { Trans. } \\
\text { Miles }\end{array}$ & $\begin{array}{l}\text { Facility } \\
\text { Abbr. }\end{array}$ & Unit Name & Comments \\
\hline \multirow[t]{4}{*}{1} & & $x 7100$ & & $\bar{a}$ & 0 & 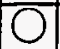 & $1.00 E+00$ & & TRANS & Transport - LLW & \\
\hline & & & & b & O) & $O$ & $1.00 E+00$ & & AMWTF & $\mathrm{CH}-$ Opening \& Sorting & \\
\hline & & & & c & 0 & $O$ & $1.00 E+00$ & & AMWTF & $\mathrm{CH}$ - Amalgamation & \\
\hline & & & & d & 0 & $\overline{0}$ & $1.00 E+00$ & & SCDF & Disposal - Contact Handled & \\
\hline
\end{tabular}

Note: Where provided, media percentages are rough estimates used to facilitate treatment planning. They do not necessarily imply an accurate knowledge of waste stream composition, and were not derived for use outside this conceptual planning exercise.

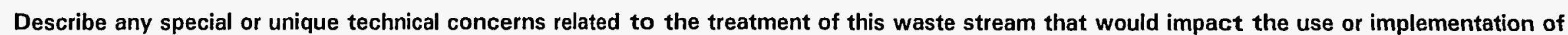
standard hazardous waste treatment methods. 


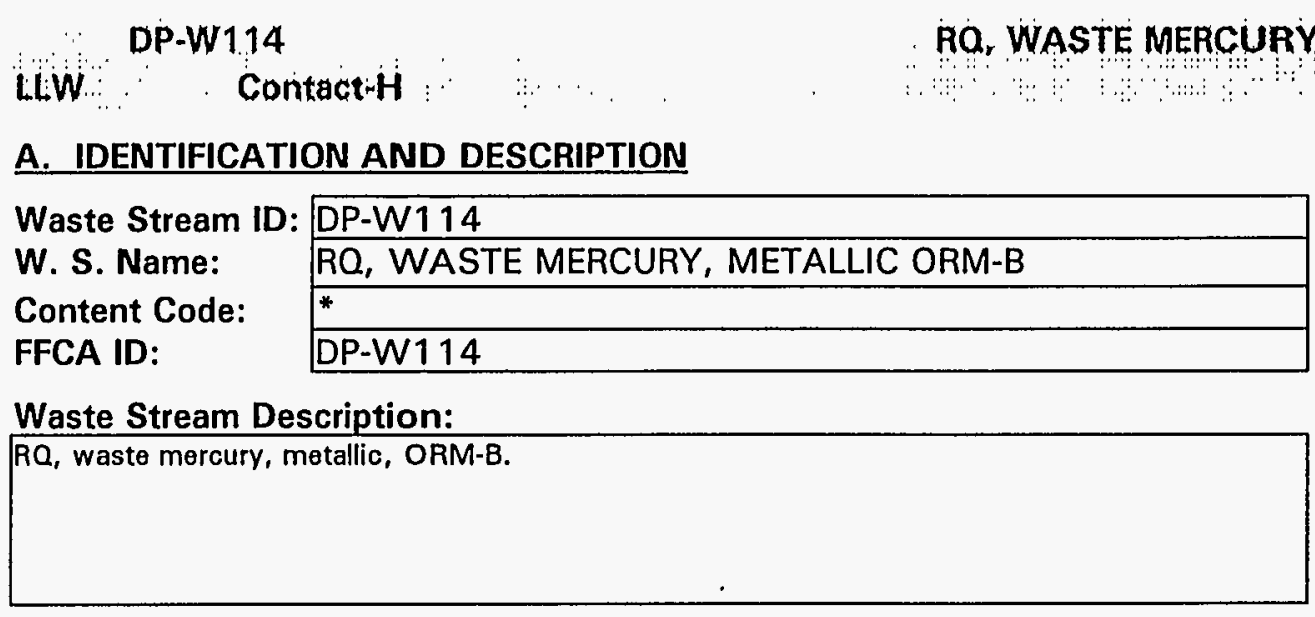

\section{B. GENERATION SITE/PROCESS DESCRIPTION}

The following questions, concerning the generation site and process, should be answered even if the waste stream is no longer generated.

Name of Site Generating Waste (e.g., INEL, Rocky Flats, etc.): ORNL

Location of activities (Area and Building):

This waste was generated at ORNL. Waste generation information is not available at the $\mathrm{K}-25$ site.

Operations (functions) performed in building:

This waste was generated at ORNL. Waste generation information is not available at the $\mathrm{K}-25$ site.

Description of Process Generating Waste:

This waste was generated at ORNL. Waste generation information is not available at the $\mathrm{K}-25$ site. 
DP

litw: ContactH

C. PROJECTED WASTE GENERATION

Is this waste stream still generated?

If yes, when is the expected generation termination date?

Current Projections
RQ WASTE MERCURY METALLIC ORM-B

OYes O No
DP.W114

Nótuèn Active

\section{Historical Projections}




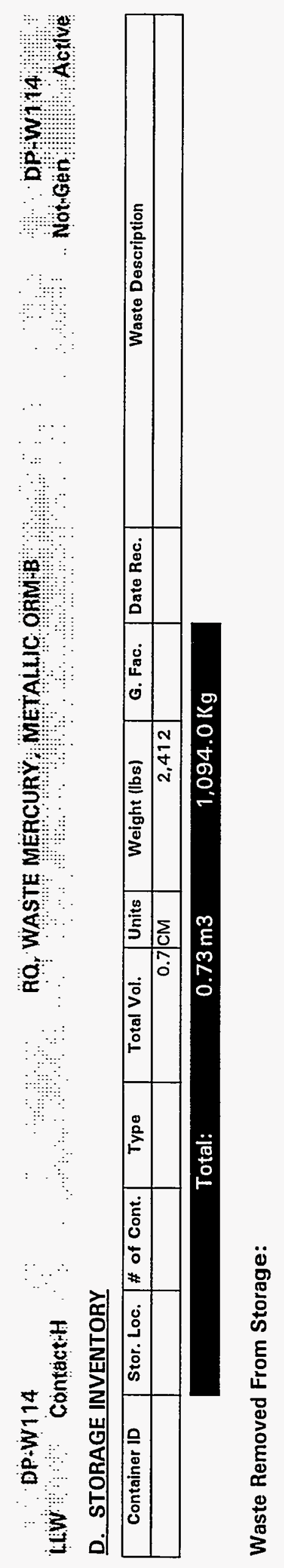




\section{DP.W 114}

RQ WASTE MERCURY METALLIC ORM B

\section{E. RADIATION CHARACTERISTICS}

Radioactive Waste Type:

\begin{tabular}{|ll|}
\hline OllW & Oa-LLW $\bigcirc$ TRU \\
OhlW & O Non-Rad \\
\hline
\end{tabular}

Mixed Waste:

Handling:

\begin{tabular}{|l|}
\hline OYes O No \\
\hline O Contact O Remote \\
\hline
\end{tabular}

Radionuclide Contamination Accessiblity:

External Surface

Internal Surface

Dispersed Through Matrix

\begin{tabular}{|ccc|}
\hline Yyes & O No & O Unknown \\
\hline OYes & O No & OUnknown \\
\hline OYes & O No & OUnknown \\
\hline
\end{tabular}

Activity Levels

Transuranic Alpha Activity:

Uranium/Thorium Alpha Activity:

Beta/Gamma Activity:

Surface Neutron Activity:

Total Activity:

\begin{tabular}{|c|c|}
\hline$<1 \mathrm{E}-8$ & Units: $\mathrm{Ci} / \mathrm{g}$ \\
\hline & Units: \\
\hline & @ surface \\
\hline & @ 1-m Units: \\
\hline & Units: \\
\hline & $\mathrm{nCi} / \mathrm{g}$ \\
\hline
\end{tabular}

\section{F. TRU ALPHA ACTIVITY DISTRIBUTION}

This waste stream is not identified as a TRU or a-LLW. 

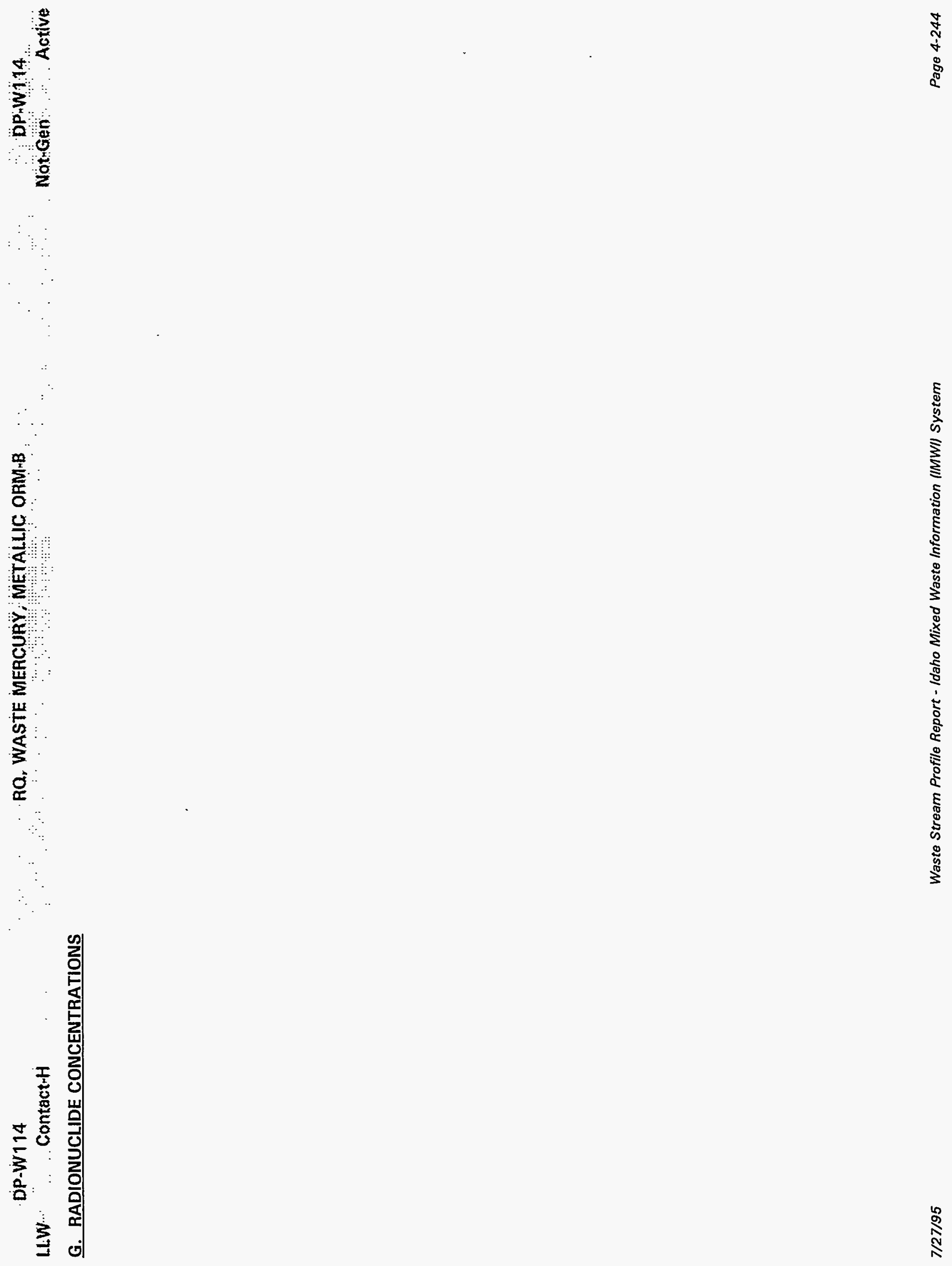
DP W 114

RQ WASTE MERCURY METALLIO ORM

Dpintia

LEW Contact H

$\because$ Q WASTEMERCURY

H. MATRIX CHARACTERISTICS

Primary Matrix Code: $\times 7100$

Description: Special Waste: Elemental Mercury: :

Combustibility: $\bigcirc$ Combustible (>90\%)

$\bigcirc$ Mixed $110 \%-90 \%)$

Noncombustible $(<10 \%)$

\section{MATRIX COMPOSITION}

\begin{tabular}{|c|c|c|c|c|c|c|}
\hline & & & \multicolumn{4}{|c|}{ Composition } \\
\hline Code & Matrix Description & Material & Typical & $\overline{L L}$ & UL & Units \\
\hline$X 7100$ & Special Waste.Elemental Mercury.. & Elemental Mercury & 100 & 100 & 100 & $\%$ vol \\
\hline
\end{tabular}


DP $\div$ W114

LLW

RQ, WASTE MERCURY METALLIC ORM

DPiWi14:

NotrGèn :.: Active

\section{J. HAZARDOUS CONSTITUENTS}

\begin{tabular}{|c|c|c|c|c|c|c|c|c|}
\hline & & & \multicolumn{4}{|c|}{ Concentration } & & \\
\hline Code & Hazardous Waste Description & Treatment Subcategory & Typical & $\mathbf{L L}$ & UL & Units & Basis & Conc. Type \\
\hline$\overline{D 008}$ & Lead & & & & & & $A ; C$ & Total \\
\hline$\overline{D 009}$ & Mercury & Elemental mercury contaminated with radioact & & & & & $A ; C$ & Total \\
\hline U151 & Mercury (High mercury $>260 \mathrm{mg} / \mathrm{kg}$ ) & & & & & & $A ; C$ & Total \\
\hline
\end{tabular}




\section{K. POLYCHLORINATED BIPHENYLS (PCBS)}

\section{Is this waste stream PCB contaminated? OYes $O$ No $O$ Unknown}

If yes, what portion of the waste is $\mathrm{PCB}$ contaminated (volume \%):

If $<100 \%$, can the PCB contaminated waste be segregated and treated separately?

What is the PCB concentration in ppm?

If only a portion of the waste is PCB contaminated, report values for contaminated portion.

\section{TREATMENT PLANS}

\begin{tabular}{|c|c|c|c|c|c|c|c|c|c|c|c|}
\hline $\begin{array}{c}\text { Opt. } \\
\#\end{array}$ & $\begin{array}{c}\text { Media Type } \\
\text { (If more than one) }\end{array}$ & $\begin{array}{c}\text { Matrix } \\
\text { Code }\end{array}$ & $\begin{array}{l}\text { Vol. \% } \\
\text { lif }\end{array}$ & Step & JIT & sc & CIF & $\begin{array}{l}\text { Trans. } \\
\text { Miles }\end{array}$ & $\begin{array}{l}\text { Facility } \\
\text { Abbr. }\end{array}$ & Unit Name & Comments \\
\hline 1 & & $x 7100$ & & a & $\mathbf{O}$ & $\mathrm{O}$ & $1.00 E+00$ & & TRANS & Transport - LLW & \\
\hline & & & & $\mathbf{b}$ & O & $\mathrm{O}$ & $1.00 \mathrm{E}+00$ & & AMWTF & $\mathrm{CH}$ - Opening \& Sorting & \\
\hline & & & & $\mathbf{c}$ & 0 & 0 & $1.00 E+00$ & & AMWTF & $\mathrm{CH}$ - Amalgamation & \\
\hline & & & & d & 0 & 0 & $1.00 E+00$ & & SCDF & Disposal - Contact Handled & \\
\hline
\end{tabular}

Note: Where provided, media percentages are rough estimates used to facilitate treatment planning. They do not necessarily imply an accurate knowledge of waste stream composition, and were not derived for use outside this conceptual planning exercise.

Describe any special or unique technical concerns related to the treatment of this waste stream that would impact the use or implementation of standard hazardous waste treatment methods. 


\begin{tabular}{l} 
ET-WO09 \\
A. IDENTIFICATION AND DESCRIPTION \\
Waste Stream ID: ET-W009 \\
W. S. Name: \\
Content Code: \\
FAINT CHIPS \\
FFCA ID: $\quad$ ET-W009 \\
Waste Stream Description: \\
\hline
\end{tabular}

\section{B. GENERATION SITE/PROCESS DESCRIPTION}

The following questions, concerning the generation site and process, should be answered even if the waste stream is no longer generated.

Name of Site Generating Waste (e.g., INEL, Rocky Flats, etc.):

Location of activities (Area and Building):

Operations (functions) performed in building:

Description of Process Generating Waste: 
ET-Woog:

HEW Contact H

C. PROJECTED WASTE GENERATION

Is this waste stream still generated?

If yes, when is the expected generation termination date?

Current Projections
PAINT CHIPS

ET:Woog

Notogen Active

OYes O No

Historical Projections 


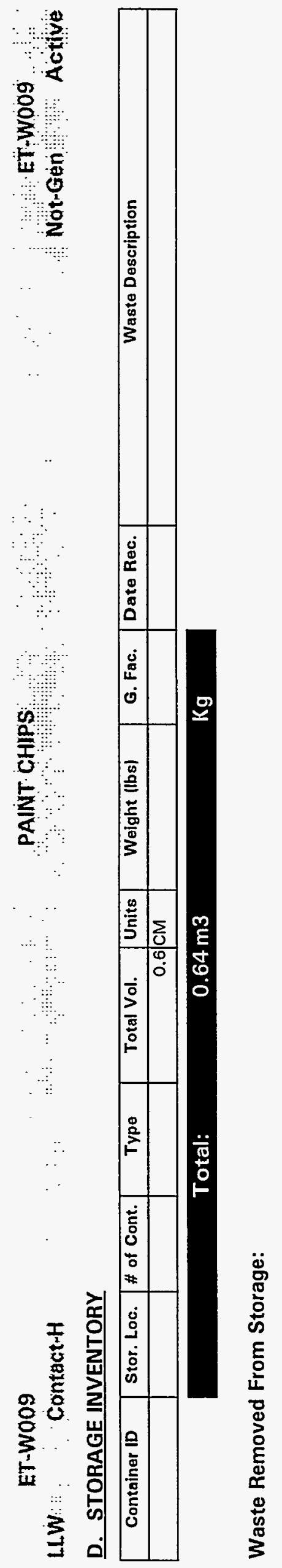

0
6
4
$\forall$
8
0

ह5 


\section{ETWOoog}

LiW Contact-H

E. RADIATION CHARACTERISTICS

Radioactive Waste Type:

\begin{tabular}{|ll}
\hline OlLW & Oa-LLW O TRU \\
O HLW & O Non-Rad
\end{tabular}

Mixed Waste:

\begin{tabular}{|l|}
\hline O Yes O No \\
\hline O Contact O Remote \\
\hline
\end{tabular}

Radionuclide Contamination Accessiblity:

\begin{tabular}{rlrl|}
\cline { 2 - 4 } External Surface & OYes & ONo & O Unknown \\
\cline { 3 - 5 } Internal Surface & OYes & ONo & O Unknown \\
\cline { 2 - 5 } &
\end{tabular}

Dispersed Through Matrix

\section{Activity Levels}

Transuranic Alpha Activity: Uranium/Thorium Alpha Activity: Beta/Gamma Activity:

Surface Neutron Activity:

Total Activity:

\begin{tabular}{l}
$\square$ Units: \\
$\square$ Units: $\square$ \\
@ surface \\
@ @ 1-m Units: $\mathrm{mR/hr}$ \\
\hline Units: \\
nCi/g
\end{tabular}

ET:WOOO9 Nöt Gen Active

\section{F. TRU ALPHA ACTIVITY DISTRIBUTION}

This waste stream is not identified as a TRU or a-LLW. 

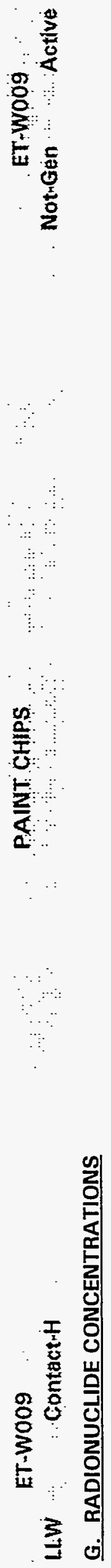
H. MATRIX CHARACTERISTICS

Primary Matrix Code: $\mathbf{S 3 1 3 1}$

Description: Homogeneous Solids: Inorganic Homogeneous Solids: Paint Waste: Paint Chips/Solids

Combustibility: $\bigcirc$ Combustible (>90\%)

Mixed $(10 \%-90 \%)$

Noncombustible $(<10 \%)$

\section{MATRIX COMPOSITION}




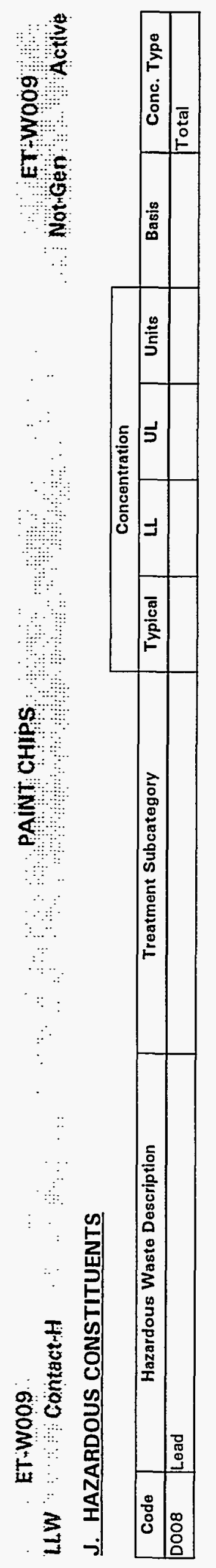

\begin{tabular}{l}
$\forall$ \\
\multirow{7}{*}{} \\
0 \\
8 \\
8
\end{tabular}

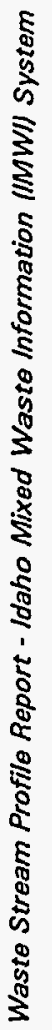




\section{A. IDENTIFICATION AND DESCRIPTION}

Waste Stream ID: ET-W019

W. S. Name:

Content Code:

FFCA ID:

$$
\text { CHROME SALT CORES }
$$

*

ET-W019

Waste Stream Description:

\section{B. GENERATION SITE/PROCESS DESCRIPTION}

The following questions, concerning the generation site and process, should be answered even if the waste stream is no longer generated.

Name of Site Generating Waste (e.g., INEL, Rocky Flats, etc.):

Location of activities (Area and Building):

Operations (functions) performed in building:

Description of Process Generating Waste: 


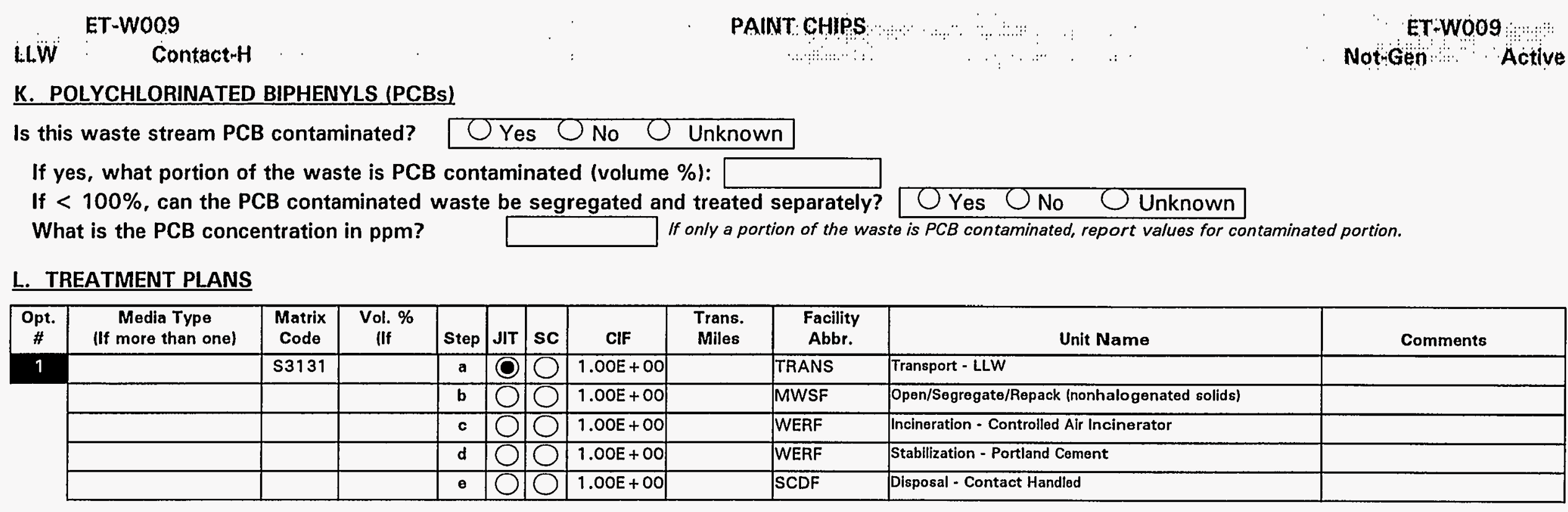

Note: Where provided, media percentages are rough estimates used to facilitate treatment planning. They do not necessarily imply an accurate knowledge of waste stream composition, and were not derived for use outside this conceptual planning exercise.

Describe any special or unique technical concerns related to the treatment of this waste stream that would impact the use or implementation of standard hazardous waste treatment methods. 


\section{PROJECTED WASTE GENERATION}

Is this waste stream still generated?

If yes, when is the expected generation termination date?

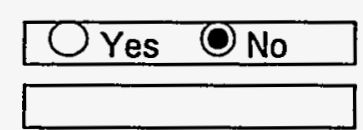

Current Projections

Historical Projections 


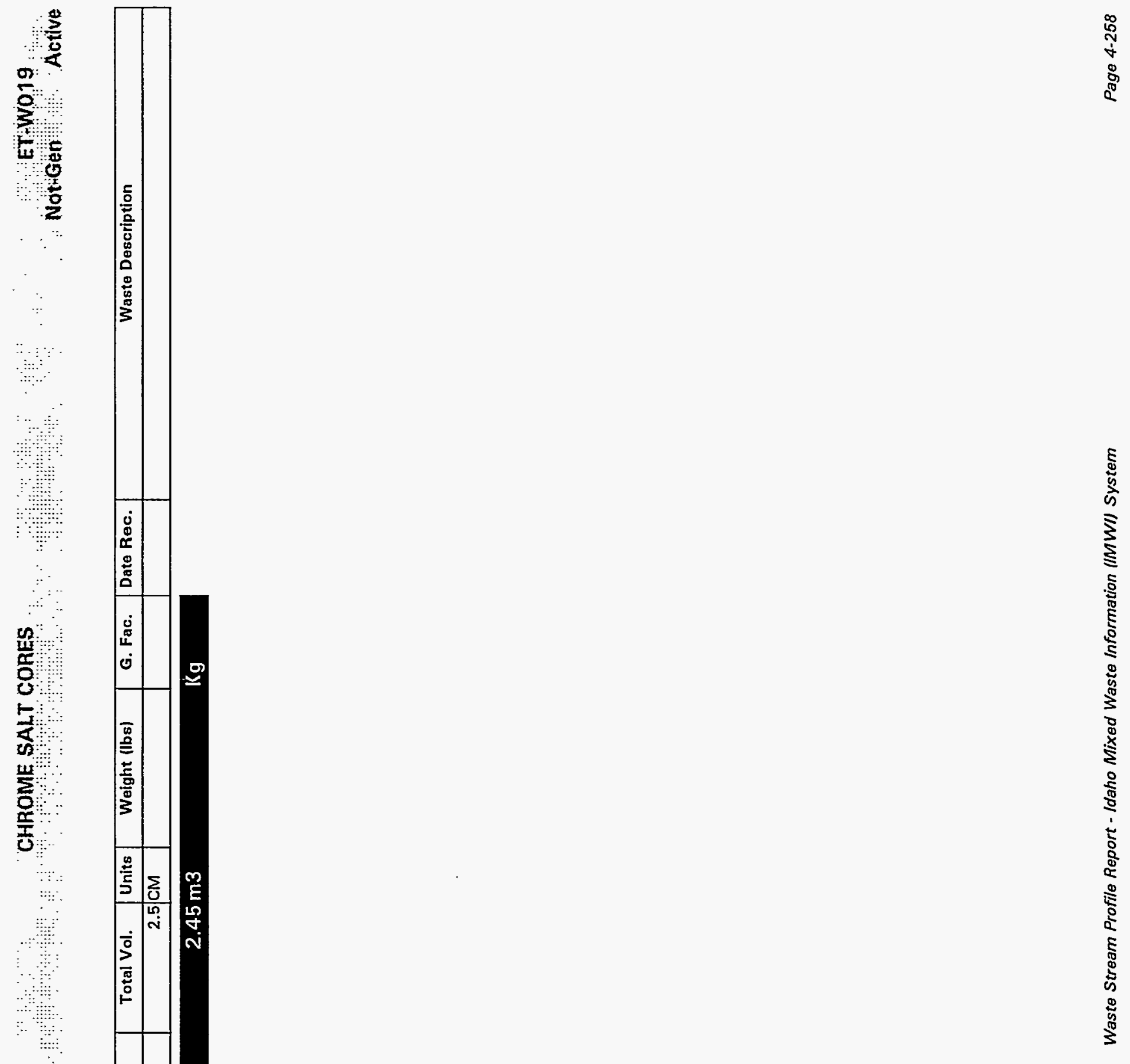




\section{F. TRU ALPHA ACTIVITY DISTRIBUTION}

Radioactive Waste Type:

\begin{tabular}{|ll|}
\hline O LLW & Oa-LLW O TRU \\
OHLW & ONon-Rad \\
\hline
\end{tabular}

This waste stream is not identified as a TRU or a-LLW.

Mixed Waste:

\begin{tabular}{l}
\hline Y Yes ONo \\
\hline O Contact \\
\hline Remote
\end{tabular}

Radionuclide Contamination Accessiblity:

External Surface

Internal Surface

Dispersed Through Matrix

\begin{tabular}{|c|c|c|}
\hline OYes & ONo & Unknown \\
\hline OYes & O No & OUnknown \\
\hline OYes & ONo & OUnknown \\
\hline
\end{tabular}

\section{Activity Levels}

Transuranic Alpha Activity:

Uranium/Thorium Alpha Activity:

Beta/Gamma Activity:

Surface Neutron Activity:

Total Activity:

\begin{tabular}{|c|c|c|}
\hline & Units: [ & \\
\hline & Units: [ & \\
\hline & @ surfa & ace \\
\hline & @ 1-m & Units: $\mathrm{mR} / \mathrm{hr}$ \\
\hline & Units: & \\
\hline & $\mathrm{nCi} / \mathrm{g}$ & \\
\hline
\end{tabular}




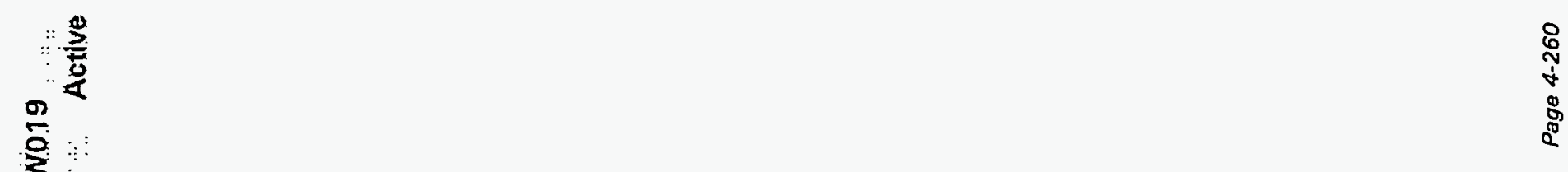




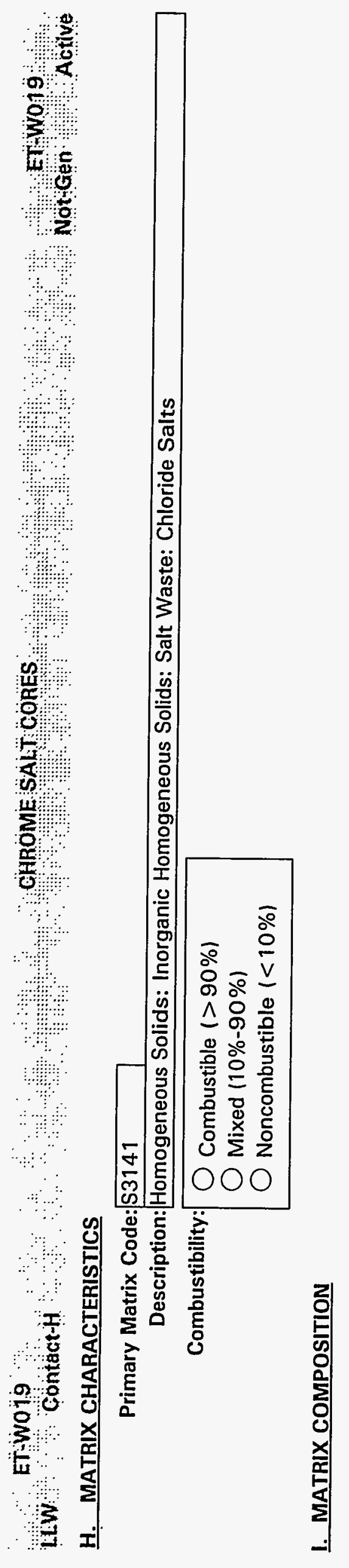

ह5

깁. 


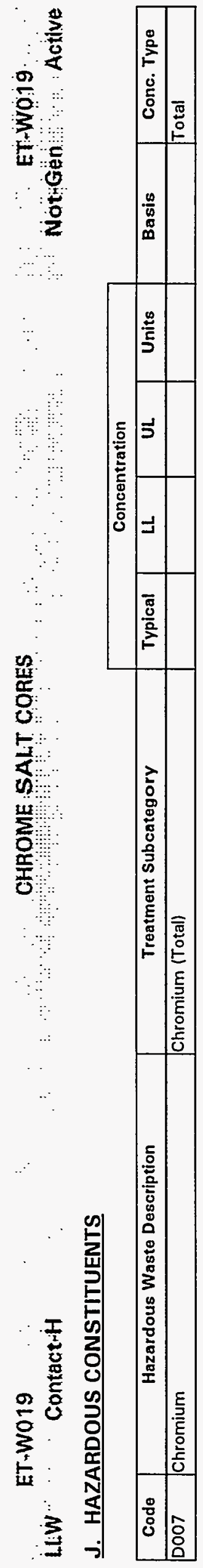




\section{K. POLYCHLORINATED BIPHENYLS (PCBS)}

\section{Is this waste stream PCB contaminated? OYes $O$ No $O$ Unknown}

If yes, what portion of the waste is PCB contaminated (volume \%):

If $<100 \%$, can the PCB contaminated waste be segregated and treated separately?

OYes O No O Unknown

What is the PCB concentration in ppm?

If only a portion of the waste is $P C B$ contaminated, report values for contaminated portion.

\section{TREATMENT PLANS}

\begin{tabular}{|c|c|c|c|c|c|c|c|c|c|c|c|}
\hline Opt. & $\begin{array}{c}\text { Media Type } \\
\text { (If more than one) }\end{array}$ & $\begin{array}{c}\text { Matrix } \\
\text { Code }\end{array}$ & $\begin{array}{l}\text { Vol. \% } \\
\text { IIf }\end{array}$ & Step & JIT & sc & CIF & $\begin{array}{l}\text { Trans. } \\
\text { Miles }\end{array}$ & $\begin{array}{l}\text { Facility } \\
\text { Abbr. }\end{array}$ & Unit Name & Comments \\
\hline \multirow[t]{3}{*}{1} & & 53141 & & a & 0 & $\mathrm{O}$ & $1.00 E+00$ & & TRANS & Transport - LLW & \\
\hline & & & & b & 0 & 0 & $1.00 E+00$ & & WERF & Stabilization - Portland Cement & \\
\hline & & & & c & 0 & 0 & $1.00 \mathrm{E}+00$ & & SCDF & Disposal - Contact Handled & \\
\hline
\end{tabular}

Note: Where provided, media percentages are rough estimates used to facilitate treatment planning. They do not necessarily imply an accurate knowledge of waste stream composition, and were not derived for use outside this conceptual planning exercise.

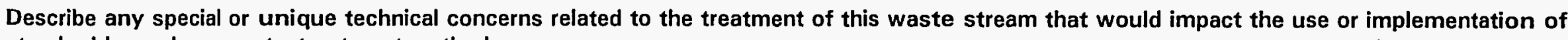
standard hazardous waste treatment methods. 


LLW $\quad$ Contact-H
A. IDENTIFICATION AND DESCRIPTION
Waste Stream ID: ET-WO20
W. S. Name:
Content Code:
LFCABARATORY ANALYTICAL REAGENT WASTE
Waste Stream Description:

\section{B. GENERATION SITE/PROCESS DESCRIPTION}

The following questions, concerning the generation site and process, should be answered even if the waste stream is no longer generated.

Name of Site Generating Waste (e.g., INEL, Rocky Flats, etc.):

\section{Location of activities (Area and Building):}

Operations (functions) performed in building:

Description of Process Generating Waste: 
If yes, when is the expected generation termination date?

Current Projections 


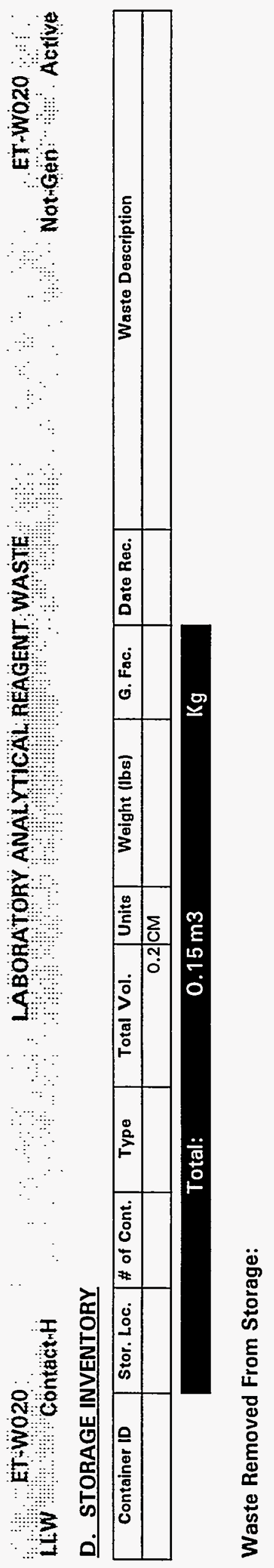


ETWÖ20

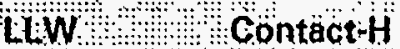

E. RADIATION CHARACTERISTICS

Radioactive Waste Type:

\begin{tabular}{|ll|}
\hline Ollw & Oa-LLW $\bigcirc$ TRU \\
OhLW & $\bigcirc$ Non-Rad \\
\hline
\end{tabular}

Mixed Waste:

\begin{tabular}{|l|}
\hline OYes ONo \\
\hline O Contact \\
\hline Remote
\end{tabular}

Radionuclide Contamination Accessiblity:

External Surface OYes ONo OUnknown

Internal Surface OYes ONo OUnknown

Dispersed Through Matrix OYes ONo OUnknown

\section{Activity Levels}

Transuranic Alpha Activity:

Uranium/Thorium Alpha Activity:

Beta/Gamma Activity:

Surface Neutron Activity:

\begin{tabular}{|c|c|c|}
\hline & Units: [ & \\
\hline & Units: [ & \\
\hline & @ surf & ace \\
\hline & @ 1-m & Units: $\mathrm{mR} / \mathrm{hr}$ \\
\hline & Units: & \\
\hline & $\mathrm{nCi} / \mathrm{g}$ & \\
\hline
\end{tabular}

Total Activity:
This waste stream is not identified as a TRU or a-LLW.

\section{F. TRU ALPHA ACTIVITY DISTRIBUTION}




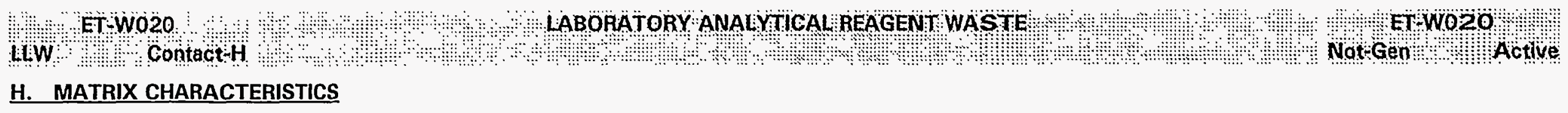

Primary Matrix Code: L2120

Description: Organic Liquids: Aqueous/Organic Liquids: Aqueous/Nonhalogenated Organic Liquids:

Combustibility:

Combustible $(>90 \%)$

Mixed $(10 \%-90 \%)$

Noncombustible $(<10 \%)$

\section{MATRIX COMPOSITION}




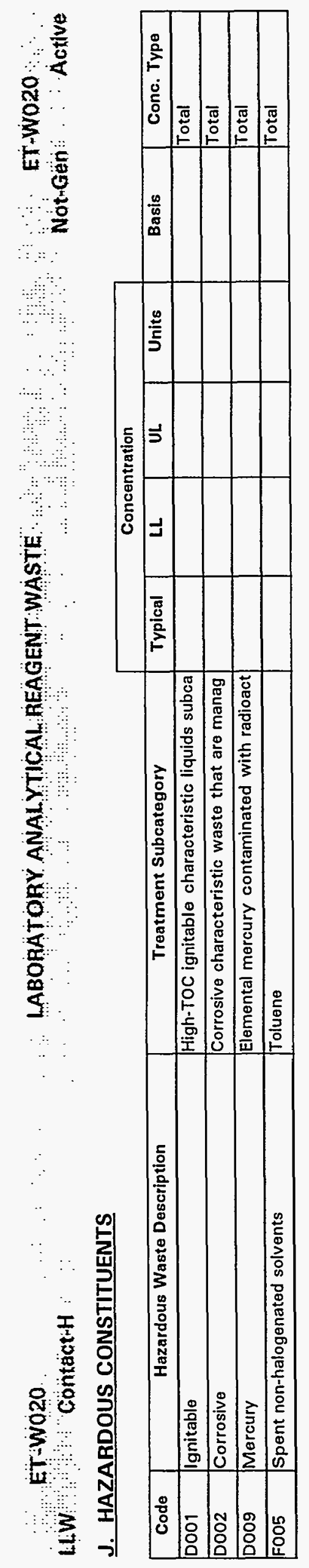




\section{ETWO20}

LABORATORY ANALYTICAL REAGENT WASTE

ETWWO20

LLW Contact H

OO Notugen in Active

K. POLYCHLORINATED BIPHENYLS (PCBS)

Is this waste stream PCB contaminated?

Yes O No $O$ Unknown

If yes, what portion of the waste is PCB contaminated (volume \%):

If $<100 \%$, can the PCB contaminated waste be segregated and treated separately?

OYes O No O Unknown

What is the PCB concentration in ppm?

If only a portion of the waste is $P C B$ contaminated, report values for contaminated portion.

\section{TREATMENT PLANS}

\begin{tabular}{|c|c|c|c|c|c|c|c|c|c|c|c|}
\hline Opt. & $\begin{array}{c}\text { Media Type } \\
\text { (If more than one) }\end{array}$ & $\begin{array}{l}\text { Matrix } \\
\text { Code }\end{array}$ & $\begin{array}{l}\text { Vol. \% } \\
\text { (If }\end{array}$ & Step & JIT & sc & CIF & $\begin{array}{l}\text { Trans. } \\
\text { Miles }\end{array}$ & $\begin{array}{l}\text { Facility } \\
\text { Abbr. }\end{array}$ & Unit Name & Comments \\
\hline 1 & & $\mathrm{~L} 2120$ & & $\bar{a}$ & $\mathbf{O}$ & 0 & $1.00 \mathrm{E}+00$ & & TRANS & Transport - LLW & \\
\hline & & & & $\mathbf{b}$ & O & 0 & $1.00 E+00$ & & MWSF & Open/Blend/Repack (Liquids \& Halogens) & \\
\hline & & & & $\bar{c}$ & 0 & 0 & $1.00 E+00$ & & WERF & Incineration - Controlled Air Incinerator & \\
\hline & & & & $\bar{d}$ & 0 & 0 & $1.00 \mathrm{E}+00$ & & WERF & Stabilization - Portland Cement & \\
\hline & & & & e & 0 & 0 & $1.00 \mathrm{E}+00$ & & SCDF & Disposal - Contact Handled & \\
\hline
\end{tabular}

Note: Where provided, media percentages are rough estimates used to facilitate treatment planning. They do not necessarily imply an accurate knowledge of waste stream composition, and were not derived for use outside this conceptual planning exercise.

Describe any special or unique technical concerns related to the treatment of this waste stream that would impact the use or implementation of standard hazardous waste treatment methods. 


\section{B. GENERATION SITE/PROCESS DESCRIPTION}

\section{A. IDENTIFICATION AND DESCRIPTION}

Waste Stream ID: ET-W023

W. S. Name:

Content Code:

ELEMENTAL MERCURY

FFCA ID:

ET-W023

Waste Stream Description:
The following questions, concerning the generation site and process, should be answered even if the waste stream is no longer generated.

Name of Site Generating Waste (e.g., INEL, Rocky Flats, etc.):

\section{Location of activities (Area and Building):}

Operations (functions) performed in building:

Description of Process Generating Waste: 


$$
\text { ETWO23 }
$$

Ulew ContactuH

C. PROJECTED WASTE GENERATION

Is this waste stream still generated?

If yes, when is the expected generation termination date?

Current Projections

OYes ONo

\section{Historical Projections}




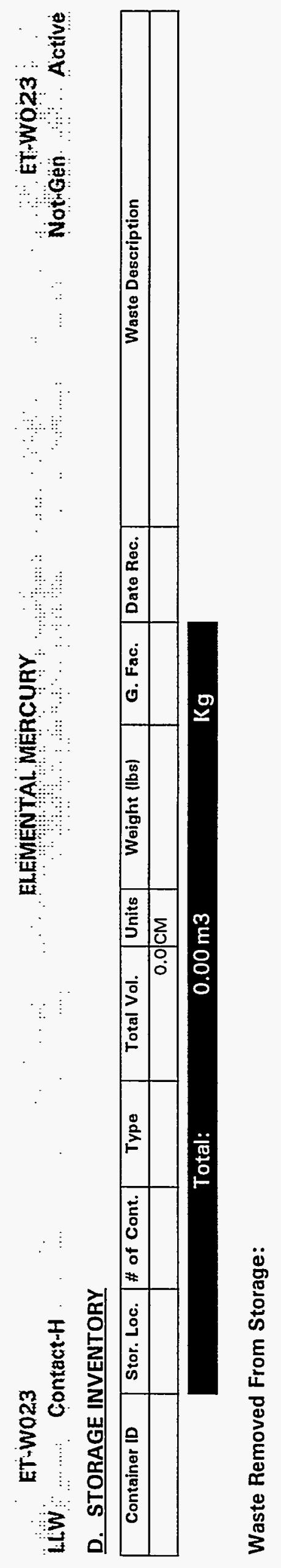

\begin{tabular}{l}
\multirow{2}{*}{} \\
\multirow{4}{*}{} \\
0 \\
0 \\
0
\end{tabular}

ह

ฌู 


\section{E. RADIATION CHARACTERISTICS}

Radioactive Waste Type:

\begin{tabular}{|ll|}
\hline O LLW & Oa-LLW O TRU \\
OHLW & O Non-Rad \\
\hline
\end{tabular}

Mixed Waste:

Handling:

\begin{tabular}{|l|}
\hline O Yes O No \\
\hline O Contact O Remote \\
\hline
\end{tabular}

Radionuclide Contamination Accessiblity:

\begin{tabular}{|c|c|c|}
\hline External Surface & OYes O No & Unknown \\
\hline Internal Surface & OYes ONo & OUnknown \\
\hline
\end{tabular}

\section{Activity Levels}

Uranium/Thorium Alpha Activity:

Beta/Gamma Activity:

Surface Neutron Activity:
Total Activity:

Surface Neutron Activity:
Total Activity:

\begin{tabular}{l}
$\square$ Units: $\square$ \\
\hline Units: $\square$ \\
\hline @ surface \\
@ @ 1-m Units: mR/hr \\
$\square$ Units: $\square$ \\
nCi/g
\end{tabular}

Transuranic Alpha Activity:
This waste stream is not identified as a TRU or a-LLW.

\section{F. TRU ALPHA ACTIVITY DISTRIBUTION}



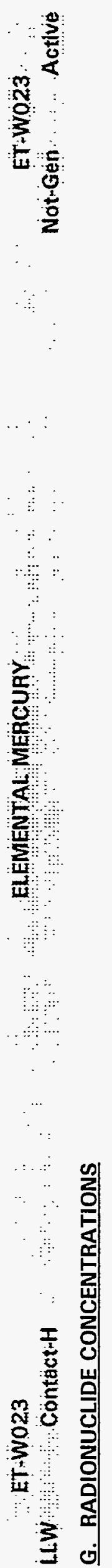
ETWWO23

H. MATRIX CHARACTERISTICS

Primary Matrix Code: $x 7100$

Description: Special Waste: Elemental Mercury: :

Combustibility: $\bigcirc$ Combustible ( $>90 \%$ )

Mixed (10\%-90\%)

Noncombustible (<10\%)

\section{MATRIX COMPOSITION}



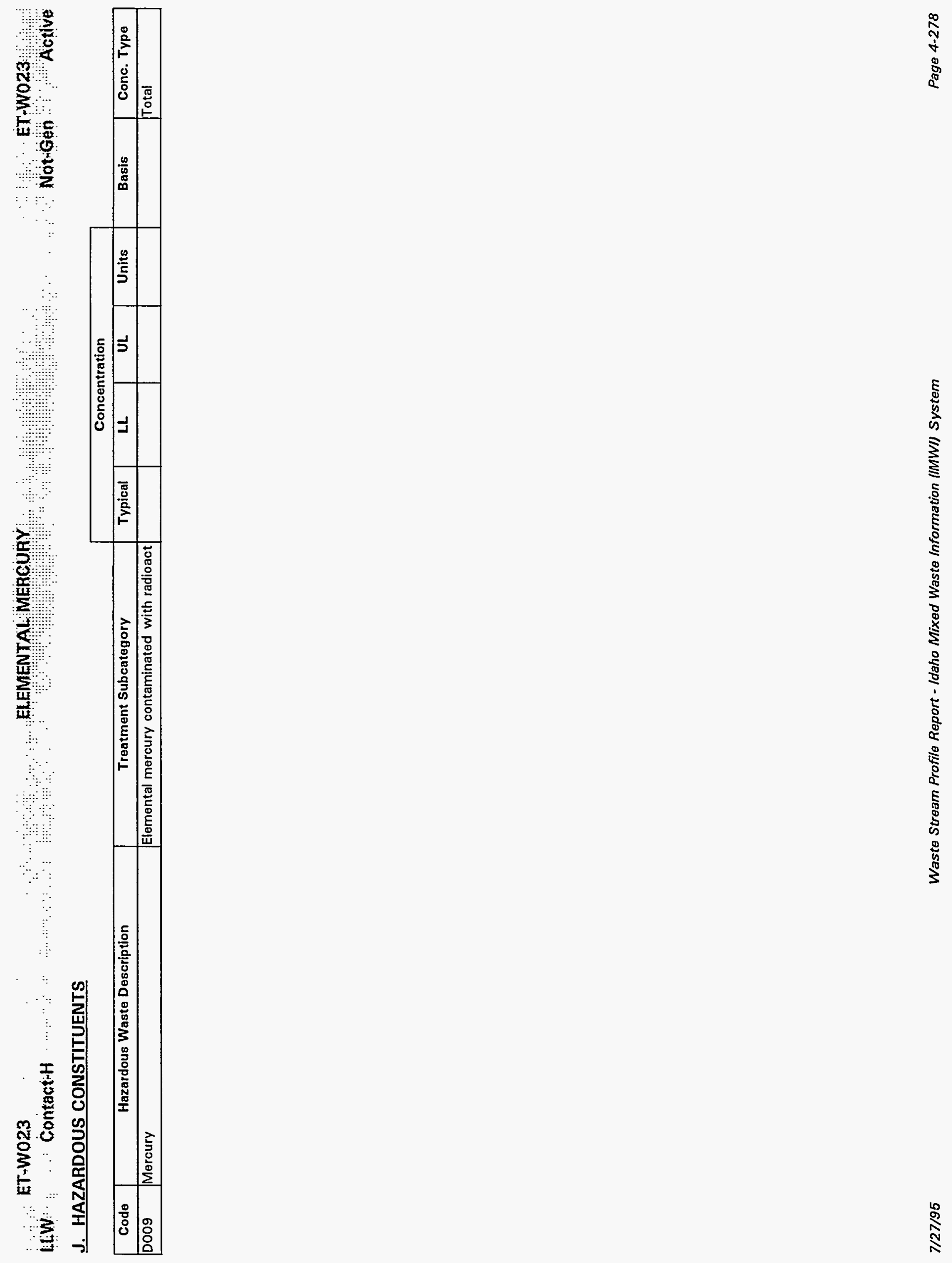


\section{K. POLYCHLORINATED BIPHENYLS (PCBS)}

Is this waste stream PCB contaminated? OYes O No $O$ Unknown

If yes, what portion of the waste is PCB contaminated (volume \%):

If $<100 \%$, can the PCB contaminated waste be segregated and treated separately?

If only a portion of the waste is $P C B$ contaminated, report values for contaminated portion.

\section{TREATMENT PLANS}

\begin{tabular}{|c|c|c|c|c|c|c|c|c|c|c|c|}
\hline $\begin{array}{c}\text { Opt. } \\
\#\end{array}$ & $\begin{array}{c}\text { Media Type } \\
\text { (If more than one) }\end{array}$ & $\begin{array}{c}\text { Matrix } \\
\text { Code }\end{array}$ & $\begin{array}{l}\text { Vol. } \% \\
\text { IIf }\end{array}$ & Step & JIT & sc & CIF & $\begin{array}{l}\text { Trans. } \\
\text { Miles }\end{array}$ & $\begin{array}{l}\text { Facility } \\
\text { Abbr. }\end{array}$ & Unit Name & Comments \\
\hline \multirow[t]{3}{*}{1} & & $\times 7100$ & & $\bar{a}$ & 0 & 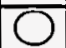 & $1.00 E+00$ & & TRANS & Transport - LLW & \\
\hline & & & & $\bar{b}$ & 0 & 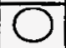 & $1.00 E+00$ & & AMWTF & $\mathrm{CH}$ - Opening \& Sorting & \\
\hline & & & & d & 0 & 0 & $1.00 E+00$ & & SCDF & Disposal - Contact Handled & \\
\hline
\end{tabular}

Note: Where provided, media percentages are rough estimates used to facilitate treatment planning. They do not necessarily imply an accurate knowledge of waste stream composition, and were not derived for use outside this conceptual planning exercise.

Describe any special or unique technical concerns related to the treatment of this waste stream that would impact the use or implementation of standard hazardous waste treatment methods. 


\section{ET-W026}

Hiw

Contäct-H

CRUSHED MERCURY LIGHT BULBS

A. IDENTIFICATION AND DESCRIPTION

Waste Stream ID: ET-W026

W. S. Name:

Content Code:

FFCA ID:

CRUSHED MERCURY LIGHT BULBS

Waste Stream Description: 
ETWO26

LWW : GontactH

C. PROJECTED WASTE GENERATION

Is this waste stream still generated?

If yes, when is the expected generation termination date?

Current Projections

Historical Projections 


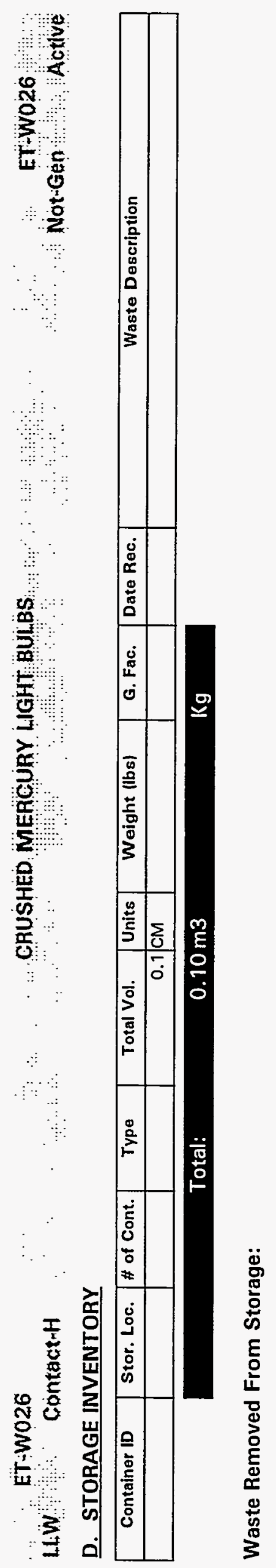




\section{HOWO26}

\section{F. TRU ALPHA ACTIVITY DISTRIBUTION}

\section{E. RADIATION CHARACTERISTICS}

Radioactive Waste Type:

\begin{tabular}{|ll|}
\hline OllW & Oa-LlW $\bigcirc$ tRU \\
OHLW & O Non-Rad \\
\hline
\end{tabular}

Mixed Waste:

Handling:

\begin{tabular}{l} 
OYes ONo \\
\hline Contact ORemote
\end{tabular}

Radionuclide Contamination Accessiblity:

External Surface

Internal Surface

Dispersed Through Matrix

\begin{tabular}{|c|c|c|}
\hline OYes & ONo & OUnknown \\
\hline OYes & $\mathrm{ONO}_{\mathrm{No}}$ & O Unknown \\
\hline$c$ & ONo & In \\
\hline
\end{tabular}

\section{Activity Levels}

Transuranic Alpha Activity:

Uranium/Thorium Alpha Activity:

Beta/Gamma Activity:

Surface Neutron Activity:

Total Activity:

\begin{tabular}{|c|c|c|}
\hline ransuranic Alpha Activity: & Units: [ & \\
\hline n/Thorium Alpha Activity: & Units: [ & \\
\hline Beta/Gamma Activity: | & @ surfa & \\
\hline & @ 1-m & Units: $\mathrm{mR} / \mathrm{hr}$ \\
\hline Surface Neutron Activity: & Units: [ & \\
\hline Total Activity: & $\mathrm{nCi} / \mathrm{g}$ & \\
\hline
\end{tabular}

This waste stream is not identified as a TRU or a-LLW. 


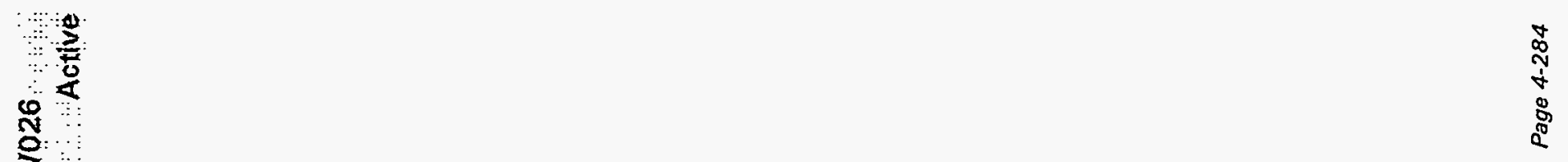

3.

世

吾

표

T.

z

8

严

III:

동

종::

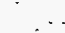

$\because$

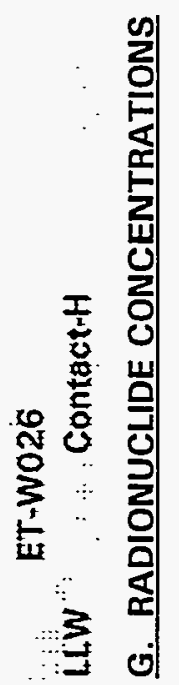


H. MATRIX CHARACTERISTICS

Primary Matrix Code: 55122

Description: Debris Waste: Inorganic Debris: Inorganic Non-Metal Debris: Glass Debris

Combustibility

\section{Combustible $(>90 \%)$}

Mixed $(10 \%-90 \%)$

Noncombustible $(<10 \%)$

\section{MATRIX COMPOSITION}




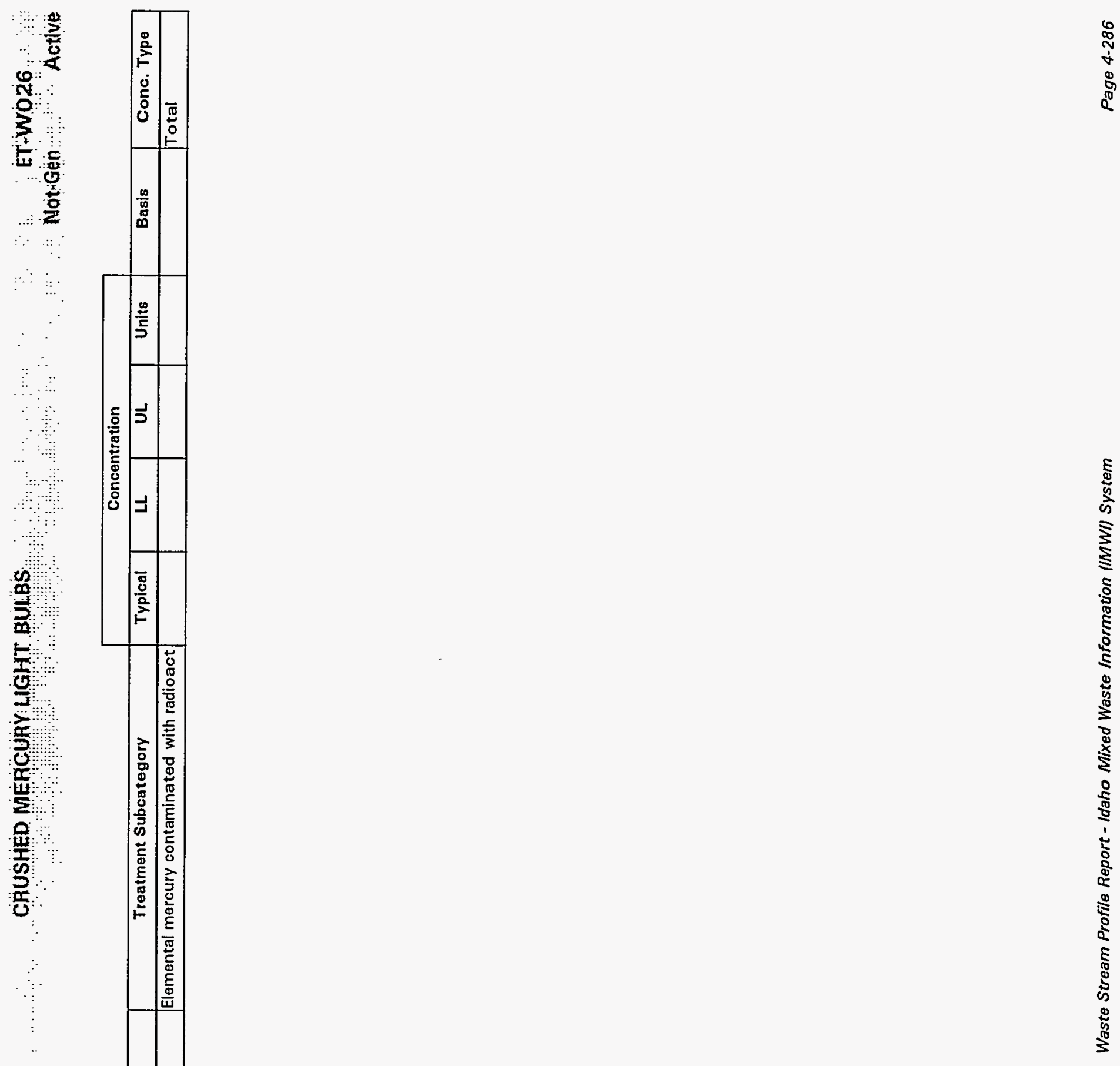

ह5 


\section{K. POLYCHLORINATED BIPHENYLS (PCBS)}

Is this waste stream PCB contaminated? OYes O No $O$ Unknown

If yes, what portion of the waste is PCB contaminated (volume \%):

If $<100 \%$, can the PCB contaminated waste be segregated and treated separately? $O$ Yes O No O Unknown

What is the PCB concentration in ppm? If only a portion of the waste is PCB contaminated, report values for contaminated portion.

\section{TREATMENT PLANS}

\begin{tabular}{|c|c|c|c|c|c|c|c|c|c|c|c|}
\hline $\begin{array}{c}\text { Opt. } \\
\#\end{array}$ & $\begin{array}{c}\text { Media Type } \\
\text { (If more than one) }\end{array}$ & $\begin{array}{c}\text { Matrix } \\
\text { Code }\end{array}$ & $\begin{array}{l}\text { Vol. \% } \\
\text { (If }\end{array}$ & Step & JIT & Sc & CIF & $\begin{array}{l}\text { Trans. } \\
\text { Miles }\end{array}$ & $\begin{array}{l}\text { Facility } \\
\text { Abbr. }\end{array}$ & Unit Name & Comments \\
\hline 1 & & S5122 & & $\mathbf{a}$ & 0 & 0 & $1.00 E+00$ & & TRANS & Transport - LLW & \\
\hline & & & & $\mathbf{b}$ & 0 & 0 & $1.00 \mathrm{E}+00$ & & WERF & Stabilization - Portland Cement & \\
\hline & & & & c & 0 & 0 & $1.00 E+00$ & & SCDF & Disposal - Contact Handled & \\
\hline
\end{tabular}

Note: Where provided, media percentages are rough estimates used to facilitate treatment planning. They do not necessarily imply an accurate knowledge of waste stream composition, and were not derived for use outside this conceptual planning exercise.

Describe any special or unique technical concerns related to the treatment of this waste stream that would impact the use or implementation of standard hazardous waste treatment methods. 


\section{$\therefore$ GAIWOO3 \\ Liw}

A. IDENTIFICATION AND DESCRIPTION

Waste Stream ID: GA-W003

W. S. Name:

Content Code:

FFCA ID:

SVA: $\mathrm{Pb}$ CONTAMINATED SLUDGE

GA-W003

Waste Stream Description:
SVÄ: Pb CONTAMINATED SLUDGE

\section{B. GENERATION SITE/PROCESS DESCRIPTION}

The following questions, concerning the generation site and process, should be answered even if the waste stream is no longer generated.

Name of Site Generating Waste (e.g., INEL, Rocky Flats, etc.):

Location of activities (Area and Building):

Operations (functions) performed in building:

Operations (functions) performed in building:

Description of Process Generating Waste: 


\section{PROJECTED WASTE GENERATION}

Is this waste stream still generated?
OYes O No

If yes, when is the expected generation termination date?

Current Projections

Historical Projections 


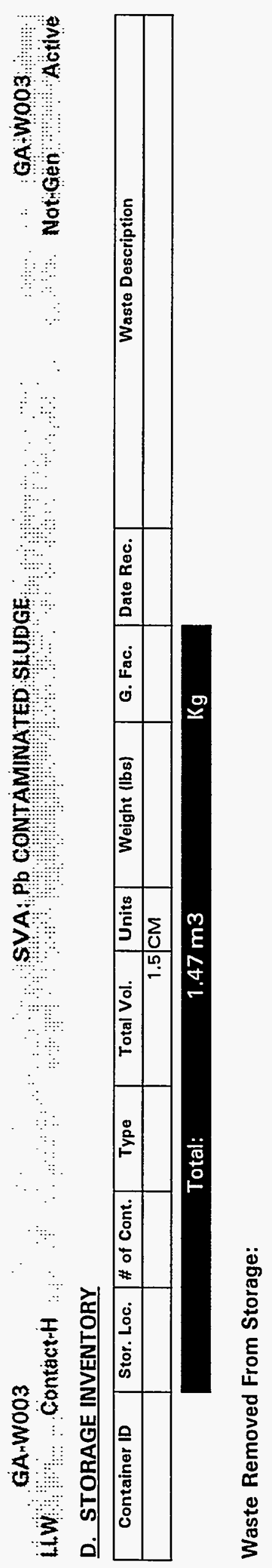




\section{G GAWOO3}

LiW Contagt-H

\section{E. RADIATION CHARACTERISTICS}

SVA: PÖ CONTAMINATED SLUUDE

\section{F. TRU ALPHA ACTIVITY DISTRIBUTION}

Radioactive Waste Type:

\begin{tabular}{|ll|}
\hline O LLW & Oa-LLW $\bigcirc$ TRU \\
O HLW & O Non-Rad \\
\hline
\end{tabular}

Mixed Waste:

\begin{tabular}{l}
\hline O Yes O No \\
\hline O Contact O Remote
\end{tabular}

Radionuclide Contamination Accessiblity:

External Surface OYes ONo O Unknown

Internal Surface OYes ONo OUnknown

Dispersed Through Matrix OYes ONo OUnknown

\section{Activity Levels}

Transuranic Alpha Activity:

Uranium/Thorium Alpha Activity:

Beta/Gamma Activity:

Surface Neutron Activity:
This waste stream is not identified as a TRU or a-LLW.

\footnotetext{
Total Activity:
}

\begin{tabular}{|l}
\hline \\
\hline \\
\hline \\
\hline \\
\hline \\
\hline
\end{tabular}

Units:

Units:

@ surface

@ 1-m Units: $\mathrm{mR} / \mathrm{hr}$

Units:

$\mathrm{nCi} / \mathrm{g}$ 


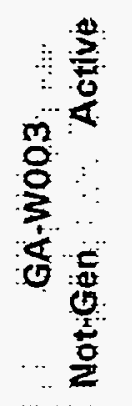

㟧

요

$\$$

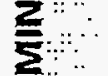

క.

8

a.

这...
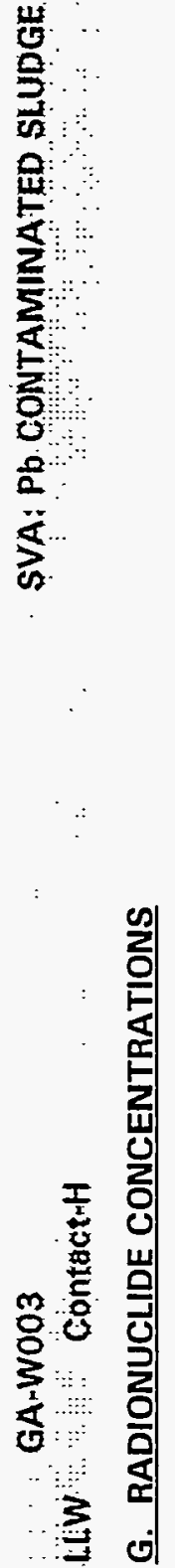

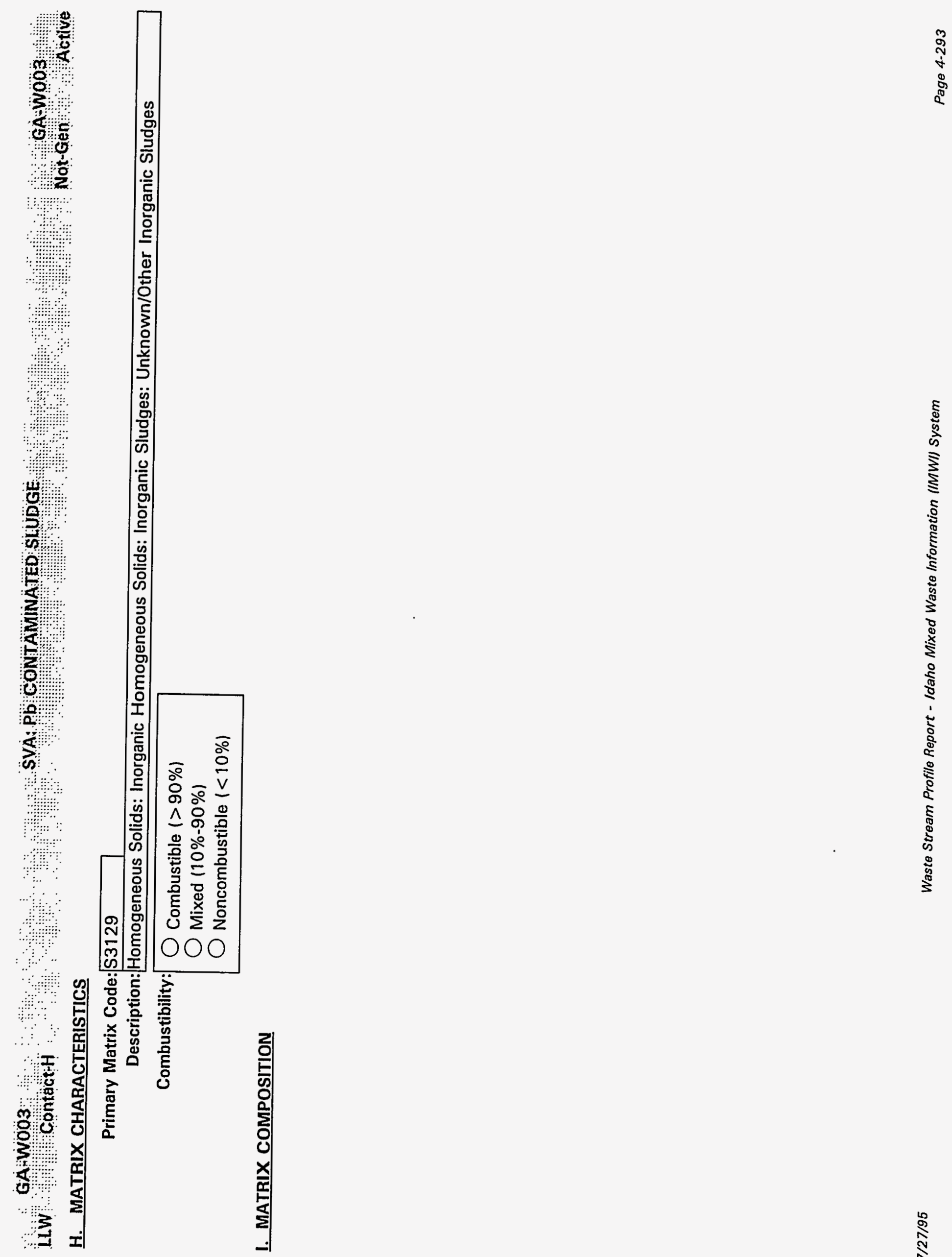
GA-W003

HLW :. Contact-H

J. HAZARDOUS CONSTITUENTS

\begin{tabular}{|c|c|c|c|c|c|c|c|c|}
\hline & & & \multicolumn{4}{|c|}{ Concentration } & & \\
\hline Code & Hazardous Waste Description & Treatment Subcategory & Typical & $\mathbf{L L}$ & UL & Units & Basis & Conc. Type \\
\hline D008 & Lead & & & & & & & Total \\
\hline
\end{tabular}




\section{K. POLYCHLORINATED BIPHENYLS (PCBS)}

Is this waste stream PCB contaminated? OYes $O$ No $O$ Unknown

If yes, what portion of the waste is PCB contaminated (volume \%):

If $<100 \%$, can the PCB contaminated waste be segregated and treated separately? OYes O No O Unknown

What is the PCB concentration in ppm? If only a portion of the waste is PCB contaminated, report values for contaminated portion.

\section{TREATMENT PLANS}

\begin{tabular}{|c|c|c|c|c|c|c|c|c|c|c|c|}
\hline $\begin{array}{c}\text { Opt. } \\
\#\end{array}$ & $\begin{array}{c}\text { Media Type } \\
\text { (If more than one) }\end{array}$ & $\begin{array}{c}\text { Matrix } \\
\text { Code }\end{array}$ & $\begin{array}{l}\text { Vol. } \% \\
\text { IIf }\end{array}$ & Step & JIT & Sc & CIF & $\begin{array}{l}\text { Trans. } \\
\text { Miles }\end{array}$ & $\begin{array}{l}\text { Facility } \\
\text { Abbr. }\end{array}$ & Unit Name & Comments \\
\hline 1 & & 53120 & & $\mathbf{a}$ & 0 & 0 & $1.00 E+00$ & & TRANS & Transport - LLW & \\
\hline & & & & b & 0 & 0 & $1.00 E+00$ & & WERF & Stabilization - Portland Cement & \\
\hline & & & & c & 0 & 0 & $1.00 E+00$ & & SCDF & Disposal - Contact Handled & \\
\hline
\end{tabular}

Note: Where provided, media percentages are rough estimates used to facilitate treatment planning. They do not necessarily imply an accurate knowledge of waste stream composition, and were not derived for use outside this conceptual planning exercise.

Describe any special or unique technical concerns related to the treatment of this waste stream that would impact the use or implementation of standard hazardous waste treatment methods. 


\section{LiW. Contact-H}

A. IDENTIFICATION AND DESCRIPTION

Waste Stream ID: GA-W007

W. S. Name: HOT CELL D\&D: Pb SHOT

Content Code:

FFCA ID:

*

GA-W007

Waste Stream Description:
HOT GELL D\&D P P SHOT

GA-WOOT NotmGen:

Active

\section{B. GENERATION SITE/PROCESS DESCRIPTION}

The following questions, concerning the generation site and process, should be answered even if the waste stream is no longer generated.

Name of Site Generating Waste (e.g., INEL, Rocky Flats, etc.):

Location of activities (Area and Building):

Operations (functions) performed in building:

Description of Process Generating Waste: 
GAWWoÖ7 LiW on Contactrit C. PROJECTED WASTE GENERATION

Is this waste stream still generated?

If yes, when is the expected generation termination date?

\section{HOT CELLO\&D PO SHOT}

GA WOO7

Nơt Gẹn lull Active

\section{Current Projections}




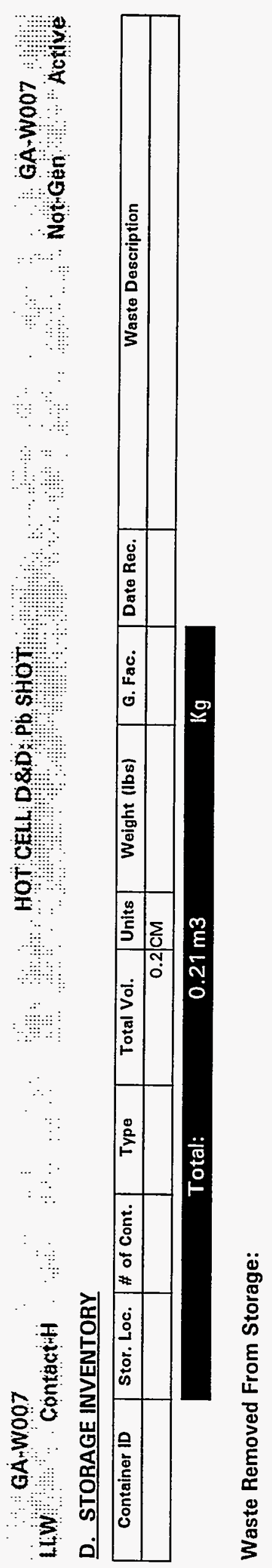


Radioactive Waste Type:

\begin{tabular}{|ll|}
\hline OlLW & Oa-LLW O TRU \\
OHLW & O Non-Rad \\
\hline
\end{tabular}

Mixed Waste:

Handling:

\begin{tabular}{|l|}
\hline O Yes ONo \\
\hline O Contact O Remote \\
\hline
\end{tabular}

Radionuclide Contamination Accessiblity:

External Surface

Internal Surface

Dispersed Through Matrix

\begin{tabular}{|c|c|c|}
\hline OYes & ONo & Unknown \\
\hline OYes & ONo & Unknown \\
\hline OYes & ONo & OUnk \\
\hline
\end{tabular}

\section{Activity Levels}

Transuranic Alpha Activity: Uranium/Thorium Alpha Activity

Beta/Gamma Activity:

Surface Neutron Activity: Total Activity:

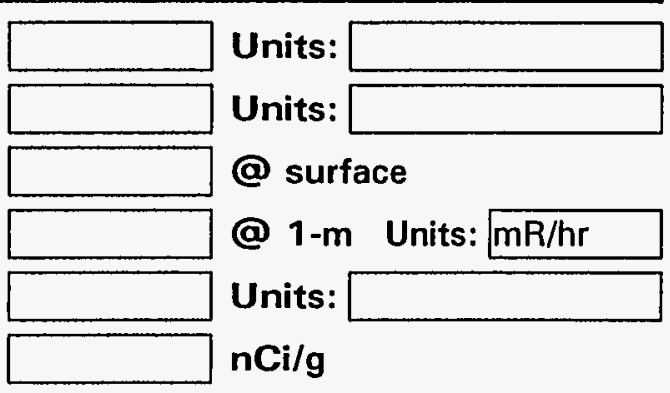

This waste stream is not identified as a TRU or a-LLW. 


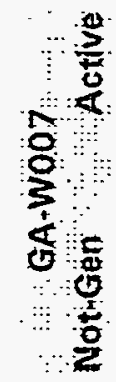

吉

을

:

i.

0

5

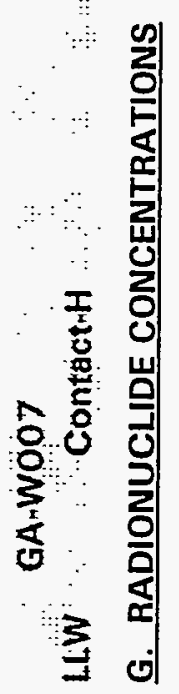


GA-WOOT:

LLW

Contacti

HOTCELL D\&DXPb SHOT

H. MATRIX CHARACTERISTICS

Primary Matrix Code: X7211

Description: Special Waste: Elemental Hazardous Metals: Elemental Lead: Non-Activated Lead

Combustibility: $\bigcirc$ Combustible $(>90 \%)$

Mixed $(10 \%-90 \%)$

Noncombustible $(<10 \%)$

\section{MATRIX COMPOSITION}




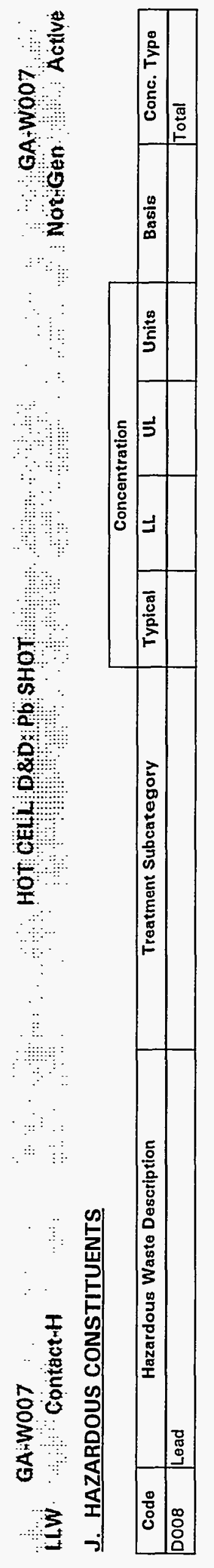

8
8
$\dot{\gamma}$
8
8
8

ह

ญุ 


\section{$\therefore$ GAWWOOOZ}

HOT GELLL D\&D $\times$ P̈ SHÖT

GAWOOZ:

HilW Contact $\because$ C

(1)

\section{K. POLYCHLORINATED BIPHENYLS (PCBS)}

Is this waste stream PCB contaminated? OYes O No $O$ Unknown

If yes, what portion of the waste is PCB contaminated (volume \%):

If $<100 \%$, can the PCB contaminated waste be segregated and treated separately?

OYes O No O Unknown

What is the PCB concentration in ppm? If only a portion of the waste is PCB contaminated, report values for contaminated portion.

\section{TREATMENT PLANS}

\begin{tabular}{|c|c|c|c|c|c|c|c|c|c|c|c|}
\hline $\begin{array}{l}\text { Opt. } \\
\#\end{array}$ & $\begin{array}{c}\text { Media Type } \\
\text { (If more than one) }\end{array}$ & $\begin{array}{c}\text { Matrix } \\
\text { Code }\end{array}$ & $\begin{array}{l}\text { Vol. \% } \\
\text { IIf }\end{array}$ & Step & JIT & sc & CIF & $\begin{array}{l}\text { Trans. } \\
\text { Miles }\end{array}$ & $\begin{array}{l}\text { Facility } \\
\text { Abbr. }\end{array}$ & Unit Name & Comments \\
\hline \multirow[t]{4}{*}{1} & & $X 7211$ & & $\bar{a}$ & O & 0 & $1.00 E+00$ & & TRANS & Transport - LLWW & \\
\hline & & & & b & 0 & 0 & $1.00 \mathrm{E}+00$ & & WROC & Sizing & \\
\hline & & & & c & 0 & 0 & $1.00 \mathrm{E}+00$ & & WROC & Macroencapsulation & \\
\hline & & & & d & 0 & J & $1.00 E+00$ & & SCDF & Disposal - Contact Handled & \\
\hline
\end{tabular}

Note: Where provided, media percentages are rough estimates used to facilitate treatment planning. They do not necessarily imply an accurate knowledge of waste stream composition, and were not derived for use outside this conceptual planning exercise.

Describe any special or unique technical concerns related to the treatment of this waste stream that would impact the use or implementation of standard hazardous waste treatment methods. 


\begin{tabular}{l} 
LiW GA-WO13 $\quad$ Contact-H \\
A. IDENTIFICATION AND DESCRIPTION \\
Waste Stream ID: \\
W. S. Name: \\
Content Code: \\
FOT CELL D\&D: Pb BRICKS \\
WFA ID: \\
Waste Stream Description: \\
\hline
\end{tabular}

BRICKS

GA-WOI3 Not Gen Actlve

\section{B. GENERATION SITE/PROCESS DESCRIPTION}

The following questions, concerning the generation site and process, should be answered even if the waste stream is no longer generated.

Name of Site Generating Waste (e.g., INEL, Rocky Flats, etc.):

Location of activities (Area and Building):

Operations (functions) performed in building:

Description of Process Generating Waste: 
Is this waste stream still generated?

OYes O No

If yes, when is the expected generation termination date?

Current Projections

\section{Historical Projections}




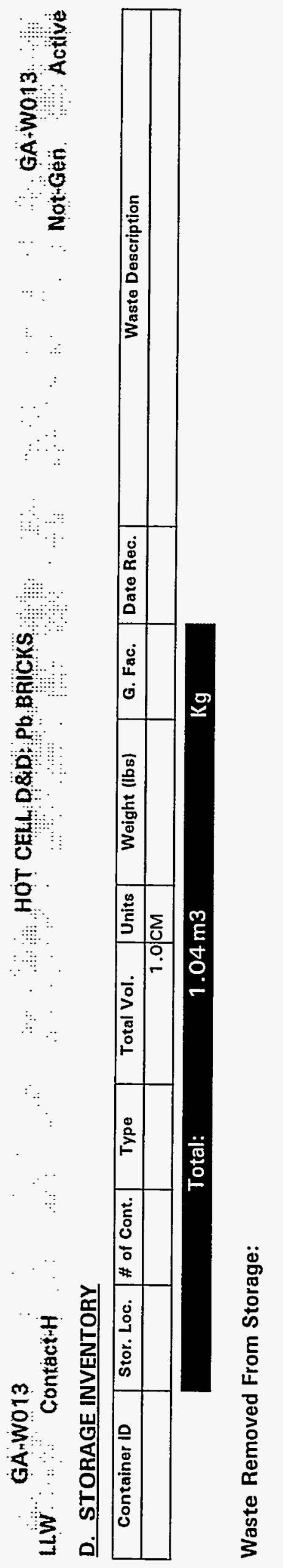

0
8
0
4
8
8
0 
GA-WO13

LiW G Contact H

E. RADIATION CHARACTERISTICS

Radioactive Waste Type:

\begin{tabular}{|ll|}
\hline OlLW & Oa-LLW O TRU \\
OHLW & O Non-Rad \\
\hline
\end{tabular}

Mixed Waste:

Handling:

\begin{tabular}{|l|}
\hline Yes ONo \\
\hline O Contact O Remote
\end{tabular}

Radionuclide Contamination Accessiblity:

\begin{tabular}{|c|c|c|c|}
\hline External Surface & Y Yes & ONo & OUnknown \\
\hline Internal Surface & OYes & ONo & OUnknown \\
\hline ispersed Through $n$ & OYes & ONo & OUnknown \\
\hline
\end{tabular}

\section{Activity Levels}

Transuranic Alpha Activity:

Uranium/Thorium Alpha Activity:

Beta/Gamma Activity:

Surface Neutron Activity:

Total Activity:

\begin{tabular}{l}
\hline \\
\hline Units: \\
\hline Units: $\square$ \\
\hline @ surface \\
\hline @ 1-m Units: mR/hr \\
Units: $\square$ \\
nCi/g
\end{tabular}

\section{F. TRU ALPHA ACTIVITY DISTRIBUTION}

This waste stream is not identified as a TRU or a-LLW. 


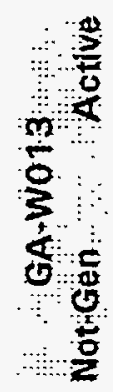


GAWOTI3

LiW: ContactH

H. MATRIX CHARACTERISTICS

.Primary Matrix Code: X7211

Description: Special Waste: Elemental Hazardous Metals: Elemental Lead: Non-Activated Lead

$$
\text { Combustibility: } \begin{aligned}
& \bigcirc \text { Combustible }(>90 \%) \\
& \bigcirc \text { Mixed }(10 \%-90 \%) \\
& \bigcirc \text { Noncombustible }(<10 \%)
\end{aligned}
$$

\section{MATRIX COMPOSITION}



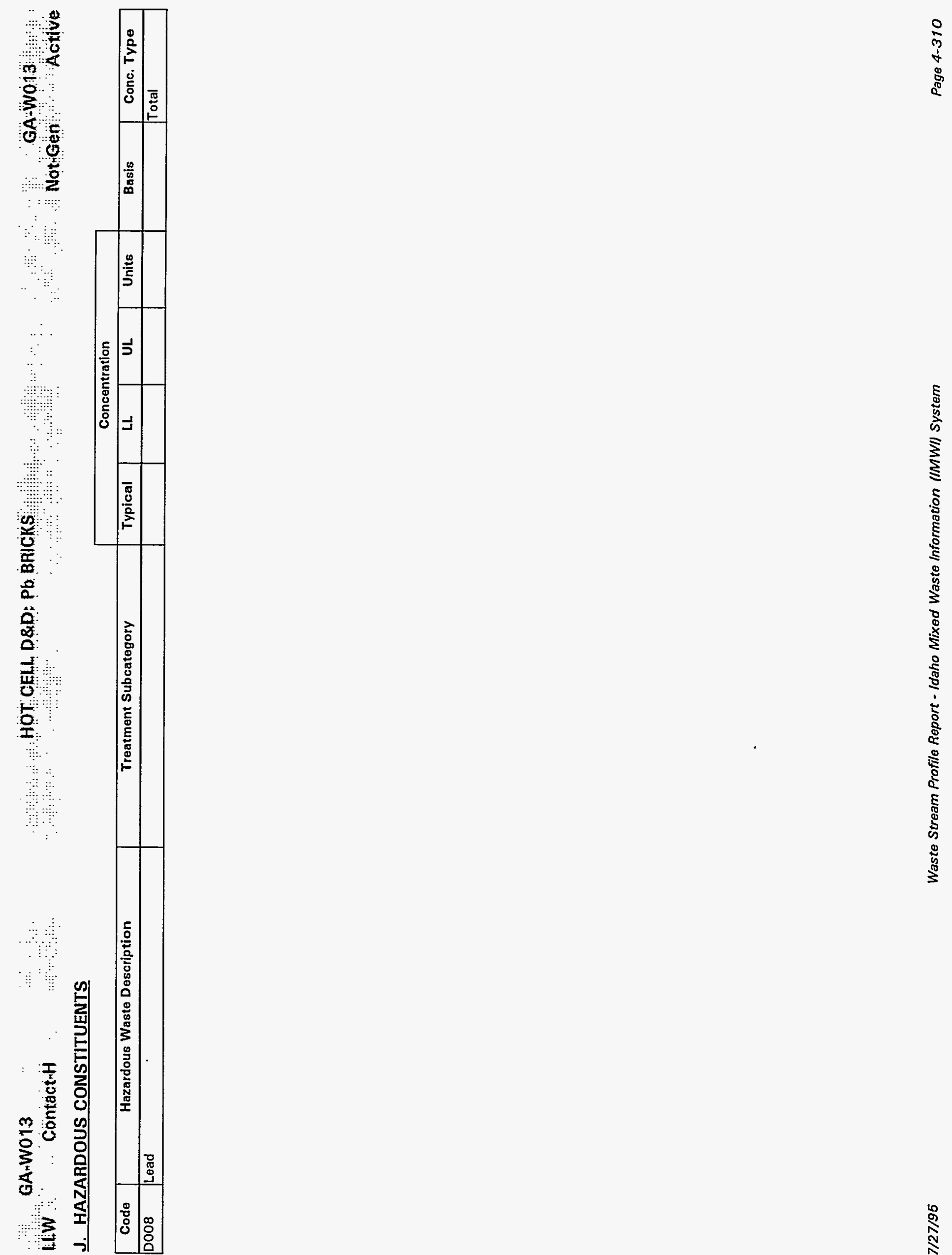
GA:WO13

\section{K. POLYCHLORINATED BIPHENYLS (PCBS)}

Is this waste stream PCB contaminated? OYes ONo O Unknown

If yes, what portion of the waste is PCB contaminated (volume \%):

If $<100 \%$, can the PCB contaminated waste be segregated and treated separately? $O$ Yes O No O Unknown

What is the PCB concentration in ppm?

If only a portion of the waste is PCB contaminated, report values for contaminated portion.

\section{TREATMENT PLANS}

\begin{tabular}{|c|c|c|c|c|c|c|c|c|c|c|c|}
\hline $\begin{array}{c}\text { Opt. } \\
\#\end{array}$ & $\begin{array}{c}\text { Media Type } \\
\text { (If more than onel }\end{array}$ & $\begin{array}{c}\text { Matrix } \\
\text { Code }\end{array}$ & $\begin{array}{l}\text { Vol. \% } \\
\text { (If }\end{array}$ & Step & JIT & sc & CIF & $\begin{array}{l}\text { Trans. } \\
\text { Miles }\end{array}$ & $\begin{array}{l}\text { Facility } \\
\text { Abbr. }\end{array}$ & Unit Name & Comments \\
\hline \multirow[t]{7}{*}{1} & & $\mathrm{X7211}$ & & a & 0 & $\bar{O}$ & $1.00 E+00$ & & TRANS & Transport - LLW & \\
\hline & & $\mathrm{X7211}$ & & $\mathbf{a}$ & 0 & O & $1.00 E+00$ & & TRANS & Transport - LLW & \\
\hline & & & & b & 0 & 0 & $1.00 E+00$ & & MWSF & Open/Segregate/Repack (nonhalogenated solids) & \\
\hline & & & & c & 0 & 0 & $1.00 E+00$ & & LEADTP & Commercial Decontamination & \\
\hline & & & & c & 0 & 0 & $1.00 E+00$ & & WROC & Sizing & \\
\hline & & & & d & 0 & 0 & $1.00 E+00$ & & WROC & Macroencapsulation & \\
\hline & & & & e & 0 & 0 & $1.00 E+00$ & & SCDF & Disposal - Contact Handled & \\
\hline
\end{tabular}

Note: Where provided, media percentages are rough estimates used to facilitate treatment planning. They do not necessarily imply an accurate knowledge of waste stream composition, and were not derived for use outside this conceptual planning exercise.

Describe any special or unique technical concerns related to the treatment of this waste stream that would impact the use or implementation of standard hazardous waste treatment methods. 


LAWWOO1
A. IDENTIFICATION AND DESCRIPTION
Waste Stream ID:
KA-W001
W. S. Name:
Content Code:
FFCA ID: LABORATORY CHEMICALS W/O METALS
Waste Stream Description:
KA-W001
Unusable laboratory chemicals. This mixed waste stream has not yet been generated. Individual
internal containers will be homogeneous. This waste stream will not be moratorium or
reclassified TRU waste.

Waste Stream ID: KA-W001

S. Name.

*

Waste Stream Description:

Unusable laboratory chemicals. This mixed waste stream has not yet been generated. Individual internal containers will be homogeneous. This waste stream will not be moratorium or reclassified TRU waste.

\section{B. GENERATION SITE/PROCESS DESCRIPTION}

The following questions, concerning the generation site and process, should be answered even if the waste stream is no longer generated.

Name of Site Generating Waste (e.g., INEL, Rocky Flats, etc.): KAPL - Knolls

Location of activities (Area and Building):

Various laboratory locations on site.

Operations (functions) performed in building:

Various laboratory operations.

Description of Process Generating Waste:

Waste will be generated through normal laboratory operations. 
KA-WOO1

MISC LABORATORY CHEMICALS WIOOMETALS

HLW Contact-H G
C. PROJECTED WASTE GENERATION

Is this waste stream still generated?

OYes O No

If yes, when is the expected generation termination date?

Current Projections

\begin{tabular}{|c|c|c|c|c|c|}
\hline Est. Date & Start Year & Interval & Vol (m3) & Mass (kg) & Source of Estimate \\
\hline $11 / 1 / 93$ & 1993 & 1 & 0.40 & 121.00 & 1993-FFCA \\
\hline $11 / 1 / 93$ & 1994 & 1 & 0.40 & 121.00 & 1993-FFCA \\
\hline $11 / 1 / 93$ & 1995 & 1 & 0.40 & 121.00 & 1993-FFCA \\
\hline $11 / 1 / 93$ & 1996 & 1 & 0.40 & 121.00 & 1993-FFCA \\
\hline $11 / 1 / 93$ & 1997 & 1 & 0.40 & 121.00 & 1993-FFCA \\
\hline $11 / 1 / 93$ & 1998 & 1 & 0.40 & 121.00 & $1993-F F C A$ \\
\hline $11 / 1 / 93$ & 1999 & 1 & 0.40 & 121.00 & 1993-FFCA \\
\hline \multicolumn{6}{|c|}{ Historical Projections } \\
\hline Est. Date & Start Year & Interval & Vol (m3) & Mass (kg) & Source of Estimate \\
\hline $11 / 1 / 93$ & 1998 & 5 & 2.00 & 605.00 & 1993-FFCA \\
\hline $11 / 1 / 93$ & 2003 & 20 & 8.00 & $2,420.00$ & 1993-FFCA \\
\hline
\end{tabular}


5

5

$\simeq$

$\because \frac{ \pm}{0}$

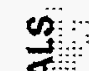

논

$\frac{0}{3}$

3

.

0

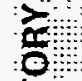

5

岁.

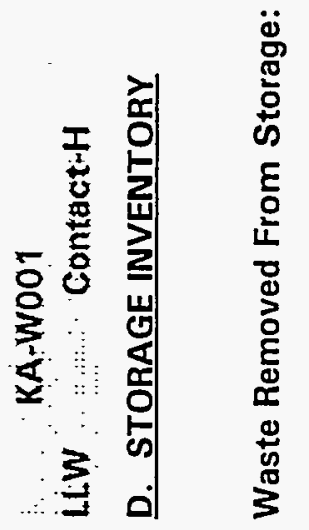


KA WOOO1

HlW Gontagth

E. RADIATION CHARACTERISTICS

Radioactive Waste Type:

\begin{tabular}{|ll|}
\hline OLLW & O a-LLW O TRU \\
O HLW & $\bigcirc_{\text {Non-Rad }}$ \\
\hline
\end{tabular}

Mixed Waste:

Handling:

OYes ONo

O Contact O Remote

Radionuclide Contamination Accessiblity:

\begin{tabular}{|c|c|c|c|}
\hline External Surface & OYes & ONo & OUnknown \\
\hline Internal Surface & OYes & ONo & OUnknown \\
\hline $\operatorname{rsed} \mathrm{T}$ & OYes & ONo & OUnknown \\
\hline
\end{tabular}

Activity Levels

Transuranic Alpha Activity:

Uranium/Thorium Alpha Activity:

Beta/Gamma Activity:

Surface Neutron Activity:

Total Activity:

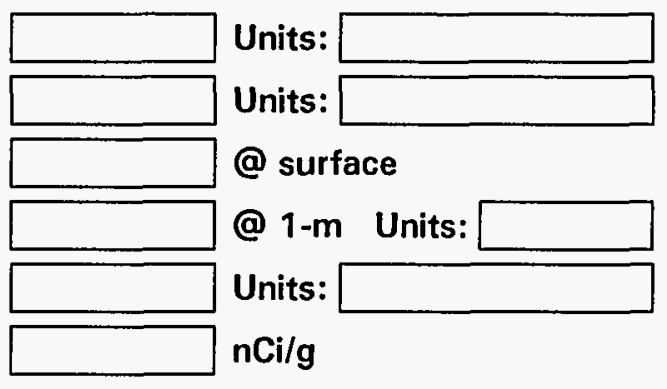

This waste stream is not identified as a TRU or a-LLW.

\section{F. TRU ALPHA ACTIVITY DISTRIBUTION}

Still-Gen U Active

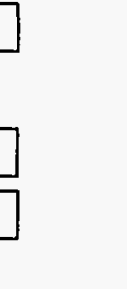



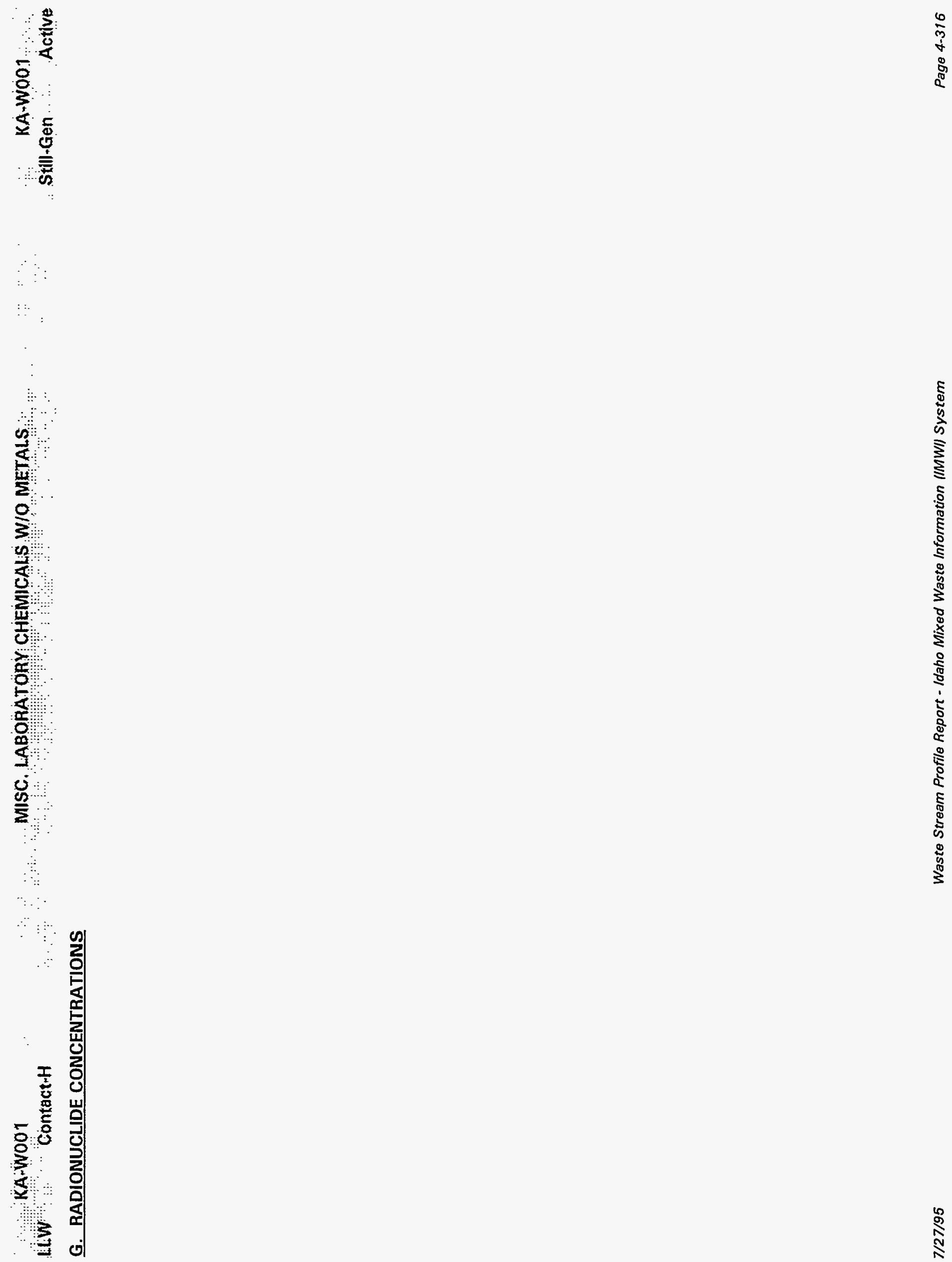


\section{H. MATRIX CHARACTERISTICS}

Primary Matrix Code: X6000

Description: Lab Packs: : :

Combustibility: $\bigcirc$ Combustible $(>90 \%)$

Mixed $(10 \%-90 \%)$

Noncombustible $(<10 \%)$

\section{MATRIX COMPOSITION}

\begin{tabular}{|c|c|c|c|c|c|c|}
\hline & & & \multicolumn{4}{|c|}{ Composittion } \\
\hline Code & Matrlx Description & Material & Typlcal & $\overline{L L}$ & $\overline{U L}$ & Units \\
\hline $\mathrm{x} 6000$ & Lab Packs... & Lab Packs & & & & \\
\hline 55313 & Debris Waste.Organic Debris.Plastic/Rubber Debris.Nonhalogenated Plastic Debris & Non-Halogenated Plastic Debris & & & & \\
\hline $\mathbf{5 5 3 1 2}$ & Debris Waste.Organic Debris.Plastic/Rubber Debris.Halogenated Plastic Debris & Halogenated Plastic Debris & & & & \\
\hline $\mathbf{5 5 1 2 2}$ & Debris Waste.Inorganic Debris.Inorganic Non-Metal Debris.Glass Debris & Glass Debris & & & & \\
\hline
\end{tabular}


UWW ContactrH

\section{J. HAZARDOUS CONSTITUENTS}

\begin{tabular}{|c|c|c|c|c|c|c|c|c|}
\hline & & & \multicolumn{4}{|c|}{ Concentration } & \multirow[b]{2}{*}{ Basis } & \multirow[b]{2}{*}{ Conc. Type } \\
\hline Code & Hazardous Waste Description & Treatment Subcategory & Typical & LL & $\mathbf{U L}$ & Units & & \\
\hline D001 & Ignitable & High-TOC ignitable characteristic liquids subca & & & & & & Total \\
\hline D002 & Corrosive & Corrosive characteristic waste that are manag & & & & & & Total \\
\hline D003 & Reactive & Other reactives based on $261.23(a)(1)$ & & & & & & Total \\
\hline 0018 & Benzene & Benzene managed in non-CWA/non-CWA-equi & & & & & & Total \\
\hline$D 035$ & Methyl ethyl ketone & Methyle ethyl ketone managed in non-CWA/no & & & & & & Total \\
\hline D036 & Nitrobenzene & Nitrobenzene managed in non-CWA/non-CWA- & & & & & & Total \\
\hline 0039 & Tetrachloroethylene & Tetrachloroethylene managed in non-CWA/non & & & & & & Total \\
\hline P015 & Beryllium powder & & & & & & & Total \\
\hline U151 & Mercury (High mercury $>260 \mathrm{mg} / \mathrm{kg}$ ) & & & & & & & Total \\
\hline 0159 & Methyl ethyl ketone & & & & & & & Total \\
\hline U220 & Toluene & & & & & & & Total \\
\hline U226 & 1,1,1-Trichloroethane & & & & & & & Total \\
\hline U228 & Trichloroethylene & & & & & & & Total \\
\hline
\end{tabular}




\section{K. POLYCHLORINATED BIPHENYLS (PCBS)}

\section{Is this waste stream PCB contaminated? $O$ Yes $O$ No $O$ Unknown}

If yes, what portion of the waste is PCB contaminated (volume \%):

If $<100 \%$, can the PCB contaminated waste be segregated and treated separately?

What is the PCB concentration in ppm?

If only a portion of the waste is PCB contaminated, report values for contaminated portion.

\section{TREATMENT PLANS}

\begin{tabular}{|c|c|c|c|c|c|c|c|c|c|c|c|}
\hline Opt. & $\begin{array}{c}\text { Media Type } \\
\text { (If more than one) }\end{array}$ & $\begin{array}{c}\text { Matrix } \\
\text { Code }\end{array}$ & $\begin{array}{l}\text { Vol. \% } \\
\text { IIf }\end{array}$ & Step & JIT & sc & CIF & $\begin{array}{l}\text { Trans. } \\
\text { Miles }\end{array}$ & $\begin{array}{l}\text { Facility } \\
\text { Abbr. }\end{array}$ & Unit Name & Comments \\
\hline 1 & & $\mathrm{X} 6000$ & & $\mathbf{a}$ & () & $\bar{O}$ & $1.00 E+00$ & & TRANS & Transport - LLW & \\
\hline & & & & b & O) & $\mathrm{O}$ & $1.00 E+00$ & & AMWTF & $\mathrm{CH}$ - Opening \& Sorting & \\
\hline & & & & $\bar{d}$ & 0 & $\mathrm{O}$ & $1.00 E+00$ & & AMWTF & $\mathrm{CH}$ - Incineration/Thermal Desorption & \\
\hline & & & & e & 0 & $\bar{O}$ & $1.00 E+00$ & & AMWTF & $\mathrm{CH}$ - Vitrification & \\
\hline & & & & $f$ & 0 & O & $1.00 E+00$ & & SCDF & Disposal - Contact Handled & \\
\hline
\end{tabular}

Note: Where provided, media percentages are rough estimates used to facilitate treatment planning. They do not necessarily imply an accurate knowledge of waste stream composition, and were not derived for use outside this conceptual planning exercise.

Describe any special or unique technical concerns related to the treatment of this waste stream that would impact the use or implementation of standard hazardous waste treatment methods. 


\section{$\therefore K A-W 015$}

LiW Contact-H

\section{A. IDENTIFICATION AND DESCRIPTION}

Waste Stream ID: KA-W015

W. S. Name:

SOILS

Content Code:

FFCA ID:

KA-W015

Waste Stream Description:

Waste soils from maintenance, D\&D, excavation, etc. This mixed waste stream has not yet been generated. This stream will not be a moratorium waste or reclassified TRU waste.

\section{sölls}

KA-WO15:

Still-Gen

\section{B. GENERATION SITE/PROCESS DESCRIPTION}

The following questions, concerning the generation site and process, should be answered even if the waste stream is no longer generated.

Name of Site Generating Waste (e.g., INEL, Rocky Flats, etc.): KAPL-Knolls

Location of activities (Area and Building):

Locations where contaminated soil may exist include areas adjacent to past and existing radiological facilities.

Operations (functions) performed in building:

Site characterization is in process.

Description of Process Generating Waste:

Excavation of soils. 
$\therefore$ KA.WÖ15

How ContactH

C. PROJECTED WASTE GENERATION

Is this waste stream still generated?

If yes, when is the expected generation termination date?

\section{OYes O No}

Current Projections

\begin{tabular}{|c|r|r|r|r|l|}
\hline Est. Date & \multicolumn{1}{|c|}{ start Year } & \multicolumn{1}{|c|}{ Interval } & \multicolumn{1}{c|}{ Vol (m3) } & Mass $(\mathbf{k g})$ & Source of Estimate \\
\hline $11 / 30 / 93$ & 1994 & 1 & 4.20 & $2,000.00$ & $1993-F F C A$ \\
\hline $11 / 30 / 93$ & 1995 & 1 & 4.20 & $2,000.00$ & $1993-F F C A$ \\
\hline $11 / 30 / 93$ & 1996 & 1 & 4.20 & $2,000.00$ & $1993-F F C A$ \\
\hline $11 / 30 / 93$ & 1997 & 1 & 4.20 & $2,000.00$ & $1993-F F C A$ \\
\hline $11 / 30 / 93$ & 1998 & 1 & 4.20 & $2,000.00$ & $1993-F F C A$ \\
\hline $11 / 30 / 93$ & 1999 & 1 & 4.20 & $2,000.00$ & $1993-F F C A$ \\
\hline
\end{tabular}

\begin{tabular}{|r|r|r|r|r|c|}
\hline Est. Date & Start Year & Interval & Vol (m) & Mass (kg) & Source of Estimate \\
\hline $11 / 30 / 93$ & 1998 & 5 & 16.80 & $8,000.00$ & $1993-F F C A$ \\
\hline $11 / 30 / 93$ & 2003 & 20 & 67.20 & $32,000.00$ & $1993-F F C A$ \\
\hline
\end{tabular}




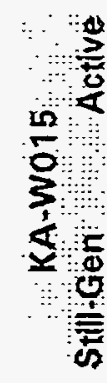

స్
+
จ

ह

$\frac{4}{8}$

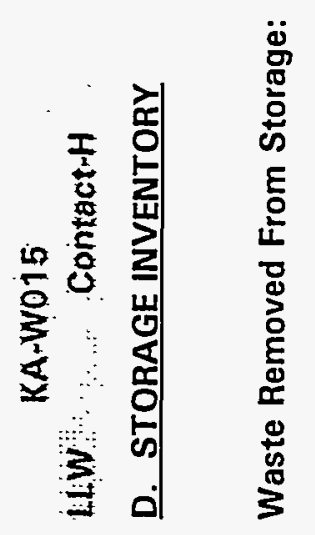




\section{F. TRU ALPHA ACTIVITY DISTRIBUTION}

Radioactive Waste Type:

\begin{tabular}{|ll|}
\hline O LLW & O a-LLW $\bigcirc$ TRU \\
OHLW & O Non-Rad \\
\hline
\end{tabular}

This waste stream is not identified as a TRU or a-LLW.

Mixed Waste:

\begin{tabular}{|l|}
\hline Yes $O$ No \\
\hline Contact \\
\hline Remote
\end{tabular}

Radionuclide Contamination Accessiblity:

\begin{tabular}{|c|c|c|}
\hline External Surface $O$ Yes & ONo & OUnknown \\
\hline Internal Surface $O$ Yes & ONo & OUnknown \\
\hline Dispersed Through Matrix $\mathrm{O}$ Yes & ONo & OUnknown \\
\hline \multicolumn{3}{|c|}{ Activity Levels } \\
\hline \multirow{3}{*}{$\begin{array}{r}\text { Transuranic Alpha Activity: } \\
\text { Uranium/Thorium Alpha Activity: } \\
\text { Beta/Gamma Activity: }\end{array}$} & & Units: \\
\hline & & Units: \\
\hline & & @ surface \\
\hline & & @ 1-m Units: $m R / h r$ \\
\hline \multicolumn{2}{|l|}{ Surface Neutron Activity: } & Units: \\
\hline Total Activity: & & $\mathrm{nCi} / \mathrm{g}$ \\
\hline
\end{tabular}



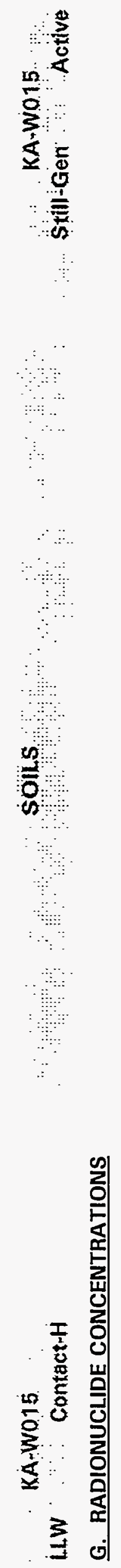
KA

\section{H. MATRIX CHARACTERISTICS}

Primary Matrix Code: $\mathbf{S 4 0 0 0}$

Description: Soil/Gravel: : :

Combustibility: $\bigcirc$ Combustible $(>90 \%)$

Mixed (10\%-90\%)

Noncombustible $(<10 \%)$

\section{MATRIX COMPOSITION}

\begin{tabular}{|c|c|c|c|c|c|c|}
\hline & & & \multicolumn{4}{|c|}{ Composition } \\
\hline Code & Matrix Description & Material & Typlcal & $\overline{L L}$ & UL & Units \\
\hline$\$ 4100$ & Soil/Gravel.Soil.. & Soils & & & & \\
\hline
\end{tabular}




\section{LLW Contact H \\ J. HAZARDOUS CONSTITUENTS}

Solits

KA-WÖ 15

$\therefore$

Still-Gen Active

\begin{tabular}{|c|c|c|c|c|c|c|c|c|}
\hline & & & \multicolumn{4}{|c|}{ Concentration } & \multirow[b]{2}{*}{ Basis } & \multirow[b]{2}{*}{ Conc. Type } \\
\hline Code & Hazardous Waste Description & Treatment Subcategory & Typical & $\mathbf{L L}$ & $\mathbf{U L}$ & Units & & \\
\hline D005 & Barium & & & & & & $\bar{A}$ & Total \\
\hline 0006 & Cadmium & & & & & & A & Total \\
\hline 0007 & Chromium & Chromium (Total) & & & & & A & Total \\
\hline$D 008$ & Lead & & & & & & A & Total \\
\hline DO09 & Mercury & Low mercury $<260 \mathrm{mg} / \mathrm{kg}$ total mercury & & & & & $\bar{A}$ & Total \\
\hline D011 & Silver & & & & & & A & Total \\
\hline D018 & Benzene & Benzene managed in non-CWA/non-CWA-equi & & & & & A & Total \\
\hline$\overline{D 035}$ & Methyl ethyl ketone & Methyle ethyl ketone managed in non-CWA/no & & & & & A & Total \\
\hline D039 & Tetrachloroethylene & Tetrachloroethylene managed in non-CWA/non & & & & & A & Total \\
\hline$\overline{D 040}$ & Trichloroethylene & Trichloroethylene managed in non-CWA/non-C & & & & & $\bar{A}$ & Total \\
\hline F001 & Spent halogenated solvents used in degreasing & Unknown & & & & & $\bar{A}$ & Total \\
\hline F002 & Spent halogenated solvents & Unknown & & & & & $\bar{A}$ & Total \\
\hline F003 & Spent non-halogenated solvents & Unknown & & & & & A & Total \\
\hline
\end{tabular}




\section{HLW: Contagth}

\section{K. POLYCHLORINATED BIPHENYLS (PCBS)}

\section{Is this waste stream PCB contaminated?}

OYes O No

O Unknown

If yes, what portion of the waste is PCB contaminated (volume \%):

If $<100 \%$, can the PCB contaminated waste be segregated and treated separately? OYes O No OUnknown

What is the PCB concentration in ppm?

If only a portion of the waste is PCB contaminated, report values for contaminated portion.

\section{TREATMENT PLANS}

\begin{tabular}{|c|c|c|c|c|c|c|c|c|c|c|c|}
\hline $\begin{array}{c}\text { Opt. } \\
\#\end{array}$ & $\begin{array}{c}\text { Media Type } \\
\text { (If more than one) }\end{array}$ & $\begin{array}{c}\text { Matrix } \\
\text { Code }\end{array}$ & $\begin{array}{l}\text { Vol. \% } \\
\text { lif }\end{array}$ & Step & JIT & sc & CIF & $\begin{array}{l}\text { Trans. } \\
\text { Miles }\end{array}$ & $\begin{array}{l}\text { Facility } \\
\text { Abbr. }\end{array}$ & Unit Name & Comments \\
\hline 1 & & $\$ 4000$ & & $\bar{a}$ & $\mathbf{0}$ & 0 & $1.00 \mathrm{E}+00$ & & TRANS & Transport - LLW & \\
\hline & & & & b & 0 & 0 & $1.00 E+00$ & & AMWTF & CH - Opening \& Sorting & \\
\hline & & & & c & ( & 0 & $1.00 \mathrm{E}+00$ & & AMWTF & $\mathrm{CH}$ - Sizing & \\
\hline & & & & d & 0 & 0 & $1.00 E+00$ & & AMWTF & CH - Incineration/Thermal Desorption & \\
\hline & & & & e & 0 & 0 & $1.00 E+00$ & & AMWTF & CH - Vitrification & \\
\hline & & & & $f$ & 0 & 0 & $1.00 E+00$ & & SCDF & Disposal - Contact Handled & \\
\hline
\end{tabular}

Note: Where provided, media percentages are rough estimates used to facilitate treatment planning. They do not necessarily imply an accurate knowledge of waste stream composition, and were not derived for use outside this conceptual planning exercise.

Describe any special or unique technical concerns related to the treatment of this waste stream that would impact the use or implementation of standard hazardous waste treatment methods. 


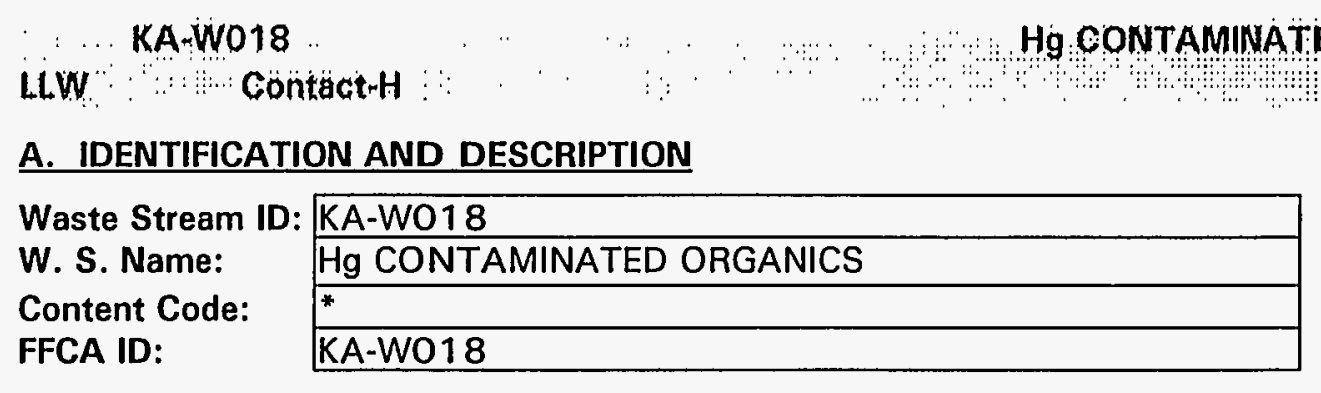

\section{Waste Stream Description:}

This waste stream consists of organic materials which have become contaminated with radioactivity and mercury greater than $260 \mathrm{ppm}$. This waste stream has not been generated. The homogeneity of this waste stream is unknown until generated. This waste stream will not be a moratorium or reclassified TRU waste.

\section{B. GENERATION SITE/PROCESS DESCRIPTION}

The following questions, concerning the generation site and process, should be answered even if the waste stream is no longer generated.

\section{Name of Site Generating Waste (e.g., INEL, Rocky Flats, etc.):} KAPL-Knolls

Location of activities (Area and Building):

Various locations on site.

Operations (functions) performed in building:

Various operations on site.

Description of Process Generating Waste:

Various laboratory activities (e.g., analysis, testing, maintenance). 
Is this waste stream still generated?

OYes ONo

If yes, when is the expected generation termination date?

Current Projections

\begin{tabular}{|c|r|r|r|r|l|}
\hline Est. Date & Start Year & Interval & \multicolumn{1}{|c|}{ Vol (m3) } & Mass $(\mathrm{kg})$ & Source of Estimate \\
\hline $11 / 15 / 94$ & 1997 & 1 & 1.00 & & $1994-D S T P$ \\
\hline
\end{tabular}

Historical Projections 


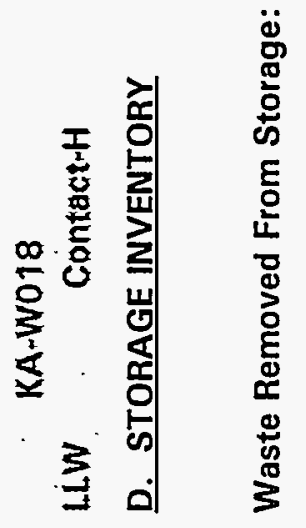


 \\ Radioactive Waste Type:

\begin{tabular}{|ll|}
\hline OllW & Oa-LlW O TRU \\
OhLW & O Non-Rad \\
\hline
\end{tabular} \\ F. TRU ALPHA ACTIVITY DISTRIBUTION \\ Mixed Waste: \\ Handling:

\begin{tabular}{|l|}
\hline O Yes O No \\
\hline O Contact O Remote \\
\hline
\end{tabular} \\ This waste stream is not identified as a TRU or a-LLW.}

Radionuclide Contamination Accessiblity:

External Surface $O$ Yes $O$ No Unknown

Internal Surface $O$ Yes $O$ No O Unknown

Dispersed Through Matrix

OYes Ono OUnknown

\section{Activity Levels}

Transuranic Alpha Activity:

Uranium/Thorium Alpha Activity:

Beta/Gamma Activity:

Surface Neutron Activity:

Total Activity:

\begin{tabular}{|c|c|c|}
\hline & Units: [ & \\
\hline & Units: [ & \\
\hline & @ surf & ace \\
\hline & @ 1-m & Units: $\mathrm{mR} / \mathrm{hr}$ \\
\hline & Units: & \\
\hline & $\mathrm{nCi} / \mathrm{g}$ & \\
\hline
\end{tabular}




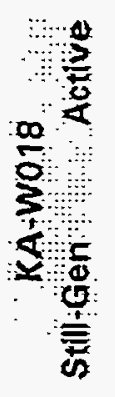

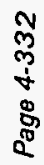

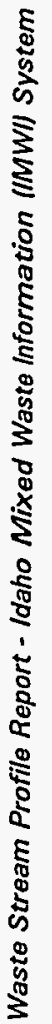

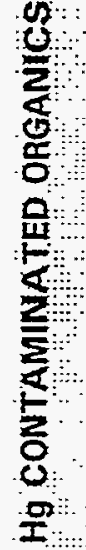

$\therefore$

$\infty$

50

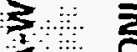

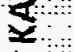

新 


\begin{tabular}{|c|c|c|c|c|c|c|}
\hline 10^\% & 001 & 001 & 001 & & 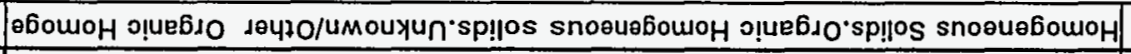 & $06 z \varepsilon s$ \\
\hline syun & 7n & רך & |EJ|dK & jецәреш & 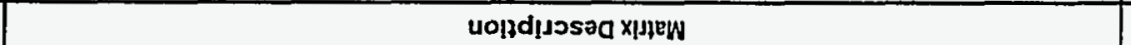 & әроO \\
\hline
\end{tabular}

\section{NOILISOdWOJ XIप्पषWW}

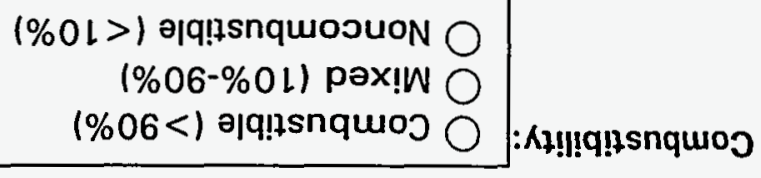

in 


\section{KA-W018}

LWW :ContactrH

\section{J. HAZARDOUS CONSTITUENTS}

\begin{tabular}{|c|c|c|c|c|c|c|c|c|}
\hline & & & \multicolumn{4}{|c|}{ Concentration } & \multirow[b]{2}{*}{ Basis } & \multirow[b]{2}{*}{ Conc. Type } \\
\hline Code & Hazardous Waste Description & Treatment Subcategory & Typical & LL & UL & Units & & \\
\hline D004 & Arsenic & & & & & & $\bar{A}$ & Total \\
\hline D005 & Barium & & & & & & $\bar{A}$ & Total \\
\hline D006 & Cadmium & & & & & & A & Total \\
\hline D007 & Chromium & Chromium (Total) & & & & & A & Total \\
\hline$D 008$ & Lead & & & & & & A & Total \\
\hline D009 & Mercury & Elemental mercury contaminated with radioact & & & & & A & Total \\
\hline D010 & Selenium & & & & & & $A$ & Total \\
\hline$D 011$ & Silver & & & & & & A & Total \\
\hline$D 018$ & Benzene & Benzene managed in non-CWA/non-CWA-equi & & & & & $\bar{A}$ & Total \\
\hline$D 035$ & Methyl ethyl ketone & Methyle ethyl ketone managed in non-CWA/no & & & & & $A$ & Total \\
\hline$D 036$ & Nitrobenzene & Nitrobenzene managed in non-CWA/non-CWA- & & & & & $A$ & Total \\
\hline D039 & Tetrachloroethylene & Tetrachloroethylene managed in non-CWA/non & & & & & $A$ & Total \\
\hline DO40 & Trichloroethylene & Trichloroethylene managed in non-CWA/non-C & & & & & A & Total \\
\hline
\end{tabular}




\section{K. POLYCHLORINATED BIPHENYLS (PCBS)}

\section{Is this waste stream PCB contaminated? OYes $O$ No $O$ Unknown}

If yes, what portion of the waste is PCB contaminated (volume \%):

If $<100 \%$, can the PCB contaminated waste be segregated and treated separately? OYes ONo OUnknown

What is the PCB concentration in ppm? If only a portion of the waste is PCB contaminated, report values for contaminated portion.

\section{TREATMENT PLANS}

\begin{tabular}{|c|c|c|c|c|c|c|c|c|c|c|c|}
\hline Opt. & $\begin{array}{c}\text { Media Type } \\
\text { (If more than one) }\end{array}$ & $\begin{array}{c}\text { Matrix } \\
\text { Code }\end{array}$ & $\begin{array}{l}\text { Vol. \% } \\
\text { Iff }\end{array}$ & Step & JIT & sc & CIF & $\begin{array}{l}\text { Trans. } \\
\text { Miles }\end{array}$ & $\begin{array}{l}\text { Facility } \\
\text { Abbr. }\end{array}$ & Unit Name & Comments \\
\hline 1 & & S5440 & & $\bar{a}$ & (O) & $\mathrm{O}$ & $1.00 E+00$ & & TRANS & Transport - LLW & \\
\hline & & & & b & $\mathrm{O}$ & $\mathrm{O}$ & $1.00 E+00$ & & WROC & Sizing & \\
\hline & & & & c & O & 0 & $1.00 E+00$ & & WROC & Hg Retort & \\
\hline & & & & d & $\overline{0}$ & 0 & $1.00 E+00$ & & WERF & Incineration - Controlled Air Incinerator & \\
\hline & & & & $\theta$ & $\mathrm{O}$ & 0 & $1.00 E+00$ & & WERF & Stabilization - Portland Cement & \\
\hline & & & & $\mathbf{f}$ & 0 & $\mathrm{O}$ & $1.00 E+00$ & & SCDF & Disposal - Contact Handled & \\
\hline
\end{tabular}

Note: Where provided, media percentages are rough estimates used to facilitate treatment planning. They do not necessarily imply an accurate knowledge of waste stream composition, and were not derived for use outside this conceptual planning exercise.

Describe any special or unique technical concerns related to the treatment of this waste stream that would impact the use or implementation of standard hazardous waste treatment methods. 


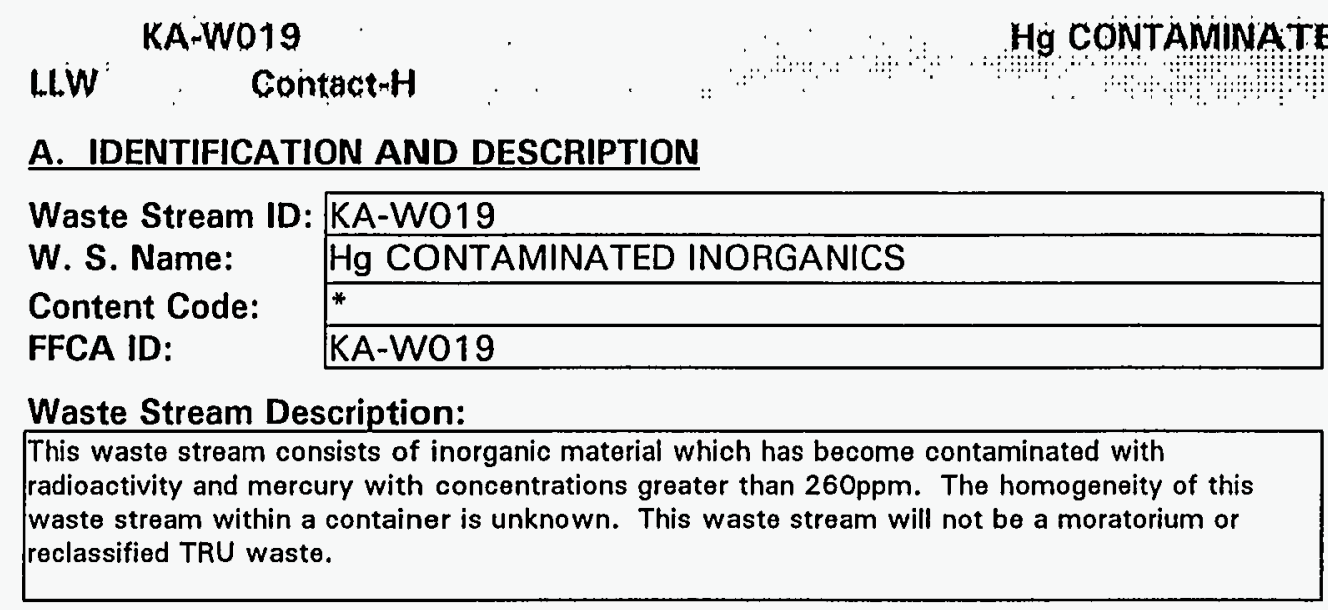

\section{B. GENERATION SITE/PROCESS DESCRIPTION}

The following questions, concerning the generation site and process, should be answered even if the waste stream is no longer generated.

Name of Site Generating Waste (e.g., INEL, Rocky Flats, etc.): KAPL-Knolls

Location of activities (Area and Building):

Various locations on site.

Operations (functions) performed in building:

Various operations on site.

Description of Process Generating Waste:

Various laboratory activities (e.g., analysis, testing, maintenance). 
KA WO19

Hg CONTAMINATED INORGANICS

LLW Contact

C. PROJECTED WASTE GENERATION

Is this waste stream still generated?

OYes ONo

If yes, when is the expected generation termination date?

Current Projections

\begin{tabular}{|c|r|r|r|r|r|}
\hline Est. Date & Start Year & Interval & \multicolumn{1}{|c|}{ Vol (m3) } & Mass $(\mathrm{kg})$ & Source of Estimate \\
\hline $11 / 15 / 94$ & 1997 & 1 & 0.30 & 240.00 & $1994-D S T P$ \\
\hline
\end{tabular}

Historical Projections 


\section{STORAGE INVENTORY}

\begin{tabular}{|l|l|l|l|r|l|r|r|r|r|}
\hline Container ID & Stor. Loc. & \# of Cont. & Type & Total Vol. & Units & Weight (lbs) & G. Fac. & Date Rec. & Waste Description \\
\hline & & & & 0.1 & CM & 176 & & & \\
\hline
\end{tabular}

Waste Removed From Storage: 


\section{KAWO19}

LW W Contacth

Hg CONTAMINATED INORGANICS

\section{E. RADIATION CHARACTERISTICS}

\section{F. TRU ALPHA ACTIVITY DISTRIBUTION}

Radioactive Waste Type:

\begin{tabular}{ll} 
OllW & Oa-llW O TRU \\
OhLW & Onon-Rad \\
\hline
\end{tabular}

This waste stream is not identified as a TRU or a-LLW.

Mixed Waste:

\begin{tabular}{|l|}
\hline Y Yes ONo \\
\hline O Contact O Remote
\end{tabular}

Radionuclide Contamination Accessiblity:

\begin{tabular}{|c|c|c|c|}
\hline External Surface & OYes & ONo & O) Unknown \\
\hline Internal Surface & OYes & ONo & () Unknown \\
\hline Dispersed Through Matrix & OYes & ONo & (O) Unknown \\
\hline
\end{tabular}

\section{Activity Levels}

Transuranic Alpha Activity:

Uranium/Thorium Alpha Activity: Beta/Gamma Activity:

Surface Neutron Activity:

$\square$
Units: Units: @ surface @ 1-m Units: $\mathrm{mR} / \mathrm{hr}$

Total Activity: $\mathrm{nCi} / \mathrm{g}$ 


\section{$\because$ KÄWO19}

Hg CONTAMINATED NORGANIOS

KA-WO19 9

HLW ContáctiH

U : StilloGen lo Active

H. MATRIX CHARACTERISTICS

Primary Matrix Code: $\$ \mathbf{S 3 1 0 0}$

Description: Homogeneous Solids: Inorganic Homogeneous Solids: :

Combustibility: $\begin{aligned} & \bigcirc \text { Combustible }(>90 \%) \\ & \bigcirc \text { Mixed }(10 \%-90 \%) \\ & \bigcirc \text { Noncombustible }(<10 \%)\end{aligned}$

\section{MATRIX COMPOSITION}

\begin{tabular}{|c|c|c|c|c|c|c|}
\hline & & & \multicolumn{4}{|c|}{ Composition } \\
\hline Code & Matrix Description & Material & Typical & LL & UL & Units \\
\hline$\$ 3190$ & Homogeneous Solids.Inorganic Homogeneous Solids. Unknown/Other Inorganic Homo & & 100 & 100 & 100 & $\%$ vol \\
\hline
\end{tabular}




\section{KA-W019}

Liw $\quad$ Contact-H

Hg CONTAMINATED INORGANICS

CONTAMINATEO NORGANICS

KA+WO19 Still-Gen Active

\section{J. HAZARDOUS CONSTITUENTS}

\begin{tabular}{|c|c|c|c|c|c|c|c|c|}
\hline & 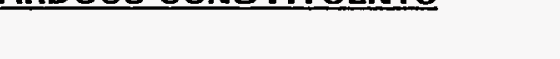 & & & & & & & \\
\hline Code & Hazardous Waste Description & Treatment Subcategory & Typical & $\mathbf{L L}$ & $\overline{\mathrm{UL}}$ & Units & Basis & Conc. Type \\
\hline$\longdiv { \mathrm { DO04 } }$ & Arsenic & & & & & & A & Total \\
\hline$\overline{D 005}$ & Barium & & & & & & A & Total \\
\hline$\overline{D 006}$ & Cadmium & & & & & & A & Total \\
\hline$\overline{D 007}$ & Chromium & Chromium (Total) & & & & & A & Total \\
\hline$\overline{D 008}$ & Lead & & & & & & A & Total \\
\hline D009 & Mercury & Elemental mercury contaminated with radioact & & & & & A & Total \\
\hline$\overline{D 010}$ & Selenium & & & & & & A & Total \\
\hline$\overline{D 011}$ & Silver & & & & & & A & Total \\
\hline
\end{tabular}




\section{KA-ẄO19}

\section{Hg CONTAMINATED INORGANICS}

LWW Contät H

\section{K. POLYCHLORINATED BIPHENYLS (PCBS)}

Is this waste stream PCB contaminated?

\section{OYes}

O No $O$ Unknown

If yes, what portion of the waste is PCB contaminated (volume \%):

If $<100 \%$, can the PCB contaminated waste be segregated and treated separately?

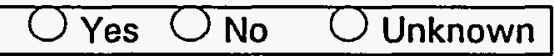

What is the PCB concentration in ppm?

If only a portion of the waste is PCB contaminated, report values for contaminated portion.

\section{TREATMENT PLANS}

\begin{tabular}{|c|c|c|c|c|c|c|c|c|c|c|c|}
\hline Opt. & $\begin{array}{c}\text { Media Type } \\
\text { (If more than one) }\end{array}$ & $\begin{array}{c}\text { Matrix } \\
\text { Code }\end{array}$ & $\begin{array}{l}\text { Vol. \% } \\
\text { IIf }\end{array}$ & Step & JIT & sc & CIF & $\begin{array}{l}\text { Trans. } \\
\text { Miles }\end{array}$ & $\begin{array}{l}\text { Facility } \\
\text { Abbr. }\end{array}$ & Unit Name & Comments \\
\hline 1 & & 55420 & & a & () & 0 & $1.00 E+00$ & & TRANS & Transport - LLW & \\
\hline & & & & b & () & 0 & $1.00 E+00$ & & WROC & Sizing & \\
\hline & & & & c & $\mathrm{O}$ & 0 & $1.00 \mathrm{E}+00$ & & WROC & Hg Retort & \\
\hline & & & & d & $\mathrm{O}$ & 0 & $1.00 E+00$ & & WERF & Stabilization - Portland Cement & \\
\hline & & & & e & $\mathrm{O}$ & 0 & $1.00 \mathrm{E}+00$ & & SCDF & Disposal - Contact Handled & \\
\hline
\end{tabular}

Note: Where provided, media percentages are rough estimates used to facilitate treatment planning. They do not necessarily imply an accurate knowledge of waste stream composition, and were not derived for use outside this conceptual planning exercise.

Describe any special or unique technical concerns related to the treatment of this waste stream that would impact the use or implementation of standard hazardous waste treatment methods. 


\section{KA-WO2O \\ LLW...: Contact-H}

A. IDENTIFICATION AND DESCRIPTION

Waste Stream ID: KA-W020

W. S. Name:

Content Code:

FFCA ID:

\begin{tabular}{|l|}
\hline ELEMENTAL Hg \\
\hline$*$ \\
\hline KA-W020 \\
\hline
\end{tabular}

\section{Waste Stream Description:}

This waste stream consists of mercury in its elemental (liquid) form contaminated with radioactivity. This waste will be homogeneous within each individual container. This waste stream will not be a moratorium or reclassified TRU waste.

\section{ELEMENTAL Hg}

\section{KA WW20}

Still-Gen Active

\section{B. GENERATION SITE/PROCESS DESCRIPTION}

The following questions, concerning the generation site and process, should be answered even if the waste stream is no longer generated.

Name of Site Generating Waste (e.g., INEL, Rocky Flats, etc.): KAPL-Knolls

Location of activities (Area and Building):

Various locations on site.

Operations (functions) performed in building:

Various operations on site.

Description of Process Generating Waste:

Waste from maintenance or testing. 
UWA-WO2O

C. PROJECTED WASTE GENERATION

Is this waste stream still generated?

OYes ONo

If yes, when is the expected generation termination date?

Current Projections

\begin{tabular}{|c|r|r|r|r|r|}
\hline Est. Date & Start Year & Interval & Vol (m3) & Mass (kg) & Source of Estlmate \\
\hline $11 / 15 / 94$ & 1997 & 1 & 0.08 & 800.00 & 1994 -DSTP \\
\hline
\end{tabular}

Historical Projections 


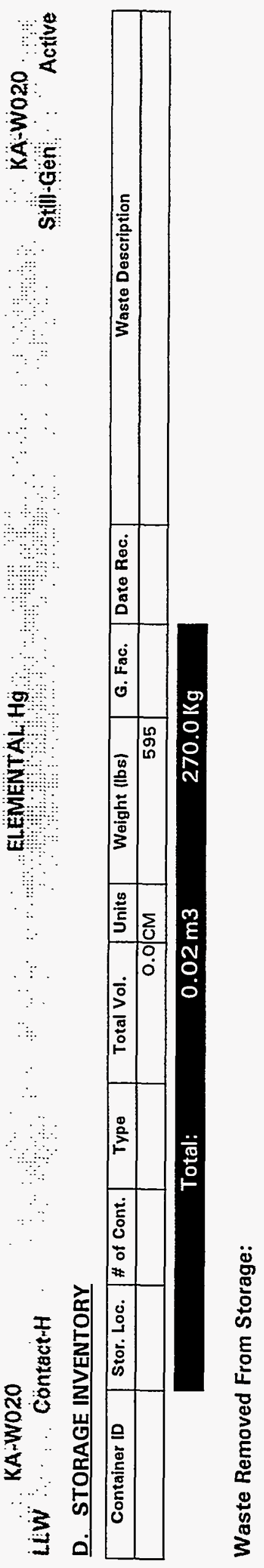

\begin{tabular}{l}
0 \\
8 \\
\multirow{2}{*}{} \\
8 \\
8 \\
0
\end{tabular}



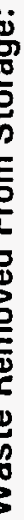




\section{E. RADIATION CHARACTERISTICS}

Radioactive Waste Type:

\begin{tabular}{ll} 
Ollw & Oa-LlW O tRU \\
OhlW & Onon-Rad \\
\hline
\end{tabular}

Mixed Waste:

Handling:

\begin{tabular}{l} 
O Yes O No \\
\hline Contact O Remote
\end{tabular}

Radionuclide Contamination Accessiblity:

\begin{tabular}{rlrl|} 
External Surface & OYes & ONo & O Unknown \\
Internal Surface & OYes & Ono & O Unknown \\
\cline { 2 - 5 } Dispersed Through Matrix & OYes & ONo & O Unknown
\end{tabular}

\section{Activity Levels}

Transuranic Alpha Activity: Uranium/Thorium Alpha Activity:

Beta/Gamma Activity:

Surface Neutron Activity: Total Activity:

$\square$
$\square$
$\square$
$\square$
$\square$
$\square$
Units: Units: @ surface @ 1-m Units: $\mathrm{mR} / \mathrm{hr}$ Units: $\mathrm{nCi} / \mathrm{g}$

\section{F. TRU ALPHA ACTIVITY DISTRIBUTION}

This waste stream is not identified as a TRU or a-LLW. 

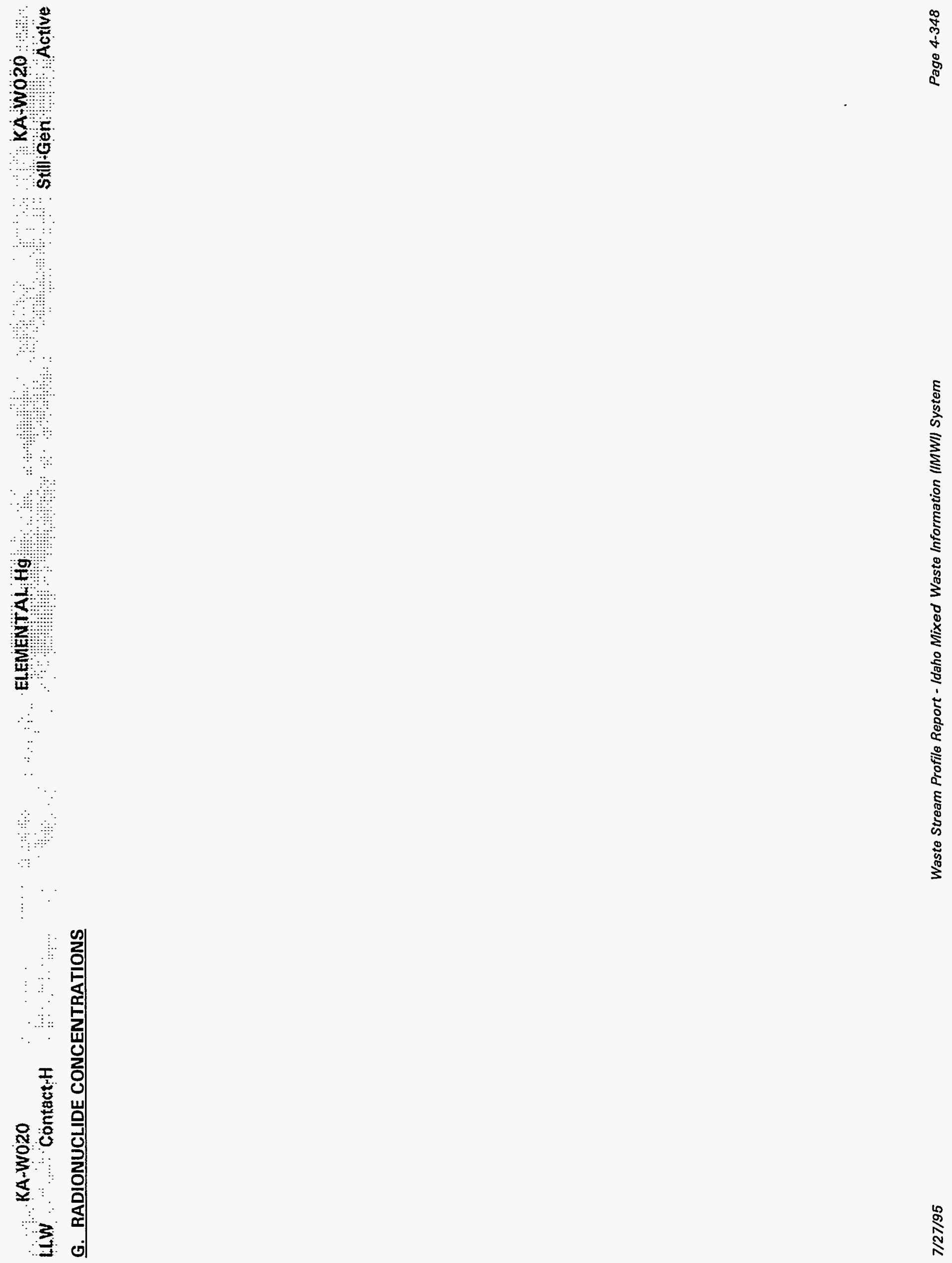

疍 


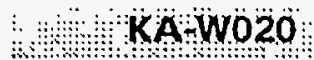

H. MATRIX CHARACTERISTICS

Primary Matrix Code: X7100

Description: Special Waste: Elemental Mercury: :

\section{Combustibility}

$$
\begin{aligned}
& \text { Combustible }(>90 \%) \\
& \text { Mixed }(10 \%-90 \%) \\
& \text { Noncombustible }(<10 \%)
\end{aligned}
$$

\begin{tabular}{|c|c|c|c|c|c|c|}
\hline & & & \multicolumn{4}{|c|}{ Composition } \\
\hline Code & Matrix Descriptlon & Material & Typlcal & LL & UL. & Units \\
\hline$X 7100$ & Special Waste.Elemental Mercury.. & & 100 & 100 & 100 & $\%$ vol \\
\hline
\end{tabular}

\section{MATRIX COMPOSITION}




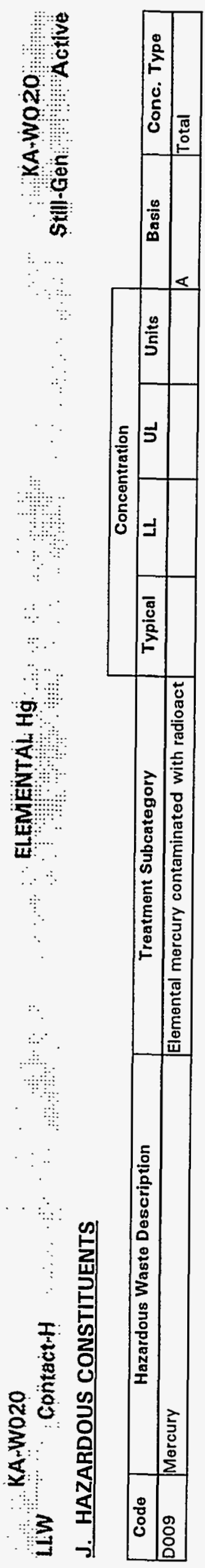

8
3
5
8
8
0

ह5 


\section{K. POLYCHLORINATED BIPHENYLS (PCBS)}

Is this waste stream PCB contaminated?

OYes

O No

Unknown

If yes, what portion of the waste is PCB contaminated (volume \%):

If $<100 \%$, can the PCB contaminated waste be segregated and treated separately?

What is the PCB concentration in ppm?

If only a portion of the waste is $P C B$ contaminated, report values for contaminated portion.

\section{TREATMENT PLANS}

\begin{tabular}{|c|c|c|c|c|c|c|c|c|c|c|c|}
\hline $\begin{array}{c}\text { Opt. } \\
\#\end{array}$ & $\begin{array}{l}\text { Media Type } \\
\text { (If more than onel }\end{array}$ & $\begin{array}{c}\text { Matrix } \\
\text { Code }\end{array}$ & $\begin{array}{l}\text { Vol. \% } \\
\text { (If }\end{array}$ & Step & JIT & sc & CIF & $\begin{array}{l}\text { Trans. } \\
\text { Miles }\end{array}$ & $\begin{array}{l}\text { Facility } \\
\text { Abbr. }\end{array}$ & Unit Name & Comments \\
\hline \multirow[t]{4}{*}{1} & & $\times 7100$ & & $\mathbf{a}$ & 0 & 0 & $1.00 E+00$ & & TRANS & Transport - LLW & \\
\hline & & & & b & $\mathbf{O}$ & 0 & $1.00 \mathrm{E}+00$ & & AMWTF & $\mathrm{CH}$ - Opening \& Sorting & \\
\hline & & & & c & 0 & 0 & $1.00 \mathrm{E}+00$ & & AMWTF & $\mathrm{CH}$ - Amalgamation & \\
\hline & & & & d & 0 & 0 & $1.00 E+00$ & & SCDF & Disposal - Contact Handled & \\
\hline
\end{tabular}

Note: Where provided, media percentages are rough estimates used to facilitate treatment planning. They do not necessarily imply an accurate knowledge of waste stream composition, and were not derived for use outside this conceptual planning exercise.

Describe any special or unique technical concerns related to the treatment of this waste stream that would impact the use or implementation of standard hazardous waste treatment methods. 


\section{KÄWO21}

LWW Contact-H

PCB CONTAMINATED WASTE

KA-WÖ21

Still-Gèn

\section{A. IDENTIFICATION AND DESCRIPTION}

Waste Stream ID: KA-W021

W. S. Name:

PCB CONTAMINATED WASTE

Content Code:

FFCA ID:

KA-W021

\section{Waste Stream Description:}

This waste stream will consist of waste contaminated with PCB's in concentrations greater than $50 \mathrm{ppm}$. This waste has not been generated. The homogeneity is unknown. This waste stream will not be a moratorium of reclassified TRU waste.

Description of Process Generating Waste:

Waste may be generated through various process. (e.g., maintenance, clean-up).

\section{B. GENERATION SITE/PROCESS DESCRIPTION}

The following questions, concerning the generation site and process, should be answered even if the waste stream is no longer generated.

Name of Site Generating Waste (e.g.. INEL, Rocky Flats, etc.): KAPL-Knolls

Location of activities (Area and Building):

Various locations on site.

Operations (functions) performed in building:

Various operations on site. 
KA WOZ21

PCB CONTAMINATED WASTE

C. PROJECTED WASTE GENERATION

Is this waste stream still generated?

OYes ONo

If yes, when is the expected generation termination date?

Current Projections

\begin{tabular}{|r|r|r|r|r|r|}
\hline Est. Date & Start Year & Interval & Vol (m3) & Mass (kg) & Source of Estimate \\
\hline $111 / 15 / 94$ & 1997 & 1 & 0.15 & 175.00 & 1994 DSTP \\
\hline
\end{tabular}

Historical Projections 

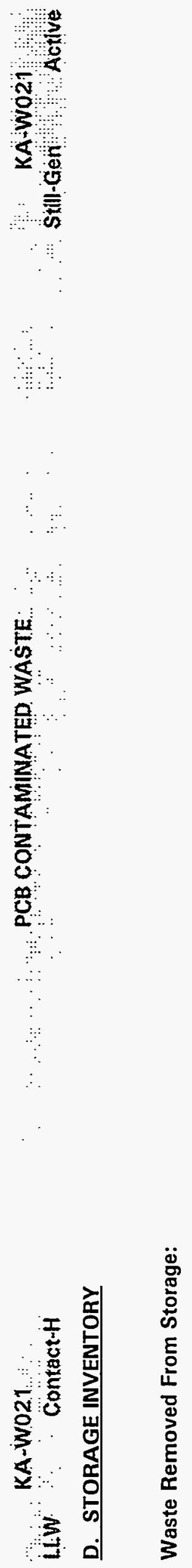


\section{KA-WO21}

PCB CONTAMINATED WASTE

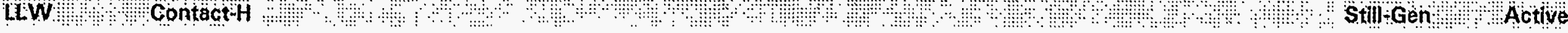

\section{E. RADIATION CHARACTERISTICS}

Radioactive Waste Type:

Mixed Waste:

Handling:

\begin{tabular}{|ll|}
\hline OLW & Oa-LLW $\bigcirc$ TRU \\
OHLW & O Non-Rad \\
\hline
\end{tabular}

Radionuclide Contamination Accessiblity:

OYes O No
O Contact
Remote

External Surface

Internal Surface

Dispersed Through Matrix

\begin{tabular}{|c|c|c|}
\hline OYes & ONo & (O) Unknown \\
\hline OYes & ONo & O Unknown \\
\hline OYes & ONo & () Unknown \\
\hline
\end{tabular}

Activity Levels

Transuranic Alpha Activity:

Uranium/Thorium Alpha Activity:

Beta/Gamma Activity:

Surface Neutron Activity:

Total Activity:

\begin{tabular}{|c|c|c|}
\hline & Units: & \\
\hline & Units: & \\
\hline & @ surfe & \\
\hline & @ 1-m & Units: $\mathrm{mR} / \mathrm{hr}$ \\
\hline & Units: [ & \\
\hline & $\mathrm{nCi} / \mathrm{g}$ & \\
\hline
\end{tabular}

This waste stream is not identified as a TRU or a-LLW. 

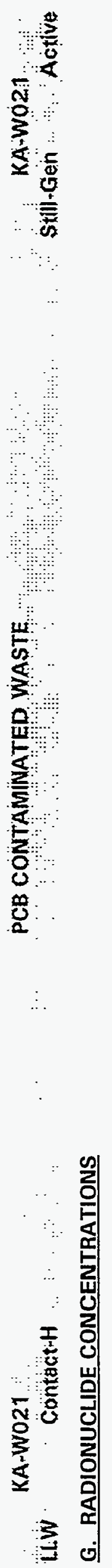
KAWO21

H. MATRIX CHARACTERISTICS

Primary Matrix Code: $\mathbf{S 3 0 0 0}$

Description: Homogeneous Solids: : :

Combustibility: \begin{tabular}{l|}
$\bigcirc$ Combustible $(>90 \%)$ \\
$\bigcirc$ Mixed $(10 \%-90 \%)$ \\
$\bigcirc$ Noncombustible $(<10 \%)$ \\
\hline
\end{tabular}

\section{MATRIX COMPOSITION}

\begin{tabular}{|c|c|c|c|c|c|c|}
\hline & & & \multicolumn{4}{|c|}{ Composition } \\
\hline Code & Matrix Description & Material & Typical & $\mathrm{LL}$ & UL & Units \\
\hline 53000 & Homogeneous Solids... & & 100 & 100 & 100 & $\%$ vol \\
\hline
\end{tabular}




\section{KA-W021 \\ LLW: : Contact-H. \\ J. HAZARDOUS CONSTITUENTS}

PCB CONTAMINATED WASTE

KA-W0211:

U

:

Still-Gen: : Active

\begin{tabular}{|c|c|c|c|c|c|c|c|c|}
\hline \multirow[b]{2}{*}{ Code } & \multirow[b]{2}{*}{ Hazardous Waste Description } & \multirow[b]{2}{*}{ Treatment Subcategory } & \multicolumn{4}{|c|}{ Concentration } & \multirow[b]{2}{*}{ Basis } & \multirow[b]{2}{*}{ Conc. Type } \\
\hline & & & Typical & LL. & $\mathbf{U L}$ & Units & & \\
\hline D004 & Arsenic & & & & & & A & Total \\
\hline 0005 & Barium & & & & & & A & Total \\
\hline D006 & Cadmium & & & & & & A & Total \\
\hline$\overline{D 007}$ & Chromium & Chromium (Total) & & & & & A & Total \\
\hline D008 & Lead & & & & & & $A$ & Total \\
\hline D009 & Mercury & Low mercury $<260 \mathrm{mg} / \mathrm{kg}$ total mercury & & & & & A & Total \\
\hline$D 010$ & Selenium & & & & & & A & Total \\
\hline D011 & Silver & & & & & & $\bar{A}$ & Total \\
\hline D018 & Benzene & Benzene managed in non-CWA/non-CWA-equi & & & & & A & Total \\
\hline D035 & Methyl ethyl ketone & Methyle ethyl ketone managed in non-CWA/no & & & & & $\bar{A}$ & Total \\
\hline$D 036$ & Nitrobenzene & Nitrobenzene managed in non-CWA/non-CWA- & & & & & $\bar{A}$ & Total \\
\hline D039 & Tetrachloroethylene & Tetrachloroethylene managed in non-CWA/non & & & & & A & Total \\
\hline$D 040$ & Trichloroethylene & Trichloroethylene managed in non-CWA/non-C & & & & & A & Total \\
\hline
\end{tabular}


WA KA WO21

LiW Contacth

\section{K. POLYCHLORINATED BIPHENYLS (PCBS)}

Is this waste stream PCB contaminated?

If yes, what portion of the waste is PCB contaminated (volume \%):

If $<100 \%$, can the PCB contaminated waste be segregated and treated separately?

What is the PCB concentration in ppm? nknown

PCB CONTAMINATED WASTE

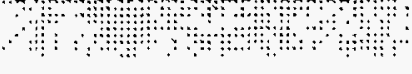

\section{TREATMENT PLANS}

\begin{tabular}{|c|c|c|c|c|c|c|c|c|c|c|c|}
\hline $\begin{array}{c}\text { Opt. } \\
\#\end{array}$ & $\begin{array}{c}\text { Media Type } \\
\text { (If more than one) }\end{array}$ & $\begin{array}{l}\text { Matrix } \\
\text { Code }\end{array}$ & $\begin{array}{l}\text { Vol. \% } \\
\text { IIf }\end{array}$ & Step & JIT & sc & CIF & $\begin{array}{l}\text { Trans. } \\
\text { Miles }\end{array}$ & $\begin{array}{l}\text { Facility } \\
\text { Abbr. }\end{array}$ & Unit Name & Comments \\
\hline 1 & & 53000 & & $a$ & O & 0 & $1.00 E+00$ & & TRANS & Transport - LLW & \\
\hline & & & & b & $\mathbf{O}$ & $O$ & $1.00 E+00$ & & AMWTF & $\mathrm{CH}$ - Opening \& Sorting & \\
\hline & & & & d & 0 & $O$ & $1.00 E+00$ & & AMWTF & $\mathrm{CH}$ - Incineration/Thermal Desorption & \\
\hline & & & & e & 0 & $\mathrm{O}$ & $1.00 \mathrm{E}+00$ & & AMWTF & $\mathrm{CH}$ - Vitrification & \\
\hline & & & & $f$ & 0 & 0 & $1.00 E+00$ & & SCDF & Disposal - Contact Handled & \\
\hline
\end{tabular}

Note: Where provided, media percentages are rough estimates used to facilitate treatment planning. They do not necessarily imply an accurate knowledge of waste stream composition, and were not derived for use outside this conceptual planning exercise.

Describe any special or unique technical concerns related to the treatment of this waste stream that would impact the use or implementation of standard hazardous waste treatment methods. 


\section{KK-WO13 \\ LLW ContactrH}

\section{A. IDENTIFICATION AND DESCRIPTION}

Waste Stream ID: KK-W013

W. S. Name:

Content Code:

FFCA ID:

$$
\text { SOILS }
$$

SOIL

$$
\text { KK-W013 }
$$

\section{Waste Stream Description:}

Waste soils from maintenance, D\&D, etc. This mixed waste stream has not yet been generated. This stream will not be a moratorium waste or reclassified TRU waste.

\section{B. GENERATION SITE/PROCESS DESCRIPTION}

The following questions, concerning the generation site and process, should be answered even if the waste stream is no longer generated.

Name of Site Generating Waste (e.g.. INEL, Rocky Flats, etc.): KAPL-Kesselring Site

Location of activities (Area and Building):

Locations where contaminated soil may exist include areas adjacent to past and existing radiological facilities.

Operations (functions) performed in building:

Site characterization is in process.

Description of Process Generating Waste:

Excavation of soils. 


\section{KK-WO13}

HWW Contact H

\section{PROJECTED WASTE GENERATION}

Is this waste stream still generated?

If yes, when is the expected generation termination date?

Current Projections

\begin{tabular}{|r|r|r|r|r|r|}
\hline Est. Date & Start Year & Interval & \multicolumn{1}{|c|}{ Vol $(\mathbf{m} 3)$} & Mass $(\mathrm{kg})$ & Source of Estimate \\
\hline $11 / 15 / 94$ & 1997 & 1 & 7.50 & & 1994-DSTP \\
\hline $11 / 30 / 93$ & 1998 & 1 & 4.20 & $2,000.00$ & 1993 -FFCA \\
\hline $11 / 30 / 93$ & 1999 & 1 & 4.20 & $2,000.00$ & $1993-$ FFCA \\
\hline
\end{tabular}

\section{Historical Projections}

\begin{tabular}{|r|r|r|r|r|l|}
\hline Est. Date & \multicolumn{1}{|c|}{ Start Year } & Interval & \multicolumn{1}{|c|}{ Vol (m3) } & \multicolumn{1}{|c|}{ Mass $(\mathrm{kg})$} & Source of Estimate \\
\hline $11 / 30 / 93$ & 1994 & 1 & 4.20 & $2,000.00$ & $1993-\mathrm{FFCA}$ \\
\hline $11 / 30 / 93$ & 1995 & 1 & 4.20 & $2,000.00$ & $1993-\mathrm{FFCA}$ \\
\hline $11 / 30 / 93$ & 1996 & 1 & 4.20 & $2,000.00$ & $1993-\mathrm{FFCA}$ \\
\hline $11 / 30 / 93$ & 1997 & 1 & 4.20 & $2,000.00$ & $1993-\mathrm{FFCA}$ \\
\hline $11 / 30 / 93$ & 1998 & 5 & 21.00 & $10,000.00$ & $1993-\mathrm{FFCA}$ \\
\hline $11 / 30 / 93$ & 2003 & 20 & 84.00 & $40,000.00$ & $1993-\mathrm{FFCA}$ \\
\hline
\end{tabular}

\section{OYes ONo}

$\square$

KR̈W013

Still-Gen Active

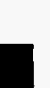


KK-W013

LLW : Contact-H

D. STORAGE INVENTORY

Waste Removed From Storage:
KK-WO13

Still-Gen Active soils 


\section{E. RADIATION CHARACTERISTICS}

Radioactive Waste Type:

\begin{tabular}{|ll|}
\hline O LLW & Oa-LLW O TRU \\
O HLW & O Non-Rad \\
\hline
\end{tabular}

Mixed Waste:

Handling:

\begin{tabular}{|l|}
\hline OYes ONo \\
\hline O Contact O Remote \\
\hline
\end{tabular}

Radionuclide Contamination Accessiblity:

\begin{aligned} External Surface & $O$ Yes & O No & O Unknown \\ Internal Surface & OYes & O No & O Unknown \\ \cline { 2 - 5 } Dispersed Through Matrix & OYes & Ono & O Unknown \end{aligned}

\section{Activity Levels}

$\begin{aligned} \text { Transuranic Alpha Activity: } & \square \text { Units: } \\ \text { Uranium/Thorium Alpha Activity: } & \square \text { Units: } \\ \text { Beta/Gamma Activity: } & \square \text { @ surface } \\ & \square \text { @ 1-m Units: } \mathrm{mR} / \mathrm{hr} \\ \text { Surface Neutron Activity: } & \square \text { Units: } \\ \text { Total Activity: } & \square \mathrm{nCi} / \mathrm{g}\end{aligned}$


吾

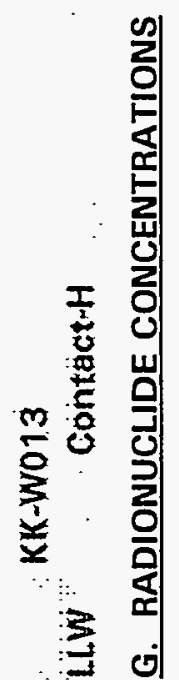


KKWO13 +Wo

LWW Contacth o

H. MATRIX CHARACTERISTICS

Primary Matrix Code: $\mathbf{S 4 0 0 0}$

Description: Soil/Gravel: : :

Combustibility: $\bigcirc$ Combustible $(>90 \%)$

Mixed $(10 \%-90 \%)$

Noncombustible $(<10 \%)$

\section{MATRIX COMPOSITION}

\begin{tabular}{|c|c|c|c|c|c|c|}
\hline & & & \multicolumn{4}{|c|}{ Composition } \\
\hline Code & Matrix Description & Material & Typical & $\overline{\mathrm{LL}}$ & UL & Units \\
\hline 54100 & Soil/Gravel.Soil.. & Soils & & & & \\
\hline
\end{tabular}




\section{KK.WO13}

\section{LLW}

\section{J. HAZARDOUS CONSTITUENTS}

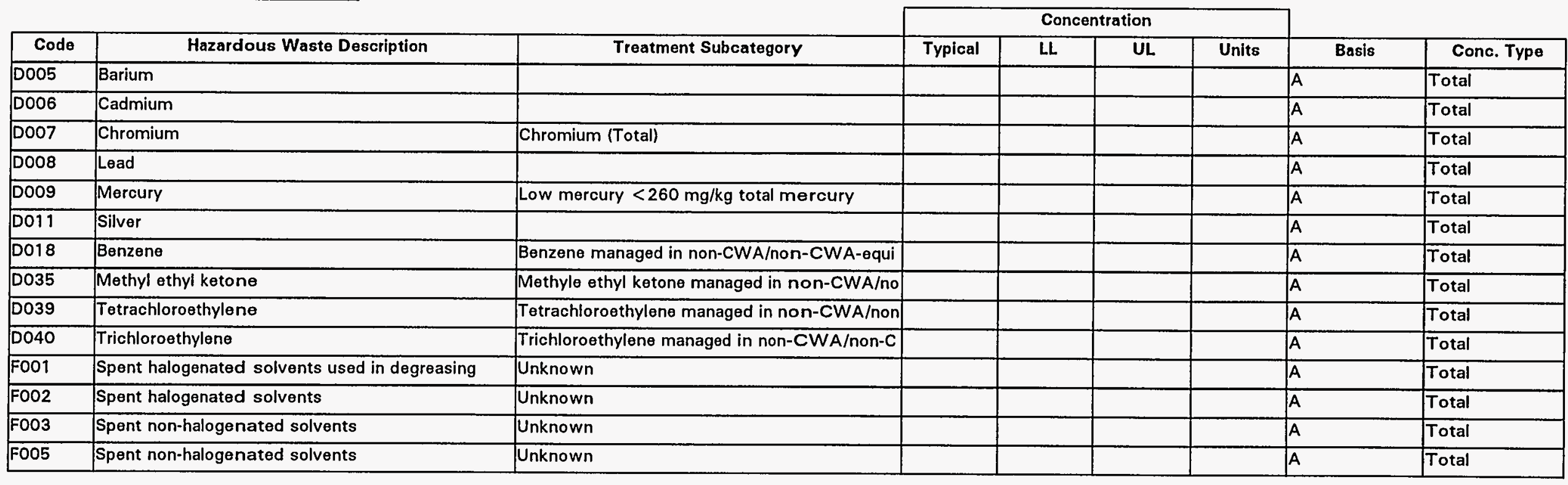




\section{LWW KKWOWO Contacth}

\section{K. POLYCHLORINATED BIPHENYLS (PCBS)}

Is this waste stream PCB contaminated?

\section{OYes O No O Unknown}

If yes, what portion of the waste is PCB contaminated (volume \%):

If $<100 \%$, can the PCB contaminated waste be segregated and treated separately?

What is the PCB concentration in ppm?

If only a portion of the waste is $P C B$ contaminated, report values for contaminated portion.

\section{TREATMENT PLANS}

\begin{tabular}{|c|c|c|c|c|c|c|c|c|c|c|c|}
\hline $\begin{array}{c}\text { Opt. } \\
\#\end{array}$ & $\begin{array}{c}\text { Media Type } \\
\text { (If more than one) }\end{array}$ & $\begin{array}{c}\text { Matrix } \\
\text { Code }\end{array}$ & $\begin{array}{l}\text { Vol. \% } \\
\text { lif }\end{array}$ & Step & JIT & sc & ClF & $\begin{array}{l}\text { Trans. } \\
\text { Miles }\end{array}$ & $\begin{array}{l}\text { Facility } \\
\text { Abbr. }\end{array}$ & Unit Name & Comments \\
\hline 1 & & 54000 & & $\bar{a}$ & () & $\bar{O}$ & $1.00 E+00$ & & TRANS & Transport - LLW & \\
\hline & & & & $\mathbf{b}$ & 0 & 0 & $1.00 E+00$ & & AMWTF & CH - Opening \& Sorting & \\
\hline & & & & $\bar{c}$ & 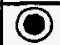 & 0 & $1.00 E+00$ & & AMWTF & $\mathrm{CH}$-Sizing & \\
\hline & & & & d & $\overline{0}$ & O & $1.00 E+00$ & & AMWTF & $\mathrm{CH}$ - Incineration/Thermal Desorption & \\
\hline & & & & e & 0 & 0 & $1.00 E+00$ & & AMWTF & $\mathrm{CH}$ - Vitrification & \\
\hline & & & & $f$ & 0 & 0 & $1.00 E+00$ & & SCDF & Disposal - Contact Handled & \\
\hline
\end{tabular}

Note: Where provided, media percentages are rough estimates used to facilitate treatment planning. They do not necessarily imply an accurate knowledge of waste stream composition, and were not derived for use outside this conceptual planning exercise.

Describe any special or unique technical concerns related to the treatment of this waste stream that would impact the use or implementation of standard hazardous waste treatment methods. 


\section{KK-WO14}

Hg CONTAMINATED ORGANICS

KK-WO14 HLW: $: \cdots:$ ContactrH

\section{A. IDENTIFICATION AND DESCRIPTION}

Waste Stream ID: KK-W014

W. S. Name:

Content Code:

FFCA ID:

\begin{tabular}{|l|}
\hline $\mathrm{Hg}$ CONTAMINATED ORGANICS \\
\hline$*$ \\
\hline$K K-W 014$ \\
\hline
\end{tabular}

\section{Waste Stream Description:}

This waste stream consists of organic materials which have become contaminated with

radioactivity and mercury greater than $260 \mathrm{ppm}$. This waste stream has not been generated.

The homogeneity of this waste stream is unknown until generated. This waste stream will not

be a moratorium or reclassified TRU waste.

\section{B. GENERATION SITE/PROCESS DESCRIPTION}

The following questions, concerning the generation site and process, should be answered even if the waste stream is no longer generated.

Name of Site Generating Waste (e.g., INEL, Rocky Flats, etc.): KAPL-Kesselring Site

Location of activities (Area and Building):

Various locations on site.

Operations (functions) performed in building:

Various operations on site.

Description of Process Generating Waste:

Various laboratory activities (e.g., analysis, testing, maintenance). 


$$
\begin{array}{r}
K K-W 0 ̈ 14 \\
\text { GWO }
\end{array}
$$

LiW ContoG:

\section{Hg CONTAMINATED ORGANICS}

C. PROJECTED WASTE GENERATION

Is this waste stream still generated? OYes O No

If yes, when is the expected generation termination date?

Current Projections

\begin{tabular}{|c|r|r|r|r|r|}
\hline Est. Date & Start Year & Interval & \multicolumn{1}{|c|}{ Vol (m3) } & Mass (kg) & Source of Estimate \\
\hline $11 / 15 / 94$ & 1997 & 1 & 0.20 & 225.00 & 1994 -DSTP \\
\hline
\end{tabular}

Historical Projections 


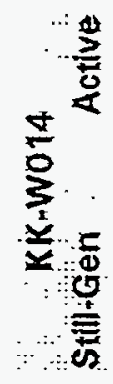




\section{E. RADIATION CHARACTERISTICS}

Radioactive Waste Type:

\begin{tabular}{|ll|}
\hline OllW & $\bigcirc$ a-llW $\bigcirc$ TRU \\
OHLW & $\bigcirc$ Non-Rad \\
\hline
\end{tabular}

Mixed Waste:

Handling:

OYes ONo

Radionuclide Contamination Accessiblity:

External Surface

Internal Surface

Dispersed Through Matrix

\begin{tabular}{|c|c|c|}
\hline OYes & ONo & OUnknown \\
\hline OYes & ONo & O Unknown \\
\hline OYes & ONo & O Unknown \\
\hline
\end{tabular}

\section{Activity Levels}

Transuranic Alpha Activity:

Uranium/Thorium Alpha Activity:

Beta/Gamma Activity:

Surface Neutron Activity:

Total Activity:

$\square$ Units: $\square$
$\square$ Units: $\square$
$\square$ @ surface
$\square$ @ $1-\mathrm{m}$ Units: $\mathrm{mR/hr}$
$\square$ Units: $\square$
$\square$ nCi/g

\section{F. TRU ALPHA ACTIVITY DISTRIBUTION}

This waste stream is not identified as a TRU or a-LLW. 


\section{KK-WWO14}

LiWW: ContactitH

G. RADIONUCLIDE CONCENTRATIONS

\section{G. RADIONUCLIDE CONCENTRATIONS}

\begin{tabular}{|c|c|c|c|c|c|c|}
\hline & \multicolumn{3}{|c|}{ Specific Activity } & \multirow[b]{2}{*}{ Units } & \multirow[b]{2}{*}{ Basis Code(s) } & \multirow[b]{2}{*}{ Basis Desc. (if other) } \\
\hline Isotope & Typical & Lower Limit & Upper Limit & & & \\
\hline C-14 & & & & & 1 & \\
\hline Co-58 & & & & & 1 & \\
\hline \multicolumn{7}{|l|}{$M n-54$} \\
\hline $\mathrm{Fe}-59$ & & & & & I & \\
\hline \multicolumn{5}{|c|}{$\begin{array}{l}\text { A. Passive-Active Neutron (PAN) } \\
\text { B. Segmented Gamma Scan (SGS) } \\
\text { C. Passive-Active Neutron/Segmented Gamma Scan (PAN/SGS) } \\
\text { D. Radiochemical Analysis } \\
\text { E. Mass Spectrometry }\end{array}$} & $\begin{array}{l}\text { Accountability } \\
\text { Calorimetry } \\
\text { Multiple Energy } \\
\text { Process Knowle } \\
\text { Other }\end{array}$ & is (MEGAS) \\
\hline
\end{tabular}


KKKWO14

HiW ContacteH

\section{H. MATRIX CHARACTERISTICS}

Primary Matrix Code: $\mathbf{S 3 2 0 0}$

Description: Homogeneous Solids: Organic Homogeneous solids: :

Combustibility: $\bigcirc$ Combustible $(>90 \%$ )

Mixed $(10 \%-90 \%)$

Noncombustible $(<10 \%)$

\section{MATRIX COMPOSITION}

\begin{tabular}{|c|c|c|c|c|c|c|}
\hline & & & \multicolumn{4}{|c|}{ Composition } \\
\hline Code & Matrix Description & Material & Typlcal & $\overline{L L}$ & UL & Unịts \\
\hline 53290 & Homogeneous Solids.Organic Homogeneous solids. Unknown/Other Organic Homoge & & 100 & 100 & 100 & $\%$ vol \\
\hline
\end{tabular}




\section{KK:W014}

\section{Hg CONTAMINATED ORGANICS}

KK-WO14

LLW :Contact+H

S Still Gen active

\section{J. HAZARDOUS CONSTITUENTS}

\begin{tabular}{|c|c|c|c|c|c|c|c|c|}
\hline \multirow[b]{2}{*}{ Code } & \multirow[b]{2}{*}{ Hazardous Waste Description } & \multirow[b]{2}{*}{ Treatment Subcategory } & \multicolumn{4}{|c|}{ Concentration } & \multirow[b]{2}{*}{ Basis } & \\
\hline & & & Typical & $\mathbf{L L}$ & UL & Units & & Conc. Typo \\
\hline D005 & Barium & & & & & & A & Total \\
\hline D006 & Cadmium & & & & & & A & Total \\
\hline$\overline{D 007}$ & Chromium & Chromium (Total) & & & & & $A$ & Total \\
\hline$\overline{\mathrm{D008}}$ & Lead & & & & & & A & Total \\
\hline D009 & Mercury & Elemental mercury contaminated with radioact & & & & & A & Total \\
\hline D011 & Silver & & & & & & A & Total \\
\hline D018 & Benzene & Benzene managed in non-CWA/non-CWA-equi & & & & & A & Total \\
\hline D035 & Methyl ethyl ketone & Methyle ethyl ketone managed in non-CWA/no & & & & & A & Total \\
\hline D039 & Tetrachloroethylene & Tetrachloroethylene managed in non-CWA/non & & & & & A & Total \\
\hline D040 & Trichloroethylene & Trichloroethylene managed in non-CWA/non-C & & & & & A & Total \\
\hline
\end{tabular}


Contact + H

\section{K. POLYCHLORINATED BIPHENYLS (PCBS)}

Is this waste stream PCB contaminated?

OYes O No O Unknown

If yes, what portion of the waste is PCB contaminated (volume \%):

If $<100 \%$, can the PCB contaminated waste be segregated and treated separately? $O$ Yes $O$ No Onknown

What is the PCB concentration in ppm?

If only a portion of the waste is $P C B$ contaminated, report values for contaminated portion.

\section{TREATMENT PLANS}

\begin{tabular}{|c|c|c|c|c|c|c|c|c|c|c|c|}
\hline $\begin{array}{l}\text { Opt. } \\
\#\end{array}$ & $\begin{array}{c}\text { Media Type } \\
\text { (If more than one) }\end{array}$ & $\begin{array}{c}\text { Matrix } \\
\text { Code }\end{array}$ & $\begin{array}{l}\text { Vol. \% } \\
\text { (If }\end{array}$ & Step & JIT & sc & CIF & $\begin{array}{l}\text { Trans. } \\
\text { Miles }\end{array}$ & $\begin{array}{l}\text { Facility } \\
\text { Abbr. }\end{array}$ & Unit Name & Comments \\
\hline 1 & & S5440 & & $a$ & 0 & $\mathrm{O}$ & $1.00 E+00$ & & TRANS & Transport - LLW & \\
\hline & & & & b & $\mathrm{O}$ & $\mathrm{O}$ & $1.00 E+00$ & & WROC & Sizing & \\
\hline & & & & c & 0 & $\bar{O}$ & $1.00 E+00$ & & WROC & Hg Retort & \\
\hline & & & & d & 0 & 0 & $1.00 \mathrm{E}+00$ & & WERF & Incineration - Controlled Air Incinerator & \\
\hline & & & & e & 0 & 0 & $1.00 E+00$ & . & WERF & Stabilization - Portland Cement & \\
\hline & & & & $f$ & 0 & O & $1.00 \mathrm{E}+00$ & & SCDF & Disposal - Contact Handled & \\
\hline
\end{tabular}

Note: Where provided, media percentages are rough estimates used to facilitate treatment planning. They do not necessarily imply an accurate knowledge of waste stream composition, and were not derived for use outside this conceptual planning exercise.

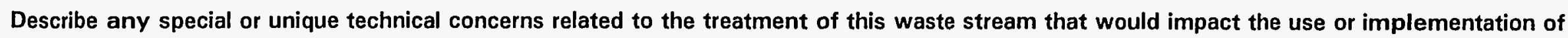
standard hazardous waste treatment methods. 


\section{KK+WÖ15}

\section{LWw: ContactrH}

\section{A. IDENTIFICATION AND DESCRIPTION}

Waste Stream ID: KK-W015

W. S. Name:

Content Code:

FFCA ID:

\begin{tabular}{|l|}
\hline KK-W015 \\
\hline Hg CONTAMINATED INORGANICS \\
\hline KK-W015 \\
\hline
\end{tabular}

\section{Waste Stream Description:}

This waste stream consists of inorganic materials which has become contaminated with radioactivity and mercury with concentrations greater than 260ppm. The homogeneity of this waste stream within a container is unknown. This waste stream will not be a moratorium or reclassified TRU waste.

\section{Hg CONTAMINATED INORGANICS}

KK̈KWO15 Still Gen Active

\section{B. GENERATION SITE/PROCESS DESCRIPTION}

The following questions, concerning the generation site and process, should be answered even if the waste stream is no longer generated.

Name of Site Generating Waste (e.g., INEL, Rocky Flats, etc.): KAPL-Kesselring Site

Location of activities (Area and Building):

Various locations on site.

Operations (functions) performed in building:

Various operations on site.

Description of Process Generating Waste:

Various laboratory activities (e.g., analysis, testing, maintenance). 
KK-WO 15

LlWW Gontáath

C. PROJECTED WASTE GENERATION

Is this waste stream still generated?

If yes, when is the expected generation termination date?

\section{Current Projections}

\begin{tabular}{|r|r|l|r|r|r|}
\hline Est. Date & Start Year & Interval & Vol (m3) & Mass (kg) & Source of Estimate \\
\hline $11 / 15 / 94$ & 1997 & 1 & 0.20 & 225.00 & $1994-D S T P$ \\
\hline \multicolumn{5}{|c|}{ Historical Proiections } \\
\hline
\end{tabular}

Hg CONTAMINATEO INORGANIOS

KKiwo 15 Still Gen Active

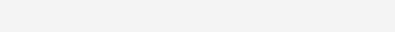

\begin{tabular}{|l|}
\hline O Yes ONo \\
\hline \\
\hline Source of Estimate \\
\hline
\end{tabular}



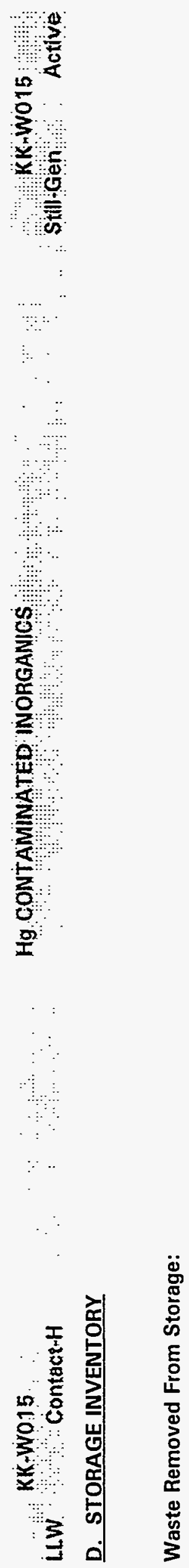


\section{E. RADIATION CHARACTERISTICS}

Radioactive Waste Type:

$\begin{array}{ll}\text { Ollw } & \text { Oa-llw OtRU } \\ \text { OhlW } & \text { O Non-Rad }\end{array}$

Mixed Waste:

Handling:

Radionuclide Contamination Accessiblity:

\begin{tabular}{rlrl|} 
External Surface & OYes & Ono & O Unknown \\
Internal Surface & OYes & O No & O Unknown \\
\cline { 2 - 5 } Dispersed Through Matrix & OYes & O No & O Unknown
\end{tabular}

\section{F. TRU ALPHA ACTIVITY DISTRIBUTION}

\section{Activity Levels}

Transuranic Alpha Activity: Uranium/Thorium Alpha Activity:

Beta/Gamma Activity

Surface Neutron Activity:

Total Activity:

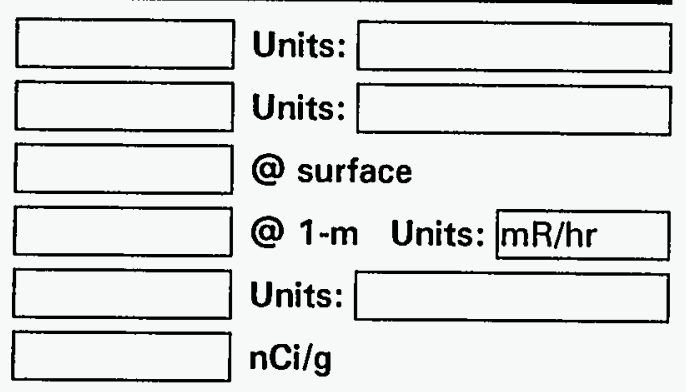




\section{KK-WO15}

\section{Hg CONTAMMINATED INORGANICS}

Ltw

Contàct-H

a $:$. . .

\section{G. RADIONUCLIDE CONCENTRATIONS}

\begin{tabular}{|c|c|c|c|c|c|c|}
\hline \multirow{2}{*}{ Isotope } & \multicolumn{3}{|c|}{ Specific Activity } & \multirow[b]{2}{*}{ Units } & \multirow[b]{2}{*}{ Basis Code(s) } & \multirow[b]{2}{*}{ Basis Desc. (if other) } \\
\hline & Typical & Lower Limit & Upper Limit & & & \\
\hline \multicolumn{7}{|l|}{ C-14 } \\
\hline $\mathrm{Ni}-63$ & & & & & 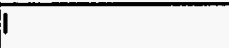 & \\
\hline \multicolumn{7}{|l|}{$\overline{C o-58}$} \\
\hline$\overline{M n-54}$ & & & & & 1 & \\
\hline Fe-59 & & & & & 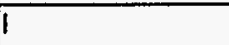 & \\
\hline Co-60 & & & & & I & \\
\hline $\begin{array}{l}\text { A. Passiv } \\
\text { B. Segm } \\
\text { C. Passi } \\
\text { D. Radio } \\
\text { E. Mass }\end{array}$ & $\begin{array}{l}\text { tive Neut' } \\
\text { Gamma } \\
\text { tive Neutr } \\
\text { ical Analy } \\
\text { trometry }\end{array}$ & $\begin{array}{l}P A N) \\
\text { (SGS) } \\
\text { Segmented } G a\end{array}$ & na Scan $(P$ & 3S) & $\begin{array}{l}\text { Accountability } \\
\text { Calorimetry } \\
\text { Multiple Energy } \\
\text { Process Knowle } \\
\text { Other }\end{array}$ & sis (MEGAS) \\
\hline
\end{tabular}


LWW ContactH

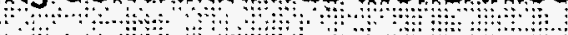

H. MATRIX CHARACTERISTICS

Primary Matrix Code: $\$ 3100$

Description: Homogeneous Solids: Inorganic Homogeneous Solids: :

Combustibility: $\begin{aligned} & \bigcirc \text { Combustible }(>90 \%) \\ & \bigcirc \text { Mixed }(10 \%-90 \%) \\ & \bigcirc \text { Noncombustible }(<10 \%)\end{aligned}$

\section{MATRIX COMPOSITION}

\begin{tabular}{|c|c|c|c|c|c|c|}
\hline & & & \multicolumn{4}{|c|}{ Composition } \\
\hline Code & Matrix Description & Material & Typical & LL & UL & Units \\
\hline 53190 & Homogeneous Solids.Inorganic Homogeneous Solids.Unknown/Other Inorganic Homo & & 100 & 100 & 100 & $\%$ vol \\
\hline
\end{tabular}


KK-WO15

LWW Contact H

\section{J. HAZARDOUS CONSTITUENTS}

\begin{tabular}{|c|c|c|c|c|c|c|c|c|}
\hline 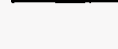 & & & & Con & & & & \\
\hline Code & Hazardous Waste Description & Treatment Subcategory & Typical & LL & UL. & Units & Basis & Conc. Type \\
\hline$\overline{D 005}$ & Barium & & & & & & A & Total \\
\hline D006 & Cadmium & & & & & & A & Total \\
\hline$\overline{D 007}$ & Chromium & Chromium (Total) & & & & & A & Total \\
\hline$\overline{D 008}$ & Lead & & & & & & A & Total \\
\hline$\overline{D 009}$ & Mercury & Elemental mercury contaminated with radioact & & & & & A & Total \\
\hline D011 & Silver & & & & & & A & Total \\
\hline
\end{tabular}


KK-WON15

LiW

\section{K. POLYCHLORINATED BIPHENYLS (PCBS)}

Is this waste stream PCB contaminated?

OYes O No O Unknown

If yes, what portion of the waste is PCB contaminated (volume \%):

If $<100 \%$, can the PCB contaminated waste be segregated and treated separately?

What is the PCB concentration in ppm?

If only a portion of the waste is PCB contaminated, report values for contaminated portion.

\section{TREATMENT PLANS}

\begin{tabular}{|c|c|c|c|c|c|c|c|c|c|c|c|}
\hline $\begin{array}{c}\text { Opt. } \\
\#\end{array}$ & $\begin{array}{c}\text { Media Type } \\
\text { (If more than one) }\end{array}$ & $\begin{array}{c}\text { Matrix } \\
\text { Code }\end{array}$ & $\begin{array}{l}\text { Vol. \% } \\
\text { IIf }\end{array}$ & Step & JIT & sc & CIF & $\begin{array}{l}\text { Trans. } \\
\text { Miles }\end{array}$ & $\begin{array}{l}\text { Facility } \\
\text { Abbr. }\end{array}$ & Unit Name & Comments \\
\hline 1 & & 55420 & & $\mathbf{a}$ & $\mathbf{O}$ & 0 & $1.00 E+00$ & & TRANS & Transport - LLW & \\
\hline & & & & b & $\mathbf{O}$ & 0 & $1.00 \mathrm{E}+00$ & & WROC & Sizing & \\
\hline & & & & d & 0 & 0 & $1.00 E+00$ & & WERF & Stabilization - Portland Cement & \\
\hline & & & & e & 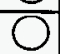 & 0 & $1.00 \mathrm{E}+00$ & & SCDF & Disposal - Contact Handled & \\
\hline
\end{tabular}

Note: Where provided, media percentages are rough estimates used to facilitate treatment planning. They do not necessarily imply an accurate knowledge of waste stream composition, and were not derived for use outside this conceptual planning exercise.

Describe any special or unique technical concerns related to the treatment of this waste stream that would impact the use or implementation of standard hazardous waste treatment methods. 


\section{A. IDENTIFICATION AND DESCRIPTION}

Waste Stream ID: KK-W016

\section{W. S. Name:}

Content Code:

FFCA ID:

\section{ELEMENTAL Hg \\ *}

\section{KK-W016}

Waste Stream Description:

This waste stream consists of mercury in its elemental (liquid) form contaminated with

radioactivity. This waste will be homogeneous within each individual container. This waste stream will not be a moratorium or reclassified TRU waste.

\section{B. GENERATION SITE/PROCESS DESCRIPTION}

The following questions, concerning the generation site and process, should be answered even if the waste stream is no longer generated.

Name of Site Generating Waste (e.g., INEL, Rocky Flats, etc.): KAPL-Kesselring Site

Location of activities (Area and Building):

Various locations on site.

Operations (functions) performed in building:

Various operations on site.

Description of Process Generating Waste:

Waste from maintenance or testing. 

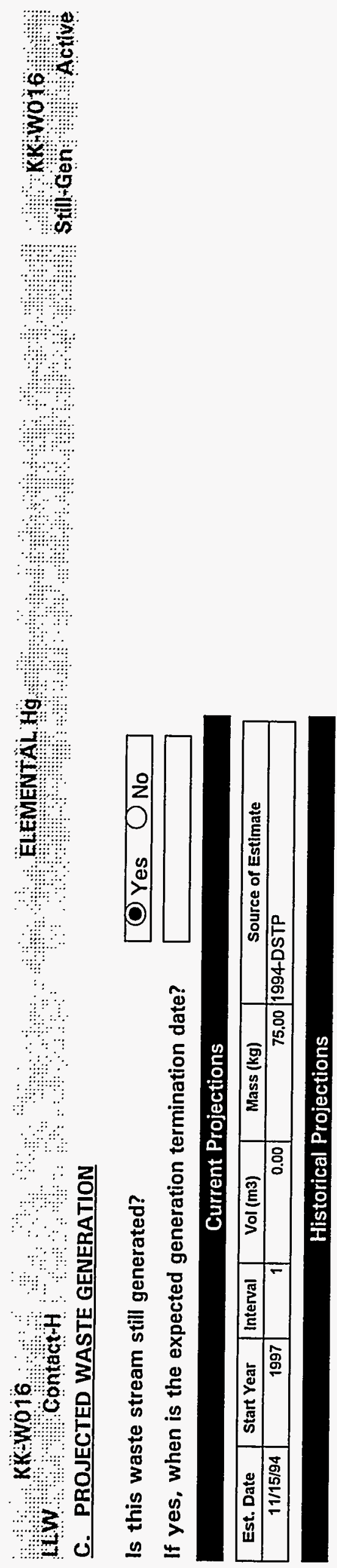

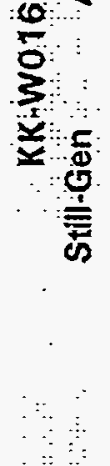

$\because$

$\because \vdots$
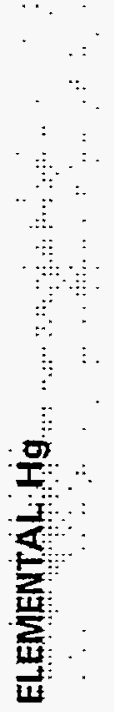

.




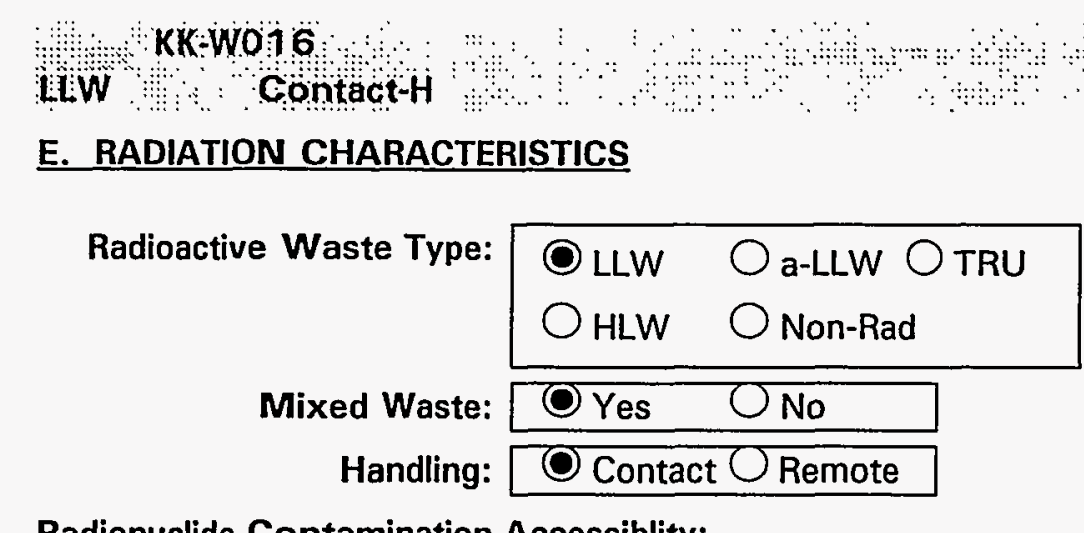

ELENENTALHO

Contact-H

\section{F. TRU ALPHA ACTIVITY DISTRIBUTION}

Radionuclide Contamination Accessiblity:

External Surface

Internal Surface

Dispersed Through Matrix

\begin{tabular}{|c|c|c|}
\hline OYes & ONo & O) Unknown \\
\hline OYes & ONo & O Unknown \\
\hline $\mathrm{OYe}_{\mathrm{Ye}}$ & No & OUn \\
\hline
\end{tabular}

This waste stream is not identified as a TRU or a-LLW.

Activity Levels

Transuranic Alpha Activity:

Uranium/Thorium Alpha Activity:

Beta/Gamma Activity:

Surface Neutron Activity:

Total Activity

\begin{tabular}{l}
\hline Units: \\
\hline Units: \\
\hline \\
\hline \\
@ surface \\
\hline @ 1-m Units: $\mathrm{mR} / \mathrm{hr}$ \\
\hline \\
Units: $\square$ \\
nCi/g
\end{tabular}




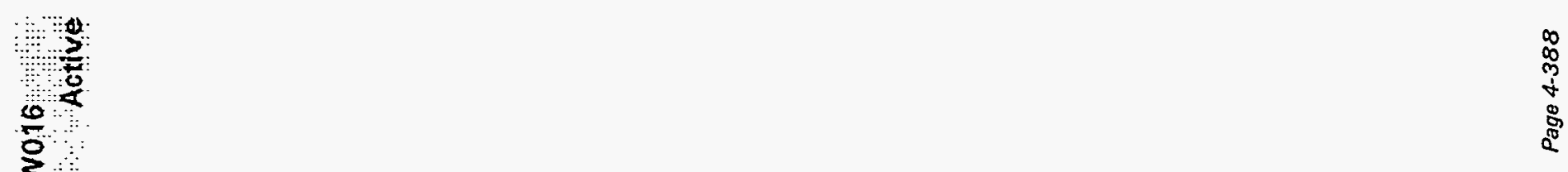

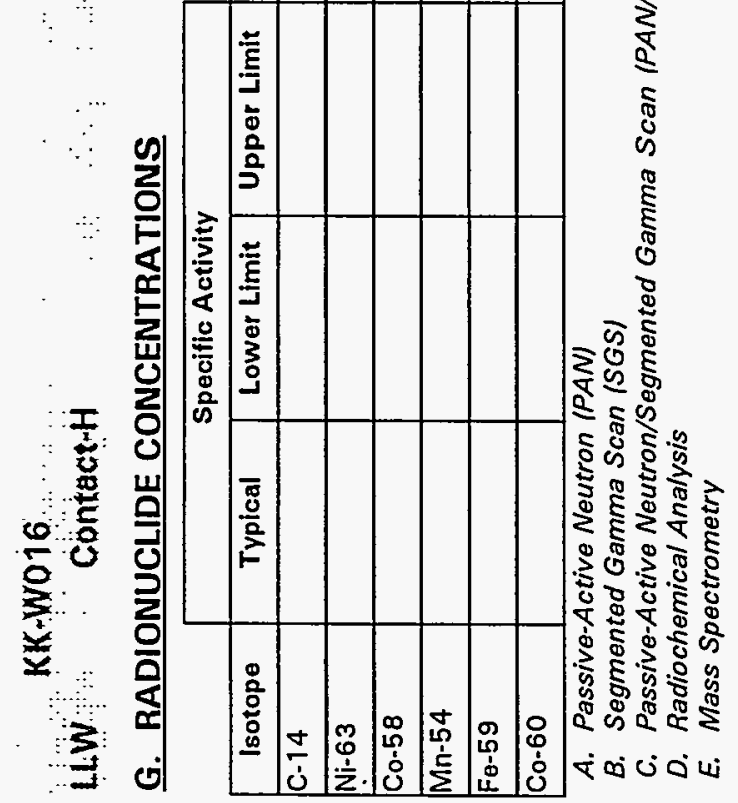


KK:WO16:

LWW

H. MATRIX CHARACTERISTICS

Primary Matrix Code: $\times 7100$

Description: Special Waste: Elemental Mercury: :

Combustibility: $\bigcirc$ Combustible (>90\%)

Mixed (10\%-90\%)

Noncombustible $(<10 \%)$

\section{MATRIX COMPOSITION}

\begin{tabular}{|c|c|c|c|c|c|c|}
\hline & & & \multicolumn{4}{|c|}{ Composition } \\
\hline Code & Matrix Description & Material & Typical & LL & UL & Units \\
\hline$\times 7100$ & Special Waste.Elemental Mercury.. & & 100 & 100 & 100 & $\%$ vol \\
\hline
\end{tabular}


KK-WO16

\section{LLW :-..: Contact+H}

J. HAZARDOUS CONSTITUENTS

\begin{tabular}{|c|c|c|c|c|c|c|c|c|}
\hline & & & \multicolumn{4}{|c|}{ Concentration } & \multirow[b]{2}{*}{ Basis } & \multirow[b]{2}{*}{ Conc. Type } \\
\hline Code & Hazardous Waste Description & Treatment Subcategory & Typical & $\mathbf{L L}$ & UL & Units & & \\
\hline$\overline{D 009}$ & Mercury & Elemental mercury contaminated with radioact & & & & & A & Total \\
\hline
\end{tabular}

KK.WÖ16

Still:Gen Active

\section{ELEMENTAL: $\mathrm{Hg}$}

ELEMENALHg 


\section{K. POLYCHLORINATED BIPHENYLS (PCBS)}

\section{Is this waste stream PCB contaminated? OYes $O$ No $O$ Unknown}

If yes, what portion of the waste is PCB contaminated (volume \%):

If $<100 \%$, can the PCB contaminated waste be segregated and treated separately?

OYes ONo O Unknown

What is the PCB concentration in ppm?

If only a portion of the waste is PCB contaminated, report values for contaminated portion.

\section{TREATMENT PLANS}

\begin{tabular}{|c|c|c|c|c|c|c|c|c|c|c|c|}
\hline $\begin{array}{c}\text { Opt. } \\
\#\end{array}$ & $\begin{array}{c}\text { Media Type } \\
\text { (If more than one) }\end{array}$ & $\begin{array}{c}\text { Matrix } \\
\text { Code }\end{array}$ & $\begin{array}{c}\text { Vol. } \% \\
\text { (lf }\end{array}$ & Step & JIT & sc & CIF & $\begin{array}{l}\text { Trans. } \\
\text { Miles }\end{array}$ & $\begin{array}{l}\text { Facility } \\
\text { Abbr. }\end{array}$ & Unit Name & Comments \\
\hline 1 & & $\times 7100$ & & a & O & 0 & $1.00 \mathrm{E}+00$ & & TRANS & Transport - LLW & \\
\hline & & & & b & 0 & 0 & $1.00 E+00$ & & AMWTF & $\mathrm{CH}$ - Opening \& Sorting & \\
\hline & & & & c & 0 & 0 & $1.00 E+00$ & & AMWTF & $\mathrm{CH}$ - Amalgamation & \\
\hline & & & & d & 0 & 0 & $1.00 E+00$ & & SCDF & Disposal - Contact Handled & \\
\hline
\end{tabular}

Note: Where provided, media percentages are rough estimates used to facilitate treatment planning. They do not necessarily imply an accurate knowledge of waste stream composition, and were not derived for use outside this conceptual planning exercise.

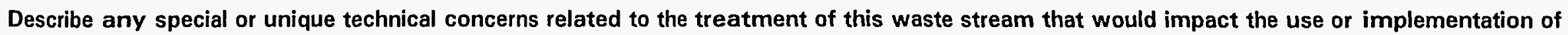
standard hazardous waste treatment methods. 


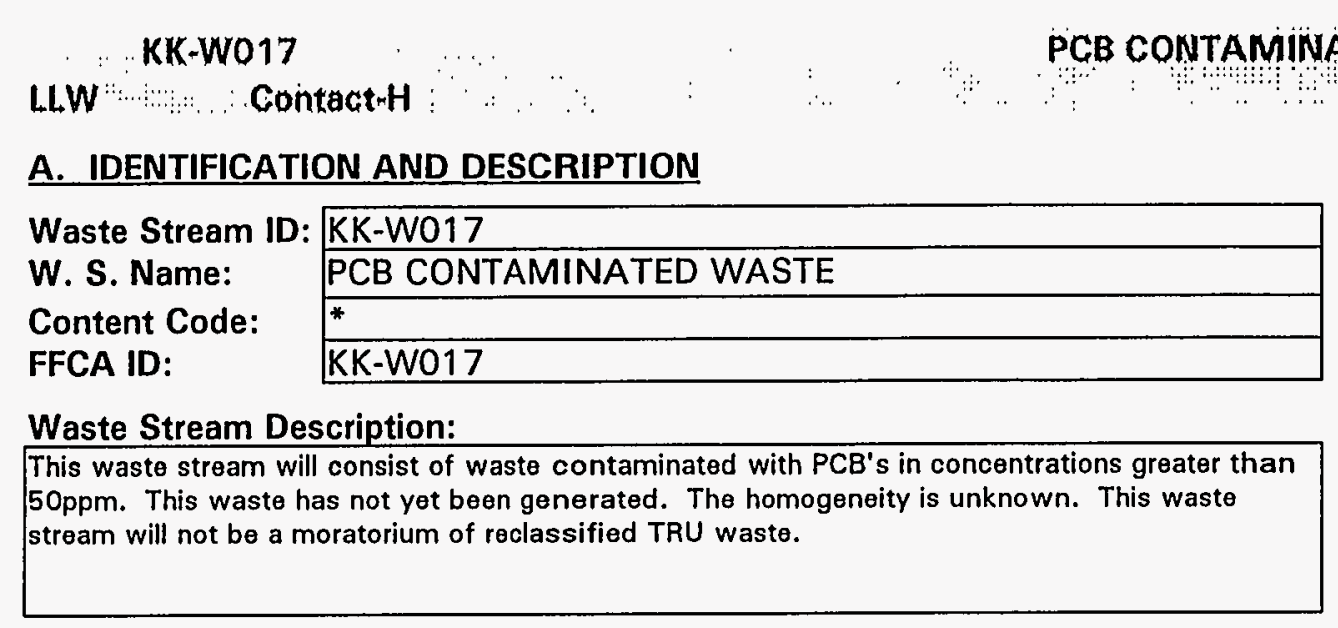

\section{B. GENERATION SITE/PROCESS DESCRIPTION}

The following questions, concerning the generation site and process, should be answered even if the waste stream is no longer generated.

Name of Site Generating Waste (e.g., INEL, Rocky Flats, etc.): KAPL-Kesselring Site

Location of activities (Area and Building):

Various locations on site.

Operations (functions) performed in building: Various operations on site.

Description of Process Generating Waste:

Waste may be generated through various processes le.g., maintenance, clean-upl. 
KK-Wo17

LlWW Contact

C. PROJECTED WASTE GENERATION

Is this waste stream still generated?

If yes, when is the expected generation termination date?

Current Projections

\begin{tabular}{|l|l|l|l|l}
\hline Est. Date & Start Year & Interval & Vol (m3) & Mass (kg) \\
\hline
\end{tabular}

\begin{tabular}{|r|r|r|}
\hline $11 / 15 / 94$ & Start Year & Interval \\
\hline
\end{tabular}

$11 / 15 / 94$

Historical Projections

PCB CONTAMINATED WASTE

\section{OYes ONo}

Source of Estimate 
D. STORAGE INVENTORY

Waste Removed From Storage: 
KK-Wo17

LiW Contact th

POB CONTÄMIINATED WASTE

KKWO17

\section{E. RADIATION CHARACTERISTICS}

Radioactive Waste Type:

$$
\begin{array}{ll}
\text { OlLW } & \text { Oa-LLW O TRU } \\
\text { OhLW } & \text { O Non-Rad }
\end{array}
$$

Mixed Waste:

Handling:

\begin{tabular}{l}
\hline O Yes O No \\
O Contact O Remote
\end{tabular}

Radionuclide Contamination Accessiblity:

External Surface

Internal Surface

\begin{tabular}{|c|c|c|}
\hline OYes & ONo & O Unknown \\
\hline OYes & ONo & OUnknown \\
\hline$T$ & $\sqrt{N}$ & 0 \\
\hline
\end{tabular}

Dispersed Through Matrix

\section{Activity Levels}

Transuranic Alpha Activity:

Uranium/Thorium Alpha Activity:

Beta/Gamma Activity:

Surface Neutron Activity:

Total Activity:

\begin{tabular}{l}
\hline \\
\hline \\
\hline \\
\hline \\
\hline \\
\hline \\
Units: @ surface \\
\\
\\
@ 1-m Units: $\mathrm{mR} / \mathrm{hr}$ \\
\hline \\
Units: \\
nCi/g
\end{tabular}

\section{F. TRU ALPHA ACTIVITY DISTRIBUTION}

This waste stream is not identified as a TRU or a-LLW. 


\section{KK-W017}

LWW Contact-H

G. RADIONUCLIDE CONCENTRATIONS

\begin{tabular}{|c|c|c|c|c|c|c|}
\hline \multirow{2}{*}{ Isotope } & \multicolumn{3}{|c|}{ Specific Activity } & \multirow[b]{2}{*}{ Units } & \multirow[b]{2}{*}{ Basis Code(s) } & \multirow[b]{2}{*}{ Basis Desc. (if other) } \\
\hline & Typical & Lower Limit & Upper Limit & & & \\
\hline \multicolumn{7}{|l|}{$\overline{C-14}$} \\
\hline \multicolumn{7}{|l|}{$\mathrm{Ni}-63$} \\
\hline \multicolumn{7}{|l|}{ Co-58 } \\
\hline \multicolumn{7}{|l|}{$M n-54$} \\
\hline \multicolumn{7}{|l|}{$\overline{F e-59}$} \\
\hline Co-60 & & & & & $!$ & \\
\hline \multicolumn{7}{|c|}{$\begin{array}{ll}\text { A. Passive-Active Neutron (PAN) } & \text { F. Accountability } \\
\text { B. Segmented Gamma Scan (SGS) } & \text { G. Calorimetry } \\
\text { C. Passive-Active Neutron/Segmented Gamma Scan (PAN/SGS) } & \text { H. Multiple Energy Gamma Analysis (MEGAS) } \\
\text { D. Radiochemical Analysis } & \text { \%. Process Knowledge } \\
\text { E. Mass Spectrometry } & \text { J. Other }\end{array}$} \\
\hline
\end{tabular}

PCB CONTAMINATED WASTE

Other
KKHWO17

Still-Gen Active 


\section{H. MATRIX CHARACTERISTICS}

Primary Matrix Code: $\$ 3000$

Description: Homogeneous Solids: : :

Combustibility: $\bigcirc$ Combustible (>90\%)

Mixed $(10 \%-90 \%)$

Noncombustible (<10\%)

\section{MATRIX COMPOSITION}

\begin{tabular}{|c|c|c|c|c|c|c|}
\hline & & & \multicolumn{4}{|c|}{ Composition } \\
\hline Code & Matrix Description & Material & Typical & LL & UL & Units \\
\hline 53000 & Homogeneous Solids... & & 100 & 100 & 100 & $\% \mathrm{vol}$ \\
\hline
\end{tabular}




\section{KK-WO17 \\ Litw: Contact-H \\ J. HAZARDOUS CONSTITUENTS}

PCB CONTAMINATEED WASTE

KK-W017

\begin{tabular}{|c|c|c|c|c|c|c|c|c|}
\hline & & & \multicolumn{4}{|c|}{ Concentration } & \multirow{2}{*}{ Basis } & \multirow{2}{*}{ Conc. Type } \\
\hline Code & Hazardous Waste Description & Treatment Subcategory & Typical & $\overline{\mathbf{L L}}$ & UL & Units & & \\
\hline D005 & Barium & & & & & & A & Total \\
\hline$\overline{D 006}$ & Cadmium & & & & & & A & Total \\
\hline 0007 & Chromium & Chromium (Total) & & & & & A & Total \\
\hline D008 & Lead & & & & & & A & Total \\
\hline D009 & Mercury & Elemental mercury contaminated with radioact & & & & & A & Total \\
\hline D011 & Silver & & & & & & A & Total \\
\hline D018 & Benzene & Benzene managed in non-CWA/non-CWA-equi & & & & & A & Total \\
\hline$\overline{D 035}$ & Methyl ethyl ketone & Methyle ethyl ketone managed in non-CWA/no & & & & & A & Total \\
\hline D039 & Tetrachloroethylene & Tetrachloroethylene managed in non-CWA/non & & & & & A & Total \\
\hline$\overline{\mathrm{DO40}}$ & Trichloroethylene & Trichloroethylene managed in non-CWA/non-C & & & & & A & Total \\
\hline
\end{tabular}




\section{KK-ẄVO17̈:}

\section{Liw Contact}

K. POLYCHLORINATED BIPHENYLS (PCBS)

Is this waste stream PCB contaminated?

O Yes O No O Unknown

If yes, what portion of the waste is PCB contaminated (volume \%): 100

If $<100 \%$, can the PCB contaminated waste be segregated and treated separately?

OYes O No O Unknown

What is the PCB concentration in ppm?

If only a portion of the waste is PCB contaminated, report values for contaminated portion.

\section{TREATMENT PLANS}

\begin{tabular}{|c|c|c|c|c|c|c|c|c|c|c|c|}
\hline $\begin{array}{c}\text { Opt. } \\
\#\end{array}$ & $\begin{array}{c}\text { Media Type } \\
\text { (If more than one) }\end{array}$ & $\begin{array}{c}\text { Matrix } \\
\text { Code }\end{array}$ & $\begin{array}{l}\text { Vol. \% } \\
\text { (If }\end{array}$ & Step & JIT & sc & CIF & $\begin{array}{l}\text { Trans. } \\
\text { Miles }\end{array}$ & $\begin{array}{l}\text { Facility } \\
\text { Abbr. }\end{array}$ & Unit Name & Comments \\
\hline 1 & & 53000 & & a & O & O & $1.00 E+00$ & & TRANS & Transport - LLW & \\
\hline & & & & b & O & O & $1.00 E+00$ & & AMWTF & CH - Opening \& Sorting & \\
\hline & & & & $\bar{c}$ & $\mathbf{O}$ & $\overline{\mathrm{O}}$ & $1.00 \mathrm{E}+00$ & & AMWTF & $\mathrm{CH}-$ Sizing & \\
\hline & & & & d & 0 & $\bar{O}$ & $1.00 \mathrm{E}+00$ & & AMWTF & CH - Incineration/Thermal Desorption & \\
\hline & & & & $\bar{e}$ & $U$ & $\bar{O}$ & $1.00 E+00$ & & AMWTF & $\mathrm{CH}$ - Vitrification & \\
\hline & & & & $f$ & 0 & $\bar{D}$ & $1.00 E+00$ & & SCDF & Disposal - Contact Handled & \\
\hline
\end{tabular}

Note: Where provided, media percentages are rough estimates used to facilitate treatment planning. They do not necessarily imply an accurate knowledge of waste stream composition, and were not derived for use outside this conceptual planning exercise.

Describe any special or unique technical concerns related to the treatment of this waste stream that would impact the use or implementation of standard hazardous waste treatment methods. 


\section{KW-WOO9}

\section{LW: Contact}

\section{A. IDENTIFICATION AND DESCRIPTION}

Waste Stream ID: KW-W009

W. S. Name:

Content Code:

FFCA ID:

$$
\text { SOILS }
$$

\begin{tabular}{ll}
\hline KW-W009 \\
\hline KW
\end{tabular}

Waste Stream Description:

Waste soils from maintenance, $D \& D$, etc. This mixed waste stream has not yet been generated. This stream will not be a moratorium waste or reclassified TRU waste.

\section{B. GENERATION SITE/PROCESS DESCRIPTION}

The following questions, concerning the generation site and process, should be answered even if the waste stream is no longer generated.

Name of Site Generating Waste (e.g., INEL, Rocky Flats, etc.): KAPL

Location of activities (Area and Building):

Locations where contaminated soil may exist include areas adjacent to past and existing radiological facilities.

Operations (functions) performed in building: Site characterization is in process.

Description of Process Generating Waste:

Excavation of soils. 
KW. Woog

soilis

\section{PROJECTED WASTE GENERATION}

Is this waste stream still generated?

OYes ONo

If yes, when is the expected generation termination date?

\begin{tabular}{|c|c|c|c|c|c|}
\hline \multicolumn{6}{|c|}{ Current Projections } \\
\hline Est. Date & Start Year & Interval & Vol (m3) & Mass (kg) & Source of Estimate \\
\hline $11 / 30 / 93$ & 1995 & 1 & 4.20 & $2,000.00$ & 1993-FFCA \\
\hline 11/30/93 & 1998 & 1 & 0.84 & 400.00 & 1993-FFCA \\
\hline $11 / 30 / 93$ & 1999 & 1] & 0.84 & 400.00 & 1993-FFCA \\
\hline \multicolumn{6}{|c|}{ Historical Projections } \\
\hline Est. Date & Start Year & Interval & Vol (m3) & Mass (kg) & Source of Estimate \\
\hline $11 / 30 / 93$ & 1998 & 5 & 4.20 & $2,000.00$ & 1993-FFCA \\
\hline $11 / 30 / 93$ & 2003 & 20 & 8.40 & $4,000.00$ & 1993-FFCA \\
\hline
\end{tabular}



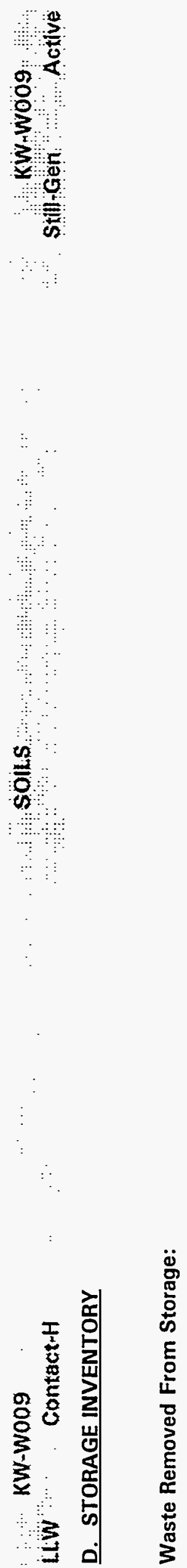


\section{KWW:Woog \\ HLW OContact-H \\ E. RADIATION CHARACTERISTICS \\ Radioactive Waste Type:

\begin{tabular}{|ll|}
\hline O lLW & OalllW $\bigcirc$ TRU \\
OHLW & O Non-Rad \\
\hline
\end{tabular} \\ Mixed Waste: \\ Handling: \\ OYes ONo \\ O Contact O Remote}

\section{F. TRU ALPHA ACTIVITY DISTRIBUTION}

Radionuclide Contamination Accessiblity:

External Surface OYes O No OUnknown

Internal Surface OYes $O$ No OUnknown

Dispersed Through Matrix

OYes ONo OUnknown

\section{Activity Levels}

Transuranic Alpha Activity:

Uranium/Thorium Alpha Activity:

Beta/Gamma Activity

Surface Neutron Activity:

Total Activity:

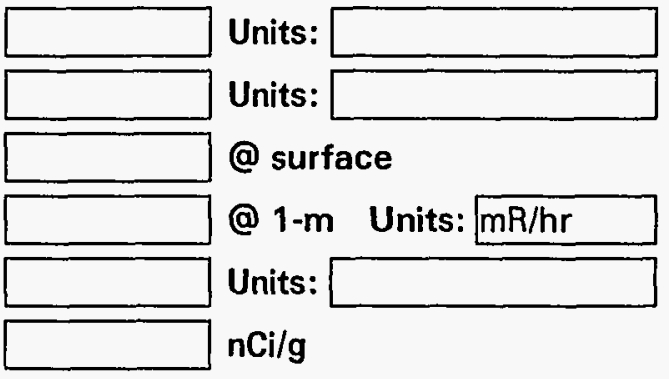

This waste stream is not identified as a TRU or a-LLW. 

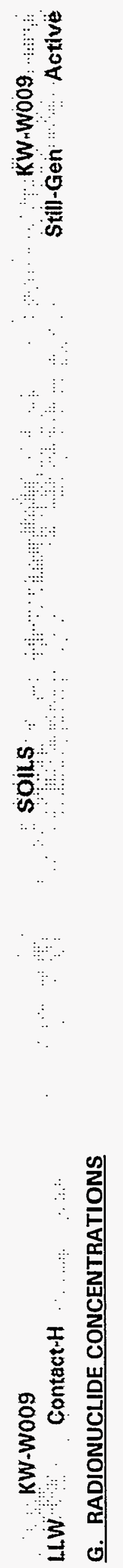
KW-Woog

soins:

LWW Contact-H

\section{H. MATRIX CHARACTERISTICS}

Primary Matrix Code: $\$ 4000$

Description: Soil/Gravel: : :

Combustibility: $\bigcirc$ Combustible (>90\%)

Mixed $(10 \%-90 \%)$

Noncombustible $(<10 \%)$

\section{MATRIX COMPOSITION}

\begin{tabular}{|c|c|c|c|c|c|c|}
\hline & & & \multicolumn{4}{|c|}{ Composition } \\
\hline Code & Matrlx Description & Material & Typlcal & LL & $\overline{U L}$ & Units \\
\hline 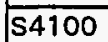 & Soil/Gravel.Soil.. & Soils & & & & \\
\hline
\end{tabular}


KW-Woog

\section{Liw : $\because$ Contact-H}

\section{J. HAZARDOUS CONSTITUENTS}

\begin{tabular}{|c|c|c|c|c|c|c|c|c|}
\hline & & & \multicolumn{4}{|c|}{ Concentration } & \multirow[b]{2}{*}{ Basis } & \multirow[b]{2}{*}{ Conc. Type } \\
\hline Code & Hazardous Waste Description & Treatment Subcategory & Typical & $\mathbf{L L}$ & UL & Units & & \\
\hline D005 & Barium & & & & & & A & Total \\
\hline D006 & Cadmium & & & & & & A & Total \\
\hline D008 & Lead & & & & & & A & Total \\
\hline 0009 & Mercury & Low mercury $<260 \mathrm{mg} / \mathrm{kg}$ total mercury & & & & & A & Total \\
\hline D011 & Silver & & & & & & A & Total \\
\hline D035 & Methyl ethyl ketone & Methyle ethyl ketone managed in non-CWA/no & & & & & A & Total \\
\hline D039 & Tetrachloroethylene & Tetrachloroethylene managed in non-CWA/non & & & & & $\bar{A}$ & Total \\
\hline 0040 & Trichloroethylene & Trichloroethylene managed in non-CWA/non-C & & & & & A & Total \\
\hline FO01 & Spent halogenated solvents used in degreasing & Unknown & & & & & A & Total \\
\hline$\overline{\mathrm{FOO2}}$ & Spent halogenated solvents & Unknown & & & & & A & Total \\
\hline F003 & Spent non-halogenated solvents & Unknown & & & & & A & Total \\
\hline
\end{tabular}


KẄ:Woog

UWW Contact-H

K. POLYCHLORINATED BIPHENYLS (PCBS)

Is this waste stream PCB contaminated?

\section{OYes O No}

ume $\%$

If yes, what portion of the waste is PCB contaminated (volume \%):

If $<100 \%$, can the PCB contaminated waste be segregated and treated separately?

What is the PCB concentration in ppm?

\section{TREATMENT PLANS}

\begin{tabular}{|c|c|c|c|c|c|c|c|c|c|c|c|}
\hline $\begin{array}{l}\text { Opt. } \\
\#\end{array}$ & $\begin{array}{c}\text { Media Type } \\
\text { (If more than one) }\end{array}$ & $\begin{array}{c}\text { Matrix } \\
\text { Code }\end{array}$ & $\begin{array}{l}\text { Vol. \% } \\
\text { (If }\end{array}$ & Step & JIT & sc & CIF & $\begin{array}{l}\text { Trans. } \\
\text { Miles }\end{array}$ & $\begin{array}{l}\text { Facility } \\
\text { Abbr. }\end{array}$ & Unit Name & Comments \\
\hline 1 & & 54000 & & a & (O) & $\overline{0}$ & $1.00 E+00$ & & TRANS & Transport-LLW & \\
\hline & & & & $\mathbf{b}$ & (O) & $\bar{O}$ & $1.00 \mathrm{E}+00$ & & AMWTF & $\mathrm{CH}$ - Opening \& Sorting & \\
\hline & & & & $\bar{c}$ & O & $\bar{O}$ & $1.00 E+00$ & & AMWTF & CH - Sizing & \\
\hline & & & & $\bar{d}$ & $\bar{O}$ & 0 & $1.00 \mathrm{E}+00$ & & AMWTF & $\mathrm{CH}$ - Incineration/Thermal Desorption & \\
\hline & & & & $\bar{\theta}$ & $\mathrm{O}$ & 0 & $1.00 E+00$ & & AMWTF & CH-Vitrification & \\
\hline & & & & $f$ & 0 & 0 & $1.00 \mathrm{E}+00$ & & SCDF & Disposal - Contact Handled & \\
\hline
\end{tabular}

Note: Where provided, media percentages are rough estimates used to facilitate treatment planning. They do not necessarily imply an accurate knowledge of waste stream composition, and were not derived for use outside this conceptual planning exercise.

Describe any special or unique technical concerns related to the treatment of this waste stream that would impact the use or implementation of standard hazardous waste treatment methods. 


\section{KW-wo10 \\ Liw: \\ Contagt-H}

\section{A. IDENTIFICATION AND DESCRIPTION}

Waste Stream ID: KW-W010

W. S. Name:

Content Code:

FFCA ID:

\begin{tabular}{|l|}
\hline Hg CONTAMINATED ORGANICS \\
\hline$*$ \\
\hline$K W-W 010$ \\
\hline
\end{tabular}

\section{Waste Stream Description:}

This waste stream consists of organic materials which have become contaminated with radioactivity and mercury greater than $260 \mathrm{ppm}$. This waste stream has not been generated.

The homogeneity of this waste stream is unknown until generated. This waste stream will not be moratorium or reclassified TRU waste.

\section{Hg CONTAMINATED ORGANIGS}

KW-WO10 Still-Gën . Active

\section{B. GENERATION SITE/PROCESS DESCRIPTION}

The following questions, concerning the generation site and process, should be answered oven if the waste stream is no longer generated.

Name of Site Generating Waste (e.g., INEL, Rocky Flats, etc.): KAPL-Windsor

\section{Location of activities (Area and Building):}

Various locations on site.

Operations (functions) performed in building:

Various operations on site.

Description of Process Generating Waste:

Various laboratory activities (e.g., analysis, testing, maintenance). 


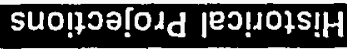

\begin{tabular}{|c|c|c|c|c|c|}
\hline$d 1 s a-666 t$ & $00^{\circ} 62$ & 500 & t & $266 \mathrm{k}$ & $66 / S L / L 6$ \\
\hline 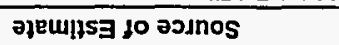 & (бy) ssew & $(\varepsilon(u) 10 \wedge$ & 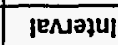 & 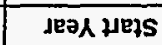 & э)ष् Is \\
\hline
\end{tabular}

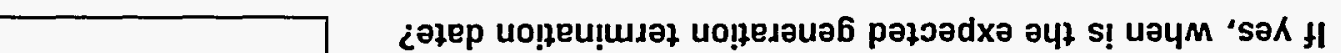

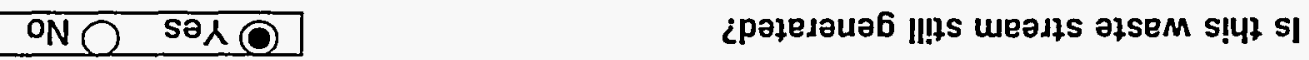

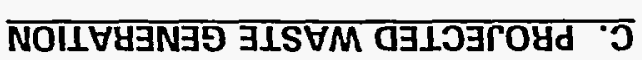
annot mog-urs

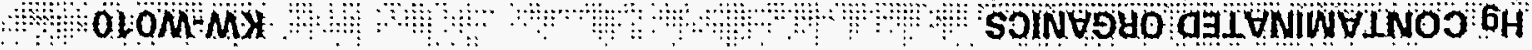


KW-W010

LLW:: : Contact-H

D. STORAGE INVENTORY

Waste Removed From Storage: 


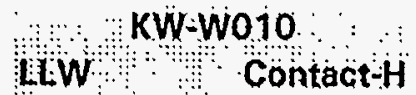

\section{E. RADIATION CHARACTERISTICS}

Radioactive Waste Type:

\begin{tabular}{|ll|}
\hline O LLW & Oa-LLW O TRU \\
OHLW & O Non-Rad \\
\hline
\end{tabular}

Mixed Waste:

Handling:

OYes ONo

$O$ Contact $O$ Remote

Radionuclide Contamination Accessiblity:

\begin{tabular}{rlrl|}
\cline { 2 - 4 } External Surface & Y Yes & ONo & O Unknown \\
Internal Surface & OYes & ONo & O Unknown \\
\cline { 2 - 5 } &
\end{tabular}

This waste stream is not identified as a TRU or a-LLW.

\section{Activity Levels}

Transuranic Alpha Activity:

Uranium/Thorium Alpha Activity:

Beta/Gamma Activity:

Surface Neutron Activity:

Total Activity:

\begin{tabular}{|c|c|}
\hline & Units: [ \\
\hline & Units: [ \\
\hline & @ surface \\
\hline & @ 1-m Units: $\mathrm{mR} / \mathrm{hr}$ \\
\hline & Units: \\
\hline & $\mathrm{nCi} / \mathrm{g}$ \\
\hline
\end{tabular}




\section{G. RADIONUCLIDE CONCENTRATIONS}

\begin{tabular}{|c|c|c|c|c|c|c|}
\hline \multirow[b]{2}{*}{ Isotope } & \multicolumn{3}{|c|}{ Specific Activity } & \multirow[b]{2}{*}{ Units } & \multirow[b]{2}{*}{ Basis Code(s) } & \multirow[b]{2}{*}{ Basis Desc. (if other) } \\
\hline & Typical & Lower Limit & Upper Limit & & & \\
\hline \multicolumn{7}{|l|}{$\mathrm{Ni}-59$} \\
\hline \multicolumn{7}{|l|}{ Fe-55 } \\
\hline \multicolumn{7}{|l|}{ C-14 } \\
\hline \multicolumn{7}{|l|}{ Co-58 } \\
\hline \multicolumn{7}{|l|}{$M n-54$} \\
\hline \multicolumn{7}{|l|}{$\mathrm{Ni}-63$} \\
\hline \multicolumn{7}{|l|}{ Co-60 } \\
\hline $\mathrm{H}-3$ & & & & & & \\
\hline
\end{tabular}

A. Passive-Active Neutron (PAN)

B. Segmented Gamma Scan (SGS)

F. Accountability

C. Passive-Active Neutron/Segmented Gamma Scan (PAN/SGS) H. Multiple Energy Gamma Analysis (MEGAS)

D. Radiochemical Analysis

E. Mass Spectrometry

j. Other 
Kพพัพั้öo

HG CONTAMINATED ORGANICS

Liw Contact-H

a

\section{H. MATRIX CHARACTERISTICS}

Primary Matrix Code: $\mathbf{S 3 2 0 0}$

Description: Homogeneous Solids: Organic Homogeneous solids: :

Combustibility: $\bigcirc$ Combustible (>90\%)

Mixed $(10 \%-90 \%)$

Noncombustible $(<10 \%)$

\section{MATRIX COMPOSITION}

\begin{tabular}{|c|c|c|c|c|c|c|}
\hline & & & \multicolumn{4}{|c|}{ Compositlon } \\
\hline Code & Matrix Description & Material & Typlcal & LL & UL & Units \\
\hline $\mathbf{S 3 2 9 0}$ & Homogeneous Solids.Organic Homogeneous solids.Unknown/Other Organic Homoge & & 100 & 100 & 100 & $\%$ vol \\
\hline
\end{tabular}


KW.WO10

LLW: $\because$ Contact-H

\section{J. HAZARDOUS CONSTITUENTS}

\begin{tabular}{|c|c|c|c|c|c|c|c|c|}
\hline & & & \multicolumn{4}{|c|}{ Concentration } & \multirow[b]{2}{*}{ Basis } & \\
\hline Code & Hazardous Waste Description & Treatment Subcategory & Typical & LL. & $\mathbf{U L}$ & Units & & Conc. Type \\
\hline$\overline{D 004}$ & Arsenic & & & & & & A & Total \\
\hline$\overline{D 005}$ & Barium & & & & & & A & Total \\
\hline D006 & Cadmium & & & & & & A & Total \\
\hline D007 & Chromium & Chromium (Total) & & & & & A & Total \\
\hline D008 & Lead & & & & & & A & Total \\
\hline D009 & Mercury & Elemental mercury contaminated with radioact & & & & & A & Total \\
\hline D010 & Selenium & & & & & & $\bar{A}$ & Total \\
\hline D011 & Silver & & & & & & A & Total \\
\hline D018 & Benzene & Benzene managed in non-CWA/non-CWA-equi & & & & & A & Total \\
\hline D035 & Methyl ethyl ketone & Methyle ethyl ketone managed in non-CWA/no & & & & & A & Total \\
\hline D036 & Nitrobenzene & 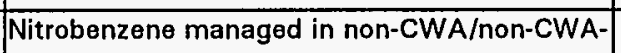 & & & & & $\bar{A}$ & Total \\
\hline D039 & Tetrachloroethylene & Tetrachloroethylene managed in non-CWA/non & & & & & $\bar{A}$ & Total \\
\hline D040 & Trichloroethylene & Trichloroethylene managed in non-CWA/non-C & & & & & A & Total \\
\hline
\end{tabular}

\section{Hg CONTAMINATED ORGANICS}

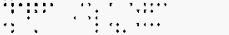

KW WWO10

Still:Gen Active 
KW-Woio

HiW ContagteH

\section{K. POLYCHLORINATED BIPHENYLS (PCBS)}

Is this waste stream PCB contaminated? OYes O No O Unknown

If yes, what portion of the waste is PCB contaminated (volume \%):

If $<100 \%$, can the PCB contaminated waste be segregated and treated separately? OYes ONo O Unknown

What is the PCB concentration in ppm?

\section{TREATMENT PLANS}

\begin{tabular}{|c|c|c|c|c|c|c|c|c|c|c|c|}
\hline $\begin{array}{c}\text { Opt. } \\
\#\end{array}$ & $\begin{array}{l}\text { Media Type } \\
\text { (If more than onel }\end{array}$ & $\begin{array}{c}\text { Matrix } \\
\text { Code }\end{array}$ & $\begin{array}{l}\text { Vol. \% } \\
\text { lif }\end{array}$ & Step & JIT & sc & CIF & $\begin{array}{l}\text { Trans. } \\
\text { Miles }\end{array}$ & $\begin{array}{l}\text { Facility } \\
\text { Abbr. }\end{array}$ & Unit Name & Comments \\
\hline \multirow[t]{5}{*}{1} & & 55440 & & a & () & 0 & $1.00 E+00$ & & TRANS & Transport - LLW & \\
\hline & & & & $\mathbf{b}$ & 0 & 0 & $1.00 E+00$ & & WROC & Sizing & \\
\hline & & & & $d$ & O & 0 & $1.00 E+00$ & & WERF & Incineration - Controlled Air Incinerator & \\
\hline & & & & e & 0 & 0 & $1.00 E+00$ & & WERF & Stabilization - Portland Cement & \\
\hline & & & & $f$ & 0 & 0 & $1.00 E+00$ & & SCDF & Disposal - Contact Handled & \\
\hline
\end{tabular}

Note: Where provided, media percentages are rough estimates used to facilitate treatment planning. They do not necessarily imply an accurate knowledge of waste stream composition, and were not derived for use outside this conceptual planning exercise.

Describe any special or unique technical concerns related to the treatment of this waste stream that would impact the use or implementation of standard hazardous waste treatment methods. 
Contact-H

\section{A. IDENTIFICATION AND DESCRIPTION}

Waste Stream ID: KW-W011

W. S. Name:

Content Code:

FFCA ID:

\begin{tabular}{|l|}
\hline Hg CONTAMINATED INORGANICS \\
\hline KW-W011 \\
\hline
\end{tabular}

\section{Waste Stream Description:}

This waste stream consists of inorganic materials which has become contaminated with

radioactivity and mercury with concentrations greater than $260 \mathrm{ppm}$. The homogeneity of this waste stream within a container is unknown. This waste stream will not be a moratorium or reclassified TRU waste.

\section{Hg CONTAMINATED INORGANICS}

KW.W011 Stiil-Gen Active

\section{B. GENERATION SITE/PROCESS DESCRIPTION}

The following questions, concerning the generation site and process, should be answered even if the waste stream is no longer generated.

Name of Site Generating Waste (e.g., INEL, Rocky Flats, etc.): KAPL-Windsor

\section{Location of activities (Area and Building):}

Various locations on site.

Operations (functions) performed in building:

Various operations on site.

Description of Process Generating Waste:

Various laboratory activities (e.g., analysis, testing, maintenance). 
MUWW011

LWW Contagt

C. PROJECTED WASTE GENERATION

Is this waste stream still generated?

If yes, when is the expected generation termination date?

Current Projections

\begin{tabular}{|r|r|r|r|r|r|}
\hline Est. Date & Start Year & Interval & \multicolumn{1}{|c|}{ Vol (m3) } & \multicolumn{1}{|c|}{ Mass (kg) } & Source of Estimate \\
\hline $11 / 15 / 94$ & 1997 & 1 & 0.50 & 79.00 & 1994 DSTP \\
\hline
\end{tabular}

Historical Projections
Hg CONTAMINATED INORGANICS

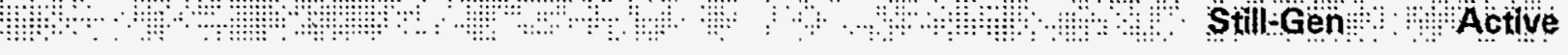

\section{OYes ONo}




\section{KW-Wo11 \\ Contact-H \\ D. STORAGE INVENTORY}

Waste Removed From Storage: 
LLW KW-WOT1 ContaGtH H GONTAMINATEDINORGANICS

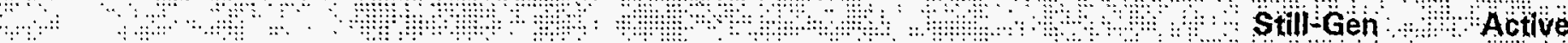

\section{E. RADIATION CHARACTERISTICS}

Radioactive Waste Type:

$\begin{array}{ll}\text { OllW } & \text { Oa-LLW } \bigcirc \text { TRU } \\ \text { OhLW } & \text { O Non-Rad }\end{array}$

Mixed Waste:

\begin{tabular}{|l|}
\hline OYes ONo \\
\hline O Contact O Remote \\
\hline
\end{tabular}

Radionuclide Contamination Accessiblity:

External Surface

Internal Surface

Dispersed Through Matrix

\begin{tabular}{|c|c|c|}
\hline OYes & ONo & OUnknown \\
\hline OYes & ONo & OUnknown \\
\hline
\end{tabular}

Activity Levels

Transuranic Alpha Activity:

Uranium/Thorium Alpha Activity:

Beta/Gamma Activity:

Surface Neutron Activity:

Total Activity:

$\square$

Units:

@ surface Units:

$\mathrm{nCi} / \mathrm{g}$

\section{F. TRU ALPHA ACTIVITY DISTRIBUTION}

This waste stream is not identified as a TRU or a-LLW.

@ 1-m Units: $\mathrm{mR} / \mathrm{hr}$ 


\section{LLW}

\section{G. RADIONUCLIDE CONCENTRATIONS}

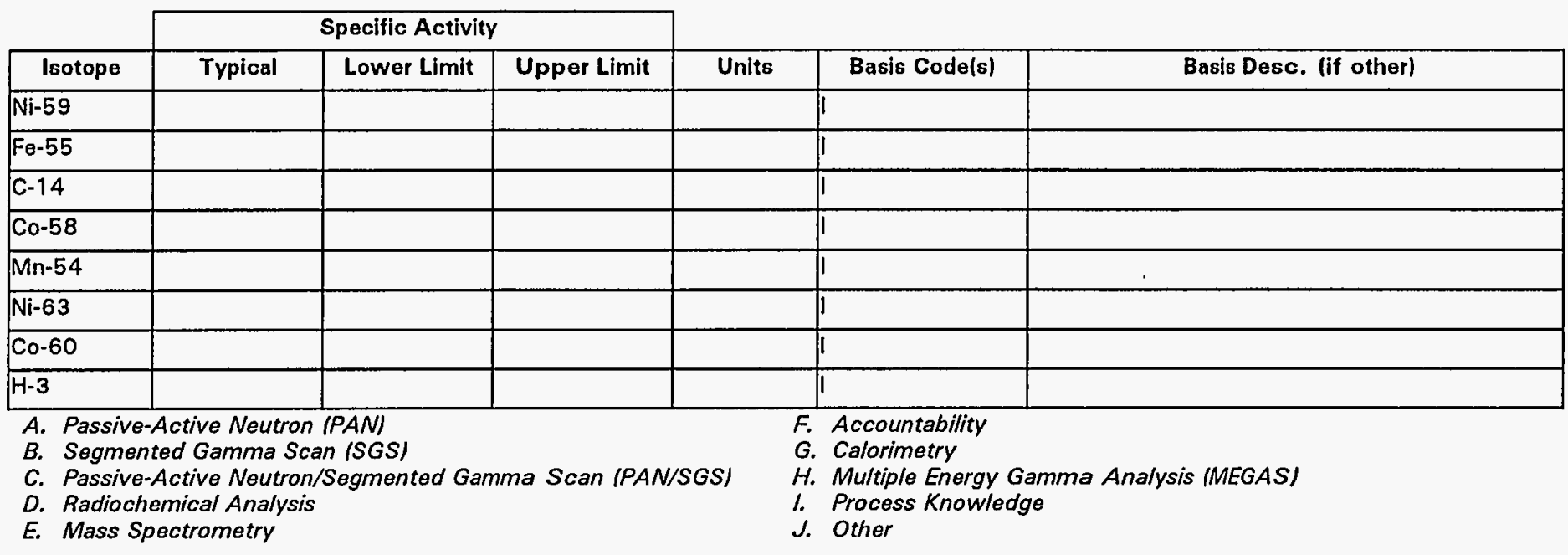




\section{$\therefore \quad K W-W 017$}

LWW Contagt H

\section{H. MATRIX CHARACTERISTICS}

Primary Matrix Code: $\mathbf{S 3 1 0 0}$

Description: Homogeneous Solids: Inorganic Homogeneous Solids: :

\section{Combustibility: $\bigcirc$ Combustible (>90\%) \\ Mixed (10\%-90\%) \\ Noncombustible $(<10 \%)$}

\section{MATRIX COMPOSITION}


KW-W011

LiW

Hg CONTAMINATED INORGANICS

KW-W011 :

Still-Gen : Active

\section{J. HAZARDOUS CONSTITUENTS}

\begin{tabular}{|c|c|c|c|c|c|c|c|c|}
\hline & & & \multicolumn{4}{|c|}{ Concentration } & & \\
\hline Code & Hazardous Waste Description & Treatment Subcategory & Typical & LL & UL & Units & Basis & Conc. Type \\
\hline$\overline{D 004}$ & Arsenic & & & & & & A & Total \\
\hline D005 & Barium & & & & & & $\bar{A}$ & Total \\
\hline$D 006$ & Cadmium & & & & & & A & Total \\
\hline$\overline{D 007}$ & Chromium & Chromium (Total) & & & & & A & Total \\
\hline$\overline{D 008}$ & Lead & & & & & & A & Total \\
\hline D009 & Mercury & Elemental mercury contaminated with radioact & & & & & A & Total \\
\hline D010 & Selenium & & & & & & A & Total \\
\hline
\end{tabular}




\section{Lit. : Contact-H}

\section{K. POLYCHLORINATED BIPHENYLS (PCBS)}

Is this waste stream PCB contaminated?

OYes

ONo Unknown

If yes, what portion of the waste is PCB contaminated (volume \%):

If $<100 \%$, can the PCB contaminated waste be segregated and treated separately? $O$ Yes O No O Unknown

What is the PCB concentration in ppm?

If only a portion of the waste is PCB contaminated, report values for contaminated portion.

\section{TREATMENT PLANS}

\begin{tabular}{|c|c|c|c|c|c|c|c|c|c|c|c|}
\hline Opt. & $\begin{array}{c}\text { Media Type } \\
\text { (If more than onel }\end{array}$ & $\begin{array}{c}\text { Matrix } \\
\text { Code }\end{array}$ & $\begin{array}{l}\text { Vol. \% } \\
\text { IIf }\end{array}$ & Step & JIT & sc & ClF & $\begin{array}{l}\text { Trans. } \\
\text { Miles }\end{array}$ & $\begin{array}{l}\text { Facility } \\
\text { Abbr. }\end{array}$ & Unit Name & Comments \\
\hline \multirow[t]{4}{*}{1} & & $\mathbf{S 5 4 2 0}$ & & $\mathbf{a}$ & $\mathbf{O}$ & 0 & $1.00 E+00$ & & TRANS & Transport - LLW & \\
\hline & & & & b & $\mathbf{O}$ & 0 & $1.00 E+00$ & & WROC & Sizing & \\
\hline & & & & d & 0 & 0 & $1.00 \mathrm{E}+00$ & & WERF & Stabilization - Portland Cement & \\
\hline & & & & e & 0 & 0 & $1.00 \mathrm{E}+00$ & & SCDF & Disposal - Contact Handled & \\
\hline
\end{tabular}

Note: Where provided, media percentages are rough estimates used to facilitate treatment planning. They do not necessarily imply an accurate knowledge of waste stream composition, and were not derived for use outside this conceptual planning exercise.

Describe any special or unique technical concerns related to the treatment of this waste stream that would impact the use or implementation of standard hazardous waste treatment methods. 


\section{KW.W012

LiW Contact-H

\section{A. IDENTIFICATION AND DESCRIPTION}

Waste Stream ID: KW-W012

W. S. Name: $\quad$ ELEMENTAL Hg

Content Code:

FFCA ID:

$$
\text { KW-W012 }
$$

\section{Waste Stream Description:}

This waste stream consists of mercury in its elemental (liquid) form contaminated with

radioactivity. This waste will be homogeneous within each individual container. This waste stream will not be a moratorium or reclassified TRU waste.

\section{B. GENERATION SITE/PROCESS DESCRIPTION}

The following questions, concerning the generation site and process, should be answered even if the waste stream is no longer generated.

Name of Site Generating Waste (e.g., INEL, Rocky Flats, etc.): KAPL-Windsor

Location of activities (Area and Building):

Various locations on site.

Operations (functions) performed in building:

Various operations on site.

Description of Process Generating Waste:

Waste from maintenance and testing. 


\section{KWWWo12}

LWW Uolontact H

C. PROJECTED WASTE GENERATION

Is this waste stream still generated?

If yes, when is the expected generation termination date?

\section{Current Projections}

\begin{tabular}{|c|r|r|r|r|r|}
\hline Est. Date & Start Year & Interval & Vol (m3) & Mass (kg) & Source of Estlmate \\
\hline $11 / 15 / 94$ & 1997 & 1 & 0.00 & 75.00 & 1994 DSTP \\
\hline
\end{tabular}

Historical Projections 
KW.W012

LiW Contact-H
D. STORAGE INVENTORY

Waste Removed From Storage:
ELEMENTAL Hg

KW.WO12

Still-Gen ... Active 
KW-Wo12 LWW ContactH

\section{E. RADIATION CHARACTERISTICS}

Radioactive Waste Type:

\begin{tabular}{|ll|}
\hline OLLW & Oa-LLW O TRU \\
OHLW & O Non-Rad \\
\hline
\end{tabular}

Mixed Waste:

\begin{tabular}{|l|}
\hline OYes O No \\
\hline O Contact O Remote \\
\hline
\end{tabular}

Radionuclide Contamination Accessiblity:

\begin{tabular}{|c|c|c|c|}
\hline External Surface & OYes & ONo & O Unknown \\
\hline rface & OYes & ONo & O) Unknown \\
\hline spersed Through & Yes & ONo & O Unknown \\
\hline
\end{tabular}

\section{Activity Levels}

Transuranic Alpha Activity:

Uranium/Thorium Alpha Activity:

Beta/Gamma Activity:

Surface Neutron Activity:

Total Activity:

\begin{tabular}{|c|c|c|}
\hline & Units: [ & \\
\hline & Units: : & \\
\hline & @ surf & ace \\
\hline & @ 1-m & Units: $\mathrm{mR} / \mathrm{hr}$ \\
\hline & Units: & \\
\hline & $\mathrm{nCi} / \mathrm{g}$ & \\
\hline
\end{tabular}

$\mathrm{nCl} / \mathrm{g}$

\section{F. TRU ALPHA ACTIVITY DISTRIBUTION}

This waste stream is not identified as a TRU or a-LLW. 
KW.W012

\section{LlW : ContactrH}

\section{G. RADIONUCLIDE CONCENTRATIONS}

\begin{tabular}{|c|c|c|c|c|c|c|}
\hline & \multicolumn{3}{|c|}{ Specific Activity } & \multirow[b]{2}{*}{ Units } & \multirow[b]{2}{*}{ Basis Code(s) } & \multirow[b]{2}{*}{ Basis Desc. (if other) } \\
\hline Isotope & Typical & Lower Limit & Upper Limit & & & \\
\hline \multicolumn{7}{|l|}{$\mathrm{Ni-59}$} \\
\hline \multicolumn{7}{|l|}{ Fe-55 } \\
\hline \multicolumn{7}{|l|}{ C-14 } \\
\hline \multicolumn{7}{|l|}{ Co-58 } \\
\hline \multicolumn{7}{|l|}{$M n-54$} \\
\hline \multicolumn{7}{|l|}{$\mathrm{Ni}-63$} \\
\hline \multicolumn{7}{|l|}{ Co-60 } \\
\hline $\mathrm{H}-3$ & & & & & $i$ & \\
\hline $\begin{array}{l}\text { A. Passiv } \\
\text { B. Segm } \\
\text { C. Passiv } \\
\text { D. Radio } \\
\text { E. Mass }\end{array}$ & $\begin{array}{l}\text { ive Neutr } \\
\text { Gamma } \\
\text { ive Neutr } \\
\text { cal Analy } \\
\text { rometry }\end{array}$ & $\begin{array}{l}\text { PAN) } \\
\text { (SGS) } \\
\text { egmented GaI }\end{array}$ & a Scan $(P$ & & $\begin{array}{l}\text { Accountability } \\
\text { Calorimetry } \\
\text { Multiple Energy } \\
\text { Process Knowlec } \\
\text { Other }\end{array}$ & is (MEGAS) \\
\hline
\end{tabular}

KWMWO12

Still-Gẹi: Active 
KW-Wo12

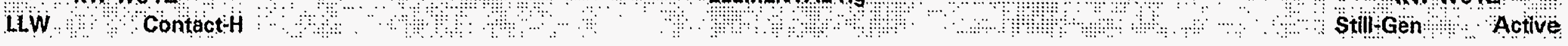

H. MATRIX CHARACTERISTICS

Primary Matrix Code: $X 7100$

Description: Special Waste: Elemental Mercury: :

Combustibility: $\begin{aligned} & \bigcirc \text { Combustible }(>90 \%) \\ & \bigcirc \text { Mixed }(10 \%-90 \%) \\ & \bigcirc \text { Noncombustible }(<10 \%)\end{aligned}$

\section{MATRIX COMPOSITION}

\begin{tabular}{|c|c|c|c|c|c|c|}
\hline & & & \multicolumn{4}{|c|}{ Composition } \\
\hline Code & Matrix Description & Material & Typlcal & $\overline{L L}$ & UL & Units \\
\hline$x 7100$ & Special Waste.Elemental Mercury.. & & 100 & 100 & 100 & $\%$ vol \\
\hline
\end{tabular}




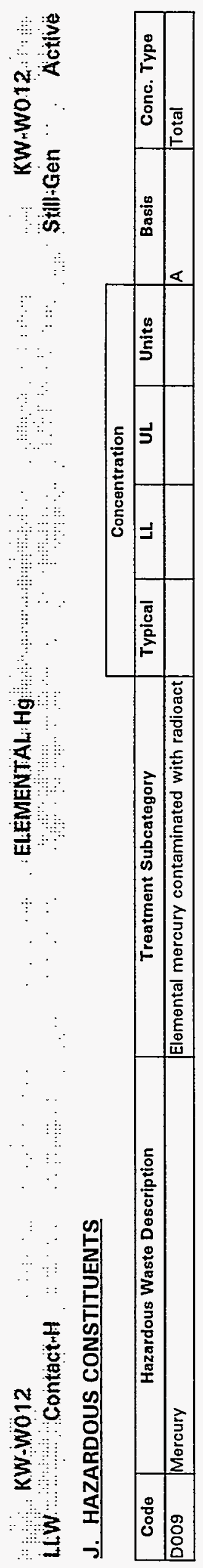




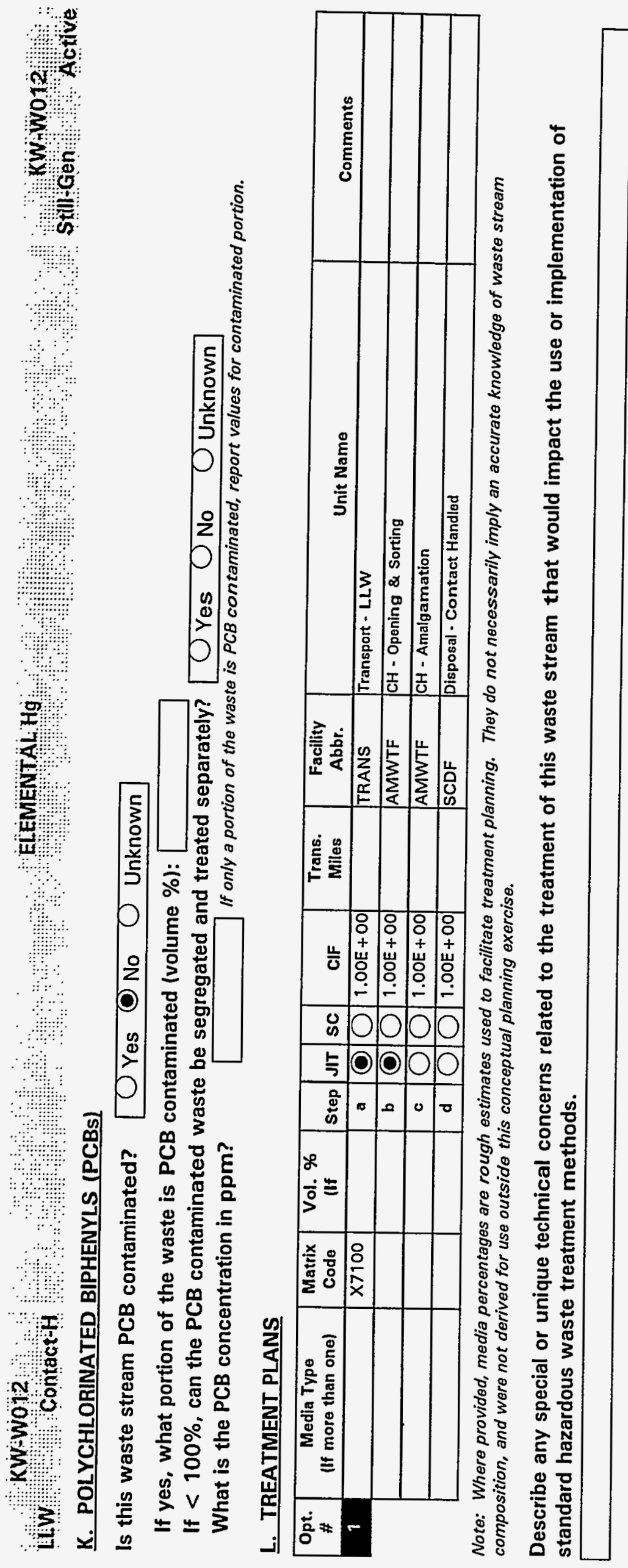

$x$
$y$
$y$
0
0
0
0

ह

กิ 


LA-W901
HLW Contact-H
A. IDENTIFICATION AND DESCRIPTION
Waste Stream ID: LA-W901
W. S. Name: IPA WASTES
Content Code:
FFCA ID:
Waste Stream Description:
This waste is an aqueous mixture of isopropyl alcohol and ammonium hydroxide.

LA:W901:

\section{B. GENERATION SITE/PROCESS DESCRIPTION}

The following questions, concerning the generation site and process, should be answered even if the waste stream is no longer generated.

Name of Site Generating Waste (e.g., INEL, Rocky Flats, etc.):

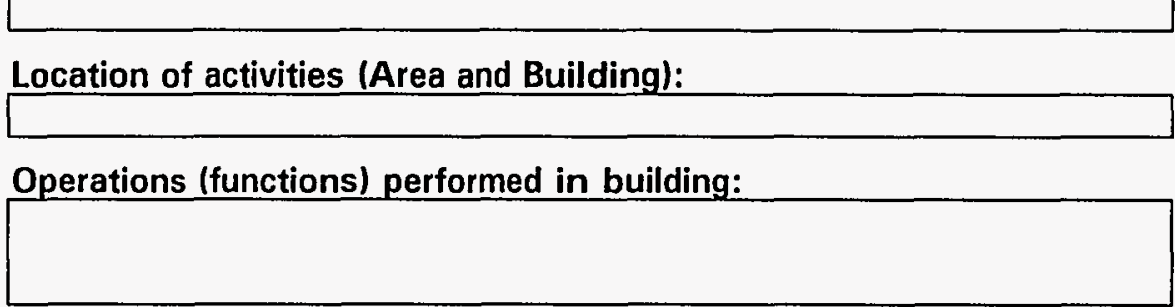

\begin{tabular}{|l|} 
Description of Process Generating Waste: \\
\hline
\end{tabular} 
$\therefore A-W 901$

LlWW ContactH

C. PROJECTED WASTE GENERATION

Is this waste stream still generated?

If yes, when is the expected generation termination date?

Current Projections

\begin{tabular}{|r|r|r|r|r|r|}
\hline Est. Date & Start Year & Interval & Vol (m3) & Mass (kg) & Source of Estimate \\
\hline $5 / 2 / 95$ & 1996 & 1 & 0.01 & & Generator Update \\
\hline
\end{tabular}

\begin{tabular}{|l|l|l|l|l|}
\hline $5 / 2 / 95$ & 1996 & 1 & 0.01 \\
\hline \multicolumn{4}{c|}{ Historical Projections } \\
\hline
\end{tabular}

\section{O Yes ONo}

Ores No

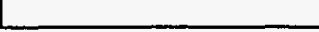

LAV WO1: Stilligen lon Active 


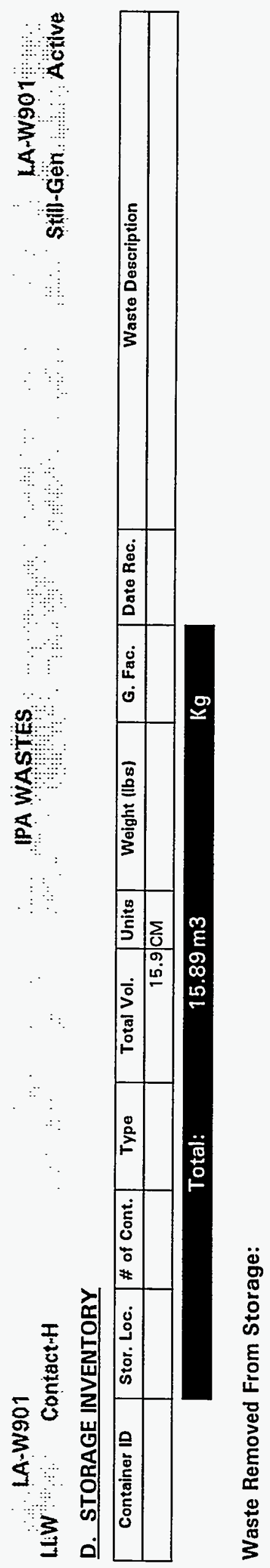


LA:W90̈1

\section{E. RADIATION CHARACTERISTICS}

Radioactive Waste Type:

\begin{tabular}{|ll|}
\hline OllW & Oa-LlW O TRU \\
OhLW & O Non-Rad \\
\hline
\end{tabular}

Mixed Waste:

Handling:

\begin{tabular}{|l|}
\hline OYes ONo \\
\hline O Contact ORemote \\
\hline
\end{tabular}

Radionuclide Contamination Accessiblity:

\begin{tabular}{rllll}
\cline { 2 - 4 } External Surface & OYes & O No & O Unknown \\
\cline { 2 - 5 } Internal Surface & OYes & O No & O Unknown \\
\cline { 2 - 5 } & &
\end{tabular}

\section{F. TRU ALPHA ACTIVITY DISTRIBUTION}

This waste stream is not identified as a TRU or a-LLW.

\section{Activity Levels}

Transuranic Alpha Activity:

Uranium/Thorium Alpha Activity:

Beta/Gamma Activity:

Surface Neutron Activity:

Total Activity:

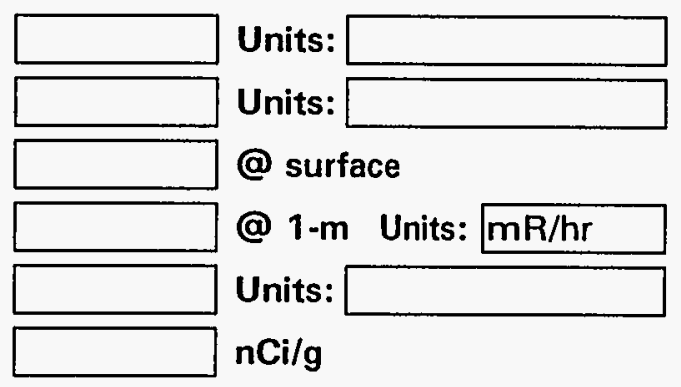




\section{G. RADIONUCLIDE CONCENTRATIONS}

\begin{tabular}{|c|c|c|c|c|c|c|}
\hline \multirow{2}{*}{ Isotope } & \multicolumn{3}{|c|}{ Specific Activity } & \multirow[b]{2}{*}{ Units } & \multirow[b]{2}{*}{ Basis Code(s) } & \multirow[b]{2}{*}{ Basis Desc. (if other) } \\
\hline & Typical & Lower Limit & Upper Limit & & & \\
\hline \multicolumn{7}{|l|}{ U-235 } \\
\hline \multicolumn{5}{|c|}{$\begin{array}{l}\text { A. Passive-Active Neutron (PAN) } \\
\text { B. Segmented Gamma Scan (SGS) } \\
\text { C. Passive-Active Neutron/Segmented Gamma Scan (PAN/SGS) } \\
\text { D. Radiochemical Analysis } \\
\text { E. Mass Spectrometry }\end{array}$} & $\begin{array}{l}\text { F. Accountability } \\
\text { G. Calorimetry } \\
\text { H. Multiple Energy } \\
\text { I. Process Knowle } \\
\text { J. Other }\end{array}$ & is (MEGAS) \\
\hline
\end{tabular}


LA-WOOO1

LWW Contact

H. MATRIX CHARACTERISTICS

Primary Matrix Code: L2100

Description: Organic Liquids: Aqueous/Organic Liquids: :

Combustibility: $\bigcirc$ Combustible (>90\%)

Mixed $(10 \%-90 \%)$

Noncombustible $(<10 \%)$

\section{MATRIX COMPOSITION}


W.W.WO1

LLW Contract

J. HAZARDOUS CONSTITUENTS

\begin{tabular}{|c|c|c|c|c|c|c|c|c|}
\hline & & & \multirow{2}{*}{\multicolumn{4}{|c|}{ Concentration }} & \multirow[b]{3}{*}{ Basis } & \multirow[b]{3}{*}{ Conc. Type } \\
\hline & & & & & & & & \\
\hline Code & Hazardous Waste Description & Treatment Subcategory & Typical & $\mathrm{LL}$ & UL & Units & & \\
\hline D001 & lgnitable & High-TOC ignitable characteristic liquids subca & & & & & A & Total \\
\hline 0009 & Mercury & Elemental mercury contaminated with radioact & & & & & A & Total \\
\hline FOO2 & Spent halogenated solvents & o-Dichlorobenzene & & & & & $A$ & Total \\
\hline F003 & Spent non-halogenated solvents & Acetone & & & & & A & Total \\
\hline F005 & Spent non-halogenated solvents & 2-Ethoxyethanol & & & & & A & Total \\
\hline
\end{tabular}

IPA WASTIES:

LA-W901

Stiil-Gen: : Active 


\section{K. POLYCHLORINATED BIPHENYLS (PCBS)}

Is this waste stream PCB contaminated? OYes O No $O$ Unknown

If yes, what portion of the waste is PCB contaminated (volume \%):

If $<100 \%$, can the PCB contaminated waste be segregated and treated separately?

If only a portion of the waste is PCB contaminated, report values for contaminated portion.

What is the PCB concentration in ppm?

\section{TREATMENT PLANS}

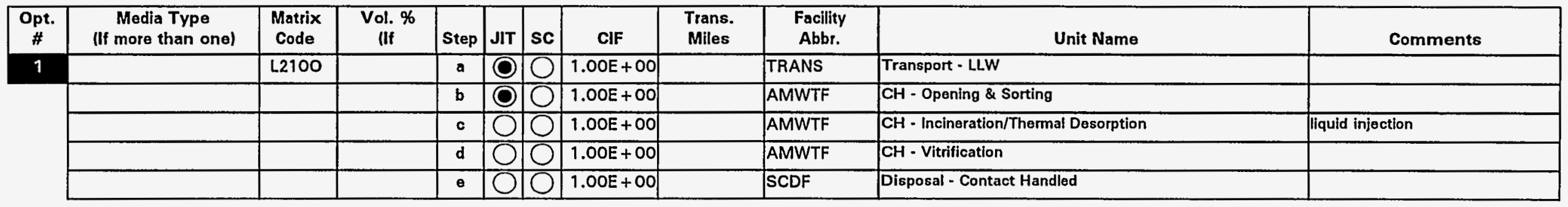

Note: Where provided, media percentages are rough estimates used to facilitate treatment planning. They do not necessarily imply an accurate knowledge of waste stream composition, and were not derived for use outside this conceptual planning exercise.

Describe any special or unique technical concerns related to the treatment of this waste stream that would impact the use or implementation of standard hazardous waste treatment methods. 


\section{$\therefore \quad$ LA:W902}

LiLW: Contact-H

A. IDENTIFICATION AND DESCRIPTION

Waste Stream ID: LA-W902

$\begin{array}{ll}\text { W. S. Name: } & \text { SCINTILLATIION VIALS } \\ \text { Wot } & \end{array}$

Content Code:

FFCA ID:

\begin{tabular}{l}
\hline LA-W902 \\
\hline LA
\end{tabular}

\section{Waste Stream Description:}

These are pseudocumene-based organics. The scintillation fluid has been removed from individual vials and consolidated in 55-gallon drums.

\section{SCINTILLATION VIALS}

LA-W902

Still-Gen Active

\section{B. GENERATION SITE/PROCESS DESCRIPTION}

The following questions, concerning the generation site and process, should be answered even if the waste stream is no longer generated.

Name of Site Generating Waste (e.g., INEL, Rocky Flats, etc.):

\section{Location of activities (Area and Building):}

Operations (functions) performed in building:

Description of Process Generating Waste: 


\section{LA-W9Ö2}

\section{LW ContaatiH \\ C. PROJECTED WASTE GENERATION}

SCINTILLATION VIALS

Is this waste stream still generated?

OYes ONo

If yes, when is the expected generation termination date?

Current Projections

\begin{tabular}{|r|r|r|r|l|l|}
\hline Est. Date & Start Year & Interval & \multicolumn{1}{|c|}{ Vol (m3) } & Mass (kg) & \multicolumn{1}{|c|}{ Source of Estimate } \\
\hline $5 / 2 / 95$ & 1995 & 1 & 0.80 & & Generator Update \\
\hline $5 / 2 / 95$ & 1996 & 1 & 0.80 & & Generator Update \\
\hline $5 / 2 / 95$ & 1997 & 1 & 0.80 & & Generator Update \\
\hline $5 / 2 / 95$ & 1998 & 1 & 0.80 & & Generator Update \\
\hline $5 / 2 / 95$ & 1999 & 1 & 0.80 & Generator Update \\
\hline \multicolumn{5}{|c|}{ Historical Projections } \\
\hline
\end{tabular}




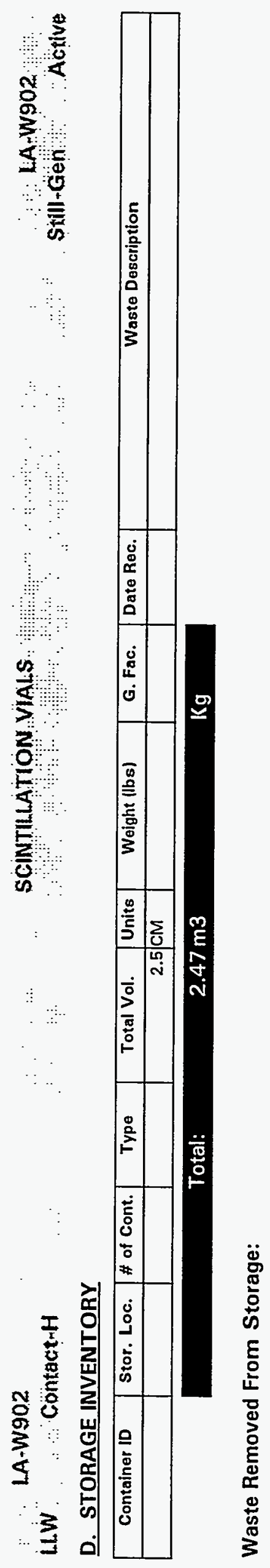


LA-W902

LW Contact

E. RADIATION CHARACTERISTICS

Radioactive Waste Type:

\begin{tabular}{|ll|}
\hline O LLW & Oa-LLW OTRU \\
OHLW & O Non-Rad \\
\hline
\end{tabular}

Mixed Waste:

Handling:

\begin{tabular}{|l|}
\hline O Yes O No \\
\hline O Contact O Remote \\
\hline
\end{tabular}

Radionuclide Contamination Accessiblity:

External Surface $O$ Yes O No O Unknown

Internal Surface OYes ONo OUnknown

Dispersed Through Matrix OYes ONo OUnknown

\section{Activity Levels}

Transuranic Alpha Activity: Uranium/Thorium Alpha Activity:

Beta/Gamma Activity:

Surface Neutron Activity:

Total Activity:

$\square$ Units:
$\square$ Units: $\square$
$\square$ @ surface
$\square$ @ 1-m Units: mR/hr
$\square$ Units: $\square$
$\square$ nCi/g

This waste stream is not identified as a TRU or a-LLW.
KA:W902

Still-Gen Active

\section{F. TRU ALPHA ACTIVITY DISTRIBUTION}



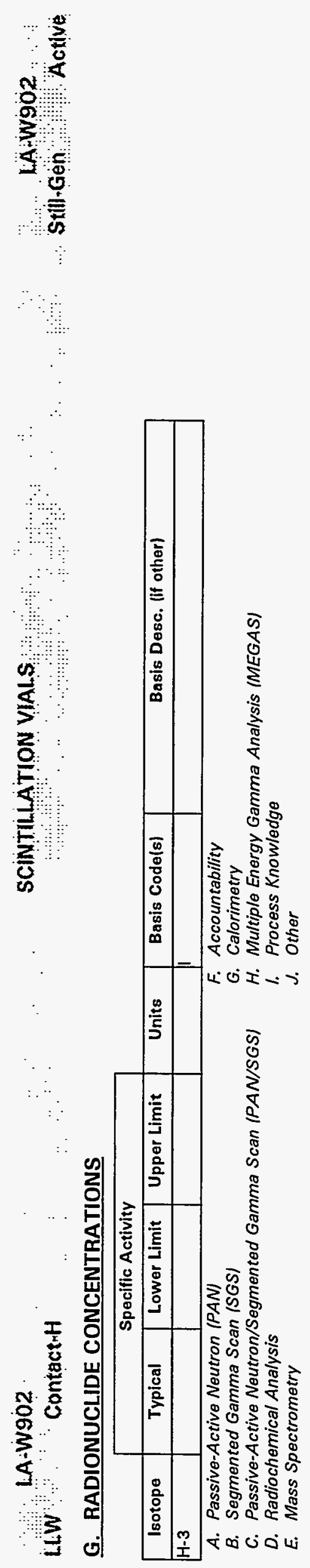


\section{H. MATRIX CHARACTERISTICS}

Primary Matrix Code: X6400

Description: Lab Packs: Scintillation Cocktails: :

Combustibility: $\bigcirc$ Combustible (>90\%)

Mixed (10\%-90\%)

Noncombustible $(<10 \%)$

\section{MATRIX COMPOSITION}




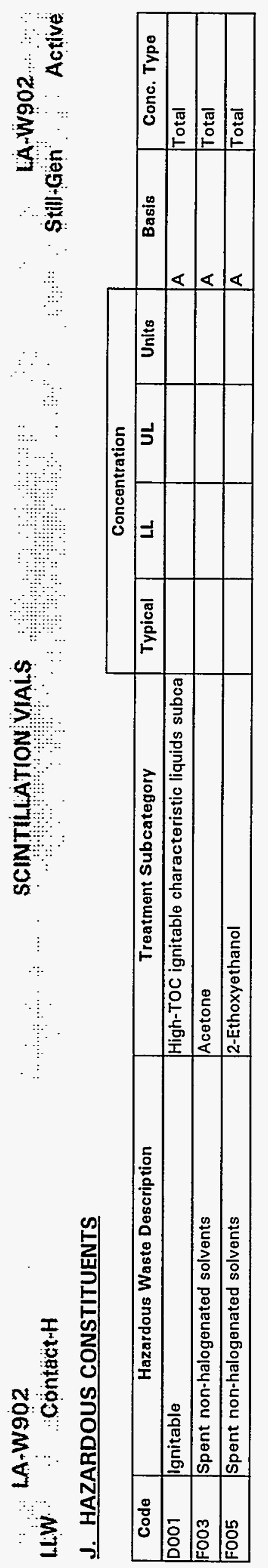


LA-W902

LWW Contactit

\section{K. POLYCHLORINATED BIPHENYLS (PCBS)}

Is this waste stream PCB contaminated? OYes O No O Unknown

If yes, what portion of the waste is PCB contaminated (volume \%):

If $<100 \%$, can the PCB contaminated waste be segregated and treated separately?

What is the PCB concentration in ppm?

If only a portion of the waste is $P C B$ contaminated, report values for contaminated portion.

\section{TREATMENT PLANS}

\begin{tabular}{|c|c|c|c|c|c|c|c|c|c|c|c|}
\hline $\begin{array}{c}\text { Opt. } \\
\#\end{array}$ & $\begin{array}{c}\text { Media Type } \\
\text { (If more than one) }\end{array}$ & $\begin{array}{c}\text { Matrix } \\
\text { Code }\end{array}$ & $\begin{array}{c}\text { Vol. } \% \\
\text { Iff }\end{array}$ & Step & JIT & sc & $\mathrm{ClF}$ & $\begin{array}{l}\text { Trans. } \\
\text { Miles }\end{array}$ & $\begin{array}{l}\text { Facility } \\
\text { Abbr. }\end{array}$ & Unit Name & Comments \\
\hline \multirow[t]{5}{*}{1} & & $\times 6400$ & & $\bar{a}$ & ( & 0 & $1.00 \mathrm{E}+00$ & & TRANS & Transport - LLW & \\
\hline & & & & $\mathbf{b}$ & (1) & 0 & $1.00 E+00$ & & AMWTF & $\mathrm{CH}$ - Opening \& Sorting & \\
\hline & & & & c & 0 & ) & $1.00 E+00$ & & AMWTF & $\mathrm{CH}$ - Incineration/Thermal Desorption & liquid injection \\
\hline & & & & d & ) & O & $1.00 E+00$ & & AMWTF & $\mathrm{CH}$ - Vitrification & \\
\hline & & & & $\bar{\theta}$ & 7 & $\bar{O}$ & $1.00 E+00$ & & SCDF & Disposal - Contact Handled & \\
\hline
\end{tabular}

Note: Where provided, media percentages are rough estimates used to facilitate treatment planning. They do not necessarily imply an accurate knowledge of waste stream composition, and were not derived for use outside this conceptual planning exercise.

Describe any special or unique technical concerns related to the treatment of this waste stream that would impact the use or implementation of standard hazardous waste treatment methods. 


LA-W904
LiW : Contact-H .
A. IDENTIFICATION AND DESCRIPTION
Waste Stream ID: LA-W904
W. S. Name:
Content Code:
FFCA ID:
Waste Stream Description:
These soils contain low levels of RCRA-regulated heavy metals. The principal contaminant is
lead.

\section{METALS}

LA-W904.

\section{B. GENERATION SITE/PROCESS DESCRIPTION}

The following questions, concerning the generation site and process, should be answered even if the waste stream is no longer generated.

Name of Site Generating Waste (e.g., INEL, Rocky Flats, etc.):

L

Location of activities (Area and Building):

Operations (functions) performed in building:

Operations (functions) performed in building:
Description of Process Generating Waste:


LA-W904

LLW COntagtH

C. PROJECTED WASTE GENERATION

Is this waste stream still generated?

If yes, when is the expected generation termination date?

\begin{tabular}{|l|r|r|r|l|l|}
\hline \multicolumn{7}{|c|}{ Current Projections } \\
\hline Est. Date & Start Year & Interval & \multicolumn{1}{c|}{ Vol (m3) } & Mass (kg) & Source of Estimate \\
\hline $5 / 2 / 95$ & 1995 & 1 & 0.40 & & Generator Update \\
\hline $5 / 2 / 95$ & 1996 & 1 & 0.40 & & Generator Update \\
\hline $5 / 2 / 95$ & 1997 & 1 & 0.40 & & Generator Update \\
\hline $5 / 2 / 95$ & 1998 & 1 & 0.40 & & Generator Update \\
\hline $5 / 2 / 95$ & 1999 & 1 & 0.40 & & Generator Update \\
\hline
\end{tabular}

Historical Projections
WA WOÖ Stili-Gen In Active 


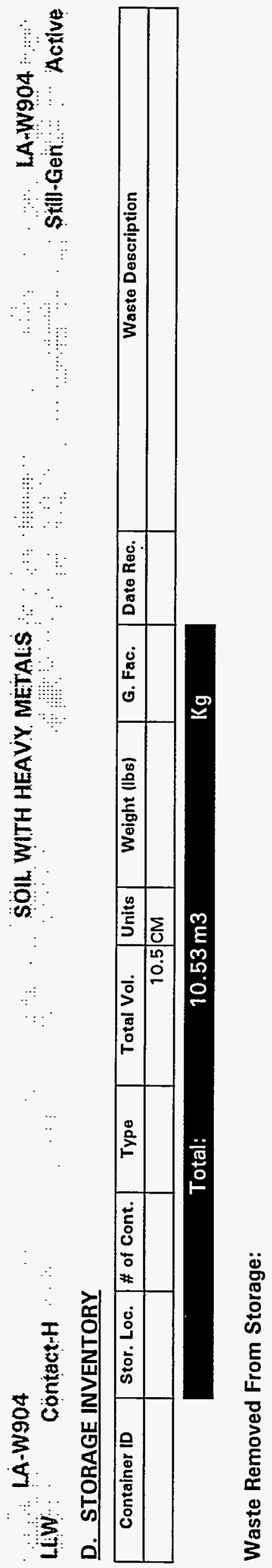




\section{E. RADIATION CHARACTERISTICS}

Radioactive Waste Type:

\begin{tabular}{|ll|}
\hline OLLW & Oa-LLW O TRU \\
O HLW & O Non-Rad \\
\hline
\end{tabular}

Mixed Waste:

Handling:

\begin{tabular}{|l|}
\hline O Yes O No \\
\hline O Contact O Remote \\
\hline
\end{tabular}

Radionuclide Contamination Accessiblity:

\begin{tabular}{|c|c|c|c|}
\hline External Surface & OYes & ONo & O Unknown \\
\hline Internal Surface & OYes & ONo & OUnknown \\
\hline ispersed Through Matrix & OYes & ONo & Unknown \\
\hline
\end{tabular}

\section{Activity Levels}

Transuranic Alpha Activity: Uranium/Thorium Alpha Activity: Beta/Gamma Activity:

Surface Neutron Activity: Total Activity:

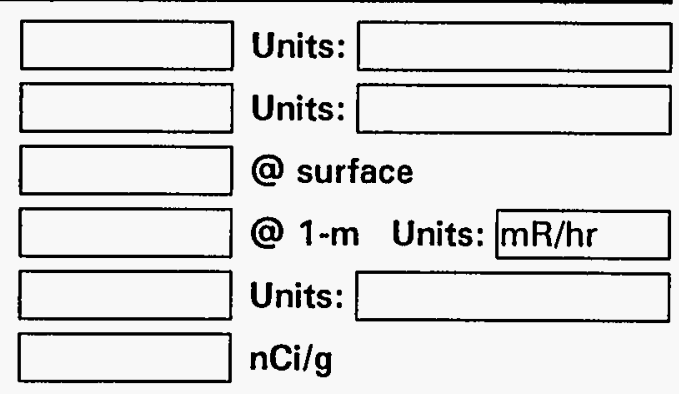

\section{F. TRU ALPHA ACTIVITY DISTRIBUTION}

This waste stream is not identified as a TRU or a-LLW. 


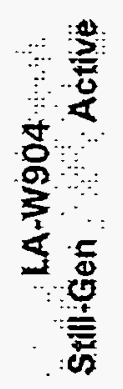


Contäct-H

Primary Matrix Code: $\mathbf{S 4 1 0 0}$

Description:Soil/Gravel: Soil: :

Combustibility: $\bigcirc$ Combustible $(>90 \%)$

Mixed $(10 \%-90 \%)$

Noncombustible $(<10 \%)$

\section{MATRIX COMPOSITION}




\section{J. HAZARDOUS CONSTITUENTS}

\begin{tabular}{|c|c|c|c|c|c|c|c|c|}
\hline Code & Hazardous Waste Description & Treatment Subcategory & Typical & $\mathbf{L L}$ & UL & Units & Basis & Conc. Type \\
\hline$\overline{D 004}$ & Arsenic & & & & & & A & Total \\
\hline DOO5 & Barium & & & & & & A & Total \\
\hline D006 & Cadmium & & & & & & A & Total \\
\hline$\overline{D 007}$ & Chromium & Chromium (Total) & & & & & A & Total \\
\hline$\overline{D 008}$ & Lead & & & & & & A & Total \\
\hline D009 & Mercury & Elemental mercury contaminated with radioact & & & & & A & Total \\
\hline D010 & Selenium & & & & & & A & Total \\
\hline$D 011$ & Silver & & & & & & A & Total \\
\hline
\end{tabular}




\section{LA-W9o4}

\section{SOIL WITH HEAVY METALS}

\section{LLWOUl Contact H}

\section{K. POLYCHLORINATED BIPHENYLS (PCBS)}

Is this waste stream PCB contaminated?

\section{OYes O No O Unknown}

If yes, what portion of the waste is PCB contaminated (volume \%):

If $<100 \%$, can the PCB contaminated waste be segregated and treated separately? OYes ONo O Unknown

What is the PCB concentration in ppm?

If only a portion of the waste is PCB contaminated, report values for contaminated portion.

\section{TREATMENT PLANS}

\begin{tabular}{|c|c|c|c|c|c|c|c|c|c|c|c|}
\hline $\begin{array}{c}\text { Opt. } \\
\#\end{array}$ & $\begin{array}{c}\text { Media Type } \\
\text { (If more than one) }\end{array}$ & $\begin{array}{c}\text { Matrix } \\
\text { Code }\end{array}$ & $\begin{array}{l}\text { Vol. \% } \\
\text { IIf }\end{array}$ & Step & JIT & sc & CIF & $\begin{array}{l}\text { Trans. } \\
\text { Miles }\end{array}$ & $\begin{array}{l}\text { Facility } \\
\text { Abbr. }\end{array}$ & Unit Name & Comments \\
\hline \multirow[t]{6}{*}{1} & & $\$ 4100$ & & $\mathbf{a}$ & O) & 0 & $1.00 E+00$ & & TRANS & Transport - LLW & \\
\hline & & & & $\mathbf{b}$ & () & 0 & $1.00 E+00$ & & AMWTF & $\mathrm{CH}$ - Opening \& Sorting & \\
\hline & & & & c & O & O & $1.00 E+00$ & & AMWTF & $\mathrm{CH}$ - Sizing & \\
\hline & & & & d & 0 & O & $1.00 E+00$ & & AMWTF & $\mathrm{CH}$ - Incineration/Thermal Desorption & \\
\hline & & & & $\vec{e}$ & 0 & 0 & $1.00 E+00$ & & AMWTF & CH - Vitrification & \\
\hline & & & & $f$ & 0 & 0 & $1.00 E+00$ & & SCDF & Disposal - Contact Handled & \\
\hline
\end{tabular}

Note: Where provided, media percentages are rough estimates used to facilitate treatment planning. They do not necessarily imply an accurate knowledge of waste stream composition, and were not derived for use outside this conceptual planning exercise.

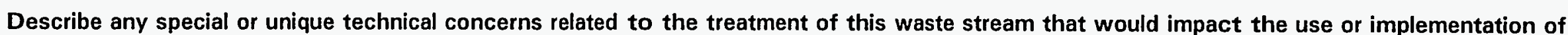
standard hazardous waste treatment methods. 


\section{LA.W905}

LiW Contact-H

\section{A. IDENTIFICATION AND DESCRIPTION}

Waste Stream ID: LA-W905

W. S. Name:

Content Code:

FFCA ID:

\begin{tabular}{|l|}
\hline LA-W905 \\
\hline ER SOILS \\
\hline LA-W905 \\
\hline
\end{tabular}

Waste Stream Description:

These are soils resulting from Environmental Restoration activities.

\section{B. GENERATION SITE/PROCESS DESCRIPTION}

The following questions, concerning the generation site and process, should be answered even if the waste stream is no longer generated.

Name of Site Generating Waste (e.g., INEL, Rocky Flats, etc.):

Location of activities (Area and Building):

Operations (functions) performed in building: (

Description of Process Generating Waste: 
$\therefore \quad$ LA WW905

LiW: Contact-

C. PROJECTED WASTE GENERATION

Is this waste stream still generated?

If yes, when is the expected generation termination date?

\section{LA.Ẅ90̈5}

Nót Gén Active

Current Projections

\section{Historical Projections}




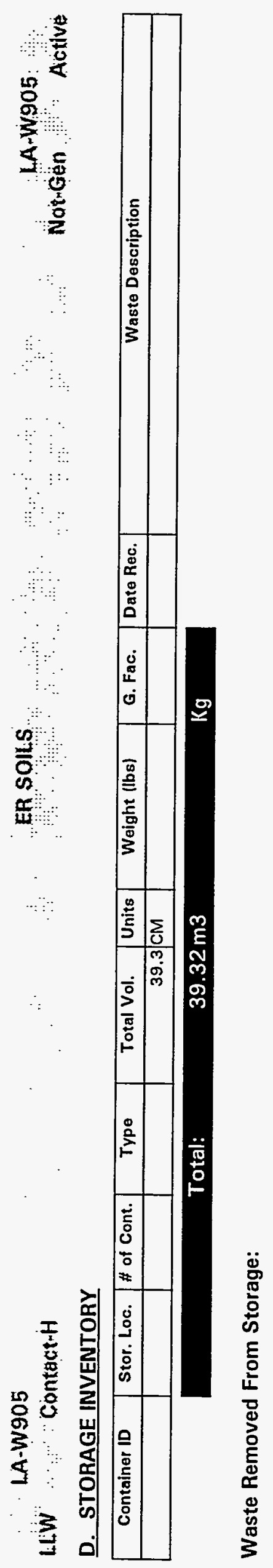




\section{LA WW905} HWW Contät

\section{E. RADIATION CHARACTERISTICS}

Radioactive Waste Type:

\begin{tabular}{|ll|}
\hline OllW & OallLw O TRU \\
OHLW & O Non-Rad \\
\hline
\end{tabular}

Mixed Waste:

\begin{tabular}{|l|}
\hline O Yes ONo \\
\hline O Contact O Remote \\
\hline
\end{tabular}

Radionuclide Contamination Accessiblity:

\begin{tabular}{rlrl|} 
External Surface & OYes & ONo & O Unknown \\
\cline { 3 - 4 } Internal Surface & OYes & ONo & O Unknown \\
\cline { 2 - 4 } Dispersed Through Matrix & OYes & ONo & O Unknown
\end{tabular}

\section{Activity Levels}

Transuranic Alpha Activity:

Uranium/Thorium Alpha Activity:

Beta/Gamma Activity:

Surface Neutron Activity:

Total Activity:

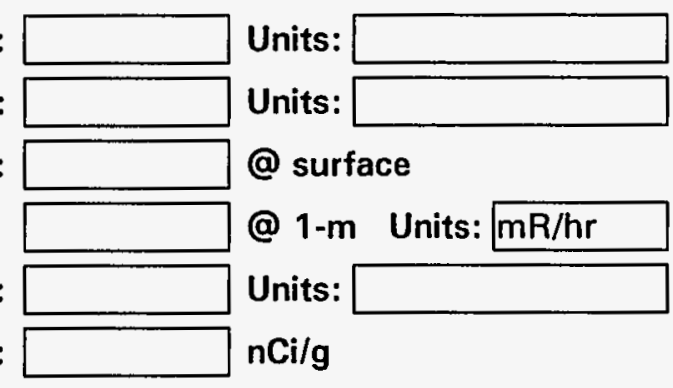

\section{ER SOils}

\section{F. TRU ALPHA ACTIVITY DISTRIBUTION}

This waste stream is not identified as a TRU or a-LLW.
KA WOÖ5 NätGen HO Actlue 

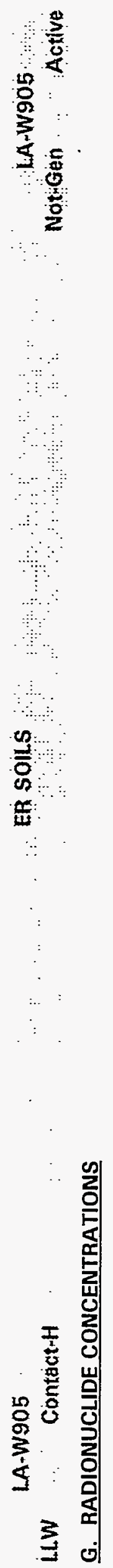


\section{LAWWOS}

\section{H. MATRIX CHARACTERISTICS}

Primary Matrix Code: $\mathbf{S 4 1 0 0}$

Description:SOil/Gravel: Soil: :

Combustibility: $\bigcirc$ Combustible (>90\%)

Mixed $(10 \%-90 \%)$

Noncombustible $(<10 \%)$

\section{MATRIX COMPOSITION}




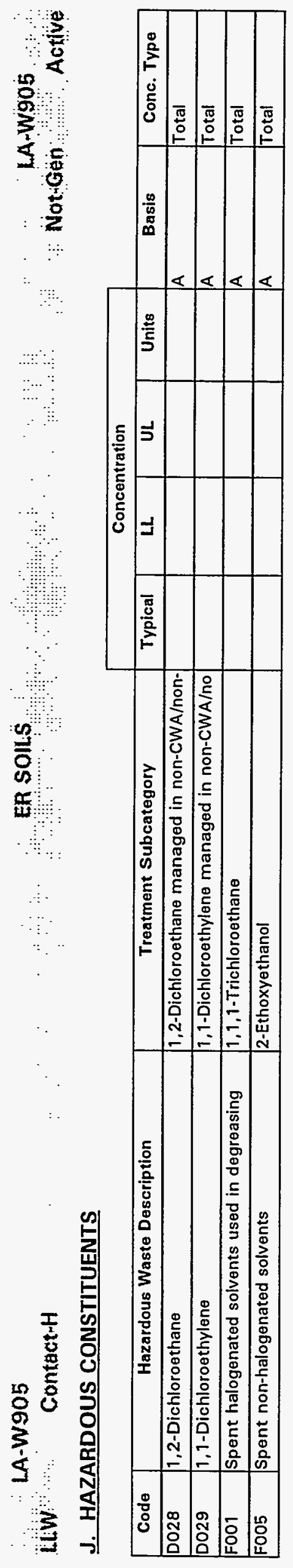




\section{LÄพพั0}

\section{ER SÖlls}

LA:Wö

UlW Contact

K. POLYCHLORINATED BIPHENYLS (PCBS)

Is this waste stream PCB contaminated?

OYes ONo O Unknown

If yes, what portion of the waste is PCB contaminated (volume \%):

If $<100 \%$, can the PCB contaminated waste be segregated and treated separately?

What is the PCB concentration in ppm?

\section{TREATMENT PLANS}

\begin{tabular}{|c|c|c|c|c|c|c|c|c|c|c|c|}
\hline $\begin{array}{c}\text { Opt. } \\
\#\end{array}$ & $\begin{array}{c}\text { Media Type } \\
\text { (If more than one) }\end{array}$ & $\begin{array}{c}\text { Matrix } \\
\text { Code }\end{array}$ & $\begin{array}{l}\text { Vol. \% } \\
\text { IIf }\end{array}$ & Step & JIT & sc & CIF & $\begin{array}{l}\text { Trans. } \\
\text { Miles }\end{array}$ & $\begin{array}{l}\text { Facility } \\
\text { Abbr. }\end{array}$ & Unit Name & Comments \\
\hline \multirow[t]{6}{*}{1} & & $\$ 4100$ & & $\mathbf{a}$ & $\mathbf{O}$ & 0 & $1.00 E+00$ & & TRANS & Transport - LLW & \\
\hline & & & & $\mathbf{b}$ & O & 0 & $1.00 E+00$ & & AMWTF & $\mathrm{CH}$ - Opening \& Sorting & \\
\hline & & & & c & ( ) & 0 & $1.00 E+00$ & & AMWTF & $\mathrm{CH}$ - Sizing & \\
\hline & & & & d & 0 & 0 & $1.00 E+00$ & & AMWTF & $\mathrm{CH}$ - Incineration/Thermal Desorption & \\
\hline & & & & e & 0 & 0 & $1.00 E+00$ & & AMWTF & $\mathrm{CH}$ - Vitrification & \\
\hline & & & & $f$ & 0 & 0 & $1.00 E+00$ & & SCDF & Disposal - Contact Handled & \\
\hline
\end{tabular}

Note: Where provided, media percentages are rough estimates used to facilitate treatment planning. They do not necessarily imply an accurate knowledge of waste stream composition, and were not derived for use outside this conceptual planning exercise.

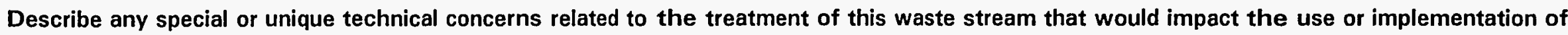
standard hazardous waste treatment methods. 


\section{LLW \\ Contact H}

A. IDENTIFICATION AND DESCRIPTION

Waste Stream ID: LA-W906

W. S. Name:

Content Code:

FFCA ID:

AQUEOUS ORGANIC WASTES

*

LA-W906

\section{Waste Stream Description:}

Aqueous organic liquids come from a large number of operations and contain a wide variety of organic contaminants. The concentration of organics in the waste is generally less than 1000 ppm. Many of these wastes also contain low concentratins of heavy metals.

\section{B. GENERATION SITE/PROCESS DESCRIPTION}

The following questions, concerning the generation site and process, should be answered even if the waste stream is no longer generated.

Name of Site Generating Waste (e.g., INEL, Rocky Flats, etc.):

Location of activities (Area and Building):

Operations (functions) performed in building:

Description of Process Generating Waste: 
C. PROJECTED WASTE GENERATION

Is this waste stream still generated?

OYes ONo

If yes, when is the expected generation termination date?

\begin{tabular}{|r|r|r|r|l|l|}
\hline \multicolumn{7}{|c|}{ Current Projections } \\
\hline Est. Date & \multicolumn{1}{|c|}{ Start Year } & Interval & \multicolumn{1}{|c|}{ Vol (m3) } & Mass (kg) & Source of Estimate \\
\hline $5 / 2 / 95$ & 1995 & 1 & 0.10 & & Generator Update \\
\hline $5 / 2 / 95$ & 1996 & 1 & 0.10 & & Generator Update \\
\hline $5 / 2 / 95$ & 1997 & 1 & 0.10 & & Generator Update \\
\hline $5 / 2 / 95$ & 1998 & 1 & 0.10 & & Generator Update \\
\hline $5 / 2 / 95$ & 1999 & 1 & 0.10 & & Generator Update \\
\hline
\end{tabular}

Historical Projections 


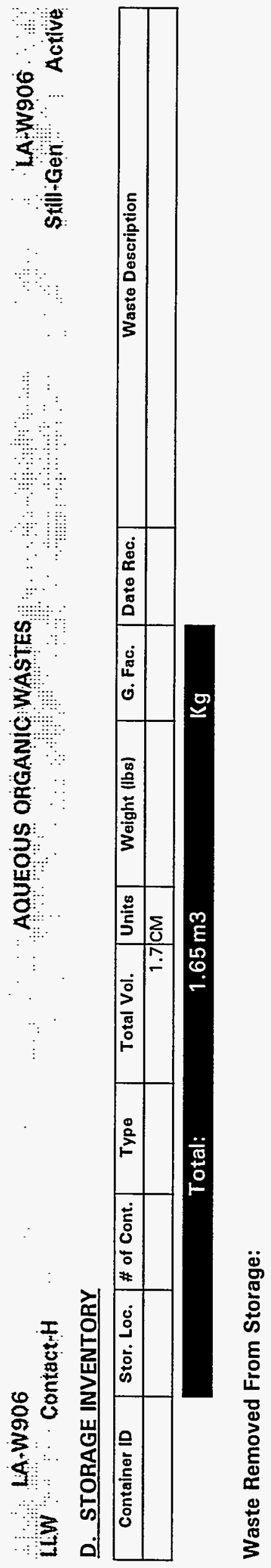


LUW $\begin{aligned} & \text { LA-W906 } \\ & \text { Contact-H }\end{aligned}$

E. RADIATION CHARACTERISTICS

Radioactive Waste Type:

\begin{tabular}{|ll|}
\hline OlLW & Oa-LLW O TRU \\
OHLW & O Non-Rad \\
\hline
\end{tabular}

Mixed Waste:

Handling:

\begin{tabular}{|l|}
\hline OYes O No \\
\hline O Contact O Remote \\
\hline
\end{tabular}

Radionuclide Contamination Accessiblity:

External Surface $O$ Yes O No OUnknown

Internal Surface OYes O No OUnknown

Dispersed Through Matrix $O$ Yes O No OUnknown

\section{Activity Levels}

Transuranic Alpha Activity:

Uranium/Thorium Alpha Activity:

Beta/Gamma Activity:

Surface Neutron Activity:

Total Activity:

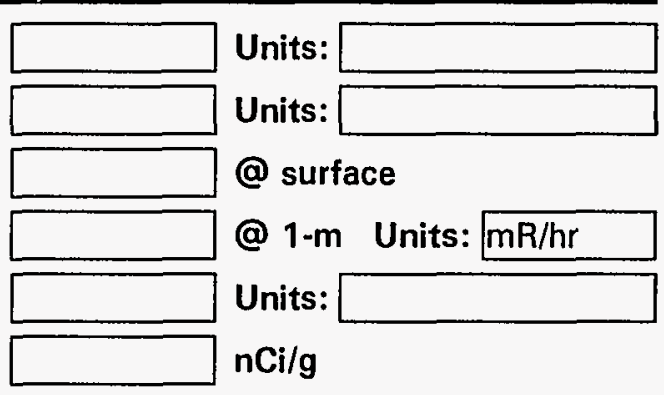

\section{F. TRU ALPHA ACTIVITY DISTRIBUTION}

This waste stream is not identified as a TRU or a-LLW. 


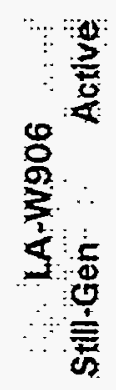

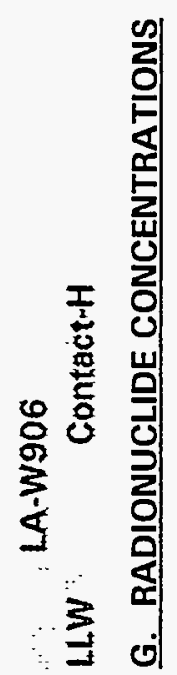


LA-W906

LWW Contätt H a

H. MATRIX CHARACTERISTICS

Primary Matrix Code: $L 1000$

Description: Aqueous Liquids/Slurries: : :

Combustibility: $\begin{aligned} & \bigcirc \text { Combustible }(>90 \%) \\ & \bigcirc \text { Mixed }(10 \%-90 \%) \\ & \bigcirc \text { Noncombustible }(<10 \%)\end{aligned}$

\section{MATRIX COMPOSITION}




\section{LA-W906}

\section{LLW: Contact-H}

\section{J. HAZARDOUS CONSTITUENTS}

\begin{tabular}{|c|c|c|c|c|c|c|c|c|}
\hline & & & & Cor & tion & & & \\
\hline Code & Hazardous Waste Description & Treatment Subcategory & Typical & LL & UL & Units & Basis & Conc. Type \\
\hline$D 001$ & Ignitable & High-TOC ignitable characteristic liquids subca & & & & & $A$ & Total \\
\hline D002 & Corrosive & Corrosive characteristic waste that are manag & & & & & A & Total \\
\hline D005 & Barium & & & & & & A & Total \\
\hline D007 & Chromium & Chromium (Total) & & & & & A & Total \\
\hline D008 & Lead & & & & & & A & Total \\
\hline D010 & Selenium & & & & & & A & Total \\
\hline$D 018$ & Benzene & Benzene managed in non-CWA/non-CWA-equi & & & & & A & Total \\
\hline D019 & Carbon tetrachioride & Carbon Tetrachloride managed in non-CWA/no & & & & & A & Total \\
\hline D022 & Chloroform & Chloroform managed in non-CWA/non-CWA-e & & & & & $\bar{A}$ & Total \\
\hline $\mathrm{DO27}$ & p-Dichlorobenzene (1,4-Dichlorobenzene) & p-Dichlorobenzene managed in non-CWA/non- & & & & & A & Total \\
\hline $\mathrm{DO28}$ & 1,2-Dichloroethane & 1,2-Dichloroethane managed in non-CWA/non- & & & & & A & Total \\
\hline D030 & 2,4-Dinitrotoluene & 2,4-Dinitrotoluene managed in non-CWA/non- & & & & & A & Total \\
\hline D032 & Hexachlorobenzene & Hexachlorobenzene managed in non-CWA/non & & & & & A & Total \\
\hline$\overline{D 036}$ & Nitrobenzene & Nitrobenzene managed in non-CWA/non-CWA- & & & & & $\bar{A}$ & Total \\
\hline D037 & Pentachlorophenol & Pentachlorophenol managed in non-CWA/non- & & & & & A & Total \\
\hline$\overline{D 038}$ & Pyridine & Pyridine managed in non-CWA/non-CWA-equiv & & & & & A & Total \\
\hline D039 & Tetrachloroethylene & Tetrachloroethylene managed in non-CWA/non & & & & & A & Total \\
\hline D041 & 2,4,5-Trichlorophenol & $2,4,5-T r i c h l o r o p h e n o l ~ m a n a g e d ~ i n ~ n o n-C W A / n$ & & & & & A & Total \\
\hline$\overline{\mathrm{DO} 42}$ & 2,4,6-Trichlorophenol & $2,4,6-$ Trichlorophenol managed in non-CWA/n & & & & & A & Total \\
\hline$\overline{D 043}$ & Vinyl chloride & Vinyl Chloride managed in non-CWA/non-CWA & & & & & A & Total \\
\hline F001 & Spent halogenated solvents used in degreasing & 1,1,1-Trichloroethano & & & & & A & Total \\
\hline F002 & Spent halogenated solvents & o-Dichlorobenzene & & & & & A & Total \\
\hline F003 & Spent non-halogenated solvents & Acetone & & & & & A & Total \\
\hline F004 & Spent non-halogenated solvents & Nitrobenzene & & & & & A & Total \\
\hline F005 & Spent non-halogenated solvents & 2-Ethoxyethanol & & & & & A & Total \\
\hline
\end{tabular}

LA-W906:

Still-Gen Active

\section{AQUEOUS ORGANIC WASTES}

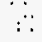




\section{LA:W906}

LiW : Contact H

\section{K. POLYCHLORINATED BIPHENYLS (PCBS)}

\section{Is this waste stream PCB contaminated? \\ OYes O No O Unknown}

If yes, what portion of the waste is PCB contaminated (volume \%):

If $<100 \%$, can the PCB contaminated waste be segregated and treated separately? $O$ Yes ONo OUnknown

What is the PCB concentration in ppm?

If only a portion of the waste is $P C B$ contaminated, report values for contaminated portion.

\section{TREATMENT PLANS}

\begin{tabular}{|c|c|c|c|c|c|c|c|c|c|c|c|}
\hline Opt. & $\begin{array}{c}\text { Media Type } \\
\text { (If more than one) }\end{array}$ & $\begin{array}{c}\text { Matrix } \\
\text { Code }\end{array}$ & $\begin{array}{l}\text { Vol. \% } \\
\text { IIf }\end{array}$ & Step & JIT & sc & CIF & $\begin{array}{l}\text { Trans. } \\
\text { Miles }\end{array}$ & $\begin{array}{l}\text { Facility } \\
\text { Abbr. }\end{array}$ & Unit Name & Comments \\
\hline 1 & & L1000 & & $\bar{a}$ & O & 0 & $1.00 E+00$ & & TRANS & Transport - LLW & \\
\hline & & & & $\bar{b}$ & $\mathbf{O}$ & 0 & $1.00 \mathrm{E}+00$ & & AMWTF & $\mathrm{CH}$ - Opening \& Sorting & 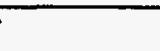 \\
\hline & & & & c & O & 0 & $1.00 E+00$ & & AMWTF & $\mathrm{CH}$ - Sizing & \\
\hline & & & & $\bar{d}$ & 0 & 0 & $1.00 E+00$ & & AMWTF & CH - Incineration/Thermal Desorption & \\
\hline & & & & e & 0 & 0 & $1.00 E+00$ & & AMWTF & $\mathrm{CH}$ - Vitrification & \\
\hline & & & & $f$ & 0 & 0 & $1.00 E+00$ & & SCDF & Disposal - Contact Handled & \\
\hline
\end{tabular}

Note: Where provided, media percentages are rough estimates used to facilitate treatment planning. They do not necessarily imply an accurate knowledge of waste stream composition, and were not derived for use outside this conceptual planning exercise.

Describe any special or unique technical concerns related to the treatment of this waste stream that would impact the use or implementation of standard hazardous waste treatment methods. 


\section{LA-W907}

Liw

Contact-H

\section{A. IDENTIFICATION AND DESCRIPTION}

Waste Stream ID: LA-W907

W. S. Name:

Content Code:

FFCA ID:

HALOGENATED ORGANIC LIQUIDS

*

Waste Stream Description:

These are generally spent solvents, laboratory chemicals, and bulk organics.

\section{HALOGENATED ORGANIC LIQUIDS}

\section{LA.W907.}

Stili:Gen

Active

\section{B. GENERATION SITE/PROCESS DESCRIPTION}

The following questions, concerning the generation site and process, should be answered even if the waste stream is no longer generated.

Name of Site Generating Waste (e.g., INEL, Rocky Flats, etc.):

Location of activities (Area and Building):

Operations (functions) performed in building:

Description of Process Generating Waste: 
LA-W907

UlW Contact H

C. PROJECTED WASTE GENERATION

Is this waste stream still generated?

If yes, when is the expected generation termination date?

Current Projections

\begin{tabular}{|c|c|c|c|c|c|}
\hline Est. Date & Start Year & Interval & Vol (m3) & Mass (kg) & Source of Estimate \\
\hline $5 / 2 / 95$ & 1995 & 1 & 1.10 & & Generator Update \\
\hline $5 / 2 / 95$ & 1996 & 1 & 1.10 & & Generator Update \\
\hline $5 / 2 / 95$ & 1997 & 1 & 1.10 & & Generator Update \\
\hline $5 / 2 / 95$ & 1998 & 1 & 1.10 & & Generator Update \\
\hline $5 / 2 / 95$ & 1999 & 1 & 1.10 & & Generator Update \\
\hline
\end{tabular}

Historical Projections
HALOGEENATED ORGANIC LIOUUIDS 


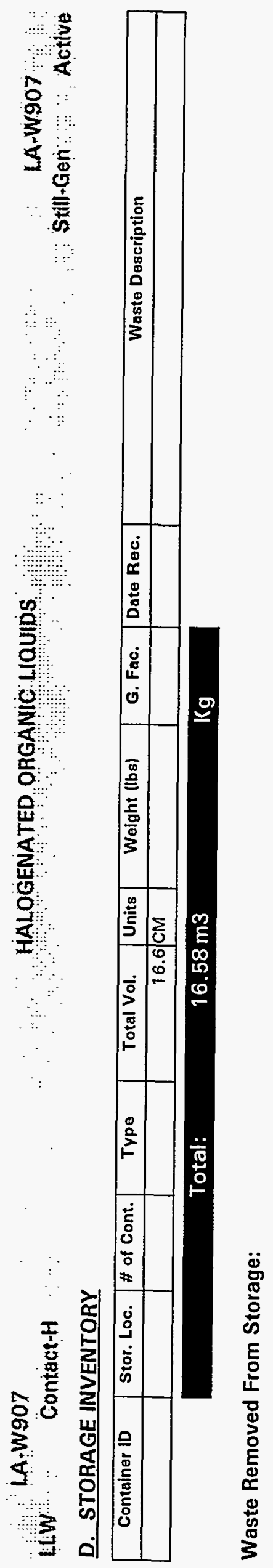


$\therefore$ LA-W907:

LLW: Contact-H

E. RADIATION CHARACTERISTICS

Radioactive Waste Type:

\begin{tabular}{|ll|}
\hline OllW & Oa-LLW O TRU \\
OHLW & O Non-Rad \\
\hline
\end{tabular}

Mixed Waste:

Handling:

OYes ONo

O Contact O Remote

Radionuclide Contamination Accessiblity:

External Surface

Internal Surface

Dispersed Through Matrix

\begin{tabular}{|ccc|}
\hline Yes & ONo & OUnknown \\
\hline OYes & ONo & O Unknown \\
\hline OYes & O No & O Unknown \\
\hline
\end{tabular}

\section{Activity Levels}

Transuranic Alpha Activity:

Uranium/Thorium Alpha Activity:

Beta/Gamma Activity:

Surface Neutron Activity:

Total Activity:

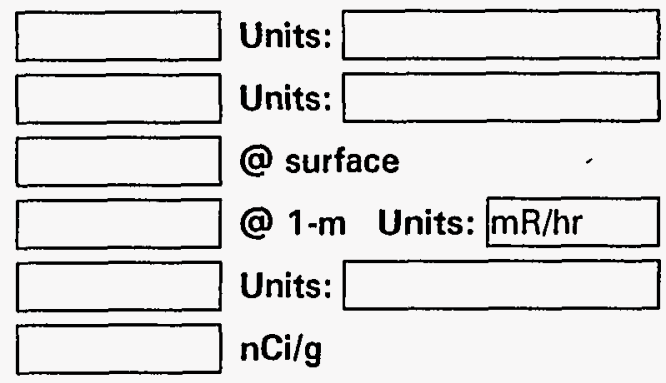

$\square$
Total Activity:

\section{F. TRU ALPHA ACTIVITY DISTRIBUTION}

This waste stream is not identified as a TRU or a-LLW. 


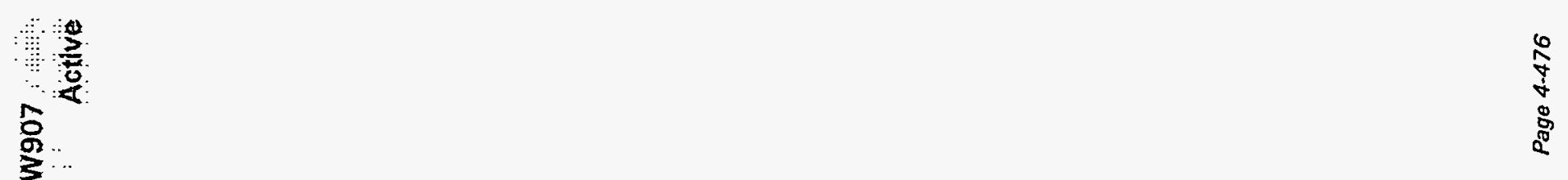

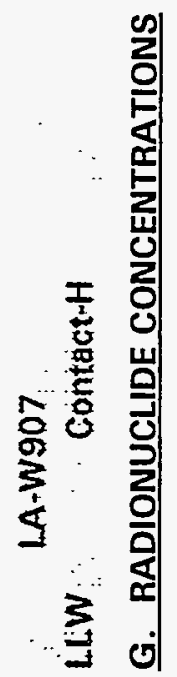


$\therefore$ LÄ-W907:

HLW Contact-H

HALOGËNATED ORGANIC LIOUUIDS

H. MATRIX CHARACTERISTICS

Primary Matrix Code: L2210

Description: Organic Liquids: Pure Organic Liquids: Halogenated Pure Organic Liquids:

Combustibility: $\bigcirc$ Combustible (>90\%)

Mixed $(10 \%-90 \%)$

Noncombustible $(<10 \%)$

\section{MATRIX COMPOSITION}




\section{$\because$ LA-W907}

\section{LLW: Contagt-H}

\section{J. HAZARDOUS CONSTITUENTS}

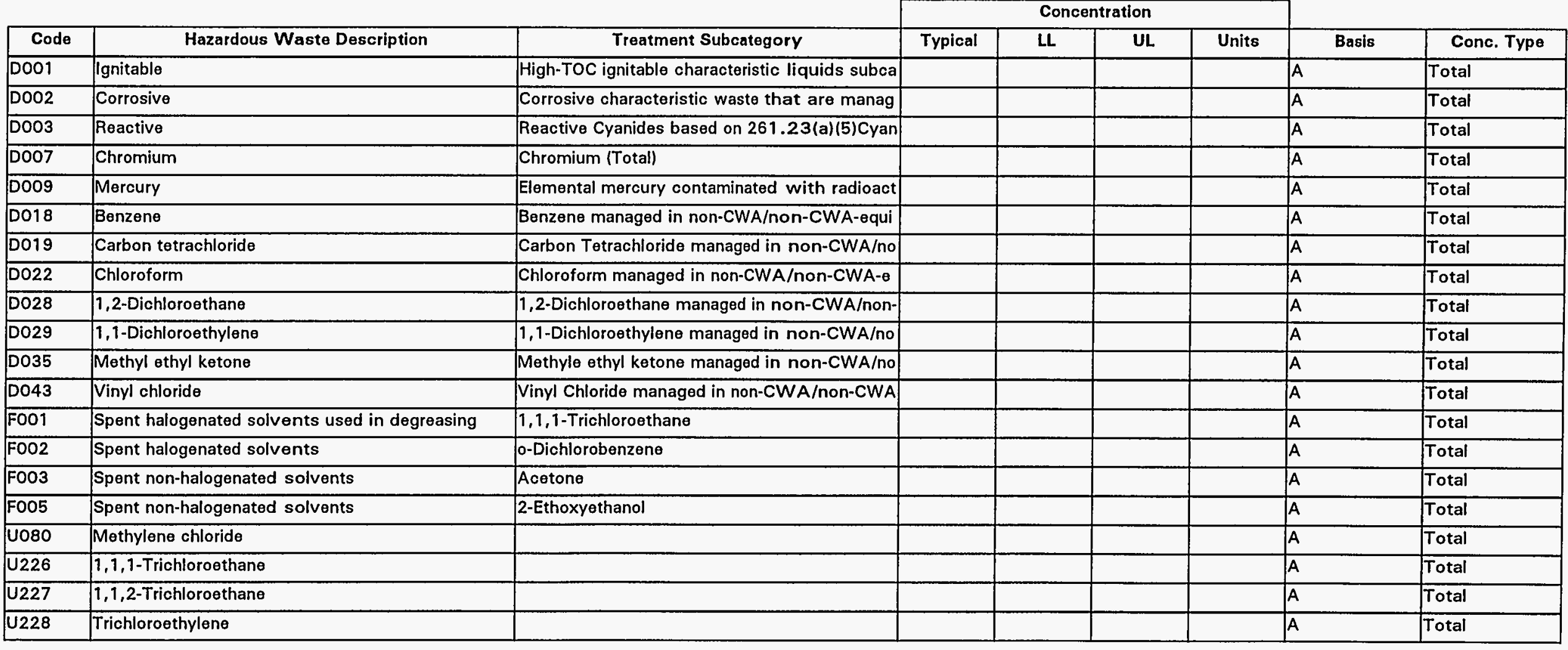

KA-W907:
St Stll Gen Active

HALOGENATED ORGANIC LIQUIDS
, 


\section{HLW Contact-H}

\section{K. POLYCHLORINATED BIPHENYLS (PCBS)}

\section{Is this waste stream PCB contaminated? OYes $O$ No $O$ Unknown}

If yes, what portion of the waste is PCB contaminated (volume \%):

If $<100 \%$, can the PCB contaminated waste be segregated and treated separately?

OYes O No O Unknown

What is the PCB concentration in ppm?

If only a portion of the waste is PCB contaminated, report values for contaminated portion.

\section{TREATMENT PLANS}

\begin{tabular}{|c|c|c|c|c|c|c|c|c|c|c|c|}
\hline $\begin{array}{c}\text { Opt. } \\
\#\end{array}$ & $\begin{array}{c}\text { Media Type } \\
\text { (If more than one) }\end{array}$ & $\begin{array}{c}\text { Matrix } \\
\text { Code }\end{array}$ & $\begin{array}{l}\text { Vol. } \% \\
\text { lif }\end{array}$ & Step & |JIT & sc & CIF & $\begin{array}{l}\text { Trans. } \\
\text { Miles }\end{array}$ & $\begin{array}{l}\text { Facility } \\
\text { Abbr. }\end{array}$ & Unit Name & Comments \\
\hline \multirow[t]{6}{*}{1} & & L2210 & & $\mathbf{a}$ & O & 0 & $1.00 E+00$ & & TRANS & Transport - LLW & \\
\hline & & & & $\mathbf{b}$ & O & 0 & $1.00 E+00$ & & AMWTF & $\mathrm{CH}$ - Opening \& Sorting & \\
\hline & & & & $\bar{c}$ & 0 & 0 & $1.00 E+00$ & & AMWTF & $\mathrm{CH}$ - Sizing & \\
\hline & & & & d & 0 & 0 & $1.00 E+00$ & & AMWTF & $\mathrm{CH}$ - Incineration/Thermal Desorption & \\
\hline & & & & e & 0 & 0 & $1.00 E+00$ & & AMWTF & $\mathrm{CH}$ - Vitrification & \\
\hline & & & & $f$ & 0 & 0 & $1.00 E+00$ & & SCDF & Disposal - Contact Handled & \\
\hline
\end{tabular}

Note: Where provided, media percentages are rough estimates used to facilitate treatment planning. They do not necessarily imply an accurate knowledge of waste stream composition, and were not derived for use outside this conceptual planning exercise.

Describe any special or unique technical concerns related to the treatment of this waste stream that would impact the use or implementation of standard hazardous waste treatment methods.

May also contain trace levels of heavy metals. 
LA.W908

ContactrH

A. IDENTIFICATION AND DESCRIPTION

Waste Stream ID: LA-W908

W. S. Name:

Content Code:

FFCA ID:

\begin{tabular}{|l|}
\hline NONHALOOGENATED ORGANIC LIQUIDS \\
\hline$*$ \\
\hline LA-W908 \\
\hline
\end{tabular}

Waste Stream Description:

These are generally spent solvents, laboratory chemicals, and bulk organics.
NONHALOGENATED ORGANIC LIQUUIDS

The following questions, concerning the generation site and process, should be answered even if the waste stream is no longer generated.

Name of Site Generating Waste (e.g., INEL, Rocky Flats, etc.):

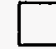

Location of activities (Area and Building):

Operations (functions) performed in building:

Description of Process Generating Waste: 


\section{LA $-W 908$}

LWW Contact H

\section{PROJECTED WASTE GENERATION}

Is this waste stream still generated?

If yes, when is the expected generation termination date?

\section{OYes ONo}

Current Projections

\begin{tabular}{|r|r|r|r|r|l|}
\hline Est. Date & \multicolumn{1}{|l|}{ Start Year } & Interval & \multicolumn{1}{|c|}{ Vol (m3) } & Mass (kg) & Source of Estimate \\
\hline $5 / 2 / 95$ & 1995 & 1 & 2.00 & & Generator Update \\
\hline $5 / 2 / 95$ & 1996 & 1 & 2.00 & & Generator Update \\
\hline $5 / 2 / 95$ & 1997 & 1 & 2.00 & & Generator Update \\
\hline $5 / 2 / 95$ & 1998 & 1 & 2.00 & & Generator Update \\
\hline $5 / 2 / 95$ & 1999 & 1 & 2.00 & & Generator Update \\
\hline
\end{tabular}

Historical Projections 


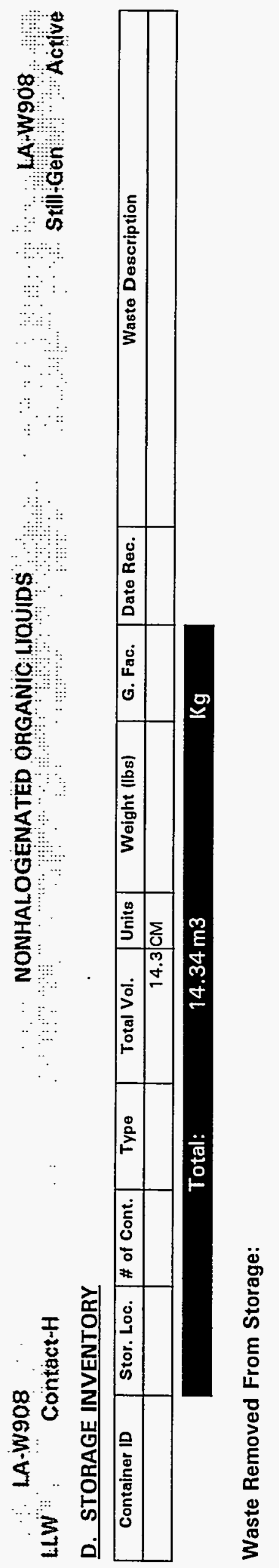




\section{E. RADIATION CHARACTERISTICS}

Radioactive Waste Type:

\begin{tabular}{|c|c|}
\hline OLLW & Oa-LLW O TRU \\
\hline OHLW & Non-Rad \\
\hline
\end{tabular}

Mixed Waste:

\begin{tabular}{|l|}
\hline OYes ONo \\
\hline O Contact O Remote \\
\hline
\end{tabular}

Radionuclide Contamination Accessiblity:

\begin{tabular}{|c|c|c|c|}
\hline External Surface & OYes & ONo & OUnknown \\
\hline Internal Surface & OYes & ONo & OUnknown \\
\hline spersed Through Matrix & OYes & ONo & OUnknown \\
\hline
\end{tabular}

\section{Activity Levels}

Transuranic Alpha Activity: Uranium/Thorium Alpha Activity: Beta/Gamma Activity:

Surface Neutron Activity:

Total Activity:

$\square$ Units:
$\square$ Units:
$\square$ @ surface
$\square$ @ 1-m Units: $\mathrm{mR} / \mathrm{hr}$
$\square$ Units: $\square$
$\square$ nci/g

\section{F. TRU ALPHA ACTIVITY DISTRIBUTION}

This waste stream is not identified as a TRU or a-LLW. 


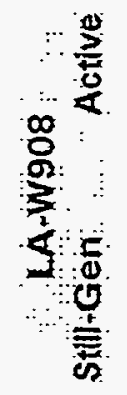

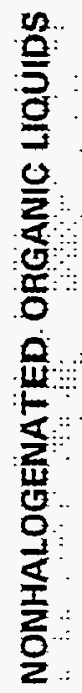

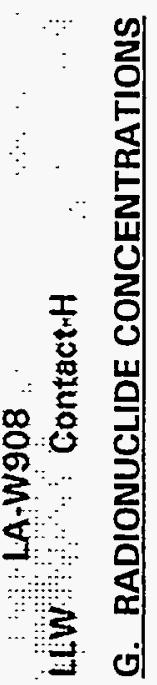


LA-W908:

LlW u Contactr

NONHALOGENATED ORGANIG GIOUUIDS

LAW9ö8

H. MATRIX CHARACTERISTICS

Primary Matrix Code: $\mathbf{L 2 2 2 0}$

Description: Organic Liquids: Pure Organic Liquids: Nonhalogenated Pure Organic Liquids:

Combustibility: $\bigcirc$ Combustible (>90\%)

Mixed (10\%-90\%)

Noncombustible (<10\%)

\section{MATRIX COMPOSITION}




\section{J. HAZARDOUS CONSTITUENTS}

\begin{tabular}{|c|c|c|c|c|c|c|c|c|}
\hline & & & & Con & & & & \\
\hline Code & Hazardous Waste Description & Treatment Subcategory & Typical & LL & UL & Units & Basis & Conc. Type \\
\hline D001 & Ignitable & High-TOC ignitable characteristic liquids subca & & & & & A & Total \\
\hline$\overline{D 002}$ & Corrosive & Corrosive characteristic waste that are manag & & & & & A & Total \\
\hline $\mathrm{D003}$ & Reactive & Reactive Cyanides based on 261.23(a)(5)Cyan & & & & & $\bar{A}$ & Total \\
\hline D004 & Arsenic & & & & & & A & Total \\
\hline$D 007$ & Chromium & Chromium (Total) & & & & & A & Total \\
\hline D008 & Lead & & & & & & A & Total \\
\hline D009 & Mercury & Elemental mercury contaminated with radioact & & & & & A & Total \\
\hline$\overline{D 011}$ & Silver & & & & & & A & Total \\
\hline D018 & Benzene & Benzene managed in non-CWA/non-CWA-equi & & & & & A & Total \\
\hline D038 & Pyridine & Pyridine managed in non-CWA/non-CWA-equiv & & & & & A & Total \\
\hline DO40 & Trichloroethylene & Trichloroethylene managed in non-CWA/non-C & & & & & A & Total \\
\hline FOO2 & Spent halogenated solvents & o-Dichlorobenzene & & & & & A & Total \\
\hline F003 & Spent non-halogenated solvents & Acetone & & & & & A & Total \\
\hline F004 & Spent non-halogenated solvents & Nitrobenzene & & & & & A & Total \\
\hline F005 & Spent non-halogenated solvents & 2-Ethoxyethanol & & & & & A & Total \\
\hline U019 & Benzene & & & & & & A & Total \\
\hline U169 & Nitrobenzene & Nitrobenzene & & & & & $\bar{A}$ & Total \\
\hline U188 & Phenol & & & & & & A & Total \\
\hline U220 & Toluene & & & & & & A & Total \\
\hline
\end{tabular}




\section{K. POLYCHLORINATED BIPHENYLS (PCBS)}

Is this waste stream PCB contaminated?

\section{OYes O No O Unknown}

If yes, what portion of the waste is PCB contaminated (volume \%):

If $<100 \%$, can the PCB contaminated waste be segregated and treated separately? $O$ Yes ONo OUnknown

What is the PCB concentration in ppm?

If only a portion of the waste is PCB contaminated, report values for contaminated portion.

\section{TREATMENT PLANS}

\begin{tabular}{|c|c|c|c|c|c|c|c|c|c|c|c|}
\hline Opt. & $\begin{array}{c}\text { Media Type } \\
\text { (If more than one) }\end{array}$ & $\begin{array}{c}\text { Matrix } \\
\text { Code }\end{array}$ & $\begin{array}{l}\text { Vol. \% } \\
\text { IIf }\end{array}$ & Step & JIT & sc & CIF & $\begin{array}{l}\text { Trans. } \\
\text { Miles }\end{array}$ & $\begin{array}{l}\text { Facility } \\
\text { Abbr. }\end{array}$ & Unit Name & Comments \\
\hline 1 & & L2220 & & a & $\mathbf{0}$ & 0 & $1.00 \mathrm{E}+00$ & & TRANS & Transport - LLW & \\
\hline & & & & b & 0 & 0 & $1.00 E+00$ & & AMWTF & $\mathrm{CH}$ - Opening \& Sorting & \\
\hline & & & & c & $\mathbf{O}$ & 0 & $1.00 E+00$ & & AMWTF & $\mathrm{CH}$ - Sizing & \\
\hline & & & & d & 0 & 0 & $1.00 E+00$ & & AMWTF & $\mathrm{CH}$ - Incineration/Thermal Desorption & \\
\hline & & & & e & 0 & 0 & $1.00 E+00$ & & AMWTF & $\mathrm{CH}$ - Vitrification & \\
\hline & & & & $f$ & 0 & 0 & $1.00 E+00$ & & SCDF & Disposal - Contact Handled & \\
\hline
\end{tabular}

Note: Where provided, media percentages are raugh estimates used to facilitate treatment planning. They do not necessarily imply an accurate knowledge of waste stream composition, and were not derived for use outside this conceptual planning exercise.

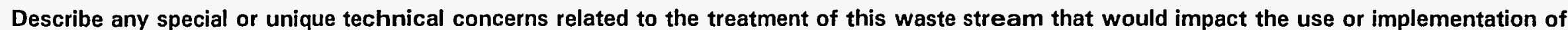
standard hazardous waste treatment methods.

May also contain trace levels of heavy metals. 


\section{$\therefore$ LA-W910 \\ HiW Contact+H}

A. IDENTIFICATION AND DESCRIPTION

Waste Stream ID: LA-W910

W. S. Name:

Content Code:

PCB WASTES WITH RCRA COMPONENTS

FFCA ID:

LA-W910

Waste Stream Description:

Generally spent solvents, laboratory chemicals, and bulk organics. 
HWW LA-W9io

Contagt-H

C. PROJECTED WASTE GENERATION

Is this waste stream still generated?

OYes ONo

If yes, when is the expected generation termination date?

Current Projections

\begin{tabular}{|r|r|r|r|l|l|}
\hline Est. Date & Start Year & Interval & Vol (m3) & Mass (kg) & \multicolumn{1}{|l|}{ Source of Estimate } \\
\hline $5 / 2 / 95$ & 1995 & 1 & 0.04 & & Generator Update \\
\hline $5 / 2 / 95$ & 1996 & 1 & 0.04 & & Generator Update \\
\hline $5 / 2 / 95$ & 1997 & 1 & 0.04 & & Generator Update \\
\hline $5 / 2195$ & 1998 & 1 & 0.04 & & Generator Update \\
\hline $5 / 2 / 95$ & 1999 & 1 & 0.04 & & Generator Update \\
\hline
\end{tabular}

Historical Projections 


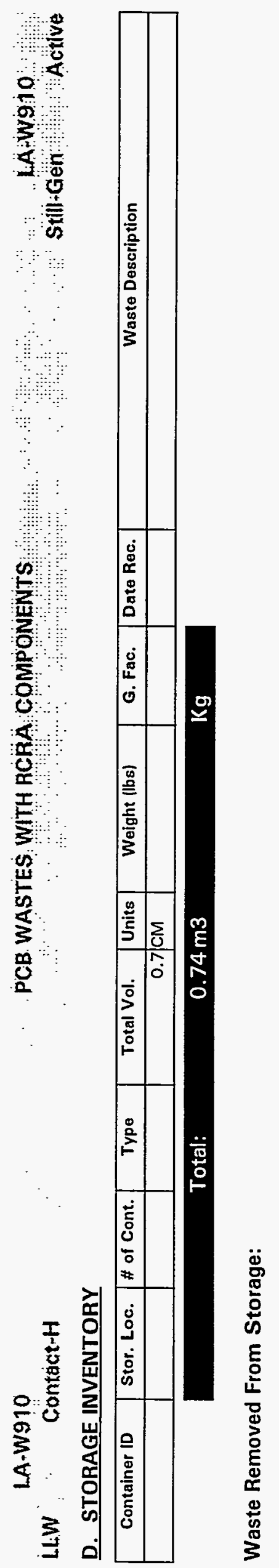




\section{E. RADIATION CHARACTERISTICS}

Radioactive Waste Type:

\begin{tabular}{|ll|}
\hline O LLW & O a-LLW $\bigcirc$ TRU \\
O HLW & O Non-Rad \\
\hline
\end{tabular}

Mixed Waste:

Handling:

\begin{tabular}{|l|}
\hline O Yes O No \\
\hline O Contact O Remote \\
\hline
\end{tabular}

Radionuclide Contamination Accessiblity:

External Surface OYes ONo OUnknown

Internal Surface $O$ Yes $O$ No $O$ Unknown

Dispersed Through Matrix OYes ONo OUnknown

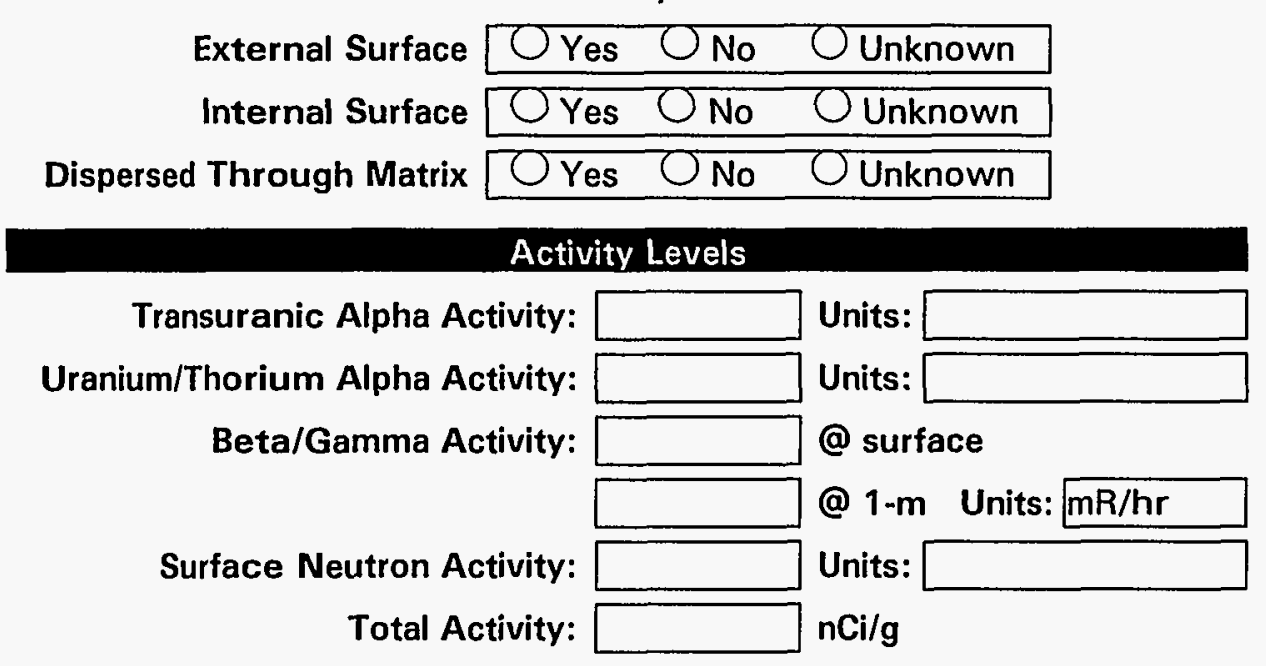

\section{F. TRU ALPHA ACTIVITY DISTRIBUTION}

This waste stream is not identified as a TRU or a-LLW. 

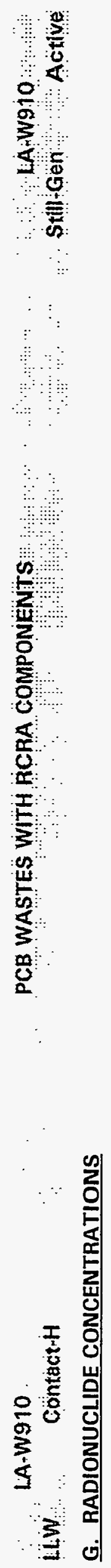
H. MATRIX CHARACTERISTICS

Primary Matrix Code:L2210

Description: Organic Liquids: Pure Organic Liquids: Halogenated Pure Organic Liquids:

$$
\begin{array}{l|l}
\text { Combustibility: } & \bigcirc \text { Combustible }(>90 \%) \\
\bigcirc \text { Mixed }(10 \%-90 \%) \\
\bigcirc \text { Noncombustible }(<10 \%)
\end{array}
$$

\section{MATRIX COMPOSITION}




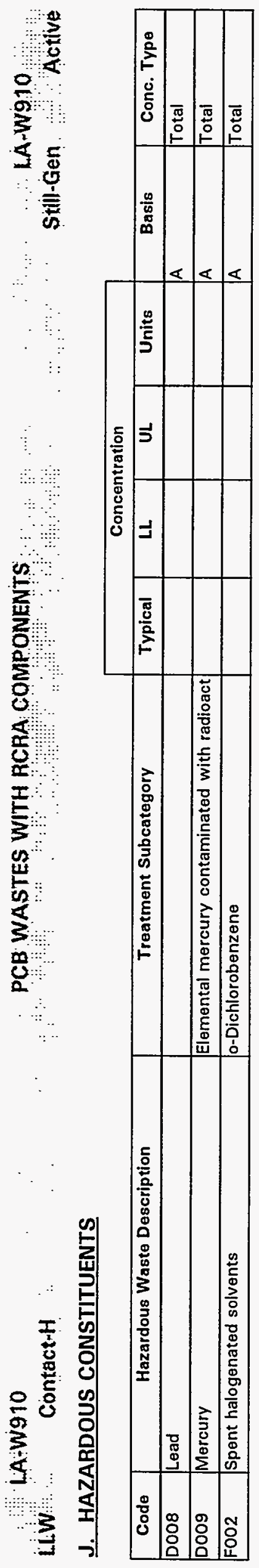


LWW Contact H

\section{K. POLYCHLORINATED BIPHENYLS (PCBS)}

Is this waste stream PCB contaminated? OYes O No $O$ Unknown

If yes, what portion of the waste is PCB contaminated (volume \%):

If $<100 \%$, can the PCB contaminated waste be segregated and treated separately? OYes ONo Onknown

What is the PCB concentration in ppm? If only a portion of the waste is PCB contaminated, report values for contaminated portion.

\section{TREATMENT PLANS}

\begin{tabular}{|c|c|c|c|c|c|c|c|c|c|c|c|}
\hline $\begin{array}{c}\text { Opt. } \\
\#\end{array}$ & $\begin{array}{c}\text { Media Type } \\
\text { (If more than one) }\end{array}$ & $\begin{array}{c}\text { Matrix } \\
\text { Code }\end{array}$ & $\begin{array}{l}\text { Vol. \% } \\
\text { (If }\end{array}$ & Step & JIT & sc & CIF & $\begin{array}{l}\text { Trans. } \\
\text { Miles }\end{array}$ & $\begin{array}{l}\text { Facility } \\
\text { Abbr. }\end{array}$ & Unit Name & Comments \\
\hline 1 & & $\mathrm{~L} 2210$ & & $\mathbf{a}$ & O & $U$ & $1.00 E+00$ & & TRANS & Transport - LLW & \\
\hline & & & & b & $\mathbf{0}$ & O & $1.00 E+00$ & & AMWTF & $\mathrm{CH}$ - Opening \& Sorting & \\
\hline & & & & d & 0 & $\bar{O}$ & $1.00 \mathrm{E}+00$ & & AMWTF & CH - Incineration/Thermal Desorption & \\
\hline & & & & e & 0 & 0 & $1.00 \mathrm{E}+00$ & & AMWTF & $\mathrm{CH}$ - Vitrification & \\
\hline & & & & $f$ & 0 & 0 & $1.00 \mathrm{E}+00$ & & SCDF & Disposal-Contact Handled & \\
\hline
\end{tabular}

Note: Where provided, media percentages are rough estimates used to facilitate treatment planning. They do not necessarily imply an accurate knowledge of waste stream composition, and were not derived for use outside this conceptual planning exercise.

Describe any special or unique technical concerns related to the treatment of this waste stream that would impact the use or implementation of standard hazardous waste treatment methods. 
LA-W911

LLW: $\quad \therefore$ Contact-H

A. IDENTIFICATION AND DESCRIPTION

Waste Stream ID: LA-W911

W. S. Name:

Content Code:

FFCA ID:

*

LA-W911

Waste Stream Description:

These are generally room trash, solvent-contaminated rags, and personnel protective equipment (PPE).

\section{ORGANIC-CONTAMINATED COMBUSTIBLE SOLIDS}

LAW

Still-Gen active

\section{B. GENERATION SITE/PROCESS DESCRIPTION}

The following questions, concerning the generation site and process, should be answered even if the waste stream is no longer generated.

Name of Site Generating Waste (e.g., INEL, Rocky Flats, etc.):

Location of activities (Area and Building):

Operations (functions) performed in building:

Description of Process Generating Waste: 
UlW Contact th

\section{ORGANICCONTAMINATED COMBUSTIBLE SOLIDS}

\section{PROJECTED WASTE GENERATION}

Is this waste stream still generated?

$$
\text { OYes ONo }
$$

If yes, when is the expected generation termination date?

\begin{tabular}{|c|c|c|c|c|c|}
\hline \multicolumn{6}{|c|}{ Current Projections } \\
\hline Est. Date & Start Year & Interval & $\operatorname{Vol}(\mathrm{m} 3)$ & Mass (kg) & Source of Estimate \\
\hline $5 / 2 / 95$ & 1995 & 1 & 1.40 & & Generator Update \\
\hline $5 / 2 / 95$ & 1996 & 1 & 1.40 & & Generator Update \\
\hline $5 / 2 / 95$ & 1997 & 1 & 1.40 & & Generator Update \\
\hline $5 / 2 / 95$ & 1998 & 1 & 1.40 & & Generator Update \\
\hline $5 / 2 / 95$ & 1999 & 1 & 1.40 & & Generator Update \\
\hline
\end{tabular}

Historical Projections 


\section{LA-W911}

Liw : $\because$ Contact-H

D. STORAGE INVENTORY

Container ID

Container ID

\begin{tabular}{|l|l|}
\hline Stor. Loc. & \# of Cont. \\
\hline
\end{tabular}

rotal:

ORGANIC CONTAMINATED COMBUSTIBLE SOLIDS

L.A.W911:

Still-Gent Active

\begin{tabular}{l|l}
\hline Type & Total Vol. \\
\hline
\end{tabular}

\begin{tabular}{l|l}
\hline 28.3 & Unit \\
\hline
\end{tabular}

Weight (lbs)

\begin{tabular}{|l|l|}
\hline G. Fac. & Date Rec. \\
\hline
\end{tabular}

$28.32 \mathrm{~m} 3$

$\mathrm{Kg}$

Waste Removed From Storage: 
HLW LAW911

ORGANIC CONTAMINATED COMBUSTIBLE SOLIDS

E. RADIATION CHARACTERISTICS

Radioactive Waste Type:

\begin{tabular}{|ll|}
\hline OlLW & Oa-LLW OTRU \\
OHLW & O Non-Rad \\
\hline
\end{tabular}

Mixed Waste:

Handling:

\begin{tabular}{|l|}
\hline OYes O No \\
\hline O Contact O Remote
\end{tabular}

Radionuclide Contamination Accessiblity:

\begin{tabular}{|c|c|c|c|}
\hline External Surface & OYes & Ono & OUnknown \\
\hline Internal Surface & OYes & ONo & OUnknown \\
\hline ispersed Through Matrix & OYes & ONo & OUnknown \\
\hline
\end{tabular}

\section{Activity Levels}

Transuranic Alpha Activity:

Uranium/Thorium Alpha Activity:

Beta/Gamma Activity:

Surface Neutron Activity:

Total Activity:

\begin{tabular}{|c|c|}
\hline & Units: \\
\hline & Units: \\
\hline & @ surface \\
\hline & @ 1-m Units: $\mathrm{mR} / \mathrm{hr}$ \\
\hline & | Units: \\
\hline & nCi/g \\
\hline
\end{tabular}

$\mathrm{nCl} / \mathrm{g}$

\section{F. TRU ALPHA ACTIVITY DISTRIBUTION}

This waste stream is not identified as a TRU or a-LLW. 

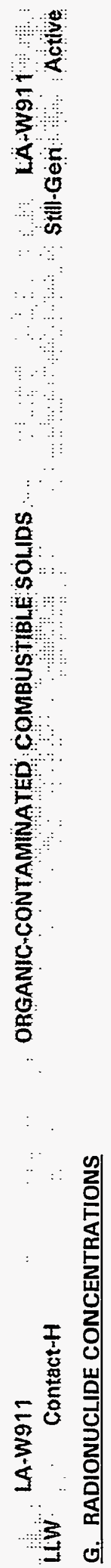
LA-W91.1

ORGANIC-CONTAMINATED COMBÜSTIBLE SOLIDS

LLW Contact H

O StilliGen Active

H. MATRIX CHARACTERISTICS

Primary Matrix Code: $\mathbf{S 5 4 0}$

Description: Debris Waste: Heterogeneous Debris: Predominantly Organic Debris:

Combustibility: $\bigcirc$ Combustible (>90\%)

Mixed $(10 \%-90 \%)$

Noncombustible (<10\%)

\section{MATRIX COMPOSITION}




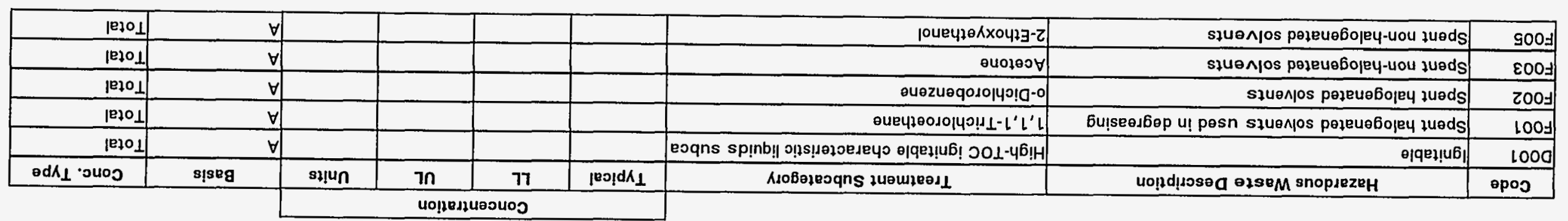




\section{K. POLYCHLORINATED BIPHENYLS (PCBS)}

\section{Is this waste stream PCB contaminated?}

OYes O No O Unknown

If yes, what portion of the waste is PCB contaminated (volume \%):

If $<100 \%$, can the PCB contaminated waste be segregated and treated separately? $O$ Yes O No O Unknown

What is the PCB concentration in ppm? If only a portion of the waste is PCB contaminated, report values for contaminated portion.

\section{TREATMENT PLANS}

\begin{tabular}{|c|c|c|c|c|c|c|c|c|c|c|c|}
\hline $\begin{array}{c}\text { Opt. } \\
\#\end{array}$ & $\begin{array}{c}\text { Media Type } \\
\text { (If more than one) }\end{array}$ & $\begin{array}{c}\text { Matrix } \\
\text { Code }\end{array}$ & $\begin{array}{l}\text { Vol. \% } \\
\text { (If }\end{array}$ & Step & JIT & sc & CIF & $\begin{array}{l}\text { Trans. } \\
\text { Miles }\end{array}$ & $\begin{array}{l}\text { Facility } \\
\text { Abbr. }\end{array}$ & Unit Name & Comments \\
\hline 1 & & $\mathbf{S 5 4 4 0}$ & & $a$ & (O) & 0 & $1.00 \mathrm{E}+00$ & & TRANS & Transport - LLW & \\
\hline & & & & b & 0 & 0 & $1.00 E+00$ & & MWSF & Open/Segregate/Repack (nonhalogenated solids) & \\
\hline & & & & $\bar{d}$ & C & O & $1.00 \mathrm{E}+00$ & & WERF & Stabilization - Portland Cement & \\
\hline & & & & e & 0 & 0 & $1.00 \mathrm{E}+00$ & & SCDF & Disposal - Contact Handled & \\
\hline
\end{tabular}

Note: Where provided, media percentages are rough estimates used to facilitate treatment planning. They do not necessarily imply an accurate knowledge of waste stream composition, and were not derived for use outside this conceptual planning exercise.

Describe any special or unique technical concerns related to the treatment of this waste stream that would impact the use or implementation of standard hazardous waste treatment methods. 


\section{LA:W912}

LiWW Contacti H

A. IDENTIFICATION AND DESCRIPTION

Waste Stream ID: LA-W912

W. S. Name: COMBUSTIBLE DEBRIS

Content Code:

FFCA ID:

LA-W912

Waste Stream Description:

The wastes in this treatability group fall under EPA's hazardous debris regulation and can be treated to waste specific standards or using a debris rule technology. 
LAWWg̈12

HLW ContacteH

\section{PROJECTED WASTE GENERATION}

Is this waste stream still generated?

OYes ONo

If yes, when is the expected generation termination date?

Current Projections

\begin{tabular}{|r|r|r|r|l|l|}
\hline Est. Date & Start Year & Interval & \multicolumn{1}{|c|}{ Vol $(\mathbf{m} 3)$} & Mass $(\mathbf{k g})$ & \multicolumn{1}{|l|}{ Source of Estimate } \\
\hline $5 / 2 / 95$ & 1995 & 1 & 0.30 & & Generator Update \\
\hline $5 / 2 / 95$ & 1996 & 1 & 0.30 & & Generator Update \\
\hline $5 / 2 / 95$ & 1997 & 1 & 0.30 & & Generator Update \\
\hline $5 / 2 / 95$ & 1998 & 1 & 0.30 & & Generator Update \\
\hline $5 / 2 / 95$ & 1999 & 1 & 0.30 & & Generator Update \\
\hline \multicolumn{5}{c|}{ Historical Projections } \\
\hline
\end{tabular}




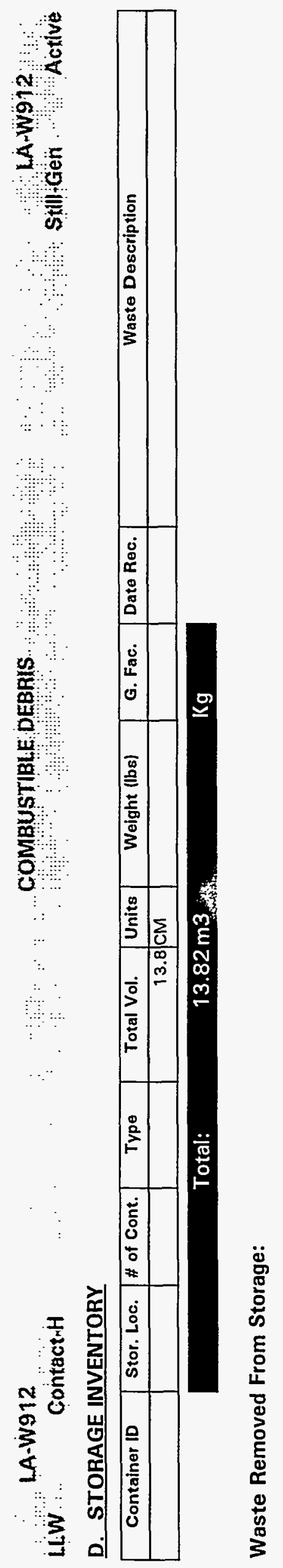




\section{LA-W912 LWW Contactio \\ E. RADIATION CHARACTERISTICS \\ F. TRU ALPHA ACTIVITY DISTRIBUTION \\ COMBUSTIBLLE DEBRIS

Radioactive Waste Type:

\begin{tabular}{|ll|}
\hline OllW & Oa-llw O tRU \\
OHLW & O Non-Rad \\
\hline
\end{tabular}

This waste stream is not identified as a TRU or a-LLW.

Mixed Waste:

Handling:

\begin{tabular}{|l|}
\hline OYes ONo \\
\hline O Contact O Remote \\
\hline
\end{tabular}

Radionuclide Contamination Accessiblity:

\begin{tabular}{|c|c|c|c|}
\hline External Surface & OYes & Ono & Unknown \\
\hline Internal Surface & OYes & ONo & OUnknown \\
\hline Dispersed Through Matrix & OYes & ONo & OUnknown \\
\hline
\end{tabular}

\section{Activity Levels}

Transuranic Alpha Activity:

Uranium/Thorium Alpha Activity:

Beta/Gamma Activity:

Surface Neutron Activity:

Total Activity:

\begin{tabular}{|c|c|c|}
\hline & Units: [ & \\
\hline & Units: [ & \\
\hline & @ surf & ace \\
\hline & @ 1-m & Units: $m R / h r$ \\
\hline & Units: [ & \\
\hline & $n C i / g$ & \\
\hline
\end{tabular}




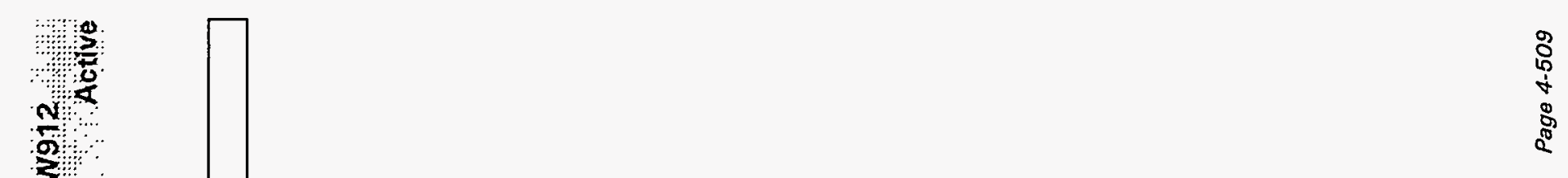


LANW912

\section{LLW .... Contact+H}

\section{COMBUSTBLE DEBRIS}

\section{J. HAZARDOUS CONSTITUENTS}

\begin{tabular}{|c|c|c|c|c|c|c|c|c|}
\hline & & & \multicolumn{4}{|c|}{ Concentration } & \multirow[b]{2}{*}{ Basis } & \multirow[b]{2}{*}{ Conc. Type } \\
\hline Code & Hazardous Waste Description & Treatment Subcategory & Typical & $\mathbf{L L}$ & UL & Units & & \\
\hline D001 & Ignitable & High-TOC ignitable characteristic liquids subca & & & & & A & Total \\
\hline$\overline{D 003}$ & Reactive & Reactive Cyanides based on 261.23(a)(5)Cyan & & & & & A & Total \\
\hline$D 005$ & Barium & & & & & & A & Total \\
\hline$\overline{D 006}$ & Cadmium & & & & & & A & Total \\
\hline$D 007$ & Chromium & Chromium (Total) & & & & & A & Total \\
\hline$D 008$ & Lead & & & & & & A & Total \\
\hline D009 & Mercury & Elemental mercury contaminated with radioact & & & & & A & Total \\
\hline D011 & Silver & & & & & & A & Total \\
\hline$\overline{D 022}$ & Chloroform & Chloroform managed in non-CWA/non-CWA-e & & & & & A & Total \\
\hline$\overline{D 035}$ & Methyl ethyl ketone & Methyle ethyl ketone managed in non-CWA/no & & & & & A & Total \\
\hline F001 & Spent halogenated solvents used in degreasing & 1,1,1-Trichloroethane & & & & & A & Total \\
\hline$\overline{F 002}$ & Spent halogenated solvents & o-Dichlorobenzene & & & & & A & Total \\
\hline$\overline{F 003}$ & Spent non-halogenated solvents & Acetone & & & & & A & Total \\
\hline F005 & Spent non-halogenated solvents & 2-Ethoxyethanol & & & & & A & Total \\
\hline
\end{tabular}




\section{K. POLYCHLORINATED BIPHENYLS (PCBS)}

Is this waste stream PCB contaminated? OYes $O$ No $O$ Unknown

If yes, what portion of the waste is $\mathrm{PCB}$ contaminated (volume \%):

If $<100 \%$, can the PCB contaminated waste be segregated and treated separately?

What is the PCB concentration in ppm?

\section{TREATMENT PLANS}

\begin{tabular}{|c|c|c|c|c|c|c|c|c|c|c|c|}
\hline $\begin{array}{c}\text { Opt. } \\
\#\end{array}$ & $\begin{array}{c}\text { Media Type } \\
\text { (If more than one) }\end{array}$ & $\begin{array}{c}\text { Matrix } \\
\text { Code }\end{array}$ & $\begin{array}{c}\text { Vol. \% } \\
\text { IIf }\end{array}$ & Step & JIT & sc & CIF & $\begin{array}{l}\text { Trans. } \\
\text { Miles }\end{array}$ & $\begin{array}{l}\text { Facility } \\
\text { Abbr. }\end{array}$ & Unit Name & Comments \\
\hline 1 & & S5400 & & $\bar{a}$ & () & 0 & $1.00 E+00$ & & TRANS & Transport - LLW & \\
\hline & & & & $\mathbf{b}$ & 0 & 0 & $1.00 \mathrm{E}+00$ & & MWSF & Open/Segregate/Repack (nonhalogenated solids) & \\
\hline & & & & c & 0 & 0 & $1.00 \mathrm{E}+00$ & & WERF & Incineration - Controlled Air Incinerator & \\
\hline & & & & d & 0 & 0 & $1.00 E+00$ & & WERF & Stabilization - Portland Cement & \\
\hline & & & & $\theta$ & 0 & 0 & $1.00 \mathrm{E}+00$ & & SCDF & Disposal - Contact Handled & \\
\hline
\end{tabular}

Note: Where provided, media percentages are rough estimates used to facilitate treatment planning. They do not necessarily imply an accurate knowledge of waste stream composition, and were not derived for use outside this conceptual planning exercise.

Describe any special or unique technical concerns related to the treatment of this waste stream that would impact the use or implementation of standard hazardous waste treatment methods. 
LA-W913

\section{LLW Contact + H}

A. IDENTIFICATION AND DESCRIPTION

Waste Stream ID: LA-W913

W. S. Name:

Content Code:

AQUEOUS WASTES WITH HEAVY METALS

FFCA ID:

LA-W913

Waste Stream Description:

These are aqueous wastes that are corrosive or that contain heavy metals, cyanides, nitrates, chromium, or arsenates.

\section{B. GENERATION SITE/PROCESS DESCRIPTION}

The following questions, concerning the generation site and process, should be answered even if the waste stream is no longer generated.

Name of Site Generating Waste (e.g., INEL, Rocky Flats, etc.):

\section{Location of activities (Area and Building):}

Operations (functions) performed in building:

Description of Process Generating Waste:

\section{LA-W913} Stili-Gen Active 
LA:W913

HWW Contact H

AQUEOUS WASTES WITH HEAVY METALS

UA W913

C. PROJECTED WASTE GENERATION

Is this waste stream still generated?

If yes, when is the expected generation termination date?

Current Projections

\begin{tabular}{|r|r|r|r|l|l|}
\hline Est. Date & \multicolumn{1}{|l|}{ Start Year } & Interval & \multicolumn{1}{|l|}{ Vol $(\mathbf{m} 3)$} & Mass $(\mathbf{k g})$ & Source of Estimate \\
\hline $5 / 2 / 95$ & 1995 & 1 & 0.20 & & Generator Update \\
\hline $5 / 2 / 95$ & 1996 & 1 & 0.20 & & Generator Update \\
\hline $5 / 2 / 95$ & 1997 & 1 & 0.20 & & Generator Update \\
\hline $5 / 2 / 95$ & 1998 & 1 & 0.20 & & Generator Update \\
\hline $5 / 2 / 95$ & 1999 & 1 & 0.20 & & Generator Update \\
\hline
\end{tabular}

Historical Projections 


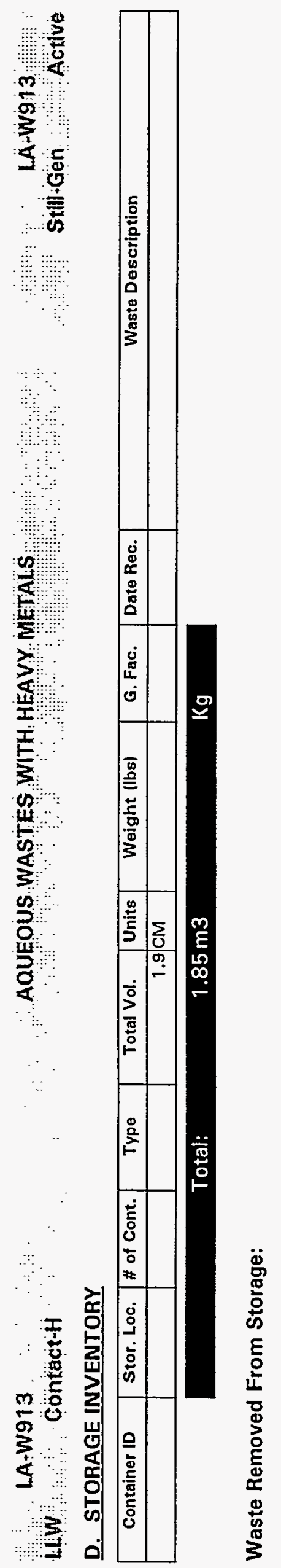




\section{LACW913 \\ LLW}

\section{E. RADIATION CHARACTERISTICS}

Radioactive Waste Type:

\begin{tabular}{|ll|}
\hline OLLW & Oa-LLW O TRU \\
OHLW & O Non-Rad \\
\hline
\end{tabular}

Mixed Waste:

Handling:

\section{F. TRU ALPHA ACTIVITY DISTRIBUTION}

This waste stream is not identified as a $T R U$ or a- $L L W$.

\begin{tabular}{|c|c|c|c|}
\hline External Surface & OYes & ONo & Onknown \\
\hline Internal Surface & OYes & ONo & OUnknown \\
\hline
\end{tabular}

\section{Activity Levels}

Transuranic Alpha Activity:

Uranium/Thorium Alpha Activity:

Beta/Gamma Activity:

Surface Neutron Activity:

Total Activity:

\begin{tabular}{l}
$\square$ \\
$\square$ Units: \\
\hline Units: \\
\hline \\
@ surface \\
@ @ 1-m Units: $\mathrm{mR} / \mathrm{hr}$ \\
\hline Units: $\square$ \\
nCi/g
\end{tabular}




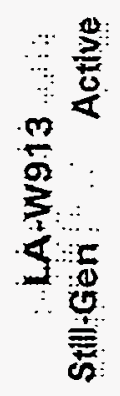

0
$\frac{5}{5}$
+
8
8
0

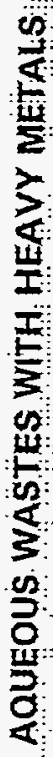

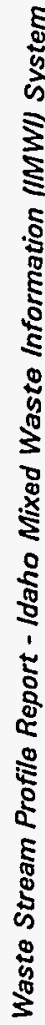

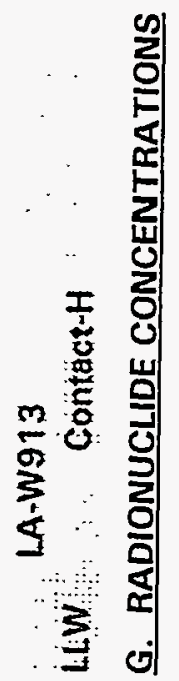


H. MATRIX CHARACTERISTICS

Primary Matrix Code: $L 1000$

Description: Aqueous Liquids/Slurries: : :

Combustibility: $\bigcirc$ Combustible (>90\%)

Mixed (10\%-90\%)

Noncombustible $(<10 \%)$

\section{MATRIX COMPOSITION}


LA.W913

\section{LWW : Contact-H}

\section{J. HAZARDOUS CONSTITUENTS}

\begin{tabular}{|c|c|c|c|c|c|c|c|c|}
\hline & & & \multicolumn{4}{|c|}{ Concentration } & & \\
\hline Code & Hazardous Waste Description & Treatment Subcategory & Typical & LL & UL & Units & Basis & Conc. Type \\
\hline 0001 & Ignitable & High-TOC ignitable characteristic liquids subca & & & & & A & Total \\
\hline$\overline{D 002}$ & Corrosive & Corrosive characteristic waste that are manag & & & & & A & Total \\
\hline 0003 & Reactive & Reactive Cyanides based on $261.23(a)(5)$ Cyan & & & & & A & Total \\
\hline$\overline{D 004}$ & Arsenic & & & & & & A & Total \\
\hline$D 005$ & Barium & & & & & & A & Total \\
\hline 0006 & Cadmium & & & & & & A & Total \\
\hline D007 & Chromium & Chromium (Total) & & & & & A & Total \\
\hline$D 008$ & Lead & & & & & & A & Total \\
\hline D009 & Mercury & Elemental mercury contaminated with radioact & & & & & A & Total \\
\hline$D 010$ & Selenium & & & & & & $A$ & Total \\
\hline D011 & Silver & & & & & & A & Total \\
\hline
\end{tabular}




\section{K. POLYCHLORINATED BIPHENYLS (PCBS)}

\section{Is this waste stream PCB contaminated? OYes O No $O$ Unknown}

If yes, what portion of the waste is PCB contaminated (volume \%):

If $<100 \%$, can the PCB contaminated waste be segregated and treated separately? $O$ Yes O No O Unknown

What is the PCB concentration in ppm?

If only a portion of the waste is PCB contaminated, report values for contaminated portion.

\section{TREATMENT PLANS}

\begin{tabular}{|c|c|c|c|c|c|c|c|c|c|c|c|}
\hline $\begin{array}{c}\text { Opt. } \\
\#\end{array}$ & $\begin{array}{c}\text { Media Type } \\
\text { (If more than one) }\end{array}$ & $\begin{array}{c}\text { Matrix } \\
\text { Code }\end{array}$ & $\begin{array}{l}\text { Vol. } \% \\
\text { (If }\end{array}$ & Step & JIT & sc & CIF & $\begin{array}{l}\text { Trans. } \\
\text { Miles }\end{array}$ & $\begin{array}{l}\text { Facility } \\
\text { Abbr. }\end{array}$ & Unit Name & Comments \\
\hline \multirow[t]{6}{*}{1} & & L1200 & & a & O & 0 & $1.00 E+00$ & & TRANS & Transport - LLW & \\
\hline & & & & b & 0 & 0 & $1.00 E+00$ & & AMWTF & $\mathrm{CH}$ - Opening \& Sorting & \\
\hline & & & & c & O & O & $1.00 \mathrm{E}+00$ & & AMWTF & $\mathrm{CH}$ - Sizing & \\
\hline & & & & d & 0 & 0 & $1.00 \mathrm{E}+00$ & & AMWTF & $\mathrm{CH}$ - Incineration/Thermal Desorption & \\
\hline & & & & $\theta$ & 0 & 0 & $1.00 E+00$ & & AMWTF & $\mathrm{CH}$ - Vitrification & \\
\hline & & & & $f$ & 0 & 0 & $1.00 E+00$ & & SCDF & Disposal · Contact Handled & \\
\hline
\end{tabular}

Note: Where provided, media percentages are rough estimates used to facilitate treatment planning. They do not necessarily imply an accurate knowledge of waste stream composition, and were not derived for use outside this conceptual planning exercise.

Describe any special or unique technical concerns related to the treatment of this waste stream that would impact the use or implementation of standard hazardous waste treatment methods. 


\section{$\therefore$ LA-W914}

LiW . Contact-H

A. IDENTIFICATION AND DESCRIPTION

Waste Stream ID: LA-W914

W. S. Name: CORROSIVE SOLUTIONS

Content Code:

FFCA ID:

\begin{tabular}{l}
$*$ \\
\hline LA-W914 \\
\hline
\end{tabular}

Waste Stream Description:

These are corrosive aqueous wastes. 


\begin{tabular}{|c|c|c|c|c|c|}
\hline \multicolumn{6}{|c|}{ C. PROJECTED WASTE GENERATION } \\
\hline \multirow{2}{*}{\multicolumn{6}{|c|}{$\begin{array}{l}\text { Is this waste stream still generated? } \\
\text { If yes, when is the expected generation termination date? }\end{array}$}} \\
\hline & & & & & \\
\hline \multicolumn{6}{|c|}{ Current Projections } \\
\hline Est. Date & Start Year & Interval & Vol (m3) & Mass (kg) & Source of Estimate \\
\hline $5 / 2 / 95$ & 1995 & 1 & 0.10 & & Generator Update \\
\hline $5 / 2 / 95$ & 1996 & 1) & 0.10 & & Generator Update \\
\hline $5 / 2 / 95$ & 1997 & 1) & 0.10 & & Generator Update \\
\hline $5 / 2 / 95$ & 1998 & 1 & 0.10 & & Generator Update \\
\hline $5 / 2 / 95$ & 1999 & 1] & 0.10 & & Generator Update \\
\hline
\end{tabular}




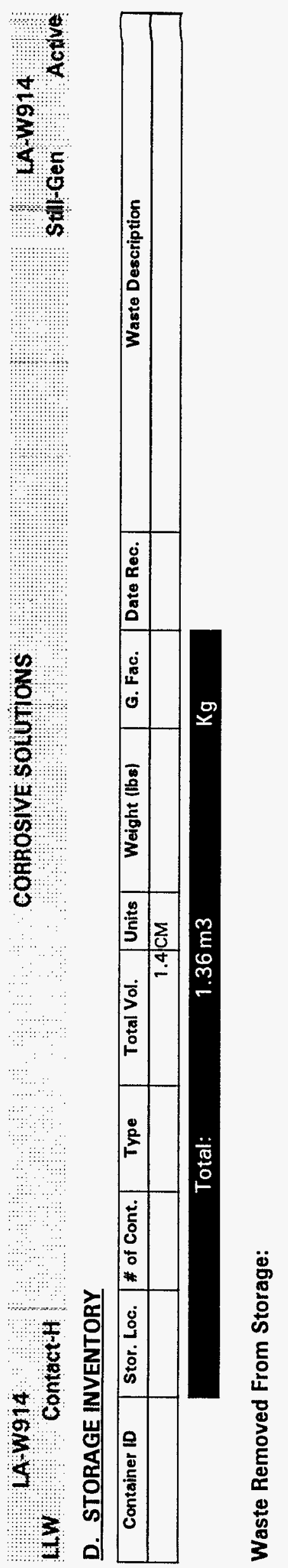




\section{E. RADIATION CHARACTERISTICS}

\section{F. TRU ALPHA ACTIVITY DISTRIBUTION}

Radioactive Waste Type:

$\begin{array}{ll}\text { Ollw } & \text { Oa-LlW O tru } \\ \text { OhlW } & \text { O Non-Rad }\end{array}$

This waste stream is not identified as a TRU or a-LLW.

Mixed Waste:

Handling:

\begin{tabular}{|l|}
\hline OYes ONo \\
\hline OContact \\
\hline Remote
\end{tabular}

Radionuclide Contamination Accessiblity:

External Surface

Internal Surface

\begin{tabular}{l} 
OYes ONo OUnknown \\
\hline OYes ONo OUnknown
\end{tabular}

Dispersed Through Matrix

OYes ONo OUnknown

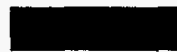

Activity Levels

\begin{tabular}{|c|c|c|}
\hline Transuranic Alpha Activity: & Units: & \\
\hline Uranium/Thorium Alpha Activity: & Units: & \\
\hline Beta/Gamma Activity: & \multicolumn{2}{|c|}{ @ surface } \\
\hline & @ 1-m & Units: $\mathrm{mR} / \mathrm{hr}$ \\
\hline Surface Neutron Activity: & Units: & \\
\hline Total Activity: & $\mathrm{nCi} / \mathrm{g}$ & \\
\hline
\end{tabular}



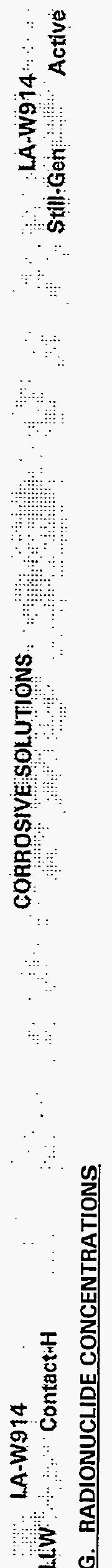
H. MATRIX CHARACTERISTICS

Primary Matrix Code: $L 2100$

Description: Organic Liquids: Aqueous/Organic Liquids: :

Combustibility: $\bigcirc$ Combustible (>90\%)

Mixed $(10 \%-90 \%)$

Noncombustible (<10\%)

\section{MATRIX COMPOSITION}


LA-W914

LLW : ContactrH

COAROSIVE SOLUTIONS

J. HAZARDOUS CONSTITUENTS

\begin{tabular}{|c|c|c|c|c|c|c|c|c|}
\hline \multirow[b]{2}{*}{ Code } & \multirow[b]{2}{*}{ Hazardous Waste Description } & \multirow[b]{2}{*}{ Treatment Subcategory } & \multicolumn{4}{|c|}{ Concentration } & \multirow[b]{2}{*}{ Basis } & \multirow[b]{2}{*}{ Conc. Type } \\
\hline & & & Typical & LL & UL & Units & & \\
\hline D001 & Ignitable & High-TOC ignitable characteristic liquids subca & & & & & A & Total \\
\hline D002 & Corrosive & Corrosive characteristic waste that are manag & & & & & A & Total \\
\hline
\end{tabular}

LA-W914: $: \cdots$ Still-Gen Active 
LWW ContactiH

\section{K. POLYCHLORINATED BIPHENYLS (PCBS)}

\section{Is this waste stream PCB contaminated? OYes $O$ No $O$ Unknown}

If yes, what portion of the waste is $\mathrm{PCB}$ contaminated (volume \%):

If $<100 \%$, can the PCB contaminated waste be segregated and treated separately? $O$ Yes ONo O Unknown

What is the PCB concentration in ppm? If only a portion of the waste is PCB contaminated, report values for contaminated portion.

L. TREATMENT PLANS

\begin{tabular}{|c|c|c|c|c|c|c|c|c|c|c|c|}
\hline $\begin{array}{c}\text { Opt. } \\
\#\end{array}$ & $\begin{array}{c}\text { Media Type } \\
\text { (If more than one) }\end{array}$ & $\begin{array}{c}\text { Matrix } \\
\text { Code }\end{array}$ & $\begin{array}{c}\text { Vol. } \% \\
\text { IIf }\end{array}$ & Step & JIT & Sc & CIF & $\begin{array}{l}\text { Trans. } \\
\text { Miles }\end{array}$ & $\begin{array}{l}\text { Facility } \\
\text { Abbr. }\end{array}$ & Unit Name & Comments \\
\hline 1 & & 1.2100 & & $\bar{a}$ & 0 & 0 & $1.00 E+00$ & & TRANS & Transport - LLW & \\
\hline & & & & b & O & 0 & $1.00 E+00$ & & AMWTF & $\mathrm{CH}$ - Opening \& Sorting & \\
\hline & & & & $\bar{c}$ & 0 & 0 & $1.00 E+00$ & & AMWTF & $\mathrm{CH}$ - Incineration/Thermal Desorption & liquid injection \\
\hline & & & & $d$ & 0 & 0 & $1.00 \mathrm{E}+00$ & & AMWTF & $\mathrm{CH}$ - Vitrification & \\
\hline & & & & e & 0 & 0 & $1.00 E+00$ & & SCDF & Disposal - Contact Handled & \\
\hline
\end{tabular}

Note: Where provided, media percentages are rough estimates used to facilitate treatment planning. They do not necessarily imply an accurate knowledge of waste stream composition, and were not derived for use outside this conceptual planning exercise.

Describe any special or unique technical concerns related to the treatment of this waste stream that would impact the use or implementation of standard hazardous waste treatment methods. 


\section{LA-W915}

LiW: Contact-H

A. IDENTIFICATION AND DESCRIPTION

Waste Stream ID: LA-W915

W. S. Name:

Content Code:

FFCA ID:

AQUEOUS CYANIDES, NITRATES, CHROMATES

*

LA-W915

\section{Waste Stream Description:}

These are aqueous solutions that contain cyanides, nitrates, chromates, or arsenates.

\section{AQUEOUS CYANIDES, NITHATES, CHROMATES}

A A W 915

Stilligen Activë

\section{B. GENERATION SITE/PROCESS DESCRIPTION}

The following questions, concerning the generation site and process, should be answered even if the waste stream is no longer generated.

Name of Site Generating Waste (e.g., INEL, Rocky Flats, etc.):

Location of activities (Area and Building):

Operations (functions) performed in building:

Description of Process Generating Waste: 
LA-W975

LLW: Contàct-H

C. PROJECTED WASTE GENERATION

Is this waste stream still generated?

If yes, when is the expected generation termination date?

Current Projections

\begin{tabular}{|c|l|l|l|l|}
\hline Est. Date & Start Year & Interval & Vol (m3) & Mass (kg) \\
\hline
\end{tabular}

O Yes ONo

Yes $\bigcirc$ No

Historical Projections 


\section{STORAGE INVENTORY}

\begin{tabular}{|c|c|c|c|c|c|c|c|c|c|}
\hline Container ID & Stor. Loc. & \# of Cont. & Type & Total Vol. & Units & Weight (lbs) & G. Fac. & Date Rec. & Waste Description \\
\hline & & & & & $\overline{C M}$ & & & & \\
\hline
\end{tabular}

Waste Removed From Storage: 


\section{LA-W9̈15}

LuWW Contact-H

AQUEOUS GYANIDES NITRATES CHROMATES

\section{LA-W915}

E. RADIATION CHARACTERISTICS

\section{F. TRU ALPHA ACTIVITY DISTRIBUTION}

Radioactive Waste Type:

\begin{tabular}{|ll|}
\hline OllW & Oa-LlW O TRU \\
O HLW & Onon-Rad \\
\hline
\end{tabular}

This waste stream is not identified as a TRU or a-LLW.

Mixed Waste:

\begin{tabular}{|l|}
\hline OYes ONo \\
\hline O Contact O Remote
\end{tabular}

Radionuclide Contamination Accessiblity:

\begin{tabular}{|c|c|c|c|}
\hline External Surface & Yyes & No & Unknown \\
\hline Internal Surface & Yes & ONo & O Unknown \\
\hline ispersed Through $\mathbf{N}$ & OYes & No & Onnknown \\
\hline
\end{tabular}

\section{Activity Levels}

Uranium/Thorium Alpha Activity:

Beta/Gamma Activity:

Surface Neutron Activity:

Total Activity:

\begin{tabular}{|c|c|c|}
\hline & Units: [ & \\
\hline & Units: & \\
\hline & \multicolumn{2}{|c|}{ @ surface } \\
\hline & @ 1-m & Units: $\mathrm{mR} / \mathrm{hr}$ \\
\hline & Units: [ & \\
\hline & $\mathrm{nCi} / \mathrm{g}$ & \\
\hline
\end{tabular}



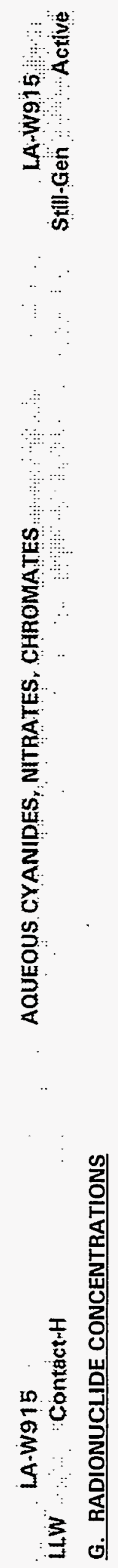


\section{H. MATRIX CHARACTERISTICS}

Primary Matrix Code: $L 1240$

Description: Aqueous Liquids/Slurries: Aqueous Slurries: Cyanide Aqueous Clurries:

Combustibility

Combustible (>90\%)

Mixed $(10 \%-90 \%)$

Noncombustible $(<10 \%)$

\section{MATRIX COMPOSITION}




\section{LA.W915}

LLW: Contact

AQUEOUS CYANIDES NITRATES CHROMATES

\section{J. HAZARDOUS CONSTITUENTS}

\begin{tabular}{|c|c|c|c|c|c|c|c|c|}
\hline \multirow[b]{2}{*}{ Code } & \multirow[b]{2}{*}{ Hazardous Waste Description } & & \multicolumn{4}{|c|}{ Concentration } & \multirow[b]{2}{*}{ Basis } & \multirow[b]{2}{*}{ Conc. Type } \\
\hline & & Treatment Subcategory & Typical & $\mathbf{L L}$ & UL & Units & & \\
\hline D001 & Ignitable & High-TOC ignitable characteristic liquids subca & & & & & A & Total \\
\hline $\mathrm{D002}$ & Corrosive & Corrosive characteristic waste that are manag & & & & & $A$ & Total \\
\hline D003 & Reactive & Reactive Cyanides based on $261.23(\mathrm{a})(5)$ Cyan & & & & & $\bar{A}$ & Total \\
\hline$\overline{D 004}$ & Arsenic & & & & & & $\bar{A}$ & Total \\
\hline D005 & Barium & & & & & & $\bar{A}$ & Total \\
\hline D006 & Cadmium & & & & & & A & Total \\
\hline D007 & Chromium & Chromium (Total) & & & & & A & Total \\
\hline$\overline{D 008}$ & Lead & & & & & & A & Total \\
\hline D009 & Mercury & Elemental mercury contaminated with radioact & & & & & A & Total \\
\hline D010 & Selenium & & & & & & A & Total \\
\hline D011 & Silver & & & & & & A & Total \\
\hline F007 & Spent cyanide plating bath solutions from electropl & Cadmium & & & & & $\bar{A}$ & Total \\
\hline P029 & Copper cyanides & Cyanide (Total) & & & & & A & Total \\
\hline P098 & Potassium cyanide & Cyanide (Total) & & & & & A & Total \\
\hline
\end{tabular}




\section{LLW : Gontact-H}

\section{A $\because$ O}

\section{K. POLYCHLORINATED BIPHENYLS (PCBS)}

Is this waste stream PCB contaminated?

OYes O No O Unknown

If yes, what portion of the waste is PCB contaminated (volume \%):

If $<100 \%$, can the PCB contaminated waste be segregated and treated separately?

What is the PCB concentration in ppm?

Y Yes $O$ No Unknown

\section{TREATMENT PLANS}

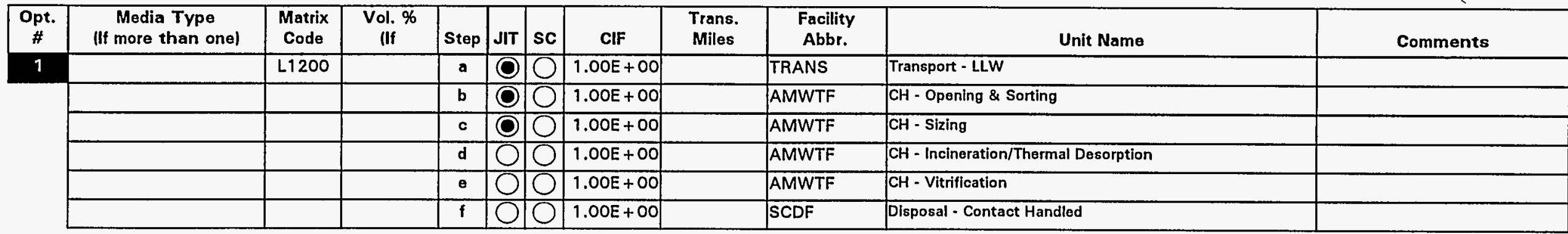

Note: Where provided, media percentages are rough estimates used to facilitate treatment planning. They do not necessarily imply an accurate knowledge of waste stream composition, and were not derived for use outside this conceptual planning exercise.

Describe any special or unique technical concerns related to the treatment of this waste stream that would impact the use or implementation of standard hazardous waste treatment methods. 


LA-W919
LWW $\quad$ Contact-H
A. IDENTIFICATION AND DESCRIPTION
Waste Stream ID: LA-W919
W. S. Name:
Content Code:
FFCA ID:
Waste Stream Description:
This group consists of a wide variety of wastes, such as organic-contaminated vermiculite,
Thich cannot be classified as debris under RCRA.

\section{B. GENERATION SITE/PROCESS DESCRIPTION}

The following questions, concerning the generation site and process, should be answered even if the waste stream is no longer generated.

Name of Site Generating Waste (e.g., INEL, Rocky Flats, etc.):

Location of activities (Area and Building):

Operations (functions) performed in building:

Operations (functions) performed in building:

Description of Process Generating Waste: 
LAW919

ORGANIC CONTAMINATED NONCOMBUSTTSOLIDS

HiW Contact-H

C. PROJECTED WASTE GENERATION

Is this waste stream still generated?

If yes, when is the expected generation termination date?

\section{Current Projections}

\begin{tabular}{|r|r|r|r|r|l|}
\hline Est. Date & Start Year & Interval & \multicolumn{1}{|c|}{ Vol (m3) } & Mass (kg) & \multicolumn{1}{|c|}{ Source of Estlmate } \\
\hline $5 / 2 / 95$ & 1995 & 1 & 1.60 & & Generator Update \\
\hline $5 / 2 / 95$ & 1996 & 1 & 1.60 & & Generator Update \\
\hline $5 / 2 / 95$ & 1997 & 1 & 1.60 & & Generator Update \\
\hline $5 / 2 / 95$ & 1998 & 1 & 1.60 & & Generator Update \\
\hline $5 / 2 / 95$ & 1999 & 1 & 1.60 & & Generator Update \\
\hline
\end{tabular}

Historical Projections 
LA-W919

LiWW. : Contact-H

ORGANIC-CONTAMINATED NONCOMBUST SOLIDS $\quad$ LA-W919

D. STORAGE INVENTORY

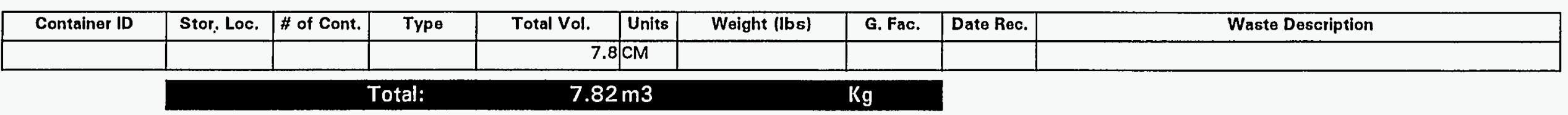

Waste Removed From Storage: 


\section{LA-W9̈19}

ORGANIC.CONTAMINATEO NONCOMBBUST SOLIDS

\section{E. RADIATION CHARACTERISTICS}

Radioactive Waste Type:

\begin{tabular}{|ll|}
\hline OLW & Oa-LLW $\bigcirc$ TRU \\
OHLW & ONon-Rad \\
\hline
\end{tabular}

Mixed Waste:

Handling:

Radionuclide Contamination Accessiblity:

External Surface

Internal Surface

Dispersed Through Matrix

\begin{tabular}{|}
\begin{tabular}{|ccc|}
\hline OYes & ONo & O Unknown \\
\hline OYes & Ono & O Unknown \\
\hline OYes & ONo & O Unknown \\
\hline
\end{tabular}
\end{tabular}

\section{Activity Levels}

Transuranic Alpha Activity:

Uranium/Thorium Alpha Activity:

Beta/Gamma Activity:

Surface Neutron Activity:

Total Activity:

$\square$ Units:
$\square$ Units: $\square$
$\square$ @ surface
$\square$ @ 1-m Units: mR/hr
$\square$ Units: $\square$
nCi/g



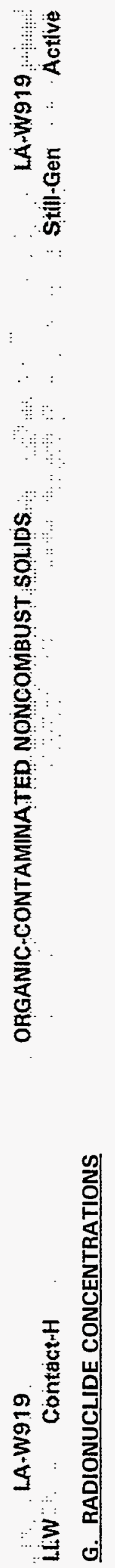
LA-W919

LLW Contact-H

H. MATRIX CHARACTERISTICS

Primary Matrix Code: $\mathbf{S 3 2 1 2}$

Description: Homogeneous Solids: Organic Homogeneous solids: Organic Particulates: Organic Absorbents

Combustibility: $\bigcirc$ Combustible (>90\%)

Mixed $(10 \%-90 \%)$

Noncombustible $(<10 \%)$

\section{MATRIX COMPOSITION}




\section{LWW Contact-H}

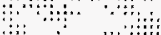

\section{J. HAZARDOUS CONSTITUENTS}

\begin{tabular}{|c|c|c|c|c|c|c|c|c|}
\hline \multirow[b]{2}{*}{ Code } & \multirow[b]{2}{*}{ Hazardous Waste Description } & \multirow[b]{2}{*}{ Treatment Subcategory } & \multicolumn{4}{|c|}{ Concentration } & \multirow[b]{2}{*}{ Basis } & \multirow[b]{2}{*}{ Conc. Type } \\
\hline & & & Typical & $\mathbf{L L}$ & UL & Units & & \\
\hline D001 & Ignitable & High-TOC ignitable characteristic liquids subca & & & & & A & Total \\
\hline D003 & Reactive & Reactive Cyanides based on $261.23(\mathrm{a})(5)$ Cyan & & & & & $A$ & Total \\
\hline D004 & Arsenic & & & & & & A & Total \\
\hline D005 & Barium & & & & & & A & Total \\
\hline D006 & Cadmium & & & & & & $\bar{A}$ & Total \\
\hline D007 & Chromium & Chromium (Total) & & & & & A & Total \\
\hline D009 & Mercury & Elemental mercury contaminated with radioact & & & & . & A & Total \\
\hline D010 & Selenium & & & & & & A & Total \\
\hline D011 & Silver & & & & & & A & Total \\
\hline $\mathrm{DO27}$ & p-Dichlorobenzene (1,4-Dichlorobenzene) & p-Dichlorobenzene managed in non-CWA/non- & & & & & A & Total \\
\hline 0030 & 2,4-Dinitrotoluene & 2,4-Dinitrotoluene managed in non-CWA/non- & & & & & $\bar{A}$ & Total \\
\hline D032 & Hexachlorobenzene & Hexachlorobenzene managed in non-CWA/non & & & & & A & Total \\
\hline D033 & Hexachlorobutadiene & Hexachlorobutadiene managed in non-CWA/no & & & & & A & Total \\
\hline D042 & 2,4,6-Trichlorophenol & 2,4,6-Trichlorophenol managed in non-CWA/n & & & & & A & Total \\
\hline D043 & Vinyl chloride & Vinyl Chloride managed in non-CWA/non-CWA & & & & & A & Total \\
\hline F001 & Spent halogenated solvents used in degreasing & 1,1,1-Trichloroethane & & & & & A & Total \\
\hline F002 & Spent halogenated solvents & o-Dichlorobenzene & & & & & A & Total \\
\hline F004 & Spent non-halogenated solvents & Nitrobenzene & & & & & A & Total \\
\hline FOO5 & Spent non-halogenated solvents & 2-Ethoxyethanol & & & & & A & Total \\
\hline
\end{tabular}




\section{LA:W919.}

LLW Contact H

\section{K. POLYCHLORINATED BIPHENYLS (PCBS)}

Is this waste stream $\mathrm{PCB}$ contaminated?

OYes O No O Unknown

If yes, what portion of the waste is PCB.contaminated (volume \%):

If $<100 \%$, can the PCB contaminated waste be segregated and treated separately?

What is the PCB concentration in ppm?

Yes $O$ No O Unknown

\section{TREATMENT PLANS}

\begin{tabular}{|c|c|c|c|c|c|c|c|c|c|c|c|}
\hline $\begin{array}{c}\text { Opt. } \\
\#\end{array}$ & $\begin{array}{c}\text { Media Type } \\
\text { (If more than one) }\end{array}$ & $\begin{array}{c}\text { Matrix } \\
\text { Code }\end{array}$ & $\begin{array}{l}\text { Vol. \% } \\
\text { lif }\end{array}$ & Step & JIT & sc & $\mathrm{CIF}$ & $\begin{array}{l}\text { Trans. } \\
\text { Miles }\end{array}$ & $\begin{array}{l}\text { Facility } \\
\text { Abbr. }\end{array}$ & Unit Name & Comments \\
\hline \multirow[t]{6}{*}{1} & & $\$ 3212$ & & $\bar{a}$ & O & 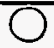 & $1.00 E+00$ & & TRANS & Transport - LLW & \\
\hline & & & & b & O & $\bar{O}$ & $1.00 E+00$ & & AMWTF & $\mathrm{CH}$ - Opening \& Sorting & \\
\hline & & & & $\bar{c}$ & $\widehat{O}$ & O & $1.00 \mathrm{E}+00$ & & AMWTF & $\mathrm{CH}$ - Sizing & \\
\hline & & & & d & 0 & 0 & $1.00 \mathrm{E}+00$ & & AMWTF & $\mathrm{CH}$ - Incineration/Thermal Dosorption & \\
\hline & & & & e & 0 & 0 & $1.00 E+00$ & & AMWTF & $\mathrm{CH}$ - Vitrification & \\
\hline & & & & $f$ & 0 & 0 & $1.00 \mathrm{E}+00$ & & SCDF & Disposal - Contact Handled & \\
\hline
\end{tabular}

Note: Where provided, media percentages are rough estimates used to facilitate treatment planning. They do not necessarily imply an accurate knowledge of waste stream composition, and were not derived for use outside this conceptual planning exercise.

Describe any special or unique technical concerns related to the treatment of this waste stream that would impact the use or implementation of standard hazardous waste treatment methods. 


\section{LLW . Contact-H}

\section{A. IDENTIFICATION AND DESCRIPTION}

Waste Stream ID: LA-W920

W. S. Name:

Content Code:

FFCA ID:

ELEMENTAL MERCURY

\begin{tabular}{l}
\hline LA-W920 \\
\hline LA-W
\end{tabular}

\section{Waste Stream Description:}

Mercury has been used historically in vacuum systems at LANL. Most of this waste in this treatability group has been reclaimed from surplus vacuum systems.

\section{B. GENERATION SITE/PROCESS DESCRIPTION}

The following questions, concerning the generation site and process, should be answered even if the waste stream is no longer generated.

Name of Site Generating Waste (e.g., INEL, Rocky Flats, etc.):

Location of activities (Area and Building):

Operations (functions) performed in building:

Description of Process Generating Waste: 


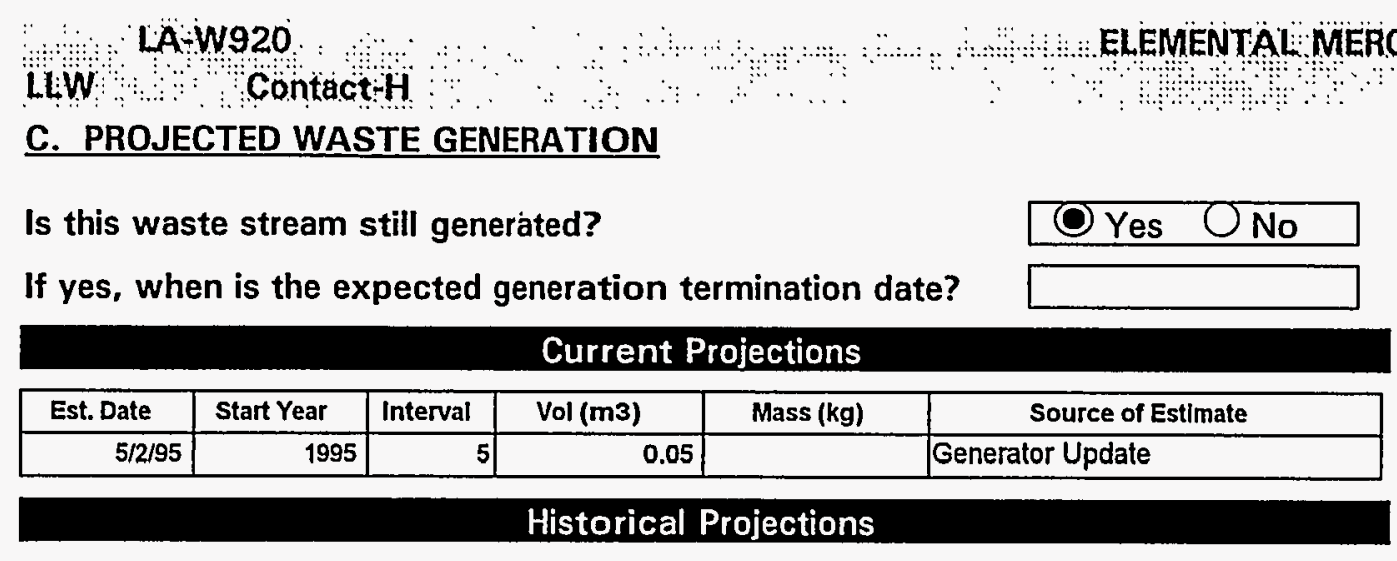

Historical Projections 


\section{LA-W920}

LWW $\quad$ ContactrH

D. STORAGE INVENTORY

\begin{tabular}{|c|c|c|}
\hline Container ID & Stor. Loc. & \# of Cont. \\
\hline & & \\
\hline
\end{tabular}

Total:

Type

\begin{tabular}{|l|l|l|}
\hline Total Vol. & Units & Weight (lbs) \\
\hline
\end{tabular}

\begin{tabular}{l|l|l|}
0.5 & $\mathrm{CM}$ \\
\hline
\end{tabular}

$0.50 \mathrm{m3}$

ELEMENTAL MERCURY

LA-W920

Stillogen Active

Waste Removed From Storage: 


\section{LLW $L$ W W 20}

E. RADIATION CHARACTERISTICS

Radioactive Waste Type:

\begin{tabular}{|ll|}
\hline OlLW & Oa-LLW O TRU \\
OHLW & O Non-Rad \\
\hline
\end{tabular}

Mixed Waste:

\begin{tabular}{|l|}
\hline O Yes $O$ No \\
\hline O Contact O Remote \\
\hline
\end{tabular}

Radionuclide Contamination Accessiblity:

\begin{tabular}{|c|c|c|}
\hline External Surface $\bigcirc$ Yes & ONo & Unknown \\
\hline Internal Surface $O$ Yes & ONo & OUnknown \\
\hline Dispersed Through Matrix $O$ Yes & ONo & Unknown \\
\hline \multicolumn{3}{|c|}{ Activity Levels } \\
\hline Transuranic Alpha Activity: & & Units: \\
\hline Uranium/Thorium Alpha Activity: & & Units: \\
\hline \multirow[t]{2}{*}{ Beta/Gamma Activity: } & & @ surface \\
\hline & & @ 1-m Units: $\mathrm{mR} / \mathrm{hr}$ \\
\hline Surface Neutron Activity: & & Units: \\
\hline Total Activity: & & $\mathrm{nCi} / \mathrm{g}$ \\
\hline
\end{tabular}

$$
\text { Handling: } O \text { Contact } O \text { Remote }
$$

\section{F. TRU ALPHA ACTIVITY DISTRIBUTION}

This waste stream is not identified as a TRU or a-LLW. 


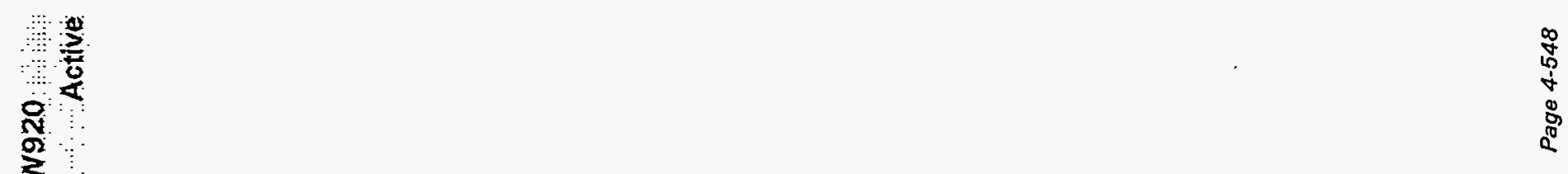


H. MATRIX CHARACTERISTICS

Primary Matrix Code: X7100

Description: Special Waste: Elemental Mercury: :

Combustibility: $\bigcirc$ Combustible ( $>90 \%$ )

Mixed $(10 \%-90 \%)$

Noncombustible $(<10 \%)$

\section{MATRIX COMPOSITION}


LA-W920

LLW:: : Contact-H

ELEMENTAL MERCUPYY

: $\therefore$ LArW920

Still-Gen : Active

\section{J. HAZARDOUS CONSTITUENTS}

\begin{tabular}{|c|c|c|c|c|c|c|c|c|}
\hline & & & \multicolumn{4}{|c|}{ Concentration } & \multirow[b]{2}{*}{ Basis } & \multirow[b]{2}{*}{ Conc. Type } \\
\hline Code & Hazardous Waste Description & Treatment Subcategory & Typical & $\overline{L L}$ & UL & Units & & \\
\hline D006 & Cadmium & & & & & & $\bar{A}$ & Total \\
\hline D009 & Mercury & Elemental mercury contaminated with radioact & & & & & A & Total \\
\hline F005 & Spent non-halogenated solvents & 2-Ethoxyethanol & & & & & $A$ & Total \\
\hline
\end{tabular}




\section{K. POLYCHLORINATED BIPHENYLS (PCBS)}

Is this waste stream PCB contaminated? OYes ONo O Unknown

If yes, what portion of the waste is PCB contaminated (volume \%):

If $<100 \%$, can the PCB contaminated waste be segregated and treated separately? OYes O No O Unknown

What is the PCB concentration in ppm?

If only a portion of the waste is PCB contaminated, report values for contaminated portion.

\section{TREATMENT PLANS}

\begin{tabular}{|c|c|c|c|c|c|c|c|c|c|c|c|}
\hline $\begin{array}{c}\text { Opt. } \\
\#\end{array}$ & $\begin{array}{c}\text { Media Type } \\
\text { (If more than one) }\end{array}$ & $\begin{array}{c}\text { Matrix } \\
\text { Code }\end{array}$ & $\begin{array}{l}\text { Vol. \% } \\
\text { (If }\end{array}$ & Step & JIT & sc & CIF & $\begin{array}{l}\text { Trans. } \\
\text { Miles }\end{array}$ & $\begin{array}{l}\text { Facility } \\
\text { Abbr. }\end{array}$ & Unit Name & Comments \\
\hline 1 & & $x 7100$ & & $\bar{a}$ & (O) & 0 & $1.00 E+00$ & & TRANS & Transport - LLW & \\
\hline \multirow[t]{2}{*}{, } & & & & $\bar{b}$ & 0 & 0 & $1.00 \mathrm{E}+00$ & & AMWTF & CH-Opening \& Sorting & \\
\hline & & & & d & 0 & $C$ & $1.00 E+00$ & & SCDF & Disposal - Contact Handled & \\
\hline
\end{tabular}

Note: Where provided, media percentages are rough estimates used to facilitate treatment planning. They do not necessarily imply an accurate knowledge of waste stream composition, and were not derived for use outside this conceptual planning exercise.

Describe any special or unique technical concerns related to the treatment of this waste stream that would impact the use or implementation of standard hazardous waste treatment methods. 


\section{LA-W921 \\ LLW: ContactrH}

A. IDENTIFICATION AND DESCRIPTION

Waste Stream ID: LA-W921

W. S. Name:

Content Code:

FFCA ID:

\begin{tabular}{|l|}
\hline ACTIVATED OR INSEPARABLE LEAD \\
\hline$*$ \\
\hline LA-W921 \\
\hline
\end{tabular}

\section{Waste Stream Description:}

This lead has been volume-contaminated with radioactivity, generally through exposure to an accelerator beam, and is not compatible with the operation of the lead decontamination trailer. 
LA-W921

LiW Contact-H

\section{PROJECTED WASTE GENERATION}

Is this waste stream still generated?

If yes, when is the expected generation termination date?

Current Projections

\begin{tabular}{|c|c|c|c|c|c|}
\hline Est. Date & Start Year & Interval & Vol (m3) & Mass (kg) & Source of Estimate \\
\hline $5 / 2 / 95$ & 1995 & 1 & 0.20 & & Generator Update \\
\hline $5 / 2 / 95$ & 1996 & 1 & 0.20 & & Generator Update \\
\hline $5 / 2 / 95$ & 1997 & 1 & 0.20 & & Generator Update \\
\hline $5 / 2 / 95$ & 1998 & 1 & 0.20 & & Generator Update \\
\hline $5 / 2 / 95$ & 1999 & 1 & 0.20 & & Generator Update \\
\hline
\end{tabular}

\section{OYes ONo}

Historical Projections
KA WO21

Still=Gen Active 


\section{LA.W921}

LWW. ContactrH

D. STORAGE INVENTORY
ACTIVATED Ö̈INSEPARABLE LEAD

L. W.W921

Still:Gën : Active

\begin{tabular}{|c|c|c|c|c|c|c|c|c|c|}
\hline Container ID & Stor. Loc. & \# of Cont. & Typo & Total Vol. & Units & Weight (lbs) & G. Fac. & Date Rec. & Waste Description \\
\hline & & & & 15. & $\mathrm{CM}$ & & & & \\
\hline
\end{tabular}

Waste Removed From Storage: 


\section{LA-W9Z̈i \\ Li.W Contact-H \\ E. RADIATION CHARACTERISTICS}

Radioactive Waste Type:

\begin{tabular}{|ll|}
\hline OllW & Oa-LLW $\bigcirc$ tRU \\
OhlW & O Non-Rad \\
\hline
\end{tabular}

Mixed Waste:

Handling:

\begin{tabular}{|l|} 
O Yes O No \\
\hline Contact \\
\hline Remote
\end{tabular}

Radionuclide Contamination Accessiblity:

External Surface OYes ONo OUnknown

Internal Surface OYes ONo OUnknown

Dispersed Through Matrix OYes ONo OUnknown

\section{Activity Levels}

Transuranic Alpha Activity:

Uranium/Thorium Alpha Activity:

Beta/Gamma Activity:

Surface Neutron Activity:

Total Activity:

\begin{tabular}{|c|c|c|}
\hline & Units: & \\
\hline & Units: [ & \\
\hline & @ surf & ace \\
\hline & @ 1-m & Units: $\mathrm{mR} / \mathrm{hr}$ \\
\hline & Units: & \\
\hline & $\mathrm{nCi} / \mathrm{g}$ & \\
\hline
\end{tabular}

\section{F. TRU ALPHA ACTIVITY DISTRIBUTION}

This waste stream is not identified as a $T R U$ or a-LLW. 


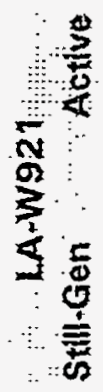


LA-Ẅ9̈21

ACTIVATED OR INSEPARÄLE LEAD

HW I Contäct

H. MATRIX CHARACTERISTICS

Primary Matrix Code: $X 7212$

Description: Special Waste: Elemental Hazardous Metals: Elemental Lead: Activated Lead

Combustibility: $\bigcirc$ Combustible (>90\%)

Mixed $(10 \%-90 \%)$

Noncombustible $(<10 \%)$

\section{MATRIX COMPOSITION}




\begin{tabular}{|c|c|c|c|c|c|c|c|c|}
\hline & & & \multicolumn{4}{|c|}{ Concentration } & & \\
\hline Code & Hazardous Waste Description & Treatment Subcategory & Typical & $\mathbf{L L}$ & UL & Units & Basis & Conc. Type \\
\hline$D 008$ & Lead & & & & & & A & Total \\
\hline
\end{tabular}




\section{LA-W921}

LiW ContäctH

\section{K. POLYCHLORINATED BIPHENYLS (PCBS)}

Is this waste stream PCB contaminated?

\section{OYes O No O Unknown}

If yes, what portion of the waste is PCB contaminated (volume \%):

If $<100 \%$, can the PCB contaminated waste be segregated and treated separately? $O$ Yes $O$ No Onknown

What is the PCB concentration in ppm?

If only a portion of the waste is PCB contaminated, report values for contaminated portion.

\section{TREATMENT PLANS}

\begin{tabular}{|c|c|c|c|c|c|c|c|c|c|c|c|}
\hline $\begin{array}{c}\text { Opt. } \\
\#\end{array}$ & $\begin{array}{c}\text { Media Type } \\
\text { (If more than one) }\end{array}$ & $\begin{array}{c}\text { Matrix } \\
\text { Code }\end{array}$ & $\begin{array}{l}\text { Vol. \% } \\
\text { lif }\end{array}$ & Step & JIT & sc & CIF & $\begin{array}{l}\text { Trans. } \\
\text { Miles }\end{array}$ & $\begin{array}{l}\text { Facility } \\
\text { Abbr. }\end{array}$ & Unit Name & Comments \\
\hline \multirow[t]{4}{*}{1} & & $\overline{X 7212}$ & & $\bar{a}$ & 0 & 0 & $1.00 E+00$ & & TRANS & Transport - LLW & \\
\hline & & & & b & 0 & 0 & $1.00 \mathrm{E}+00$ & & WROC & Sizing & \\
\hline & & & & c & 0 & 0 & $1.00 E+00$ & & WROC & Macroencapsulation & \\
\hline & & & & $\bar{d}$ & 0 & 0 & $1.00 E+00$ & & SCDF & Disposal - Contact Handled & \\
\hline
\end{tabular}

Note: Where provided, media percentages are rough estimates used to facilitate treatment planning. They do not necessarily imply an accurate knowledge of waste stream composition, and were not derived for use outside this conceptual planning exercise.

Describe any special or unique technical concerns related to the treatment of this waste stream that would impact the use or implementation of standard hazardous waste treatment methods.

This material has not been treated. It is not compatible with lead decontamination trailer due to the volume contamination. 


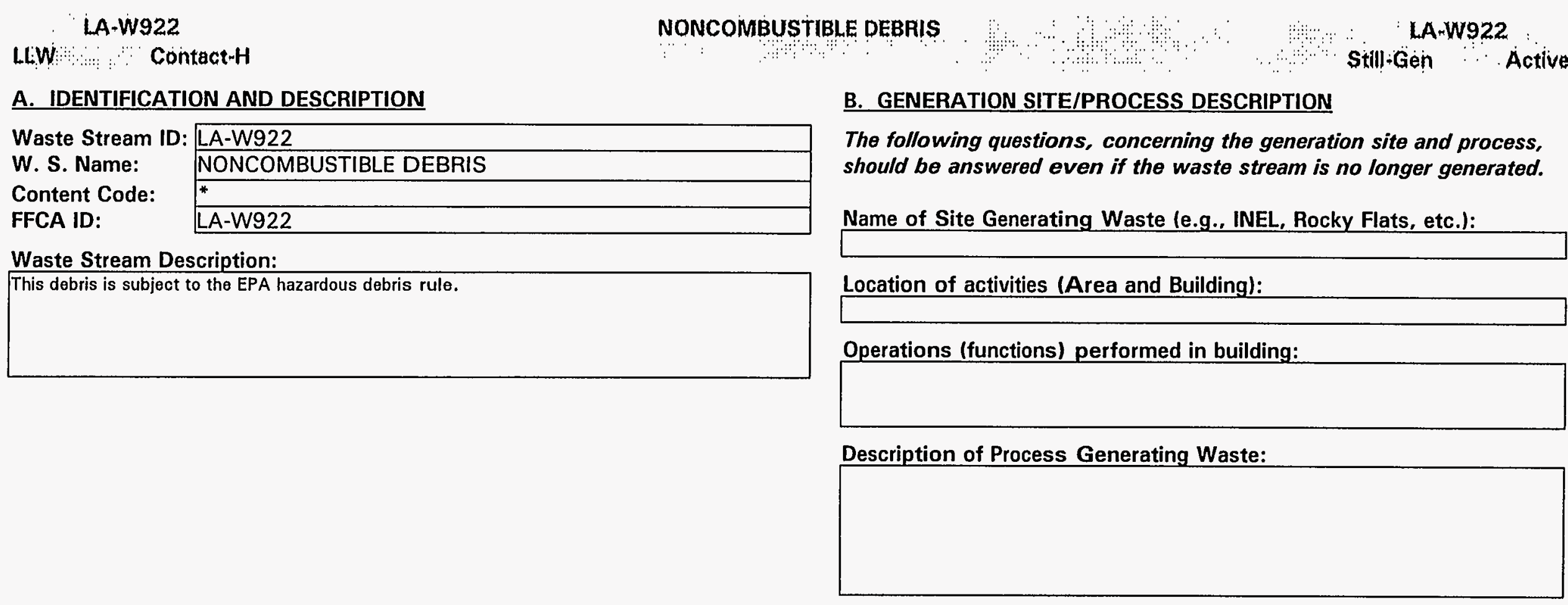




\section{PROJECTED WASTE GENERATION}

Is this waste stream still generated?

$$
\text { OYes ONo }
$$

If yes, when is the expected generation termination date?

\begin{tabular}{|r|r|r|r|l|l|}
\hline \multicolumn{7}{|c|}{ Current Projections } \\
\hline Est. Date & \multicolumn{1}{|c|}{ Start Year } & Interval & \multicolumn{1}{|c|}{ Vol (m3) } & Mass (kg) & \multicolumn{1}{|c|}{ Source of Estlmate } \\
\hline $5 / 2 / 95$ & 1995 & 1 & 0.60 & & Generator Update \\
\hline $5 / 2 / 95$ & 1996 & 1 & 0.60 & & Generator Update \\
\hline $5 / 2 / 95$ & 1997 & 1 & 0.60 & Generator Update \\
\hline $5 / 2 / 95$ & 1998 & 1 & 0.60 & Generator Update \\
\hline $5 / 2195$ & 1999 & 1 & 0.60 & Generator Update \\
\hline \multicolumn{7}{|c|}{ Historical Projections } \\
\hline
\end{tabular}




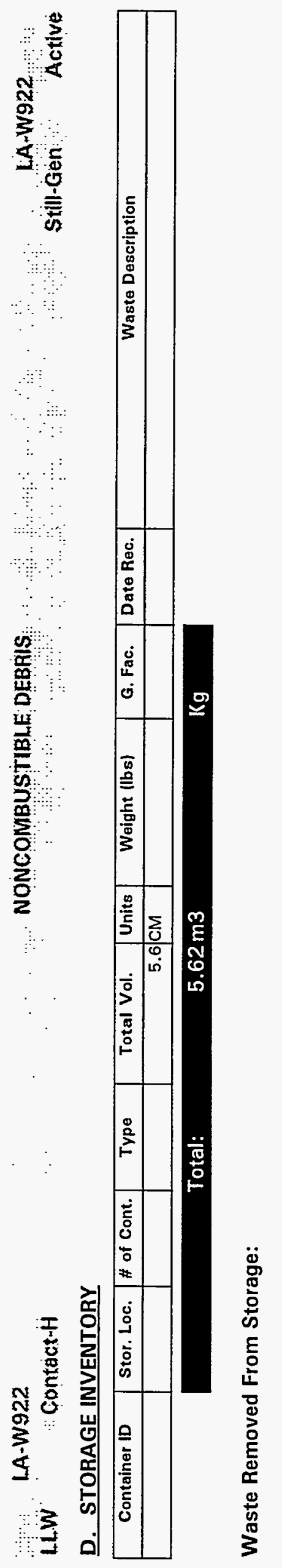




\section{E. RADIATION CHARACTERISTICS}

Radioactive Waste Type:

\begin{tabular}{ll|} 
OlLW & Oa-LLW $\bigcirc$ TRU \\
OHLW & O Non-Rad \\
\hline
\end{tabular}

Mixed Waste:

\begin{tabular}{|l|}
\hline O Yes O No \\
\hline O Contact O Remote \\
\hline
\end{tabular}

Radionuclide Contamination Accessiblity:

\begin{tabular}{|c|c|c|c|}
\hline External Surface & OYes & ONo & OUnknown \\
\hline Internal Surface & OYes & ONo & O Unknown \\
\hline Through Matrix & les & $\longdiv { \text { No } }$ & 00 \\
\hline
\end{tabular}

Dispersed Through Matrix

\section{Activity Levels}

Transuranic Alpha Activity:

Uranium/Thorium Alpha Activity:

Beta/Gamma Activity:

Surface Neutron Activity:

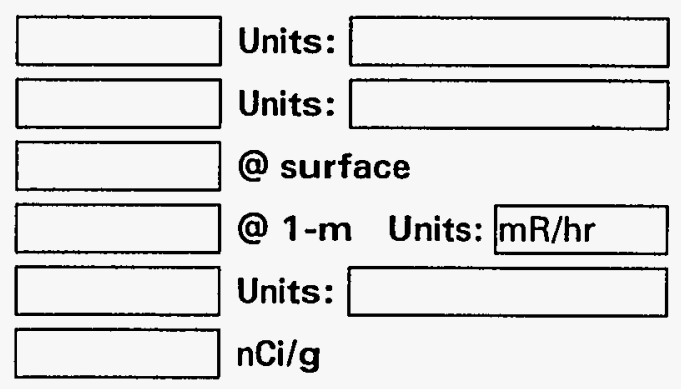

Total Activity:

\section{F. TRU ALPHA ACTIVITY DISTRIBUTION}

This waste stream is not identified as a TRU or a-LLW.

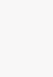



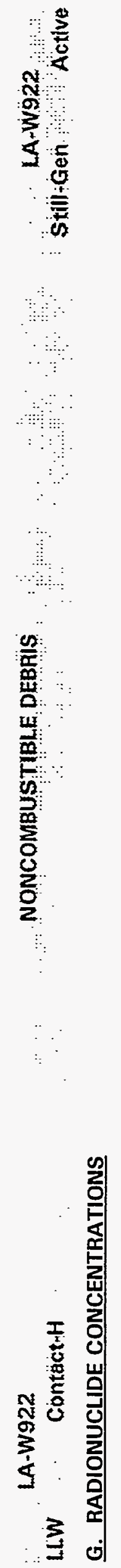
H. MATRIX CHARACTERISTICS

Primary Matrix Code: $\mathbf{5 5 0 0 0}$

Description: Debris Waste: : :

Combustibility: $\bigcirc$ Combustible (>90\%)

Mixed (10\%-90\%)

Noncombustible $(<10 \%)$

\section{MATRIX COMPOSITION}


LA-W922

NONCOMBUSTIBLE DEBÄIS

LA-W922

LLW $\because$ Contact-H

\section{a}

Stili-Gen Active

\section{J. HAZARDOUS CONSTITUENTS}

\begin{tabular}{|c|c|c|c|c|c|c|c|c|}
\hline \multirow{2}{*}{ Code } & \multirow[b]{2}{*}{ Hazardous Waste Description } & \multirow[b]{2}{*}{ Treatment Subcategory } & \multicolumn{4}{|c|}{ Concentration } & \multirow[b]{2}{*}{ Basis } & \multirow[b]{2}{*}{ Conc. Type } \\
\hline & & & Typical & $\mathbf{L L}$ & UL & Units & & \\
\hline$D 001$ & Ignitable & High-TOC ignitable characteristic liquids subca & & & & & $A$ & Total \\
\hline D004 & Arsenic & & & & & & $A$ & Total \\
\hline 0005 & Barium & & & & & & A & Total \\
\hline$\overline{D 006}$ & Cadmium & & & & & & A & Total \\
\hline$\overline{D 007}$ & Chromium & Chromium (Total) & & & & & $A$ & Total \\
\hline$\overline{D 008}$ & Lead & & & & & & A & Total \\
\hline 0009 & Mercury & Elemental mercury contaminated with radioact & & & & & A & Total \\
\hline D010 & Selenium & & & & & & A & Total \\
\hline D011 & Silver & & & & & & $A$ & Total \\
\hline
\end{tabular}




\section{$\therefore$ LA-W922}

HWW Contactit

\section{K. POLYCHLORINATED BIPHENYLS (PCBS)}

Is this waste stream PCB contaminated?

OYes O No O Unknown

If yes, what portion of the waste is PCB contaminated (volume \%):

If $<100 \%$, can the PCB contaminated waste be segregated and treated separately? OYes O No OUnknown

What is the PCB concentration in ppm? If only a portion of the waste is PCB contaminated, report values for contaminated portion.

\section{TREATMENT PLANS}

\begin{tabular}{|c|c|c|c|c|c|c|c|c|c|c|c|}
\hline Opt. & $\begin{array}{c}\text { Media Typo } \\
\text { (If more than one) }\end{array}$ & $\begin{array}{c}\text { Matrix } \\
\text { Code }\end{array}$ & $\begin{array}{l}\text { Vol. \% } \\
\text { lif }\end{array}$ & Step & JIT & sc & CIF & $\begin{array}{l}\text { Trans. } \\
\text { Miles }\end{array}$ & $\begin{array}{l}\text { Facility } \\
\text { Abbr. }\end{array}$ & Unit Name & Comments \\
\hline \multirow[t]{4}{*}{1} & & 55000 & & $\bar{a}$ & $\mathbf{O}$ & 0 & $1.00 E+00$ & & TRANS & Transport - LLW & \\
\hline & & & & b & 0 & 0 & $1.00 E+00$ & & WROC & Sizing & \\
\hline & & & & c & 0 & 0 & $1.00 E+00$ & & WROC & Macroencapsulation & \\
\hline & & & & d & 0 & 0 & $1.00 \mathrm{E}+00$ & & SCDF & Disposal - Contact Handled & \\
\hline
\end{tabular}

Note: Where provided, media percentages are rough estimates used to facilitate treatment planning. They do not necessarily imply an accurate knowledge of waste stream composition, and were not derived for use outside this conceptual planning exercise.

Describe any special or unique technical concerns related to the treatment of this waste stream that would impact the use or implementation of standard hazardous waste treatment methods. 


LiWW-W923
A. IDENTIFICATION AND DESCRIPTION
Waste Stream ID: LA-W923
W. S. Name:
Content Code:
FFCA ID:
Waste Stream Description:
LA-W923
This group is primarily uranium and thorium nitrate and magnesium perchlorate wastes. Most of
this waste is laboratory chemicals. The magnesium perchlorate is contaminated with trace
amounts of plutonium and americium.

\section{OXIDIZERS:}

LÄ-W923 Still-Gen .." "Active

\section{B. GENERATION SITE/PROCESS DESCRIPTION}

The following questions, concerning the generation site and process, should be answered even if the waste stream is no longer generated.

Name of Site Generating Waste (e.g., INEL, Rocky Flats, etc.):

Location of activities (Area and Bullingl:

Location of activities (Area and Building):

Operations (functions) performed in building:

Description of Process Generating Waste: 
C. PROJECTED WASTE GENERATION

Is this waste stream still generated?

OYes ONo

If yes, when is the expected generation termination date?

Current Projections

\begin{tabular}{|r|r|r|r|r|l|}
\hline Est. Date & Start Year & interval & \multicolumn{1}{|c|}{ Vol (m3) } & Mass (kg) & Source of Estimate \\
\hline $5 / 2 / 95$ & 1995 & 1 & 0.05 & & Generator Update \\
\hline
\end{tabular}

Historical Projections 


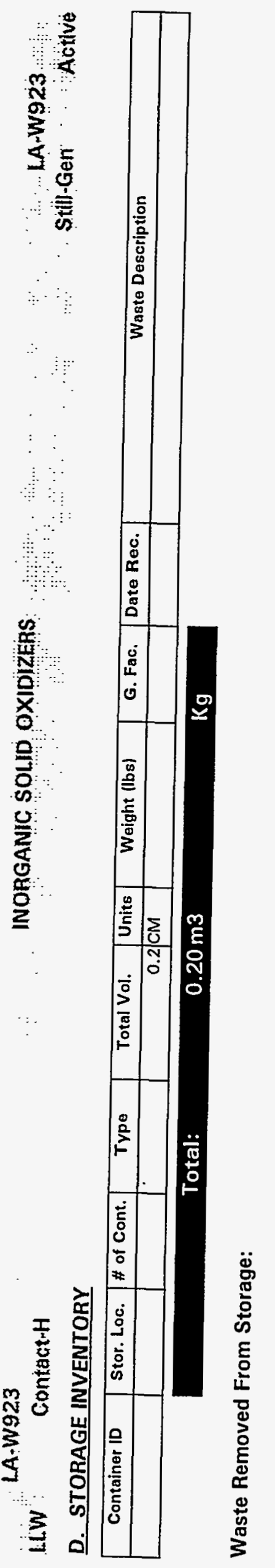




\section{LA-W923}

.

LLW Contact

\section{E. RADIATION CHARACTERISTICS}

\section{F. TRU ALPHA ACTIVITY DISTRIBUTION}

Radioactive Waste Type:

$\begin{array}{ll}\text { O LLW } & \bigcirc \text { a-LLW } \bigcirc \text { TRU } \\ \text { OHLW } & \bigcirc \text { Non-Rad }\end{array}$

This waste stream is not identified as a TRU or a-LLW.

Mixed Waste:

Handling:

\begin{tabular}{|l|}
\hline Yes O No \\
\hline O Contact \\
\hline Remote
\end{tabular}

Radionuclide Contamination Accessiblity:

External Surface

\begin{tabular}{l} 
OYes ONo OUnknown \\
\hline OYes O No OUnknown
\end{tabular}

Dispersed Through Matrix

OYes ONo OUnknown

\section{Activity Levels}

$\begin{aligned} & \text { Transuranic Alpha Activity: } \\ & \text { Uranium/Thorium Alpha Activity: } \text { Units: } \\ & \text { Beta/Gamma Activity: } \text { Units: } \\ & \\ & \text { @ surface } \\ & \text { Surface Neutron Activity: } \square \text { @ 1-m Units: } \mathrm{mR/hr} \\ & \text { Total Activity: } \text { Units: } \\ &\end{aligned}$



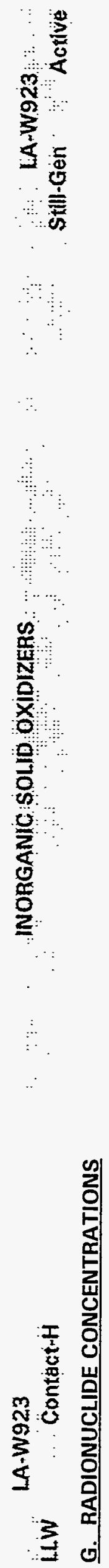


\section{LiWW Contact}

\section{H. MATRIX CHARACTERISTICS}

Primary Matrix Code: X6300

Description: Lab Packs: Solid Lab Packs: :

Combustibility: $\bigcirc$ Combustible (>90\%)

Mixed $(10 \%-90 \%)$

Noncombustible (<10\%)

\section{MATRIX COMPOSITION}




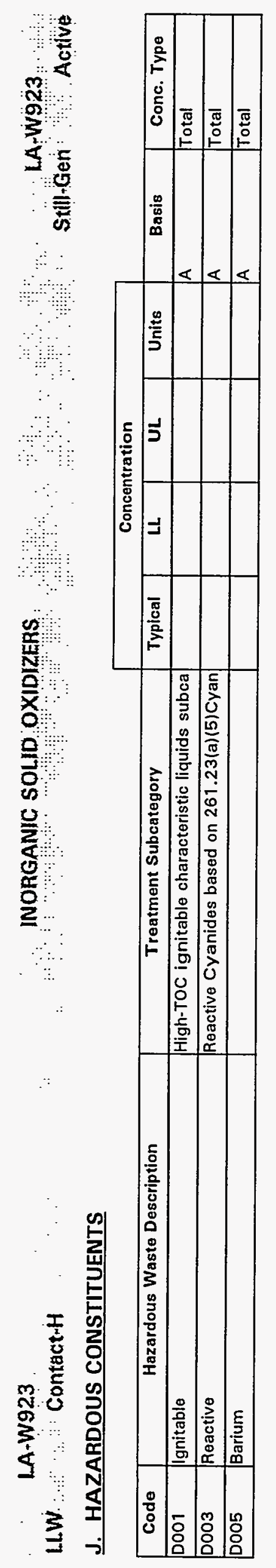

7
5
$\frac{7}{8}$
8
0

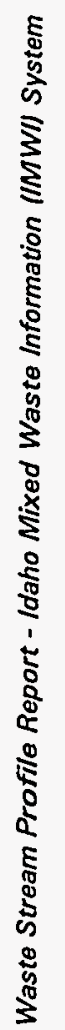

\begin{tabular}{l}
10 \\
\multirow{N}{N}{}
\end{tabular} 


\section{K. POLYCHLORINATED BIPHENYLS (PCBS)}

\section{Is this waste stream PCB contaminated? OYes O No O Unknown}

If yes, what portion of the waste is PCB contaminated (volume \%):

If $<100 \%$, can the PCB contaminated waste be segregated and treated separately? $O$ Yes ONo O Unknown

What is the PCB concentration in ppm?

If only a portion of the waste is PCB contaminated, report values for contaminated portion.

\section{TREATMENT PLANS}

\begin{tabular}{|c|c|c|c|c|c|c|c|c|c|c|c|}
\hline $\begin{array}{c}\text { Opt. } \\
\#\end{array}$ & $\begin{array}{c}\text { Media Type } \\
\text { (If more than one) }\end{array}$ & $\begin{array}{c}\text { Matrix } \\
\text { Code }\end{array}$ & $\begin{array}{l}\text { Vol. \% } \\
\text { IIf }\end{array}$ & Step & JIT & sc & $\mathrm{CIF}$ & $\begin{array}{l}\text { Trans. } \\
\text { Miles }\end{array}$ & $\begin{array}{l}\text { Facility } \\
\text { Abbr. }\end{array}$ & Unit Name & Comments \\
\hline 1 & & $\times 6300$ & & $\bar{a}$ & 0 & O & $1.00 \mathrm{E}+00$ & & TRANS & Transport - LLW & \\
\hline & & & & $\bar{b}$ & (O) & O & $1.00 \mathrm{E}+00$ & & AMWTF & $\mathrm{CH} \cdot$ Opening \& Sorting & \\
\hline & & & & c & 0 & $\overline{0}$ & $1.00 E+00$ & & AMWTF & $\mathrm{CH}$ - Sizing & \\
\hline & & & & d & D & O & $1.00 E+00$ & & AMWTF & $\mathrm{CH}$ - Incineration/Thermal Desorption & \\
\hline & & & & e & $\mathrm{O}$ & 0 & $1.00 E+00$ & & AMWTF & $\mathrm{CH}$ - Vitrification & \\
\hline & & & & $f$ & $\bar{O}$ & 0 & $1.00 E+00$ & & SCDF & Disposal - Contact Handled & \\
\hline
\end{tabular}

Note: Where provided, media percentages are rough estimates used to facilitate treatment planning. They do not necessarily imply an accurate knowledge of waste stream composition, and were not derived for use outside this conceptual planning exercise.

Describe any special or unique technical concerns related to the treatment of this waste stream that would impact the use or implementation of standard hazardous waste treatment methods. 


LLW LA-W925
A. IDENTIFICATION AND DESCRIPTION
Waste Stream ID:
W. S. Name:
Content Code:
FFCA ID:
Waste Stream Description:
This waste was generated between 1/1/93 and 9/30/94 and was outside the scope of the,
improved characterization activity.

TES TRO

LA-W925

\section{B. GENERATION SITE/PROCESS DESCRIPTION}

The following questions, concerning the generation site and process, should be answered even if the waste stream is no longer generated.

Name of Site Generating Waste (e.g., INEL, Rocky Flats, etc.):

Location of activities (Area and Building):

Operations (functions) performed in building:

\begin{tabular}{l} 
Operations (functions) performed in building: \\
Description of Process Generating Waste: \\
\hline
\end{tabular}




\section{PROJECTED WASTE GENERATION}

Is this waste stream still generated?

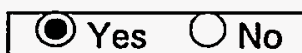

If yes, when is the expected generation termination date?

\begin{tabular}{|c|c|c|c|c|c|}
\hline \multicolumn{6}{|c|}{ Current Projections } \\
\hline Est. Date & Start Year & Interval & Vol (m3) & Mass $(\mathrm{kg})$ & Source of Estimate \\
\hline $5 / 2 / 95$ & 1995 & 1 & 5.10 & & Generator Update \\
\hline $5 / 2 / 95$ & 1996 & 1 & 5.10 & & Generator Update \\
\hline $5 / 2 / 95$ & 1997 & 1 & 5.10 & & Generator Update \\
\hline $5 / 2 / 95$ & 1998 & 1 & 5.10 & . & Generator Update \\
\hline $5 / 2 / 95$ & 1999 & 1 & 5.10 & & Generator Update \\
\hline
\end{tabular}




\section{STORAGE INVENTORY}

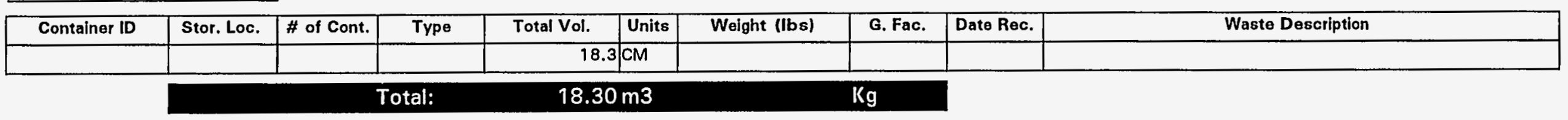

Waste Removed From Storage: 


\section{LÄ-W925:}

Hiw Contact-H

E. RADIATION CHARACTERISTICS

Radioactive Waste Type:

\begin{tabular}{|ll}
\hline Llw & Oa-llW O TRU \\
O HLW & O Non-Rad
\end{tabular}

Mixed Waste:

Handling:

\begin{tabular}{|l|}
\hline O Yes ONo \\
\hline O Contact O Remote \\
\hline
\end{tabular}

Radionuclide Contamination Accessiblity:

External Surface

Internal Surface

Dispersed Through Matrix

\begin{tabular}{|c|c|c|}
\hline OYes & ONo & O Unknown \\
\hline OYes & ONo & Unknown \\
\hline OYes & ONo & $\widetilde{J}$ \\
\hline
\end{tabular}

\section{Activity Levels}

Transuranic Alpha Activity: Uranium/Thorium Alpha Activity:

Beta/Gamma Activity:

Surface Neutron Activity:

Total Activity:

\begin{tabular}{l}
\hline \\
\hline Units: \\
Units: \\
@ @ surface \\
@ $1-\mathrm{m}$ Units: $\mathrm{mR} / \mathrm{hr}$ \\
\hline Units: \\
\hline nCi/g
\end{tabular}

This waste stream is not identified as a TRU or a-LLW.

\section{F. TRU ALPHA ACTIVITY DISTRIBUTION}




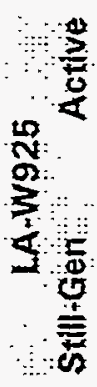

8
8
5
+
8
8
0

हूँ

粂

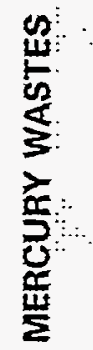

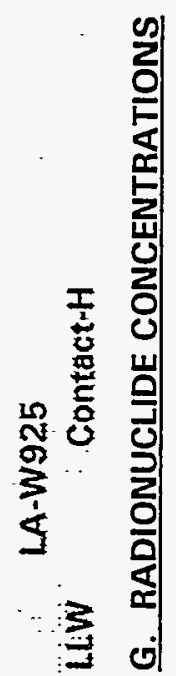


LA-W925

HWW ContactH

H. MATRIX CHARACTERISTICS

Primary Matrix Code: $\mathbf{5 4 0 0}$

Description: Debris Waste: Heterogeneous Debris: :

Combustibility:

Combustible (>90\%)

Mixed (10\%-90\%)

Noncombustible $(<10 \%)$

\section{MATRIX COMPOSITION}




\section{J. HAZARDOUS CONSTITUENTS}

\begin{tabular}{|c|c|c|c|c|c|c|c|c|}
\hline & & & \multicolumn{4}{|c|}{ Concentration } & \multirow[b]{2}{*}{ Basis } & \multirow[b]{2}{*}{ Conc. Type } \\
\hline Code & Hazardous Waste Description & Treatment Subcategory & Typical & $\mathbf{L L}$ & UL & Units & & \\
\hline D007 & Chromium & Chromium (Total) & & & & & A & Total \\
\hline D008 & Lead & & & & & & $\bar{A}$ & Total \\
\hline D009 & Mercury & Elemental mercury contaminated with radioact & & & & & A & Total \\
\hline F001 & Spent halogenated solvents used in degreasing & 1,1,1-Trichloroethane & & & & & A & Total \\
\hline
\end{tabular}




\section{LA-W925}

MERCURY WÄSTES - TBD

LA.W925

HW ContactiH

\section{K. POLYCHLORINATED BIPHENYLS (PCBS)}

Is this waste stream PCB contaminated?

OYes O No O Unknown

If yes, what portion of the waste is PCB contaminated (volume \%):

If $<100 \%$, can the PCB contaminated waste be segregated and treated separately?

What is the PCB concentration in ppm?

If only a portion of the waste is PCB contaminated, report values for contaminated portion.

\section{TREATMENT PLANS}

\begin{tabular}{|c|c|c|c|c|c|c|c|c|c|c|c|}
\hline $\begin{array}{l}\text { Opt. } \\
\#\end{array}$ & $\begin{array}{c}\text { Media Type } \\
\text { (If more than one) }\end{array}$ & $\begin{array}{c}\text { Matrix } \\
\text { Code }\end{array}$ & $\begin{array}{l}\text { Vol. \% } \\
\text { IIf }\end{array}$ & Step & JIT & sc & CIF & $\begin{array}{l}\text { Trans. } \\
\text { Miles }\end{array}$ & $\begin{array}{l}\text { Facility } \\
\text { Abbr. }\end{array}$ & Unit Name & Comments \\
\hline 1 & & $\$ 5400$ & & $\mathbf{a}$ & O) & O & $1.00 E+00$ & & TRANS & Transport - LLW & \\
\hline & & & & $\mathbf{b}$ & O) & O & $1.00 E+00$ & & AMWTF & $\mathrm{CH}$ - Opening \& Sorting & \\
\hline & & & & c & () & 0 & $1.00 E+00$ & & AMWTF & $\mathrm{CH}$ - Sizing & \\
\hline & & & & d & 0 & 0 & $1.00 \mathrm{E}+00$ & & AMWTF & $\mathrm{CH}$ - Incineration/Thermal Desorption & \\
\hline & & & & e & 0 & 0 & $1.00 E+00$ & & AMWTF & $\mathrm{CH}$ - Vitrification & \\
\hline & & & & $f$ & $O$ & O & $1.00 E+00$ & & SCDF & Disposal - Contact Handled & \\
\hline
\end{tabular}

Note: Where provided, media percentages are rough estimates used to facilitate treatment planning. They do not necessarily imply an accurate knowledge of waste stream composition, and were not derived for use outside this conceptual planning exercise.

Describe any special or unique technical concerns related to the treatment of this waste stream that would impact the use or implementation of standard hazardous waste treatment methods.

Additional characterization information will be obtained through generator interviewa, and the waste will be assigned to appropriate treatability groups. 


LBWW002
A. IDENTIFICATION AND DESCRIPTION
Waste Stream ID: LB-WO02
W. S. Name:
Content Code:
FFCA ID:
Waste Stream Description:
Lab packed solutions and solids with metals and radionuclides. Alpha contamination less than or
equal to 10 nCi/g. This waste stream consists of lab packed acidic aqueous solutions with
radionuclides. These wastes are generated from several research laboratories.

Code:

Waste Stream Description:

ab packed solutions and solids with metals and radionuclides. Alpha contamination less than or qual to $10 \mathrm{nCi} / \mathrm{g}$. This waste stream consists of lab packed acidic aqueous solutions with adionuclides. These wastes are generated from several research laboratories.

\section{B. GENERATION SITE/PROCESS DESCRIPTION}

The following questions, concerning the generation site and process, should be answered even if the waste stream is no longer generated.

Name of Site Generating Waste (e.g., INEL, Rocky Flats, etc.): LBL

Location of activities (Area and Building):

Legacy waste.

Operations (functions) performed in building: Research and development.

Description of Process Generating Waste:

Various research laboratory procedures. 


\section{LBWOOOZ}

ulW Contact H

C. PROJECTED WASTE GENERATION

Is this waste stream still generated?

If yes, when is the expected generation termination date?

BASIC AQUEOUS LIQUIDS - LOW ALPHA

Current Projections

\begin{tabular}{|r|r|r|r|r|r|}
\hline Est. Date & Start Year & Interval & \multicolumn{1}{|c|}{ Vol (m3) } & Mass (kg) & Source of Estlmate \\
\hline $4 / 14 / 94$ & 1997 & 1 & 0.81 & & $1993-F F C A$ \\
\hline
\end{tabular}

\begin{tabular}{|r|r|r|r|r|l|}
\hline Est. Date & \multicolumn{1}{|c|}{ Start Year } & \multicolumn{1}{|c|}{ Interval } & \multicolumn{1}{|c|}{ Vol $(\mathbf{m} 3)$} & Mass $(\mathrm{kg})$ & \multicolumn{1}{|c|}{ Source of Estimate } \\
\hline $11 / 30 / 93$ & 1993 & 1 & 0.50 & & $1993-F F C A$ \\
\hline $11 / 30 / 93$ & 1994 & 1 & 0.50 & & $1993-F F C A$ \\
\hline $11 / 30 / 93$ & 1995 & 1 & 0.50 & & $1993-F F C A$ \\
\hline $11 / 30 / 93$ & 1996 & 1 & 0.50 & & $1993-F F C A$ \\
\hline $11 / 30 / 93$ & 1997 & 1 & 0.50 & & $1993-F F C A$ \\
\hline $11 / 30 / 93$ & 1998 & 5 & 2.50 & & $1993-F F C A$ \\
\hline $11 / 30 / 93$ & 2003 & 20 & 10.00 & & $1993-F F C A$ \\
\hline
\end{tabular}




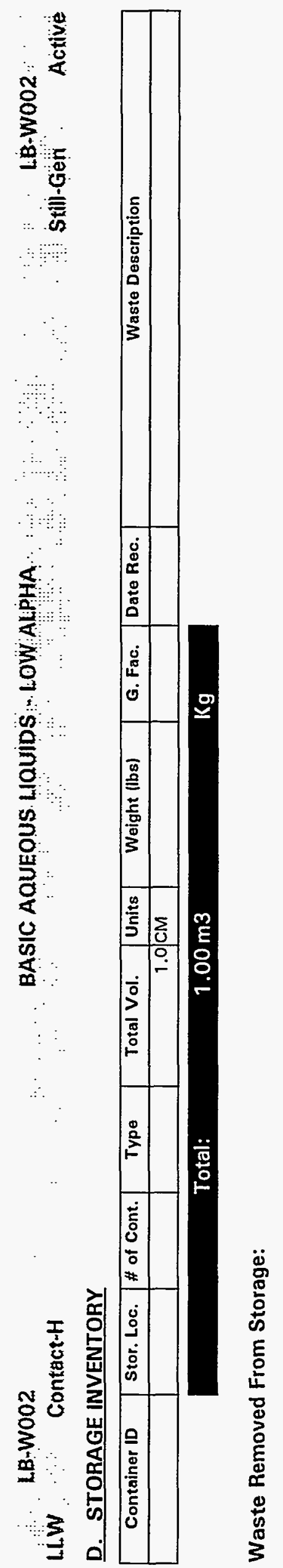




\section{E. RADIATION CHARACTERISTICS}

Radioactive Waste Type:

$\begin{array}{ll}\text { OllW } & \text { Oa-LLW } \bigcirc \text { TRU } \\ \text { OHLW } & \bigcirc \text { Non-Rad }\end{array}$

Mixed Waste:

Handling:

\begin{tabular}{|l|}
\hline OYes ONo \\
\hline O Contact O Remote \\
\hline
\end{tabular}

Radionuclide Contamination Accessiblity:

\begin{tabular}{|c|c|c|c|}
\hline External Surface & OYes & ONo & OUnknown \\
\hline Internal Surface & OYes & $\mathrm{ONo}$ & OUnknown \\
\hline spersed Through Matrix & OYes & ONo & OUnknown \\
\hline
\end{tabular}

\section{Activity Levels}

Transuranic Alpha Activity: Uranium/Thorium Alpha Activity: Beta/Gamma Activity:

Surface Neutron Activity: Total Activity:

\begin{tabular}{|c|c|c|}
\hline & Units: & \\
\hline & Units: & \\
\hline & @ surf & ce \\
\hline & @ 1-m & Units: $\mathrm{mR} / \mathrm{hr}$ \\
\hline & Units: & \\
\hline
\end{tabular}
$1.4 \mathrm{nCi} / \mathrm{g}$

\section{F. TRU ALPHA ACTIVITY DISTRIBUTION}

This waste stream is not identified as a TRU or a-LLW. 


\section{$\because \quad \cdots \quad L B+W 002$}

LLW

ContactrH

BASIC AQUEOUS LIQUIDS LOW ALPHA

LB-WOO2 Stilitgen Active

\section{G. RADIONUCLIDE CONCENTRATIONS}

\begin{tabular}{|c|c|c|c|c|c|c|}
\hline \multirow{2}{*}{ Isotope } & \multicolumn{3}{|c|}{ Specific Activity } & \multirow[b]{2}{*}{ Units } & \multirow[b]{2}{*}{ Basis Code(s) } & \multirow[b]{2}{*}{ Basis Desc. (if other) } \\
\hline & Typical & Lower Limit & Upper Limit & & & \\
\hline$A m-241$ & $1.1000 E+00$ & $1.1000 E+00$ & $1.1000 E+00$ & $\mathrm{Ci} / \mathrm{g}$ & $D$ & \\
\hline $\begin{array}{l}\text { A. Passiv } \\
\text { B. Segme } \\
\text { C. Passiv } \\
\text { D. Radioc } \\
\text { E. Mass }\end{array}$ & $\begin{array}{l}\text { lctive Neutron } \\
\text { 9d Gamma Sca } \\
\text { lctive Neutron/ } \\
\text { mical Analysis } \\
\text { ctrometry }\end{array}$ & $\begin{array}{l}\text { PAN) } \\
\text { (SGS) } \\
\text { Segmented Gam }\end{array}$ & ma Scan IPAN & GS) & $\begin{array}{l}\text { Accountability } \\
\text { 7. Calorimetry } \\
\text { 1. Multiple Energy } \\
\text { Process Knowlec } \\
\text { Other }\end{array}$ & is (MEGAS) \\
\hline
\end{tabular}




\section{H. MATRIX CHARACTERISTICS}

Primary Matrix Code: $L 1120$

Description: Aqueous Liquids/Slurries: Wastewaters: Basic Wastewaters:

Combustibility: $\bigcirc$ Combustible $(>90 \%)$

Mixed (10\%-90\%)

Noncombustible (<10\%)

\section{MATRIX COMPOSITION}

\begin{tabular}{|c|c|c|c|c|c|c|}
\hline & & & \multicolumn{4}{|c|}{ Composition } \\
\hline Code & Matrix Description & Material & Typical & LL & UL & Units \\
\hline 55313 & Debris Waste.Organic Debris.Plastic/Rubber Debris.Nonhalogenated Plastic Debris & Plastic containers & & & & \\
\hline 55122 & Debris Waste.Inorganic Debris.Inorganic Non-Metal Debris.Glass Debris & Glass containers & & & & \\
\hline 53113 & Homogeneous Solids.Inorganic Homogeneous Solids.Inorganic Particulates.Inorganic & Inorganic particulate absorbents & & & & \\
\hline$L 1210$ & Aqueous Liquids/Slurries.Aqueous Slurries.Acidic Aqueous Slurries. & Acidic aqueous liquids & & & & \\
\hline
\end{tabular}




\section{LB:W002}

\section{LLW $\because$ ContactrH}

\section{BASIC AQUEOUS LIOUIDS - LOW ALPHA}

LB-W002

\section{J. HAZARDOUS CONSTITUENTS}

\begin{tabular}{|c|c|c|c|c|c|c|c|c|}
\hline & & & \multicolumn{4}{|c|}{ Concentration } & \multirow[b]{2}{*}{ Basis } & \multirow[b]{2}{*}{ Conc. Type } \\
\hline Code & Hazardous Waste Description & Treatment Subcategory & Typical & $\mathbf{L L}$ & UL & Units & & \\
\hline$\overline{D 002}$ & Corrosive & Corrosive characteristic waste that are manag & & & & & C1 & Total \\
\hline$\overline{D 004}$ & Arsenic & & & & & & A & Total \\
\hline$\overline{D 005}$ & Barium & & & & & & A & Total \\
\hline$\overline{D 006}$ & Cadmium & & & & & & A & Total \\
\hline$\overline{D 007}$ & Chromium & Chromium (Total) & & & & & A & Total \\
\hline$\overline{D 008}$ & Lead & & & & & & A & Total \\
\hline$\overline{D 009}$ & Mercury & Elemental mercury contaminated with radioact & & & & & A & Total \\
\hline$\overline{D 010}$ & Selenium & & & & & & A & Total \\
\hline$D 011$ & Silver & & & & & & A & Total \\
\hline$\overline{0022}$ & Chloroform & Chloroform managed in non-CWA/non-CWA-e & & & & & A & Total \\
\hline$\overline{D 035}$ & Methyl ethyl ketone & Methyle ethyl ketone managed in non-CWA/no & & & & & A & Total \\
\hline$\overline{0039}$ & Tetrachloroethylene & Tetrachloroethylene managed in non-CWA/non & & & & & A & Total \\
\hline F005 & Spent non-halogenated solvents & Toluene & & & & & & Total \\
\hline
\end{tabular}




\section{K. POLYCHLORINATED BIPHENYLS (PCBS)}

Is this waste stream PCB contaminated? OYes O No O Unknown

If yes, what portion of the waste is PCB contaminated (volume \%):

If $<100 \%$, can the PCB contaminated waste be segregated and treated separately?

What is the PCB concentration in ppm?

If only a portion of the waste is PCB contaminated, report values for contaminated portion.

\section{TREATMENT PLANS}

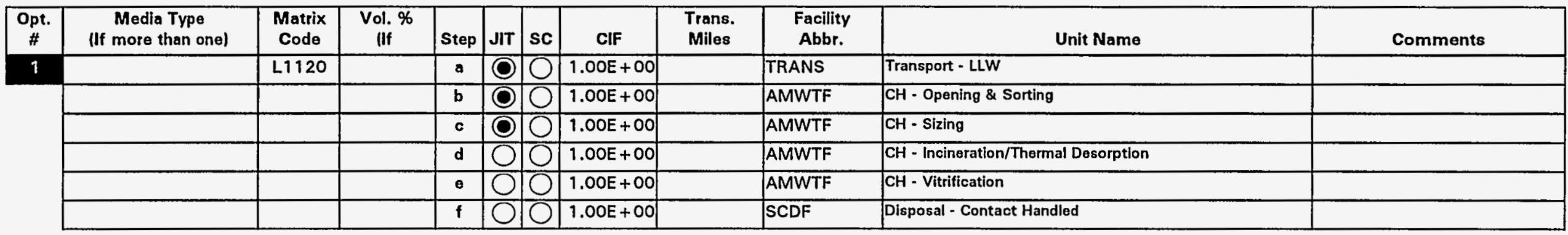

Note: Where provided, media percentages are rough estimates used to facilitate treatment planning. They do not necessarily imply an accurate knowledge of waste stream composition, and were not derived for use outside this conceptual planning exercise.

Describe any special or unique technical concerns related to the treatment of this waste stream that would impact the use or implementation of standard hazardous waste treatment methods. 


\section{$\therefore \quad$ LBWW005}

LLW $\quad$ Contact-H

\section{A. IDENTIFICATION AND DESCRIPTION}

Waste Stream ID: LB-W005

W. S. Name:

Content Code:

FFCA ID:

BLOCKS \& SHEET LEAD - CONTAMINATED

*

LB-W005

Waste Stream Description:

\section{B. GENERATION SITE/PROCESS DESCRIPTION}

The following questions, concerning the generation site and process, should be answered even if the waste stream is no longer generated.

Name of Site Generating Waste (e.g., INEL, Rocky Flats, etc.): LBL

Location of activities (Area and Building):

Legacy waste.

Operations (functions) performed in building:

Research and development.

Description of Process Generating Waste:

Various research laboratory procedures. 
LBWOOD

WW: Contact H

C. PROJECTED WASTE GENERATION

Is this waste stream still generated?

If yes, when is the expected generation termination date?

Current Projections

\section{Historical Projections}

\begin{tabular}{|r|r|r|r|r|l|}
\hline Est. Date & \multicolumn{1}{|c|}{ Start Year } & \multicolumn{1}{|c|}{ Interval } & \multicolumn{1}{c|}{ Vol $(\mathbf{m} 3)$} & Mass $(\mathbf{k g})$ & \multicolumn{1}{|c|}{ Source of Estimate } \\
\hline $11 / 30 / 93$ & 1993 & 1 & 0.40 & & $1993-F F C A$ \\
\hline $11 / 30 / 93$ & 1994 & 1 & 0.40 & & $1993-F F C A$ \\
\hline $11 / 30 / 93$ & 1995 & 1 & 0.40 & & $1993-F F C A$ \\
\hline $11 / 30 / 93$ & 1996 & 1 & 0.40 & & $1993-F F C A$ \\
\hline $11 / 30 / 93$ & 1997 & 1 & 0.40 & & $1993-F F C A$ \\
\hline $11 / 30 / 93$ & 1998 & 5 & 2.00 & & $1993-F F C A$ \\
\hline $11 / 30 / 93$ & 2003 & 20 & 8.00 & & $1993-F F C A$ \\
\hline
\end{tabular}


$\therefore$ LB-W005

i.i. : Contact-H

D. STORAGE INVENTORY

\begin{tabular}{|l|l|}
\hline Container ID & Stor Loc.
\end{tabular}

\begin{tabular}{|l|l|}
\hline Stor. Loc. & \# of Cont \\
\hline
\end{tabular}

Total:
BLOCKS \& SHEET LEAD ..CONTAMINATED
LB-W005

Nót-Gên.

Active

\begin{tabular}{l|c|c|c|c|c|c|}
\hline Type & Total Vol. & Units & Weight (lbs) & G. Fac. & Date Rec. \\
\hline & \multicolumn{7}{|c|}{0.4} & $\mathrm{CM}$ & & & \\
\hline
\end{tabular}

Waste Description

Waste Removed From Storage: 
WW LWOO5

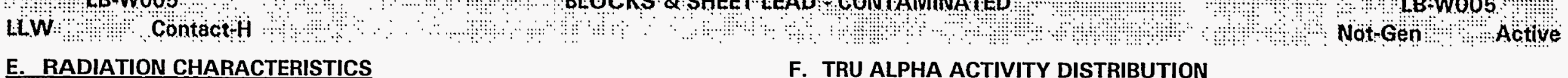

\section{E. RADIATION CHARACTERISTICS}

\section{F. TRU ALPHA ACTIVITY DISTRIBUTION}

Radioactive Waste Type:

$\begin{array}{ll}\text { OllW } & \text { Oa-LlW O TRU } \\ \text { OHLW } & \text { O Non-Rad }\end{array}$

This waste stream is not identified as a TRU or a-LLW.

Mixed Waste:

\begin{tabular}{|l|}
\hline O Yes O No \\
\hline O Contact O Remote \\
\hline
\end{tabular}

Radionuclide Contamination Accessiblity:

External Surface

Internal Surface

\begin{tabular}{|c|c|c|}
\hline OYes & O No & OUnknown \\
\hline OYes & O No & OUnknown \\
\hline & c & 01 \\
\hline
\end{tabular}

Dispersed Through Matrix

\section{Activity Levels}

Transuranic Alpha Activity:

Uranium/Thorium Alpha Activity:

Beta/Gamma Activity:

Surface Neutron Activity:

$\square$
$\square$
$\square$
$\square$
$\square$

Units: Units: @ surface @ 1-m Units: $\mathrm{mR} / \mathrm{hr}$ Units: $\mathrm{nCi} / \mathrm{g}$

Total Activity: 


\section{......W-W005}

\section{LLW ContäctrH}

\section{G. RADIONUCLIDE CONCENTRATIONS}

\begin{tabular}{|l|c|c|c|c|l|l|}
\cline { 2 - 7 } \multicolumn{1}{c|}{} & \multicolumn{3}{c|}{ Specific Activity } & \multicolumn{1}{c|}{ Units } & Basis Code(s) & Basis Desc. (if other) \\
\hline Isotope & Typical & Lower Limit & Upper Limit & Units & \\
\hline U-238 & $7.0000 \mathrm{E}-10$ & & $1.4000 \mathrm{E}-09$ & $\mathrm{Ci} / \mathrm{ml}$ & $\mathrm{D}$ & \\
\hline $\mathrm{C}-14$ & $1.1200 \mathrm{E}-10$ & & $2.2400 \mathrm{E}-10$ & $\mathrm{Ci} / \mathrm{ml}$ & $\mathrm{D}$ & \\
\hline $\mathrm{H}-3$ & & & & & $\mathrm{D}$ & \\
\hline
\end{tabular}

A. Passive-Active Neutron (PAN)

B. Segmented Gamma Scan (SGS)

F. Accountability

. Passive-Active Neutron/Segmented Gamma Scan (PAN/SGS)

D. Radiochemical Analysis

E. Mass Spectrometry

H. Multiple Energy Gamma Analysis (MEGAS)

1. Process Knowledge

J. Other
BLOCKS \& SHEET LEAD CONTAMINATED

LB-WOOS

Notgen : Active 


\begin{tabular}{|c|c|c|c|c|c|c|}
\hline & & & & 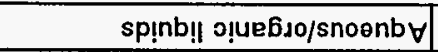 & 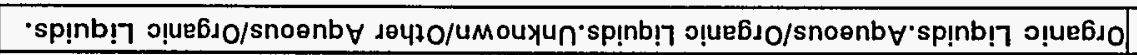 & 06127 \\
\hline & & & & 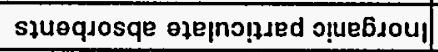 & 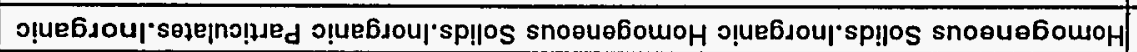 & $\varepsilon\llcorner L \varepsilon S$ \\
\hline & & & & sjou!̣ejuos ssej|g & 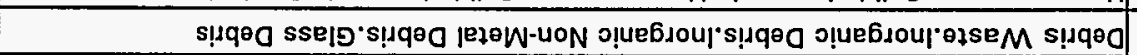 & ZZLSS \\
\hline & & & & słau!̣ejuoo o!̣se|d & 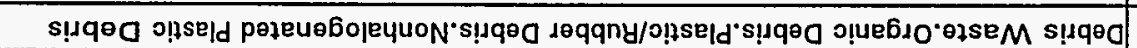 & 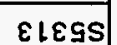 \\
\hline syun & $7 \mathrm{n}$ & 77 & 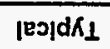 & 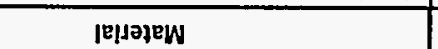 & 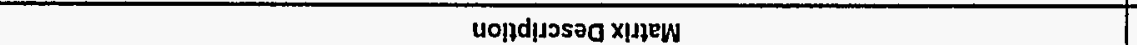 & әро5 \\
\hline
\end{tabular}

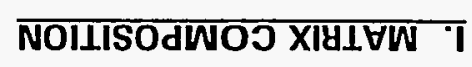

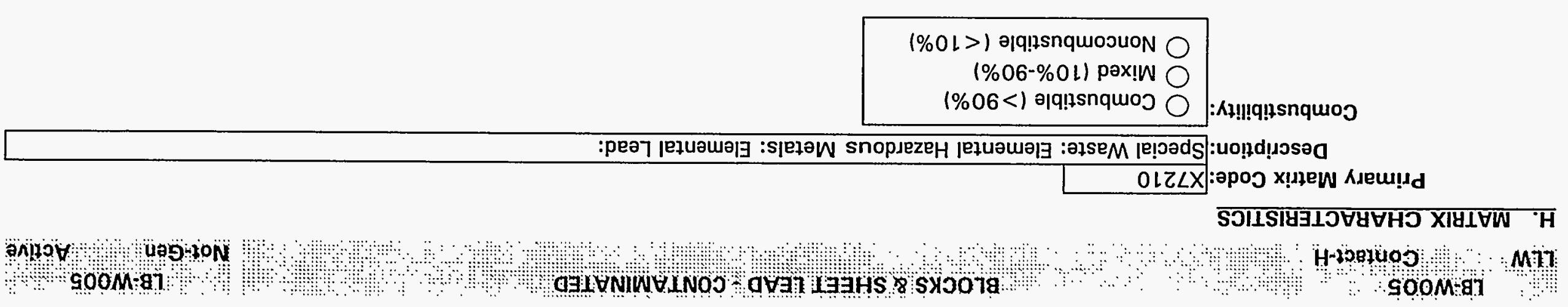




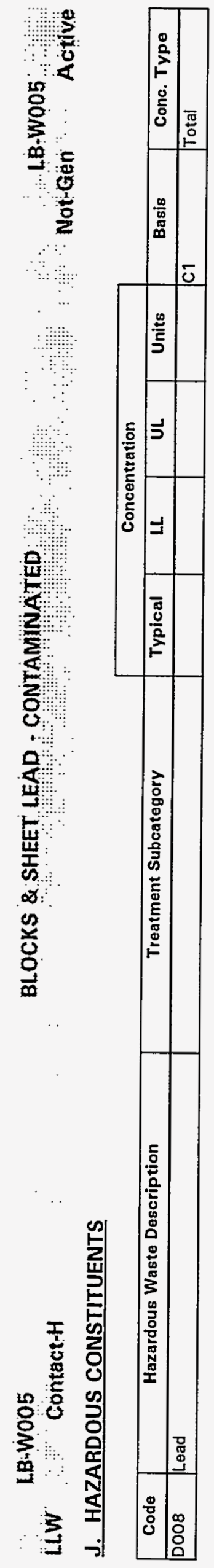

व 


\section{K. POLYCHLORINATED BIPHENYLS (PCBS)}

Is this waste stream PCB contaminated?

O Yes O No O Unknown

If yes, what portion of the waste is PCB contaminated (volume \%):

If $<100 \%$, can the PCB contaminated waste be segregated and treated separately? $O$ Yes O No O Unknown

What is the PCB concentration in ppm?

If only a portion of the waste is $P C B$ contaminated, report values for contaminated portion.

\section{TREATMENT PLANS}

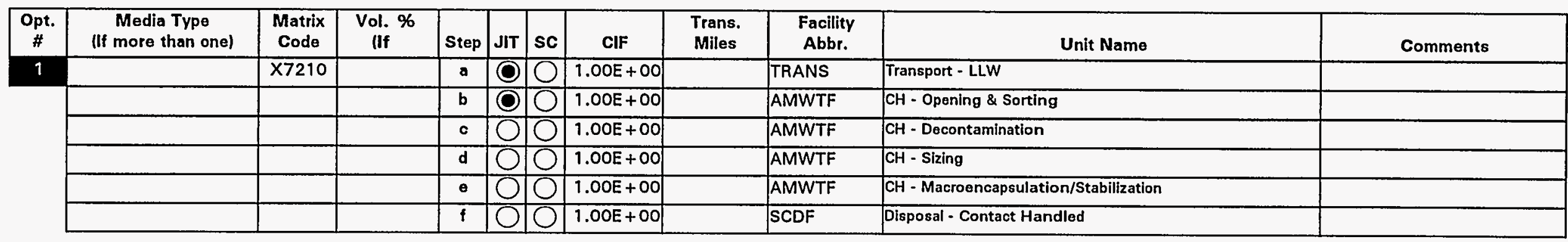

Note: Where provided, media percentages are rough estimates used to facilitate treatment planning. They do not necessarily imply an accurate knowledge of waste stream composition, and were not derived for use outside this conceptual planning exercise.

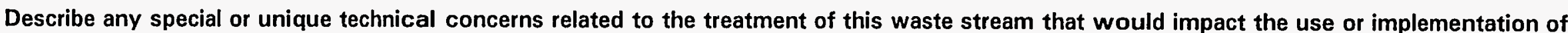
standard hazardous waste treatment methods. 


\section{LB.W006}

LIQUID INDUCED MERCURY

LB-WODG

Liw Contact-H

A. IDENTIFICATION AND DESCRIPTION

Waste Stream ID: LB-W006

W. S. Name:

LIQUID INDUCED MERCURY

Content Code:

FFCA ID:

LB-W006

\section{B. GENERATION SITE/PROCESS DESCRIPTION}

The following questions, concerning the generation site and process, should be answered even if the waste stream is no longer generated.

Name of Site Generating Waste (e.g., INEL, Rocky Flats, etc.):

Waste Stream Description:

Induced mercury waste.

Location of activities (Area and Building):

Operations (functions) performed in building:

Operations (functions) performed in building:

Description of Process Generating Waste: 


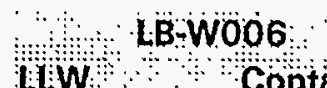
LLW Contact-H

LIQUID INDUCED MERCUAYY

C. PROJECTED WASTE GENERATION

Is this waste stream still generated?

OYes O No

If yes, when is the expected generation termination date?

Current Projections

\section{Historical Projections}




\section{LB-W006}

LLW. ContactrH

D. STORAGE INVENTORY

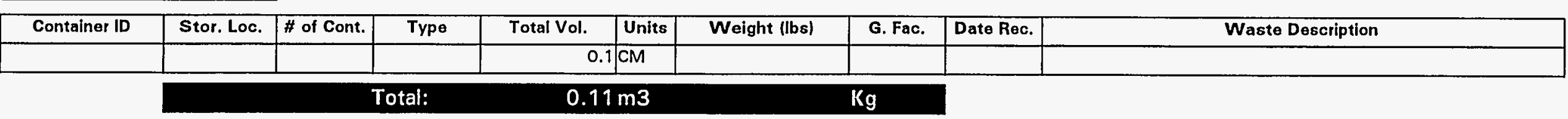

Waste Removed From Storage:

\section{LIQUID INDUCED MERCURY}

L.B.W006: Nót-Gèn

Active 


\section{E. RADIATION CHARACTERISTICS}

Radioactive Waste Type:

\begin{tabular}{ll} 
OllW & Oa-LLW $\bigcirc$ tRU \\
OhlW & O Non-Rad \\
\hline
\end{tabular}

Mixed Waste:

Handling:

\begin{tabular}{|l|}
\hline O Yes O No \\
\hline O Contact O Remote \\
\hline
\end{tabular}

Radionuclide Contamination Accessiblity:

External Surface OYes O No OUnknown

Internal Surface OYes O No OUnknown

Dispersed Through Matrix OYes O No O Unknown

\section{Activity Levels}

Transuranic Alpha Activity: Uranium/Thorium Alpha Activity: Beta/Gamma Activity:

Surface Neutron Activity: Total Activity:

\begin{tabular}{|c|c|c|}
\hline & Units: & \\
\hline & Units: & \\
\hline & @ surf: & \\
\hline & @ 1-m & Units: $\mathrm{mR} / \mathrm{hr}$ \\
\hline & Units: & \\
\hline & $\mathrm{nCi} / \mathrm{g}$ & \\
\hline
\end{tabular}

\section{F. TRU ALPHA ACTIVITY DISTRIBUTION}

This waste stream is not identified as a TRU or a-LLW. 

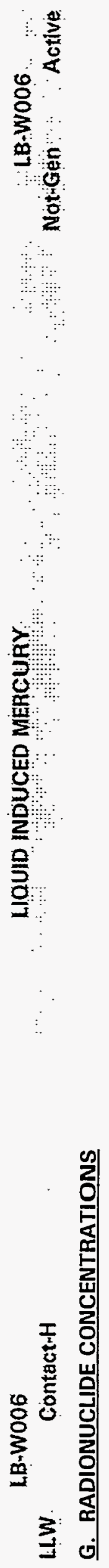
LB-WOOG

Contactit

LOUID INDUCED MERCURY

LBWWOOO

H. MATRIX CHARACTERISTICS

Primary Matrix Code: X7100

Description: Special Waste: Elemental Mercury: :

Combustibility: $\bigcirc$ Combustible (>90\%)

Mixed $(10 \%-90 \%)$

Noncombustible $(<10 \%)$

\section{MATRIX COMPOSITION}


$\therefore \quad$ LB-W006

LLW: : ContactrH

J. HAZARDOUS CONSTITUENTS

\begin{tabular}{|c|c|c|c|c|c|c|c|c|}
\hline & & & \multicolumn{4}{|c|}{ Concentration } & & \\
\hline Code & Hazardous Waste Description & Treatment Subcategory & Typical & LL & UL & Units & Basis & Conc. Type \\
\hline D009 & Mercury & Elemental mercury contaminated with radioact & & & & & & Total \\
\hline
\end{tabular}




\section{K. POLYCHLORINATED BIPHENYLS (PCBS)}

Is this waste stream PCB contaminated?

\section{OYes O No O Unknown}

If yes, what portion of the waste is PCB contaminated (volume \%):

If $<100 \%$, can the PCB contaminated waste be segregated and treated separately?

If only a portion of the waste is PCB contaminated, report values for contaminated portion.

What is the PCB concentration in ppm?

\section{TREATMENT PLANS}

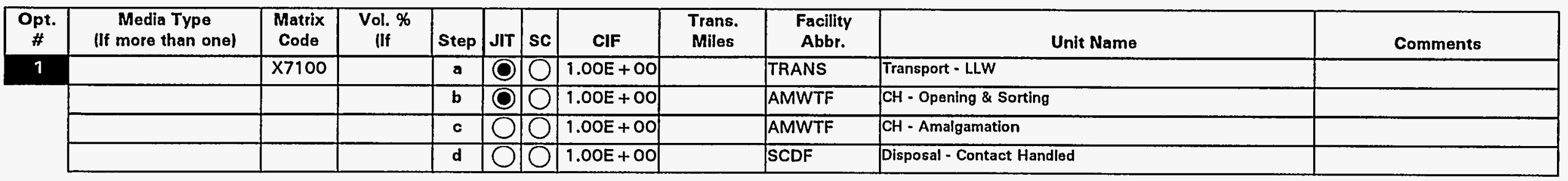

Note: Where provided, media percentages are rough estimates used to facilitate treatment planning. They do not necessarily imply an accurate knowledge of waste stream composition, and were not derived for use outside this conceptual planning exercise.

Describe any special or unique technical concerns related to the treatment of this waste stream that would impact the use or implementation of standard hazardous waste treatment methods. 


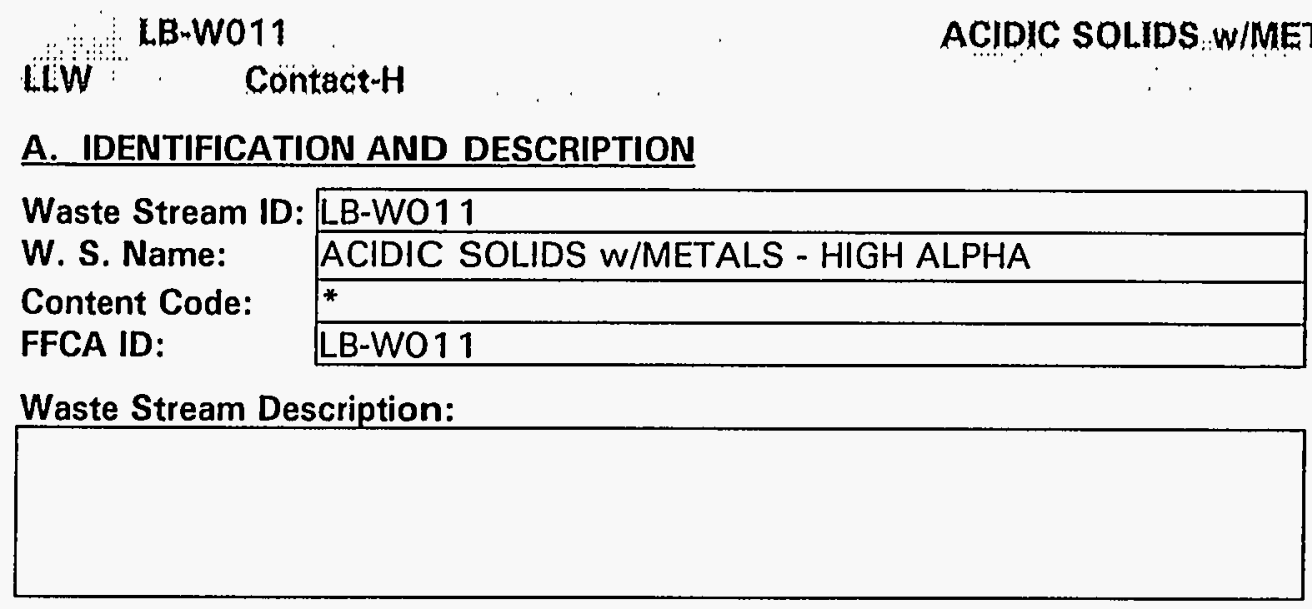

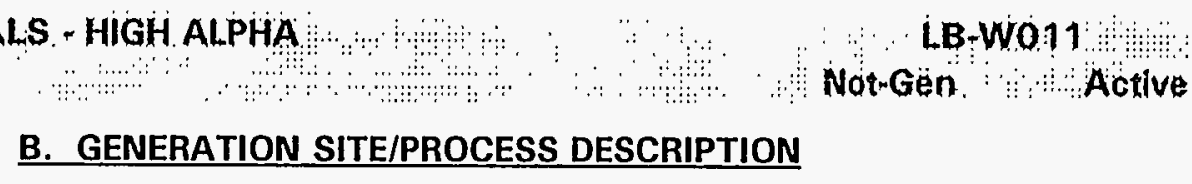

The following questions, concerning the generation site and process, should be answered even if the waste stream is no longer generated.

Name of Site Generating Waste (e.g., INEL, Rocky Flats, etc.): LBL

Location of activities (Area and Building): Legacy waste.

Operations (functions) performed in building: Research and Development.

Description of Process Generating Waste: 
LE.W011 LiWW Contact-H

ACIDIC SOLIDS W/METALS HIGH ALPIAA

C. PROJECTED WASTE GENERATION

Is this waste stream still generated?

If yes, when is the expected generation termination date?

Current Projections

Historical Projections 


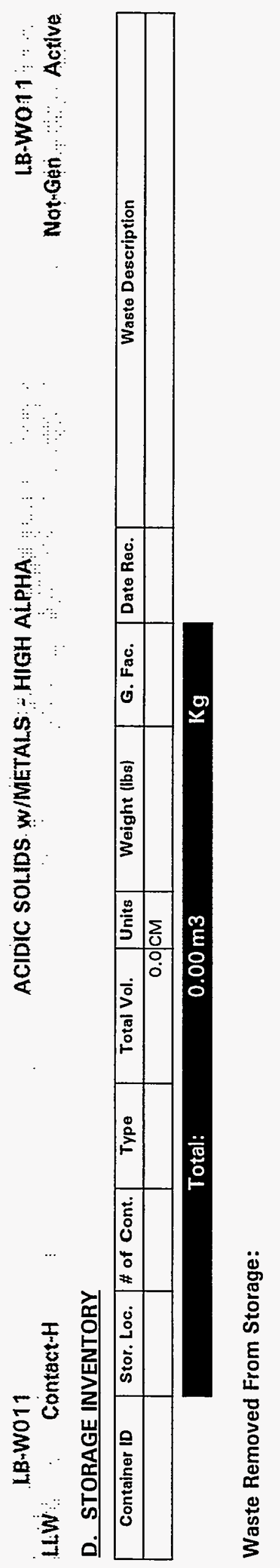

0
6
$\dot{7}$
0
0
0

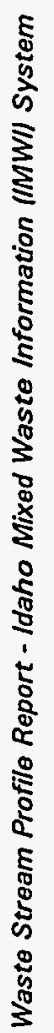

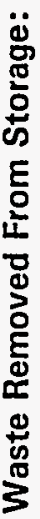


LB-Wo11

LlW

E. RADIATION CHARACTERISTICS

Radioactive Waste Type:

\begin{tabular}{|ll|}
\hline OllW & Oa-LLW O TRU \\
OHLW & O Non-Rad \\
\hline
\end{tabular}

Mixed Waste:

Handling:

OYes O No

Radionuclide Contamination Accessiblity:

\begin{tabular}{rlrlr|}
\cline { 2 - 4 } External Surface & OYes & O No & O Unknown \\
\cline { 2 - 4 } Internal Surface & OYes & Ono & O Unknown \\
\cline { 2 - 5 } Dispersed Through Matrix & OYes & ONo & O Unknown
\end{tabular}

\section{Activity Levels}

Transuranic Alpha Activity:

Uranium/Thorium Alpha Activity

Beta/Gamma Activity:

Surface Neutron Activity:

Total Activity:

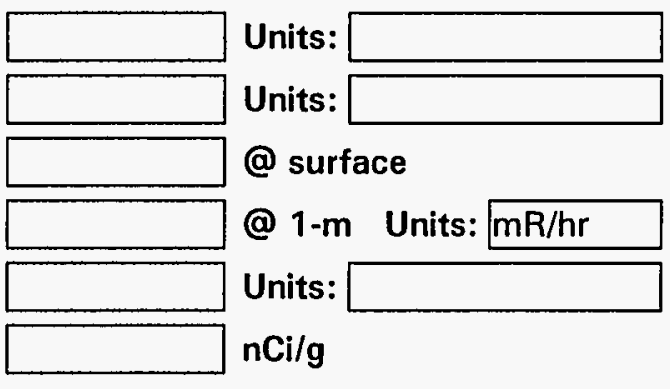

\section{F. TRU ALPHA ACTIVITY DISTRIBUTION}

This waste stream is not identified as a TRU or a- $L L W$.
LB+WOI1

Not Gen Active 


\section{LLW: Contact-H \\ G. RADIONUCLIDE CONCENTRATIONS}

\begin{tabular}{|c|c|c|c|c|c|c|}
\hline & \multicolumn{3}{|c|}{ Specific Activity } & \multirow[b]{2}{*}{ Units } & \multirow[b]{2}{*}{ Basis Code(s) } & \multirow[b]{2}{*}{ Basis Desc. (if other) } \\
\hline Isotope & Typical & Lower Limit & Upper Limit & & & \\
\hline Po-212 & & & & & 1 & \\
\hline \multicolumn{7}{|l|}{$B i-212$} \\
\hline $\begin{array}{l}\text { A. Passiv } \\
\text { B. Segm } \\
\text { C. Passiv } \\
\text { D. Radio } \\
\text { E. Mass }\end{array}$ & $\begin{array}{l}\text { tive Neutr } \\
\text { Gamma } \\
\text { tive Neutr } \\
\text { cal Analy } \\
\text { trometry }\end{array}$ & $\begin{array}{l}P A N) \\
\text { (SGS) } \\
\text { Segmented Ga }\end{array}$ & ma Scan IPA & & $\begin{array}{l}\text { Accountability } \\
\text { Calorimetry } \\
\text { Multiple Energy } \\
\text { Process Know/e } \\
\text { Other }\end{array}$ & sis (MEGAS) \\
\hline
\end{tabular}




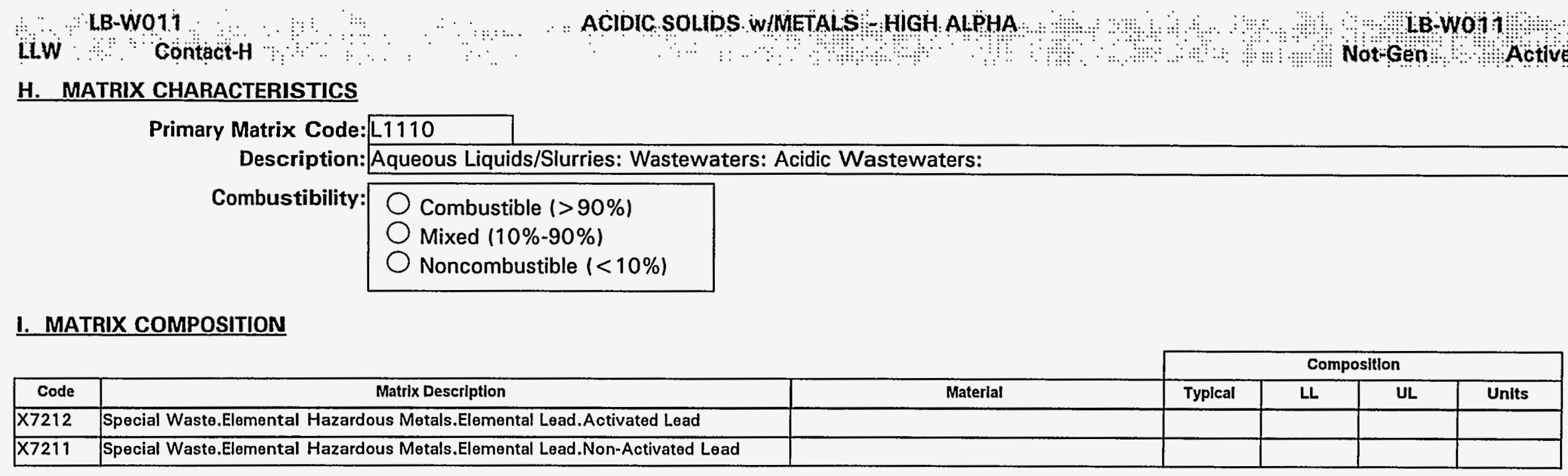


LB-W011

\section{LiW: : Contact-H}

\section{J. HAZARDOUS CONSTITUENTS}

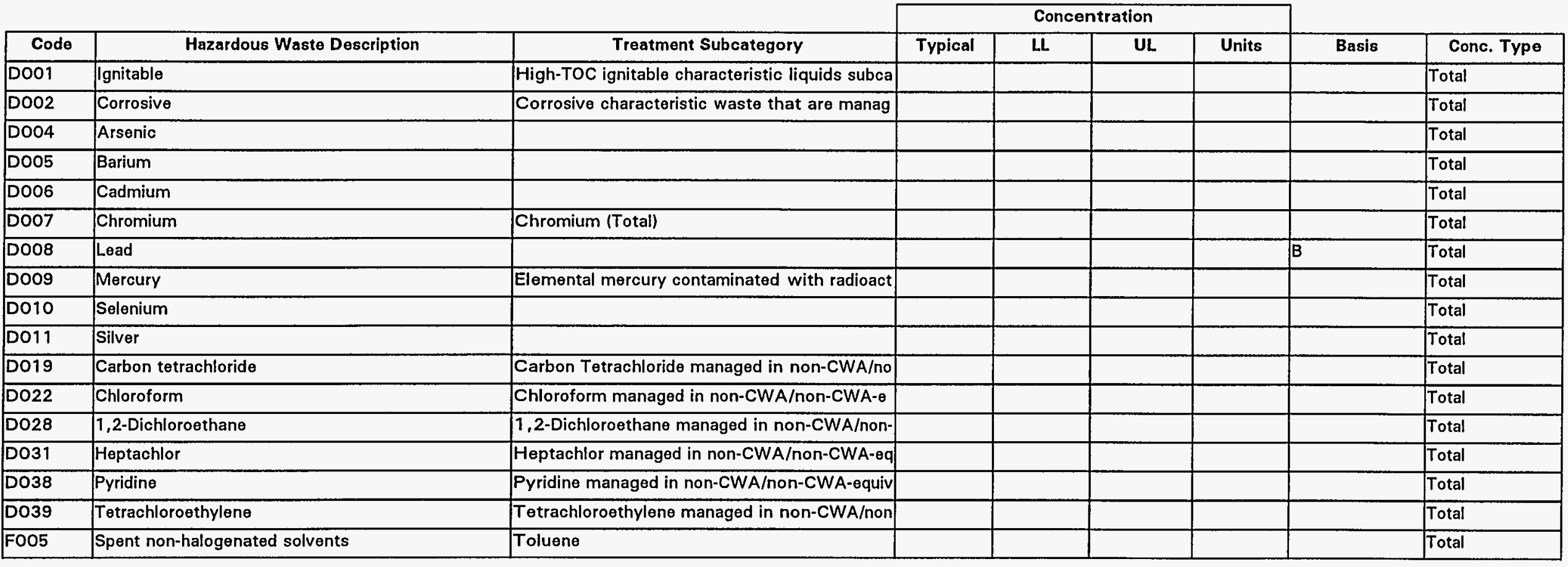




\section{LLW: Contact}

\section{K. POLYCHLORINATED BIPHENYLS (PCBS)}

Is this waste stream PCB contaminated? OYes $O$ No $O$ Unknown

If yes, what portion of the waste is PCB contaminated (volume \%):

If $<100 \%$, can the PCB contaminated waste be segregated and treated separately?

What is the PCB concentration in ppm?

If only a portion of the waste is PCB contaminated, report values for contaminated portion.

\section{TREATMENT PLANS}

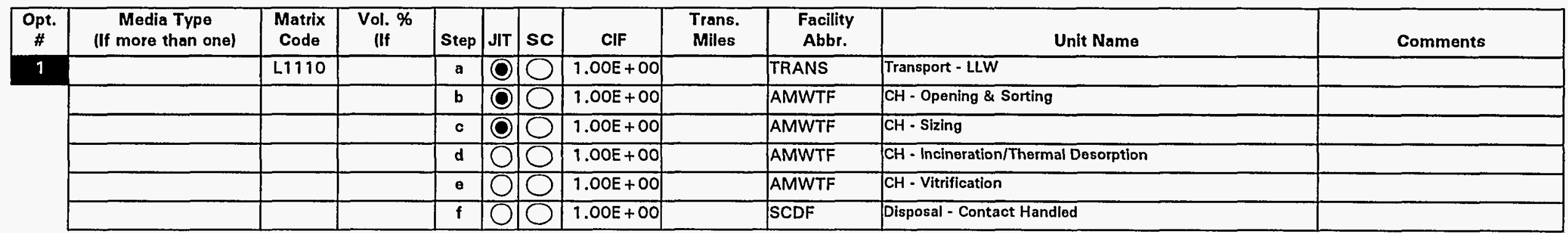

Note: Where provided, media percentages are rough estimates used to facilitate treatment planning. They do not necessarily imply an accurate knowledge of waste stream composition, and were not derived for use outside this conceptual planning exercise.

Describe any special or unique technical concerns related to the treatment of this waste stream that would impact the use or implementation of standard hazardous waste treatment methods. 


LiW
A. IDENTIFICATION AND DESCRIPTION
Waste Stream ID: LB-W014
W. S. Name:
Content Code:
FFCA ID:
Waste Stream Description:
Lab-W014
Labpacks with contaminated acid solutions, bases, oils, solvents, cyanides, asbestos, or PCBs.
Some contaminants are non-RCRA. The waste in this stream have not undergone sufficient
initial characterization to enable assignment to one of the other LBL streams. As characterization
continues, the waste in this stream will be assigned to another LBL stream.

IG SOLVENTS \& OIL

L.B-W014:

Nötmen Active

\section{B. GENERATION SITE/PROCESS DESCRIPTION}

The following questions, concerning the generation site and process, should be answered even if the waste stream is no longer generated.

Name of Site Generating Waste (e.g., INEL, Rocky Flats, etc.): LBL

Location of activities (Area and Building):

Legacy waste.

Operations (functions) performed in building:

Research and Development.

Description of Process Generating Waste:

Various research projects. 
Contáct

C. PROJECTED WASTE GENERATION

Is this waste stream still generated?

$$
\text { OYes O No }
$$

If yes, when is the expected generation termination date? Current Projections

Historical Projections 
CB-W014

LLW Contact-H

LIQUID/SOLIDS CONTAININGG SOLVENTS \& OIL

D. STORAGE INVENTORY

Container ID

Container ID

Stor. Loc. \# of Cont.

Type

Total:

Waste Removed From Storage: 


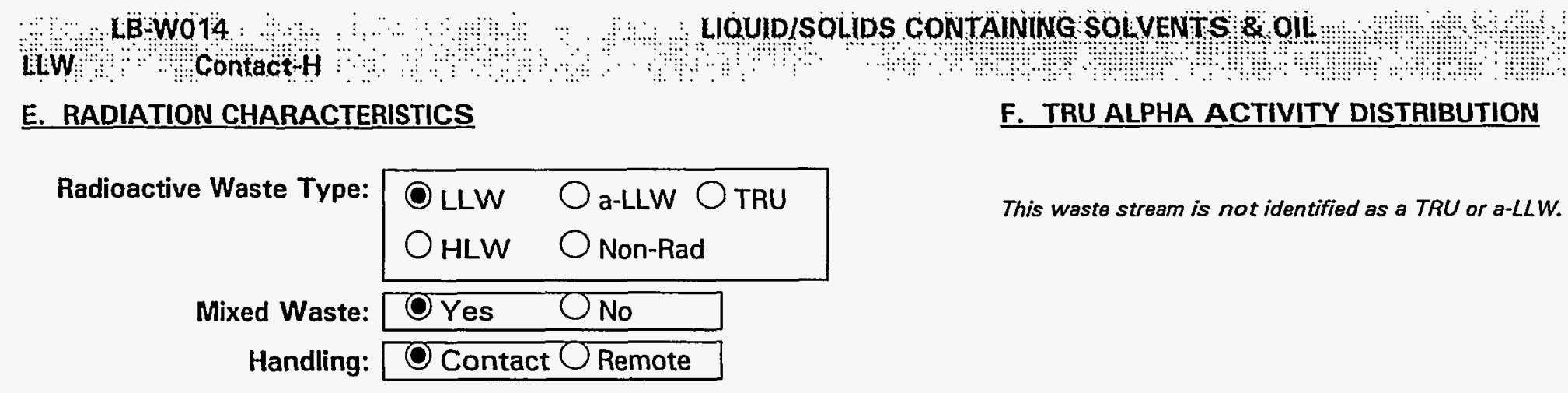

Radionuclide Contamination Accessiblity:

\begin{tabular}{|c|c|c|}
\hline External Surface OYes & ONo & O Unknown \\
\hline Internal Surface $\longdiv { O }$ Yes & ONo & (O) Unknown \\
\hline Dispersed Through Matrix OYes & ONo & O Unknown \\
\hline \multicolumn{3}{|c|}{ Activity Levels } \\
\hline Transuranic Alpha Activity: & & Units: \\
\hline Uranium/Thorium Alpha Activity: & & Units: \\
\hline \multirow[t]{2}{*}{ Beta/Gamma Activity: } & & @ surface \\
\hline & & @ 1-m Units: $m R / h r$ \\
\hline Surface Neutron Activity: & & Units: \\
\hline Total Activity: & & $\mathrm{nCi} / \mathrm{g}$ \\
\hline
\end{tabular}




\section{L.B-WO14}

\section{LLW: Contact-H}

LIQUID/SOLIDS CONTAINING SOLVENTS \& OIL:

\section{G. RADIONUCLIDE CONCENTRATIONS}

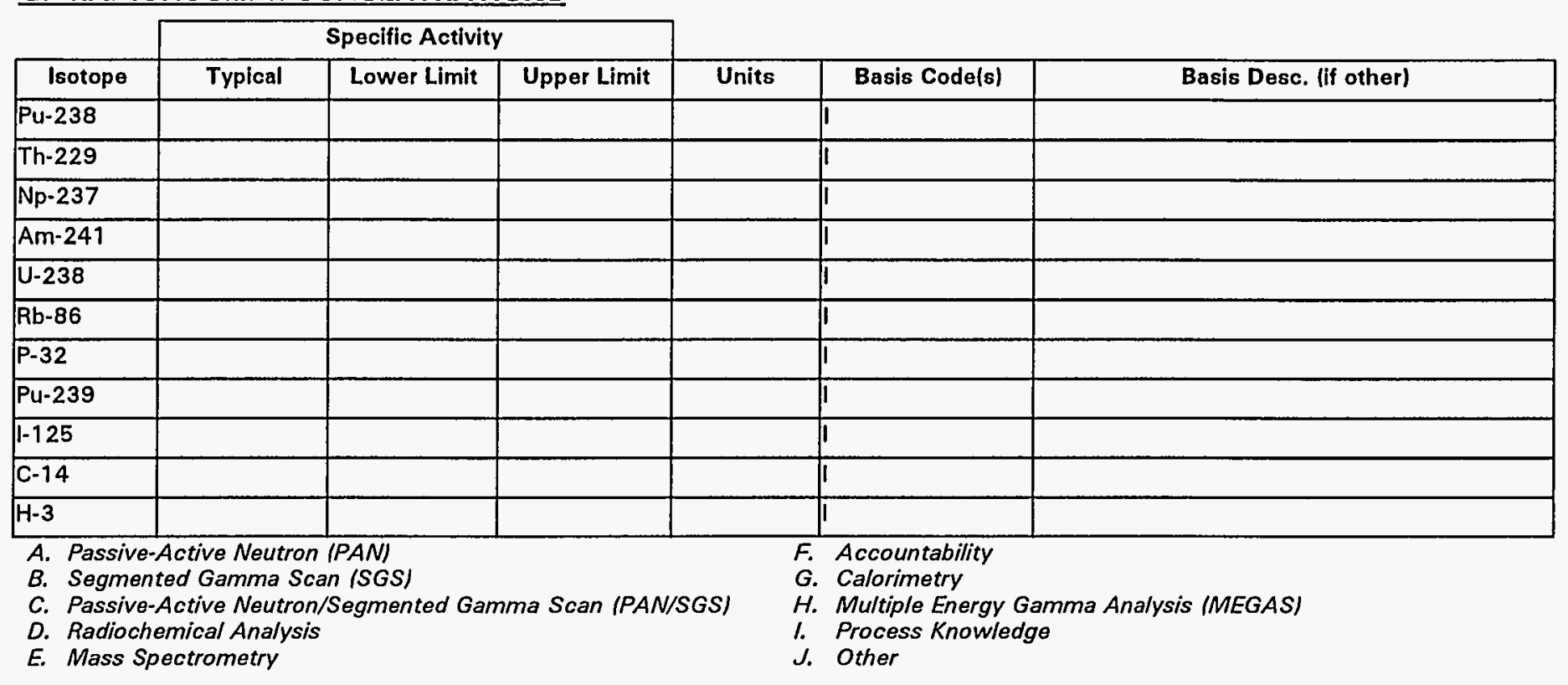

$\because \quad$ LB-WO14 Nót-Gen Active 


\section{$\therefore \quad$ HBW 14}

LiW Contact+H

\section{H. MATRIX CHARACTERISTICS}

Primary Matrix Code: L2900

Description: Organic Liquids: Unknown/Other Organic Liquids: :

\section{Combustibility: Combustible (>90\%) \\ Mixed (10\%-90\%) \\ Noncombustible $(<10 \%)$}

\section{MATRIX COMPOSITION}

\begin{tabular}{|c|c|c|c|c|c|c|}
\hline & & & \multicolumn{4}{|c|}{ Compositton } \\
\hline Code & Matrix Description & Material & Typlcal & LL & UL. & Units \\
\hline$\times 6900$ & Lab Packs.Unknown/Other Lab Packs.. & & & & & \\
\hline$x 6300$ & Lab Packs.Solid Lab Packs.. & & & & & \\
\hline$\times 6200$ & Lab Packs.Aqueous Lab Packs.. & & & & & \\
\hline$\times 6100$ & Lab Packs.Organio Lab Packs.. & & & & & \\
\hline
\end{tabular}


AB-WO14

LLW: Contact-H

LIQUID/SOLIDS CONTAINING SOLVENTS \& OIL

LB:W014

Nat-Gen

Active

\section{J. HAZARDOUS CONSTITUENTS}

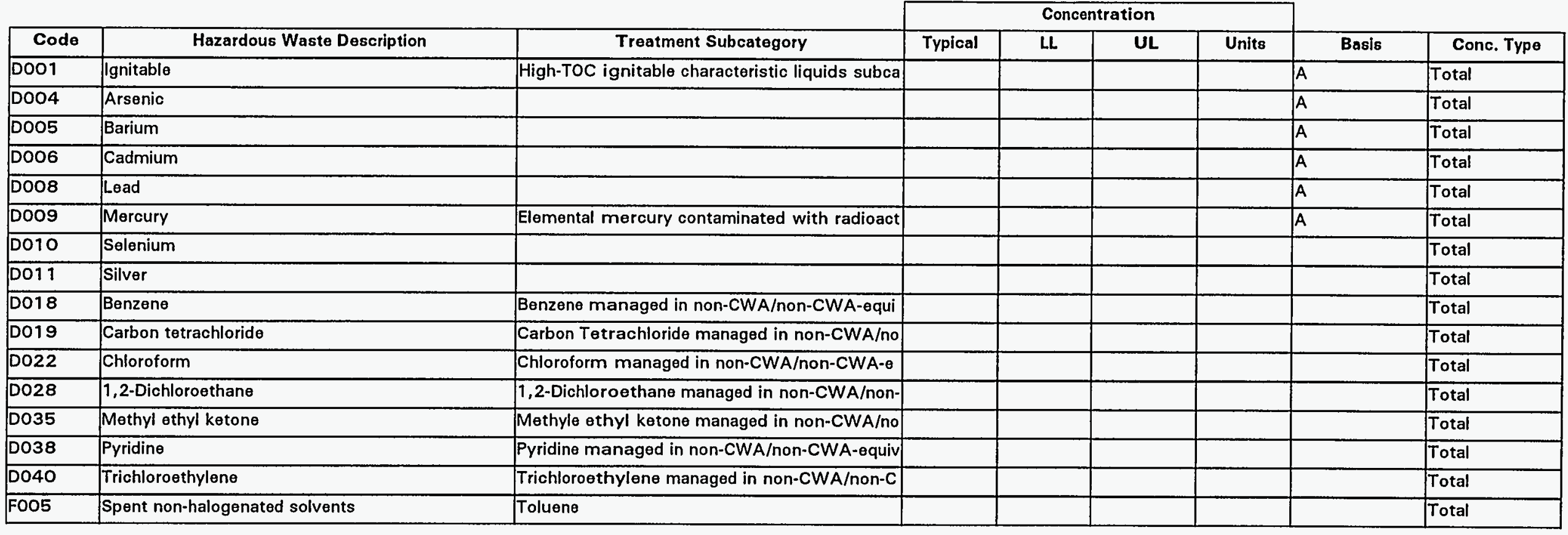




\title{
LBWo14
}

LLW

\section{K. POLYCHLORINATED BIPHENYLS (PCBS)}

Is this waste stream PCB contaminated?

\author{
OYes O No
}

Unknown

If yes, what portion of the waste is PCB contaminated (volume \%):

If $<100 \%$, can the PCB contaminated waste be segregated and treated separately?

What is the PCB concentration in ppm?

If only a portion of the waste is $P C B$ contaminated, report values for contaminated portion.

\section{TREATMENT PLANS}

\begin{tabular}{|c|c|c|c|c|c|c|c|c|c|c|c|}
\hline Opt. & $\begin{array}{c}\text { Media Type } \\
\text { (If more than one) }\end{array}$ & $\begin{array}{c}\text { Matrix } \\
\text { Code }\end{array}$ & $\begin{array}{l}\text { Vol. \% } \\
\text { lif }\end{array}$ & Step & JIT & sc & CIF & $\begin{array}{l}\text { Trans. } \\
\text { Miles }\end{array}$ & $\begin{array}{l}\text { Facility } \\
\text { Abbr. }\end{array}$ & Unit Name & Comments \\
\hline \multirow[t]{6}{*}{1} & & $\mathrm{~L} 2100$ & & a & $\mathbf{0}$ & 0 & $1.00 E+00$ & & TRANS & Transport - LLW & \\
\hline & & & & $\mathbf{b}$ & O & 0 & $1.00 E+00$ & & AMWTF & $\mathrm{CH}$ - Opening \& Sorting & \\
\hline & & & & $c$ & $\mathbf{O}$ & 0 & $1.00 E+00$ & & AMWTF & $\mathrm{CH}$ - Sizing & \\
\hline & & & & d & 0 & 0 & $1.00 E+00$ & & AMWTF & $\mathrm{CH}$ - Incineration/Thermal Desorption & \\
\hline & & & & e & 0 & 0 & $1.00 E+00$ & & AMWTF & CH - Vitrification & \\
\hline & & & & $f$ & O & 0 & $1.00 E+00$ & & SCDF & Disposal - Contact Handled & \\
\hline
\end{tabular}

Note: Where provided, media percentages are rough estimates used to facilitate treatment planning. They do not necessarily imply an accurate knowledge of waste stream composition, and were not derived for use outside this conceptual planning exercise.

Describe any special or unique technical concerns related to the treatment of this waste stream that would impact the use or implementation of standard hazardous waste treatment methods. 


\begin{tabular}{l} 
LiW $\stackrel{\text { LBWW017 }}{\text { Contact-H }}$ \\
A. IDENTIFICATION AND DESCRIPTION \\
Waste Stream ID: LB-W017 \\
W. S. Name: \\
Content Code: \\
FFCA ID: \\
Waste Stream Description: \\
LB-W017 SCINTILLATION FLUIDS- HIGH ALPHA \\
\hline
\end{tabular}

FLUIDS HIGH ALPHA

LB:W017

\section{B. GENERATION SITE/PROCESS DESCRIPTION}

The following questions, concerning the generation site and process, should be answered even if the waste stream is no longer generated.

Name of Site Generating Waste (e.g., INEL, Rocky Flats, etc.):

Location of activities (Area and Building):

Operations (functions) performed in building:

Description of Process Generating Waste:

\begin{tabular}{|l|} 
Description of Process Generating Waste: \\
\hline
\end{tabular}




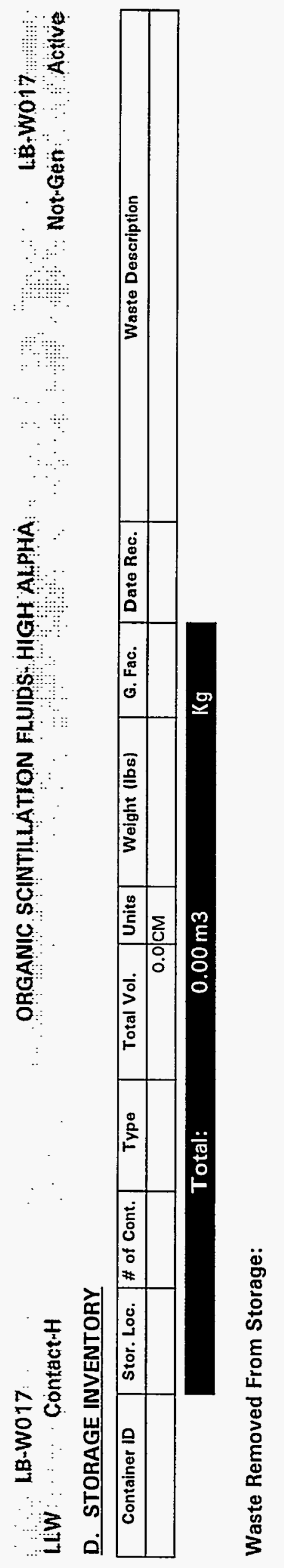




\section{E. RADIATION CHARACTERISTICS}

Radioactive Waste Type:

\begin{tabular}{|ll|}
\hline OllW & $\bigcirc$ a-llw $\bigcirc$ TRU \\
OHLW & $\bigcirc$ Non-Rad \\
\hline
\end{tabular}

Mixed Waste:

Handling:

\begin{tabular}{|l|}
\hline OYes O No \\
\hline O Contact \\
\hline Remote \\
\hline
\end{tabular}

Radionuclide Contamination Accessiblity:

External Surface

Internal Surface

Dispersed Through Matrix

\begin{tabular}{|c|c|c|}
\hline OYes & ONo & OUnknown \\
\hline OYes & ONo & OUnknown \\
\hline OYes & No & C \\
\hline
\end{tabular}

\section{Activity Levels}

Transuranic Alpha Activity:

Uranium/Thorium Alpha Activity:

Beta/Gamma Activity:

Surface Neutron Activity:

Total Activity:

$\square$ Units:
$\square$ Units: $\square$
$\square$ @ surface
$\square$ @ 1-m Units: $\mathrm{mR/hr}$
$\square$ Units: $\square$
$\square$ nCi/g

\section{F. TRU ALPHA ACTIVITY DISTRIBUTION}

This waste stream is not identified as a TRU or a-LLW. 


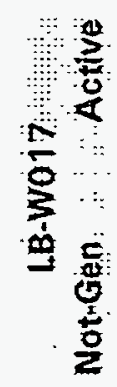

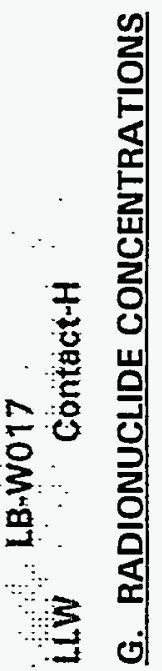


H. MATRIX CHARACTERISTICS

Primary Matrix Code: L2900

Description: Organic Liquids: Unknown/Other Organic Liquids: :

\section{Combustibility: $\bigcirc$ Combustible (>90\%) \\ Mixed $(10 \%-90 \%)$ \\ Noncombustible $(<10 \%)$}

\section{MATRIX COMPOSITION}




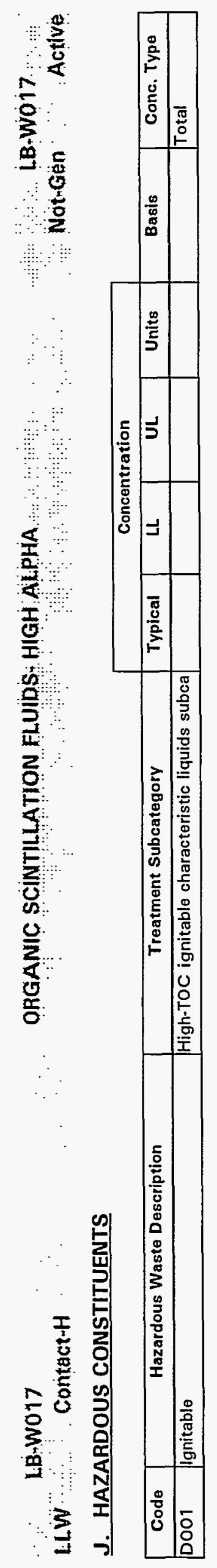




\section{LLW Contact-H}

\section{K. POLYCHLORINATED BIPHENYLS (PCBS)}

Is this waste stream PCB contaminated? OYes O No O Unknown

If yes, what portion of the waste is $\mathrm{PCB}$ contaminated (volume \%):

If $<100 \%$, can the PCB contaminated waste be segregated and treated separately? $O$ Yes O No O Unknown

What is the PCB concentration in ppm? If only a portion of the waste is PCB contaminated, report values for contaminated portion.

\section{TREATMENT PLANS}

\begin{tabular}{|c|c|c|c|c|c|c|c|c|c|c|c|}
\hline Opt. & $\begin{array}{c}\text { Media Type } \\
\text { (If more than one) }\end{array}$ & $\begin{array}{c}\text { Matrix } \\
\text { Code }\end{array}$ & $\begin{array}{l}\text { Vol. \% } \\
\text { lif }\end{array}$ & Step & JIT & sc & ClF & $\begin{array}{l}\text { Trans. } \\
\text { Miles }\end{array}$ & $\begin{array}{l}\text { Facility } \\
\text { Abbr. }\end{array}$ & Unit Name & Comments \\
\hline \multirow[t]{5}{*}{1} & & 1.2100 & & $\bar{a}$ & $\mathbf{O}$ & 0 & $1.00 E+00$ & & TRANS & Transport - LLW & \\
\hline & & & & $\mathbf{b}$ & (O) & O & $1.00 E+00$ & & AMWTF & $\mathrm{CH}$ - Opening \& Sorting & \\
\hline & & & & d & 0 & O & $1.00 E+00$ & & AMWTF & $\mathrm{CH}$ - Incineration/Thermal Desorption & \\
\hline & & & & e & 0 & O & $1.00 E+00$ & & AMWTF & $\mathrm{CH}$ - Vitrification & \\
\hline & & & & $t$ & 0 & O & $1.00 E+00$ & & SCDF & Disposal - Contact Handled & \\
\hline
\end{tabular}

Note: Where provided, media percentages are rough estimates used to facilitate treatment planning. They do not necessarily imply an accurate knowledge of waste stream composition, and were not derived for use outside this conceptual planning exercise.

Describe any special or unique technical concerns related to the treatment of this waste stream that would impact the use or implementation of standard hazardous waste treatment methods. 


\section{Liw Contact H \\ A. IDENTIFICATION AND DESCRIPTION}

Waste Stream ID: LB-W018

W. S. Name:

Content Code:

FFCA ID:

AQUEOUS/SOLID OXIDIZERS - HIGH ALPHA

LB-W018

Waste Stream Description:

\section{AQUEOUS/SOLID OXIDIZERS - HIGHALPHA}

LB-W018 NötGën:: : Active

\section{B. GENERATION SITE/PROCESS DESCRIPTION}

The following questions, concerning the generation site and process, should be answered even if the waste stream is no longer generated.

Name of Site Generating Waste (e.g., INEL, Rocky Flats, etc.):

Location of activities (Area and Building):

Operations (functions) performed in building:

Description of Process Generating Waste: 


\section{PROJECTED WASTE GENERATION}

Is this waste stream still generated?

If yes, when is the expected generation termination date?

Current Projections

\section{Historical Projections}




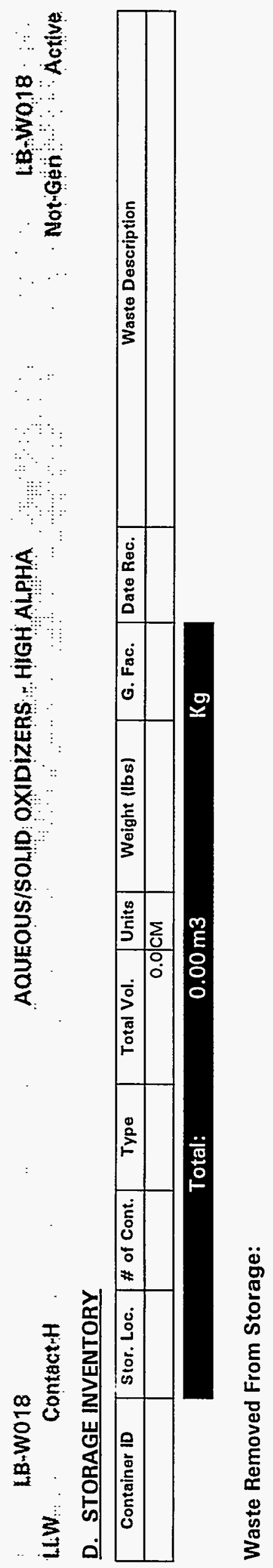




\section{: LBWO18}

HiW Contacti

\section{E. RADIATION CHARACTERISTICS}

Radioactive Waste Type:

\begin{tabular}{|ll|}
\hline OLLW & $\bigcirc$ a-LLW $\bigcirc$ TRU \\
OHLW & $\bigcirc$ Non-Rad \\
\hline
\end{tabular}

Mixed Waste:

Handling:

OYes O No

Radionuclide Contamination Accessiblity:

\begin{tabular}{|c|c|c|c|}
\hline External Surface & OYes & ONo & Unknown \\
\hline Inter & OYes & ONo & OUnknown \\
\hline persed Through Matrix & OYes & $\mathrm{No}$ & own \\
\hline
\end{tabular}

\section{Activity Levels}

Transuranic Alpha Activity:

Uranium/Thorium Alpha Activity:

Beta/Gamma Activity:

Surface Neutron Activity: Total Activity:

\begin{tabular}{|c|c|c|}
\hline & Units: & \\
\hline & Units: & \\
\hline & @ surf & ace \\
\hline & @ 1-m & Units: $\mathrm{mR} / \mathrm{hr}$ \\
\hline & Units: [ & \\
\hline & $\mathrm{nCi} / \mathrm{g}$ & \\
\hline
\end{tabular}



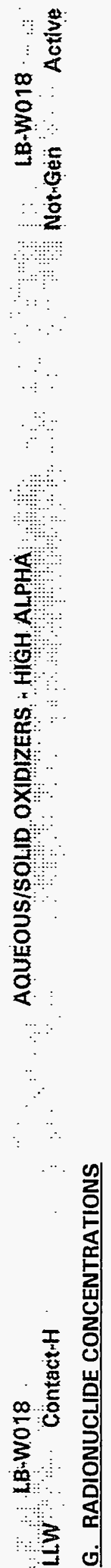


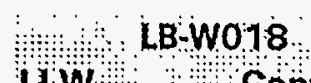

H. MATRIX CHARACTERISTICS

Primary Matrix Code: X6200

Description: Lab Packs: Aqueous Lab Packs: :

Combustibility: $\bigcirc$ Combustible (>90\%)

Mixed $(10 \%-90 \%)$

Noncombustible $(<10 \%)$

\section{MATRIX COMPOSITION}


LB-W018

LiW: " : Contact-H

J. HAZARDOUS CONSTITUENTS

\begin{tabular}{|c|c|c|c|c|c|c|c|c|}
\hline & & & \multicolumn{4}{|c|}{ Concentration } & & \\
\hline Code & Hazardous Waste Description & Treatment Subcategory & Typical & LL & UL & Units & Basis & Conc. Type \\
\hline$\overline{D 001}$ & Ignitable & High-TOC ignitable characteristic liquids subca & & & & & & Total \\
\hline
\end{tabular}

AQUEOUS/SOLID OXIDIZERS HIGHALPHA H AB-WO18

NotGen Active 


\section{CB-Wo18}

LLW ContactiH

\section{A}

\section{K. POLYCHLORINATED BIPHENYLS (PCBS)}

Is this waste stream PCB contaminated? $O$ Yes $O$ No $O$ Unknown

If yes, what portion of the waste is PCB contaminated (volume \%):

If $<100 \%$, can the PCB contaminated waste be segregated and treated separately? $O$ Yes $O$ No $O$ Unknown

What is the PCB concentration in ppm? If only a portion of the waste is PCB contaminated, report values for contaminated portion.

\section{TREATMENT PLANS}

\begin{tabular}{|c|c|c|c|c|c|c|c|c|c|c|c|}
\hline $\begin{array}{c}\text { Opt. } \\
\#\end{array}$ & $\begin{array}{c}\text { Media Type } \\
\text { (If more than one) }\end{array}$ & $\begin{array}{c}\text { Matrix } \\
\text { Code }\end{array}$ & $\begin{array}{l}\text { Vol. \% } \\
\text { Iff }\end{array}$ & Step & JIT & sc & ClF & $\begin{array}{l}\text { Trans. } \\
\text { Miles }\end{array}$ & $\begin{array}{l}\text { Facility } \\
\text { Abbr. }\end{array}$ & Unit Name & Comments \\
\hline 1 & & $\times 6200$ & & $\bar{a}$ & (O) & 10 & $1.00 E+00$ & & TRANS & Transport - LLW & \\
\hline & & & & b & 0 & 0 & $1.00 E+00$ & & AMWTF & $\mathrm{CH}$ - Opening \& Sorting & \\
\hline & & & & c & O & 0 & $1.00 E+00$ & & AMWTF & $\mathrm{CH} \cdot$ Sizing & \\
\hline & & & & d & $\overline{0}$ & 0 & $1.00 E+00$ & & AMWTF & $\mathrm{CH}$ - Incineration/Thermal Desorption & \\
\hline & & & & $\theta$ & 0 & 0 & $1.00 E+00$ & & AMWTF & CH - Vitrification & \\
\hline & & & & $f$ & 0 & 0 & $1.00 E+00$ & & SCDF & Disposal - Contact Handled & \\
\hline
\end{tabular}

Note: Where provided, media percentages are rough estimates used to facilitate treatment planning. They do not necessarily imply an accurate knowledge of waste stream composition, and were not derived for use outside this conceptual planning exercise.

Describe any special or unique technical concerns related to the treatment of this waste stream that would impact the use or implementation of standard hazardous waste treatment methods. 

AB-W019
HLW ContactrH

\section{A. IDENTIFICATION AND DESCRIPTION}

Waste Stream ID: LB-W019

W. S. Name:

Content Code:

FFCA ID:

DEBRIS CONTAMINATED W/ORGANIC-HIGH ALPHA

Waste Stream Description:

\begin{tabular}{|l|} 
Waste Stream Description: \\
\hline \\
\end{tabular}

\section{DEBRIS CONTAMINATED WIORGANIC HIG̈H ÄL̈̈HA}

LB-WO19

Not-Gen Active

\section{B. GENERATION SITE/PROCESS DESCRIPTION}

The following questions, concerning the generation site and process, should be answered even if the waste stream is no longer generated.

Name of Site Generating Waste (e.g., INEL, Rocky Flats, etc.):

Location of activities (Area and Building):

Operations (functions) performed in building:

Description of Process Generating Waste: 
WBWO19 DEBRIS CONTAMINATED WIORGANICHIGHALPHA

Lit W Contact $-H$

C. PROJECTED WASTE GENERATION

Is this waste stream still generated?

If yes, when is the expected generation termination date?

OYes ONo

Current Projections

Historical Projections 


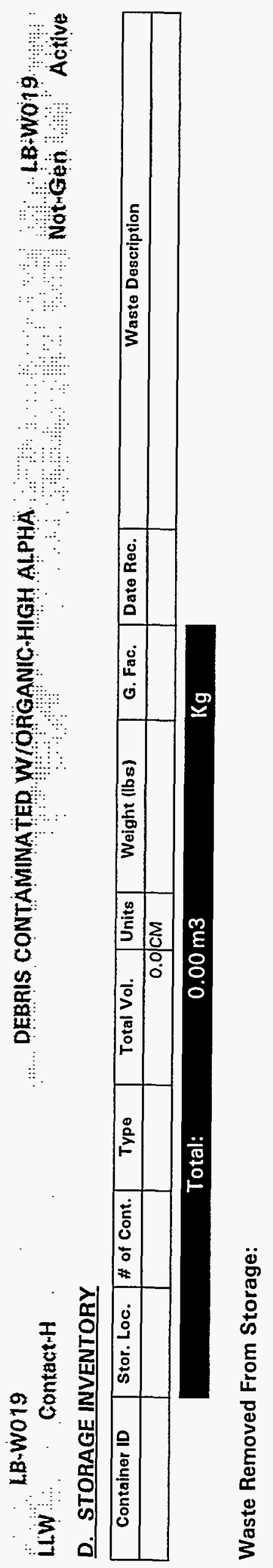

\begin{tabular}{l}
\multirow{7}{*}{} \\
$\dot{0}$ \\
8 \\
8 \\
0
\end{tabular} 


\section{LBWO19 \\ LWW ContactH}

E. RADIATION CHARACTERISTICS

DEBRIS CONTAMINATED WIORGANIC HIGH ALPHA

LB:Wojg

Nöt-Gen Active

\section{F. TRU ALPHA ACTIVITY DISTRIBUTION}

Radioactive Waste Type:

\begin{tabular}{|ll|}
\hline OllW & Oa-LlW O TRU \\
OHLW & O Non-Rad \\
\hline
\end{tabular}

Mixed Waste:

Handling:

\begin{tabular}{|l|}
\hline OYes O No \\
\hline O Contact O Remote \\
\hline
\end{tabular}

Radionuclide Contamination Accessiblity:

\begin{tabular}{|c|c|c|c|}
\hline External Surface & OYes & ONo & Unknown \\
\hline Internal Surface & OYes & No & OUnknown \\
\hline ersed Through Matrix & OYes & ONo & OUnknown \\
\hline
\end{tabular}

\section{Activity Levels}

Transuranic Alpha Activity:

Uranium/Thorium Alpha Activity:

Beta/Gamma Activity:

Surface Neutron Activity:

\begin{tabular}{|c|c|c|}
\hline & Units: [ & \\
\hline & Units: & \\
\hline & @ surf & ace \\
\hline & @ 1-m & Units: $\mathrm{mR} / \mathrm{hr}$ \\
\hline & Units: [ & \\
\hline & $\mathrm{nCi} / \mathrm{g}$ & \\
\hline
\end{tabular}

This waste stream is not identified as a TRU or a.LLW.
Total Activity: $\mathrm{nCi} / \mathrm{g}$ 

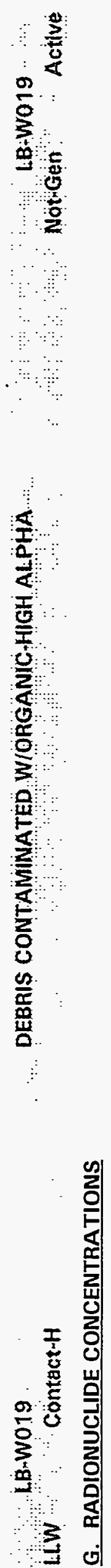


\section{NOIIISOdWOJ XIY्dIEW $\cdot$}

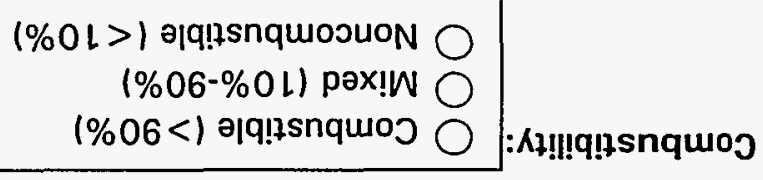




\section{(........B.WO19}

LLW ... Contact-H

\section{J. HAZARDOUS CONSTITUENTS}

\begin{tabular}{|c|c|c|c|c|c|c|c|c|}
\hline & & & \multicolumn{4}{|c|}{ Concentration } & \multirow[b]{2}{*}{ Basis } & \multirow[b]{2}{*}{ Conc. Type } \\
\hline Code & Hazardous Waste Description & Treatment Subcategory & Typical & LL & UL & Units & & \\
\hline$\overline{D 001}$ & Ignitable & High-TOC ignitable characteristic liquids subca & & & & & & Total \\
\hline$\overline{D 004}$ & Arsenic & & & & & & & Total \\
\hline D005 & Barium & & & & & & & Total \\
\hline$D 006$ & Cadmium & & & & & & & Total \\
\hline D007 & Chromium & Chromium (Total) & & & & & & Total \\
\hline 0008 & Lead & & & & & & & Total \\
\hline$\overline{D 009}$ & Mercury & Elemental mercury contaminated with radioact & & & & & & Total \\
\hline D010 & Selenium & & & & & & & Total \\
\hline$\overline{D 011}$ & Silver & & & & & & & Total \\
\hline
\end{tabular}

DEBRIS CONTAMINATED WIORGANICHIGH ALPHA

\section{DERIS CONTAM MATED W \\ $\because$

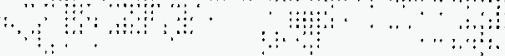

$\vdots$
$\cdots$
$\cdots$

LB-WO19

Natugen Active 


\section{LBWO19}

\section{DEBRIS CONTAMINATED WIORGANICHIGH ALPHA}

\section{LWW Contacti}

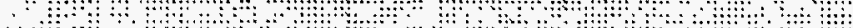

\section{K. POLYCHLORINATED BIPHENYLS (PCBS)}

Is this waste stream PCB contaminated? OYes $O$ No $O$ Unknown

If yes, what portion of the waste is PCB contaminated (volume \%):

If $<100 \%$, can the PCB contaminated waste be segregated and treated separately?

OYes ONo O Unknown

What is the PCB concentration in ppm?

If only a portion of the waste is PCB contaminated, report values for contaminated portion.

\section{TREATMENT PLANS}

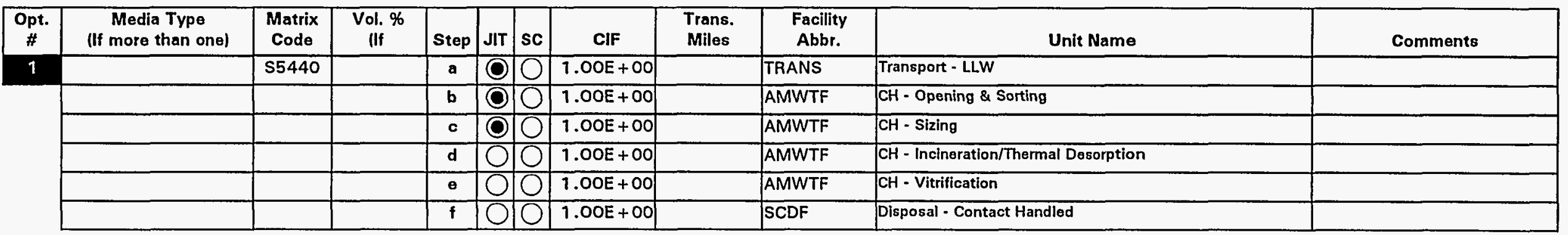

Note: Where provided, media percentages are rough estimates used to facilitate treatment planning. They do not necessarily imply an accurate knowledge of waste stream composition, and were not derived for use outside this conceptual planning exercise.

Describe any special or unique technical concerns related to the treatment of this waste stream that would impact the use or implementation of standard hazardous waste treatment methods. 


\begin{tabular}{l} 
LWW-WIO1 \\
A. IDENTIFICATION AND DESCRIPTION \\
Waste Stream ID: LB-W101 \\
W. S. Name: \\
Content Code: \\
FFCA ID: \\
Waste Stream Description: \\
LB-W101 \\
\hline
\end{tabular}

\section{G:LIQUIDS \\ LB-W10.1 \\ B. GENERATION SITE/PROCESS DESCRIPTION}

The following questions, concerning the generation site and process, should be answered even if the waste stream is no longer generated.

Name of Site Generating Waste (e.g.. INEL, Rocky Flats, etc.):

Location of activities (Area and Building):

Operations (functions) performed in building:

Description of Process Generating Waste: 
Is this waste stream still generated?

OYes O No

If yes, when is the expected generation termination date?

Current Projections

Historical Projections 


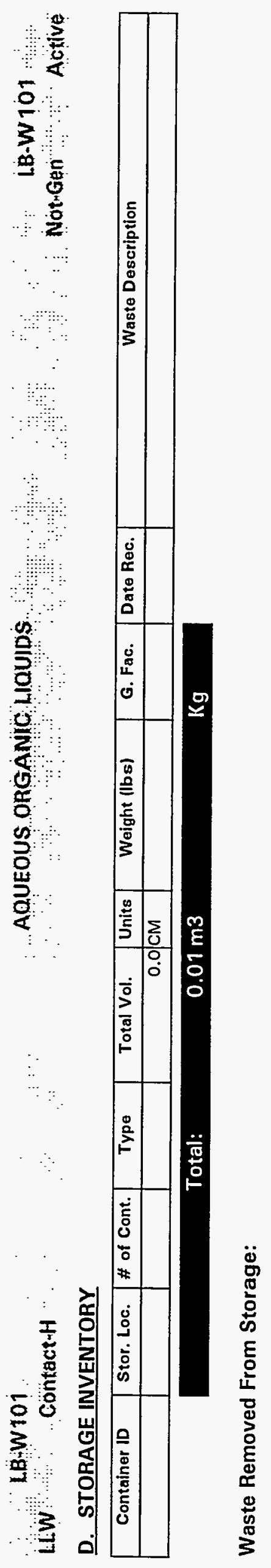




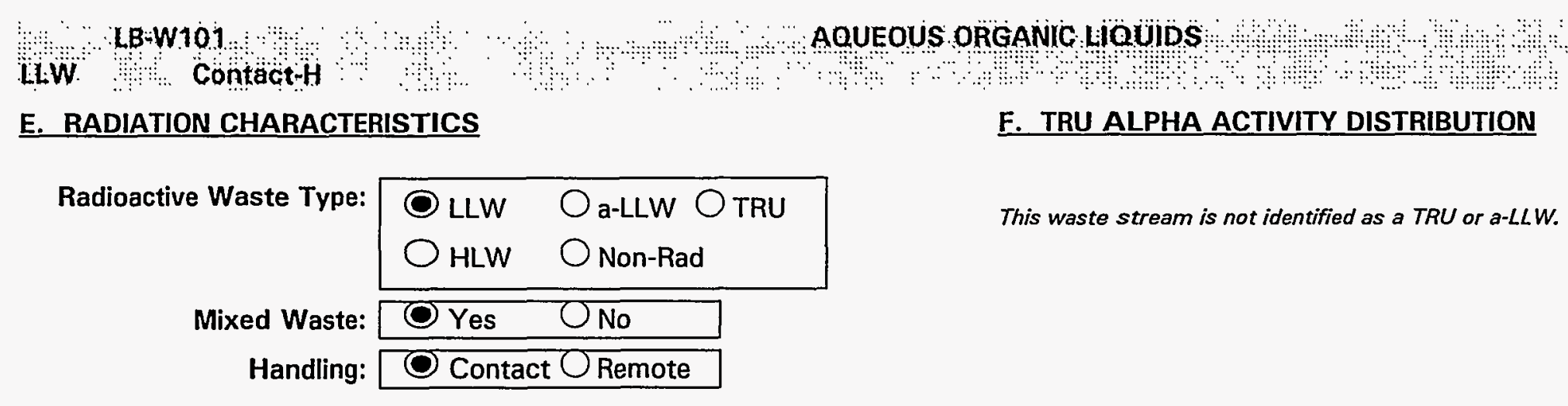

Radionuclide Contamination Accessiblity:

\begin{tabular}{|c|c|c|}
\hline 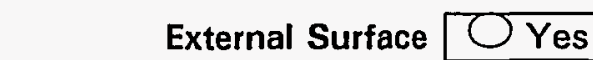 & ONo & Unknown \\
\hline Internal Surface $\longdiv { O Y e s }$ & ONo & OUnknown \\
\hline Dispersed Through Matrix $\bigcirc$ Yes & ONo & Onknown \\
\hline \multicolumn{3}{|c|}{ Activity Levels } \\
\hline \multirow{2}{*}{$\begin{array}{l}\text { Transuranic Alpha Activity: } \\
\text { Uranium/Thorium Alpha Activity: }\end{array}$} & & Units: \\
\hline & & Units: \\
\hline \multirow[t]{2}{*}{ Beta/Gamma Activity: } & & @ surface \\
\hline & & @ 1-m Units: $m R / h r$ \\
\hline \multicolumn{2}{|l|}{ Surface Neutron Activity: } & Units: \\
\hline Total Activity: & & $\mathrm{nCi} / \mathrm{g}$ \\
\hline
\end{tabular}


$\sum^{\frac{3}{0}}$

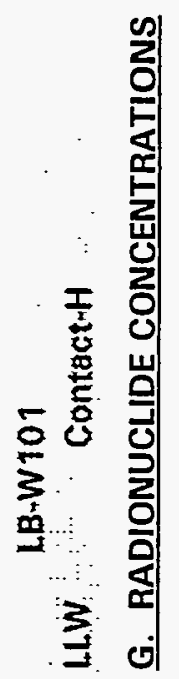




\section{LBWTO1}

LWW Contact-H

H. MATRIX CHARACTERISTICS

Primary Matrix Code: $\mathbf{L 2 1 0 0}$

Description: Organic Liquids: Aqueous/Organic Liquids: :

Combustibility

Combustible $(>90 \%)$

Mixed $(10 \%-90 \%)$

Noncombustible $(<10 \%)$

\section{MATRIX COMPOSITION}



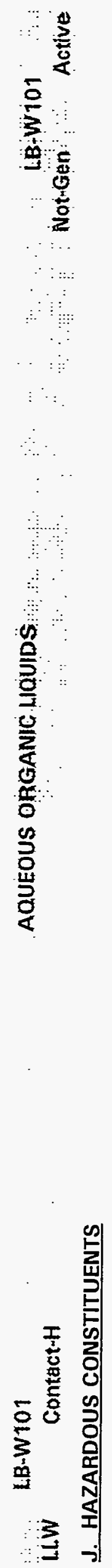


\section{AB-W101}

LWW Contact H

\section{K. POLYCHLORINATED BIPHENYLS (PCBS)}

\section{Is this waste stream PCB contaminated? OYes O No O Unknown}

If yes, what portion of the waste is PCB contaminated (volume \%):

If $<100 \%$, can the PCB contaminated waste be segregated and treated separately?

What is the PCB concentration in ppm?

\section{TREATMENT PLANS}

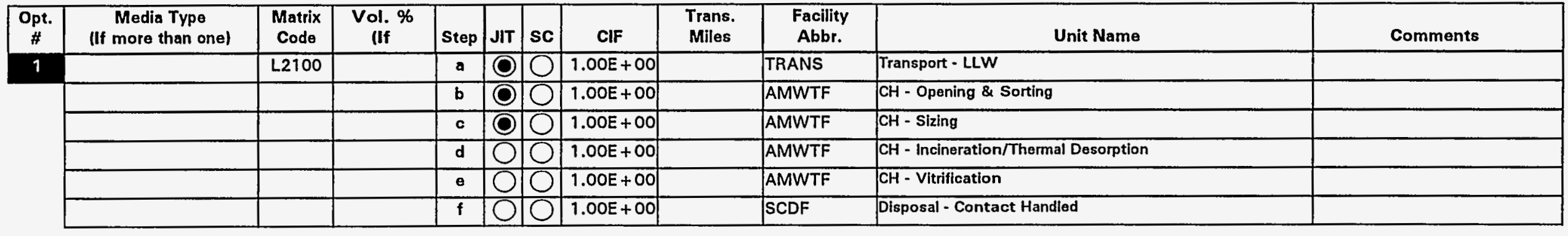

Note: Where provided, media percentages are rough estimates used to facilitate treatment planning. They do not necessarily imply an accurate knowledge of waste stream composition, and were not derived for use outside this conceptual planning exercise.

Describe any special or unique technical concerns related to the treatment of this waste stream that would impact the use or implementation of standard hazardous waste treatment methods. 
LBWW111:

\section{LWW ContactrH}

\section{A. IDENTIFICATION AND DESCRIPTION}

Waste Stream ID: LB-W111

W. S. Name:

Content Code:

FFCA ID:

\begin{tabular}{|l|}
\hline LB-W111 \\
\hline *QUEOUS LIQUIDS \\
\hline LB-W111 \\
\hline
\end{tabular}

Waste Stream Description:

\section{AQUEÖUS LIQUIDS}

$\therefore:$

\section{B. GENERATION SITE/PROCESS DESCRIPTION}

The following questions, concerning the generation site and process, should be answered even if the waste stream is no longer generated.

Name of Site Generating Waste (e.g., INEL, Rocky Flats, etc.):

Location of activities (Area and Building):

Operations (functions) performed in building: 
LWWT1

AQUEEÖUS LIQUIDS

LB:W111

Contact-H

C. PROJECTED WASTE GENERATION

Is this waste stream still generated?

OYes O No

If yes, when is the expected generation termination date?

Current Projections

Historical Projections 


\begin{abstract}
เลืพา 11
\end{abstract}
LiW ContactrH

D. STORAGE INVENTORY

\begin{tabular}{|c|c|}
\hline Container ID & Stor. Loc. \\
\hline
\end{tabular}

\begin{tabular}{|l|l|l|}
\hline Container ID & Stor. Loc. & \# of Cont. \\
\hline
\end{tabular}

Total:

$\ldots \quad \therefore \quad$ AQUEOUS LQUIDS

LB-W111

Notigen : Active

Total:

Waste Removed From Storage: 
LB-W111

LlW Contactin

AQÜEOUS KLUUIDS

\section{E. RADIATION CHARACTERISTICS}

Radioactive Waste Type:

\begin{tabular}{|ll}
\hline OllW & $\bigcirc$ a-llw $\bigcirc$ tRU \\
OhlW & $\bigcirc$ Non-Rad
\end{tabular}

Mixed Waste:

\begin{tabular}{l} 
O Yes $O$ No \\
\hline Contact \\
\hline Remote
\end{tabular}

Radionuclide Contamination Accessiblity:

External Surface

Internal Surface

Dispersed Through Matrix

\begin{tabular}{|c|c|c|}
\hline OYes & ONo & OUnknown \\
\hline OYes & ONo & OUnknown \\
\hline C & Vo & \\
\hline
\end{tabular}

Activity Levels

Transuranic Alpha Activity:

Uranium/Thorium Alpha Activity:

Beta/Gamma Activity:

Surface Neutron Activity:

$\square$ Units: $\square$
$\square$ Units: $\square$
$\square$ @ surface
@ 1-m Units: mR/hr
$\square$ Units: $\square$
nci/g

Total Activity: $\mathrm{nCi} / \mathrm{g}$

\section{F. TRU ALPHA ACTIVITY DISTRIBUTION}

This waste stream is not identified as a TRU or a-LLW. 


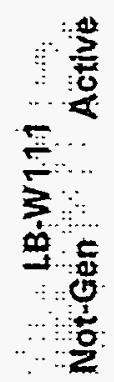

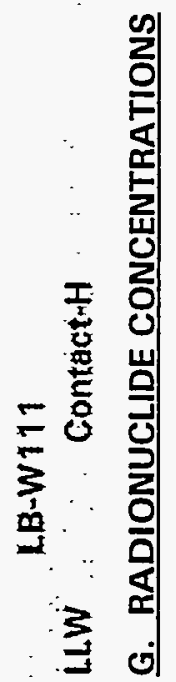


LB+W111

Contactit

AQUEOUS LQUIDS

H. MATRIX CHARACTERISTICS

Primary Matrix Code: L1000

Description: Aqueous Liquids/Slurries: : :

Combustibility: $\bigcirc$ Combustible (>90\%)

Mixed $170 \%-90 \%)$

Noncombustible (<10\%)

\section{MATRIX COMPOSITION}




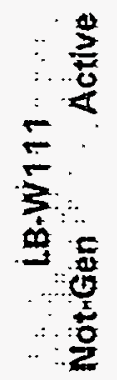

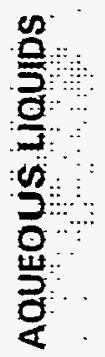

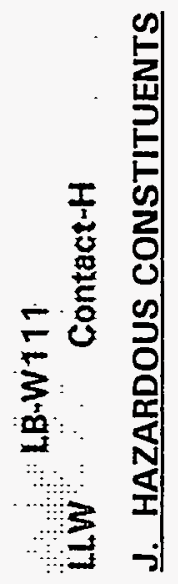




\section{UWW Contact-H}

\section{K. POLYCHLORINATED BIPHENYLS (PCBS)}

Is this waste stream PCB contaminated?

OYes O No O Unknown

If yes, what portion of the waste is PCB contaminated (volume \%):

If $<100 \%$, can the PCB contaminated waste be segregated and treated separately? $O$ Yes O No O Unknown

What is the PCB concentration in ppm?

If only a portion of the waste is PCB contaminated, report values for contaminated portion.

\section{TREATMENT PLANS}

\begin{tabular}{|c|c|c|c|c|c|c|c|c|c|c|c|}
\hline $\begin{array}{c}\text { Opt. } \\
\#\end{array}$ & $\begin{array}{c}\text { Media Type } \\
\text { (If more than one) }\end{array}$ & $\begin{array}{c}\text { Matrix } \\
\text { Code }\end{array}$ & $\begin{array}{l}\text { Vol. \% } \\
\text { (If }\end{array}$ & Step & JIT & sc & CIF & $\begin{array}{l}\text { Trans. } \\
\text { Miles }\end{array}$ & $\begin{array}{l}\text { Facility } \\
\text { Abbr. }\end{array}$ & Unit Name & Comments \\
\hline 1 & & L2100 & & $\bar{a}$ & O & $\bar{O}$ & $1.00 \mathrm{E}+00$ & & TRANS & Transport - LLW & \\
\hline & & & & $\mathbf{b}$ & 0 & $\mathrm{O}$ & $1.00 E+00$ & & AMWTF & $\mathrm{CH}$ - Opening \& Sorting & \\
\hline & & & & c & $\mathbf{O}$ & $\bar{O}$ & $1.00 E+00$ & & AMWTF & $\mathrm{CH}$ - Sizing & \\
\hline & & & & d & 0 & 0 & $1.00 E+00$ & & AMWTF & $\mathrm{CH}$ - Incineration/Thermal Desorption & \\
\hline & & & & e & 0 & 0 & $1.00 E+00$ & & AMWTF & $\mathrm{CH}$ - Vitrification & \\
\hline & & & & $f$ & 0 & 0 & $1.00 E+00$ & & SCDF & Disposal - Contact Handled & \\
\hline
\end{tabular}

Note: Where provided, media percentages are rough estimates used to facilitate treatment planning. They do not necessarily imply an accurate knowledge of waste stream composition, and were not derived for use outside this conceptual planning exercise.

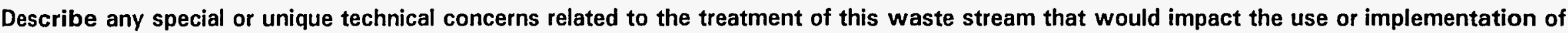
standard hazardous waste treatment methods. 


\section{LL.WOOH \\ Contact-H}

\section{A. IDENTIFICATION AND DESCRIPTION}

Waste Stream ID: LL-W003

W. S. Name:

Content Code:

FFCA ID:

\section{LOW LEVEL MIXED INORGANIC TRASH-1}

Waste Stream Description:

Inorganic trash received from on-site laboratory research and maintenance operations. This waste consists of sludge, paper coveralls, Kemwipes, gloves, booties, wood, low level radioactivity, and chromium. Generated from changing on-site research and development activities where trash is derived from clean up and used safety equipment such as coveralls, paper, gloves, and booties.
Description of Process Generating Waste:

Generated from changing on-site research and development activities where trash derived from clean up and used safety equipment such as coveralls, paper, gloves, and booties.
Still-Gen..... Active

\section{B. GENERATION SITE/PROCESS DESCRIPTION}

The following questions, concerning the generation site and process, should be answered even if the waste stream is no longer generated.

Name of Site Generating Waste (e.g., INEL, Rocky Flats, etc.): LLNL

Location of activities (Area and Building):

Sitewide. Buildings include but are not limited to $377,361,292$, $151,331,366,2312,226$.

Operations (functions) performed in building:
Site wide R\&D and characterization activities. 
LLWOOS

LOW LEVEL MIXED INORGANIC TRASHI

HEW: Contact H

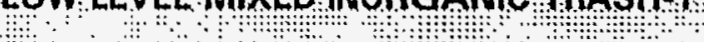

\section{PROJECTED WASTE GENERATION}

Is this waste stream still generated?

\section{OYes ONo}

If yes, when is the expected generation termination date?

Current Projections

\begin{tabular}{|r|r|r|r|r|l|}
\hline Est. Date & Start Year & interval & Vol (m3) & Mass (kg) & Source of Estimate \\
\hline $3 / 30 / 95$ & 1995 & 5 & 7.00 & & Generator Update \\
\hline
\end{tabular}

\begin{tabular}{|c|c|c|c|c|c|}
\hline \multicolumn{6}{|c|}{ Historical Projections } \\
\hline Est. Date & Start Year & Interval & Vol (m3) & Mass (kg) & Source of Estlmate \\
\hline $11 / 30 / 93$ & 1993 & 1 & 0.40 & 396.00 & 1993-FFCA \\
\hline $11 / 30 / 93$ & 1994 & 1 & 0.40 & 396.00 & 1993-FFCA \\
\hline $11 / 30 / 93$ & 1995 & 1 & 0.40 & 396.00 & 1993-FFCA \\
\hline $11 / 30 / 83$ & 1996 & 1 & 0.40 & 396.00 & 1993-FFCA \\
\hline $11 / 30 / 93$ & 1997 & 1 & 0.40 & 396.00 & 1993-FFCA \\
\hline $11 / 30 / 93$ & 1998 & 1 & 0.40 & 396.00 & 1993-FFCA \\
\hline $11 / 30 / 93$ & 1999 & 1 & 0.40 & 396.00 & 1993-FFCA \\
\hline $11 / 30 / 93$ & 2000 & 1 & 0.40 & 396.00 & 1993-FFCA \\
\hline 11/30/93 & 2001 & 1 & 0.40 & 396.00 & 1993-FFCA \\
\hline $11 / 30 / 93$ & 2002 & 1 & 0.40 & 396.00 & 1993-FFCA \\
\hline $11 / 30 / 93$ & 2003 & 10 & 7.60 & $7,524.00$ & 1993-FFCA \\
\hline
\end{tabular}




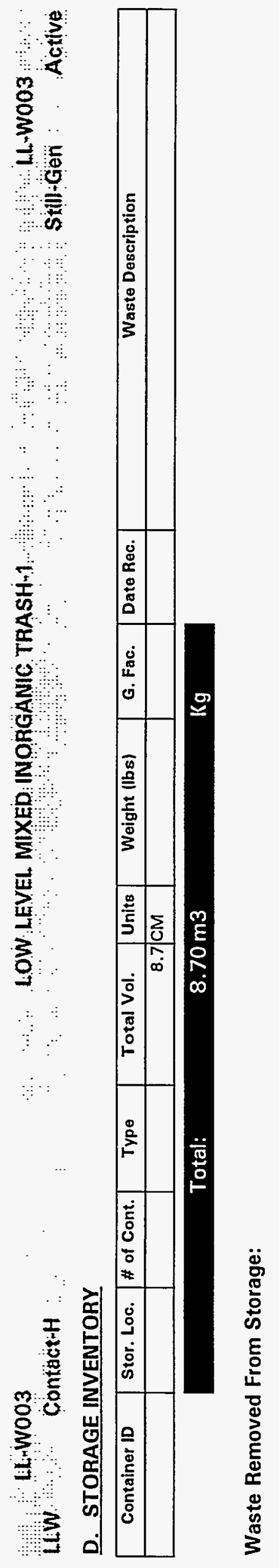


LLWOO3

LLW Contáct H

\section{E. RADIATION CHARACTERISTICS}

Radioactive Waste Type:

\begin{tabular}{|ll|}
\hline O lLW & O a-LLW O TRU \\
OHLW & O Non-Rad \\
\hline
\end{tabular}

Mixed Waste:

Handling:

\begin{tabular}{|l|}
\hline OYes O No \\
\hline O Contact O Remote \\
\hline
\end{tabular}

Radionuclide Contamination Accessiblity:

\begin{tabular}{|c|c|c|c|}
\hline External Surface & OYes & ONo & OUnknown \\
\hline Internal : & OYes & O No & OUnknown \\
\hline rsed Through & (O) Yes & ONo & O Unknown \\
\hline
\end{tabular}

\section{Activity Levels}

Transuranic Alpha Activity:

Uranium/Thorium Alpha Activity:

Beta/Gamma Activity:

Surface Neutron Activity:

Total Activity:

\begin{tabular}{|c|c|c|}
\hline & Units: [ & \\
\hline & Units: [ & \\
\hline & @ surfe & ace \\
\hline & @ 1-m & Units: $\mathrm{mR} / \mathrm{hr}$ \\
\hline & Units: [ & \\
\hline 9262.28 & $\mathrm{nCi} / \mathrm{g}$ & \\
\hline
\end{tabular}

This waste stream is not identified as a TRU or a-LLW.

\section{F. TRU ALPHA ACTIVITY DISTRIBUTION}

LOWWLEVEL MIXED INORGANIC TRASH I 


\section{LLW Contact-H}

\section{G. RADIONUCLIDE CONCENTRATIONS}

\begin{tabular}{|c|c|c|c|c|c|c|}
\hline & & Specific Activity & & & & \\
\hline Isotope & Typical & Lower Limit & Upper Limit & Units & Basis Code(s) & Basis Desc. (if other) \\
\hline Sb-124 & & $0.0000 E+00$ & $2.1000 \mathrm{E}-01$ & $n \mathrm{ni} / \mathrm{g}$ & $J$ & \\
\hline $\mathrm{H}-3$ & & $0.0000 \mathrm{E}+00$ & $2.8251 E+03$ & $\mathrm{nCi} / \mathrm{g}$ & J & \\
\hline Co-60 & & $0.0000 E+00$ & $2.2000 \mathrm{E}-01$ & $n \mathrm{ni} / \mathrm{g}$ & $\mathrm{j}$ & \\
\hline$M n-54$ & & $0.0000 E+00$ & $2.6000 \mathrm{E}-03$ & $n \mathrm{ni} / \mathrm{g}$ & $J$ & \\
\hline $\mathrm{U}-235$ & & $0.0000 E+00$ & $3.7000 \mathrm{E}-01$ & $\mathrm{nCi} / \mathrm{g}$ & $\mathrm{J}$ & \\
\hline$C-14$ & & $0.0000 E+00$ & $7.3420 E+01$ & $\mathrm{nCi} / \mathrm{g}$ & $J$ & \\
\hline Cr-51 & & $0.0000 E+00$ & $2.5173 E+02$ & $\mathrm{nCi} / \mathrm{g}$ & j & \\
\hline Am-243 & & $0.0000 E+00$ & $3.7000 \mathrm{E}-03$ & $\widehat{n C i / g}$ & $\mathrm{j}$ & \\
\hline Ce-141 & & $0.0000 E+00$ & $6.2900 E+00$ & $\mathrm{nCi} / \mathrm{g}$ & 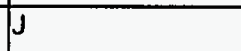 & \\
\hline Eu-152 & & $0.0000 E+00$ & $1.3000 \mathrm{E}-01$ & $\mathrm{nCi} / \mathrm{g}$ & j & \\
\hline$K-40$ & & $0.0000 E+00$ & $3.8000 \mathrm{E}-03$ & $n \mathrm{nCi} / \mathrm{g}$ & $j$ & \\
\hline $\mathrm{Nb}-95$ & & $0.0000 E+00$ & $6.2940 E+01$ & $n \mathrm{ni} / \mathrm{g}$ & j & \\
\hline U-dep & & $0.0000 E+00$ & $2.2000 \mathrm{E}-01$ & $n \mathrm{Ci} / \mathrm{g}$ & j & \\
\hline Pu-239 & & $0.0000 E+00$ & $4.4100 E+00$ & $n \mathrm{nCi} / \mathrm{g}$ & j & \\
\hline W-181 & & $0.0000 E+00$ & $7.4000 \mathrm{E}-01$ & $\mathrm{nCi} / \mathrm{g}$ & j & \\
\hline Sr-90 & & $0.0000 E+00$ & $8.8106 E+02$ & $\mathrm{nCi} / \mathrm{g}$ & $\sqrt{3}$ & \\
\hline Th-232 & & $0.0000 E+00$ & $1.5000 \mathrm{E}-02$ & $\mathrm{nCi} / \mathrm{g}$ & 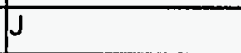 & \\
\hline $2 r-95$ & & $0.0000 E+00$ & $1.1300 E+00$ & $n \mathrm{ni} / \mathrm{g}$ & J & \\
\hline$A m-241$ & & $0.0000 E+00$ & $2.2300 E+00$ & $n \mathrm{nCi} / \mathrm{g}$ & $J$ & \\
\hline$B e-7$ & & $0.0000 E+00$ & $2.2000 \mathrm{E}-02$ & $\mathrm{nCi} / \mathrm{g}$ & $j$ & \\
\hline $\mathrm{Cm}-244$ & & $0.0000 E+00$ & $2.2000 \mathrm{E}-02$ & $\mathrm{nCi} / \mathrm{g}$ & $j$ & \\
\hline Co-57 & & $0.0000 E+00$ & $2.2000 \mathrm{E}-02$ & $\mathrm{nCi} / \mathrm{g}$ & $J$ & \\
\hline Co-58 & & $0.0000 E+00$ & $2.2000 \mathrm{E}-02$ & $\mathrm{nCi} / \mathrm{g}$ & $J$ & \\
\hline Cs-137 & & $0.0000 E+00$ & $4.0000 \mathrm{E}-03$ & $\mathrm{nCi} / \mathrm{g}$ & $J$ & \\
\hline MFP & & $0.0000 E+00$ & $5.4386 \mathrm{E}+03$ & $\mathrm{nCi} / \mathrm{g}$ & $J$ & \\
\hline $\mathrm{Rb}-83$ & & $0.0000 E+00$ & $2.2000 \mathrm{E}-02$ & $n \mathrm{ni} / \mathrm{g}$ & $\mathrm{J}$ & \\
\hline Sc-46 & & $0.0000 E+00$ & $2.2000 \mathrm{E}-02$ & $\mathrm{nCi} / \mathrm{g}$ & $J$ & \\
\hline Th-228 & & $0.0000 E+00$ & $7.0000 \mathrm{E}-04$ & $n \mathrm{Ci} / \mathrm{g}$ & $J$ & \\
\hline$P m-147$ & & $0.0000 E+00$ & $3.5300 E+00$ & $n \mathrm{ni} / \mathrm{g}$ & $j$ & \\
\hline
\end{tabular}

A. Passive-Active Neutron (PAN)

B. Segmented Gamma Scan (SGS)

F. Accountability

C. Passive-Active Neutron/Segmented Gamma Scan (PAN/SGS)

D. Radiochemical Analysis

E. Mass Spectrometry
LOW LEVEL MIXED INOARGANIC TRASH-1

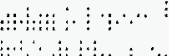

Still-Gen Active 
H. MATRIX CHARACTERISTICS

Primary Matrix Code: $\mathbf{5 5 3 1 0}$

Description: Debris Waste: Organic Debris: Plastic/Rubber Debris:

Combustibility: $\bigcirc$ Combustible (>90\%)

Mixed $(10 \%-90 \%)$

Noncombustible $(<10 \%)$

\section{MATRIX COMPOSITION}

\begin{tabular}{|c|c|c|c|c|c|c|}
\hline & & & \multicolumn{4}{|c|}{ Composition } \\
\hline Code & Matrix Description & Material & Typlcal & LL & UL & Units \\
\hline $\mathbf{5 5 3 1 0}$ & Debris Waste.Organic Debris.Plastic/Rubber Debris. & & 100 & & & $\%$ vol \\
\hline
\end{tabular}


LL. WÖO03

LWW ContactrH

\section{J. HAZARDOUS CONSTITUENTS}

\begin{tabular}{|c|c|c|c|c|c|c|c|c|}
\hline & \multicolumn{4}{|c|}{ Concentration } & \multirow[b]{2}{*}{ Basis } & \multirow[b]{2}{*}{ Conc. Type } \\
\hline Code & Hazardous Waste Description & Treatment Subcategory & Typical & LL & UL & Units & & \\
\hline $\mathrm{D003}$ & Reactive & Reactive Cyanides based on $261.23(a)(5)$ Cyan & & 0 & \multicolumn{2}{|c|}{$250 \mathrm{ppm}$} & $F$ & Total \\
\hline D004 & Arsenic & & & o & 100 & $\mathrm{ppm}$ & $F$ & Total \\
\hline$\overline{D 005}$ & Barium & & & 0 & 1100 & $\mathrm{ppm}$ & $F$ & Total \\
\hline 0006 & Cadmium & & & 0 & & $\mathrm{ppm}$ & $F$ & Total \\
\hline D007 & Chromium & Chromium (Total) & & 아 & 1370 & $\mathrm{ppm}$ & $F$ & Total \\
\hline D008 & Lead & & & 0 & 130 & $\mathrm{ppm}$ & $F$ & Total \\
\hline D009 & Mercury & Elemental mercury contaminated with radioact & & 0 & 500 & $\mathrm{ppm}$ & $F$ & Total \\
\hline D010 & Selenium & & & 0 & 100 & $\mathrm{ppm}$ & $F$ & Total \\
\hline D011 & Silver & & & 0 & 79 & $\mathrm{ppm}$ & $F$ & Total \\
\hline D018 & Benzene & Benzene managed in non-CWA/non-CWA-equi & & 0 & 200 & $\mathrm{ppm}$ & $F$ & Total \\
\hline $\mathrm{D022}$ & Chloroform & Chloroform managed in non-CWA/non-CWA-e & & 0 & & ppm & $F$ & Total \\
\hline F001 & Spent halogenated solvents used in degreasing & Trichloroethylene & & & & & $B$ & Total \\
\hline F003 & Spent non-halogenated solvents & Xylenes - mixed isomers & & 0 & 1800 & $\mathrm{ppm}$ & $B$ & Total \\
\hline P015 & Beryllium powder & & & & & & $F$ & Total \\
\hline
\end{tabular}




\section{K. POLYCHLORINATED BIPHENYLS (PCBS)}

\section{Is this waste stream PCB contaminated?

OYes O No O Unknown

If yes, what portion of the waste is PCB contaminated (volume \%):

If $<100 \%$, can the PCB contaminated waste be segregated and treated separately?

What is the PCB concentration in ppm?

If only a portion of the waste is $P C B$ contaminated, report values for contaminated portion.

\section{TREATMENT PLANS}

\begin{tabular}{|c|c|c|c|c|c|c|c|c|c|c|c|}
\hline $\begin{array}{l}\text { Opt. } \\
\#\end{array}$ & $\begin{array}{c}\text { Media Type } \\
\text { (If more than one) }\end{array}$ & $\begin{array}{c}\text { Matrix } \\
\text { Code }\end{array}$ & $\begin{array}{l}\text { Vol. \% } \\
\text { (If }\end{array}$ & Step & JIT & sc & CIF & $\begin{array}{l}\text { Trans. } \\
\text { Miles }\end{array}$ & $\begin{array}{l}\text { Facility } \\
\text { Abbr. }\end{array}$ & Unit Name & Comments \\
\hline \multirow[t]{6}{*}{1} & & $\$ 5310$ & & $\bar{a}$ & () & 0 & $1.00 E+00$ & & TRANS & Transport - LLW & \\
\hline & & & & b & O & O & $1.00 E+00$ & & AMWTF & $\mathrm{CH}$ - Opening \& Sorting & \\
\hline & & & & c & $\mathbf{O}$ & 0 & $1.00 E+00$ & & AMWTF & CH - Sizing & \\
\hline & & & & $\bar{d}$ & 0 & 0 & $1.00 E+00$ & & AMWTF & $\mathrm{CH}$ - Incineration/Thermal Desorption & \\
\hline & & & & e & 0 & 0 & $1.00 E+00$ & & AMWTF & $\mathrm{CH}$ - Vitrification & \\
\hline & & & & $\bar{f}$ & 0 & $O$ & $1.00 E+00$ & & SCDF & Disposal - Contact Handled & \\
\hline
\end{tabular}

Note: Where provided, media percentages are rough estimates used to facilitate treatment planning. They do not necessarily imply an accurate knowledge of waste stream composition, and were not derived for use outside this conceptual planning exercise.

Describe any special or unique technical concerns related to the treatment of this waste stream that would impact the use or implementation of standard hazardous waste treatment methods. 


LLWWOO6
A. IDENTIFICATION AND DESCRIPTION
Waste Stream ID:
LL-W006
W. S. Name:
Content Code:
FFCA ID:

\section{Waste Stream Description:}

Low level radioactive scrap metal from on-site research and maintenance, including laboratory clean up. Includes lead bricks, metal buttons, pumps, and stainless steel vessels. Used and decommisssioned contaminated equipment and metal parts from changing research and development activities.

\section{B. GENERATION SITE/PROCESS DESCRIPTION}

The following questions, concerning the generation site and process, should be answered even if the waste stream is no longer generated.

Name of Site Generating Waste (e.g.. INEL, Rocky Flats, etc.): LLNL

Location of activities (Area and Building):

Sitewide. Buildings include but are not limited to $377,361,292$,

$151,331,366,2312,226$

Operations (functions) performed in building:

Site wide $R \& D$ and characterization activities.

Description of Process Generating Waste:

Generated from on-site research and maintenance, including laboratory clean up.
Li-W0.W6 Active 


\section{Lixwo06}

LOW LEVEL MIXED SCRAP METAL

Contact-H

\section{PROJECTED WASTE GENERATION}

Is this waste stream still generated?

If yes, when is the expected generation termination date?

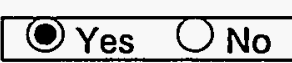

\begin{tabular}{|}
\begin{tabular}{|r|r|r|r|r|c|}
\hline Est. Date & Start Year & Interval & Vol (m3) & Mass (kg) & Source of Estimate \\
\hline $3 / 30 / 95$ & 1995 & 5 & 5.00 & & Generator Update \\
\hline
\end{tabular}
\end{tabular}

\begin{tabular}{|r|r|r|r|r|r|}
\hline \multicolumn{7}{|c|}{ Historical Projections } \\
\hline \multicolumn{1}{|c|}{ Est. Date } & Start Year & \multicolumn{1}{|c|}{ Interval } & Vol (m3) & \multicolumn{1}{c|}{ Mass $(\mathrm{kg})$} & Source of EstImate \\
\hline $11 / 30 / 93$ & 1993 & 1 & 6.00 & $6,029.00$ & 1993 -FFCA \\
\hline $11 / 30 / 93$ & 1994 & 1 & 6.00 & $6,029.00$ & $1993-F F C A$ \\
\hline $11 / 30 / 93$ & 1995 & 1 & 6.00 & $6,029.00$ & $1993-F F C A$ \\
\hline $11 / 30 / 93$ & 1996 & 1 & 6.00 & $6,029.00$ & $1993-F F C A$ \\
\hline $11 / 30 / 93$ & 1997 & 1 & 6.00 & $6,029.00$ & $1993-F F C A$ \\
\hline $11 / 30 / 93$ & 1998 & 1 & 6.00 & $6,029.00$ & $1993-F F C A$ \\
\hline $11 / 30 / 93$ & 1999 & 1 & 6.00 & $6,029.00$ & $1993-F F C A$ \\
\hline $11 / 30 / 93$ & 2000 & 1 & 6.00 & $6,029.00$ & $1993-F F C A$ \\
\hline $11 / 30 / 93$ & 2001 & 1 & 6.00 & $6,029.00$ & $1993-F F C A$ \\
\hline $11 / 30 / 93$ & 2002 & 1 & 6.00 & $6,029.00$ & $1993-F F C A$ \\
\hline $11 / 30 / 93$ & 2003 & 20 & 114.00 & $114,551.00$ & $1993-F F C A$ \\
\hline
\end{tabular}




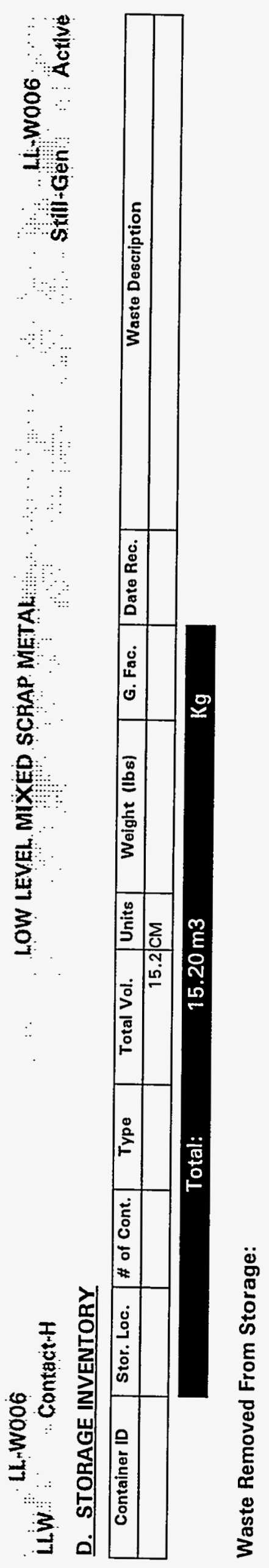

7
$\frac{7}{4}$
0
8
0

ह 
linWoo6 UWW Contagth

E. RADIATION CHARACTERISTICS

LOW LEVEL MIXED SCRAP METAL

Radioactive Waste Type:

\begin{tabular}{|ll|}
\hline O lLW & Oa-LLW $\bigcirc$ TRU \\
O HLW & $\bigcirc$ Non-Rad \\
\hline
\end{tabular}

Mixed Waste:

\begin{tabular}{|l|}
\hline OYes O No \\
\hline O Contact O Remote \\
\hline
\end{tabular}

Handling: $O$ Contact $O$ Remote

Radionuclide Contamination Accessiblity:

\begin{tabular}{|c|c|c|c|}
\hline External Surface & OYes & ONo & OUnknown \\
\hline Internal Surface & OYes & O No & OUnknown \\
\hline arsed Through Matrix & O Yes & No & OUnknown \\
\hline
\end{tabular}

\section{Activity Levels}

Transuranic Alpha Activity:

Uranium/Thorium Alpha Activity:

Beta/Gamma Activity:

\begin{tabular}{|c|c|c|}
\hline & Units: [ & \\
\hline & Units: & \\
\hline 11.2 & @ surf & \\
\hline 5.0 & @ 1-m & Units: $\mathrm{mR} / \mathrm{hr}$ \\
\hline & Units: & \\
\hline
\end{tabular}

Surface Neutron Activity:

Total Activity:

$$
2864.21 \mathrm{nCi} / \mathrm{g}
$$

\section{F. TRU ALPHA ACTIVITY DISTRIBUTION}

This waste stream is not identified as a TRU or a-LLW. 


\section{G. RADIONUCLIDE CONCENTRATIONS}

\section{Specific Activity}

\begin{tabular}{|c|c|c|c|c|c|c|}
\hline Isotope & Typical & Lower Limit & Upper Limit & Units & Basis Code(s) & Basis Desc. (if other) \\
\hline C-14 & & $0.0000 E+00$ & $3.6710 \mathrm{E}-01$ & $\mathrm{nCi} / \mathrm{g}$ & $\mathrm{J}$ & \\
\hline Pu-239 & & $0.0000 E+00$ & $5.1400 E-02$ & $\mathrm{nCi} / \mathrm{g}$ & $J$ & \\
\hline$\overline{\mathrm{Na}-22}$ & & $0.0000 E+00$ & $1.2780 \mathrm{E}-01$ & $\mathrm{nCi} / \mathrm{g}$ & $J$ & \\
\hline Eu-154 & & $0.0000 \mathrm{E}+00$ & $1.4680 \mathrm{E}-01$ & $n \mathrm{Ci} / \mathrm{g}$ & $\mathrm{J}$ & \\
\hline Cs-137 & & $0.0000 E+00$ & $2.3906 E+02$ & $\mathrm{nCi} / \mathrm{g}$ & $J$ & \\
\hline Cd-109 & & $0.0000 E+00$ & $1.3000 \mathrm{E}-03$ & $n \mathrm{Ci} / \mathrm{g}$ & $J$ & \\
\hline Am-241 & & $0.0000 E+00$ & $1.4479 E+03$ & $n \mathrm{Ci} / \mathrm{g}$ & $\hat{\jmath}$ & \\
\hline U-dep & & $0.0000 E+00$ & 6.0000 E-04 & $\mathrm{nCi} / \mathrm{g}$ & $j$ & \\
\hline Sr-90 & & $0.0000 \mathrm{E}+00$ & $1.0012 E+03$ & $\mathrm{nCi} / \mathrm{g}$ & 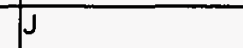 & \\
\hline Th-232 & & $0.0000 E+00$ & $1.5000 \mathrm{E}-03$ & $\mathrm{nCi} / \mathrm{g}$ & $j$ & \\
\hline $\mathrm{H}-3$ & & $0.0000 E+00$ & $9.9800 E+01$ & $\mathrm{nCi} / \mathrm{g}$ & $j$ & \\
\hline $\mathrm{U}-238$ & & $0.0000 E+00$ & $7.6280 \mathrm{E}+01$ & $\mathrm{nCi} / \mathrm{g}$ & 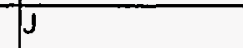 & \\
\hline \multicolumn{5}{|c|}{$\begin{array}{l}\text { A. Passive-Active Neutron (PAN) } \\
\text { B. Segmented Gamma Scan (SGS) } \\
\text { C. Passive-Active Neutron/Segmented Gamma Scan (PAN/SGS) } \\
\text { D. Radiochemical Analysis } \\
\text { E. Mass Spectrometry }\end{array}$} & \multicolumn{2}{|c|}{$\begin{array}{l}\text { F. Accountability } \\
\text { G. Calorimetry } \\
\text { H. Multiple Energy Gamma Analysis (MEGAS) } \\
\text { I. Process Knowledge } \\
\text { J. Other }\end{array}$} \\
\hline
\end{tabular}




\section{H. MATRIX CHARACTERISTICS}

Primary Matrix Code: $\mathbf{S 1 1 2}$

Description: Debris Waste: Inorganic Debris: Metal Debris: Metal Debris w/Pb

\section{Combustibility: $\bigcirc$ Combustible (>90\%) \\ Mixed (10\%-90\%) \\ O Noncombustible $(<10 \%)$}

\section{MATRIX COMPOSITION}

\begin{tabular}{|c|c|c|c|c|c|c|}
\hline & & & \multicolumn{4}{|c|}{ Composition } \\
\hline Code & Matrlx Description & Materlal & Typlcal & LL & UL & Units \\
\hline $\mathbf{S 5 1 1 2}$ & Debris Waste.Inorganic Debris.Metal Debris.Metal Debris w/Pb & & 100 & & & $\% \mathrm{vol}$ \\
\hline
\end{tabular}


LitWOOG

Liw Contact-H

J. HAZARDOUS CONSTITUENTS

\begin{tabular}{|c|c|c|c|c|c|c|c|c|}
\hline & , & & & Concen & tion & & & \\
\hline Code & Hazardous Waste Description & Treatment Subcategory & Typical & LL & UL & Units & Basis & Conc. Type \\
\hline D004 & Arsenic & & & 0 & 100 & $\mathrm{ppm}$ & $F$ & Total \\
\hline D007 & Chromium & Chromium (Total) & & of & 32000 & $\mathrm{ppm}$ & $F$ & Total \\
\hline 0008 & Lead & Radioactive lead solids & & 0 & 180000 & ppm & $F$ & Total \\
\hline$D 009$ & Mercury & High mercury $>=260 \mathrm{mg} / \mathrm{kg}$ total mercury & & & & & $F$ & Total \\
\hline D010 & Selenium & & & 0 & 100 & $\mathrm{ppm}$ & $F$ & Total \\
\hline P015 & Beryllium powder & & & 이 & 1200 & $\mathrm{ppm}$ & $\bar{B}$ & Total \\
\hline
\end{tabular}


K. POLYCHLORINATED BIPHENYLS (PCBS)

Is this waste stream PCB contaminated?

\section{OYes O No O Unknown}

If yes, what portion of the waste is PCB contaminated (volume \%):

If $<100 \%$, can the PCB contaminated waste be segregated and treated separately? OYes O No O Unknown

What is the PCB concentration in ppm?

If only a portion of the waste is PCB contaminated, report values for contaminated portion.

\section{TREATMENT PLANS}

\begin{tabular}{|c|c|c|c|c|c|c|c|c|c|c|c|}
\hline $\begin{array}{c}\text { Opt. } \\
\#\end{array}$ & $\begin{array}{c}\text { Media Type } \\
\text { (If more than one) }\end{array}$ & $\begin{array}{c}\text { Matrix } \\
\text { Code }\end{array}$ & $\begin{array}{c}\text { Vol. \% } \\
\text { IIf }\end{array}$ & Step & JIT & sc & ClF & $\begin{array}{l}\text { Trans. } \\
\text { Miles }\end{array}$ & $\begin{array}{l}\text { Facility } \\
\text { Abbr. }\end{array}$ & Unit Name & Comments \\
\hline \multirow[t]{6}{*}{1} & & S5110 & & a & O) & $\mathrm{O}$ & $1.00 E+00$ & & TRANS & Transport - LLW & \\
\hline & & & & $b$ & O & $\mathrm{O}$ & $1.00 E+00$ & & WROC & Hg Retort & \\
\hline & & & & c & 0 & 0 & $1.00 E+00$ & & AMWTF & $\mathrm{CH}$ - Sizing & \\
\hline & & & & c & D & O & $1.00 \mathrm{E}+00$ & & AMWTF & $\mathrm{CH}$ - Docontamination & \\
\hline & & & & e & O & $\mathrm{O}$ & $1.00 \mathrm{E}+00$ & & AMWTF & CH - Macroencapsulation/Stabilization & \\
\hline & & & & $f$ & 0 & $\bar{O}$ & $1.00 E+00$ & & SCDF & Disposal - Contact Handled & \\
\hline
\end{tabular}

Note: Where provided, media percentages are rough estimates used to facilitate treatment planning. They do not necessarily imply an accurate knowledge of waste stream composition, and were not derived for use outside this conceptual planning exercise.

Describe any special or unique technical concerns related to the treatment of this waste stream that would impact the use or implementation of standard hazardous waste treatment methods. 


LLWWOT
LLW
A. IDENTIFICATION AND DESCRIPTION
Waste Stream ID: LL-W007
W. S. Name:
ELEMENTAL LEAD
FFCA ID:

Waste Stream Description:

\section{Still-Ger Active \\ B. GENERATION SITE/PROCESS DESCRIPTION}

The following questions, concerning the generation site and process, should be answered even if the waste stream is no longer generated.

Name of Site Generating Waste (e.g., INEL, Rocky Flats, etc.):

\begin{tabular}{l}
\hline Location of activities (Area and Building): \\
\hline \\
Operations (functions) performed in building: \\
\hline
\end{tabular}

Description of Process Generating Waste: 
Is this waste stream still generated?

If yes, when is the expected generation termination date?

Current Projections

\begin{tabular}{|r|r|r|r|l|l|}
\hline Est. Date & \multicolumn{1}{|c|}{ Start Year } & \multicolumn{1}{|l|}{ Interval } & \multicolumn{1}{|c|}{ Vol $(\mathbf{m} 3)$} & Mass $(\mathrm{kg})$ & Source of Estimate \\
\hline $3 / 30 / 95$ & 1995 & 1 & 1.00 & & Generator Update \\
\hline $3 / 30 / 95$ & 1996 & 1 & 1.00 & & Generator Update \\
\hline $3 / 30 / 95$ & 1997 & 1 & 1.00 & & Generator Update \\
\hline $3 / 30 / 95$ & 1998 & 1 & 1.00 & & Generator Update \\
\hline $3 / 30 / 95$ & 1999 & 1 & 1.00 & & Generator Update \\
\hline
\end{tabular}

Historical Projections 


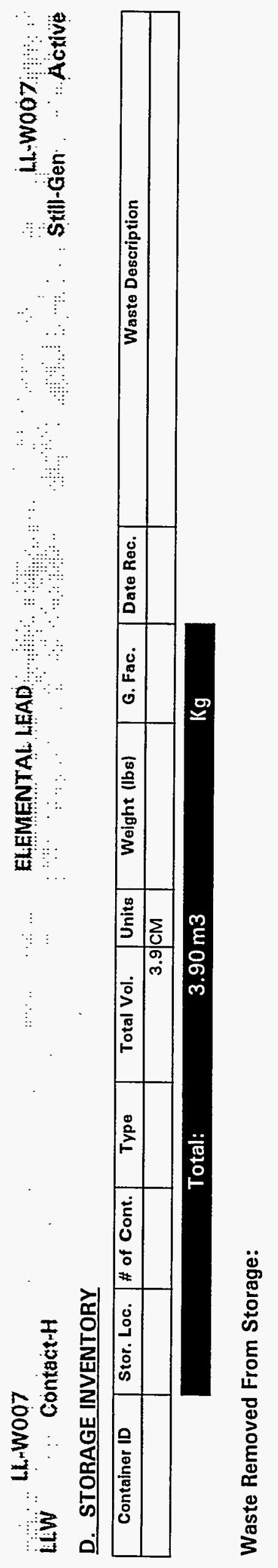




\section{$\therefore \quad$ LW-WOOT}

LlWW Contäct-H

\section{E. RADIATION CHARACTERISTICS}

\section{ELEMENTAL LEAD}

Radioactive Waste Type:

\begin{tabular}{|ll|}
\hline Ollw & Oa-LLW $\bigcirc$ TRU \\
OhLW & ONon-Rad \\
\hline
\end{tabular}

Mixed Waste:

Handling:

\begin{tabular}{|l|}
\hline OYes ONo \\
\hline O Contact ORemote \\
\hline
\end{tabular}

Radionuclide Contamination Accessiblity:

External Surface

Internal Surface

Dispersed Through Matrix

\begin{tabular}{|c|c|c|}
\hline OYes & $\mathrm{ONo}_{\mathrm{No}}$ & OUnknown \\
\hline OYes & ONo & O Unknown \\
\hline OYes & ONo & OUnknown \\
\hline
\end{tabular}

\section{Activity Levels}

Transuranic Alpha Activity:

Uranium/Thorium Alpha Activity:

Beta/Gamma Activity:

Surface Neutron Activity:

$\square$
$\square$
$\square$
$\square$
$\square$
$\square$

Units:

Units:

@ surface

@ 1-m Units: $\mathrm{mR} / \mathrm{hr}$

Total Activity:

Units:

$\mathrm{nCi} / \mathrm{g}$

Total Activity: $\mathrm{nCi} / \mathrm{g}$

\section{F. TRU ALPHA ACTIVITY DISTRIBUTION}

This waste stream is not identified as a TRU or a-LLW. 


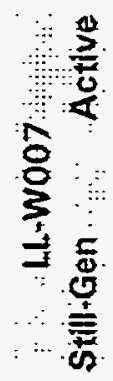

8
8
$\vdots$
+
8
8

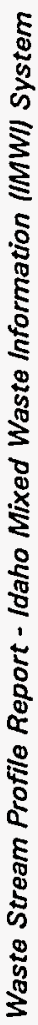

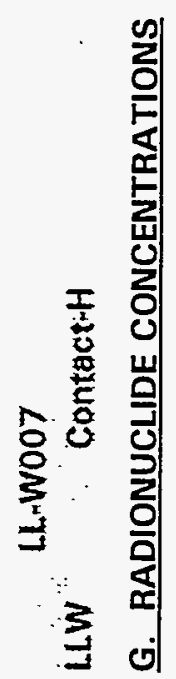


H. MATRIX CHARACTERISTICS

Primary Matrix Code: $X 7210$

Description: Special Waste: Elemental Hazardous Metals: Elemental Lead:

Combustibility: $\bigcirc$ Combustible (>90\%)

Mixed (10\%-90\%)

Noncombustible $(<10 \%)$

\section{MATRIX COMPOSITION}




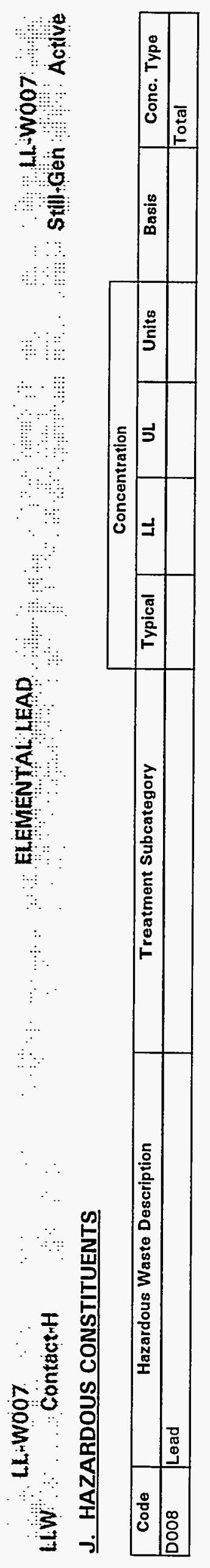

0
0
0
$\dot{8}$
0
8
0
0

ह⿱ 


\section{LLWWOOT \\ LW Contact H}

ELEMENTAL LEAD

Li-Wöoo7

K. POLYCHLORINATED BIPHENYLS (PCBS)

Is this waste stream PCB contaminated?

If yes, what portion of the waste is PCB contaminated (volume \%):

If $<100 \%$, can the PCB contaminated waste be segregated and treated separately?

What is the PCB concentration in ppm?

If only a portion of the waste is PCB contaminated, report values for contaminated portion.

\section{TREATMENT PLANS}

\begin{tabular}{|c|c|c|c|c|c|c|c|c|c|c|c|}
\hline $\begin{array}{c}\text { Opt. } \\
\#\end{array}$ & $\begin{array}{c}\text { Media Type } \\
\text { (If more than one) }\end{array}$ & $\begin{array}{c}\text { Matrix } \\
\text { Code }\end{array}$ & $\begin{array}{l}\text { Vol. \% } \\
\text { (If }\end{array}$ & Step & JIT & sc & CIF & $\begin{array}{l}\text { Trans. } \\
\text { Miles }\end{array}$ & $\begin{array}{l}\text { Facility } \\
\text { Abbr. }\end{array}$ & Unit Name & Comments \\
\hline \multirow[t]{6}{*}{1} & & $\times 7210$ & & $\mathbf{a}$ & O & 0 & $1.00 E+00$ & & TRANS & Transport - LLW & \\
\hline & & & & $\bar{b}$ & 0 & 0 & $1.00 \mathrm{E}+00$ & & AMWTF & $\mathrm{CH} \cdot$ Opening \& Sorting & \\
\hline & & & & c & 0 & 0 & $1.00 E+00$ & & AMWTF & $\mathrm{CH}$ - Decontamination & \\
\hline & & & & $d$ & 0 & 0 & $1.00 E+00$ & & AMWTF & $\mathrm{CH}$ - Sizing & \\
\hline & & & & $\theta$ & 0 & 0 & $1.00 E+00$ & & AMWTF & $\mathrm{CH}$ - Macroencapsulation/Stabilization & \\
\hline & & & & $f$ & 0 & 0 & $1.00 E+00$ & & SCDF & Disposal - Contact Handled & \\
\hline
\end{tabular}

Note: Where provided, media percentages are rough estimates used to facilitate treatment planning. They do not necessarily imply an accurate knowledge of waste stream composition, and were not derived for use outside this conceptual planning exercise.

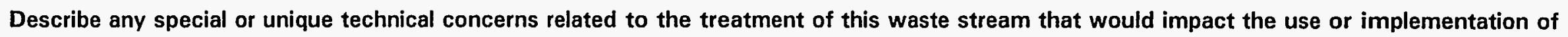
standard hazardous waste treatment methods. 


LLWWWI5
Contact-H
A. IDENTIFICATION AND DESCRIPTION
Waste Stream ID: LL-W015
W. S. Name:
Content Code: INORGANIC DEBRIS
FFCA ID:
Waste Stream Description:
LL-W015

\section{B. GENERATION SITE/PROCESS DESCRIPTION}

The following questions, concerning the generation site and process, should be answered even if the waste stream is no longer generated.

Name of Site Generating Waste (e.g., INEL, Rocky Flats, etc.):

Location of activities (Area and Building):

Operations (functions) performed in building:

Description of Process Generating Waste: 
LLW W P ContactiH

\section{PROJECTED WASTE GENERATION}

Is this waste stream still generated?

If yes, when is the expected generation termination date?

Current Projections

\begin{tabular}{|r|r|r|r|l|l|}
\hline Est. Date & Start Year & Interval & \multicolumn{1}{|l|}{ Vol (m3) } & Mass (kg) & Source of Estimate \\
\hline $3 / 30 / 95$ & 1995 & 1 & 3.00 & & Generator Update \\
\hline $3 / 30 / 95$ & 1996 & 1 & 3.00 & & Generator Update \\
\hline $3 / 30 / 95$ & 1997 & 1 & 3.00 & & Generator Update \\
\hline $3 / 30 / 95$ & 1998 & 1 & 3.00 & & Generator Update \\
\hline $3 / 30 / 95$ & 1999 & 1 & 3.00 & & Generator Update \\
\hline
\end{tabular}

Historical Projections 


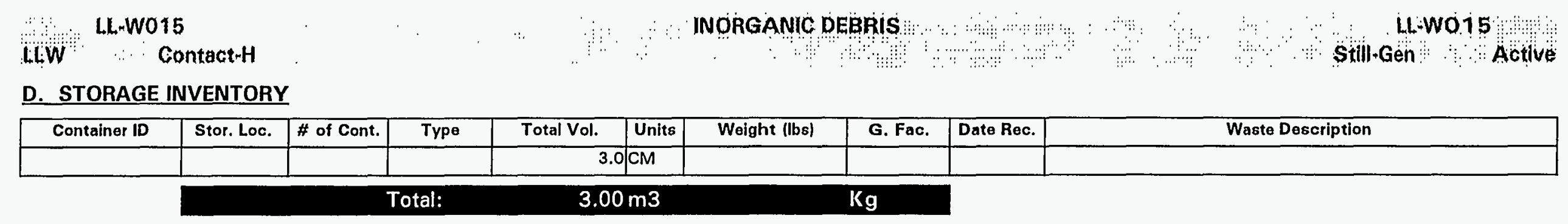

Waste Removed From Storage: 


\section{F. TRU ALPHA ACTIVITY DISTRIBUTION}

Radioactive Waste Type:

\begin{tabular}{|ll|}
\hline OlLW & Oa-LLW $\bigcirc$ TRU \\
O HLW & $\bigcirc$ Non-Rad
\end{tabular}

This waste stream is not identified as a TRU or a-LLW.

Mixed Waste:

Handling:

O Contact O Remote

Radionuclide Contamination Accessiblity:

External Surface

\begin{tabular}{|c|c|c|}
\hline OYes & ONo & OUnknown \\
\hline OYes & ONo & OUnknown \\
\hline OYes & ONo & O \\
\hline
\end{tabular}

Dispersed Through Matrix

\section{Activity Levels}

Transuranic Alpha Activity: Uranium/Thorium Alpha Activity:

Beta/Gamma Activity:

Surface Neutron Activity:

Total Activity:

\begin{tabular}{|c|c|c|}
\hline & Units: [ & \\
\hline & Units: [ & \\
\hline & @ surfe & face \\
\hline & @ 1-m & Units: $\mathrm{mR} / \mathrm{hr}$ \\
\hline & Units: [ & \\
\hline & $\mathrm{nCi} / \mathrm{g}$ & \\
\hline
\end{tabular}



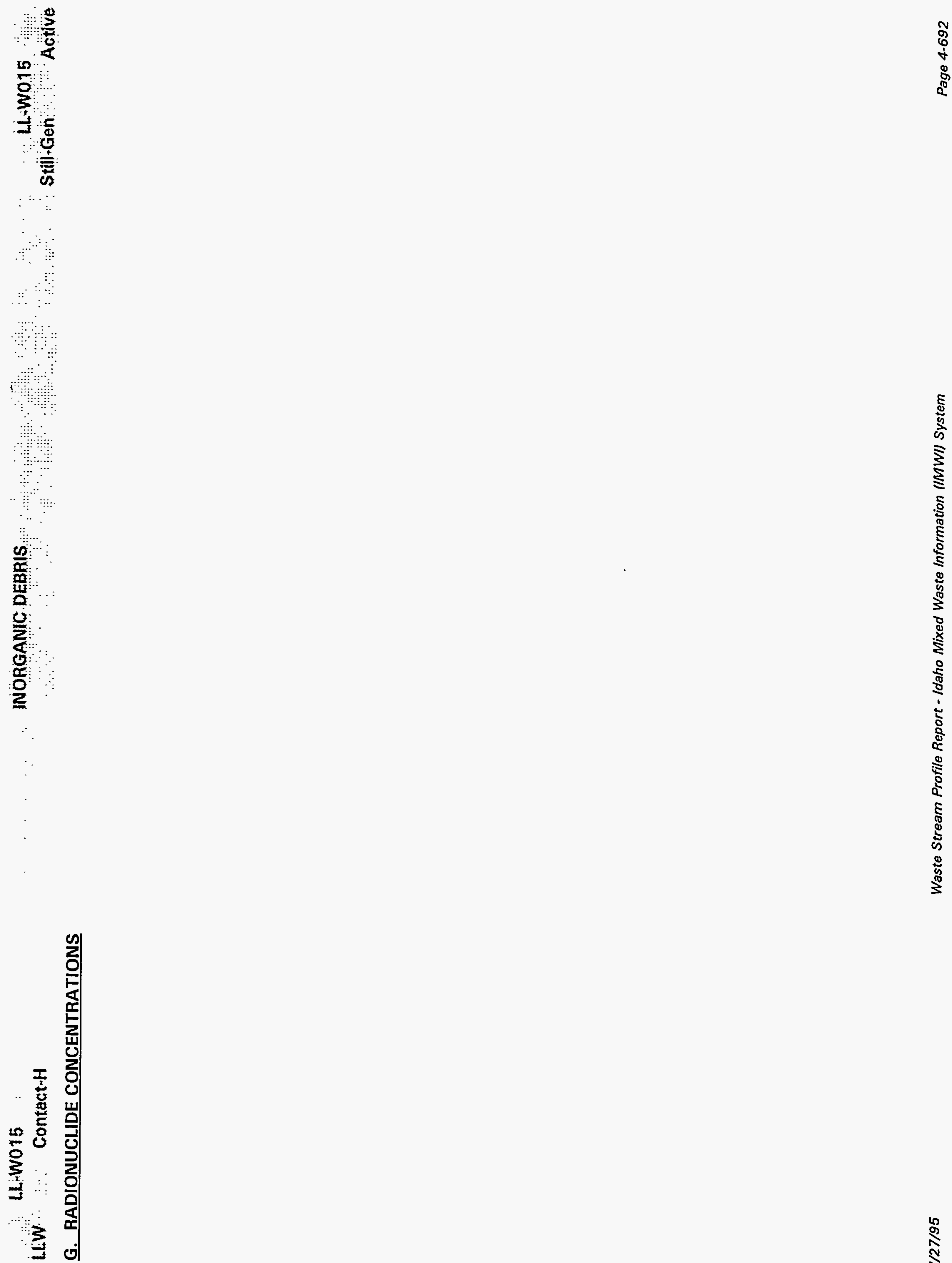
LWW LWWO15 ContaGt-H INORGANIC OEBRIS
H. MATRIX CHARACTERISTICS H. MATRIX CHARACTERISTICS

Primary Matrix Code: 55410

Description: Debris Waste: Heterogeneous Debris: Composite Filter Debris:

Combustibility: $\begin{aligned} & \bigcirc \text { Combustible (>90\%) } \\ & \bigcirc \text { Mixed }(10 \%-90 \%) \\ & \bigcirc \text { Noncombustible }(<10 \%)\end{aligned}$

\section{MATRIX COMPOSITION}




\section{LLWO15}

LiW Cöntäct-H

\section{J. HAZARDOUS CONSTITUENTS}

\begin{tabular}{|c|c|c|c|c|c|c|c|c|}
\hline & \multicolumn{4}{|c|}{ Concentration } & \multirow[b]{2}{*}{ Basis } & \multirow[b]{2}{*}{ Conc. Type } \\
\hline Code & Hazardous Waste Description & Treatment Subcategory & Typical & LL & UL & Units & & \\
\hline D004 & Arsenic & & & & & & & Total \\
\hline D005 & Barium & & & & & & & Total \\
\hline 0006 & Cadmium & & & & & & & Total \\
\hline D007 & Chromium & Chromium (Total) & & & & & & Total \\
\hline D008 & Lead & & & & & & & Total \\
\hline 0009 & Mercury & Elemental mercury contaminated with radioact & . & & & & & Total \\
\hline D011 & Silver & & & & & & & Total \\
\hline D015 & Toxaphene & & & & & & & Total \\
\hline$\overline{D 018}$ & Benzene & Benzene managed in non-CWA/non-CWA-equi & & & & & & Total \\
\hline$\overline{D 028}$ & 1,2-Dichloroethane & 1,2-Dichloroethane managed in non-CWA/non- & & & & & & Total \\
\hline 0039 & Tetrachloroethylene & Tetrachloroethylene managed in non-CWA/non & & & & & & Total \\
\hline$\overline{0040}$ & Trichloroethylene & Trichloroethylene managed in non-CWA/non-C & & & & & & Total \\
\hline F005 & Spent non-halogenated solvents & Toluene & & & & & & Total \\
\hline
\end{tabular}




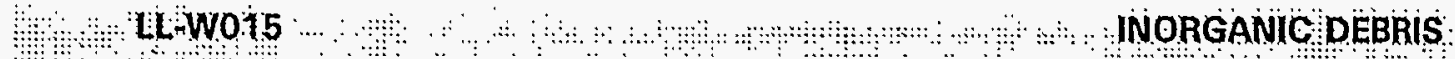

\section{HLW Contact-H}

\section{K. POLYCHLORINATED BIPHENYLS (PCBS)}

Is this waste stream PCB contaminated? OYes O No $O$ Unknown

If yes, what portion of the waste is PCB contaminated (volume \%):

If $<100 \%$, can the PCB contaminated waste be segregated and treated separately? $O$ Yes $O$ No O Unknown

What is the PCB concentration in ppm? If only a portion of the waste is PCB contaminated, report values for contaminated portion.

\section{TREATMENT PLANS}

\begin{tabular}{|c|c|c|c|c|c|c|c|c|c|c|c|}
\hline $\begin{array}{c}\text { Opt. } \\
\#\end{array}$ & $\begin{array}{c}\text { Media Type } \\
\text { (If more than one) }\end{array}$ & $\begin{array}{c}\text { Matrix } \\
\text { Code }\end{array}$ & $\begin{array}{l}\text { Vol. \% } \\
\text { (If }\end{array}$ & Step & JIT & sc & CIF & $\begin{array}{l}\text { Trans. } \\
\text { Miles }\end{array}$ & $\begin{array}{l}\text { Facility } \\
\text { Abbr. }\end{array}$ & Unit Name & Comments \\
\hline 1 & & 55410 & & $\bar{a}$ & O & 0 & $1.00 \mathrm{E}+00$ & & TRANS & Transport - LLW & \\
\hline & & & & b & $O$ & 0 & $1.00 E+00$ & & MWSF & Open/Segregate/Repack (nonhalogenated solids) & \\
\hline & & & & d & O & $\mathrm{O}$ & $1.00 E+00$ & & WERF & Stabillzation - Portland Cement & \\
\hline & & & & $\theta$ & O & 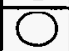 & $1.00 E+00$ & & SCDF & Disposal - Contact Handled & \\
\hline
\end{tabular}

Note: Where provided, media percentages are rough estimates used to facilitate treatment planning. They do not necessarily imply an accurate knowledge of waste stream composition, and were not derived for use outside this conceptual planning exercise.

Describe any special or unique technical concerns related to the treatment of this waste stream that would impact the use or implementation of standard hazardous waste treatment methods. 


LiW: LL-W017
A. IDENTIFICATION AND DESCRIPTION
Waste Stream ID:
W. S. Name:
Content Code:
FFCA ID:
WOW LEVEL MIXED INORGANIC TRASH-3
$\begin{aligned} & \text { Waste Stream Description: } \\ & \text { Low level radioactive inorganic trash generated by on-site research and laboratory clean-up } \\ & \text { activities. This waste includes a mixture of the following: pipettes, funnels, beakers, gloves, } \\ & \text { paper, filters, plastics, sponges, floor dry, and other laboratory trash. These may be } \\ & \text { contaminated with: beryllium, lead, mercury, and/or low level radioactive materials. Waste } \\ & \text { stream generated from changing research and development activities on site at LLNL. Wastes } \\ & \text { consists of dry solid trash. }\end{aligned}$

\section{A. IDENT}

Content Code:

FFCA ID:

*

LL-W017

Low level radioactive inorganic trash generated by on-site research and laboratory clean-up activities. This waste includes a mixture of the following: pipettes, funnels, beakers, gloves,

consists of dry solid trash.

\section{B. GENERATION SITE/PROCESS DESCRIPTION}

The following questions, concerning the generation site and process, should be answered even if the waste stream is no longer generated.

Name of Site Generating Waste (e.g., INEL, Rocky Flats, etc.): LLNL

Location of activities (Area and Building):

Sitewide. Buildings include but are not limited to $377,361,292$,

$151,331,366,2312,226$.

Operations (functions) performed in building:

Site wide R\&D and characterization activities.

Description of Process Generating Waste:

Generated from changing research and laboratory clean-up activities. 
un LL-W01:

HWW Contagt

C. PROJECTED WASTE GENERATION

Is this waste stream still generated?

If yes, when is the expected generation termination date?

Current Projections

\begin{tabular}{|r|r|r|r|r|r|}
\hline Est. Date & \multicolumn{1}{|c|}{ Start Year } & Interval & Vol (m3) & Mass (kg) & Source of Estimate \\
\hline $3 / 30 / 95$ & 1995 & 5 & 50.00 & & Generator Update \\
\hline
\end{tabular}

\section{Historical Projections}

\begin{tabular}{|c|c|c|c|c|c|}
\hline Est. Date & Start Year & Interval & Vol (m3) & Mass (kg) & Source of Estimate \\
\hline $11 / 30 / 93$ & 1993 & 1 & 25.00 & $12,251.00$ & 1993-FFCA \\
\hline 11/30/93 & 1994 & 1 & 25.00 & $12,251.00$ & 1993-FFCA \\
\hline $11 / 30 / 93$ & 1995 & 1 & 25.00 & $12,251.00$ & 1993-FFCA \\
\hline $11 / 30 / 93$ & 1996 & 1 & 25.00 & $12,251,00$ & 1993-FFCA \\
\hline $11 / 30 / 93$ & 1997 & 1 & 25.00 & $12,251.00$ & 1993-FFCA \\
\hline $11 / 30 / 93$ & 1998 & 1 & 25.00 & $12,251.00$ & 1993-FFCA \\
\hline $11 / 30 / 93$ & 1999 & 1 & 25.00 & $12,251.00$ & 1993-FFCA \\
\hline $11 / 30 / 93$ & 2000 & 1 & 25.00 & $12,251.00$ & 1993-FFCA \\
\hline $11 / 30 / 93$ & 2001 & 1 & 25.00 & $12,251.00$ & 1993-FFCA \\
\hline $11 / 30 / 93$ & 2002 & 1 & 25.00 & $12,251.00$ & 1993-FFCA \\
\hline $11 / 30 / 93$ & 2003 & 20 & 475.00 & $232,770,00$ & 1993-FFCA \\
\hline
\end{tabular}


LikW017

HWW ContactrH

D. STORAGE INVENTORY

Container ID

\# of Cont

Total:

LOW LEVEL MIXED INQRGANIC TRASH 3

LLFO17:

Still-Gern Active

\begin{tabular}{|l|l}
\hline Container ID & Stor. Loc. \\
\hline
\end{tabular}

Total Vol.

\begin{tabular}{|l|l|l|}
\hline Units & Weight (lbs) \\
\hline 0.7 & $\mathrm{CM}$ & \\
\hline
\end{tabular}

G. Fac. Date Rec.

.

\section{$50.70 \mathrm{~m} 3$}

$\mathrm{Kg}$

Waste Removed From Storage: 
E. RADIATION CHARACTERISTICS

Radioactive Waste Type:

\begin{tabular}{|ll|}
\hline OLLW & Oa-LLW O TRU \\
OHLW & O Non-Rad \\
\hline
\end{tabular}

F. TRU ALPHA ACTIVITY DISTRIBUTION

This waste stream is not identified as a TRU or a-LLW.

Mixed Waste:

Handling:

\begin{tabular}{|l|}
\hline OYes O No \\
\hline O Contact O Remote \\
\hline
\end{tabular}

Radionuclide Contamination Accessiblity:

\begin{tabular}{rllll|}
\cline { 2 - 4 } External Surface & OYes & O No & O Unknown \\
\cline { 2 - 4 } Internal Surface & OYes & O No & O Unknown \\
\cline { 2 - 5 } & &
\end{tabular}

Dispersed Through Matrix

\section{Activity Levels}

Transuranic Alpha Activity:

Uranium/Thorium Alpha Activity:

Beta/Gamma Activity:

Surface Neutron Activity:

$\square$
Units:

Units:

\begin{tabular}{l}
\hline 1.0 @ surface \\
$\square$ @ 1-m Units: mR/hr \\
\hline Units: $\square$
\end{tabular}

Total Activity: $6690.05 \mathrm{nCi} / \mathrm{g}$ 


\section{LL-WO17 \\ LiW" Contact-H \\ G. RADIONUCLIDE CONCENTRATIONS}

LOW LEVEL MIXED INORGANIC TRASH 3

Still-Gen H.W17

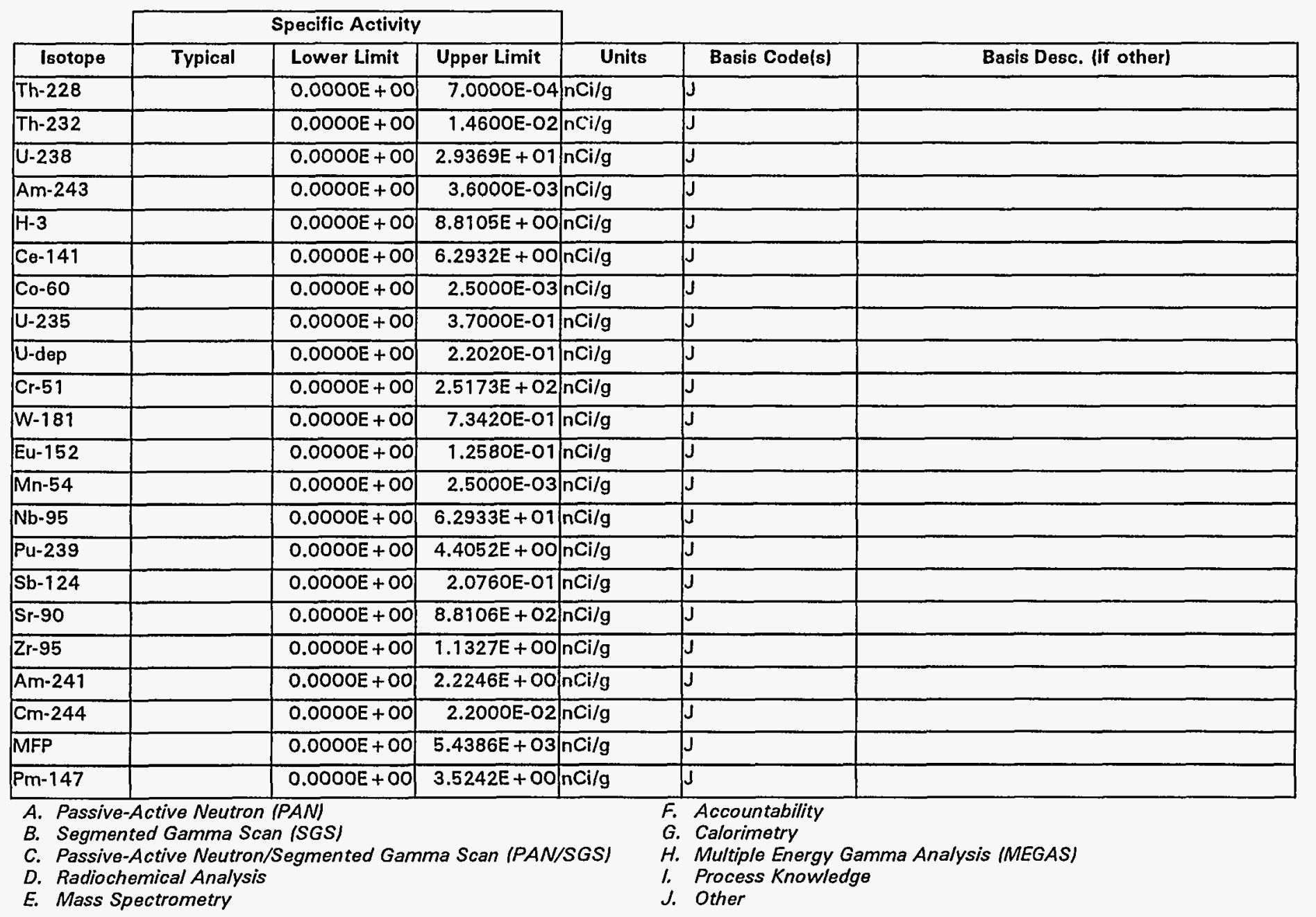




\section{LINWO17}

LlW Contact

LOW LEVEL MIXED INORGANIC TRASH

Li-WOTl

H. MATRIX CHARACTERISTICS

Primary Matrix Code: 55400

Description: Debris Waste: Heterogeneous Debris: :

Combustibility: $\bigcirc$ Combustible (>90\%)

Mixed (10\%-90\%)

Noncombustible $(<10 \%)$

\section{MATRIX COMPOSITION}

\begin{tabular}{|c|c|c|c|c|c|c|}
\hline \multirow[b]{2}{*}{ Code } & \multirow[b]{2}{*}{ Matrix Description } & \multirow[b]{2}{*}{ Materlal } & \multicolumn{4}{|c|}{ Composition } \\
\hline & & & Typlcal & LL & UL & Units \\
\hline$\$ 4100$ & Soil/Gravel.Soil.. & Soil & 10 & 0 & & $\%$ vol \\
\hline$\$ 5122$ & Debris Waste.Inorganic Debris.Inorganic Non-Metal Debris.Glass Debris & Glass & 10 & 0 & & $\%$ vol \\
\hline $\mathbf{5 5 3 2 0}$ & Debris Waste.Organic Debris.Wood Debris. & Paper wipes/Clothing & 20 & 0 & & $\%$ vol \\
\hline $\mathbf{5 5 3 1 0}$ & Debris Waste.Organic Debris.Plastic/Rubber Debris. & Plastic containers & 10 & 0 & & $\%$ vol \\
\hline$\$ 55400$ & Debris Waste.Heterogeneous Debris.. & & 50 & 0 & 100 & $\%$ vol \\
\hline
\end{tabular}




\begin{tabular}{|c|c|c|c|c|c|c|c|c|c|}
\hline \multicolumn{2}{|c|}{$\therefore \quad$ LLWO17 } & \multicolumn{3}{|c|}{ LOW LEVEL MIXED INORGANIC TRASH 3} & $\therefore$ & \multirow{2}{*}{\multicolumn{2}{|c|}{ ": }} & \\
\hline Liw & $\therefore \quad$ Contact-H & $\begin{array}{llll}\cdots & \vdots & \cdots\end{array}$ & $\because \vdots$ & : & & & & \multicolumn{2}{|c|}{$\begin{array}{l}\text { Sh-WO17 } \\
\text { Still-Gen }\end{array}$} \\
\hline \multicolumn{10}{|c|}{ J. HAZARDOUS CONSTITUENTS } \\
\hline & & & \multicolumn{4}{|c|}{ Concentration } & & & \\
\hline Code & Hazardous Waste Description & Treatment Subcategory & Typical & LL & UL & Units & & Basis & Conc. Type \\
\hline$\overline{D 004}$ & Arsenic & & & 0 & 100 & $\mathrm{pm}$ & $F$ & & Total \\
\hline$\overline{D 005}$ & Barium & & & 0 & & $\mathrm{pm}$ & $\mathrm{F}$ & & Total \\
\hline$\overline{\mathrm{DO06}}$ & Cadmium & & & 0 & & $\overline{p m}$ & $F$ & & Total \\
\hline$\overline{0007}$ & Chromium & Chromium (Total) & & 0 & $\overline{310}$ & $\overline{\mathrm{pm}}$ & $F$ & & Total \\
\hline$\overline{D 008}$ & Lead & & & 0 & 130 & $\mathrm{spm}$ & $F$ & & Total \\
\hline$\overline{D 009}$ & Mercury & Elemental mercury contaminated with radioact & & 0 & 500 & $\mathrm{spm}$ & $F$ & & Total \\
\hline D010 & Selenium & & & 0 & 100 & $\mathrm{pm}$ & $F$ & & Total \\
\hline$\overline{D 011}$ & Silver & & & 0 & & $\overline{p m}$ & $F$ & & Total \\
\hline F005 & Spent non-halogenated solvents & Toluene & & & & & $B$ & & Total \\
\hline$\overline{P 015}$ & Beryllium powder & & & & & & $\bar{B}$ & & Total \\
\hline
\end{tabular}




\section{$\therefore \quad$ Liwo17}

LLW Contagt-H

\section{K. POLYCHLORINATED BIPHENYLS (PCBS)}

Is this waste stream PCB contaminated?

\section{OYes}

O No

\section{Unknown}

If yes, what portion of the waste is PCB contaminated (volume \%):

If $<100 \%$, can the PCB contaminated waste be segregated and treated separately?

What is the PCB concentration in ppm?

If only a portion of the waste is PCB contaminated, report values for contaminated portion.

\section{TREATMENT PLANS}

\begin{tabular}{|c|c|c|c|c|c|c|c|c|c|c|c|}
\hline $\begin{array}{c}\text { Opt. } \\
\#\end{array}$ & $\begin{array}{c}\text { Media Type } \\
\text { (If more than one) }\end{array}$ & $\begin{array}{c}\text { Matrix } \\
\text { Code }\end{array}$ & $\begin{array}{l}\text { Vol. } \% \\
\text { (If }\end{array}$ & Step & JIT & sc & CIF & $\begin{array}{l}\text { Trans. } \\
\text { Miles }\end{array}$ & $\begin{array}{l}\text { Facility } \\
\text { Abbr. }\end{array}$ & Unit Name & Comments \\
\hline \multirow[t]{6}{*}{1} & & 55440 & & $a$ & 0 & $\mathrm{O}$ & $1.00 E+00$ & & TRANS & Transport - LLW & \\
\hline & & & & $\mathbf{b}$ & $\overline{0}$ & $\bar{O}$ & $1.00 E+00$ & & AMWTF & $\mathrm{CH}$ - Opening \& Sorting & \\
\hline & & & & c & 0 & 0 & $1.00 E+00$ & & AMWTF & $\mathrm{CH}$ - Sizing & \\
\hline & & & & d & O & 0 & $1.00 E+00$ & & AMWTF & $\mathrm{CH}$ - Incineration/Thermal Desorption & \\
\hline & & & & e & 0 & 0 & $1.00 E+00$ & & AMWTF & $\mathrm{CH}$ - Vitrification & \\
\hline & & & & $f$ & 0 & 0 & $1.00 E+00$ & & SCDF & Disposal - Contact Handled & \\
\hline
\end{tabular}

Note: Where provided, media percentages are rough estimates used to facilitate treatment planning. They do not necessarily imply an accurate knowledge of waste stream composition, and were not derived for use outside this conceptual planning exercise.

Describe any special or unique technical concerns related to the treatment of this waste stream that would impact the use or implementation of standard hazardous waste treatment methods. 


LLW
A. IDENTIFICATION AND DESCRIPTION
Waste Stream ID: LL-W021
W. S. Name:
Content Code:
FFCA ID:
Waste Stream Description:
$\begin{aligned} & \text { Waste is lab packed waste contaminated with TC metals. Waste is one or more small containers } \\ & \text { of free liquids or solids surrounded by solid materials (virgin or waste materials) within a larger } \\ & \text { container, includes scintillation fluids packaged with vials. }\end{aligned}$

\section{MTALS \\ B. GENERATION SITE/PROCESS DESCRIPTION}

LiLiWO21

The following questions, concerning the generation site and process, should be answered even if the waste stream is no longer generated.

Name of Site Generating Waste (e.g., INEL, Rocky Flats, etc.): LLNL

Location of activities (Area and Building):

Sitewide. Buildings include but are not limited to 377, 361, 292, $151,331,366,2312,226$.

Operations (functions) performed in building:

Site wide R\&D and characterization activities.

Description of Process Generating Waste:

Generated from CMID and on-site research and development activities.

(


ALWWO21

Hew Contact

C. PROJECTED WASTE GENERATION

Is this waste stream still generated?

If yes, when is the expected generation termination date?

Current Projections

\begin{tabular}{|r|r|r|r|r|l|}
\hline Est. Date & \multicolumn{1}{|c|}{ Start Year } & \multicolumn{1}{|c|}{ Interval } & \multicolumn{1}{|c|}{ Vol (m3) } & Mass (kg) & Source of Estlmate \\
\hline $11 / 30 / 93$ & 1993 & 1 & 0.30 & 212.00 & $1993-F F C A$ \\
\hline $11 / 30 / 93$ & 1994 & 1 & 0.30 & 212.00 & $1993-F F C A$ \\
\hline $11 / 30 / 93$ & 1995 & 1 & 0.30 & 212.00 & $1993-F F C A$ \\
\hline $11 / 30 / 93$ & 1996 & 1 & 0.30 & 212.00 & $1993-F F C A$ \\
\hline $11 / 30 / 93$ & 1997 & 1 & 0.30 & 212.00 & $1993-F F C A$ \\
\hline $11 / 30 / 93$ & 1998 & 1 & 0.30 & 212.00 & $1993-F F C A$ \\
\hline $11 / 30 / 93$ & 1999 & 1 & 0.30 & 212.00 & $1993-F F C A$ \\
\hline
\end{tabular}

Historical Projections

\begin{tabular}{|r|r|r|r|r|l|}
\hline Est. Date & \multicolumn{1}{|c|}{ Start Year } & \multicolumn{1}{|l|}{ Interval } & \multicolumn{1}{|c|}{ Vol (m3) } & \multicolumn{1}{c|}{ Mass (kg) } & \multicolumn{1}{c|}{ Source of Estimate } \\
\hline $11 / 30 / 93$ & 2000 & 1 & 0.30 & 212.00 & $1993-\mathrm{FFCA}$ \\
\hline $11 / 30 / 93$ & 2001 & 1 & 0.30 & 212.00 & $1993-\mathrm{FFCA}$ \\
\hline $11 / 30 / 93$ & 2002 & 1 & 0.30 & 212.00 & $1993-\mathrm{FFCA}$ \\
\hline $11 / 30 / 93$ & 2003 & 20 & 5.70 & $4,028.00$ & $1993-\mathrm{FFCA}$ \\
\hline
\end{tabular}

OYes ONo

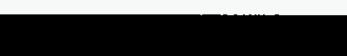

$$
\text { . }
$$

Li-WOZZ1

Still-Gen Active

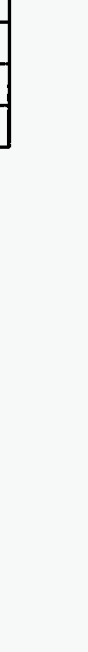

$$
\text { to }
$$$$
\text { . }
$$ 


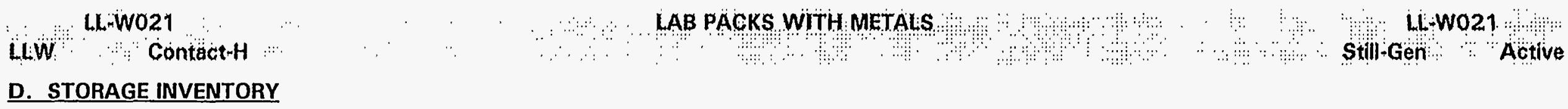

D. STORAGE INVENTORY

\begin{tabular}{|c|c|c|c|c|c|c|c|c|c|}
\hline Container ID & Stor. Loc. & \# of Cont. & Type & Total Vol. & Units & Weight (lbs) & G. Fac. & Date Rec. & Waste Description \\
\hline & & & & & $\overline{C M}$ & & & & \\
\hline
\end{tabular}

Waste Removed From Storage: 
LLW Contátith

\section{E. RADIATION CHARACTERISTICS}

Radioactive Waste Type:

\begin{tabular}{|ll|}
\hline OlLW & Oa-LLW $\bigcirc$ TRU \\
OhLW & O Non-Rad \\
\hline
\end{tabular}

Mixed Waste:

Handling:

Radionuclide Contamination Accessiblity:

External Surface OYes O No OUnknown

Internal Surface OYes O No OUnknown

Dispersed Through Matrix

OYes ONo OUnknown

\section{Activity Levels}

Transuranic Alpha Activity: Uranium/Thorium Alpha Activity:

Beta/Gamma Activity:

Surface Neutron Activity:

Total Activity:

\begin{tabular}{l}
$\square$ Units: $\square$ \\
\hline Units: $\square$ \\
\hline
\end{tabular}

\section{F. TRU ALPHA ACTIVITY DISTRIBUTION}

This waste stream is not identified as a TRU or a-LLW. 


\section{$\therefore \quad$ LLWWO21}

Contact-H

LAB PACKS WITH METALS

LLWWOZ2!

\section{G. RADIONUCLIDE CONCENTRATIONS}

\section{Specific Activity}

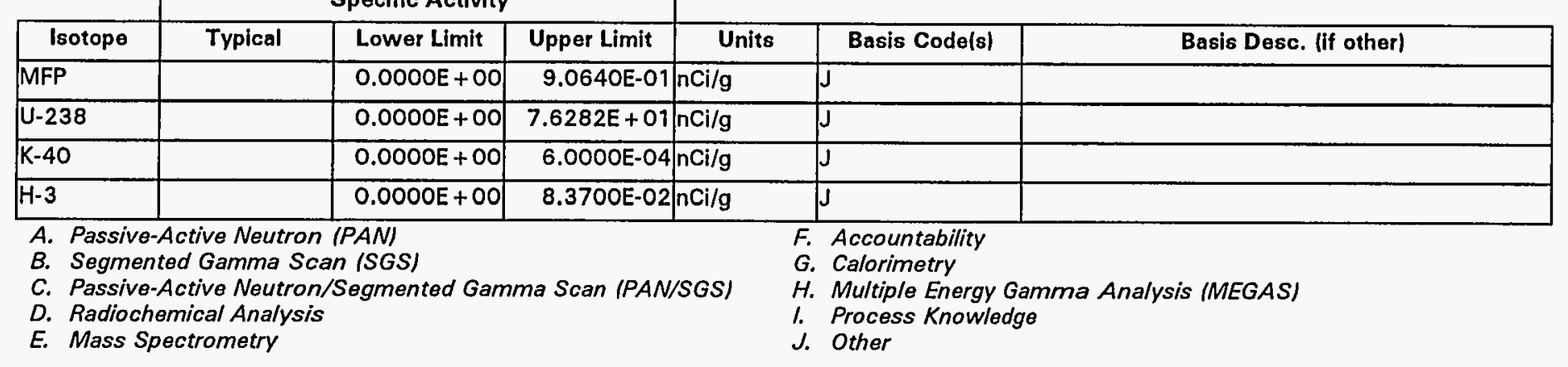


H. MATRIX CHARACTERISTICS

Primary Matrix Code: X6000

Description:Lab Packs: : :

Combustibility: $\bigcirc$ Combustible (>90\%)

Mixed $(10 \%-90 \%)$

Noncombustible (<10\%)

\section{MATRIX COMPOSITION}

\begin{tabular}{|c|c|c|c|c|c|c|}
\hline & & & \multicolumn{4}{|c|}{ Compositton } \\
\hline Code & Matrlx Description & Material & Typlcal & LL & UL & Units \\
\hline X6000 & Lab Packs... & Lab packs with metals & 100 & & & $\%$ vol \\
\hline
\end{tabular}




\section{LL.WO21}

L.W

Contact-H

J. HAZARDOUS CONSTITUENTS

\begin{tabular}{|c|c|c|c|c|c|c|c|c|}
\hline & & & \multicolumn{4}{|c|}{ Concentration } & \multirow[b]{2}{*}{ Basis } & \multirow[b]{2}{*}{ Conc. Type } \\
\hline Code & Hazardous Waste Description & Treatment Subcategory & Typical & $\mathbf{L L}$ & UL & Units & & \\
\hline$\overline{D 002}$ & Corrosive & Corrosive characteristic wastes that are mana & & & & & $F$ & Total \\
\hline$D 009$ & Mercury & Elemental mercury contaminated with radioact & & & & $\mathrm{g} / \mathrm{kg}$ & $F$ & Total \\
\hline
\end{tabular}

\section{LAB PACKS WITH METALS}

LLWWOZ21 till:Gen Active 
K. POLYCHLORINATED BIPHENYLS (PCBS)

Is this waste stream PCB contaminated? OYes $O$ No $O$ Unknown

If yes, what portion of the waste is PCB contaminated (volume \%):

If $<100 \%$, can the PCB contaminated waste be segregated and treated separately?

OYes ONo OUnknown

What is the PCB concentration in ppm?

If only a portion of the waste is PCB contaminated, report values for contaminated portion.

\section{TREATMENT PLANS}

\begin{tabular}{|c|c|c|c|c|c|c|c|c|c|c|c|}
\hline $\begin{array}{l}\text { Opt. } \\
\#\end{array}$ & $\begin{array}{c}\text { Media Type } \\
\text { (If more than one) }\end{array}$ & $\begin{array}{c}\text { Matrix } \\
\text { Code }\end{array}$ & $\begin{array}{l}\text { Vol. \% } \\
\text { IIf }\end{array}$ & Step & JIT & SC & CIF & $\begin{array}{l}\text { Trans. } \\
\text { Miles }\end{array}$ & $\begin{array}{l}\text { Facility } \\
\text { Abbr. }\end{array}$ & Unit Name & Comments \\
\hline \multirow[t]{6}{*}{1} & & $\times 6000$ & & $\mathbf{a}$ & 0 & $\overline{0}$ & $1.00 \mathrm{E}+00$ & & TRANS & Transport-LLW & \\
\hline & & & & $\bar{b}$ & 0 & 0 & $1.00 E+00$ & & AMWTF & $\mathrm{CH}$ - Opening \& Sorting & \\
\hline & & & & c & O & 0 & $1.00 E+00$ & & AMWTF & $\mathrm{CH}$ - Sizing & \\
\hline & & & & $\bar{d}$ & 0 & 0 & $1.00 E+00$ & & AMWTF & $\mathrm{CH}$ - Incineration/Thermal Desorption & \\
\hline & & & & e & 0 & 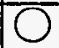 & $1.00 E+00$ & & AMWTF & $\mathrm{CH}$ - Vitrification & \\
\hline & & & & $f$ & 0 & $\overline{0}$ & $1.00 E+00$ & & SCDF & Disposal - Contact Handled & \\
\hline
\end{tabular}

Note: Where provided, media percentages are rough estimates used to facilitate treatment planning. They do not necessarily imply an accurate knowledge of waste stream composition, and were not derived for use outside this conceptual planning exercise.

Describe any special or unique technical concerns related to the treatment of this waste stream that would impact the use or implementation of standard hazardous waste treatment methods. 


LiWWOW24
A. IDENTIFICATION AND DESCRIPTION
Waste Stream ID: LL-W024
W. S. Name:
Content Code:
FFCA ID:

Waste Stream Description:

This waste contains liquid mercury contained in flasks and small containers resulting from equipment maintenance.

\section{WASTE}

\section{LiL-Wo24}

Still-Gen Active

\section{B. GENERATION SITE/PROCESS DESCRIPTION}

The following questions, concerning the generation site and process, should be answered even if the waste stream is no longer generated.

Name of Site Generating Waste (e.g., INEL, Rocky Flats, etc.): LLNL

Location of activities (Area and Building):

Sitewide. Buildings include but are not limited to 377, 361, 292, $151,331,366,2312,266$.

Operations (functions) performed in building: Site wide $R \& D$ and charaterization activities.

Description of Process Generating Waste:

Waste resulting from equipment maintenance. 
LiW Contägt H

O

\section{PROJECTED WASTE GENERATION}

Is this waste stream still generated?

O Yes ONo

If yes, when is the expected generation termination date?

Current Projections

\begin{tabular}{|r|r|r|r|r|l|}
\hline Est. Date & \multicolumn{1}{|c|}{ Start Year } & \multicolumn{1}{l|}{ Interval } & \multicolumn{1}{c|}{ Vol (m3) } & \multicolumn{1}{c|}{ Mass (kg) } & Source of Estimate \\
\hline $11 / 30 / 93$ & 1993 & 1 & 0.01 & 9.00 & $1993-F F C A$ \\
\hline $11 / 30 / 93$ & 1994 & 1 & 0.01 & 9.00 & $1993-F F C A$ \\
\hline $11 / 30 / 93$ & 1995 & 1 & 0.01 & 9.00 & $1993-F F C A$ \\
\hline $11 / 30 / 93$ & 1996 & 1 & 0.01 & 9.00 & $1993-F F C A$ \\
\hline $11 / 30 / 93$ & 1997 & 1 & 0.01 & 9.00 & $1993-F F C A$ \\
\hline $11 / 30 / 93$ & 1998 & 1 & 0.01 & 9.00 & $1993-F F C A$ \\
\hline $11 / 30 / 93$ & 1999 & 1 & 0.01 & 9.00 & $1993-F F C A$ \\
\hline \multicolumn{7}{|c|}{ Historical Projections } \\
\hline Est. Date & Start Year & Interval & Vol (m3) & Mass (kg) & Source of Estimate \\
\hline $11 / 30 / 93$ & 1998 & 5 & 0.04 & 36.00 & $1993-F F C A$ \\
\hline $11 / 30 / 93$ & 2003 & 20 & 0.00 & 171.00 & $1993-F F C A$ \\
\hline
\end{tabular}




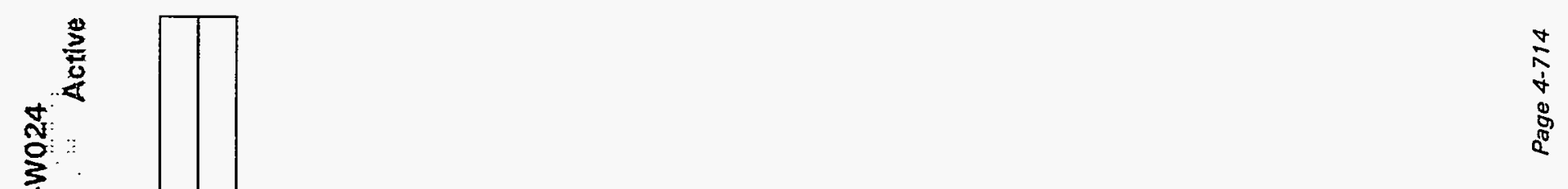



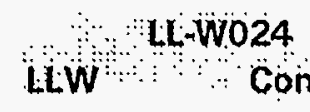

Radioactive Waste Type:

\begin{tabular}{|ll|}
\hline OLLW & Oa-LLW $\bigcirc$ TRU \\
OhLW & $\bigcirc$ Non-Rad \\
\hline
\end{tabular}

This waste stream is not identified as a TRU or a-LLW.

Mixed Waste:

\begin{tabular}{|l|}
\hline O Yes O No \\
\hline O Contact O Remote \\
\hline
\end{tabular}

Radionuclide Contamination Accessiblity:

\begin{tabular}{rlrl|}
\cline { 2 - 4 } External Surface & OYes & O No & O Unknown \\
\cline { 3 - 5 } Internal Surface & OYes & O No & O Unknown \\
\cline { 2 - 5 } Dispersed Through Matrix & O Yes & O No & O Unknown
\end{tabular}

\section{Activity Levels}

Transuranic Alpha Activity:

Uranium/Thorium Alpha Activity:

Beta/Gamma Activity:

\begin{tabular}{l}
$\square$ Units: $\square$ \\
\hline Units: \\
\hline
\end{tabular}

(a)

Surface Neutron Activity: 1.0 @ surface

1.0 @ 1-m Units: $\mathrm{mR} / \mathrm{hr}$

Total Activity: Units: $\mathrm{nCi} / \mathrm{g}$ 


\section{$\therefore$ LLWWO24}

\section{HiW: Contact-H}

\section{G. RADIONUCLIDE CONCENTRATIONS}

\begin{tabular}{|c|c|c|c|c|c|c|}
\hline \multirow{2}{*}{ Isotope } & \multicolumn{3}{|c|}{ Specific Activity } & \multirow[b]{2}{*}{ Units } & \multirow[b]{2}{*}{ Basis Code(s) } & \multirow[b]{2}{*}{ Basis Desc. (if other) } \\
\hline & Typical & Lower Limit & Upper Limit & & & \\
\hline $\mathrm{U}-238$ & & & & & J & \\
\hline $\mathrm{Nb}-95$ & & & & & j & \\
\hline$K-40$ & & & & & $J$ & \\
\hline$A m-241$ & & & & & J & \\
\hline
\end{tabular}

1. Process Knowledge
LL.WO24
LIQUID MERCURYY WASTE

\section{Still-Gen $\because$ Active}




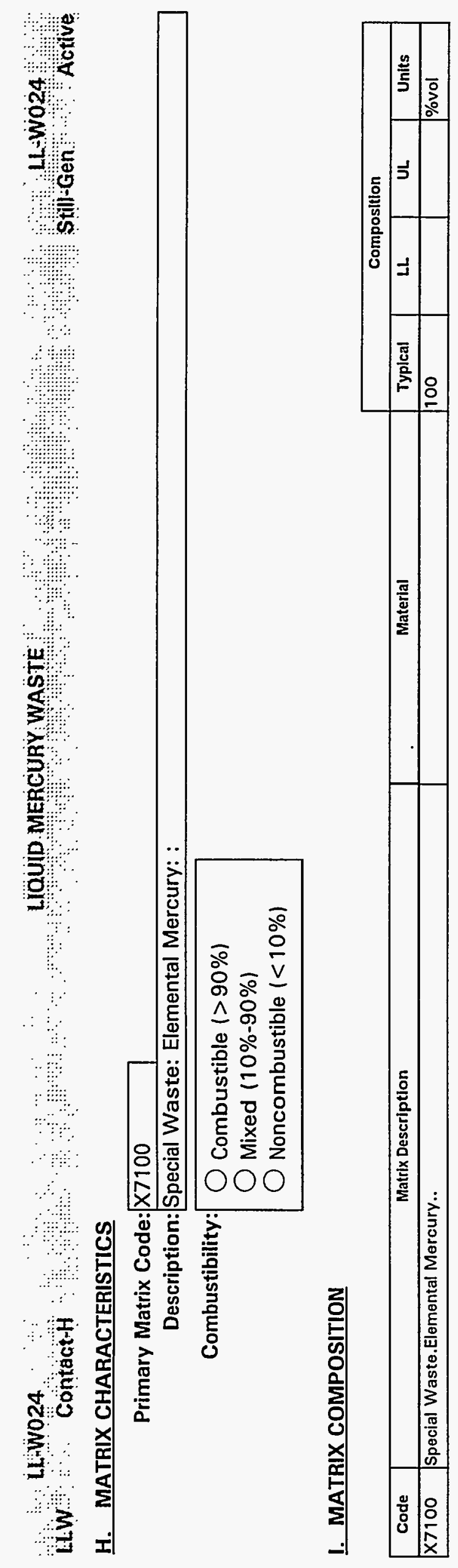

裹

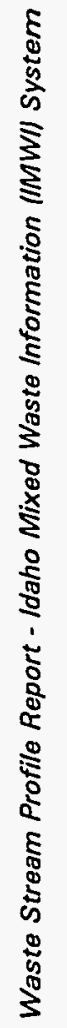




\section{LWW Contact \\ J. HAZARDOUS CONSTITUENTS}

\begin{tabular}{|c|c|c|c|c|c|c|c|c|}
\hline & & & \multicolumn{4}{|c|}{ Concentration } & & \\
\hline Code & Hazardous Waste Description & Treatment Subcategory & Typical & $\overline{\mathrm{LL}}$ & UL & Units & Basis & Conc. Type \\
\hline D009 & Mercury & Low mercury $<260 \mathrm{mg} / \mathrm{kg}$ total mercury & 90 & & & vol & & Total \\
\hline
\end{tabular}




\section{LWW ôno contati}

\section{K. POLYCHLORINATED BIPHENYLS (PCBS)}

Is this waste stream PCB contaminated?

\section{OYes}

No

\section{Unknown}

If yes, what portion of the waste is PCB contaminated (volume \%):

If $<100 \%$, can the PCB contaminated waste be segregated and treated separately?

What is the PCB concentration in ppm?

\section{TREATMENT PLANS}

\begin{tabular}{|c|c|c|c|c|c|c|c|c|c|c|c|}
\hline Opt. & $\begin{array}{c}\text { Media Type } \\
\text { (If more than onel }\end{array}$ & $\begin{array}{c}\text { Matrix } \\
\text { Code }\end{array}$ & $\begin{array}{l}\text { Vol. \% } \\
\text { (If }\end{array}$ & Step & JIT & sc & CIF & $\begin{array}{l}\text { Trans. } \\
\text { Miles }\end{array}$ & $\begin{array}{l}\text { Facility } \\
\text { Abbr. }\end{array}$ & Unit Name & Comments \\
\hline \multirow[t]{4}{*}{1} & & $x 7100$ & & $\bar{a}$ & 0 & 0 & $1.00 \mathrm{E}+00$ & & TRANS & Transport - LLW & \\
\hline & & & & b & O & 0 & $1.00 E+00$ & & AMWTF & $\mathrm{CH}$ - Opening \& Sorting & \\
\hline & & & & c & 0 & 0 & $1.00 E+00$ & & AMWTF & CH-Amalgamation & \\
\hline & & & & $d$ & 0 & 0 & $1.00 E+00$ & & SCDF & Disposal - Contact Handled & \\
\hline
\end{tabular}

Note: Where provided, media percentages are rough estimates used to facilitate treatment planning. They do not necessarily imply an accurate knowledge of waste stream composition, and were not derived for use outside this conceptual planning exercise.

Describe any special or unique technical concerns related to the treatment of this waste stream that would impact the use or implementation of standard hazardous waste treatment methods. 


\section{A. IDENTIFICATION AND DESCRIPTION}

Waste Stream ID: MI-W011

W. S. Name:

Content Code:

FFCA ID:

\section{MATERIALS CONTAINING PCBS}

*

MI-W011

\section{Waste Stream Description:}

Cloth and paper debris containing PCBs. This is not a RCRA waste, but is regulated by the State of California as a hazardous waste. The waste will be of similar physical form from container to container. This waste stream will not be moratorium waste or will not be reclassified TRU waste. The individual sizes range from 6 to 36 inches squared.

\section{B. GENERATION SITE/PROCESS DESCRIPTION}

The following questions, concerning the generation site and process, should be answered even if the waste stream is no longer generated.

Name of Site Generating Waste (e.g., INEL, Rocky Flats, etc.): Mare Island Naval Shipyards

Location of activities (Area and Building):

Naval vessels undergoing maintenance activities. Radiological work and waste handling facilities.

Operations (functions) performed in building:

Repair, overhaul, and maintenance of Naval Nuclear Propulsion

systems and components, waste handling. Only activation products, primarily Co-60, are present in radioactive material. Use of hazardous material is not normally permitted or required at the worksites.

However, during maintenance on oil lubricated machinery, sometimes PCB contaminants may be encountered in some of this oil.

Description of Process Generating Waste:

Maintenance on equipment with PCB contaminated oil is present, solid waste is cloth and plastic debris. 


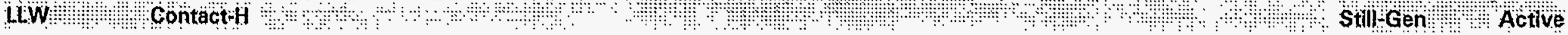

C. PROJECTED WASTE GENERATION

Is this waste stream still generated?

If yes, when is the expected generation termination date?

Current Projections

\begin{tabular}{|r|r|r|r|r|r|}
\hline Est. Date & Start Year & Interval & Vol (m3) & Mass $(\mathrm{kg})$ & Source of Estlmate \\
\hline $11 / 15 / 94$ & 1996 & 1 & 0.62 & & 1994-DSTP \\
\hline
\end{tabular}

Historical Projections 


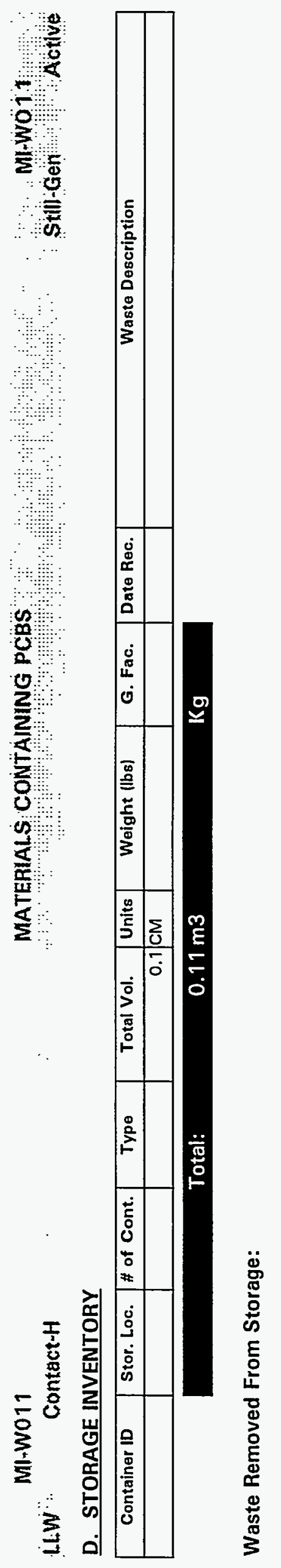

\begin{tabular}{l}
\multirow{1}{*}{} \\
$\dot{7}$ \\
8 \\
0 \\
0
\end{tabular} 


\section{F. TRU ALPHA ACTIVITY DISTRIBUTION}

Radioactive Waste Type:

\begin{tabular}{|ll|}
\hline O LLW & Oa-LLW $\bigcirc$ TRU \\
O HLW & O Non-Rad \\
\hline
\end{tabular}

This waste stream is not identified as a TRU or a-LLW.

Mixed Waste:

Handling:

\begin{tabular}{|l|}
\hline O Yes O No \\
\hline O Contact \\
\hline Remote
\end{tabular}

Radionuclide Contamination Accessiblity:

External Surface $O$ Yes $O$ No OUnknown

Internal Surface $O$ Yes O No OUnknown

Dispersed Through Matrix $O$ Yes O No OUnknown

\section{Activity Levels}

Transuranic Alpha Activity:

Uranium/Thorium Alpha Activity:

Beta/Gamma Activity:

\begin{tabular}{|c|c|}
\hline 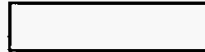 & Units: \\
\hline & Units: \\
\hline
\end{tabular}

0.02 @ surface

$<0.02 @ 1-m$ Units: $\mathrm{mR} / \mathrm{hr}$

Surface Neutron Activity: Units:

Total Activity: $5.7 \mathrm{E}-2 \mathrm{nCi} / \mathrm{g}$ 


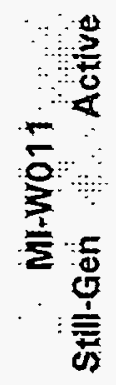

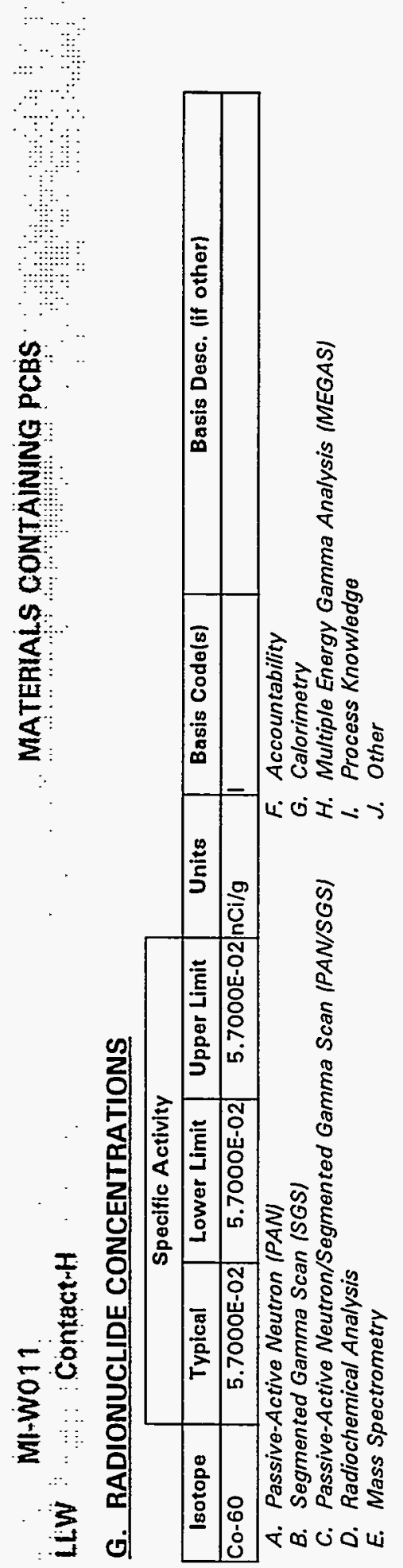




\section{MI:Wö11}

\section{LlW $\because$ ContactiH}

\section{MATERIALS CONTAINING PCBS}

\section{H. MATRIX CHARACTERISTICS}

Primary Matrix Code: $\mathbf{S 5 3 9 0}$

Description: Debris Waste: Organic Debris: Unknown/Other Organic Debris:

Combustibility:

Combustible (>90\%)

Mixed $(10 \%-90 \%)$

Noncombustible $(<10 \%)$

\section{MATRIX COMPOSITION}

\begin{tabular}{|c|c|c|c|c|c|c|}
\hline & & & \multicolumn{4}{|c|}{ Composition } \\
\hline Code & Matrix Description & Material & Typical & LL & UL & Units \\
\hline $\mathbf{5 5 3 9 0}$ & Debris Waste.Organic Debris.Unknown/Other Organic Debris. & Cloth & 50 & 30 & 70 & $\%$ vol \\
\hline$\$ 5390$ & Debris Waste.Organic Debris.Unknown/Other Organic Debris. & Plastic & 50 & 30 & 70 & $\%$ vol \\
\hline
\end{tabular}



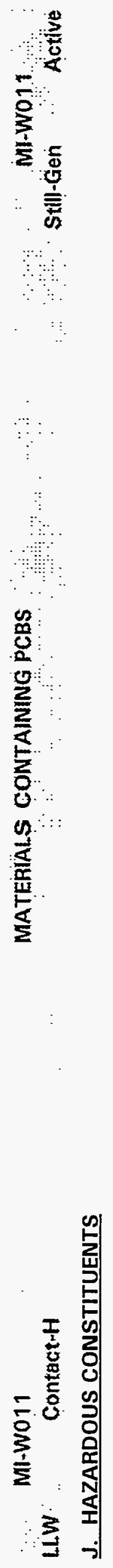


\section{$\because: \quad$ MII-WO11}

\section{LiW Contäct-H}

\section{MATERIALS CONTAINING PCBSS}

K. POLYCHLORINATED BIPHENYLS (PCBS)

Is this waste stream PCB contaminated?

\section{O Yes O No O Unknown}

If yes, what portion of the waste is PCB contaminated (volume \%):

If $<100 \%$, can the PCB contaminated waste be segregated and treated separately?

OYes O No O Unknown

What is the PCB concentration in ppm? If only a portion of the waste is PCB contaminated, report values for contaminated portion.

\section{TREATMENT PLANS}

\begin{tabular}{|c|c|c|c|c|c|c|c|c|c|c|c|}
\hline $\begin{array}{c}\text { Opt. } \\
\#\end{array}$ & $\begin{array}{c}\text { Media Type } \\
\text { (If more than one) }\end{array}$ & $\begin{array}{c}\text { Matrix } \\
\text { Code }\end{array}$ & $\begin{array}{l}\text { Vol. \% } \\
\text { lif }\end{array}$ & Step & JIT & sc & $\mathrm{CJF}$ & $\begin{array}{l}\text { Trans. } \\
\text { Miles }\end{array}$ & $\begin{array}{l}\text { Facility } \\
\text { Abbr. }\end{array}$ & Unit Name & Comments \\
\hline 1 & & 55330 & & $\bar{a}$ & (O) & $\bar{O}$ & $1.00 \mathrm{E}+00$ & & TRANS & Transport - LLW & \\
\hline & & & & b & 0 & $\mathrm{O}$ & $1.00 E+00$ & & AMWTF & $\mathrm{CH}$ - Opening \& Sorting & \\
\hline & & & & $\bar{c}$ & $\mathbf{O}$ & $\overline{0}$ & $1.00 \mathrm{E}+00$ & & AMWTF & $\mathrm{CH}$ - Sizing & \\
\hline & & & & $\bar{d}$ & $\overline{0}$ & $\overline{0}$ & $1.00 E+00$ & & AMWTF & CH - Incineration/Thermal Desorption & \\
\hline & & & & $\bar{\theta}$ & 0 & $\overline{0}$ & $1.00 E+00$ & & AMWTF & $\mathrm{CH}$ - Vitrification & \\
\hline & & & & $f$ & 0 & 0 & $1.00 E+00$ & & SCDF & Disposal - Contact Handled & \\
\hline
\end{tabular}

Note: Where provided, media percentages are rough estimates used to facilitate treatment planning. They do not necessarily imply an accurate knowledge of waste stream composition, and were not derived for use outside this conceptual planning exercise.

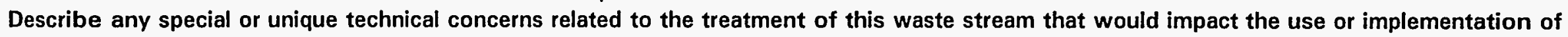
standard hazardous waste treatment methods. 


\section{A. IDENTIFICATION AND DESCRIPTION}

Waste Stream ID: MI-W013

W. S. Name:

ORGANIC PROCESS RESIDUES

Content Code:

FFCA ID:

MI-W013

\section{Waste Stream Description:}

Solidified Sludges and associated liquors with chemical residues containing chromates, metal sludges, and steam generator cleaning chemicals. The waste will be of a similar form from container to container. The waste stream will not be a moratorium waste or reclassified TRU waste.

\section{B. GENERATION SITE/PROCESS DESCRIPTION}

The following questions, concerning the generation site and process, should be answered even if the waste stream is no longer generated.

Name of Site Generating Waste (e.g., INEL, Rocky Flats, etc.): Mare Island Naval Shipyards

Location of activities (Area and Building):

Naval vessels undergoing maintenance activities. Radiological work and waste handling facilities.

Operations (functions) performed in building: Repair, overhaul, and maintenance of Naval Nuclear Propulsion systems and components, waste handling. Only activation products, primarily Co-60, are present in radioactive material. Use of hazardous material is not normally permitted at worksites. However, some nuclear ships system operation and maintenance procedures involve chemical cleaning or corrosion inhibition.

Description of Process Generating Waste:

These materials were generated from the decontamination of equipment used to process or collect various mixed material streams resulting from chemicals used during ships system maintenance. 


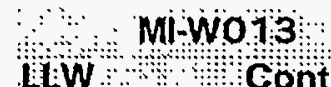

HWW ou Gontact H

C. PROJECTED WASTE GENERATION

Is this waste stream still generated?

If yes, when is the expected generation termination date?

ORGANIC PRÖOCESS RESIDUES

\begin{tabular}{|c|c|r|r|r|r|}
\hline \multicolumn{7}{|c|}{ Current Projections } \\
\hline Est. Date & Start Year & Interval & Vol (m3) & Mass (kg) & Source of Estimate \\
\hline $11 / 15 / 94$ & 1995 & 5 & 1.06 & 1994 -DSTP \\
\hline \multicolumn{7}{|c|}{ Historical Projections } \\
\hline
\end{tabular}




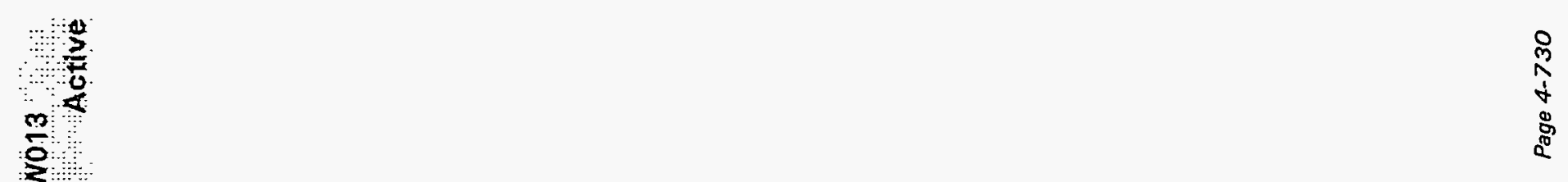




\section{E. RADIATION CHARACTERISTICS}

Radioactive Waste Type:

\begin{tabular}{|ll|}
\hline OllW & Oa-llw $\bigcirc$ tRU \\
OhlW & O Non-Rad
\end{tabular}

Mixed Waste:

Handling:

\begin{tabular}{|l|}
\hline OYes O No \\
\hline O Contact O Remote \\
\hline
\end{tabular}

Radionuclide Contamination Accessiblity:

External Surface

Internal Surface

Dispersed Through Matrix

\begin{tabular}{|}
\begin{tabular}{|lll|}
\hline Yres & O No & O Unknown \\
\hline OYes & O No & OUnknown \\
\hline OYes & O No & OUnknown \\
\hline
\end{tabular}
\end{tabular}

\section{Activity Levels}

Transuranic Alpha Activity:

Uranium/Thorium Alpha Activity:

Beta/Gamma Activity:

\begin{tabular}{|c|c|}
\hline ( & Units: \\
\hline & Units: \\
\hline
\end{tabular}

\begin{tabular}{r|}
\hline 150 \\
\hline
\end{tabular} @ surface

0.2 @ 1-m Units: mR/hr

Surface Neutron Activity:

Total Activity:

$\square$

Units: $\mathrm{nCi} / \mathrm{g}$

\section{F. TRU ALPHA ACTIVITY DISTRIBUTION}

This waste stream is not identified as a TRU or a-LLW. 


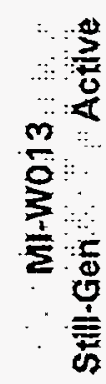

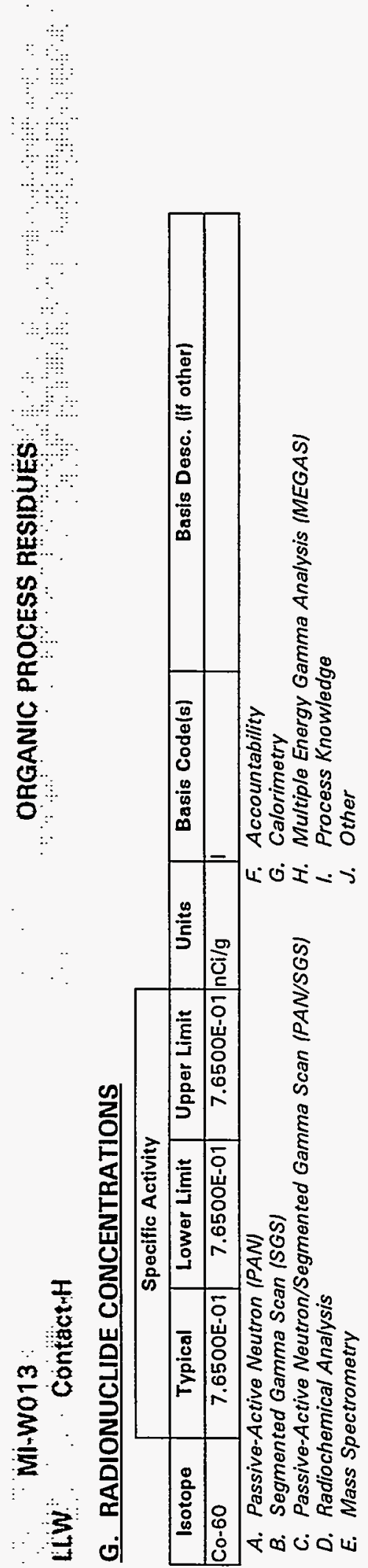


H. MATRIX CHARACTERISTICS

Primary Matrix Code: $\mathbf{S 3 1 5 0}$

Description: Homogeneous Solids: Inorganic Homogeneous Solids: Solidified Homogeneous Solids:

\begin{tabular}{l|l} 
Combustibility: & $\bigcirc$ Combustible $(>90 \%)$ \\
$\bigcirc$ Mixed $(10 \%-90 \%)$ \\
$\bigcirc$ Noncombustible $(<10 \%)$
\end{tabular}

\section{MATRIX COMPOSITION}

\begin{tabular}{|c|c|c|c|c|c|c|}
\hline & & & \multicolumn{4}{|c|}{ Composition } \\
\hline Code & Matrix Description & Material & Typical & LL & UL & Units \\
\hline 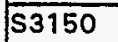 & Homogeneous Solids.Inorganic Homogeneous Solids.Solidified Homogeneous Solids. & (Cement) & 100 & 100 & 100 & $\%$ vol \\
\hline
\end{tabular}




\section{J. HAZARDOUS CONSTITUENTS}

\begin{tabular}{|c|c|c|c|c|c|c|c|c|}
\hline & & & \multicolumn{4}{|c|}{ Concentration } & & \\
\hline Code & Hazardous Waste Description & Treatment Subcategory & Typical & LL & UL & Units & Basis & Conc. Type \\
\hline 0007 & Chromium & Chromium (Total) & & & & & A & Total \\
\hline
\end{tabular}




\section{LiWW Contact H}

\section{K. POLYCHLORINATED BIPHENYLS (PCBS)}

Is this waste stream PCB contaminated?

\section{OYes O No O Unknown}

If yes, what portion of the waste is $\mathrm{PCB}$ contaminated (volume \%):

If $<100 \%$, can the PCB contaminated waste be segregated and treated separately?

What is the PCB concentration in ppm?

If only a portion of the waste is $P C B$ contaminated, report values for contaminated portion.

\section{TREATMENT PLANS}

\begin{tabular}{|c|c|c|c|c|c|c|c|c|c|c|c|}
\hline $\begin{array}{l}\text { Opt. } \\
\#\end{array}$ & $\begin{array}{c}\text { Media Type } \\
\text { (If more than one) }\end{array}$ & $\begin{array}{c}\text { Matrix } \\
\text { Code }\end{array}$ & $\begin{array}{l}\text { Vol. } \% \\
\text { (If }\end{array}$ & Step & |JIT & SC & CIF & $\begin{array}{l}\text { Trans. } \\
\text { Miles }\end{array}$ & $\begin{array}{l}\text { Facility } \\
\text { Abbr. }\end{array}$ & Unit Name & Comments \\
\hline 1 & & 53150 & & $\mathbf{a}$ & $\mathbf{O}$ & 0 & $1.00 E+00$ & & TRANS & Transport - LLW & \\
\hline & & & & b & $\mathbf{0}$ & 0 & $1.00 \mathrm{E}+00$ & & AMWTF & $\mathrm{CH}$ - Opening \& Sorting & \\
\hline & & & & c & $\mathbf{O}$ & 0 & $1.00 E+00$ & & AMWTF & $\mathrm{CH}$ - Sizing & \\
\hline & & & & d & C & O & $1.00 E+00$ & & AMWTF & $\mathrm{CH}$ - Incineration/Thermal Desorption & \\
\hline & & & & e & 0 & 0 & $1.00 E+00$ & & AMWTF & $\mathrm{CH}$ - Vitrification & \\
\hline & & & & $f$ & 0 & 0 & $1.00 \mathrm{E}+00$ & & SCDF & Disposal - Contact Handled & \\
\hline
\end{tabular}

Note: Where provided, media percentages are rough estimates used to facilitate treatment planning. They do not necessarily imply an accurate knowledge of waste stream composition, and were not derived for use outside this conceptual planning exercise.

Describe any special or unique technical concerns related to the treatment of this waste stream that would impact the use or implementation of standard hazardous waste treatment methods. 


LLW
A. IDENTIFICATION AND DESCRIPTION
Waste Stream ID: MU-W001
W. S. Name:
Content Code:
FFCA ID:
Waste Stream Description:
$\begin{aligned} & \text { MLLW Heterogeneous Debris. The radioactive wastes generated on the project will come first } \\ & \text { from normal operations and second from the D\&D of the facility at the end of the project. } \\ & \text { Radioactive wastes from normal operations will consist of the following: HEPA filters from the } \\ & \text { glove box; HEPA filters from offgas and room filtration systems; paper wipes from periodic } \\ & \text { cleaning of the glove boxes; used sample bottles; damaged glove box gloves; used crucibles, } \\ & \text { tubes, and wires. }\end{aligned}$

\section{A. IDENTIFICATION AND DESCRIPTION}

Content Code:

FFCA ID:

from normal operations and second from the D\&D of the facility at the ond of the project.

Radioactive wastes from normal operations will consist of the following: HEPA filters from the

gleaning, the

tubes, and wires.
MU-WOO1.

Still-Gen: $\therefore$ Active

\section{B. GENERATION SITE/PROCESS DESCRIPTION}

The following questions, concerning the generation site and process, should be answered even if the waste stream is no longer generated.

Name of Site Generating Waste (e.g., INEL, Rocky Flats, etc.): University of Missouri Research Reactor (MURR)

\section{Location of activities (Area and Building):}

MURR Alpha Room

Operations (functions) performed in building:

The TRUMP-S test program uses three glove boxes located in the Alpha Lab: the argon box, the air box, and the ICP box. The three boxes are equipped with various pieces of test equipment.

\section{Description of Process Generating Waste:}

The radioactive wastes generated on the project will come first from normal operations and second from the D\&D of the facility at the end of the project. Radioactive wastes from normal operation will consist of the following: HEPA filters from the glove box; HEPA filters from offgas and room filtration systems; paper wipes from periodic cleaning of the glove boxes; used sample bottles; damaged glove box gloves; used crucibles, tubes, and wires. 
MU:W001

LLW Contact H

C. PROJECTED WASTE GENERATION

Is this waste stream still generated?

If yes, when is the expected generation termination date?
OYes O No

$12 / 31 / 98$

\section{Current Projections}

\begin{tabular}{|r|r|r|r|r|l|}
\hline Est. Date & Start Year & \multicolumn{1}{|l|}{ Interval } & Vol (m3) & Mass (kg) & \multicolumn{1}{|c|}{ Source of Estimate } \\
\hline $11 / 30 / 93$ & 1995 & 1 & 0.80 & & Generator Update \\
\hline $11 / 30 / 93$ & 1996 & 1 & 0.80 & & Generator Update \\
\hline $11 / 30 / 93$ & 1997 & 1 & 0.80 & & Generator Update \\
\hline $11 / 30 / 93$ & 1998 & 1 & 0.80 & & Generator Update \\
\hline $11 / 30 / 93$ & 1999 & 1 & 0.80 & & Generator Update \\
\hline
\end{tabular}

Historical Projections

\begin{tabular}{|r|r|r|r|r|r|}
\hline Est. Date & \multicolumn{1}{|c|}{ Start Year } & \multicolumn{1}{|c|}{ Interval } & \multicolumn{1}{c|}{ Vol (m3) } & \multicolumn{1}{c|}{ Mass $(\mathrm{kg})$} & Source of Estimate \\
\hline $11 / 30 / 93$ & 1993 & 1 & 0.17 & 30.00 & $1993-\mathrm{FFCA}$ \\
\hline $11 / 30 / 93$ & 1994 & 1 & 0.17 & 30.00 & $1993-\mathrm{FFCA}$ \\
\hline
\end{tabular}




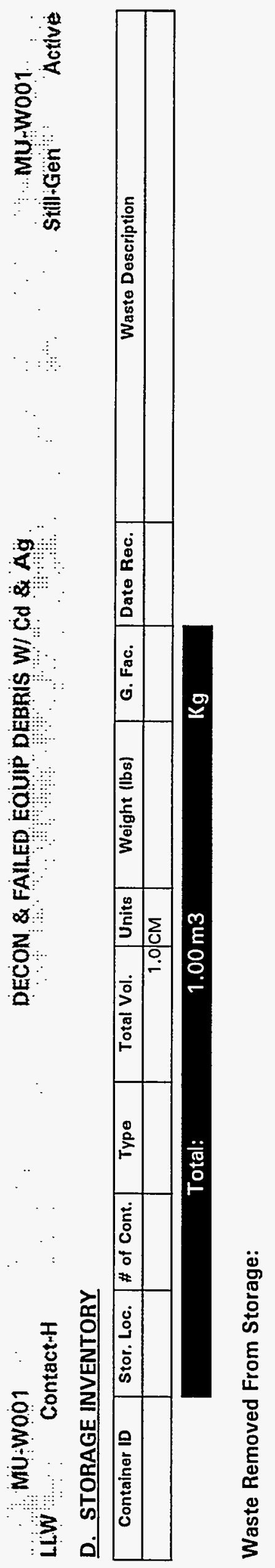




\section{E. RADIATION CHARACTERISTICS}

Radioactive Waste Type:

$\begin{array}{ll}\text { OllW } & \text { Oa-llw } \bigcirc \text { TRU } \\ \text { OhLW } & \text { Onon-Rad }\end{array}$

Mixed Waste:

Handling:

\section{F. TRU ALPHA ACTIVITY DISTRIBUTION}

This waste stream is not identified as a TRU or a-LLW.

\begin{tabular}{|c|c|c|c|}
\hline External Surface & OYes & Ono & OUnknown \\
\hline Internal Surface & OYes & ONo & (O) Unknown \\
\hline
\end{tabular}

\section{Activity Levels}

Transuranic Alpha Activity:

Uranium/Thorium Alpha Activity:

Beta/Gamma Activity:

Surfe Neutron Activity:

Total Activity:

\begin{tabular}{l}
$\square$ Units: $\square$ \\
\hline Units: $\square$ \\
\hline
\end{tabular}

3.1 @ surface

0.5 @ 1-m Units: $\mathrm{mR} / \mathrm{hr}$

:

$12 \mathrm{nCi} / \mathrm{g}$ 


\section{Liw: ContactrH \\ G. RADIONUCLIDE CONCENTRATIONS}

DECON \& FAILED EQUIP DEBRIS W/ Cd \& Ag

MU WOOT

\begin{tabular}{|c|c|c|c|c|c|c|}
\hline & \multicolumn{3}{|c|}{ Specific Activity } & \multirow[b]{2}{*}{ Units } & \multirow[b]{2}{*}{ Basis Code(s) } & \multirow[b]{2}{*}{ Basis Desc. (if other) } \\
\hline Isotope & Typical & Lower Limit & Upper Limit & & & \\
\hline Np-237 & & $2.6000 \mathrm{E}-12$ & $4.0000 E-11$ & $\mathrm{Ci} / \mathrm{g}$ & $F$ & \\
\hline U-238 & & $3.5000 \mathrm{E}-12$ & $5.4000 \mathrm{E}-12$ & $\mathrm{Ci} / \mathrm{g}$ & $F$ & \\
\hline Am-241 & & $3.5000 E-09$ & $1.2000 \mathrm{E}-08$ & $\mathrm{Ci} / \mathrm{g}$ & $F$ & \\
\hline $\begin{array}{l}\text { A. Passiv } \\
\text { B. Segm } \\
\text { C. Passiv } \\
\text { D. Radioc } \\
\text { E. Mass }\end{array}$ & $\begin{array}{l}\text { tive Neutr } \\
\text { Gamma } \\
\text { tive Neutr } \\
\text { ical Analy } \\
\text { trometry }\end{array}$ & $\begin{array}{l}A N \text { ) } \\
\text { (SGSI) } \\
\text { ggmented Ga }\end{array}$ & a Scan $(P A$ & $(S G S)$ & $\begin{array}{l}\text { Accountability } \\
\text { Calorimetry } \\
\text { Multiple Energy } \\
\text { Process Knowle } \\
\text { Other }\end{array}$ & sis (MEGAS) \\
\hline
\end{tabular}




\section{H. MATRIX CHARACTERISTICS}

Primary Matrix Code: $\mathbf{S 5 4 0}$

Description: Debris Waste: Heterogeneous Debris: Predominantly Organic Debris:

Combustibility: $\bigcirc$ Combustible $(>90 \%)$

Mixed $(10 \%-90 \%)$

Noncombustible $(<10 \%)$

\section{MATRIX COMPOSITION}

\begin{tabular}{|c|c|c|c|c|c|c|}
\hline \multirow[b]{2}{*}{ Code } & \multirow[b]{2}{*}{ Matrix Description } & \multirow[b]{2}{*}{ Material } & \multicolumn{4}{|c|}{ Composition } \\
\hline & & & Typlcal & $\mathbf{L L}$ & UL & Units \\
\hline$\$ \mathbf{S 5 1 1 3}$ & Debris Waste.Inorganic Debris.Metal Debris.Metal Debris w/Cd & Metal cans & 2 & & & $\%$ vol \\
\hline $\mathbf{S 5 1 2 2}$ & Debris Waste.Inorganic Debris.Inorganic Non-Metal Debris.Glass Debris & Glass beakers, rods, vials & 20 & & & \%vol \\
\hline$\overline{\mathbf{5 5 3 1 0}}$ & Debris Waste.Organic Debris.Plastic/Rubber Debris. & Rubber gloves, plastic gloves, bags & 26 & & & $\%$ vol \\
\hline $\mathbf{S 5 3 3 0}$ & Debris Waste.Organic Debris.Paper/Cloth Debris. & Paper towels & 12 & & & $\%$ vol \\
\hline $\mathbf{5 5 3 1 2}$ & Debris Waste.Organic Debris.Plastic/Rubber Debris.Halogenated Plastic Debris & Plastic vials, containers & 40 & & & $\%$ vol \\
\hline
\end{tabular}


MU.W001

DECON \& FAILED EQUIP DEBRIS W/ CA \& Ag

MU:-W001

LlWW , Contact-H

Still-Gen Active

J. HAZARDOUS CONSTITUENTS

\begin{tabular}{|c|c|c|c|c|c|c|c|c|}
\hline & & & \multicolumn{4}{|c|}{ Concentration } & & \\
\hline Code & Hazardous Waste Description & Treatment Subcategory & Typical & $\mathbf{L L}$ & UL & Units & Basis & Conc. Type \\
\hline$\overline{D 006}$ & Cadmium & & & & & & A & Total \\
\hline$\overline{D 011}$ & Silver & & & & & & A & Total \\
\hline
\end{tabular}


K. POLYCHLORINATED BIPHENYLS (PCBS)

Is this waste stream PCB contaminated?

OYes

O No O Unknown

If yes, what portion of the waste is PCB contaminated (volume \%):

If $<100 \%$, can the PCB contaminated waste be segregated and treated separately?

What is the PCB concentration in ppm?

\section{TREATMENT PLANS}

\begin{tabular}{|c|c|c|c|c|c|c|c|c|c|c|c|}
\hline $\begin{array}{l}\text { Opt. } \\
\#\end{array}$ & $\begin{array}{c}\text { Media Type } \\
\text { (If more than onel }\end{array}$ & $\begin{array}{c}\text { Matrix } \\
\text { Code }\end{array}$ & $\begin{array}{l}\text { Vol. \% } \\
\text { (If }\end{array}$ & Step & JIT & sc & CIF & $\begin{array}{l}\text { Trans. } \\
\text { Miles }\end{array}$ & $\begin{array}{l}\text { Facility } \\
\text { Abbr. }\end{array}$ & Unit Name & Comments \\
\hline 1 & & $\mathbf{S 5 4 4 0}$ & & a & $\mathbf{0}$ & 0 & $1.00 E+00$ & & TRANS & Transport - LLW & \\
\hline & & & & b & $\mathrm{O}$ & 0 & $1.00 \mathrm{E}+00$ & & MWSF & Open/Segregate/Repack (nonhalogenated solids) & \\
\hline & & & & c & 0 & $\bar{O}$ & $1.00 E+00$ & & WERF & Incineration - Controlled Air Incinerator & \\
\hline & & & & d & 0 & O & $1.00 E+00$ & & WERF & Stabilization - Portland Cement & \\
\hline & & & & e & 0 & 0 & $1.00 E+00$ & & SCDF & Disposal - Contact Handled & \\
\hline
\end{tabular}

Note: Where provided, media percentages are rough estimates used to facilitate treatment planning. They do not necessarily imply an accurate knowledge of waste stream composition, and were not derived for use outside this conceptual planning exercise.

Describe any special or unique technical concerns related to the treatment of this waste stream that would impact the use or implementation of standard hazardous waste treatment methods. 


\section{OR:WOOZ \\ LWW \\ Contact-H}

A. IDENTIFICATION AND DESCRIPTION

Waste Stream ID: OR-W007

W. S. Name:

Content Code:

FFCA ID:

BULK LIQUID MERCURY

B

OR-W007

Waste Stream Description:

This waste stream grouping consists of bulk liquid mercury. The physical form is liquid and may be in a lab-pack configuration (matrix code 7100 ).
BULK LIQUID MERCUAY

\section{OR+W007}

Still-Gén Active

\section{B. GENERATION SITE/PROCESS DESCRIPTION}

The following questions, concerning the generation site and process, should be answered even if the waste stream is no longer generated.

Name of Site Generating Waste (e.g., INEL, Rocky Flats, etc.): ORNL site

Location of activities (Area and Building):

The information on the source building is not compiled.

Operations (functions) performed in building:

The information on the operation at the source building is not compiled.

Description of Process Generating Waste:

The information on the process(es) generating the waste is not compiled. 
$\therefore \quad \therefore$ OROWOOZ

LLW Contact H

C. PROJECTED WASTE GENERATION

Is this waste stream still generated?

If yes, when is the expected generation termination date?

\footnotetext{
Current Projections
}

BULK LIQUID MËRCURY

Historical Projections 

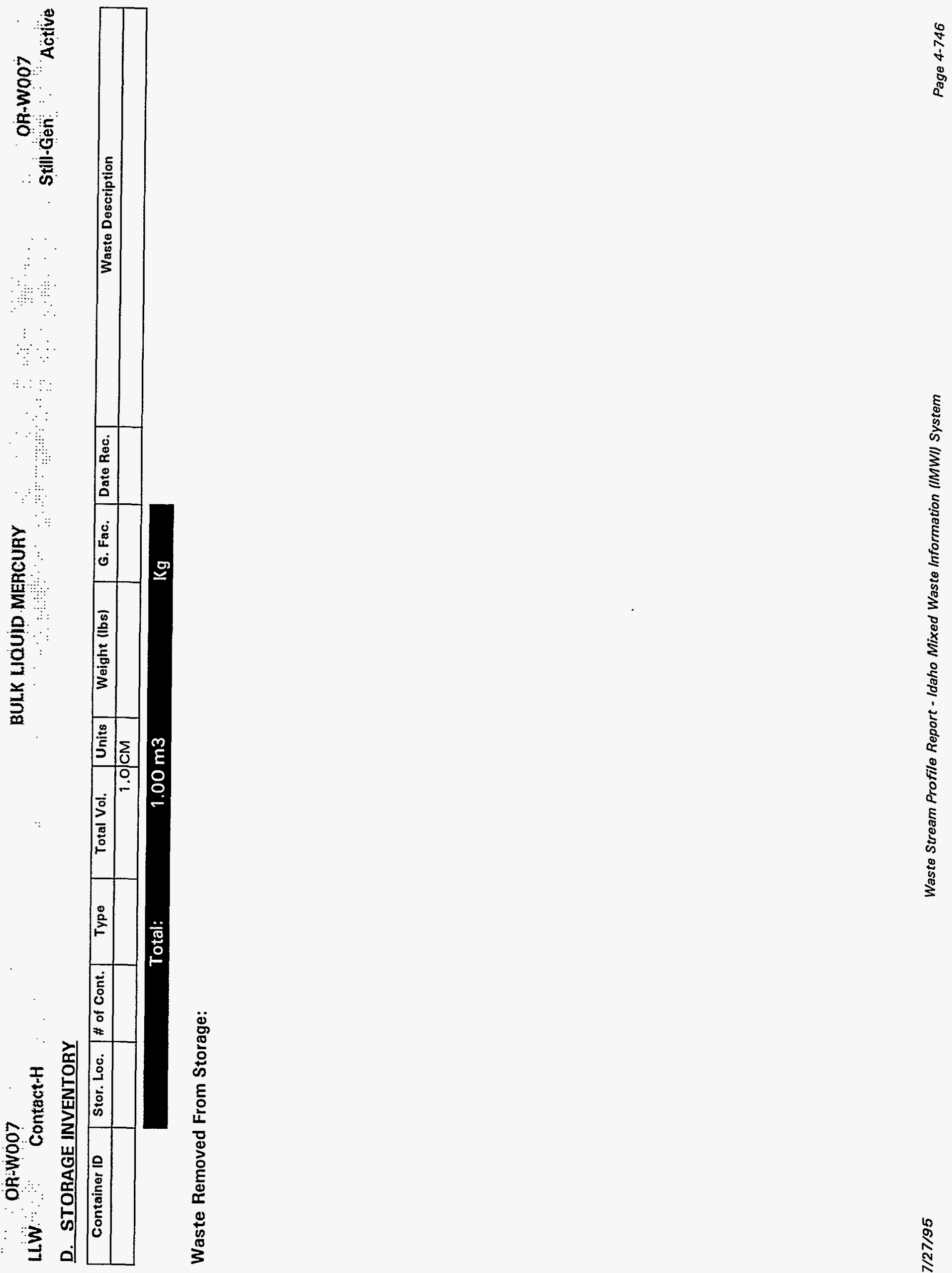


\section{OR-WOOZ7}

Lew Contact-H

\section{F. TRU ALPHA ACTIVITY DISTRIBUTION}

\section{E. RADIATION CHARACTERISTICS}

Radioactive Waste Type:

\begin{tabular}{ll} 
OllW & Oa-LlW $\bigcirc$ TRU \\
OHLW & O Non-Rad \\
\hline
\end{tabular}

Mixed Waste:

Handling:

\begin{tabular}{|l|}
\hline OYes O No \\
\hline O Contact O Remote \\
\hline
\end{tabular}

Radionuclide Contamination Accessiblity:

External Surface $O$ Yes $O$ No OUnknown

Internal Surface $O$ Yes $O$ No OUnknown

Dispersed Through Matrix

OYes ONo OUnknown

\section{Activity Levels}

Transuranic Alpha Activity:

Uranium/Thorium Alpha Activity:

Beta/Gamma Activity:

Surface Neutron Activity:

Total Activity:

\begin{tabular}{|c|c|c|}
\hline & Units: & \\
\hline & Units: & \\
\hline & @ surf & ace \\
\hline & @ 1-m & Units: \\
\hline & Units: [ & \\
\hline & $\mathrm{nCi} / \mathrm{g}$ & \\
\hline
\end{tabular}

This waste stream is not identified as a TRU or a-LLW. 

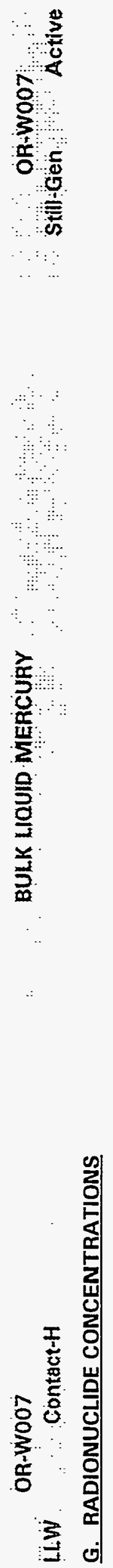


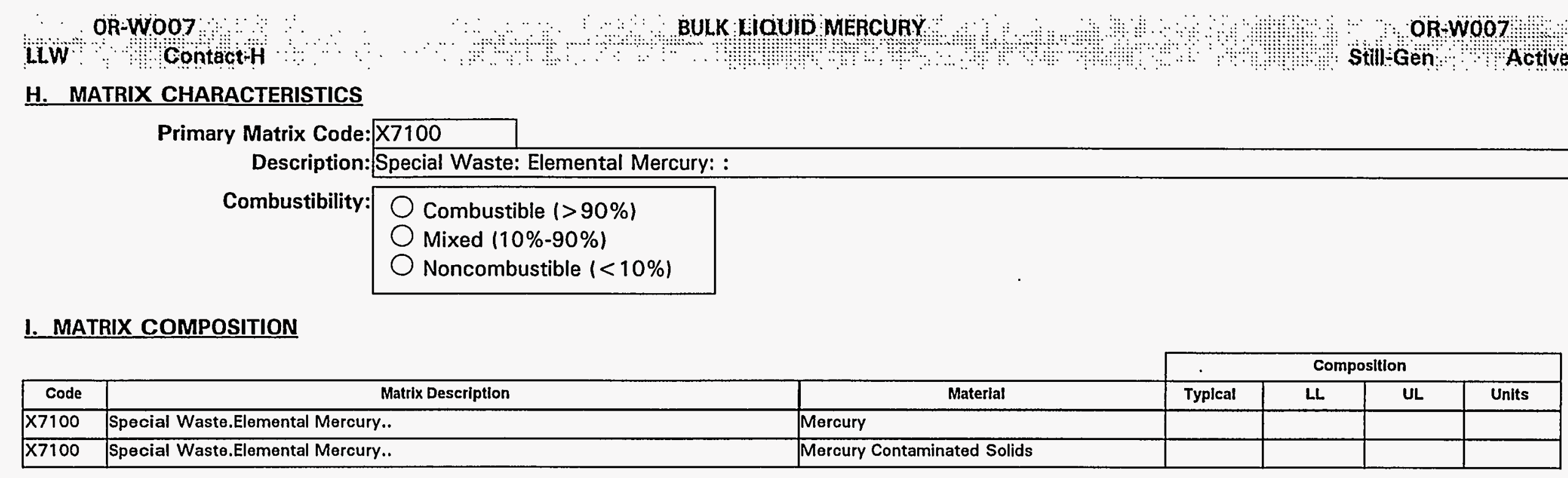




\section{J. HAZARDOUS CONSTITUENTS}

\begin{tabular}{|c|c|c|c|c|c|c|c|c|}
\hline & & & \multicolumn{4}{|c|}{ Concentration } & \multirow[b]{2}{*}{ Basis } & \multirow[b]{2}{*}{ Conc. Type } \\
\hline Code & Hazardous Waste Description & Treatment Subcategory & Typical & LL & UL & Units & & \\
\hline D005 & Barium & & & & & & $A$ & Total \\
\hline D007 & Chromium & Chromium (Total) & & & & & $\bar{A}$ & Total \\
\hline D009 & Mercury & Elemental mercury contaminated with radioact & & & & & $A$ & Total \\
\hline P012 & Arsenic trioxide & & & & & & $A$ & Total \\
\hline U151 & Mercury (Low mercury $<260 \mathrm{mg} / \mathrm{kg}$ ) & & & & & & A & Total \\
\hline
\end{tabular}




\section{K. POLYCHLORINATED BIPHENYLS (PCBS)}

Is this waste stream PCB contaminated?

\section{OYes O No O Unknown}

If yes, what portion of the waste is PCB contaminated (volume \%):

If $<100 \%$, can the PCB contaminated waste be segregated and treated separately?

What is the PCB concentration in ppm?

If only a portion of the waste is $P C B$ contaminated, report values for contaminated portion.

\section{TREATMENT PLANS}

\begin{tabular}{|c|c|c|c|c|c|c|c|c|c|c|c|}
\hline $\begin{array}{l}\text { Opt. } \\
\#\end{array}$ & $\begin{array}{c}\text { Media Type } \\
\text { (If more than one) }\end{array}$ & $\begin{array}{c}\text { Matrix } \\
\text { Code }\end{array}$ & $\begin{array}{c}\text { Vol. \% } \\
\text { (If }\end{array}$ & Step & JIT & sc & CIF & $\begin{array}{l}\text { Trans. } \\
\text { Miles }\end{array}$ & $\begin{array}{l}\text { Facility } \\
\text { Abbr. }\end{array}$ & Unit Name & Comments \\
\hline 1 & & $x 7100$ & & $\bar{a}$ & $\mathbf{0}$ & 0 & $1.00 \mathrm{E}+00$ & & TRANS & Transport - LLW & \\
\hline & & & & $\bar{b}$ & 0 & 0 & $1.00 \mathrm{E}+00$ & & AMWTF & $\mathrm{CH}$ - Opening \& Sorting & \\
\hline & & & & c & 0 & 0 & $1.00 E+00$ & & AMWTF & $\mathrm{CH}$ - Amalgamation & \\
\hline & & & & $\bar{d}$ & $\overline{0}$ & $\bar{O}$ & $1.00 E+00$ & & SCDF & Disposal - Contact Handled & \\
\hline
\end{tabular}

Note: Where provided, media percentages are rough estimates used to facilitate treatment planning. They do not necessarily imply an accurate knowledge of waste stream composition, and were not derived for use outside this conceptual planning exercise.

Describe any special or unique technical concerns related to the treatment of this waste stream that would impact the use or implementation of standard hazardous waste treatment methods. 


\section{PO-WODG \\ HiW. Contact-H}

\section{A. IDENTIFICATION AND DESCRIPTION}

Waste Stream ID: PO-W006

\section{W. S. Name: $\quad$ WASTE HG, METALLIC}

Content Code:

FFCA ID: PO-W006

\section{Waste Stream Description:}

This stream is generated in the switchyards, cascade, cooling tower pumphouse, water

treatment facility, and the decontamination facility. Waste is elemental mercury from manometer or pump cleaning and/or replacement.

\section{WASTE HG, METALLIC}

\section{PO-WO06} Still-Gen : Active

\section{B. GENERATION SITE/PROCESS DESCRIPTION}

The following questions, concerning the generation site and process, should be answered even if the waste stream is no longer generated.

Name of Site Generating Waste (e.g., INEL, Rocky Flats, etc.): Portsmouth Gaseous Diffusion Plant

Location of activities (Area and Building):

Building X-720 and the Cascade Area in Buildings $X-330, X-333$, and X326.

Operations (functions) performed in building:

Building 720 - maintanance; cascade area - uranium enrichment.

Possible chemical contaminants: heavy metals, solvents. Possible radiological contaminants: U-234, U-235, U-238, Tc-99.

\section{Description of Process Generating Waste:}

Manometer and pump cleaning and/or replacement. 
$\therefore$ PO-WOOG

HiW contătt

C. PROJECTED WASTE GENERATION

Is this waste stream still generated?

If yes, when is the expected generation termination date?

Current Projections

\begin{tabular}{|r|r|r|r|r|l|}
\hline Est. Date & \multicolumn{1}{|c|}{ Start Year } & \multicolumn{1}{|c|}{ Interval } & \multicolumn{1}{|c|}{ Vol $(\mathrm{m} 3)$} & \multicolumn{1}{c|}{ Mass $(\mathbf{k g})}$. & Source of Estimate \\
\hline $11 / 30 / 93$ & 1993 & 1 & 0.21 & 170.00 & $1993-F F C A$ \\
\hline $11 / 30 / 93$ & 1994 & 1 & 0.21 & 170.00 & $1993-F F C A$ \\
\hline $11 / 30 / 93$ & 1995 & 1 & 0.21 & 170.00 & $1993-F F C A$ \\
\hline $11 / 30 / 93$ & 1996 & 1 & 0.21 & 170.00 & $1993-F F C A$ \\
\hline $11 / 30 / 93$ & 1997 & 1 & 0.21 & 170.00 & $1993-F F C A$ \\
\hline $11 / 30 / 93$ & 1998 & 1 & 0.21 & 170.00 & $1993-F F C A$ \\
\hline $11 / 30 / 93$ & 1999 & 1 & 0.21 & 170.00 & $1993-F F C A$ \\
\hline
\end{tabular}

Historical Projections

\begin{tabular}{|c|r|r|r|r|l|}
\hline Est. Date & \multicolumn{1}{|c|}{ Start Year } & Interval & \multicolumn{1}{|c|}{ Vol (m3) } & \multicolumn{1}{c|}{ Mass $(\mathrm{kg})$} & \multicolumn{1}{c|}{ Source of Estimate } \\
\hline $11 / 30 / 93$ & 1998 & 5 & 1.05 & 850.00 & $1993-\mathrm{FFCA}$ \\
\hline $11 / 30 / 93$ & 2003 & 20 & 4.20 & 3.400 .00 & $1993-\mathrm{FFCA}$ \\
\hline
\end{tabular}




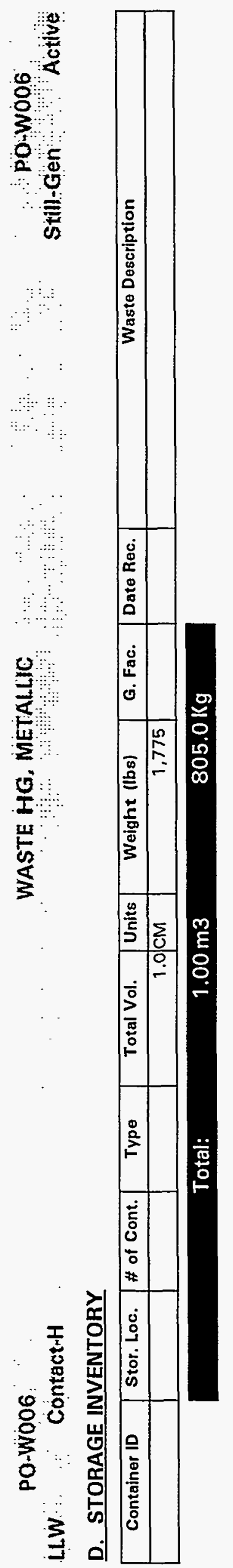


POWOOO6

LiW : Contact

E. RADIATION CHARACTERISTICS

WASTE: HG METÄLLIIG

\section{Po-woo 6}

Still-Gen Active

F. TRU ALPHA ACTIVITY DISTRIBUTION

Radioactive Waste Type:

\begin{tabular}{|ll|}
\hline OLLW & Oa-LLW $\bigcirc$ TRU \\
OHLW & O Non-Rad \\
\hline
\end{tabular}

Mixed Waste:

Handling:

\begin{tabular}{|l|}
\hline O Yes O No \\
\hline O Contact $O$ Remote \\
\hline
\end{tabular}

Radionuclide Contamination Accessiblity:

\begin{tabular}{rlrl|}
\cline { 2 - 4 } External Surface & OYes & ONo & O Unknown \\
\cline { 2 - 4 } Internal Surface & OYes & ONo & O Unknown \\
\cline { 2 - 4 } O O Yes & O No & O Unknown
\end{tabular}

Dispersed Through Matrix

\section{Activity Levels}

Transuranic Alpha Activity:

Uranium/Thorium Alpha Activity: Beta/Gamma Activity:

Surface Neutron Activity:

$\square$
$\square$
$\square$
$\square$
$\square$

Units: Units:

@ surface

@ 1-m Units: $m R / h r$

Total Activity: Units: $\mathrm{nCi} / \mathrm{g}$ 


\begin{tabular}{|c|c|c|c|c|c|c|}
\hline$: \mathbf{P}$ & 006 & & & & WASTI & AlulC \\
\hline HLW & Contac & & & & & $\therefore \quad$ \\
\hline G. RAD & JCLIDE & NCENTRAT & DNS & & & \\
\hline & & pecific Activi & & & & \\
\hline Isotope & Typical & Lower Limit & Upper Limit & Units & Basis Code(s) & Basis Desc. lif other) \\
\hline Tc-99 & & & & & & \\
\hline $\begin{array}{l}\text { A. Passi } \\
\text { B. Segm } \\
\text { C. Passi } \\
\text { D. Radio } \\
\text { E. Mass }\end{array}$ & $\begin{array}{l}\text { tive Neutr } \\
\text { Gamma } \\
\text { tive Neut } \\
\text { cal Analy } \\
\text { trometry }\end{array}$ & $\begin{array}{l}\text { PANI } \\
\text { (SGS) } \\
\text { segmented }\end{array}$ & Scan & & $\begin{array}{l}\text { Accountability } \\
\text { Calorimetry } \\
\text { Multiple Energy } \\
\text { Process Knowle } \\
\text { Other }\end{array}$ & sis (MEGAS) \\
\hline
\end{tabular}


H. MATRIX CHARACTERISTICS

Primary Matrix Code: X7100

Description: Special Waste: Elemental Mercury: :

Combustibility: $\bigcirc$ Combustible (>90\%)

Mixed (10\%-90\%)

Noncombustible $(<10 \%)$

\section{MATRIX COMPOSITION}

\begin{tabular}{|c|c|c|c|c|c|c|}
\hline & & & \multicolumn{4}{|c|}{ Composition } \\
\hline Code & Matrix Description & Material & Typical & LL & UL & Units \\
\hline$\times 7100$ & Special Waste.Elemental Mercury.. & Liquid mercury & & & & \\
\hline
\end{tabular}



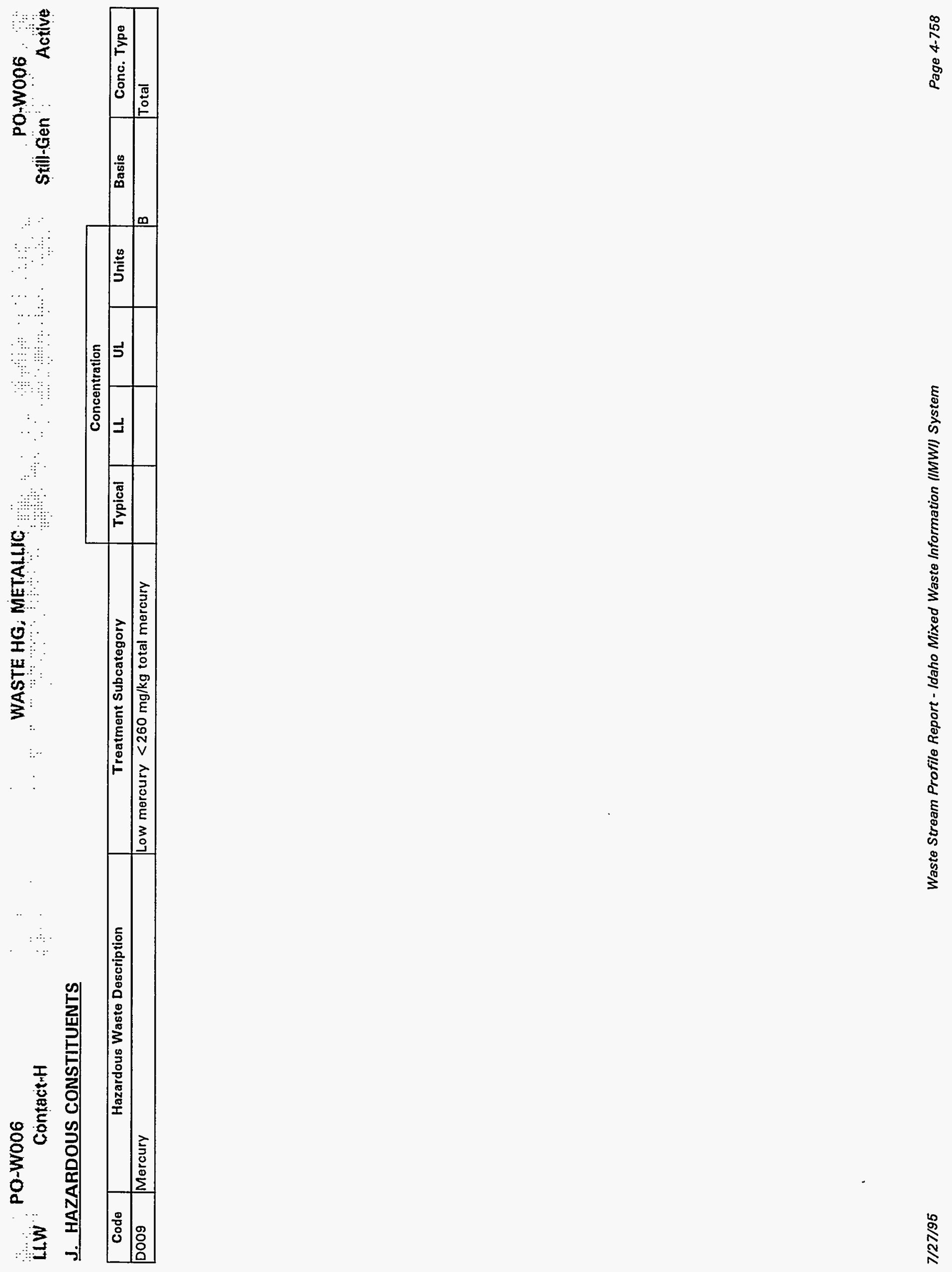


\section{Po-WOOO}

LLW $\mathrm{W}$ Contact $\mathrm{H}$

WASTE HG, METALIIC

pow wook

\section{K. POLYCHLORINATED BIPHENYLS (PCBS)}

Is this waste stream PCB contaminated?

OYes

No

Unknown

If yes, what portion of the waste is PCB contaminated (volume \%):

If $<100 \%$, can the PCB contaminated waste be segregated and treated separately?

What is the PCB concentration in ppm?

If only a portion of the waste is PCB contaminated, report values for contaminated portion.

\section{TREATMENT PLANS}

\begin{tabular}{|c|c|c|c|c|c|c|c|c|c|c|c|}
\hline $\begin{array}{c}\text { Opt. } \\
\#\end{array}$ & $\begin{array}{c}\text { Media Type } \\
\text { (If more than one) }\end{array}$ & $\begin{array}{c}\text { Matrix } \\
\text { Code }\end{array}$ & $\begin{array}{l}\text { Vol. \% } \\
\text { (If }\end{array}$ & Step & JIT & sc & CIF & $\begin{array}{l}\text { Trans. } \\
\text { Miles }\end{array}$ & $\begin{array}{l}\text { Facility } \\
\text { Abbr. }\end{array}$ & Unit Name & Comments \\
\hline \multirow[t]{4}{*}{1} & & $\times 7100$ & & $\mathbf{a}$ & O & 0 & $1.00 E+00$ & & TRANS & Transport - LLW & \\
\hline & & & & $\mathbf{b}$ & 0 & 0 & $1.00 E+00$ & & AMWTF & CH - Opening \& Sorting & \\
\hline & & & & c & 0 & 0 & $1.00 E+00$ & & AMWTF & CH - Amalgamation & \\
\hline & & & & d & 0 & 0 & $1.00 E+00$ & & SCDF & Disposal - Contact Handled & \\
\hline
\end{tabular}

Note: Where provided, media percentages are rough estimates used to facilitate treatment planning. They do not necessarily imply an accurate knowledge of waste stream composition, and were not derived for use outside this conceptual planning exercise.

Describe any special or unique technical concerns related to the treatment of this waste stream that would impact the use or implementation of standard hazardous waste treatment methods. 


PO-W061
LLW $: \because$ Contact-H
A. IDENTIFICATION AND DESCRIPTION
Waste Stream ID: PO-W061
W. S. Name:
Content Code:
FFCA ID:

\section{Waste Stream Description:}

Waste stream consists of materials that are contaminated with mercury. Materials include scrap plastic, gloves, dust, equipment (metal and glass), and floor sweepings. Waste also includes mercury spill clean-up and containers of liquid waste contaminated with mercury.

\section{PO-W061}

Still-Gen . Active

\section{B. GENERATION SITE/PROCESS DESCRIPTION}

The following questions, concerning the generation site and process, should be answered even if the waste stream is no longer generated.

Name of Site Generating Waste (e.g., INEL, Rocky Flats, etc.): Portsmouth Gaseous Diffusion Plant

Location of activities (Area and Building):

$\mathrm{X}-770, \mathrm{X}-608$, Process buildings, Pumphouses, $\mathrm{X}-720$.

Operations (functions) performed in building:

Wquipment maintenance and testing, uranium enrichment, pumping of cooling water, and wellfield.

\section{Description of Process Generating Waste:}

Manometers are dismantled and cleaned, and the waste or spilled

mercury and mercury contaminated materials are part of this waste

stream. In 1989, the \#6B well pump mercury seal failed and released mercury into the well. Waste oil, water, sand, and mercury

generated from this accident make up the remainder of this waste

stream. 
HWW Gontact H

(a)

\section{PROJECTED WASTE GENERATION}

Is this waste stream still generated?

If yes, when is the expected generation termination date?

Current Projections

\begin{tabular}{|r|r|r|r|r|l|}
\hline Est. Date & \multicolumn{1}{|c|}{ Start Year } & Interval & \multicolumn{1}{c|}{ Vol (m3) } & \multicolumn{1}{c|}{ Mass $(\mathbf{k g})$} & Source of Estlmate \\
\hline $11 / 30 / 93$ & 1993 & 1 & 2.00 & 750.00 & $1993-F F C A$ \\
\hline $11 / 30 / 93$ & 1994 & 1 & 2.00 & 750.00 & $1993-F F C A$ \\
\hline $11 / 30 / 93$ & 1995 & 1 & 2.00 & 750.00 & $1993-F F C A$ \\
\hline $11 / 30 / 93$ & 1996 & 1 & 2.00 & 750.00 & $1993-F F C A$ \\
\hline $11 / 30 / 93$ & 1997 & 1 & 2.00 & 750.00 & $1993-F F C A$ \\
\hline $11 / 30 / 93$ & 1998 & 1 & 2.00 & 750.00 & $1993-F F C A$ \\
\hline $11 / 30 / 93$ & 1999 & 1 & 2.00 & 750.00 & $1993-F F C A$ \\
\hline
\end{tabular}

Historical Projections

\begin{tabular}{|r|r|r|r|r|r|}
\hline Est. Date & \multicolumn{1}{|c|}{ Start Year } & Interval & Vol (m3) & \multicolumn{1}{c|}{ Mass (kg) } & Source of Estimate \\
\hline $11 / 30 / 93$ & 1998 & 5 & 10.00 & $3,750.00$ & $1993-F F C A$ \\
\hline $11 / 30 / 93$ & 2003 & 20 & 40.00 & $15,000.00$ & $1993-F F C A$ \\
\hline
\end{tabular}




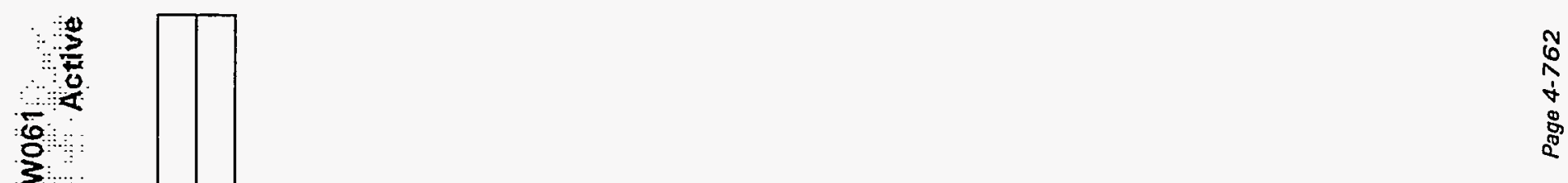


PO-WO61

HiW Contact H

E. RADIATION CHARACTERISTICS

Radioactive Waste Type:

\begin{tabular}{|ll|}
\hline Ollw & Oa-LlW O TRU \\
OhlW & O Non-Rad
\end{tabular}

Mixed Waste:

Handling:

\begin{tabular}{|l|}
\hline O Yes ONo \\
\hline O Contact O Remote \\
\hline
\end{tabular}

Radionuclide Contamination Accessiblity:

External Surface

Internal Surface

Dispersed Through Matrix

\begin{tabular}{|c|c|c|}
\hline OYes & ONo & OUnknown \\
\hline OYes & ONo & O Unknown \\
\hline Y Yes & ONo & OUnknown \\
\hline
\end{tabular}

\section{Activity Levels}

Transuranic Alpha Activity:

Uranium/Thorium Alpha Activity:

Beta/Gamma Activity:

Surface Neutron Activity:

Total Activity:

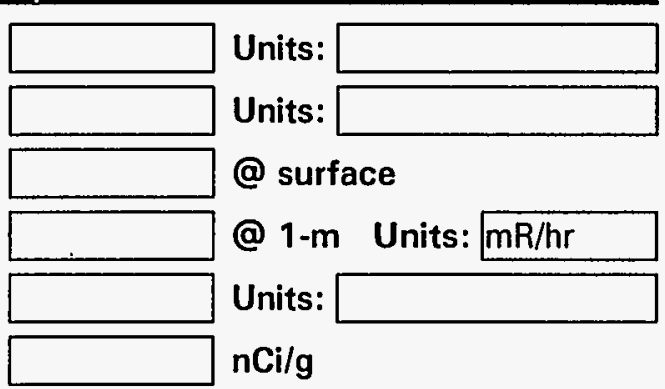

Pö Wo6

\section{F. TRU ALPHA ACTIVITY DISTRIBUTION}

This waste stream is not identified as a TRU or a-LLW. 

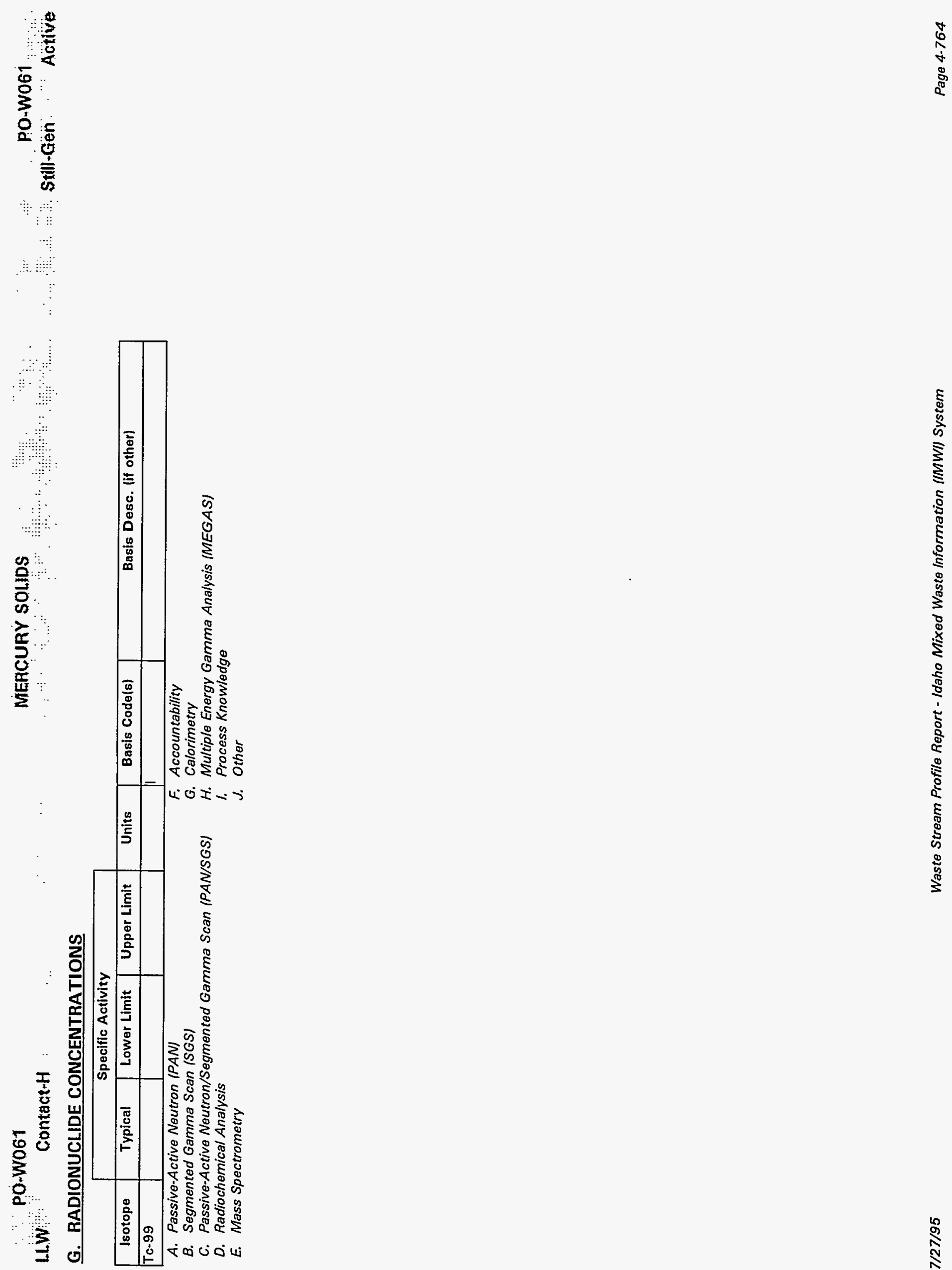

ह5 
PO-WO61

\section{MERCURY SÖLIDS}

PO-W061

LlW Contact H

H. MATRIX CHARACTERISTICS

Primary Matrix Code: $\$ 5490$

Description: Debris Waste: Heterogeneous Debris: Unknown/Other Heterogeneous Debris:

Combustibility: $\bigcirc$ Combustible (>90\%)

Mixed $(10 \%-90 \%)$

Noncombustible $(<10 \%)$

\section{MATRIX COMPOSITION}

\begin{tabular}{|c|c|c|c|c|c|c|}
\hline \multirow[b]{2}{*}{ Code } & \multirow[b]{2}{*}{ Matrix Description } & \multirow[b]{2}{*}{ Material } & \multicolumn{4}{|c|}{ Composition } \\
\hline & & & Typical & $L$ & UL & Units \\
\hline L2190 & Organic Liquids.Aqueous/Organic Liquids.Unknown/Other Aqueous/Organic Liquids. & & & & & \\
\hline$\$ 5490$ & Debris Waste.Heterogeneous Debris.Unknown/Other Heterogeneous Debris. & & & & & \\
\hline$\$ 5390$ & Debris Waste.Organic Debris.Unknown/Other Organic Debris. & & & & & \\
\hline 55129 & Debris Waste.Inorganic Debris.Inorganic Non-Metal Debris.Unknown/Other Inorganic & & & & & \\
\hline $\mathbf{5 5 3 1 9}$ & Debris Waste.Organic Debris.Plastic/Rubber Debris.Unknown/Other Plastic/Rubber D & & & & & \\
\hline $\mathbf{S 5 1 1 0}$ & Debris Waste.Inorganic Debris.Metal Debris. & & & & & \\
\hline
\end{tabular}


PO-W061

Po.W061

LLW _. Contact-H

Stili-Gen Active

J. HAZARDOUS CONSTITUENTS

\begin{tabular}{|c|c|c|c|c|c|c|c|c|}
\hline & & & \multicolumn{4}{|c|}{ Concentration } & & \\
\hline Code & Hazardous Waste Description & Treatment Subcategory & Typical & $\mathbf{L L}$ & UL & Units & Basis & Conc. Type \\
\hline$D 009$ & Mercury & Elemental mercury contaminated with radioact & & & & & $B$ & Total \\
\hline
\end{tabular}


LEW $\quad$ Contagt

\section{K. POLYCHLORINATED BIPHENYLS (PCBS)}

Is this waste stream PCB contaminated?

OYes O No O Unknown

If yes, what portion of the waste is PCB contaminated (volume \%):

If $<100 \%$, can the PCB contaminated waste be segregated and treated separately?

What is the PCB concentration in ppm?

If only a portion of the waste is PCB contaminated, report values for contaminated portion.

\section{TREATMENT PLANS}

\begin{tabular}{|c|c|c|c|c|c|c|c|c|c|c|c|}
\hline $\begin{array}{c}\text { Opt. } \\
\#\end{array}$ & $\begin{array}{c}\text { Media Type } \\
\text { (If more than one) }\end{array}$ & $\begin{array}{c}\text { Matrix } \\
\text { Code }\end{array}$ & $\begin{array}{l}\text { Vol. \% } \\
\text { llf }\end{array}$ & Step & JIT & sc & CIF & $\begin{array}{l}\text { Trans. } \\
\text { Miles }\end{array}$ & $\begin{array}{l}\text { Facility } \\
\text { Abbr. }\end{array}$ & Unit Name & Comments \\
\hline 1 & & $\mathbf{5 5 4 9 0}$ & & $\bar{a}$ & 0 & $\overline{0}$ & $1.00 E+00$ & & TRANS & Transport - LLW & \\
\hline & & & & $\mathbf{b}$ & (O) & $\bar{O}$ & $1.00 \mathrm{E}+00$ & & AMWTF & $\mathrm{CH}$ - Opening \& Sorting & \\
\hline & & & & d & 0 & 0 & $1.00 E+00$ & & AMWTF & $\mathrm{CH}$ - Incineration/Thermal Desorption & \\
\hline & & & & e & 0 & 0 & $1.00 E+00$ & & AMWTF & $\mathrm{CH}$ - Vitrification & \\
\hline & & & & $f$ & 0 & $\bar{O}$ & $1.00 E+00$ & & $S C D F$ & Disposal - Contact Handled & \\
\hline
\end{tabular}

Note: Where provided, media percentages are rough estimates used to facilitate treatment planning. They do not necessarily imply an accurate knowledge of waste stream composition, and were not derived for use outside this conceptual planning exercise.

Describe any special or unique technical concerns related to the treatment of this waste stream that would impact the use or implementation of standard hazardous waste treatment methods. 


\section{PS-W004}

ContactrH

\section{A. IDENTIFICATION AND DESCRIPTION}

Waste Stream ID: PS-W004

W. S. Name:

Content Code:

FFCA ID:

\section{LIQUID WITH F-LISTED SOLVENTS}

*

PS-W004

\section{Waste Stream Description:}

$1,1,1$-Trichloroethane solution used for chemical analysis. Contained in poly bottles. Waste is homogeneous between containers. This waste is not a moratorium or reclassified TRU waste.

\section{LIQUID WITH F-LISTED SOLVENTS}

\section{$\ddot{\mathbf{p S}} \ddot{\mathbf{W}} \mathbf{1 0 0 4}$}

Notrgen Active

\section{B. GENERATION SITE/PROCESS DESCRIPTION}

The following questions, concerning the generation site and process, should be answered even if the waste stream is no longer generated.

Name of Site Generating Waste (e.g., INEL, Rocky Flats, etc.): Puget Sound Naval Shipyard

Location of activities (Area and Building):

Radiochemistry Trailer, Building 839

Operations (functions) performed in building:

Radiochemistry (e.g. titration)

Description of Process Generating Waste:

$1,1,1$-Trichloroethane is one of the many chemicals used for radiochemistry analysis of coolant during criticality testing. 


\section{PS-Wood}

LWW Contact

LIQUID WITH FELISTED SOLVENTTS

C. PROJECTED WASTE GENERATION

Is this waste stream still generated?

If yes, when is the expected generation termination date?
Current Projections
(ats,

PSWWOOH

Not-Gën Active 


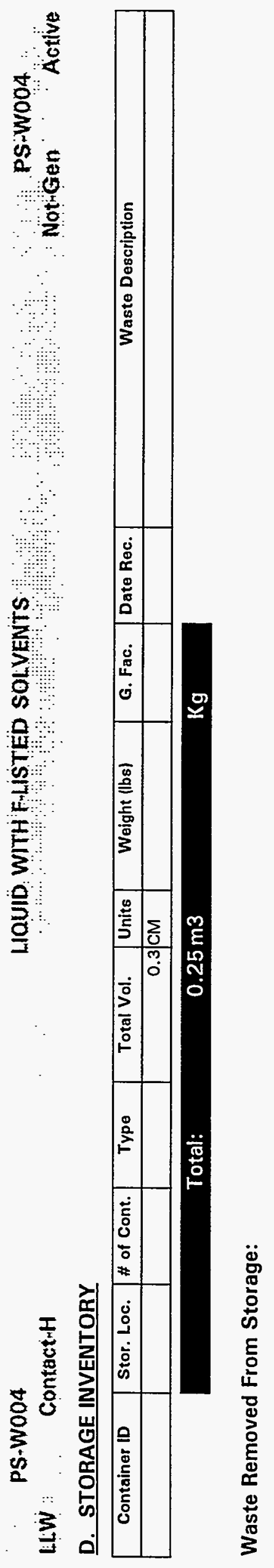


PS-WOO4 U LUDUD WITH FLLISTED SOLVENTS

\section{E. RADIATION CHARACTERISTICS}

Radioactive Waste Type:

$\begin{array}{ll}\text { O llW } & \bigcirc \text { a-llw } \bigcirc \text { TRU } \\ \text { OhlW } & \bigcirc \text { Non-Rad }\end{array}$

Mixed Waste:

\begin{tabular}{|l|}
\hline O Yes O No \\
\hline O Contact O Remote
\end{tabular}

Radionuclide Contamination Accessiblity:

External Surface

Internal Surface

Dispersed Through Matrix

\begin{tabular}{|c|c|c|}
\hline OYes & O No & OUnknown \\
\hline Yes & (C) No & OUnknown \\
\hline OYes & $O$ No & O Ut \\
\hline
\end{tabular}

\section{Activity Levels}

Transuranic Alpha Activity:

Uranium/Thorium Alpha Activity:

Beta/Gamma Activity:

\begin{tabular}{|r|}
\hline \\
\hline \\
\hline$\quad 0.2$ \\
\hline \\
\hline \\
\hline
\end{tabular}

Units:

Units:

F. TRU ALPHA ACTIVITY DISTRIBUTION

This waste stream is not identified as a TRU or a-LLW.

Surface Neutron Activity:

Total Activity:

$0.2 @$ surface

<0.05 @ 1-m Units: mR/hr Units: $\mathrm{nCi} / \mathrm{g}$ 


\section{PS-W004}

Liw : Cöntact+H

LIQUID WITH F-LISTED SOLVENTS

PS-WOOA

\section{G. RADIONUCLIDE CONCENTRATIONS}

\begin{tabular}{|c|c|c|c|c|c|c|}
\hline & \multicolumn{3}{|c|}{ Specific Activity } & \multirow[b]{2}{*}{ Units } & \multirow[b]{2}{*}{ Basis Code(s) } & \multirow[b]{2}{*}{ Basis Desc. (if other) } \\
\hline Isotope & Typical & Lower Limit & Upper Limit & & & \\
\hline \multicolumn{7}{|l|}{ Tc-99 } \\
\hline \multicolumn{7}{|l|}{ C-14 } \\
\hline \multicolumn{7}{|l|}{$\mathrm{Ni}-63$} \\
\hline \multicolumn{7}{|l|}{ Co-58 } \\
\hline \multicolumn{7}{|l|}{$\mathrm{Fe}-55$} \\
\hline \multicolumn{7}{|l|}{ Co-60 } \\
\hline $\begin{array}{l}\text { A. Passiv } \\
\text { B. Segme } \\
\text { C. Passiv } \\
\text { D. Radioc } \\
\text { E. Mass }\end{array}$ & $\begin{array}{l}\text { tive Neutr } \\
\text { Gamma } \\
\text { tive Neutr } \\
\text { cal Analy } \\
\text { trometry }\end{array}$ & $\begin{array}{l}A N I \\
\text { (SGS) } \\
\text { egmented } G \text {. }\end{array}$ & a Scan I & & $\begin{array}{l}\text { Accountability } \\
\text { Calorimetry } \\
\text { Multiple Energy } \\
\text { Process Knowled } \\
\text { Other }\end{array}$ & is (MEGAS) \\
\hline
\end{tabular}


H. MATRIX CHARACTERISTICS

Primary Matrix Code: $L 2210$

Description: Organic Liquids: Pure Organic Liquids: Halogenated Pure Organic Liquids:

Combustibility: $\bigcirc$ Combustible (>90\%)

Mixed $(10 \%-90 \%)$

Noncombustible $(<10 \%)$

\section{MATRIX COMPOSITION}

\begin{tabular}{|c|c|c|c|c|c|c|}
\hline & & & \multicolumn{4}{|c|}{ Composition } \\
\hline Code & Matrix Description & Material & Typical & LL & UL & Units \\
\hline$\lcm{2210}$ & Organic Liquids.Pure Organic Liquids. Halogenated Pure Organic Liquids. & Halogenated Organic Liquid & 100 & 100 & 100 & $\%$ vol \\
\hline
\end{tabular}




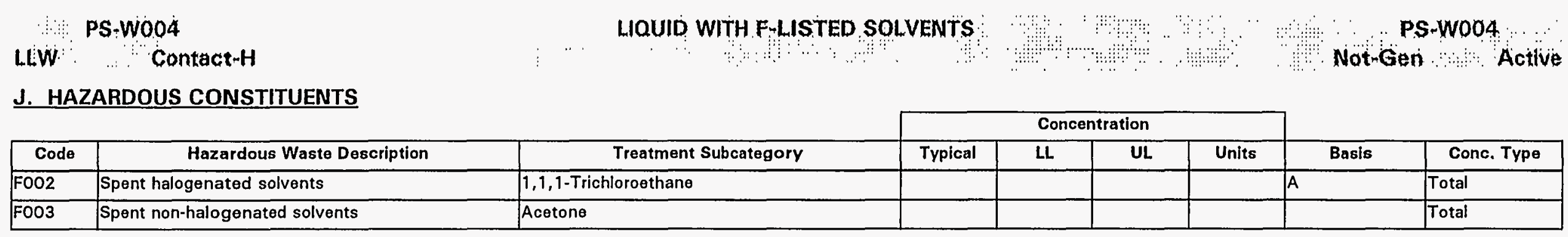




\section{PS-Wo04}

LIQUID WITH FLLISTED SÖLVENTS

LiW Contact-H

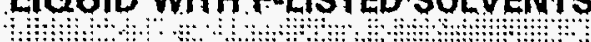

K. POLYCHLORINATED BIPHENYLS (PCBS)

Is this waste stream PCB contaminated?

\section{OYes O No O Unknown}

If yes, what portion of the waste is PCB contaminated (volume \%):

If $<100 \%$, can the PCB contaminated waste be segregated and treated separately?

\section{OYes O No O Unknown}

What is the PCB concentration in ppm? If only a portion of the waste is PCB contaminated, report values for contaminated portion.

\section{TREATMENT PLANS}

\begin{tabular}{|c|c|c|c|c|c|c|c|c|c|c|c|}
\hline $\begin{array}{l}\text { Opt. } \\
\#\end{array}$ & $\begin{array}{c}\text { Media Type } \\
\text { (If more than one) }\end{array}$ & $\begin{array}{l}\text { Matrix } \\
\text { Code }\end{array}$ & $\begin{array}{l}\text { Vol. } \% \\
\text { lIf }\end{array}$ & Step & JIT & sc & CIF & $\begin{array}{l}\text { Trans. } \\
\text { Miles }\end{array}$ & $\begin{array}{l}\text { Facility } \\
\text { Abbr. }\end{array}$ & Unit Name & Comments \\
\hline \multirow[t]{6}{*}{1} & & L2210 & & $\bar{a}$ & O) & 0 & $1.00 E+00$ & & TRANS & Transport - LLW & \\
\hline & & & & $\bar{b}$ & 0 & 0 & $1.00 E+00$ & & AMWTF & $\mathrm{CH}$ - Opening \& Sorting & \\
\hline & & & & c & O) & 0 & $1.00 \mathrm{E}+00$ & & AMWTF & $\mathrm{CH}$ - Sizing & \\
\hline & & & & d & 0 & 0 & $1.00 \mathrm{E}+\mathrm{OO}$ & & AMWTF & $\mathrm{CH}$ - Incineration/Thermal Desorption & \\
\hline & & & & e & $O$ & O & $1.00 E+00$ & & AMWTF & $\mathrm{CH}$ - Vitrification & \\
\hline & & & & $f$ & 0 & O & $1.00 E+00$ & & SCDF & Disposal - Contact Handled & \\
\hline
\end{tabular}

Note: Where provided, media percentages are rough estimates used to facilitate treatment planning. They do not necessarily imply an accurate knowledge of waste stream composition, and were not derived for use outside this conceptual planning exercise.

Describe any special or unique technical concerns related to the treatment of this waste stream that would impact the use or implementation of standard hazardous waste treatment methods. 


\section{$\therefore$ PS-WO06 \\ Contact-H \\ A. IDENTIFICATION AND DESCRIPTION}

Waste Stream ID: PS-W006

W. S. Name:

Content Code:

FFCA ID:

\begin{tabular}{|l|}
\hline SOLIDIFIED LIQUID WITH ACETONE \\
\hline PS-W006 \\
\hline
\end{tabular}

Waste Stream Description:

Solidified water containing acetone. (State-specific waste)

The water contained acetone, methanol and/or EDTA. The liquid was solidified using portland cement and sodium silicule.

No internal shielding is added.

The percent acetone in each drum is not known.

\section{SOLIDIFIED LIQUID WITH ACETONE}

PS-WOOO06

Not-Gen : :...: Active

\section{B. GENERATION SITE/PROCESS DESCRIPTION}

The following questions, concerning the generation site and process, should be answered even if the waste stream is no longer generated.

Name of Site Generating Waste (e.g., INEL, Rocky Flats, etc.): Puget Sound Naval Shipyard

Location of activities (Area and Building):

Building 839 decontamination sink

Operations (functions) performed in building:

Decontamination of reactor plant components, including ultra sonic sink use.

Description of Process Generating Waste:

Radioactive components are wiped and/or immersed in

decontamination liquids. An ultrasonic sink is used, where items are immersed in the liquid. The liquids are discarded when they become radioactive or chemically depleted. 


\section{PSWWOO6}

HW ContactH

C. PROJECTED WASTE GENERATION

Is this waste stream still generated?

If yes, when is the expected generation termination date?

\section{Historical Projections}




\section{Li.W Contact-H \\ D. STORAGE INVENTORY}

SOLIDIFIED LIQUID WITH ACETONIE

PSiW006:

\begin{tabular}{|l|l|l|l|r|l|l|l|l|l|}
\hline Container ID & Stor. Loc. & \# of Cont. & Type & Total Vol. & Units & Weight (lbs) & G. Fac. & Date Rec. & Waste Description \\
\hline & & \multicolumn{7}{|c|}{0.8} & \multicolumn{7}{c|}{$0.84 \mathrm{m3}$} & & & & \\
\hline
\end{tabular}

Waste Removed From Storage: 


\section{F. TRU ALPHA ACTIVITY DISTRIBUTION}

Radioactive Waste Type:

\begin{tabular}{ll} 
OllW & Oa-LLW $\bigcirc$ TRU \\
OHLW & O Non-Rad \\
\hline
\end{tabular}

Mixed Waste:

Handling:

\begin{tabular}{|l|}
\hline OYes O No \\
\hline O Contact O Remote \\
\hline
\end{tabular}

Radionuclide Contamination Accessiblity:

\begin{tabular}{rlrlr|} 
External Surface & OYes & O No & O Unknown \\
\cline { 3 - 5 } Internal Surface & OYes & O No & O Unknown \\
\cline { 2 - 5 } Dispersed Through Matrix & OYes & O No & O Unknown
\end{tabular}

\section{Activity Levels}

Transuranic Alpha Activity:

Uranium/Thorium Alpha Activity:

Beta/Gamma Activity:

Surface Neutron Activity:

Total Activity:

\begin{tabular}{|c|c|c|}
\hline & Units: [ & \\
\hline & Units: [ & \\
\hline 0.23 & @ surfa & ace \\
\hline$<0.05$ & @ 1-m & Units: $\mathrm{mR} / \mathrm{hr}$ \\
\hline & Units: [ & \\
\hline & $\mathrm{nCi} / \mathrm{g}$ & \\
\hline
\end{tabular}

This waste stream is not identified as a TRU or a-LLW. 


\section{PS-W006}

\section{LLW $\because$ Contact-H}

\section{G. RADIONUCLIDE CONCENTRATIONS}

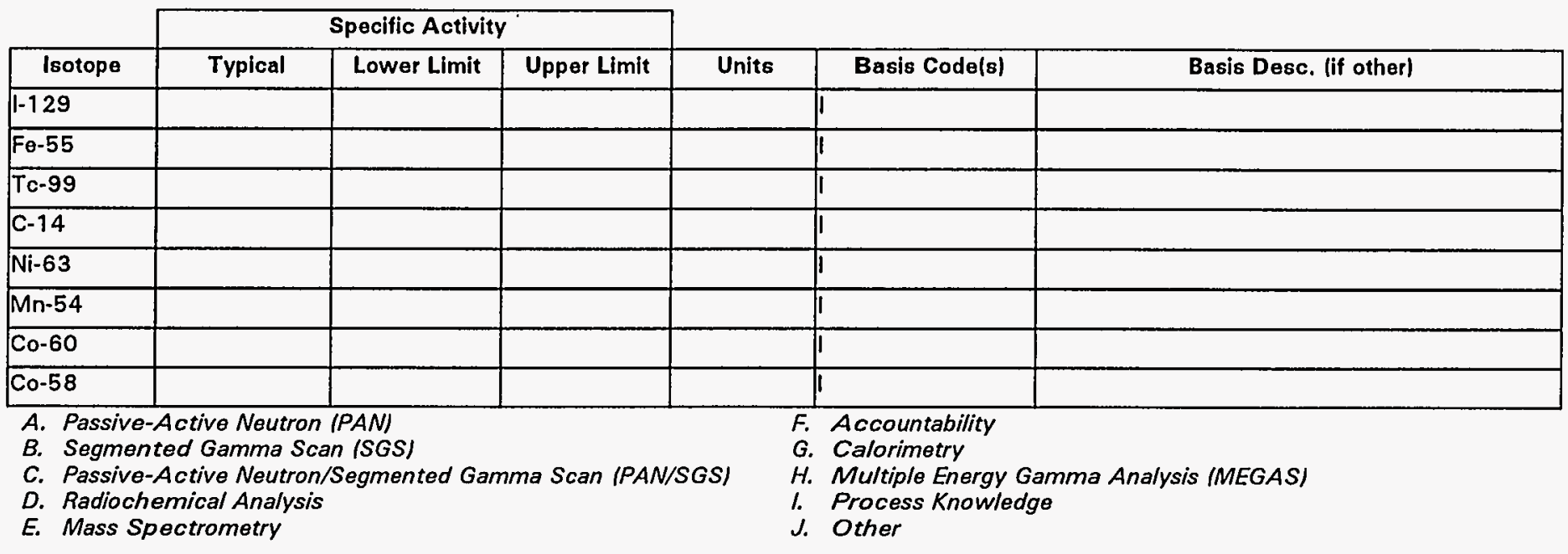

PSWWOOO6 Notmgèn Active 


\section{LLW ContactiH}

\section{SOL}

\section{H. MATRIX CHARACTERISTICS}

Primary Matrix Code: $\widehat{\text { S3150 }}$

Description: Homogeneous Solids: Inorganic Homogeneous Solids: Solidified Homogeneous Solids:

Combustibility: $\bigcirc$ Combustible (>90\%)

Mixed $(10 \%-90 \%)$

Noncombustible $(<10 \%)$

\section{MATRIX COMPOSITION}

\begin{tabular}{|c|c|c|c|c|c|c|}
\hline & & & \multicolumn{4}{|c|}{ Composition } \\
\hline Code & Matrix Description & Material & Typlcal & LL & UL & Units \\
\hline $\mathbf{S 3 1 5 0}$ & Homogeneous Solids.Inorganic Homogeneous Solids.Solidified Homogeneous Solids. & Cemented solids & & & & \\
\hline 53150 & Homogeneous Solids.Inorganic Homogeneous Solids.Solidified Homogeneous Solids. & Aqueous/Non HOC liquids & & & & \\
\hline
\end{tabular}


PS:W006

SOLIDIFIED LIQUID WITH ACETONE

PS-W006

\section{HWW : Contact-H}

Nat-Gen Active

\section{J. HAZARDOUS CONSTITUENTS}

\begin{tabular}{|c|c|c|c|c|c|c|c|c|}
\hline & & & \multicolumn{4}{|c|}{ Concentration } & & \\
\hline Code & Hazardous Waste Description & Treatment Subcategory & Typical & $\mathbf{L L}$ & UL & Units & Basis & Conc. Type \\
\hline$F 003$ & Spent non-halogenated solvents & Acetone & & & & & $A$ & Tota! \\
\hline FO05 & Spent non-halogenated solvents & 2-Ethoxyethanol & & & & & & Total \\
\hline
\end{tabular}




\section{K. POLYCHLORINATED BIPHENYLS (PCBS)}

Is this waste stream PCB contaminated?

\section{OYes O No O Unknown}

If yes, what portion of the waste is PCB contaminated (volume \%): $<50$

If $<100 \%$, can the PCB contaminated waste be segregated and treated separately? OYes O No O Unknown

What is the PCB concentration in ppm? If only a portion of the waste is PCB contaminated, report values for contaminated portion.

\section{TREATMENT PLANS}

\begin{tabular}{|c|c|c|c|c|c|c|c|c|c|c|c|}
\hline $\begin{array}{c}\text { Opt. } \\
\#\end{array}$ & $\begin{array}{c}\text { Media Type } \\
\text { (If more than one) }\end{array}$ & $\begin{array}{l}\text { Matrix } \\
\text { Code }\end{array}$ & $\begin{array}{l}\text { Vol. \% } \\
\text { (If }\end{array}$ & Step & JIT & sc & CIF & $\begin{array}{l}\text { Trans. } \\
\text { Miles }\end{array}$ & $\begin{array}{l}\text { Facility } \\
\text { Abbr. }\end{array}$ & Unit Name & Comments \\
\hline 1 & & $\mathbf{S 3 1 5 0}$ & & $\bar{a}$ & $\mathbf{O}$ & 0 & $1.00 E+00$ & & TRANS & Transport - LLW & \\
\hline & & & & b & $\mathbf{0}$ & 0 & $1.00 E+00$ & & AMWTF & CH. Opening \& Sorting & \\
\hline & & & & $\bar{c}$ & $\mathbf{0}$ & 0 & $1.00 E+00$ & & AMWTF & $\mathrm{CH} \cdot$ Sizing & \\
\hline & & & & $\bar{d}$ & 0 & 0 & $1.00 E+00$ & & AMWTF & $\mathrm{CH}$ - Incineration/Thermal Desorption & \\
\hline & & & & e & 0 & 0 & $1.00 E+00$ & & AMWTF & $\mathrm{CH} \cdot$ Vitrification & \\
\hline & & & & $f$ & 0 & 0 & $1.00 E+00$ & & SCDF & Disposal - Contact Handled & \\
\hline
\end{tabular}

Note: Where provided, media percentages are rough estimates used to facilitate treatment planning. They do not necessarily imply an accurate knowledge of waste stream composition, and were not derived for use outside this conceptual planning exercise.

Describe any special or unique technical concerns related to the treatment of this waste stream that would impact the use or implementation of standard hazardous waste treatment methods. 


\section{LLW Contactr \\ A. IDENTIFICATION AND DESCRIPTION}

Waste Stream ID: PS-W007

W. S. Name:

\section{DEBRIS WITH HEAVY METALS AND PCB}

Content Code:

FFCA ID:

$*$

PS-W007

\section{Waste Stream Description:}

Debris containing PCB's, lead, cadmium, and/or chromium (cloth, plastic, small components,

etc.). Includes electrical components - such as wire, transformers, current adjusters/controllers, capacitors and solenoids. Includes valves with rubber parts (o-rings). This waste stream varies

in homogeneity between containers. Containers may contain only one hazardous constituent and

others may contain all identified hazardous constituents of this waste stream. This waste is not a moratorium waste or reclassified TRU waste.
PS-WOOT:

Still:Gen Active

\section{B. GENERATION SITE/PROCESS DESCRIPTION}

The following questions, concerning the generation site and process, should be answered even if the waste stream is no longer generated.

Name of Site Generating Waste (e.g., INEL, Rocky Flats, etc.): Puget Sound Naval Shipyard

Location of activities (Area and Building):

Ships in drydocks and at piers.

Operations (functions) performed in building:

Disassembly of ships for decommissioning.

Description of Process Generating Waste:

Valves/electrical components are removed from the ship as

interference to decommissioning/disposal. 
ULW

\section{PROJECTED WASTE GENERATION}

Is this waste stream still generated?

OYes ONo

If yes, when is the expected generation termination date?

Current Projections

\begin{tabular}{|r|r|r|r|r|r|}
\hline Est. Date & \multicolumn{1}{|c|}{ Start Year } & Interval & \multicolumn{1}{|l|}{ Vol (m) } & Mass $(\mathrm{kg})$ & Source of Estimate \\
\hline $11 / 15 / 94$ & 1997 & 1 & 0.50 & & 1994 DSTP \\
\hline
\end{tabular}

Historical Projections

\begin{tabular}{|r|r|r|r|r|l|}
\hline Est. Date & \multicolumn{1}{|c|}{ Start Year } & \multicolumn{1}{|c|}{ Interval } & \multicolumn{1}{|c|}{ Vol (m3) } & \multicolumn{1}{c|}{ Mass (kg) } & Source of Estlmate \\
\hline $11 / 1 / 93$ & 1994 & 1 & 1.80 & 340.20 & $1993-F F C A$ \\
\hline $11 / 1 / 93$ & 1995 & 1 & 1.80 & 340.20 & $1993-F F C A$ \\
\hline $11 / 1 / 93$ & 1996 & 1 & 1.80 & 340.20 & $1993-F F C A$ \\
\hline $11 / 1 / 93$ & 1997 & 1 & 1.80 & 340.20 & $1993-F F C A$ \\
\hline
\end{tabular}


PS.W007

LiW "Contact-H

\section{STORAGE INVENTORY}

\begin{tabular}{|c|c|c|c|c|c|c|c|c|c|}
\hline Container ID & Stor. Loc. & \# of Cont. & Type & Total Vol. & Units & Weight (lbs) & G. Fac. & \begin{tabular}{|l|} 
Date Rec. \\
\end{tabular} & Waste Description \\
\hline & & & & & CM & & & & \\
\hline
\end{tabular}

Waste Removed From Storage: 


\section{EWW \\ PS-W007 \\ E. RADIATION CHARACTERISTICS}

Radioactive Waste Type:

\begin{tabular}{|ll|}
\hline OllW & Oa-LlW O TRU \\
O hLW & Onon-Rad \\
\hline
\end{tabular}

Mixed Waste:

\begin{tabular}{|l|}
\hline O Yes O No \\
\hline O Contact O Remote \\
\hline
\end{tabular}

Handling: $O$ Contact $O$ Remote

Radionuclide Contamination Accessiblity:

\begin{tabular}{|c|c|c|c|}
\hline External Surface & OYes & O No & Unknown \\
\hline Inte & OYes & (C) No & OUnknown \\
\hline
\end{tabular}

Dispersed Through Matrix $O$ Yes ONo OUnknown

\section{Activity Levels}

Transuranic Alpha Activity:

Uranium/Thorium Alpha Activity:

Beta/Gamma Activity:

\begin{tabular}{|c|c|c|}
\hline & Units: & \\
\hline & Units: [ & \\
\hline$<0.05$ & @ surfa & \\
\hline$<0.05$ & @ 1-m & Units: $m R / h r$ \\
\hline & Units: & \\
\hline & $\mathbf{n C i} / \mathrm{g}$ & \\
\hline
\end{tabular}

Surface Neutron Activity: Total Activity: $\mathrm{nCi} / \mathrm{g}$

\section{DEBRIS WITH HEÁVY METALS ÄND̈ PCB}

\section{F. TRU ALPHA ACTIVITY DISTRIBUTION}

This waste stream is not identified as a TRU or a-LLW. 


\section{LLW .... Contact-H}

\section{G. RADIONUCLIDE CONCENTRATIONS}

\begin{tabular}{|c|c|c|c|c|c|c|}
\hline & \multicolumn{3}{|c|}{ Specific Activity } & \multirow[b]{2}{*}{ Units } & \multirow[b]{2}{*}{ Basis Code(s) } & \multirow[b]{2}{*}{ Basis Desc. (if other) } \\
\hline Isotope & Typical & Lower Limit & Upper Limit & & & \\
\hline Tc-99 & & & & & I & \\
\hline \multicolumn{7}{|l|}{ C-14 } \\
\hline \multicolumn{7}{|l|}{$\overline{N i-63}$} \\
\hline \multicolumn{7}{|l|}{ Co-58 } \\
\hline \multicolumn{7}{|l|}{$F_{\theta} \cdot 55$} \\
\hline \multicolumn{7}{|l|}{ Co-60 } \\
\hline $\begin{array}{l}\text { A. Passiv } \\
\text { B. Segme } \\
\text { C. Passiv } \\
\text { D. Radioc } \\
\text { E. Mass }\end{array}$ & $\begin{array}{l}\text { ive Neutr } \\
\text { Gamma } \\
\text { ive Neutr } \\
\text { cal Analy } \\
\text { rometry }\end{array}$ & $\begin{array}{l}\text { PAN) } \\
\text { (SGS) } \\
\text { egmented G }\end{array}$ & Scan $(P A$ & ) & $\begin{array}{l}\text { Accountability } \\
\text { Calorimetry } \\
\text { Multiple Energy } \\
\text { Process Knowleo } \\
\text { Other }\end{array}$ & is (MEGAS) \\
\hline
\end{tabular}


H. MATRIX CHARACTERISTICS

Primary Matrix Code: $\$ 5420$

Description: Debris Waste: Heterogeneous Debris: Predominantly Inorganic Debris:

\section{Combustibility: $\bigcirc$ Combustible (>90\%) \\ Mixed $(10 \%-90 \%)$ \\ Noncombustible $<<10 \%)$}

\section{MATRIX COMPOSITION}

\begin{tabular}{|c|c|c|c|c|c|c|}
\hline & & & \multicolumn{4}{|c|}{ Composition } \\
\hline Code & Matrix Description & Materlal & Typical & LL & UL & Units \\
\hline$\$ 5330$ & Debris Waste.Organic Debris.Paper/Cloth Debris. & Cloth & & & & \\
\hline 55310 & Debris Waste.Organic Debris.Plastic/Rubber Debris. & Plastic/Rubber & & & & \\
\hline 55110 & Debris Waste.Inorganic Debris.Metal Debris. & Metal Components & & & & \\
\hline
\end{tabular}




\section{J. HAZARDOUS CONSTITUENTS}

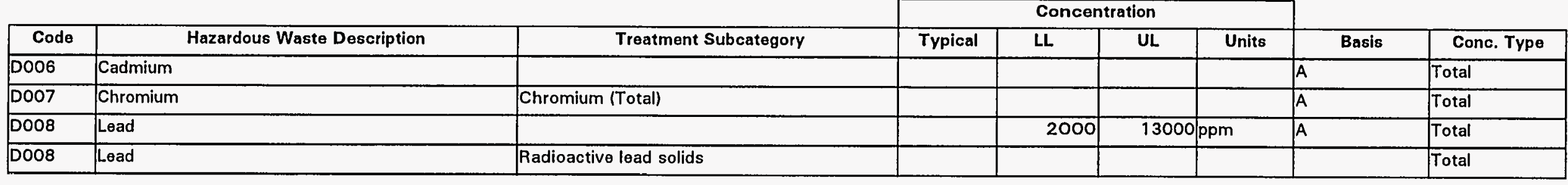




\section{K. POLYCHLORINATED BIPHENYLS (PCBS)}

Is this waste stream PCB contaminated? OYes O No O Unknown

If yes, what portion of the waste is PCB contaminated (volume \%): 25-100

If $<100 \%$, can the PCB contaminated waste be segregated and treated separately?

What is the PCB concentration in ppm? If only a portion of the waste is PCB contaminated, report values for contaminated portion.

\section{TREATMENT PLANS}

\begin{tabular}{|c|c|c|c|c|c|c|c|c|c|c|c|}
\hline $\begin{array}{c}\text { Opt. } \\
\#\end{array}$ & $\begin{array}{c}\text { Media Type } \\
\text { (If more than one) }\end{array}$ & $\begin{array}{c}\text { Matrix } \\
\text { Code }\end{array}$ & $\begin{array}{l}\text { Vol. \% } \\
\text { IIf }\end{array}$ & Step & |JIT & sc & CIF & $\begin{array}{l}\text { Trans. } \\
\text { Miles }\end{array}$ & $\begin{array}{l}\text { Facility } \\
\text { Abbr. }\end{array}$ & Unit Name & Comments \\
\hline 1 & & 55420 & & $\bar{a}$ & O) & 0 & $1.00 E+00$ & & TRANS & Transport - LLW & \\
\hline & & & & $\bar{b}$ & (O) & $\overline{0}$ & $1.00 E+00$ & & AMWTF & $\mathrm{CH}$ - Opening \& Sorting & \\
\hline & & & & $\bar{c}$ & (O) & 0 & $1.00 E+00$ & & AMWTF & CH-Sizing & \\
\hline & & & & d & O & 0 & $1.00 E+00$ & & AMWTF & $\mathrm{CH}$ - Incineration/Thermal Desorption & \\
\hline & & & & $\theta$ & 0 & 0 & $1.00 E+00$ & & AMWTF & CH - Vitrification & \\
\hline & & & & $f$ & 0 & 0 & $1.00 \mathrm{E}+00$ & & SCDF & Disposal - Contact Handled & \\
\hline
\end{tabular}

Note: Where provided, media percentages are rough estimates used to facilitate treatment planning. They do not necessarily imply an accurate knowledge of waste stream composition, and were not derived for use outside this conceptual planning exercise.

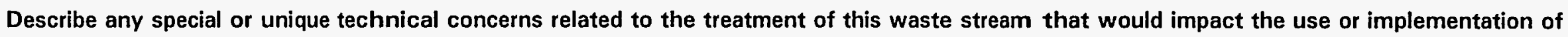
standard hazardous waste treatment methods. 


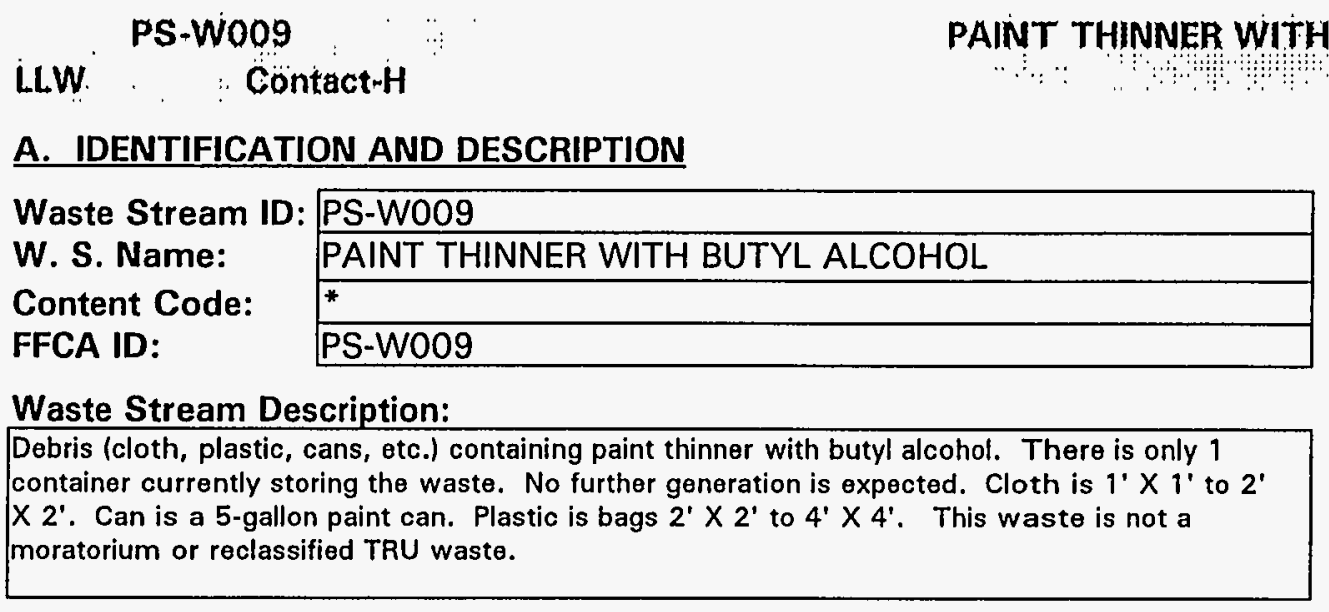

Waste Stream ID: PS-W009

W. S. Name:

Content Code:

Waste Stream Description:

Debris (cloth, plastic, cans, etc.) containing paint thinner with butyl alcohol. There is only 1

$X 2^{\prime}$. Can is a 5-gallon paint can. Plastic is bags $2^{\prime} \times 2^{\prime}$ to $4^{\prime} \times 4^{\prime}$. This waste is not a

moratorium or reclassified TRU waste.

\section{B. GENERATION SITE/PROCESS DESCRIPTION}

The following questions, concerning the generation site and process, should be answered even if the waste stream is no longer generated.

Name of Site Generating Waste (e.g., INEL, Rocky Flats, etc.): Puget Sound Naval Shipyard

Location of activities (Area and Building):

Ship in dry dock.

Operations (functions) performed in building:

Painting radioactive tank (cleaning paint sprayers).

Description of Process Generating Waste:

Paint was applied in a radioactive tank. Paint sprayers were cleaned using butyl alcohol. 
LlEW ContactH A Actue C. PROJECTED WASTE GENERATION

Is this waste stream still generated?

If yes, when is the expected generation termination date?

OYes O No

Current Projections

Historical Projections 


\section{Pי̈.WOOg}

LlWW: Contact-H

D. STORAGE INVENTORY

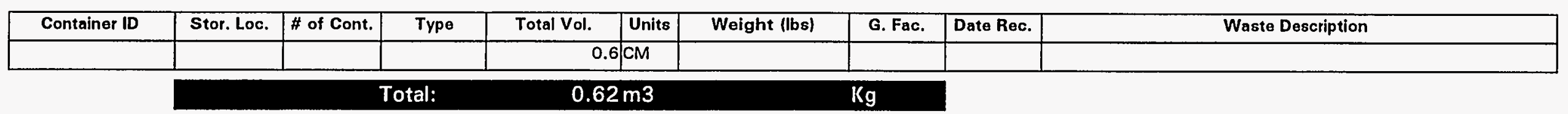

Waste Removed From Storage:

\section{PAINT THINNER WITH BUTYL ALCOHOL}

PSWWÖ09

Notigen Active
MER W... BUTYLALCOHOL.? 


\section{PS-Woog} HLW Contact H

\section{E. RADIATION CHARACTERISTICS}

PAINT THINNER WITH BUTYL ALCOHOL

PSTWOÖg

Radioactive Waste Type:

\begin{tabular}{|ll|}
\hline OlLW & Oa-LLW O TRU \\
O HLW & O Non-Rad \\
\hline
\end{tabular}

Mixed Waste:

\begin{tabular}{|l|}
\hline OYes O No \\
\hline O Contact O Remote \\
\hline
\end{tabular}

Radionuclide Contamination Accessiblity:

External Surface

Internal Surface

Dispersed Through Matrix

\begin{tabular}{|c|c|c|}
\hline OYes & ONo & OUnknown \\
\hline OYes & O No & O Unknown \\
\hline OYes & ONo & OUnknown \\
\hline
\end{tabular}

\section{Activity Levels}

Transuranic Alpha Activity:

Uranium/Thorium Alpha Activity: Beta/Gamma Activity:

\begin{tabular}{l}
$\square$ Units: $\square$ \\
$\square$ Units: $\square$ \\
\hline
\end{tabular}

\section{F. TRU ALPHA ACTIVITY DISTRIBUTION}

This waste stream is not identified as a TRU or a-LLW.

<0.05 @ 1-m Units: mR/hr

Surface Neutron Activity: Units:

Total Activity: $\mathrm{nCi} / \mathrm{g}$ 


\section{LLW Contact-H}

\section{G. RADIONUCLIDE CONCENTRATIONS}

\begin{tabular}{|c|c|c|c|c|c|c|}
\hline & \multicolumn{3}{|c|}{ Specific Activity } & \multirow[b]{2}{*}{ Units } & \multirow[b]{2}{*}{ Basis Code(s) } & \multirow[b]{2}{*}{ Basis Desc. (if other) } \\
\hline Isotope & Typical & Lower Limit & Upper Limit & & & \\
\hline \multicolumn{7}{|l|}{ Tc-99 } \\
\hline \multicolumn{7}{|l|}{$1-129$} \\
\hline \multicolumn{7}{|l|}{$C-14$} \\
\hline \multicolumn{7}{|l|}{$\mathrm{Ni}-63$} \\
\hline \multicolumn{7}{|l|}{ Co-58 } \\
\hline \multicolumn{7}{|l|}{$M n-54$} \\
\hline \multicolumn{7}{|l|}{ Fe-55 } \\
\hline \multicolumn{7}{|l|}{ Co-60 } \\
\hline $\begin{array}{l}\text { A. Passiv } \\
\text { B. Segme } \\
\text { C. Passiv } \\
\text { D. Radioc } \\
\text { E. Mass }\end{array}$ & $\begin{array}{l}\text { ive Neutr } \\
\text { GammaS } \\
\text { ive Neutr } \\
\text { cal Ana/v } \\
\text { rometry }\end{array}$ & $\begin{array}{l}\text { ANI } \\
\text { (SGS) } \\
\text { egmented }\end{array}$ & la Scan If & SI & $\begin{array}{l}\text { Accountability } \\
\text { Calorimetry } \\
\text { Multiple Energy } \\
\text { Process Knowle } \\
\text { Other }\end{array}$ & sis (MEGAS) \\
\hline
\end{tabular}




\section{H. MATRIX CHARACTERISTICS}

Primary Matrix Code: S5490

Description: Debris Waste: Heterogeneous Debris: Unknown/Other Heterogeneous Debris:

\section{Combustibility: $\bigcirc$ Combustible (>90\%) \\ Mixed (10\%-90\%) \\ Noncombustible (<10\%)}

\section{MATRIX COMPOSITION}

\begin{tabular}{|c|c|c|c|c|c|c|}
\hline & & & \multicolumn{4}{|c|}{ Composition } \\
\hline Code & Matrix Description & Material & Typlcal & LL & UL & Units \\
\hline$\$ 5330$ & Debris Waste.Organic Debris.Paper/Cloth Debris. & Paper/Cloth Debris & & & & \\
\hline 55313 & Debris Waste.Organic Debris.Plastic/Rubber Debris.Nonhalogenated Plastic Debris & Non-Halogenated Plastic & & & & \\
\hline 55110 & Debris Waste.Inorganic Debris.Metal Debris. & Metal Debris/Cans & & & & \\
\hline 53130 & Homogeneous Solids.Inorganic Homogeneous Solids.Paint Waste. & Paint Waste & & & & \\
\hline
\end{tabular}




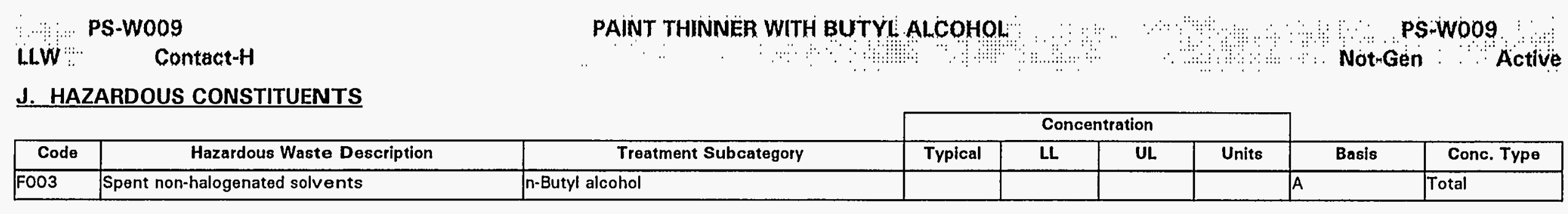


LLW Contact H

\section{K. POLYCHLORINATED BIPHENYLS (PCBS)}

Is this waste stream PCB contaminated?

O Yes O No O Unknown

If yes, what portion of the waste is PCB contaminated (volume \%):

If $<100 \%$, can the PCB contaminated waste be segregated and treated separately?

What is the PCB concentration in ppm?

$$
<50
$$

\section{TREATMENT PLANS}

\begin{tabular}{|c|c|c|c|c|c|c|c|c|c|c|c|}
\hline $\begin{array}{c}\text { Opt. } \\
\#\end{array}$ & $\begin{array}{c}\text { Media Type } \\
\text { (If more than one) }\end{array}$ & $\begin{array}{c}\text { Matrix } \\
\text { Code }\end{array}$ & $\begin{array}{l}\text { Vol. } \% \\
\text { IIf }\end{array}$ & Step & JIT & sc & CIF & $\begin{array}{l}\text { Trans. } \\
\text { Miles }\end{array}$ & $\begin{array}{l}\text { Facility } \\
\text { Abbr. }\end{array}$ & Unit Name & Comments \\
\hline 1 & & $\$ 5490$ & & a & $\mathbf{0}$ & $\bar{O}$ & $1.00 E+00$ & & TRANS & Transport - LLW & \\
\hline & & & & $\mathbf{b}$ & $\mathbf{O}$ & O & $1.00 \mathrm{E}+00$ & & AMWTF & $\mathrm{CH}$ - Opening \& Sorting & \\
\hline & & & & c & O & 0 & $1.00 E+00$ & & AMWTF & $\mathrm{CH}$ - Sizing & \\
\hline & & & & d & 0 & $\overline{\mathrm{O}}$ & $1.00 E+00$ & & AMWTF & $\mathrm{CH}$ - Incineration/Thermal Desorption & \\
\hline & & & & e & 0 & 0 & $1.00 E+00$ & & AMWTF & $\mathrm{CH}$ - Vitrification & \\
\hline & & & & $f$ & 0 & 0 & $1.00 E+00$ & & SCDF & Disposal - Contact Handled & \\
\hline
\end{tabular}

Note: Where provided, media percentages are rough estimates used to facilitate treatment planning. They do not necessarily imply an accurate knowledge of waste stream composition, and were not derived for use outside this conceptual planning exercise.

Describe any special or unique technical concerns related to the treatment of this waste stream that would impact the use or implementation of standard hazardous waste treatment methods. 


\begin{tabular}{|c|c|}
\hline \multicolumn{2}{|l|}{ PS-W011 } \\
\hline \multicolumn{2}{|c|}{ ContactrH } \\
\hline \multicolumn{2}{|c|}{ A. IDENTIFICATION AND DESCRIPTION } \\
\hline \multirow{4}{*}{$\begin{array}{l}\text { Waste Stream ID: } \\
\text { W. S. Name: } \\
\text { Content Code: } \\
\text { FFCA ID: }\end{array}$} & PS-W011 \\
\hline & DEBRIS WITH TCLP METALS/F LISTED SOLVENT \\
\hline & \\
\hline & PS-W011 \\
\hline \multicolumn{2}{|c|}{ Waste Stream Description: } \\
\hline \multicolumn{2}{|c|}{$\begin{array}{l}\text { Debris containing lead, chromium, cadmium, and acetone. Waste stream is stored in a single } \\
\text { container. Waste consists of cloth } 1^{\prime} \times 1^{\prime} \text { to } 4^{\prime} \times 4^{\prime} \text {, miscellaneous metal items up to } 12^{\prime \prime} \text { in } \\
\text { length and plastic from } 1^{\prime} \times 1^{\prime} \text { to } 4^{\prime} \times 4^{\prime} \text {. This waste is not a moratorium waste or reclassified } \\
\text { TRU waste. }\end{array}$} \\
\hline
\end{tabular}

\section{B. GENERATION SITE/PROCESS DESCRIPTION}

The following questions, concerning the generation site and process, should be answered even if the waste stream is no longer generated.

Name of Site Generating Waste (e.g., INEL, Rocky Flats, etc.): Puget Sound Naval Shipyard

Location of activities (Area and Building):

Ships in dry dock and at piers, building 839 .

Operations (functions) performed in building:

Cleaning and decontamination of Naval Nuclear Propulsion system and components using rags.

Description of Process Generating Waste:

Rags were used to decontaminate components and clean tanks. Liquid was generated by dewatering rags. 


\section{PROJECTED WASTE GENERATION}

Is this waste stream still generated?

If yes, when is the expected generation termination date?

Current Projections

Historical Projections 


\section{. . .PS-W011 \\ LLW Contact H
D. STORAGE INVENTORY}

DEBRIS WITH TCLP METALS/F LISTED SOLVENT

PS-W011

\begin{tabular}{|c|c|c|c|c|c|c|c|c|c|}
\hline Container ID & Stor. Loc. & \# of Cont. & Type & Total Vol. & Units & Weight (lbs) & G. Fac. & \begin{tabular}{|l|} 
Date Rec. \\
\end{tabular} & Waste Description \\
\hline & & & & \multicolumn{3}{|c|}{\begin{tabular}{l|l}
0.2 & $\mathrm{CM}$ \\
\end{tabular}} & & & \\
\hline & \multicolumn{6}{|c|}{ Total: } & & & \\
\hline
\end{tabular}

Waste Removed From Storage: 


\section{E. RADIATION CHARACTERISTICS}

Radioactive Waste Type:

\begin{tabular}{|ll|}
\hline OllW & Oa-lLW $\bigcirc$ TRU \\
OhlW & Onon-Rad \\
\hline
\end{tabular}

Mixed Waste:

Handling:

O Yes ONo

Radionuclide Contamination Accessiblity:

\begin{tabular}{rlrl|}
\cline { 3 - 4 } External Surface & OYes & O No & O Unknown \\
Internal Surface & OYes & O No & O Unknown \\
\cline { 2 - 4 } Dispersed Through Matrix & OYes & O No & O Unknown \\
\cline { 2 - 3 } & &
\end{tabular}

\section{Activity Levels}

Transuranic Alpha Activity: Uranium/Thorium Alpha Activity: Beta/Gamma Activity:

Surface Neutron Activity: Total Activity:

\begin{tabular}{l}
\hline \\
\hline Units: \\
\hline Units: \\
\hline 0.3 @ surface \\
\hline$<0.05$ @ 1-m Units: mR/hr \\
\hline Units: \\
\hline nCi/g
\end{tabular}

\section{F. TRU ALPHA ACTIVITY DISTRIBUTION}

This waste stream is not identified as a TRU or a-LLW. 
PS-W011

\section{LLW Contact-H}

\section{G. RADIONUCLIDE CONCENTRATIONS}

\begin{tabular}{|c|c|c|c|c|c|c|}
\hline & \multicolumn{3}{|c|}{ Specific Activity } & \multirow[b]{2}{*}{ Units } & \multirow[b]{2}{*}{ Basis Code(s) } & \multirow[b]{2}{*}{ Basis Desc. (if other) } \\
\hline Isotope & Typical & Lower Limit & Upper Limit & & & \\
\hline \multicolumn{7}{|l|}{ Tc-99 } \\
\hline \multicolumn{7}{|l|}{$1-129$} \\
\hline \multicolumn{7}{|l|}{ C-14 } \\
\hline \multicolumn{7}{|l|}{$\mathrm{Ni}-63$} \\
\hline \multicolumn{7}{|l|}{ Co-58 } \\
\hline \multicolumn{7}{|l|}{$M n-54$} \\
\hline \multicolumn{7}{|l|}{$F \theta-55$} \\
\hline \multicolumn{7}{|l|}{ Co-60 } \\
\hline $\begin{array}{l}\text { A. Passiv } \\
\text { B. Segm } \\
\text { C. Passiv } \\
\text { D. Radioc } \\
\text { E. Mass }\end{array}$ & $\begin{array}{l}\text { ive Neutr } \\
\text { GammaS } \\
\text { ive Neutr } \\
\text { cal Analy } \\
\text { rometry }\end{array}$ & $\begin{array}{l}A N \text { ) } \\
\text { (SGS) } \\
\text { egmented } G\end{array}$ & a Scan & & $\begin{array}{l}\text { Accountability } \\
\text { Calorimetry } \\
\text { Multiple Energy } \\
\text { Process Knowlec } \\
\text { Other }\end{array}$ & is (MEGAS) \\
\hline
\end{tabular}

1. Process Knowledge
PS-WO11

Notmen

\section{DEBRIS WITH TCLP METALS/F LISTED SOLVENT}

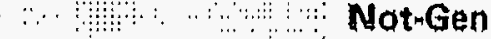


PS-WO11

HLW Contact $-H$

DEBRIS WITH TCLP̈ METALSSIF LISTED SOLVENT

PS-WVOT1

Not-Gen : Active

\section{H. MATRIX CHARACTERISTICS}

Primary Matrix Code: 55490

Description: Debris Waste: Heterogeneous Debris: Unknown/Other Heterogeneous Debris:

Combustibility:

Combustible $(>90 \%)$

Mixed (10\%-90\%)

Noncombustible $(<10 \%)$

\section{MATRIX COMPOSITION}

\begin{tabular}{|c|c|c|c|c|c|c|}
\hline & & & \multicolumn{4}{|c|}{ Compositton } \\
\hline Code & Matrix Description & Materlal & Typical & LL & UL & Units \\
\hline 55330 & Debris Waste.Organic Debris.Paper/Cloth Debris. & Paper/Cloth Debris & & & & \\
\hline 55313 & Debris Waste.Organic Debris.Plastic/Rubber Debris.Nonhalogenated Plastic Debris & Non-Halogenated Plastic Debris & & & & \\
\hline$\$ 5110$ & Debris Waste.Inorganic Debris.Metal Debris. & Metal Debris & & & & \\
\hline
\end{tabular}




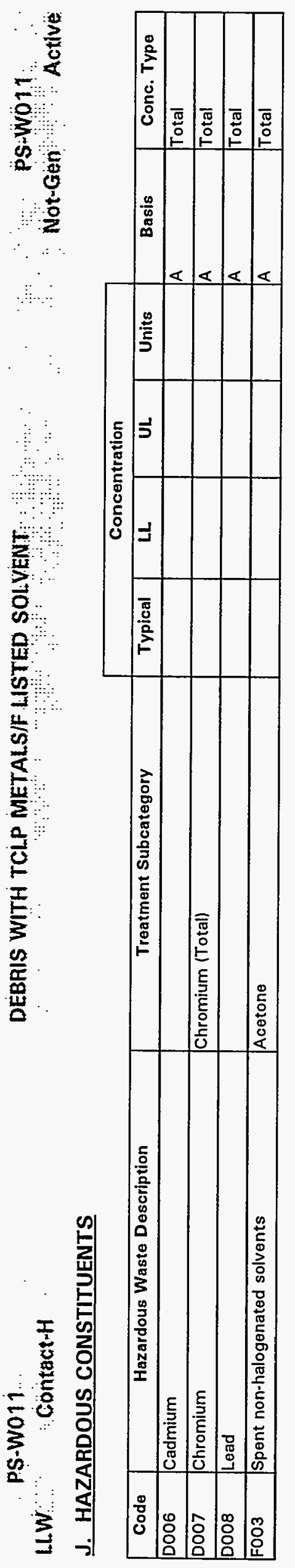




\section{PS:Wo11}

\section{DEBÄIS WITH TCLP MIETÄLS/F LISTED SOLVENT}

\section{LLW Contact}

\section{K. POLYCHLORINATED BIPHENYLS (PCBS)}

Is this waste stream PCB contaminated?

OYes O No O Unknown

If yes, what portion of the waste is PCB contaminated (volume \%):

If $<100 \%$, can the PCB contaminated waste be segregated and treated separately? OYes O No O Unknown

What is the PCB concentration in ppm? If only a portion of the waste is PCB contaminated, report values for contaminated portion.

\section{TREATMENT PLANS}

\begin{tabular}{|c|c|c|c|c|c|c|c|c|c|c|c|}
\hline $\begin{array}{c}\text { Opt. } \\
\#\end{array}$ & $\begin{array}{c}\text { Media Type } \\
\text { (If more than one) }\end{array}$ & $\begin{array}{c}\text { Matrix } \\
\text { Code }\end{array}$ & $\begin{array}{l}\text { Vol. \% } \\
\text { (If }\end{array}$ & Step & JiT & sc & CIF & $\begin{array}{l}\text { Trans. } \\
\text { Miles }\end{array}$ & $\begin{array}{l}\text { Facility } \\
\text { Abbr. }\end{array}$ & Unit Name & Comments \\
\hline \multirow[t]{5}{*}{1} & & S5490 & & $\bar{a}$ & 0 & O & $1.00 \mathrm{E}+00$ & & TRANS & Transport - LLW & \\
\hline & & & & $\bar{b}$ & O & O & $1.00 E+00$ & & AMWTF & $\mathrm{CH}$ - Opening \& Sorting & \\
\hline & & & & d & 0 & $\mathrm{O}$ & $1.00 E+00$ & & AMWTF & $\mathrm{CH}$ - Incineration/Thermal Desorption & \\
\hline & & & & e & $\bar{O}$ & $\bar{O}$ & $1.00 \mathrm{E}+00$ & & AMWTF & CH - Vitrification & \\
\hline & & & & $f$ & O & $\mathrm{O}$ & $1.00 E+00$ & & SCDF & Disposal - Contact Handled & \\
\hline
\end{tabular}

Note: Where provided, media percentages are rough estimates used to facilitate treatment planning. They do not necessarily imply an accurate knowledge of waste stream composition, and were not derived for use outside this conceptual planning exercise.

Describe any special or unique technical concerns related to the treatment of this waste stream that would impact the use or implementation of standard hazardous waste treatment methods. 


\section{A. IDENTIFICATION AND DESCRIPTION}

Waste Stream ID: PS-W012

W. S. Name:

Content Code: FFCA ID:

\section{CHROMIUM \& LEAD BASED PAINT CHIPS W/PCB}

\section{Waste Stream Description:}

Paint removed from radioactive surfaces. This mixed waste stream has not been generated. However, based on similar non-hazardous radioactive waste streams, this stream will consist of paint chips and dust less than $1 \mathrm{~cm}$ long and less than $0.1 \mathrm{~cm}$ thick. The waste stream will be of a similar physical form from container to container. This waste will not be a moratorium waste or reclassified TRU waste.

\section{B. GENERATION SITE/PROCESS DESCRIPTION}

The following questions, concerning the generation site and process, should be answered even if the waste stream is no longer generated.

Name of Site Generating Waste (e.g., INEL, Rocky Flats, etc.): Puget Sound Naval Shipyard

\section{Location of activities (Area and Building):}

Naval vessels undergoing maintenance activities. Radiological work and waste handling facilities.

Operations (functions) performed in building:

Repair, overhaul, and maintenance of Naval Nuclear Propulsion systems and components, waste handling. Only activation products, primarily Co-60, are present in radioactive material. Use of hazardous materials containing chemical contaminants is not normally permitted or required at the worksite. However, lead and chromium and PCB's are sometimes in paint removed at the worksite.

Description of Process Generating Waste:

Painted surfaces are stripped prior to repainting. Paint is removed by mechanical means. Removed paint is collected by hand and placed in plastic bags, or vacuum cleaners. 
Ps:wo12

LlW Contact

\section{PROJECTED WASTE GENERATION}

Is this waste stream still generated?

OYes ONo

If yes, when is the expected generation termination date?

Current Projections

\begin{tabular}{|r|r|r|r|r|r|}
\hline Est. Date & Start Year & Interval & Vol (m3) & Mass (kg) & Source of EstImate \\
\hline $11 / 15 / 94$ & 1997 & 1 & 0.23 & & 1994 DSTP \\
\hline
\end{tabular}
\begin{tabular}{|r|r|r|r|r|r|}
\hline Est. Date & Start Year & Interval & Vol (m3) & Mass (kg) & Source of Estlmate \\
\hline $11 / 1 / 93$ & 1994 & 1 & 0.50 & 113.40 & $1993-F F C A$ \\
\hline $11 / 1 / 93$ & 1995 & 1 & 0.50 & 113.40 & $1993-F F C A$ \\
\hline $11 / 1 / 93$ & 1996 & 1 & 0.50 & 113.40 & $1993-F F C A$ \\
\hline $11 / 1 / 93$ & 1997 & 1 & 0.50 & 113.40 & $1993-F F C A$ \\
\hline
\end{tabular}


OM A LEAD BASED PAN CHIPS W/PCB

D. STORAGE INVENTORY

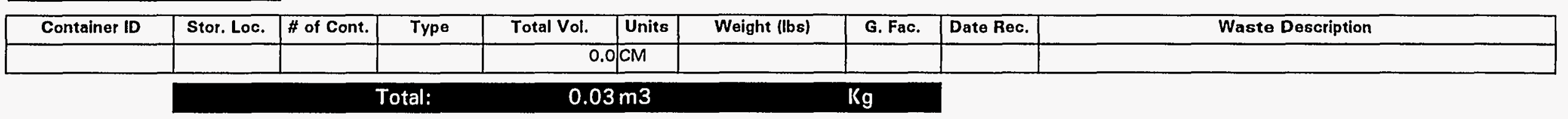

Waste Removed From Storage: 


\section{F. TRU ALPHA ACTIVITY DISTRIBUTION}

Radioactive Waste Type:

\begin{tabular}{|ll|}
\hline OllW & Oa-LLW $\bigcirc$ TRU \\
O HLW & O Non-Rad \\
\hline
\end{tabular}

Mixed Waste:

Handling:

\begin{tabular}{|l|}
\hline O Yes O No \\
\hline O Contact O Remote \\
\hline
\end{tabular}

Radionuclide Contamination Accessiblity:

\begin{tabular}{|c|c|c|c|}
\hline External Surface & OYes & (O) No & OUnknown \\
\hline Internal Surface & OYes & ONo & OUnknown \\
\hline spersed Through Matrix & OYes & ONo & OUnknown \\
\hline
\end{tabular}

\section{Activity Levels}

$\begin{aligned} \text { Transuranic Alpha Activity: } & \\ \text { Uranium/Thorium Alpha Activity: } & \\ \text { Beta/Gamma Activity: } & \\ & \text { Units: } \\ & \\ \text { Surface Neutron Activity: } & \square 0.05 \text { @ surface } \\ \text { Total Activity: } & \text { Units: } \square \text { Units: } \mathrm{mR} / \mathrm{hr} \\ & \mathrm{nCi} / \mathrm{g}\end{aligned}$

This waste stream is not identified as a TRU or a-LLW. 


\section{LLW Contactri}

\section{CHROMIUM LEAD BASED PAIN CHIPS WHCB}

\section{G. RADIONUCLIDE CONCENTRATIONS}

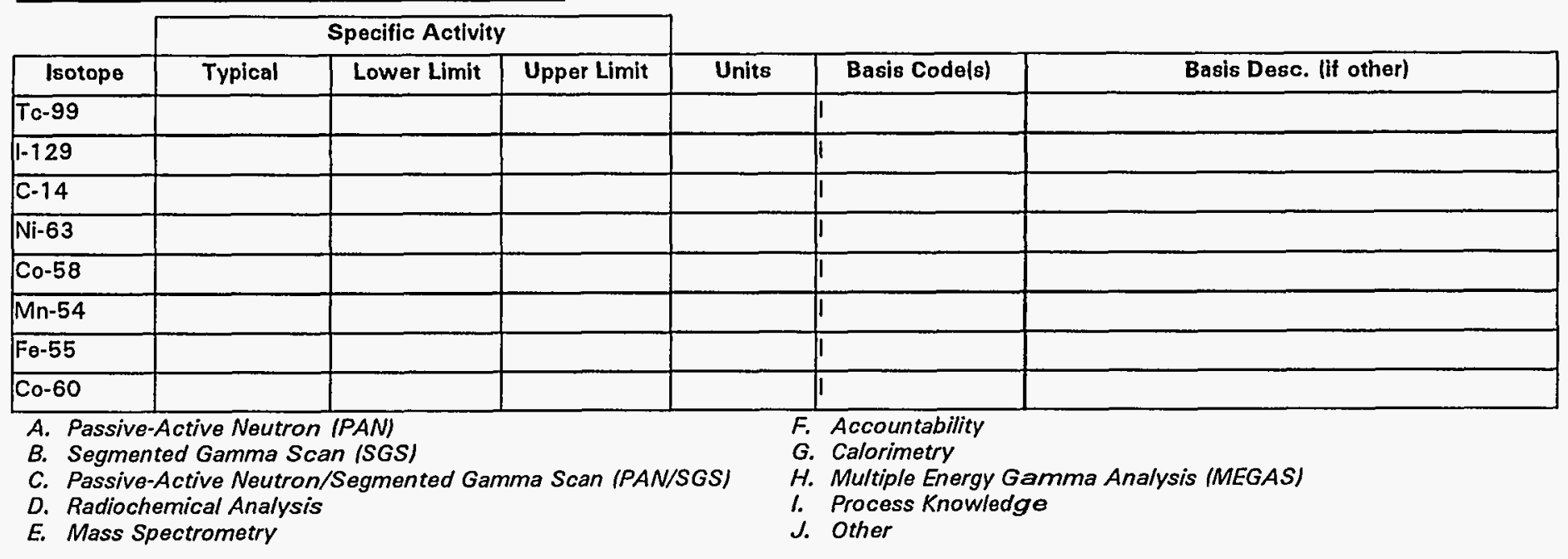




\section{H. MATRIX CHARACTERISTICS}

Primary Matrix Code: S3131

Description: Homogeneous Solids: Inorganic Homogeneous Solids: Paint Waste: Paint Chips/Solids

\section{Combustibility: $\bigcirc$ Combustible (>90\%) \\ Mixed $(10 \%-90 \%)$ \\ Noncombustible $(<10 \%)$}

\section{MATRIX COMPOSITION}

\begin{tabular}{|c|c|c|c|c|c|c|}
\hline & & & \multicolumn{4}{|c|}{ Composition } \\
\hline Code & Matrix Description & Materlal & Typical & LL & UL & Units \\
\hline$\$ 3131$ & Homogeneous Solids.Inorganic Homogeneous Solids.Paint Waste.Paint Chips/Solids & Paint Chips/Solids & 100 & 100 & 100 & $\%$ vol \\
\hline
\end{tabular}




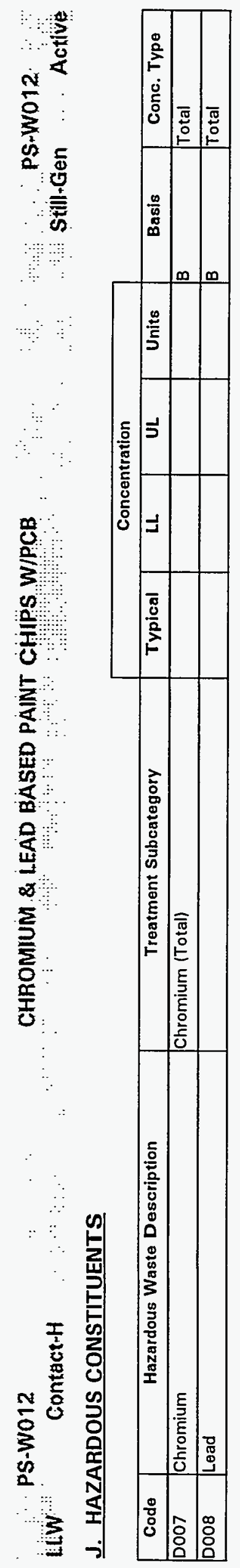

$\forall$
0
$\vdots$
0
0
0
0

ह5 


\section{K. POLYCHLORINATED BIPHENYLS (PCBS)}

Is this waste stream PCB contaminated?

O Yes O No O Unknown

If yes, what portion of the waste is PCB contaminated (volume \%): 100

If $<100 \%$, can the PCB contaminated waste be segregated and treated separately?

Y Yes O No O Unknown

What is the PCB concentration in ppm?

$50-500$

If only a portion of the waste is PCB contaminated, report values for contaminated portion.

\section{TREATMENT PLANS}

\begin{tabular}{|c|c|c|c|c|c|c|c|c|c|c|c|}
\hline $\begin{array}{c}\text { Opt. } \\
\#\end{array}$ & $\begin{array}{c}\text { Media Type } \\
\text { (If more than one) }\end{array}$ & $\begin{array}{c}\text { Matrix } \\
\text { Code }\end{array}$ & $\begin{array}{l}\text { Vol. } \% \\
\text { (If }\end{array}$ & Step & JIT & sc & CIF & $\begin{array}{l}\text { Trans. } \\
\text { Miles }\end{array}$ & $\begin{array}{l}\text { Facility } \\
\text { Abbr. }\end{array}$ & Unit Name & Comments \\
\hline 1 & & 53131 & & $\bar{a}$ & $\mathbf{O}$ & O & $1.00 \mathrm{E}+00$ & & TRANS & Transport - LLW & \\
\hline & & & & b & O & O & $1.00 E+00$ & & AMWTF & $\mathrm{CH}$ - Opening \& Sorting & \\
\hline & & & & $\bar{d}$ & $\bar{O}$ & $\overline{0}$ & $1.00 E+00$ & & AMWTF & $\mathrm{CH}$ - Incineration/Thermal Desorption & \\
\hline & & & & e & 0 & O & $1.00 E+00$ & & AMWTF & $\mathrm{CH}$ - Vitrification & \\
\hline & & & & $f$ & 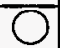 & $\bar{O}$ & $1.00 E+00$ & & SCDF & Disposal - Contact Handled & \\
\hline
\end{tabular}

Note: Where provided, media percentages are rough estimates used to facilitate treatment planning. They do not necessarily imply an accurate knowledge of waste stream composition, and were not derived for use outside this conceptual planning exercise.

Describe any special or unique technical concerns related to the treatment of this waste stream that would impact the use or implementation of standard hazardous waste treatment methods. 


\section{PS-W019 \\ Contact-H}

\section{A. IDENTIFICATION AND DESCRIPTION}

Waste Stream ID: PS-W019

W. S. Name:

Content Code:

FFCA ID:

\section{FILTERS WIASBESTOS AND DIOCTYL PHTHALATE}

Waste Stream Descrip

Waste filters and filter media. Contains Di(ethylexyl)phthalate. This is not a RCRA waste, but is regulated by Washington State as a carcinogenic characteristic dangerous waste (State waste code WCO2). Also contains asbestos. Filters range in size from 6" diameter by $6^{\prime \prime}$ to $2^{\prime} \times 2$ '. This waste stream is not a moratorium waste or reclassified TRU waste. 
PSwojg

FILTERS WIASBESTOS AND DIOCTYL PHTTAALATE

HWW Contact H

C. PROJECTED WASTE GENERATION

Is this waste stream still generated?

OYes ONo

If yes, when is the expected generation termination date?

Current Projections

\begin{tabular}{|r|r|r|r|r|r|}
\hline Est. Date & Start Year & Interval & Vol (m3) & Mass (kg) & Source of Estimate \\
\hline $11 / 15 / 94$ & 1997 & 1 & 2.18 & & 1994-DSTP \\
\hline
\end{tabular}

Historical Projections 


\section{PS:W019 \\ LLW : Contact-H \\ D. STORAGE INVENTORY}

FILTERS WIASBESTOS AND DIOCTYL PHTHALATE

PS-WO19

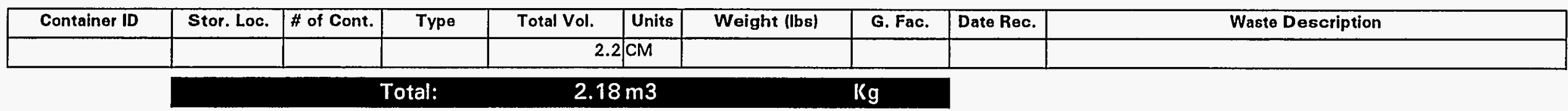

Waste Removed From Storage: 


\section{F. TRU ALPHA ACTIVITY DISTRIBUTION}

Radioactive Waste Type:

\begin{tabular}{ll} 
O llW & Oa-LlW O TRU \\
OhLW & O Non-Rad \\
\hline
\end{tabular}

This waste stream is not identified as a TRU or a-LLW.

Mixed Waste:

\begin{tabular}{|l|}
\hline OYes ONo \\
\hline O Contact O Remote \\
\hline
\end{tabular}

Radionuclide Contamination Accessiblity:

\begin{tabular}{|c|c|c|c|}
\hline External Surface & OYes & ONo & OUnknown \\
\hline Internal Surface & OYes & ONo & O Unknown \\
\hline Through Ma & & No & $n$ \\
\hline
\end{tabular}

Dispersed Through Matrix Activity Levels

Transuranic Alpha Activity:

Uranium/Thorium Alpha Activity:

Beta/Gamma Activity:

Surface Neutron Activity:

Total Activity:

\begin{tabular}{|c|c|c|}
\hline & Units: & \\
\hline & Units: & \\
\hline 0.7 & \multicolumn{2}{|c|}{ @ surface } \\
\hline$<0.05$ & @ 1-m & Units: $\mathrm{mR} / \mathrm{hr}$ \\
\hline & Units: [ & \\
\hline & $\mathrm{nCi} / \mathrm{g}$ & \\
\hline
\end{tabular}




\section{LiW Contact-H}

U

G. RADIONUCLIDE CONCENTRATIONS

\begin{tabular}{|c|c|c|c|c|c|c|}
\hline & \multicolumn{3}{|c|}{ Specific Activity } & \multirow[b]{2}{*}{ Units } & \multirow[b]{2}{*}{ Basis Code(s) } & \multirow[b]{2}{*}{ Basis Desc. (if other) } \\
\hline Isotope & Typical & Lower Limit & Upper Limit & & & \\
\hline Co-60 & & & & & $\pi$ & \\
\hline $\begin{array}{l}\text { A. Passiv } \\
\text { B. Segme } \\
\text { C. Passiv } \\
\text { D. Radioc } \\
\text { E. Mass }\end{array}$ & $\begin{array}{l}\text { tive Neutr } \\
\text { I Gamma } \\
\text { tive Neutr } \\
\text { ical Analy } \\
\text { trometry }\end{array}$ & $\begin{array}{l}A N) \\
(S G S) \\
\text { gmented } G\end{array}$ & na Scan IPAI & & $\begin{array}{ll}\text { F. Accountability } \\
\text { G. Calorimetry } \\
\text { H. Multiple Energy } \\
\text { I. Process Knowle } \\
\text { J. Other }\end{array}$ & sis (MEGAS) \\
\hline
\end{tabular}


LLW

\section{H. MATRIX CHARACTERISTICS}

Primary Matrix Code: 55410

Description: Debris Waste: Heterogeneous Debris: Composite Filter Debris:

\section{Combustibility: $\bigcirc$ Combustible $(>90 \%)$ \\ Mixed $(10 \%-90 \%)$ \\ Noncombustible $(<10 \%)$}

\section{MATRIX COMPOSITION}

\begin{tabular}{|c|c|c|c|c|c|c|}
\hline & & & \multicolumn{4}{|c|}{ Composition } \\
\hline Code & Matrix Description & Material & Typical & $\mathbf{L L}$ & UL & Units \\
\hline 10 & Debris Waste.Heterageneous Debris & & & & & \\
\hline
\end{tabular}



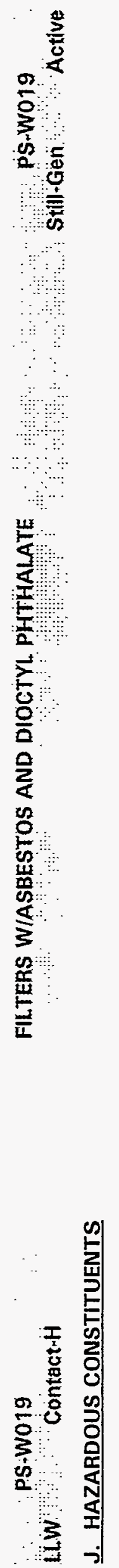
LLW Contact-H

\section{K. POLYCHLORINATED BIPHENYLS (PCBS)}

Is this waste stream PCB contaminated?

OYes O No O Unknown

If yes, what portion of the waste is PCB contaminated (volume \%):

If $<100 \%$, can the PCB contaminated waste be segregated and treated separately?

What is the PCB concentration in ppm?

$<50$

If only a portion of the waste is $P C B$ contaminated, report values for contaminated portion.

\section{TREATMENT PLANS}

\begin{tabular}{|c|c|c|c|c|c|c|c|c|c|c|c|}
\hline $\begin{array}{c}\text { Opt. } \\
\#\end{array}$ & $\begin{array}{l}\text { Media Type } \\
\text { (If more than one) }\end{array}$ & $\begin{array}{c}\text { Matrix } \\
\text { Code }\end{array}$ & $\begin{array}{l}\text { Vol. \% } \\
\text { IIf }\end{array}$ & Step & JIT & sc & CIF & $\begin{array}{l}\text { Trans. } \\
\text { Miles }\end{array}$ & $\begin{array}{l}\text { Facility } \\
\text { Abbr. }\end{array}$ & Unit Name & Comments \\
\hline \multirow[t]{6}{*}{1} & & 55410 & & a & (O) & 0 & $1.00 E+00$ & & TRANS & Transport - LLW & \\
\hline & & & & b & O) & 0 & $1.00 E+00$ & & AMWTF & $\mathrm{CH}$ - Opening \& Sorting & \\
\hline & & & & c & () & $\mathrm{O}$ & $1.00 E+00$ & & AMWTF & $\mathrm{CH}$ - Sizing & \\
\hline & & & & d & O & $\mathrm{O}$ & $1.00 E+00$ & & AMWTF & $\mathrm{CH}$ - Incineration/Thermal Desorption & \\
\hline & & & & e & O & $\mathrm{O}$ & $1.00 \mathrm{E}+00$ & & AMWTF & $\mathrm{CH}$ - Vitrification & \\
\hline & & & & $f$ & 0 & $\mathrm{O}$ & $1.00 \mathrm{E}+00$ & & SCDF & Disposal - Contact Handled & \\
\hline
\end{tabular}

Note: Where provided, media percentages are rough estimates used to facilitate treatment planning. They do not necessarily imply an accurate knowledge of waste stream composition, and were not derived for use outside this conceptual planning exercise.

Describe any special or unique technical concerns related to the treatment of this waste stream that would impact the use or implementation of standard hazardous waste treatment methods. 


\section{$\therefore$ PS+WO2O \\ Liw ContactrH}

\section{A. IDENTIFICATION AND DESCRIPTION}

Waste Stream ID: PS-W020

W. S. Name:

Content Code:

FFCA ID:

\section{COMPRESSED FILTERS W/DIOCTYL PHTHALATES}

WeAste St

\section{Waste Stream Description:}

Waste filter media, removed from high efficency particulate air filters. Contains dioctylphthalate, a state only (nonRCRA) waste. State code WCO2, for carcinogens. Media has been compress into 55 -gallon carbon steel drums. This waste stream is not a moratorium waste or reclassified TRU waste.

\section{B. GENERATION SITE/PROCESS DESCRIPTION}

The following questions, concerning the generation site and process, should be answered oven if the waste stream is no longer generated.

Name of Site Generating Waste (e.g., INEL, Rocky Flats, etc.): Puget Sound Naval Shipyard

Location of activities (Area and Building):

Ships in dry dock or piers, radiological work facilities including Buildings 839,856 , and 880 .

Operations (functions) performed in building:

Refueling of reactor plants, repair of reactor plant components, processing waste materials. Vacuum cleaners are used for containment clean up, the containments minimize the spread of contamination. The building has radioactive tanks holding liquids awaiting processing.

Description of Process Generating Waste:

Filters are used to remove radioactive particles from the air. May be used for localized work area ventilization or general building

ventililation. Also used in vacuum cleaners, tanks, and containments. 
PS-WO2O

HLW.: Contact+H

C. PROJECTED WASTE GENERATION

Is this waste stream still generated?

If yes, when is the expected generation termination date?

\section{COMPRESSED FILTERS WIDIOCTYYLPHTHALATES}

Current Projections

Historical Projections 


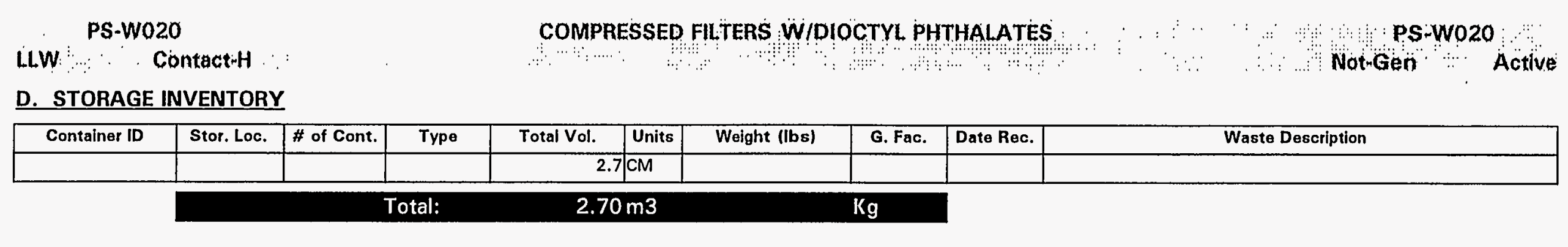

Waste Removed From Storage: 


\section{:PS-WOZZO}

HLW Contact H

\section{E. RADIATION CHARACTERISTICS}

Radioactive Waste Type:

\begin{tabular}{|ll|}
\hline OlLW & Oa-LLW O TRU \\
OHLW & O Non-Rad \\
\hline
\end{tabular}

Mixed Waste:

Handling:

\begin{tabular}{|l|}
\hline O Yes O No \\
\hline O Contact O Remote \\
\hline
\end{tabular}

Radionuclide Contamination Accessiblity:

External Surface OYes O No OUnknown

Internal Surface OYes O No OUnknown

Dispersed Through Matrix

OYes ONo OUnknown

\section{Activity Levels}

Transuranic Alpha Activity: Uranium/Thorium Alpha Activity:

Beta/Gamma Activity:

Surface Neutron Activity:

Total Activity:

\begin{tabular}{|c|c|c|}
\hline & \multirow{2}{*}{$\begin{array}{l}\text { Units: } \\
\text { Units: }\end{array}$} & \\
\hline & & \\
\hline 0.7 & \multicolumn{2}{|c|}{ @ surface } \\
\hline$<0.05$ & @ 1-m & Units: $\mathrm{mR} / \mathrm{hr}$ \\
\hline & Units: & \\
\hline & $\mathrm{nCi} / \mathrm{g}$ & \\
\hline
\end{tabular}

\section{F. TRU ALPHA ACTIVITY DISTRIBUTION}

This waste stream is not identified as a TRU or a-LLW. 


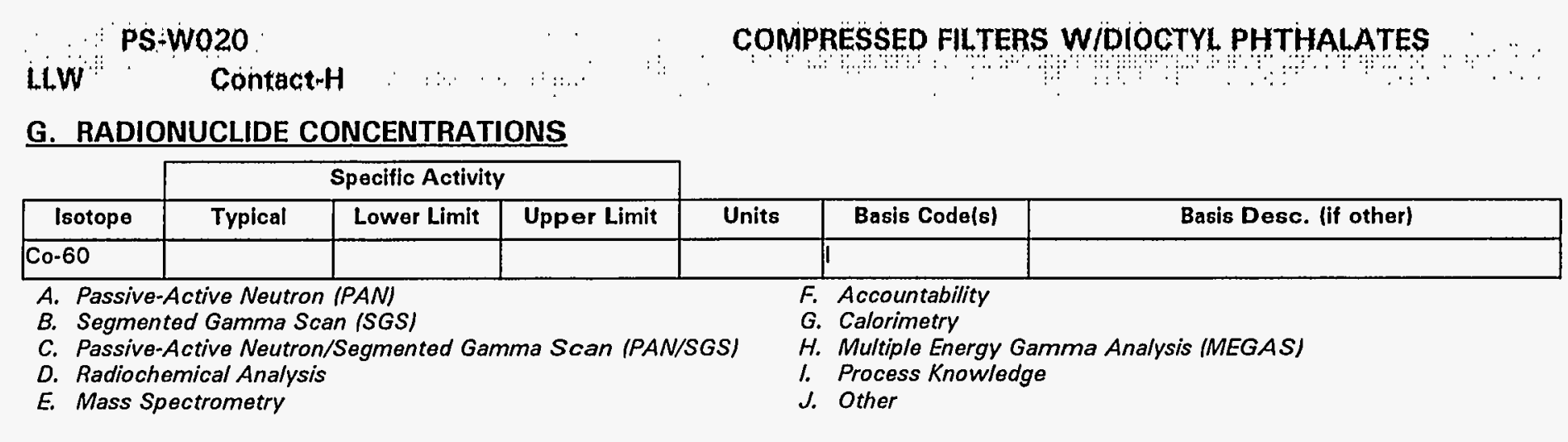

G. RADIONUCLIDE CONCENTRATIONS

E. Mass Spectrometry 
PS-WOZ20

LWW ContactH

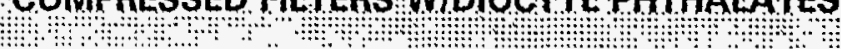

H. MATRIX CHARACTERISTICS

Primary Matrix Code: 55410

Description: Debris Waste: Heterogeneous Debris: Composite Filter Debris:

\section{Combustibility: $O$ Combustible (>90\%)}

Mixed $(10 \%-90 \%)$

Noncombustible (<10\%)

\section{MATRIX COMPOSITION}

\begin{tabular}{|c|c|c|c|c|c|c|}
\hline & & & \multicolumn{4}{|c|}{ Composition } \\
\hline Code & Matrix Description & Material & Typical & LL & UL & Units \\
\hline$\$ 5410$ & Debris Waste.Heterogeneous Debris.Composite Filter Debris. & & & & & \\
\hline
\end{tabular}



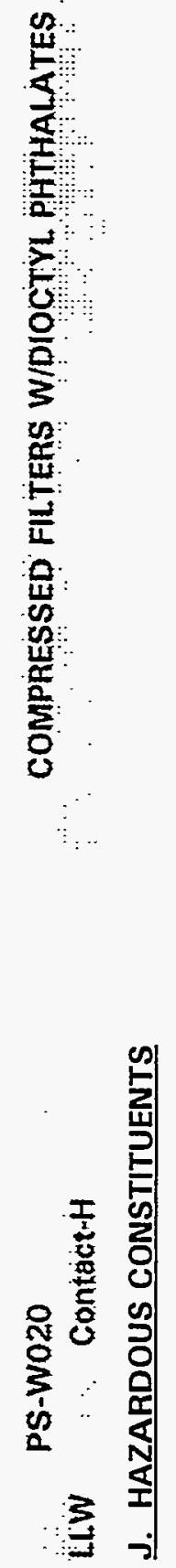
LLW Oontáct

K. POLYCHLORINATED BIPHENYLS (PCBS)

Is this waste stream PCB contaminated?

OYes ONo O Unknown

If yes, what portion of the waste is PCB contaminated (volume \%):

If $<100 \%$, can the $\mathrm{PCB}$ contaminated waste be segregated and treated separately?

What is the PCB concentration in ppm?

$<50$

If only a portion of the waste is PCB contaminated, report values for contaminated portion.

\section{TREATMENT PLANS}

\begin{tabular}{|c|c|c|c|c|c|c|c|c|c|c|c|}
\hline $\begin{array}{c}\text { Opt. } \\
\#\end{array}$ & $\begin{array}{c}\text { Media Type } \\
\text { (If more than one) }\end{array}$ & $\begin{array}{c}\text { Matrix } \\
\text { Code }\end{array}$ & $\begin{array}{l}\text { Vol. \% } \\
\text { lif }\end{array}$ & Step & JIT & sc & CIF & $\begin{array}{l}\text { Trans. } \\
\text { Miles }\end{array}$ & $\begin{array}{l}\text { Facility } \\
\text { Abbr. }\end{array}$ & Unit Name & Comments \\
\hline 1 & & S5410 & & $\bar{a}$ & O & $\mathrm{O}$ & $1.00 E+00$ & & TRANS & Transport - LLWW & \\
\hline & & & & b & $\mathbf{O}$ & O & $1.00 E+00$ & & AMWTF & $\mathrm{CH}-$ Opening \& Sorting & \\
\hline & & & & d & 0 & O & $1.00 E+00$ & & AMWTF & $\mathrm{CH}$ - Incineration/Thermal Desorption & \\
\hline & & & & e & 0 & 0 & $1.00 E+00$ & & AMWTF & $\mathrm{CH}$ - Vitrification & \\
\hline & & & & $\mathbf{f}$ & C & $\bar{O}$ & $1.00 E+00$ & & SCDF & Disposal - Contact Handled & \\
\hline
\end{tabular}

Note: Where provided, media percentages are rough estimates used to facilitate treatment planning. They do not necessarily imply an accurate knowledge of waste stream composition, and were not derived for use outside this conceptual planning exercise.

Describe any special or unique technical concerns related to the treatment of this waste stream that would impact the use or implementation of standard hazardous waste treatment methods. 
LWW RF WO17

PCB LIQUIDSTLLM

\section{A. IDENTIFICATION AND DESCRIPTION}

Waste Stream ID: RF-W017

\section{W. S. Name:}

Content Code:

FFCA ID:

\section{PCB LIQUIDS/LLM}

\section{Waste Stream Description:}

This waste stream is transformer oil and hydaulic fluid contaminated with PCBs. IDC No. N/A

PCB liquid waste was generated during removal of PCB-contaminated electrical or hydraulic equipment, including transformers and capacitors. The waste varied from high percentage transformer oil to hydraulic fluids contaminated with comparable low concentrations of PCB.

Liquid PCB waste is stored in 55-gallon drums placed in 85 -gallon overpacks.

The source of the hazardous constituents associated with this waste form is two drums of halogenated degreasing solvents.

\section{B. GENERATION SITE/PROCESS DESCRIPTION}

The following questions, concerning the generation site and process, should be answered even if the waste stream is no longer generated.

Name of Site Generating Waste (e.g., INEL, Rocky Flats, etc.): Rocky Flats Plant

\section{Location of activities (Area and Building):}

Various locations throughout RFP.

\section{Operations (functions) performed in building:}

Description of Process Generating Waste:

PCB liquid waste was generated from removal of PCB contaminated items including transformers, capacitors, and hydraulic systems. This waste has been generated at various locations. No further generation of this waste is anticipated. 
RF-W017

LiW ContactH

C. PROJECTED WASTE GENERATION

Is this waste stream still generated?

If yes, when is the expected generation termination date?

\section{RF. WO 17}

NötGen Ho Active

Current Projections

Historical Projections 

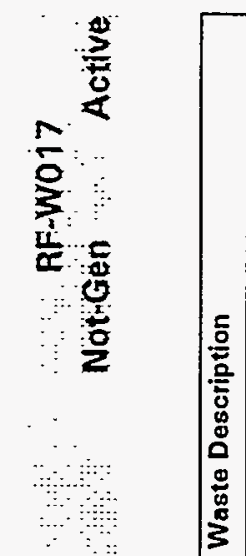

ले
0
+
5
8

ह

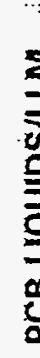


HW RFWOI7

\section{E. RADIATION CHARACTERISTICS}

Radioactive Waste Type:

\begin{tabular}{|ll|}
\hline OllW & Oa-LLW $\bigcirc$ TRU \\
O HLW & $\bigcirc$ Non-Rad \\
\hline
\end{tabular}

Mixed Waste:

Handling:

\begin{tabular}{|l|}
\hline OYes O No \\
\hline O Contact O Remote \\
\hline
\end{tabular}

Radionuclide Contamination Accessiblity:

\begin{tabular}{|c|c|c|c|}
\hline External Surface & OYes & ONo & OUnknown \\
\hline Internal Surface & OYes & O No & O Unknown \\
\hline spersed Through Matrix & OYes & ONo & OUnknown \\
\hline
\end{tabular}

\section{Activity Levels}

Transuranic Alpha Activity: Uranium/Thorium Alpha Activity:

Beta/Gamma Activity:

Surface Neutron Activity:

Total Activity:

\begin{tabular}{l}
\hline$<1 \mathrm{E}-7$ Units: $\mathrm{Ci} / \mathrm{g}$ \\
\hline \\
\hline \\
\hline \\
\hline \\
Units: @ surface \\
\hline \\
@ 1-m Units: mR/hr \\
Units: \\
\hline \\
nCi/g
\end{tabular}

\section{F. TRU ALPHA ACTIVITY DISTRIBUTION}

This waste stream is not identified as a TRU or a-LLW. 


\section{RF-W017}

LLW: Gontact-H

\section{G. RADIONUCLIDE CONCENTRATIONS}

\begin{tabular}{|c|c|c|c|c|c|c|}
\hline & \multicolumn{3}{|c|}{ Specific Activity } & \multirow[b]{2}{*}{ Units } & \multirow[b]{2}{*}{ Basis Code(s) } & \multirow[b]{2}{*}{ Basis Desc. (if other) } \\
\hline Isotope & Typical & Lower Limit & Upper Limit & & & \\
\hline Pu-240 & & & & & $B$ & \\
\hline Pu-239 & & & & & $B$ & \\
\hline $\begin{array}{l}\text { A. Passiv } \\
\text { B. Segm } \\
\text { C. Passiv } \\
\text { D. Radio } \\
\text { E. Mass }\end{array}$ & $\begin{array}{l}\text { Ge Neut } \\
\text { Gamma } \\
\text { ive Neut } \\
\text { cal Analy } \\
\text { rometry }\end{array}$ & $\begin{array}{l}\text { PANI } \\
\text { (SGS) } \\
\text { egmented } G\end{array}$ & ma Scan IPA & & $\begin{array}{l}\text { Accountability } \\
\text { Calorimetry } \\
\text { Multiple Energy } \\
\text { Process Knowle } \\
\text { Other }\end{array}$ & sis (MEGAS) \\
\hline
\end{tabular}




\section{RFWoj7}

LLW Contạct-H

\section{H. MATRIX CHARACTERISTICS}

Primary Matrix Code: $\lcm{L 2210}$

Description: Organic Liquids: Pure Organic Liquids: Halogenated Pure Organic Liquids:

\section{Combustibility: $\bigcirc$ Combustible (>90\%) \\ Mixed $(10 \%-90 \%)$ \\ Noncombustible $(<10 \%)$}

\section{MATRIX COMPOSITION}

\begin{tabular}{|c|c|c|c|c|c|c|}
\hline & & & \multicolumn{4}{|c|}{ Composition } \\
\hline Code & Matrix Description & Material & Typical & LL & UL & Units \\
\hline $\mathbf{L 2 2 1 0}$ & Organic Liquids.Pure Organic Liquids. Halogenated Pure Organic Liquids. & Hydraulic oil & & & & \\
\hline $\mathbf{L 2 2 1 0}$ & Organic Liquids.Pure Organic Liquids. Halogenated Pure Organic Liquids. & Transformer oil & & & & \\
\hline
\end{tabular}


RF-W017

\section{J. HAZARDOUS CONSTITUENTS}

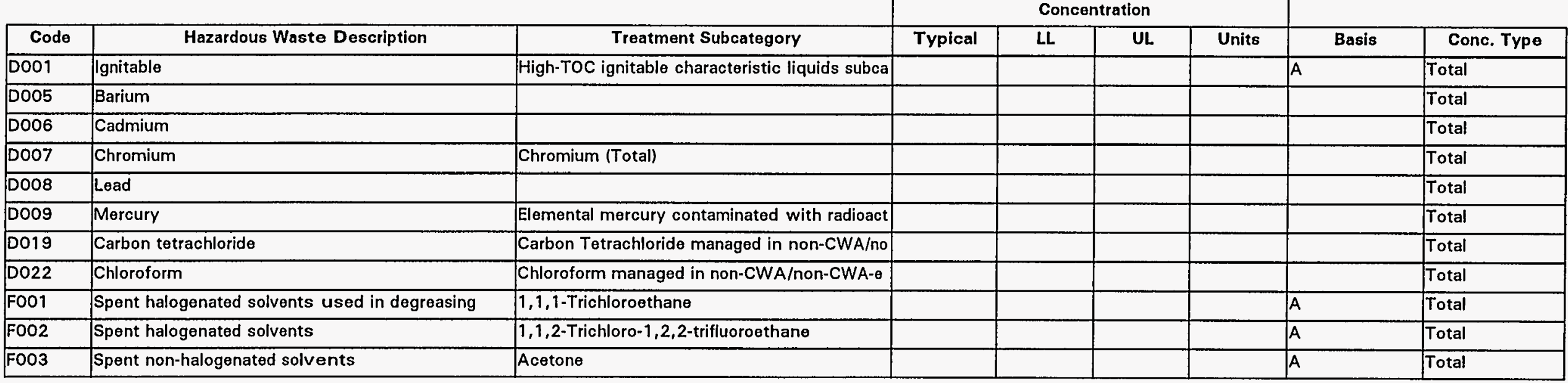


LLW: Contact H

K. POLYCHLORINATED BIPHENYLS (PCBS)

Is this waste stream PCB contaminated?

\section{O Yes $O$ No $O$ Unknown}

If yes, what portion of the waste is PCB contaminated (volume \%): 100

If $<100 \%$, can the PCB contaminated waste be segregated and treated separately? OYes O No O Unknown

What is the PCB concentration in ppm? If only a portion of the waste is PCB contaminated, report values for contaminated portion.

\section{TREATMENT PLANS}

\begin{tabular}{|c|c|c|c|c|c|c|c|c|c|c|c|}
\hline $\begin{array}{c}\text { Opt. } \\
\#\end{array}$ & $\begin{array}{c}\text { Media Type } \\
\text { (If more than one) }\end{array}$ & $\begin{array}{c}\text { Matrix } \\
\text { Code }\end{array}$ & $\begin{array}{l}\text { Vol. \% } \\
\text { IIf }\end{array}$ & Step & JIT & sc & CIF & $\begin{array}{l}\text { Trans. } \\
\text { Miles }\end{array}$ & $\begin{array}{l}\text { Facility } \\
\text { Abbr. }\end{array}$ & Unit Name & Comments \\
\hline 1 & & $\mathbf{L 2 2 1 0}$ & & $\bar{a}$ & (O) & 0 & $1.00 \mathrm{E}+00$ & & TRANS & Transport - LLWW & \\
\hline & & & & b & O) & 0 & $1.00 \mathrm{E}+00$ & & AMWTF & CH - Opening \& Sorting & \\
\hline & & & & d & 0 & 0 & $1.00 E+00$ & & AMWTF & $\mathrm{CH}$ - Vitrification & \\
\hline & & & & e & $\mathrm{O}$ & 0 & $1.00 E+00$ & & SCDF & Disposal - Contact Handled & \\
\hline
\end{tabular}

Note: Where provided, media percentages are rough estimates used to facilitate treatment planning. They do not necessarily imply an accurate knowledge of waste stream composition, and were not derived for use outside this conceptual planning exercise.

Describe any special or unique technical concerns related to the treatment of this waste stream that would impact the use or implementation of standard hazardous waste treatment methods. 


\section{RF.W027 \\ ContactrH}

\section{A. IDENTIFICATION AND DESCRIPTION}

Waste Stream ID: RF-W027

W. S. Name: PAINTS/LLM

Content Code:

FFCA ID:

RF-W027

Waste Stream Description:

This waste consists of oil-base paint and thinners. This waste stream was called "Paints and

Cyanides/LLW Mixed." The cyanide waste has been separated out to a new waste stream.

Waste oil-base paint and thinners are generated by maintenance operations throughout the plant

site. These wastes are radiologically contaminated with plutonium or uranium, depending on

areas in which operations are performed. The waste is packaged in 55-gallon drums.

Characterization of paint is based on lab analysis (Lab Nos. E87-4501, E88-2836, and 89ENV.00297).
This waste form was generated from production and maintenance operations both inside and outside of the P.A.

\section{RF.W027.}

Not-Gen. Active

\section{B. GENERATION SITE/PROCESS DESCRIPTION}

The following questions, concerning the generation site and process,

Name of Site Generating Waste (e.g.. INEL, Rocky Flats, etc.):

Rocky Flats Plant

Location of activities (Area and Building):

Numerous locations throughout RFP.

Operations (functions) performed in building:

\section{Description of Process Generating Waste:}


RF $-W 027$ LLW ContactiH C. PROJECTED WASTE GENERATION

Is this waste stream still generated?

If yes, when is the expected generation termination date?

Current Projections

\section{PAINTSIILLM}

OYes ONo

\section{Historical Projections}




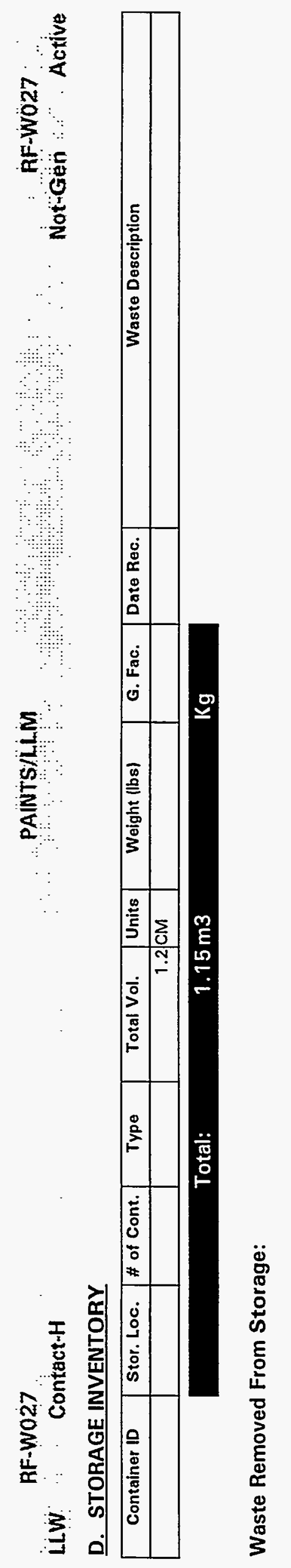

\begin{tabular}{l}
2 \\
\multirow{2}{*}{} \\
$\vdots$ \\
8 \\
8 \\
0
\end{tabular}

है 
Radioactive Waste Type:

\begin{tabular}{ll} 
Ollw & Oa-llw $\bigcirc$ tRU \\
OhlW & Onon-Rad \\
\hline
\end{tabular}

This waste stream is not identified as a TRU or a-LLW.

Mixed Waste:

\begin{tabular}{|l|}
\hline OYes ONo \\
\hline O Contact O Remote \\
\hline
\end{tabular}

Radionuclide Contamination Accessiblity:

\begin{tabular}{rlrlr|} 
External Surface & $O$ Yes & O No & O Unknown \\
\cline { 3 - 5 } Internal Surface & OYes & O No & O Unknown \\
\cline { 2 - 5 } Dispersed Through Matrix & $O$ Yes & O No & OUnknown
\end{tabular}

\section{Activity Levels}

Transuranic Alpha Activity:

Uranium/Thorium Alpha Activity:

\begin{tabular}{|c|c|}
\hline$<1 \mathrm{E}-7$ & Units: $\mathrm{Ci} / \mathrm{g}$ \\
\hline & Units: \\
\hline & @ surface \\
\hline & @ 1-m Units: \\
\hline & Units: \\
\hline & $\mathrm{nCi} / \mathrm{g}$ \\
\hline
\end{tabular}


LWW Contact-H

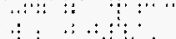

G. RADIONUCLIDE CONCENTRATIONS

\begin{tabular}{|c|c|c|c|c|c|c|}
\hline & \multicolumn{3}{|c|}{ Specific Activity } & \multirow[b]{2}{*}{ Units } & \multirow[b]{2}{*}{ Basis Code(s) } & \multirow[b]{2}{*}{ Basis Desc. (if other) } \\
\hline Isotope & Typical & Lower Limit & Upper Limit & & & \\
\hline \multicolumn{7}{|l|}{$\mathrm{U}-238$} \\
\hline Pu-240 & & & & & 3 & \\
\hline Pu-239 & & & & & 3 & \\
\hline
\end{tabular}

A. Passive-Active Neutron (PAN)

F. Accountability

B. Segmented Gamma Scan (SGS)

G. Calorimetry

C. Passive-Active Neutron/Segmented Gamma Scan (PAN/SGS)

D. Radiochemical Analysis

H. Multiple Energy Gamma Analysis (MEGAS)

E. Mass Spectrometry

1. Process Knowledge

J. Other 


\section{H. MATRIX CHARACTERISTICS}

Primary Matrix Code: $\mathbf{5 3 1 3 2}$

Description: Homogeneous Solids: Inorganic Homogeneous Solids: Paint Waste: Paint Sludges

Combustibility:

$$
\begin{aligned}
& \text { Combustible (>90\%) } \\
& \text { Mixed }(10 \%-90 \%) \\
& \text { Noncombustible }(<10 \%)
\end{aligned}
$$

\begin{tabular}{|c|c|c|c|c|c|c|}
\hline & & & \multicolumn{4}{|c|}{ Composition } \\
\hline Code & Matrix Description & Material & Typical & $\overline{L L}$ & UL & Units \\
\hline $\mathbf{5 3 1 3 2}$ & Homogeneous Solids.Inorganic Homogeneous Solids.Paint Waste.Paint Sludges & Paint Liquid/Sludges & 100 & & & \%vol \\
\hline
\end{tabular}

\section{MATRIX COMPOSITION}




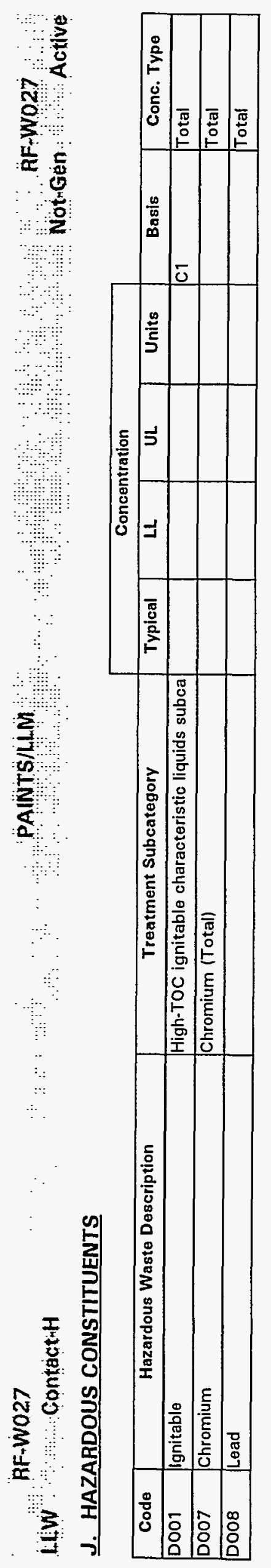




\section{K. POLYCHLORINATED BIPHENYLS (PCBS)}

\section{Is this waste stream PCB contaminated?}

OYes O No O Unknown

If yes, what portion of the waste is PCB contaminated (volume \%):

If $<100 \%$, can the PCB contaminated waste be segregated and treated separately?

What is the PCB concentration in ppm?

If only a portion of the waste is PCB contaminated, report values for contaminated portion.

\section{TREATMENT PLANS}

\begin{tabular}{|c|c|c|c|c|c|c|c|c|c|c|c|}
\hline $\begin{array}{l}\text { Opt. } \\
\#\end{array}$ & $\begin{array}{c}\text { Media Type } \\
\text { (If more than one) }\end{array}$ & $\begin{array}{c}\text { Matrix } \\
\text { Code }\end{array}$ & $\begin{array}{l}\text { Vol. \% } \\
\text { IIf }\end{array}$ & Step & JIT & sc & CIF & $\begin{array}{l}\text { Trans. } \\
\text { Miles }\end{array}$ & $\begin{array}{l}\text { Facility } \\
\text { Abbr. }\end{array}$ & Unit Name & Comments \\
\hline \multirow[t]{5}{*}{1} & & $\$ 3132$ & & $\overline{\mathbf{a}}$ & 0 & 0 & $1.00 E+00$ & & TRANS & Transport - LLW & \\
\hline & & & & $\mathbf{b}$ & $\mathbf{O}$ & 0 & $1.00 E+00$ & & AMWTF & $\mathrm{CH} \cdot$ Opening \& Sorting & \\
\hline & & & & $\mathbf{c}$ & 0 & 0 & $1.00 E+00$ & & AMWTF & $\mathrm{CH}$ - Incineration/Thermal Desorption & liquid injection \\
\hline & & & & d & 0 & 0 & $1.00 E+00$ & & AMWTF & $\mathrm{CH}$ - Vitrification & \\
\hline & & & & $\mathbf{e}$ & 0 & 0 & $1.00 \mathrm{E}+00$ & & SCDF & Disposal - Contact Handled & \\
\hline
\end{tabular}

Note: Where provided, media percentages are rough estimates used to facilitate treatment planning. They do not necessarily imply an accurate knowledge of waste stream composition, and were not derived for use outside this conceptual planning exercise.

Describe any special or unique technical concerns related to the treatment of this waste stream that would impact the use or implementation of standard hazardous waste treatment methods. 


\section{RF-W049 \\ LLW: Contäct-H}

\section{A. IDENTIFICATION AND DESCRIPTION}

Waste Stream ID: RF-W049

W. S. Name:

Content Code:

FFCA ID:

\begin{tabular}{|l|}
\hline MISCELLANEOUS LIQUIDS/LLM \\
\hline * \\
\hline RF-W049 \\
\hline
\end{tabular}

\section{Waste Stream Description:}

This waste stream contains various miscellaneous liquids that do not fit the category of any

other previously mentioned liquids. This waste includes materials such as a mixture of

toluene/DDCP/nitric acid/water and a drum containing stripper and floor glue. Waste

characterization is based upon process knowledge. No IDC.

\section{MISCELLANEOUS LIOUIDSILKM}

RF-WOAg

Natugen Active

\section{B. GENERATION SITE/PROCESS DESCRIPTION}

The following questions, concerning the generation site and process, should be answered even if the waste stream is no longer generated.

Name of Site Generating Waste (e.g., INEL, Rocky Flats, etc.): Rocky Flats Plant

Location of activities (Area and Building):

Numerous locations throughout RFP.

\section{Operations (functions) performed in building:}

Description of Process Generating Waste:

This waste form is not associated with any process routinely performed at RFP. The waste form encompasses various miscellaneous liquids that do not fit the categories of other previously mentioned liquids. 


\begin{tabular}{|c|c|c|c|c|c|}
\hline 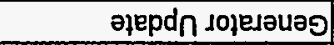 & & $\varepsilon \tau \angle Z$ & $s$ & s66t & $S 6 / L \varepsilon / \varepsilon$ \\
\hline 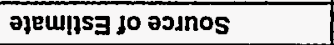 & (6y) Ssew & (عU) $10 \wedge$ & 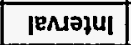 & JEכ人 2 HETS & 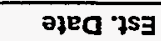 \\
\hline
\end{tabular}

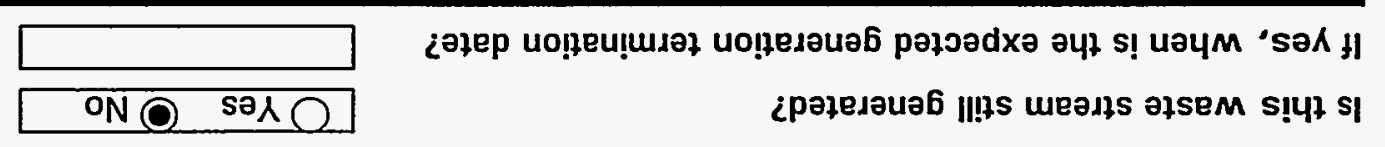

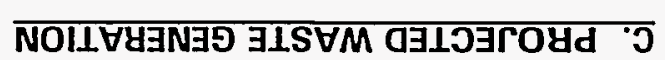




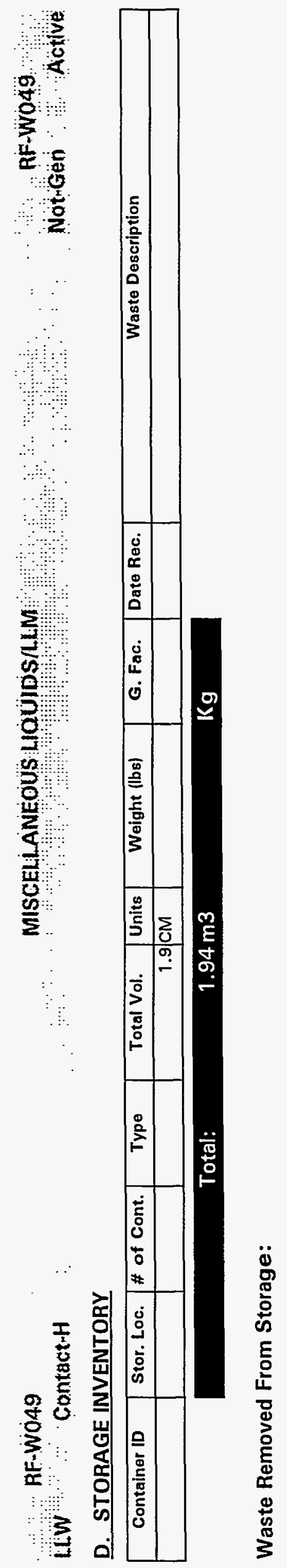




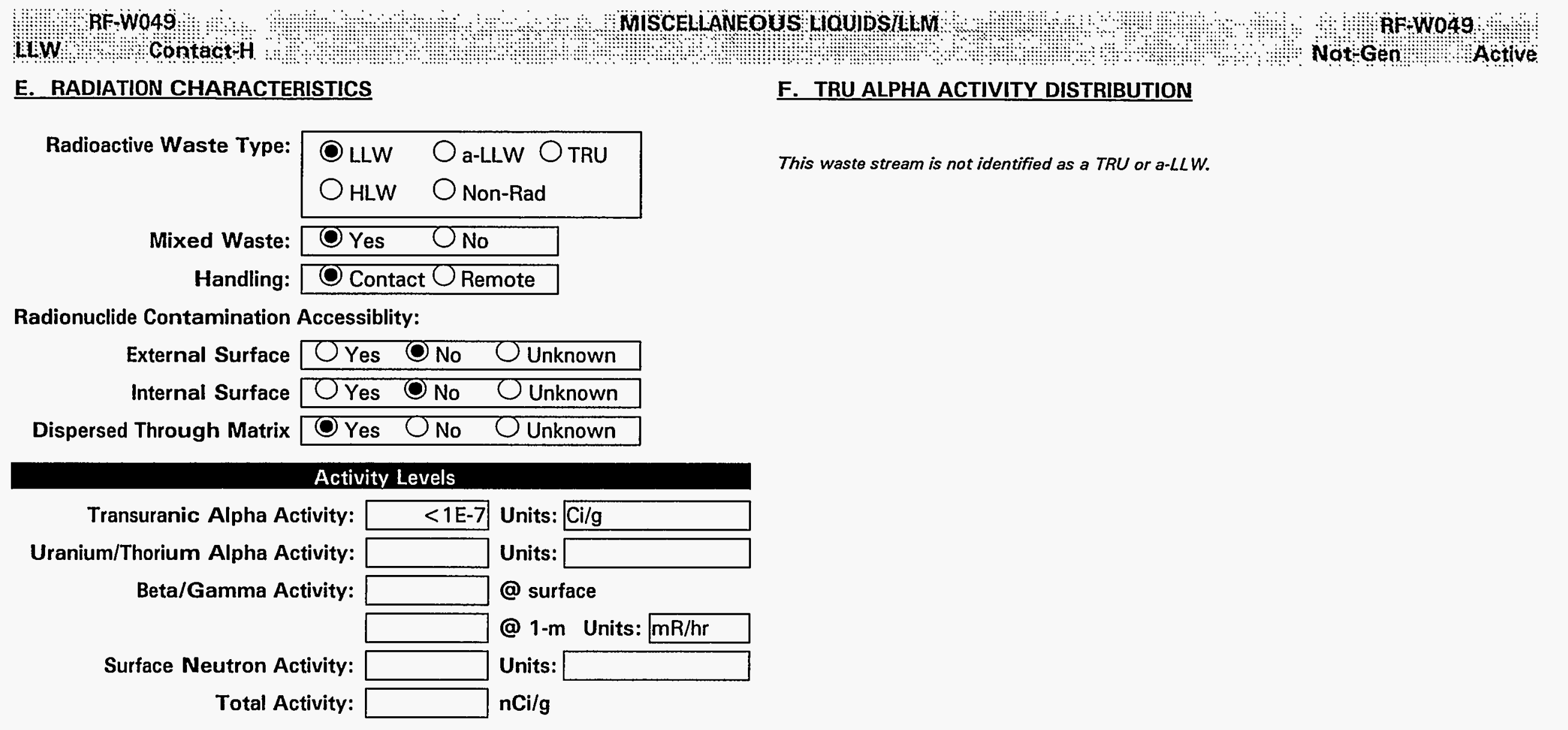




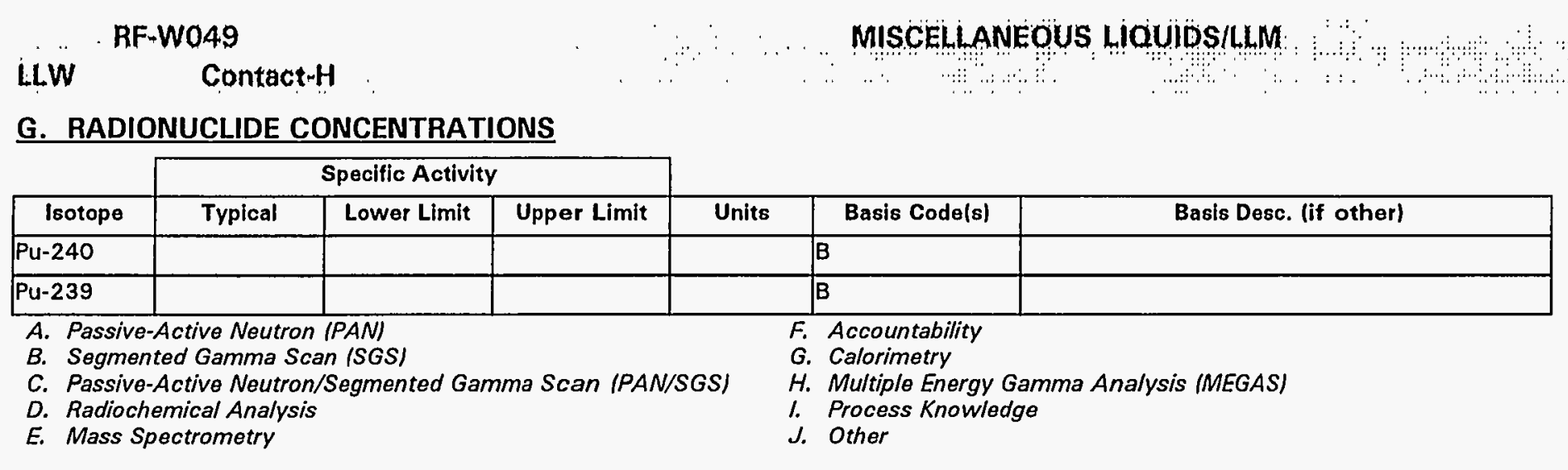

G. RADIONUCLIDE CONCENTRATIONS 


\section{LWW "Contact H}

\section{H. MATRIX CHARACTERISTICS}

Primary Matrix Code: L2100

Description: Organic Liquids: Aqueous/Organic Liquids:

\section{Combustibility: $\bigcirc$ Combustible (>90\%) \\ Mixed (10\%-90\%) \\ Noncombustible (<10\%)}

\section{MATRIX COMPOSITION}

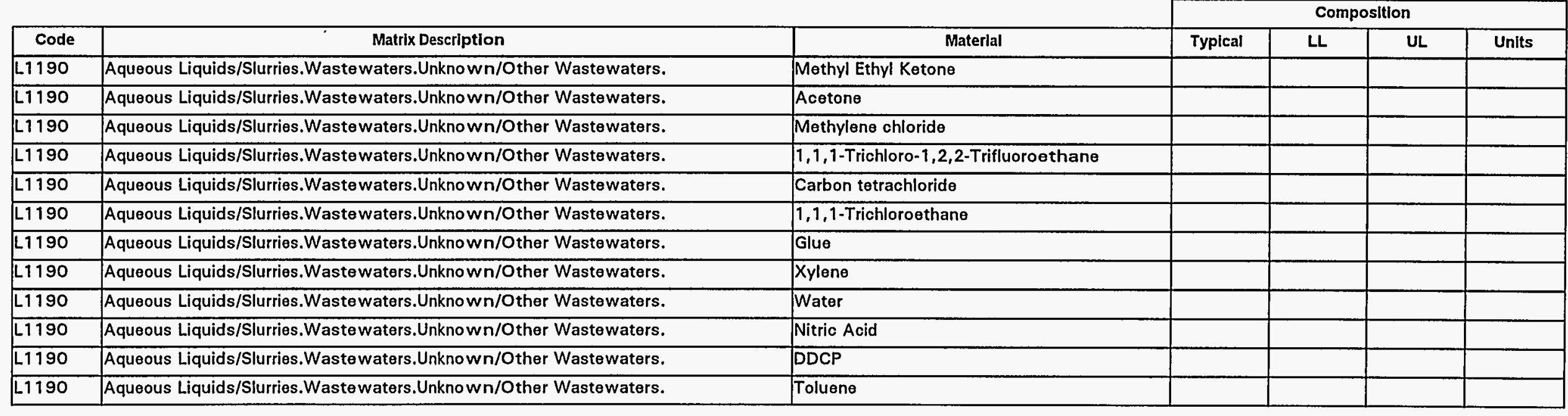




\section{J. HAZARDOUS CONSTITUENTS}

\begin{tabular}{|c|c|c|c|c|c|c|c|c|}
\hline \multirow[b]{2}{*}{ Code } & \multirow[b]{2}{*}{ Hazardous Waste Description } & \multirow[b]{2}{*}{ Treatment Subcategory } & \multicolumn{4}{|c|}{ Concentration } & \multirow[b]{2}{*}{ Basis } & \multirow[b]{2}{*}{ Conc. Type } \\
\hline & & & Typical & $\mathbf{L L}$ & $\mathbf{U L}$ & Units & & \\
\hline Do01 & Ignitable & High-TOC ignitable characteristic liquids subca & & & & & & Total \\
\hline $\mathrm{DO02}$ & Corrosive & Corrosive characteristic wastes that are mana & & & & & A & Total \\
\hline D006 & Cadmium & & & & & & & Total \\
\hline Do07 & Chromium & Chromium (Total) & & & & & & Total \\
\hline D008 & Lead & & & & & & & Total \\
\hline$\overline{D 011}$ & Silver & & & & & & & Total \\
\hline D035 & Methyl ethyl ketone & Methyle ethyl ketone managed in non-CWA/no & & & & & & Total \\
\hline F001 & Spent halogenated solvents used in degreasing & 1,1,1-Trichloroethane & & & & & A & Total \\
\hline Fo01 & Spent halogenated solvents used in degreasing & Carbon tetrachloride & & & & & A & Total \\
\hline FOO2 & Spent halogenated solvents & 1,1,2-Trichloro-1,2,2-trifluoroethan $\theta$ & & & & & A & Total \\
\hline FOO2 & Spent halogenated solvents & Methylene chloride & & & & & A & Total \\
\hline F003 & Spent non-halogenated solvents & Acetone & & & & & A & Total \\
\hline F003 & Spent non-halogenated solvents & Xylenes - mixed isomers & & & & & A & Total \\
\hline F005 & Spent non-halogenated solvents & Methyl ethyl ketone & & & & & A & Total \\
\hline F005 & Spent non-halogenated solvents & Toluene & & & & & A & Total \\
\hline
\end{tabular}




\section{K. POLYCHLORINATED BIPHENYLS (PCBS)}

Is this waste stream PCB contaminated?

OYes

O No O Unknown

If yes, what portion of the waste is PCB contaminated (volume \%):

If $<100 \%$, can the PCB contaminated waste be segregated and treated separately? $O$ Yes O No O Unknown

What is the PCB concentration in ppm?

If only a portion of the waste is PCB contaminated, report values for contaminated portion.

\section{TREATMENT PLANS}

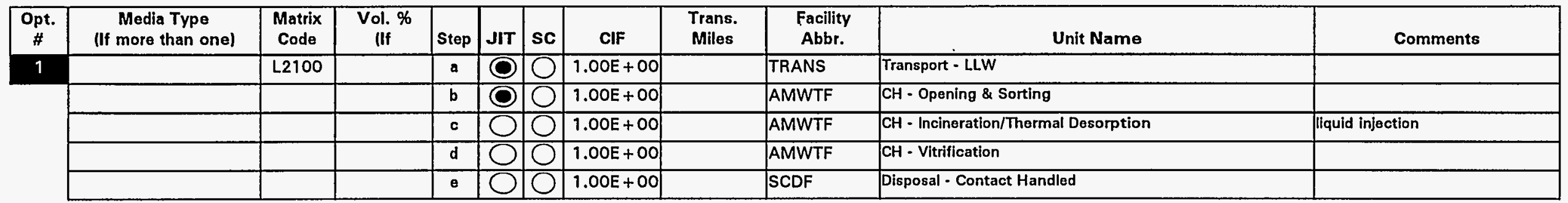

Note: Where provided, media percentages are rough estimates used to facilitate treatment planning. They do not necessarily imply an accurate knowledge of waste stream composition, and were not derived for use outside this conceptual planning exercise.

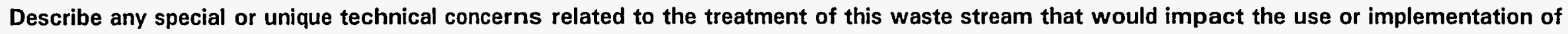
standard hazardous waste treatment methods. 


LiW : RF-W083
A. IDENTIFICATION AND DESCRIPTION
Waste Stream ID:
RF-W083
W. S. Name:
Content Code:
FFCA ID:
Waste Stream Description:
Rab-W083
Rab packs, excess chemicals. This waste form is packaged into drums in various buildings at
Rocky Flats and is still generated on a continuous basis.

\section{RF-W083}

\section{B. GENERATION SITE/PROCESS DESCRIPTION}

The following questions, concerning the generation site and process, should be answered even if the waste stream is no longer generated.

Name of Site Generating Waste (e.g., INEL, Rocky Flats, etc.): Rocky Flats

Location of activities (Area and Building):

Various buildings

Operations (functions) performed in building:

Description of Process Generating Waste:

The lab packs and excess chemicals are generated throughout Rocky Flats site as the chemicals reach their expiration date or become unneeded as Rocky Flats mission changes. All radioactive wastes in this category are described as excess chemicals at this time. 
LLW Contagt-H

\section{PROJECTED WASTE GENERATION}

Is this waste stream still generated?

OYes ONo

If yes, when is the expected generation termination date?

Current Projections

\begin{tabular}{|c|c|c|c|c|c|}
\hline Est. Date & Start Year & Interval & Vol (m3) & Mass (kg) & Source of Estimate \\
\hline $3 / 31 / 95$ & 1995 & 5 & 13.49 & & Generator Update \\
\hline
\end{tabular}
\begin{tabular}{|c|r|r|r|r|r|}
\hline Est. Date & Start Year & Interval & Vol (m3) & Mass (kg) & Source of Estlmate \\
\hline $11 / 1 / 93$ & 1997 & 1 & 10.12 & & $1993-F F C A$ \\
\hline
\end{tabular}


D. STORAGE INVENTORY

\begin{tabular}{|c|c|c|c|c|c|c|c|c|c|}
\hline Container ID & Stor. Loc. & \# of Cont. & Type & Total Vol. & Units & Weight (lbs) & G. Fac. & \begin{tabular}{|l|} 
Date Rec. \\
\end{tabular} & Waste Description \\
\hline & & & & \multicolumn{2}{|c|}{$\begin{array}{l}40.5 \mathrm{CM} \\
\end{array}$} & & & & \\
\hline & \multicolumn{3}{|c|}{ Total: } & \multicolumn{2}{|c|}{$40.46 \mathrm{m3}$} & & & & \\
\hline
\end{tabular}

Waste Removed From Storage: 


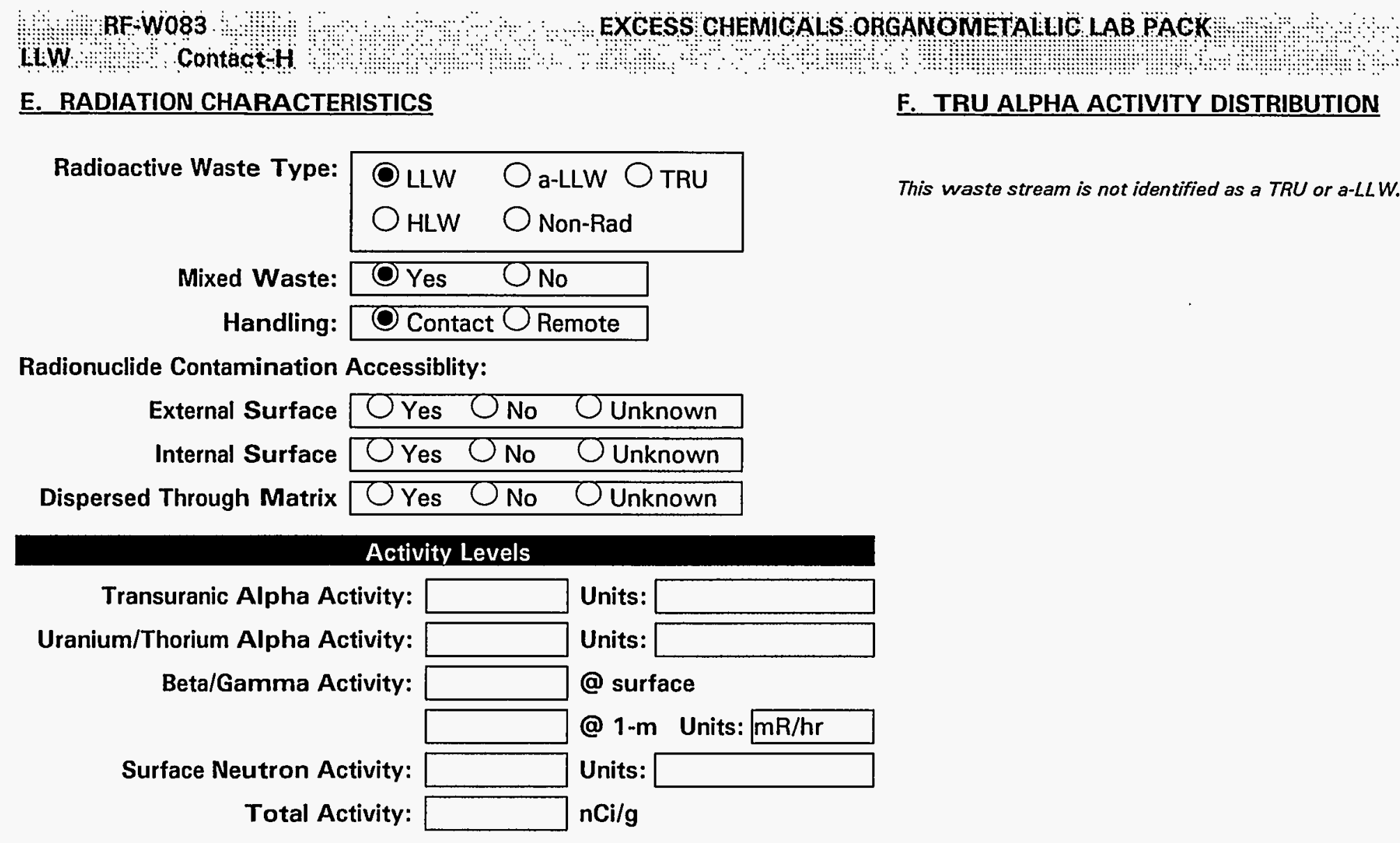

$\begin{array}{ll}\text { O LLW } & \text { Oa-LLW } \bigcirc \text { TRU } \\ \text { OHLW } & \text { O Non-Rad }\end{array}$

Wed Waste:

\begin{tabular}{l} 
O Yes O No \\
\hline Contact $O$ Remote
\end{tabular}

Radionuclide Contamination Accessiblity:

External Surface

Internal Surface $\mathrm{nCi} / \mathrm{g}$ 


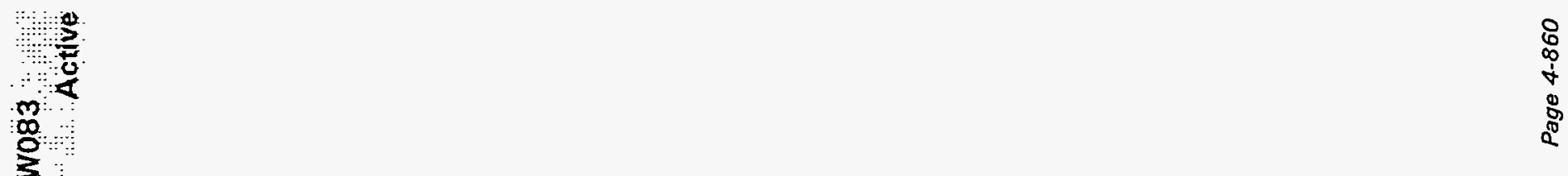

范: : 竞

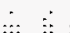

$\because$

$\vdots$

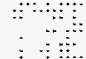

$\because$

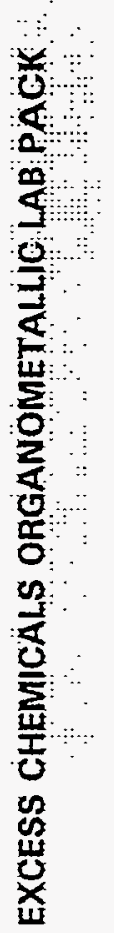

ह5

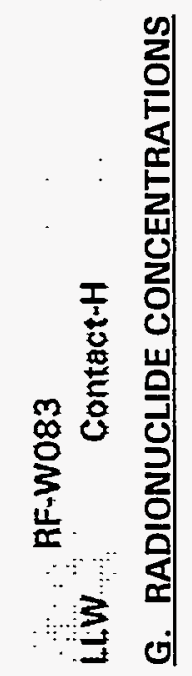


HLW ofolontact-H

H. MATRIX CHARACTERISTICS

Primary Matrix Code: X6900

Description: Lab Packs: Unknown/Other Lab Packs: :

Combustibility: $\bigcirc$ Combustible (>90\%)

Mixed $(10 \%-90 \%)$

Noncombustible $(<10 \%)$

\section{MATRIX COMPOSITION}




\section{J. HAZARDOUS CONSTITUENTS}

\begin{tabular}{|c|c|c|c|c|c|c|c|c|}
\hline & & & & Con & tion & & & \\
\hline Code & Hazardous Waste Description & Treatment Subcategory & Typical & LL & UL & Units & Basis & Conc. Type \\
\hline 0001 & Ignitable & High-TOC ignitable characteristic liquids subca & & & & & A & Total \\
\hline D002 & Corrosive & Corrosive characteristic waste that are manag & & & & & A & Total \\
\hline D003 & Reactive & Reactive Cyanides based on $261.23(a)(5)$ Cyan & & & & & A & Total \\
\hline D004 & Arsenic & & & & & & A & Total \\
\hline D005 & Barium & & & & & & A & Total \\
\hline D006 & Cadmium & & & & & & A & Total \\
\hline D007 & Chromium & Chromium (Total) & & & & & A & Total \\
\hline$D 008$ & Lead & & & & & & $A$ & Total \\
\hline D009 & Mercury & Elemental mercury contaminated with radioact & & & & & A & Total \\
\hline D010 & Selenium & & & & & & A & Total \\
\hline D011 & Silver & & & & & & A & Total \\
\hline D016 & 2,4-D & 2,4-D (2,4-Dichlorophenoxyacetic acid) & & & & & A & Total \\
\hline D017 & 2,4,5-TP (Silvex) & & & & & & A & Total \\
\hline 0019 & Carbon tetrachloride & Carbon Tetrachloride managed in non-CWA/no & & & & & A & Total \\
\hline$D 021$ & Chlorobenzene & Chlorobenzene managed in non-CWA/non-CW & & & & & A & Total \\
\hline$\overline{D 022}$ & Chloroform & Chloroform managed in non-CWA/non-CWA-e & & & & & A & Total \\
\hline $\mathrm{DO} 24$ & m-Cresol & m-Cresol managed in non-CWA/non-CWA-equi & & & & & A & Total \\
\hline $\mathrm{DO25}$ & p-Cresol & p-Cresol managed in non-CWA/non-CWA-equi & & & & & A & Total \\
\hline 0027 & p-Dichlorobenzene (1,4-Dichlorobenzene) & p-Dichlorobenzene managed in non-CWA/non- & & & & & A & Total \\
\hline 0028 & 1,2-Dichloroethane & 1,2-Dichloroethane managed in non-CWA/non- & & & & & A & Total \\
\hline $\mathrm{D029}$ & 1,1-Dichloroethylene & 1,1-Dichloroethylene managed in non-CWA/no & & & & & A & Total \\
\hline D033 & Hexachlorobutadiene & Hexachlorobutadiene managed in non-CWA/no & & & & & A & Total \\
\hline D035 & Methyl ethyl ketone & Methyle ethyl ketone managed in non-CWA/no & & & & & A & Total \\
\hline D036 & Nitrobenzene & Nitrobenzene managed in non-CWA/non-CWA- & & & & & A & Total \\
\hline D037 & Pentachlorophenol & Pentachlorophenol managed in non-CWA/non- & & & & & A & Total \\
\hline 0038 & Pyridine & Pyridine managed in non-CWA/non-CWA-equiv & & & & & A & Total \\
\hline D039 & Tetrachloroethylene & Tetrachloroethylene managed in non-CWA/non & & & & & $\bar{A}$ & Total \\
\hline D042 & 2,4,6-Trichlorophenol & 2,4,6-Trichlorophenol managed in non-CWA/n & & & & & A & Total \\
\hline$\overline{P 022}$ & Carbon disulfide & & & & & & $\bar{A}$ & Total \\
\hline $\mathrm{PO24}$ & p-Chloroaniline & & & & & & A & Total \\
\hline
\end{tabular}




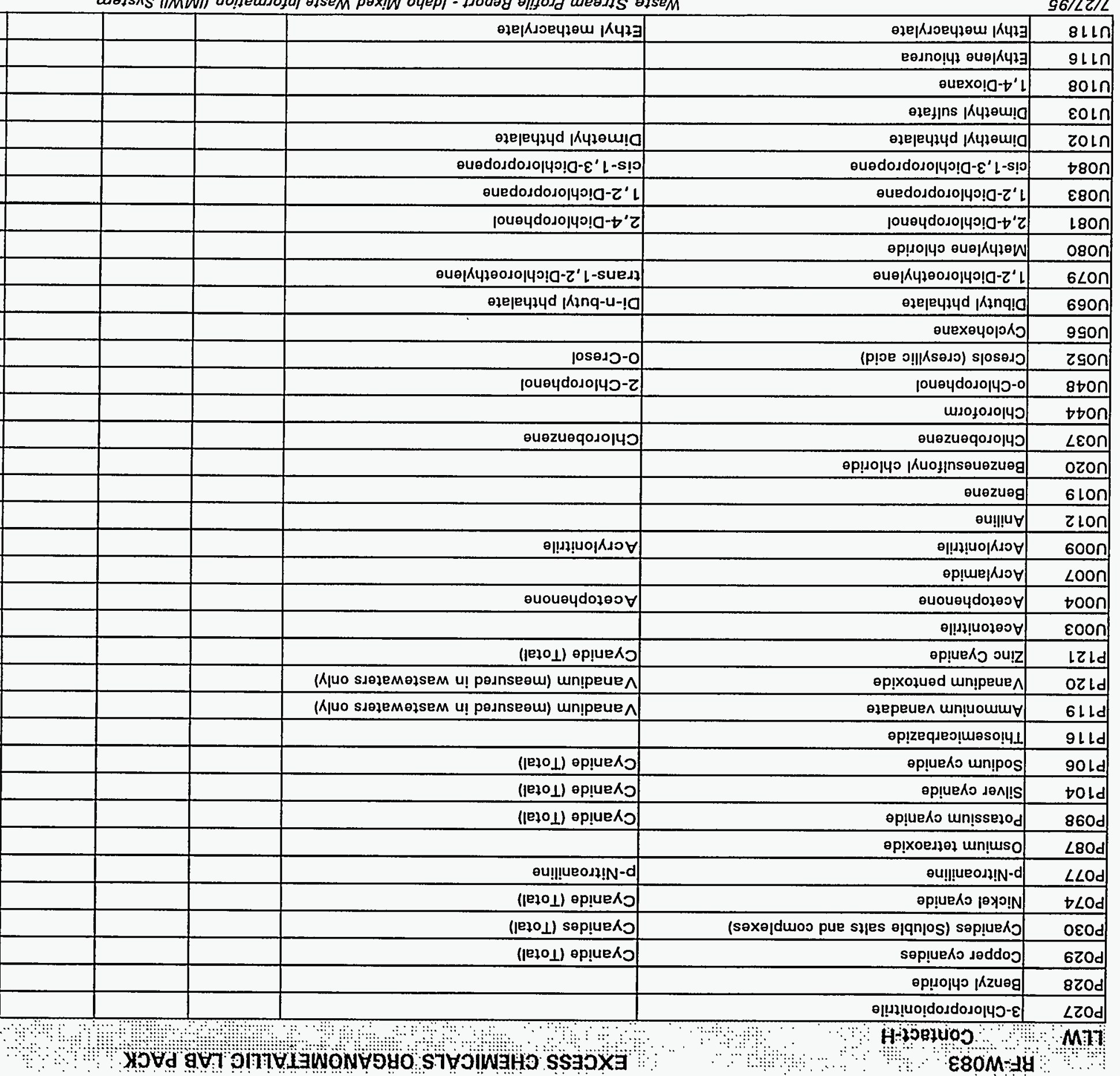




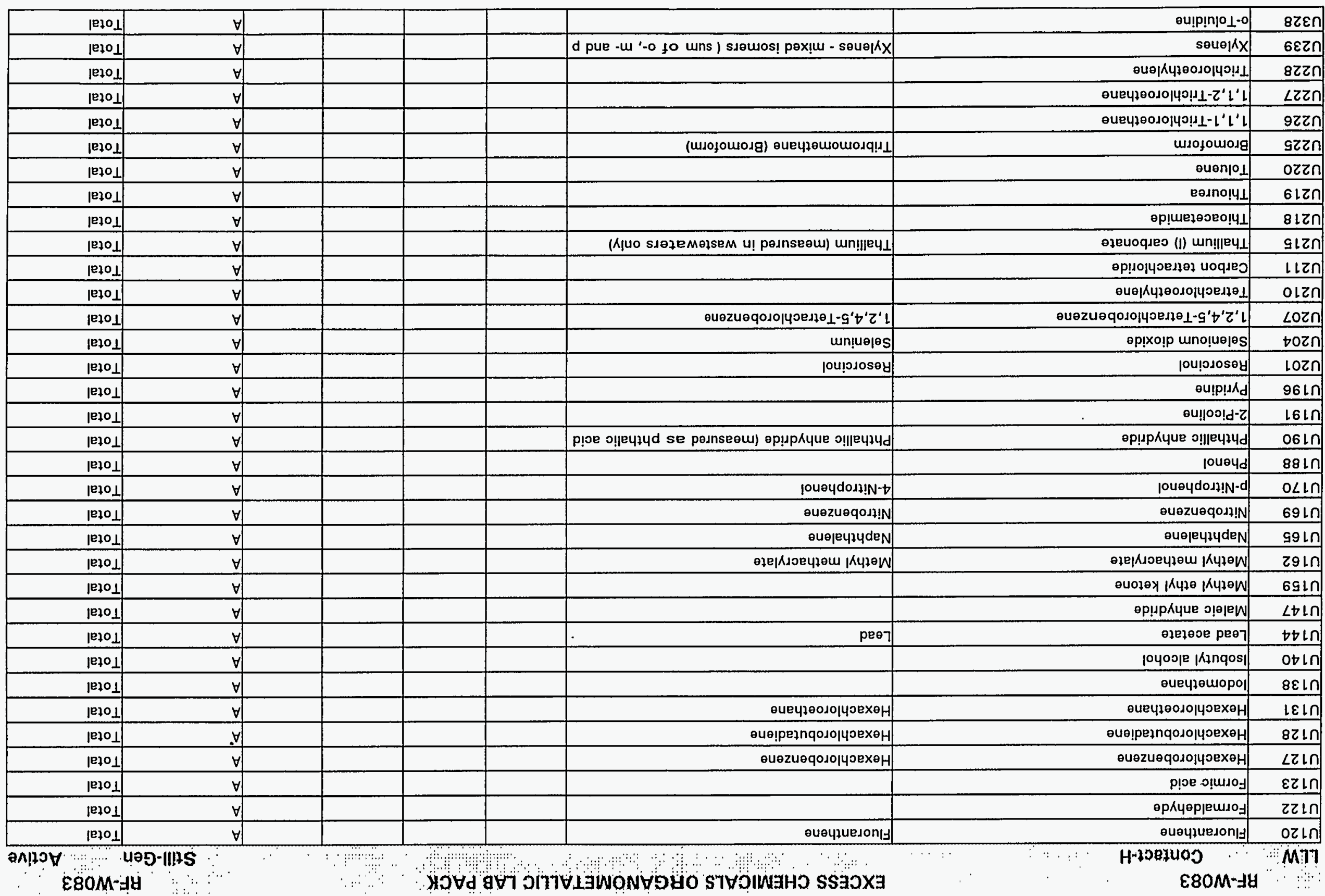




\section{K. POLYCHLORINATED BIPHENYLS (PCBS)}

Is this waste stream PCB contaminated? OYes $O$ No $O$ Unknown

If yes, what portion of the waste is PCB contaminated (volume \%):

If $<100 \%$, can the PCB contaminated waste be segregated and treated separately?

What is the PCB concentration in ppm?

If only a portion of the waste is $P C B$ contaminated, report values for contaminated portion.

\section{TREATMENT PLANS}

\begin{tabular}{|c|c|c|c|c|c|c|c|c|c|c|c|}
\hline $\begin{array}{l}\text { Opt. } \\
\#\end{array}$ & $\begin{array}{l}\text { Media Type } \\
\text { (If more than onel }\end{array}$ & $\begin{array}{c}\text { Matrix } \\
\text { Code }\end{array}$ & $\begin{array}{l}\text { Vol. \% } \\
\text { Iff }\end{array}$ & Step & JIT & SC & CIF & $\begin{array}{l}\text { Trans. } \\
\text { Miles }\end{array}$ & $\begin{array}{l}\text { Facility } \\
\text { Abbr. }\end{array}$ & Unit Name & Comments \\
\hline 1 & & $\times 6100$ & & $\bar{a}$ & 0 & 0 & $1.00 E+00$ & & TRANS & Transport - LLW & \\
\hline & & & & b & $\mathbf{O}$ & 0 & $1.00 E+00$ & & AMWTF & $\mathrm{CH}$ - Opening \& Sorting & \\
\hline & & & & c & O & $\mathrm{O}$ & $1.00 E+00$ & & AMWTF & $\mathrm{CH}-\operatorname{sizing}$ & \\
\hline & & & & $d$ & 0 & 0 & $1.00 \mathrm{E}+00$ & & AMWTF & $\mathrm{CH}$ - Incineration/Thermal Desorption & \\
\hline & & & & e & 0 & 0 & $1.00 \mathrm{E}+00$ & & AMWTF & $\mathrm{CH}$ - Vitrification & \\
\hline & & & & $f$ & 0 & 0 & $1.00 E+00$ & & SCDF & Disposal - Contact Handled & \\
\hline
\end{tabular}

Note: Where provided, media percentages are rough estimates used to facilitate treatment planning. They do not necessarily imply an accurate knowledge of waste stream composition, and were not derived for use outside this conceptual planning exercise.

Describe any special or unique technical concerns related to the treatment of this waste stream that would impact the use or implementation of standard hazardous waste treatment methods. 


\section{RF-W085}

LiWW Contact-H

\section{A. IDENTIFICATION AND DESCRIPTION}

Waste Stream ID: RF-W085

W. S. Name:

Content Code:

FFCA ID:

EXCESS CHEMICALS NON-LABPACKS W/D009/LLM

*

RF-W085

Waste Stream Description:

This waste form is packaged into drums in various buildings at Rocky Flats.

\section{EXCESS CHEMICALS NON-LABPACKS W/DOO9/LLM}

RF-WOSE Still-Gen Active

\section{B. GENERATION SITE/PROCESS DESCRIPTION}

The following questions, concerning the generation site and process, should be answered even if the waste stream is no longer generated.

Name of Site Generating Waste (e.g., INEL, Rocky Flats, etc.): Rocky Flats

Location of activities (Area and Building):

Various buildings.

Operations (functions) performed in building:

Description of Process Generating Waste:

The lab packs and excess chemicals are generated throughout Rocky Flats site as the chemicals reach their expiration date or become unneeded as Rocky Flats' mission is changed. All of the radioactive wastes in this category are described as excess chemicals at this time. 
RFWOO85

LWW Contact H

C. PROJECTED WASTE GENERATION

Is this waste stream still generated?

If yes, when is the expected generation termination date?
RF-WO85

Still-Gen Active

Current Projections

\begin{tabular}{|r|r|r|r|r|r|}
\hline Est. Date & Start Year & Interval & Vol (m3) & Mass (kg) & Source of Estlmate \\
\hline $111 / 1 / 94$ & 1997 & 1 & 13.49 & & \\
\hline
\end{tabular}

Historical Projections 


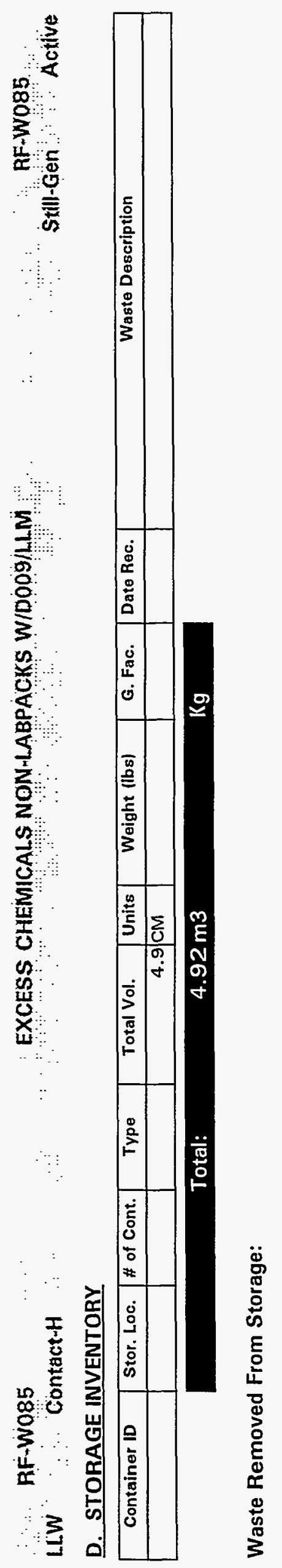




\section{E. RADIATION CHARACTERISTICS}

Radioactive Waste Type:

\begin{tabular}{|ll|}
\hline OllW & Oa-llW $\bigcirc$ TRU \\
OhlW & O Non-Rad
\end{tabular}

Mixed Waste:

Handling:

\begin{tabular}{|l|}
\hline OYes ONo \\
\hline O Contact O Remote \\
\hline
\end{tabular}

Radionuclide Contamination Accessiblity:

External Surface $O$ Yes O No OUnknown

Internal Surface OYes O No OUnknown

Dispersed Through Matrix OYes O No OUnknown

\section{Activity Levels}

Transuranic Alpha Activity:

Uranium/Thorium Alpha Activity:

Beta/Gamma Activity:

Surface Neutron Activity:

Total Activity:

\begin{tabular}{|c|c|c|}
\hline & \multirow{2}{*}{$\begin{array}{l}\text { Units: } \\
\text { Units: }\end{array}$} & \\
\hline & & \\
\hline & \multicolumn{2}{|c|}{ @ surface } \\
\hline & @ 1-m & Units: $\mathrm{mR} / \mathrm{hr}$ \\
\hline & Units: & \\
\hline & $\mathrm{nCi} / \mathrm{g}$ & \\
\hline
\end{tabular}

\section{F. TRU ALPHA ACTIVITY DISTRIBUTION}

This waste stream is not identified as a TRU or a-LLW. 


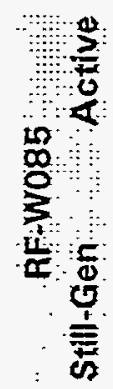

5 竞

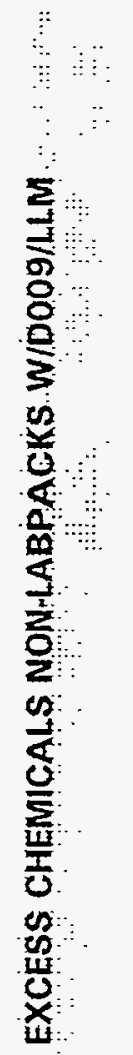

崖

요융

눈 은

$\because$ द

$\prod_{3}^{3}$ 


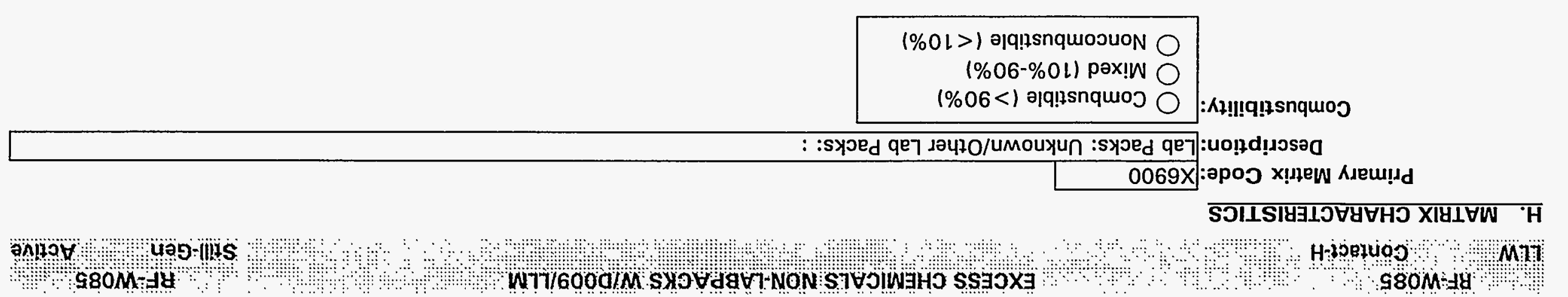




\section{LLW *. Contact-H}

\section{J. HAZARDOUS CONSTITUENTS}

\begin{tabular}{|c|c|c|c|c|c|c|c|c|}
\hline & & & \multicolumn{4}{|c|}{ Concentration } & \multirow[b]{2}{*}{ Basis } & \multirow[b]{2}{*}{ Conc. Type } \\
\hline Code & Hazardous Waste Description & Treatment Subcategory & Typical & LL. & UL & Units & & \\
\hline 0001 & Ignitable & High-TOC ignitable characteristic liquids subca & & & & & A & Total \\
\hline$\overline{\mathrm{DOO2}}$ & Corrosive & Corrosive characteristic waste that are manag & & & & & A & Total \\
\hline$\overline{D 003}$ & Reactive & Reactive Cyanides based on $261.23(a)(5)$ Cyan & & & & & $\bar{A}$ & Total \\
\hline DOO4 & Arsenic & & & & & & A & Total \\
\hline D005 & Barium & & & & & & A & Total \\
\hline 0006 & Cadmium & & & & & & A & Total \\
\hline D007 & Chromium & Chromium (Total) & & & & & A & Total \\
\hline D008 & Lead & Radioactive high level wastes generated durin & & & & & A & Total \\
\hline 0009 & Mercury & Elemental mercury contaminated with radioact & & & & & A & Total \\
\hline 0010 & Selenium & & & & & & A & Total \\
\hline D011 & Silver & & & & & & A & Total \\
\hline$\overline{0012}$ & Endrin & & & & & & A & Total \\
\hline D015 & Toxaphene & & & & & & A & Total \\
\hline D019 & Carbon tetrachloride & Carbon Tetrachloride managed in non-CWA/no & & & & & A & Total \\
\hline D022 & Chloroform & Chloroform managed in non-CWA/non-CWA-e & & & & & A & Total \\
\hline$\overline{D 025}$ & p-Cresol & p-Cresol managed in non-CWA/non-CWA-equi & & & & & A & Total \\
\hline D039 & Tetrachloroethylene & Tetrachloroethylene managed in non-CWA/non & & & & & A & Total \\
\hline 0040 & Trichloroethylene & Trichloroethylene managed in non-CWA/non-C & & & & & A & Total \\
\hline D043 & Vinyl chloride & Vinyl Chloride managed in non-CWA/non-CWA & & & & & A & Total \\
\hline P012 & Arsenic trioxide & & & & & & A & Total \\
\hline$P 015$ & Beryllium powder & & & & & & A & Total \\
\hline P030 & Cyanides (Soluble salts and complexes) & Cyanides (Total) & & & & & A & Total \\
\hline P074 & Nickel cyanide & Cyanide (Total) & & & & & A & Total \\
\hline P087 & Osmium tetraoxide & & & & & & A & Total \\
\hline P113 & Thallic oxide & Thallium (measured in wastewaters only) & & & & & A & Total \\
\hline 0080 & Methylene chloride & & & & & & $\bar{A}$ & Total \\
\hline U123 & Formic acid & & & & & & A & Total \\
\hline U127 & Hexachlorobenzene & Hexachlorobenzene & & & & & A & Total \\
\hline U151 & Mercury (High mercury $>260 \mathrm{mg} / \mathrm{kg}$ ) & & & & & & A & Total \\
\hline U170 & p-Nitrophenol & 4-Nitrophenol & & & & & A & Total \\
\hline U188 & Phenol & & & & & & A & Total \\
\hline U201 & Resorcinol & Resorcinol & & & & & A & Total \\
\hline U204 & Selenioum dioxide & Selenium & & & & & A & Total \\
\hline
\end{tabular}




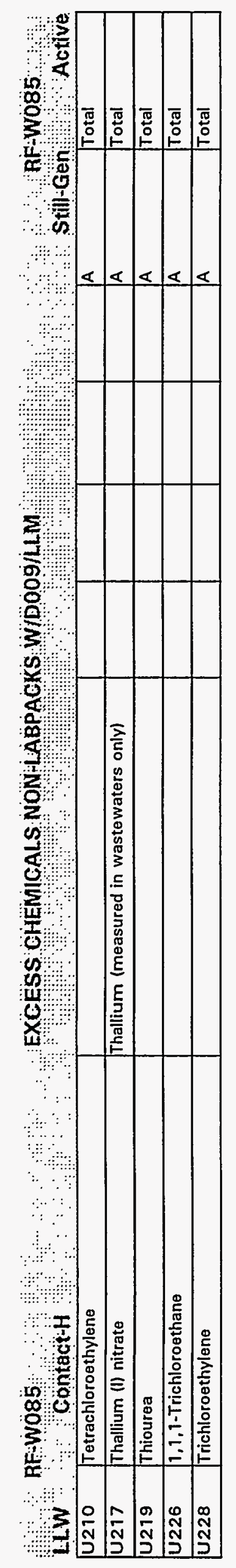

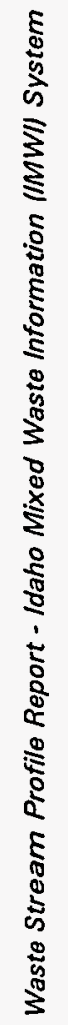




\section{RF-W085 \\ L.W …... Contact-H \\ K. POLYCHLORINATED BIPHENYLS (PCBS)}

Is this waste stream PCB contaminated? OYes $O$ No $O$ Unknown

If yes, what portion of the waste is PCB contaminated (volume \%):

If $<100 \%$, can the PCB contaminated waste be segregated and treated separately?

If only a portion of the waste is PCB contaminated, report values for contaminated portion.

What is the PCB concentration in ppm?

\section{TREATMENT PLANS}

\begin{tabular}{|c|c|c|c|c|c|c|c|c|c|c|c|}
\hline $\begin{array}{c}\text { Opt. } \\
\#\end{array}$ & $\begin{array}{c}\text { Media Type } \\
\text { (If more than one) }\end{array}$ & $\begin{array}{l}\text { Matrix } \\
\text { Code }\end{array}$ & $\begin{array}{l}\text { Vol. \% } \\
\text { lif }\end{array}$ & Step & JIT & sc & CIF & $\begin{array}{l}\text { Trans. } \\
\text { Miles }\end{array}$ & $\begin{array}{l}\text { Facility } \\
\text { Abbr. }\end{array}$ & Unit Name & Comments \\
\hline 1 & & $\times 6000$ & & $\bar{a}$ & (O) & 0 & $1.00 E+00$ & & TRANS & Transport - LLW & \\
\hline & & & & b & (1) & 0 & $1.00 \mathrm{E}+00$ & & AMWTF & $\mathrm{CH}$ - Opening \& Sorting & \\
\hline & & & & c & (0) & 0 & $1.00 E+00$ & & AMWTF & $\mathrm{CH} \cdot$ Sizing & \\
\hline & & & & $d$ & 0 & 0 & $1.00 \mathrm{E}+00$ & & AMWTF & $\mathrm{CH}$ - Incineration/Thermal Desorption & \\
\hline & & & & $\theta$ & 0 & 0 & $1.00 E+00$ & & AMWTF & $\mathrm{CH}$ - Vitrification & \\
\hline & & & & $f$ & 0 & 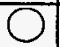 & $1.00 E+00$ & & SCDF & Disposal - Contact Handled & \\
\hline
\end{tabular}

Note: Where provided, media percentages are rough estimates used to facilitate treatment planning. They do not necessarily imply an accurate knowledge of waste stream composition, and were not derived for use outside this conceptual planning exercise.

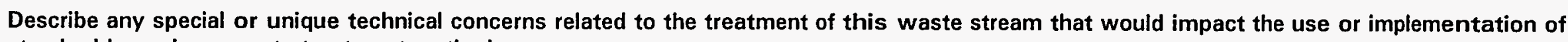
standard hazardous waste treatment methods. 


\section{A. IDENTIFICATION AND DESCRIPTION}

Waste Stream ID: RF-W086

\section{W. S. Name:}

Content Code:

FFCA ID:

EXCESS CHEMICALS NON-LAB PACKS-OTHER/LLM

\section{Waste Stream Description:}

Lab packs, excess chemicals. This waste form is packaged into drums in various buildings in

Rocky Flats and is generated on a continuous basis.

\section{B. GENERATION SITE/PROCESS DESCRIPTION}

The following questions, concerning the generation site and process, should be answered even if the waste stream is no longer generated.

Name of Site Generating Waste (e.g., INEL, Rocky Flats, etc.): Rocky Flats

Location of activities (Area and Building):

Various builgings.

Operations (functions) performed in building:

Description of Process Generating Waste:

The lab packs and excess chemicals are generated throughout Rocky Flats site as the chemicals reach their expiration date or become unneeded as Rocky Flats' mission is changed. All radioactive wastes in this category are described as excess chemicals at this time. 
RF-W086

Lilw ContactrH

C. PROJECTED WASTE GENERATION

Is this waste stream still generated?

If yes, when is the expected generation termination date?

Current Projections

\begin{tabular}{|r|r|r|r|r|l|}
\hline Est. Date & Start Year & Interval & Vol (m3) & Mass (kg) & Source of EstImate \\
\hline $3 / 31 / 95$ & 1995 & 5 & 13.49 & & Generator Update \\
\hline \multicolumn{7}{|c|}{ Historical Projections } \\
\hline Est. Date & Start Year & Interval & Vol (m3) & Mass (kg) & Source of Estimate \\
\hline $11 / 1 / 93$ & 1997 & 1 & 4.73 & & $1993-F F C A$ \\
\hline
\end{tabular}

OYes ONo

RF-W086

Still-Gen

Active 


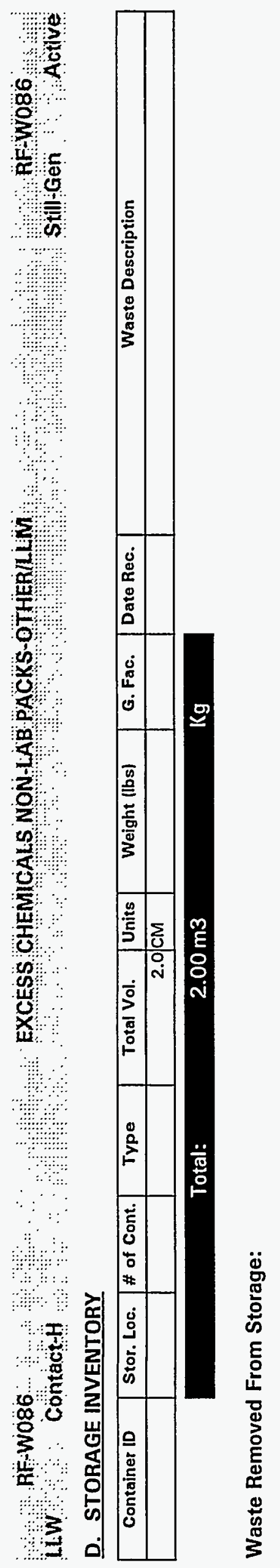




\section{LLW Contact-H \\ E. RADIATION CHARACTERISTICS}

Radioactive Waste Type:

$\begin{array}{ll}\text { OlLW } & \bigcirc \text { a-LLW } \bigcirc \text { TRU } \\ \text { OHLW } & \bigcirc \text { Non-Rad }\end{array}$

Mixed Waste:

\begin{tabular}{|l|}
\hline O Yes ONo \\
\hline O Contact $O_{\text {Remote }}$
\end{tabular}

Radionuclide Contamination Accessiblity:

External Surface $O$ Yes ONo OUnknown

Internal Surface OYes ONo O Unknown

Dispersed Through Matrix $O$ Yes $O$ No $O$ Unknown

\section{Activity Levels}

Transuranic Alpha Activity:

Uranium/Thorium Alpha Activity:

Beta/Gamma Activity:

Surface Neutron Activity:

Total Activity:

\begin{tabular}{|c|c|c|}
\hline & Units: & \\
\hline & Units: [ & \\
\hline & @ surf & face \\
\hline & @ 1-m & Units: $m R / h r$ \\
\hline & Units: [ & \\
\hline & $\mathrm{nCi} / \mathrm{g}$ & \\
\hline
\end{tabular}

RF-W086

Still:Gen

Active

\section{F. TRU ALPHA ACTIVITY DISTRIBUTION}

This waste stream is not identified as a TRU or a-LLW. 


\section{$\mathrm{RFWO} 86$ \\ G. RADIONUCLIDE CONCENTRATIONS}


RF-W086

EXCESS CHEMICALS NON-LAB PACKS OTHER/LM

RF $-W 086$

LLW $\cdots$ ContactrH

$\because$ O O

H. MATRIX CHARACTERISTICS

Primary Matrix Code: X6900

Description: Lab Packs: Unknown/Other Lab Packs: :

Combustibility: $\bigcirc$ Combustible (>90\%)

Mixed $(10 \%-90 \%)$

Noncombustible $(<10 \%)$

\section{MATRIX COMPOSITION}




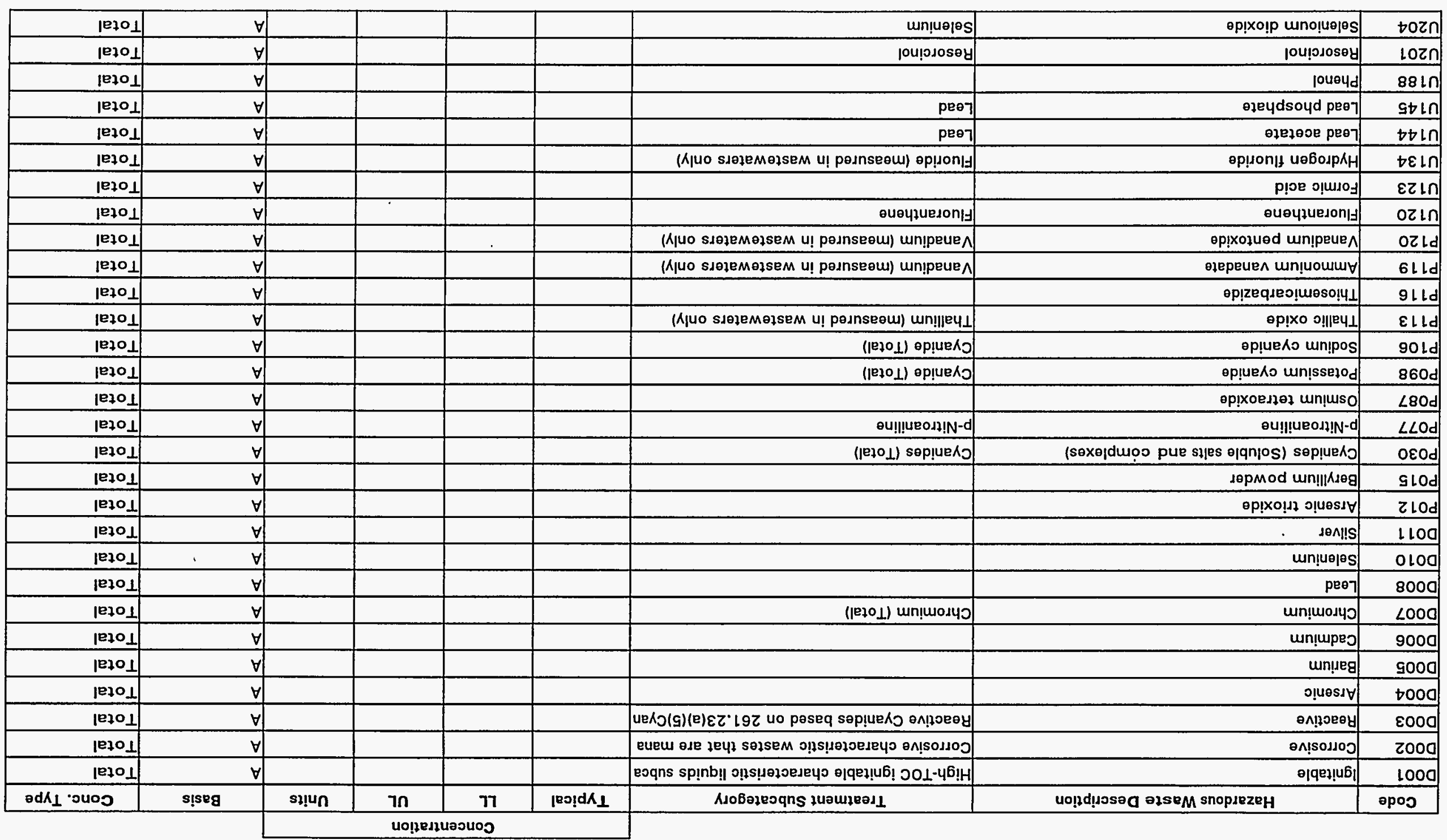




\section{Lilw Contact-H}

EXCESS CHEMICALS NON-LAB PACKS-OTHERILLM

RF-W086

\section{K. POLYCHLORINATED BIPHENYLS (PCBS)}

Is this waste stream PCB contaminated?

OYes

(O) No

If yes, what portion of the waste is PCB contaminated (volume \%):

If $<100 \%$, can the PCB contaminated waste be segregated and treated separately?

What is the PCB concentration in ppm?

If only a portion of the waste is PCB contaminated, report values for contaminated portion.

\section{TREATMENT PLANS}

\begin{tabular}{|c|c|c|c|c|c|c|c|c|c|c|c|}
\hline $\begin{array}{l}\text { Opt. } \\
\#\end{array}$ & $\begin{array}{c}\text { Media Type } \\
\text { (If more than one) }\end{array}$ & $\begin{array}{c}\text { Matrix } \\
\text { Code }\end{array}$ & $\begin{array}{l}\text { Vol. \% } \\
\text { (If }\end{array}$ & Step & JIT & sc & CIF & $\begin{array}{l}\text { Trans. } \\
\text { Miles }\end{array}$ & $\begin{array}{l}\text { Facility } \\
\text { Abbr. }\end{array}$ & Unit Name & Comments \\
\hline \multirow[t]{6}{*}{1} & & $\times 6000$ & & $\bar{a}$ & () & 0 & $1.00 \mathrm{E}+00$ & - & TRANS & Transport - LLW & \\
\hline & & & & $\mathbf{b}$ & O & 0 & $1.00 E+00$ & & AMWTF & $\mathrm{CH}$ - Opening \& Sorting & \\
\hline & & & & c & () & 0 & $1.00 E+00$ & & AMWTF & $\mathrm{CH} \cdot$ Sizing & \\
\hline & & & & d & 0 & 0 & $1.00 \mathrm{E}+00$ & & AMWTF & $\mathrm{CH}$ - Incineration/Thermal Desorption & \\
\hline & & & & e & 0 & $\bar{O}$ & $1.00 E+00$ & & AMWTF & $\mathrm{CH} \cdot$ Vitrification & \\
\hline & & & & $f$ & 0 & 0 & $1.00 E+00$ & & SCDF & Disposal - Contact Handled & \\
\hline
\end{tabular}

Note: Where provided, media percentages are rough estimates used to facilitate treatment planning. They do not necessarily imply an accurate knowledge of waste stream composition, and were not derived for use outside this conceptual planning exercise.

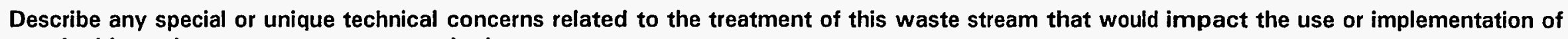
standard hazardous waste treatment methods. 


\section{LEW \\ A. IDENTIFICATION AND DESCRIPTION \\ Waste Stream ID: SR-W014 \\ W. S. Name: \\ Content Code: \\ FFCA ID:

\begin{tabular}{|l|}
\hline SR-WITIATED MERCURY \\
\hline$*$ \\
\hline SR-W014 \\
\hline
\end{tabular}

\section{Waste Stream Description:}

Elemental mercury with dissolved metal contaminants derived from leaching/erosion of stainlesssteel pump housings and piping (HWl\# 002 ).

The steel-component contaminants ( $\mathrm{Fe}, \mathrm{C}, \mathrm{Cr}$, etc.) are mostly present as metal

oxides/hydroxides. Because of the limited solubility of these contaminants in mercury, they are present mostly as a separate slag/cruds phase, floating on the bulk liquid.

While mercury is inert to and immiscible with hydrogen (tritium), direct contact with process gases has resulted in tritium contamination, which is associated with the metal-oxide

contaminants. Studies of similar material (performed at Lawrence Livermore National

Laboratory - LLNL) have shown that the majority of this tritium $(>>90 \%)$ is associated with

floating cruds $(10-3 \mathrm{Ci} / \mathrm{g})$. The remainder of the tritium (typically $2.5 \%$ ) is associated with the

dissolved contaminants in the bulk liquid $(10-9 \mathrm{Ci} / \mathrm{g})$.
SR WOY 14

Stillogen alo Active

\section{B. GENERATION SITE/PROCESS DESCRIPTION}

The following questions, concerning the generation site and process, should be answered even if the waste stream is no longer generated.

Name of Site Generating Waste (e.g., INEL, Rocky Flats, etc.): SRS

Location of activities (Area and Building): Tritium Facility (Bldgs 232-H and 234-H)

Operations (functions) performed in building:

Purification and packaging of tritium-containing gas mixtures.

Description of Process Generating Waste:

Process gases are transferred through the facility either by direct entrainment with mercury mists or by pressure differentials created by evaporation/condensation of mercury. Direct contact between the motive-fluid mercury and the tritium-containing process gases result. 


\section{: SR+W014 \\ LLW ...... Contactr \\ C. PROJECTED WASTE GENERATION}

TRITIATED MERCURY

$\sin +$ WO14

Still-Gën

Active

Is this waste stream still generated?

O Yes ONo

If yes, when is the expected generation termination date?

Current Projections

\begin{tabular}{|r|r|r|r|l|l|}
\hline Est. Date & Start Year & Interval & Vol (m3) & Mass (kg) & Source of Estimate \\
\hline $11 / 15 / 94$ & 1997 & 1 & 0.10 & & 1994-DSTP \\
\hline \multicolumn{6}{|c|}{ Historical Projections } \\
\hline
\end{tabular}

\begin{tabular}{|c|r|r|r|c|c|}
\hline Est. Date & \multicolumn{1}{|c|}{ Start Year } & Interval & Vol (m3) & Mass (kg) & Source of Estimate \\
\hline $11 / 1 / 93$ & 1998 & 5 & 0.05 & 721.00 & $1993-F F C A$ \\
\hline
\end{tabular}



LlWW ContacteH

\section{STORAGE INVENTORY}

\begin{tabular}{|c|c|c|c|c|}
\hline Container ID & Stor. Loc. & \# of Cont. & Type & Total Vol. \\
\hline & & & & 0.3 \\
\hline
\end{tabular}

Total:
SR Wo14

TRITIATED MIERCURY

SÄWO14

Waste Removed From Storage: 


\section{F. TRU ALPHA ACTIVITY DISTRIBUTION}

Radioactive Waste Type:

\begin{tabular}{|ll} 
OllW & Oa-LlW O TRU \\
OhlW & O Non-Rad
\end{tabular}

Mixed Waste:

Handling:

Radionuclide Contamination Accessiblity:

External Surface $O$ Yes $O$ No O Unknown

Internal Surface OYes O No OUnknown

Dispersed Through Matrix $O$ Yes O No O Unknown

This waste stream is not identified as a TRU or a- $L L W$.

\section{Activity Levels}

$\begin{aligned} & \text { Transuranic Alpha Activity: } \\ & \text { Uranium/Thorium Alpha Activity: } \text { Units: } \\ & \text { Beta/Gamma Activity: } \text { Units: } \\ & \\ & \text { Surface Neutron Activity: } \text { @ surface } \\ & \text { Total Activity: } \text { @ 1-m Units: } \\ & \text { Units: } \\ &\end{aligned}$


SR-WO14

LEW Contact H

\section{G. RADIONUCLIDE CONCENTRATIONS}

\begin{tabular}{|c|c|c|c|c|c|c|}
\hline & \multicolumn{3}{|c|}{ Specific Activity } & \multirow[b]{2}{*}{ Units } & \multirow[b]{2}{*}{ Basis Code(s) } & \multirow[b]{2}{*}{ Basis Desc. lif other) } \\
\hline Isotope & Typical & Lower Limit & Upper Limit & & & \\
\hline $\mathrm{H}-3$ & $3.5000 E+02$ & & & $n C i / g$ & 1 & \\
\hline $\begin{array}{l}\text { A. Passiv } \\
\text { B. Segme } \\
\text { C. Passiv } \\
\text { D. Radioc } \\
\text { E. Mass }\end{array}$ & $\begin{array}{l}\text { 4ctive Neutron } \\
\text { ed Gamma Scal } \\
\text { 4ctive Neutron/ } \\
\text { mical Ana/ysis } \\
\text { ectrometry }\end{array}$ & $\begin{array}{l}\text { PAN) } \\
\text { (SGS) } \\
\text { Segmented Ga }\end{array}$ & na Scan IPAA & (SGSI & $\begin{array}{ll}\text { F. Accountability } \\
\text { G. Calorimetry } \\
\text { H. Multiple Energy } \\
\text { I. Process Knowlec } \\
\text { J. Other }\end{array}$ & is (MEGAS) \\
\hline
\end{tabular}




\section{SR-W014}

TRITIATED MERCURY

SR-WO14

LLW Contact

\section{THITIATED MERCURY}

Still-Gen

Active

H. MATRIX CHARACTERISTICS

Primary Matrix Code: X7100

Description: Special Waste: Elemental Mercury: :

Combustibility: $\bigcirc$ Combustible (>90\%)

Mixed (10\%-90\%)

Noncombustible $(<10 \%)$

\section{MATRIX COMPOSITION}

\begin{tabular}{|c|c|c|c|c|c|c|}
\hline & & & \multicolumn{4}{|c|}{ Composition } \\
\hline Code & Matrix Description & Material & Typical & LL & UL & Units \\
\hline$\overline{X 7100}$ & Special Waste.Elemental Mercury.. & Liquid Mercury & 97 & & & $\% w t$ \\
\hline $\mathbf{5 3 1 1 0}$ & Homogeneous Solids.Inorganic Homogeneous Solids.Inorganic Particulates. & Metal-Oxide Slag & 3 & & & $\% w t$ \\
\hline
\end{tabular}




\section{J. HAZARDOUS CONSTITUENTS}

\begin{tabular}{|c|c|c|c|c|c|c|c|c|}
\hline & & & \multicolumn{4}{|c|}{ Concentration } & & \\
\hline Code & Hazardous Waste Description & Treatment Subcategory & Typical & $\mathbf{L L}$ & UL & Units & Basis & Conc. Type \\
\hline D009 & Mercury & Elemental mercury contaminated with radioact & 100 & & & $\%$ vol & B & Total \\
\hline
\end{tabular}




\section{LiW SR-W014 \\ K. POLYCHLORINATED BIPHENYLS (PCBS)}

Is this waste stream PCB contaminated?

Yes

.6.

Unknown

If yes, what portion of the waste is PCB contaminated (volume \%):

If $<100 \%$, can the PCB contaminated waste be segregated and treated separately?

What is the PCB concentration in ppm?

TRITIATED MER̈CURY

SR-WO14

Still-Gen Active

\section{TREATMENT PLANS}

\begin{tabular}{|c|c|c|c|c|c|c|c|c|c|c|c|}
\hline $\begin{array}{c}\text { Opt. } \\
\#\end{array}$ & $\begin{array}{c}\text { Media Type } \\
\text { (If more than one) }\end{array}$ & $\begin{array}{c}\text { Matrix } \\
\text { Code }\end{array}$ & $\begin{array}{l}\text { Vol. \% } \\
\text { IIf }\end{array}$ & Step & JIT & sc & CIF & $\begin{array}{l}\text { Trans. } \\
\text { Miles }\end{array}$ & $\begin{array}{l}\text { Facility } \\
\text { Abbr. }\end{array}$ & Unit Name & Comments \\
\hline \multirow[t]{4}{*}{1} & & $\times 7100$ & & $\bar{a}$ & $\overline{0}$ & 0 & $1.00 E+00$ & & TRANS & Transport - LLW & \\
\hline & & & & $\mathbf{b}$ & 0 & 0 & $1.00 E+00$ & & AMWTF & CH - Opening \& Sorting & \\
\hline & & & & $\mathbf{c}$ & 0 & 0 & $1.00 E+00$ & & AMWTF & $\mathrm{CH}$-Amalgamation & \\
\hline & & & & $\bar{d}$ & 0 & 0 & $1.00 E+00$ & & SCDF & Disposal - Contact Handled & \\
\hline
\end{tabular}

Note: Where provided, media percentages are rough estimates used to facilitate treatment planning. They do not necessarily imply an accurate knowledge of waste stream composition, and were not derived for use outside this conceptual planning exercise.

Describe any special or unique technical concerns related to the treatment of this waste stream that would impact the use or implementation of standard hazardous waste treatment methods. 


\section{B. GENERATION SITE/PROCESS DESCRIPTION}

\section{A. IDENTIFICATION AND DESCRIPTION}

Waste Stream ID: SR-W068

W. S. Name:

LIQUID ELEMENTAL MERCURY

Content Code:

FFCA ID:

$$
\text { SR-W068 }
$$

Waste Stream Description:
The following questions, concerning the generation site and process, should be answered even if the waste stream is no longer generated.

Name of Site Generating Waste (e.g., INEL, Rocky Flats, etc.):

Location of activities (Area and Building):

Operations (functions) performed in building:

Description of Process Generating Waste: 


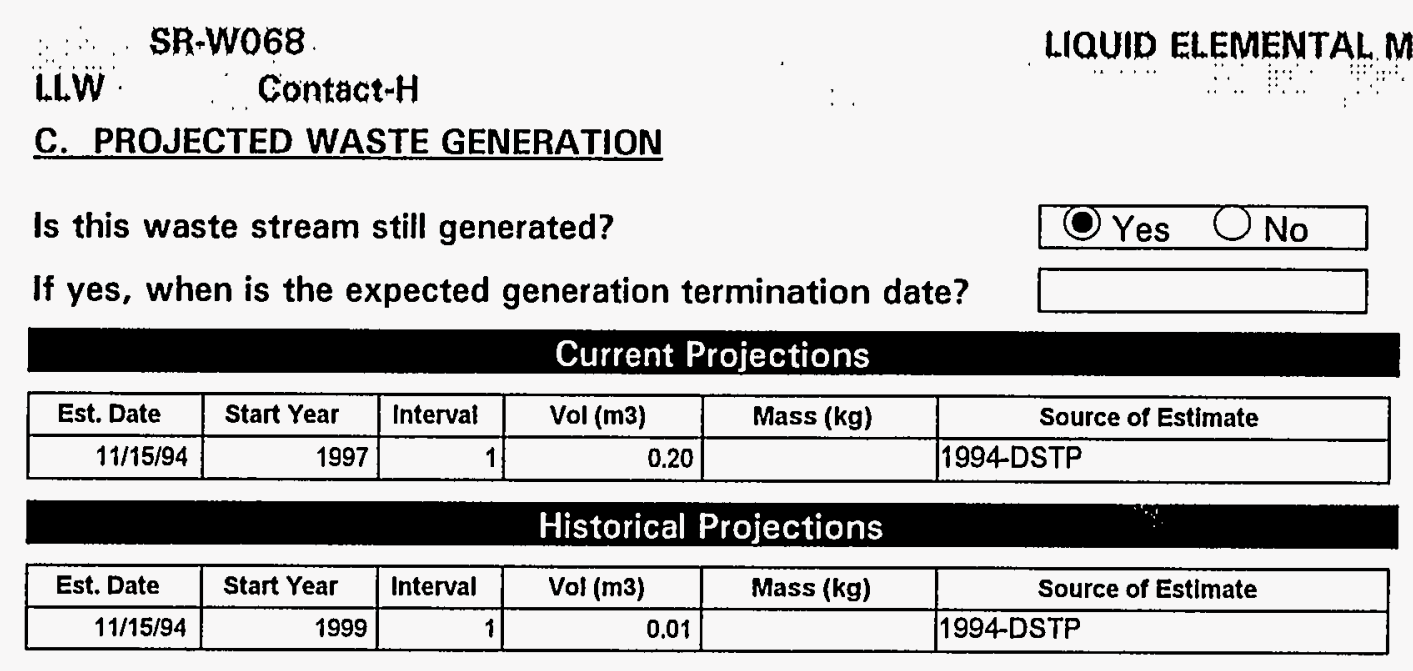


D. STORAGE INVENTORY

\begin{tabular}{|l|c|c|c|c|c|c|c|c|c|}
\hline Container ID & Stor. Loc. & \# of Cont. & Type & Total Vol. & Units & Weight (lbs) & G. Fac. & Date Rec. & Waste Description \\
\hline & & \multicolumn{7}{c|}{0.1} & \multicolumn{7}{c|}{$0.10 \mathrm{~m} 3$} & & & & \\
\hline
\end{tabular}

Waste Removed From Storage: 
SR:W068

LIQUID ELEMENTAL MERCURY

SR-W068

Liw

\section{F. TRU ALPHA ACTIVITY DISTRIBUTION}

Radioactive Waste Type:

\begin{tabular}{|ll|} 
OlLW & Oa-LLW $\bigcirc$ TRU \\
OhLW & ONon-Rad \\
\hline
\end{tabular}

Mixed Waste:

Handling:

\begin{tabular}{|l|}
\hline Yes O No \\
\hline O Contact O Remote \\
\hline
\end{tabular}

Radionuclide Contamination Accessiblity:

External Surface OYes ONo OUnknown

Internal Surface OYes ONo OUnknown

Dispersed Through Matrix OYes ONo OUnknown

\section{Activity Levels}

Transuranic Alpha Activity: Uranium/Thorium Alpha Activity: Beta/Gamma Activity:

Surface Neutron Activity: Total Activity:

\begin{tabular}{|c|c|c|}
\hline & Units: [ & \\
\hline & Units: [ & \\
\hline & \multicolumn{2}{|c|}{ @ surface } \\
\hline & @ 1-m & Units: $\mathrm{mR} / \mathrm{hr}$ \\
\hline & Units: [ & \\
\hline & $\mathrm{nCi} / \mathrm{g}$ & \\
\hline
\end{tabular}

This waste stream is not identified as a TRU or a-LLW. 
H. MATRIX CHARACTERISTICS

Primary Matrix Code: X7100

Description: Special Waste: Elemental Mercury: :

$$
\begin{array}{l|l}
\text { Combustibility: } & \bigcirc \text { Combustible }(>90 \%) \\
\bigcirc \text { Mixed }(10 \%-90 \%) \\
\bigcirc \text { Noncombustible }(<10 \%)
\end{array}
$$

\section{MATRIX COMPOSITION}




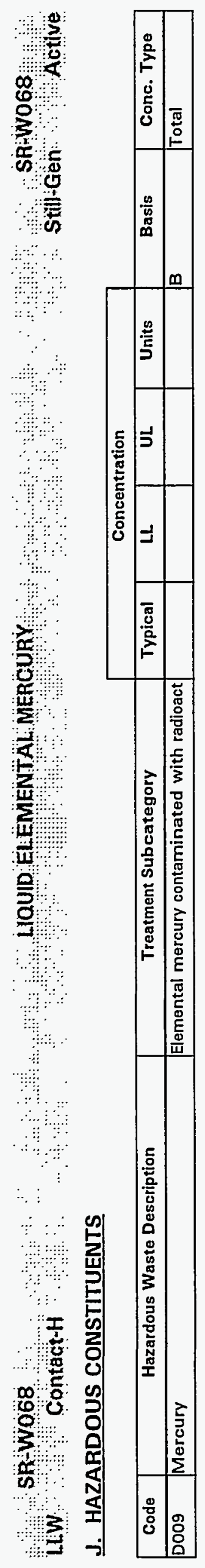




\section{SR-W068 \\ LiW Contact-H}

LQUUID ELEMENTAL MERCUURY

SRWW068

K. POLYCHLORINATED BIPHENYLS (PCBS)

Is this waste stream PCB contaminated? OYes 0 No $O$ Unknown

If yes, what portion of the waste is PCB contaminated (volume \%):

If $<100 \%$, can the PCB contaminated waste be segregated and treated separately?

What is the PCB concentration in ppm?

If only a portion of the waste is PCB contaminated, report values for contaminated portion.

L. TREATMENT PLANS

\begin{tabular}{|c|c|c|c|c|c|c|c|c|c|c|c|}
\hline Opt. & $\begin{array}{c}\text { Media Type } \\
\text { (If more than one) }\end{array}$ & $\begin{array}{c}\text { Matrix } \\
\text { Code }\end{array}$ & $\begin{array}{l}\text { Vol. \% } \\
\text { IIf }\end{array}$ & Step & JIT & sc & CIF & $\begin{array}{l}\text { Trans. } \\
\text { Miles }\end{array}$ & $\begin{array}{l}\text { Facility } \\
\text { Abbr. }\end{array}$ & Unit Name & Comments \\
\hline \multirow[t]{4}{*}{1} & & $\times 7100$ & & a & O) & 0 & $1.00 E+00$ & & TRANS & Transport - LLW & \\
\hline & & & & $\mathbf{b}$ & () & 0 & $1.00 E+00$ & & AMWTF & $\mathrm{CH}$ - Opening \& Sorting & \\
\hline & & & & c & 0 & 0 & $1.00 E+00$ & & AMWTF & $\mathrm{CH}$ - Amalgamation & \\
\hline & & & & d & $O$ & 0 & $1.00 E+00$ & & SCDF & Disposal - Contact Handled & \\
\hline
\end{tabular}

Note: Where provided, media percentages are rough estimates used to facilitate treatment planning. They do not necessarily imply an accurate knowledge of waste stream composition, and were not derived for use outside this conceptual planning exercise.

Describe any special or unique technical concerns related to the treatment of this waste stream that would impact the use or implementation of standard hazardous waste treatment methods. 


\section{B. GENERATION SITE/PROCESS DESCRIPTION}

\section{A. IDENTIFICATION AND DESCRIPTION}

Waste Stream ID: LB-W012

W. S. Name:

Content Code:

FFCA ID:

BASIC SOLIDS w/METALS - HIGH ALPHA

\section{LB-W012}

\section{Waste Stream Description:}

Lab packed solutions and solids with metals and radionuclides. Alpha contamination greater than $10 \mathrm{nCi} / \mathrm{g}$ but less than or equal to $100 \mathrm{nCi} / \mathrm{g}$.
The following questions, concerning the generation site and process, should be answered even if the waste stream is no longer generated.

Name of Site Generating Waste (e.g., INEL, Rocky Flats, etc.):

LBL

Location of activities (Area and Building):

Legacy waste.

Operations (functions) performed in building:

Research and development.

Description of Process Generating Waste:

Cleanup during research activities, eg. wipes, gloves, etc. 


OYes O No

If yes, when is the expected generation termination date?

Current Projections 


\section{STORAGE INVENTORY}

Waste Removed From Storage: 


\section{LB-W012

a-LiW Contact-H \\ E. RADIATION CHARACTERISTICS}

Radioactive Waste Type:

Mixed Waste:

Handling:

\begin{tabular}{|ll|}
\hline OLLW & Oa-LLW $\bigcirc$ TRU \\
OHLW & $\bigcirc$ Non-Rad \\
\hline
\end{tabular}

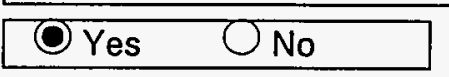

Radionuclide Contamination Accessiblity:

External Surface

Internal Surface

Dispersed Through Matrix

\begin{tabular}{|}
\begin{tabular}{|ccc|}
\hline OYes & O No & O Unknown \\
\hline OYes & O No & O Unknown \\
\hline O Yes & O No & OUnknown \\
\hline
\end{tabular}
\end{tabular}

\section{Activity Levels}

Transuranic Alpha Activity:

Uranium/Thorium Alpha Activity: Beta/Gamma Activity:

Surface Neutron Activity:

Total Activity:

\begin{tabular}{l}
$\square$ \\
$\square$ Units: \\
\hline Units: \\
\hline \\
@ surface \\
@ 1-m Units: $\mathrm{mR} / \mathrm{hr}$ \\
\hline \\
\hline
\end{tabular}

BASIC SOLIDS W/METALS + HIGH ALPHA

LB-Wo 12

Nät-Gen :

\section{F. TRU ALPHA ACTIVITY DISTRIBUTION}




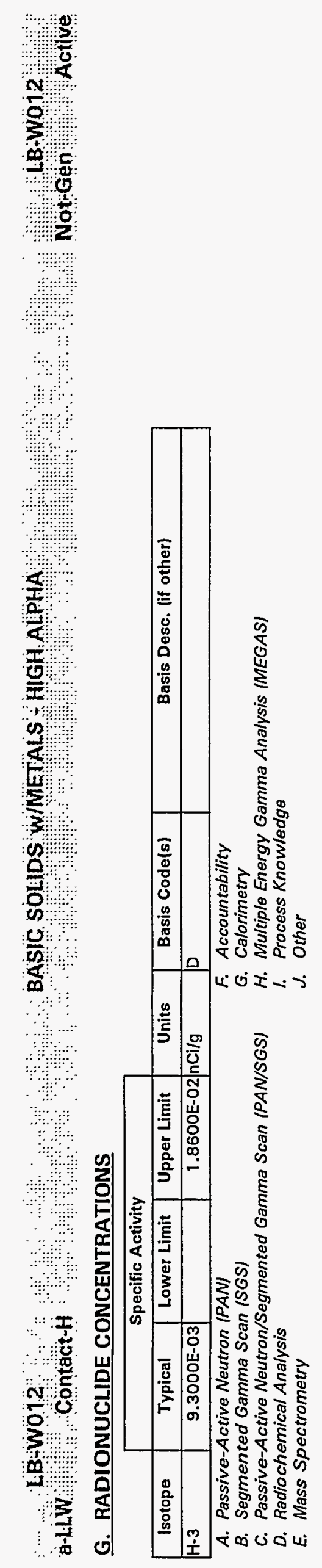




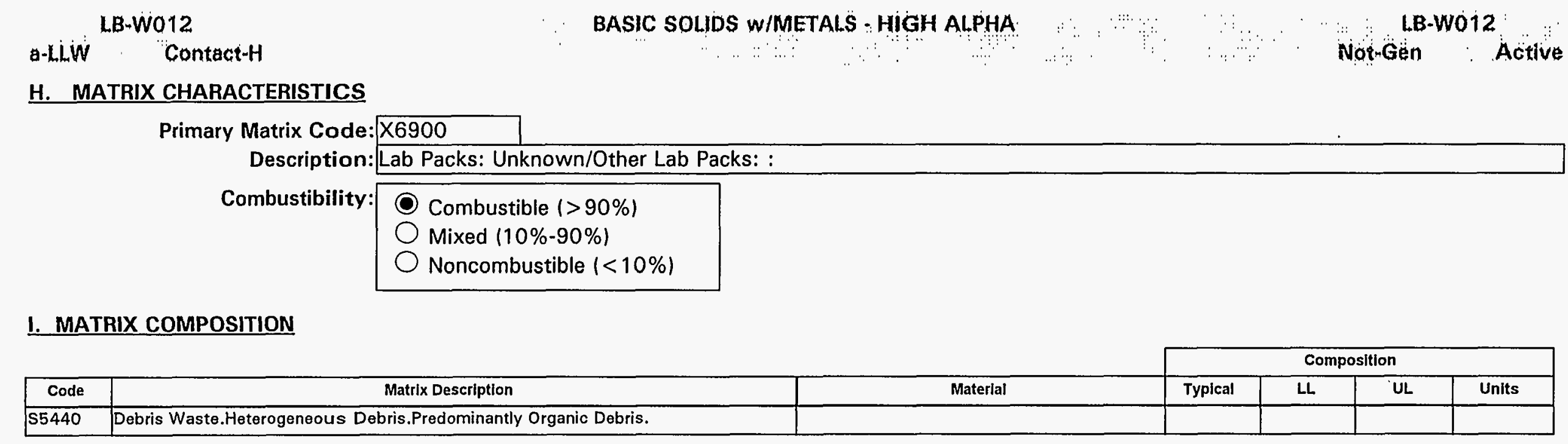


LBWO12

a-kiW Contact-H

\section{J. HAZARDOUS CONSTITUENTS}




LBWWO12
a-KLW : $\quad \because$ ContactH
K. POLYCHLORINATED BIPHENYLS (PCBS)

BASIC SOLIDS W/METAL̈S - HIGH ALPHA

LB-WO12 Notmen Active
Is this waste stream PCB contaminated?

OYes
O No
Unknown

If yes, what portion of the waste is PCB contaminated (volume \%):

If $<100 \%$, can the PCB contaminated waste be segregated and treated separately?

What is the PCB concentration in ppm?

\section{TREATMENT PLANS}

\begin{tabular}{|c|c|c|c|c|c|c|c|c|c|c|c|}
\hline $\begin{array}{c}\text { Opt. } \\
\#\end{array}$ & $\begin{array}{c}\text { Media Type } \\
\text { (If more than one) }\end{array}$ & $\begin{array}{l}\text { Matrix } \\
\text { Code }\end{array}$ & $\begin{array}{l}\text { Vol. } \% \\
\text { lif }\end{array}$ & Step & JIT & Sc & CIF & $\begin{array}{l}\text { Trans. } \\
\text { Miles }\end{array}$ & $\begin{array}{l}\text { Facility } \\
\text { Abbr. }\end{array}$ & Unit Name & Comments \\
\hline \multirow[t]{6}{*}{1} & & S5440 & & a & 0 & 0 & $1.00 E+00$ & & TRANS & Transport - LLW & \\
\hline & & & & b & ( & 0 & $1.00 E+00$ & & AMWTF & $\mathrm{CH}$ - Opening \& Sorting & \\
\hline & & & & c & 0 & 0 & $1.00 E+00$ & & AMWTF & $\mathrm{CH}$ - Sizing & \\
\hline & & & & d & 0 & 0 & $1.00 E+00$ & & AMWTF & $\mathrm{CH}$ - Incineration/Thermal Desorption & \\
\hline & & & & e & O & $\bar{O}$ & $1.00 E+00$ & & AMWTF & $\mathrm{CH}$ - Vitrification & \\
\hline & & & & $f$ & 0 & O & $1.00 E+00$ & & SCDF & Disposal - Contact Handled & \\
\hline
\end{tabular}

Note: Where provided, media percentages are rough estimates used to facilitate treatment planning. They do not necessarily imply an accurate knowledge of waste stream composition, and were not derived for use outside this conceptual planning exercise.

Describe any special or unique technical concerns related to the treatment of this waste stream that would impact the use or implementation of standard hazardous waste treatment methods. 


\section{REFERENCES}

1. U.S. Department of Energy, Radiological, Physical, and Chemical Characterization of Low-Level Alpha-Contaminated Wastes Stored at the Idaho National Engineering Laboratory for Use in Conducting Feasibility Studies for Treatment Services, April 5, 1994.

2. U.S. Department of Energy, Radiological, Physical, and Chemical Characterization of Transuranic Wastes Stored at the Idaho National Engineering Laboratory for Use in Conducting Feasibility Studies for Treatment Services, April 5, 1994.

3. U.S. Department of Energy, Idaho National Engineering Laboratory Proposed Site Treatment Plan, DOE/ID-10493, March 30, 1995.

4. U.S. Department of Energy, DOE Waste Treatability Group Guidance, Radioactive Waste Technical Support Program, DOE-LLW-217, January 1995. 